
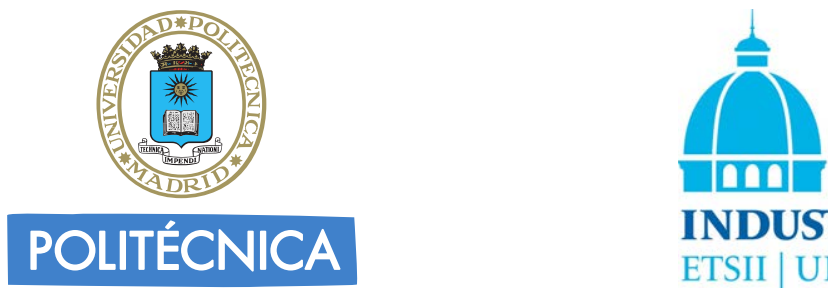

INDUSTRIALES

ETSII | UPM

Universidad Politécnica de Madrid

Escuela Técnica Superior de Ingenieros Industriales

\title{
Vision-BASED AUTONOMOUS NAVIGATION of Multirotor Micro Aerial Vehicles
}

\author{
Ph.D. Thesis
}

\author{
Jesús Pestana Puerta \\ M.Sc. in Automation and Robotics UPM \\ Ingeniero Industrial UPM \\ Master Recherche spécialité ATSI Supélec \\ Ingénieur Supélec
}




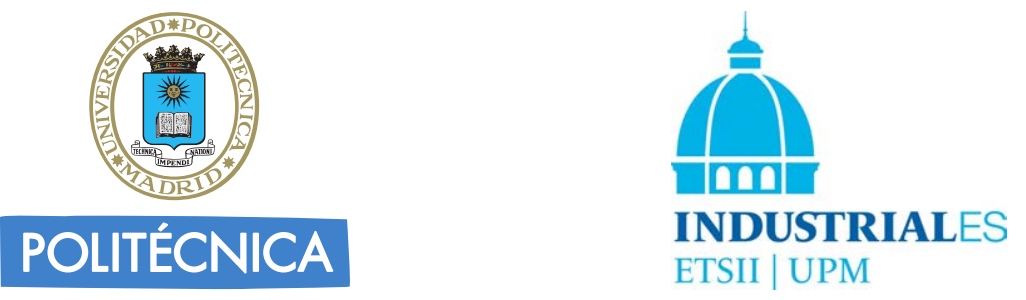

Departamento de Automática, Ingeniería Eléctrica y Electrónica

e Informatica Industrial

Escuela Técnica Superior de Ingenieros Industriales

Universidad Politécnica de Madrid

\title{
Vision-BASED AUtonomous Navigation of Multirotor Micro Aerial Vehicles
}

A thesis submitted for the degree of Doctor of Philosophy in Automation and Robotics

\author{
Author: \\ Jesús Pestana Puerta \\ M.Sc. in Automation and Robotics UPM \\ Ingeniero Industrial UPM \\ Master Recherche spécialité ATSI Supélec \\ Ingénieur Supélec
}

Advisors:

Dr. Pascual Campoy Cervera

Full Professor UPM

Dr. Sergio Domínguez Cabrerizo

Professor UPM

Madrid, July 2017 

Título:

Vision-Based Autonomous Navigation of Multirotor Micro Aerial Vehicles

\author{
Autor: \\ Jesús Pestana Puerta \\ M.Sc. in Automation and Robotics UPM \\ Ingeniero Industrial UPM \\ Master Recherche spécialité ATSI Supélec \\ Ingénieur Supélec \\ Directores: \\ Dr. Pascual Campoy Cervera \\ Full Professor UPM \\ Dr. Sergio Domínguez Cabrerizo \\ Professor UPM
}

Tribunal nombrado por el Mgfco. y Excmo. Sr Rector de la Universidad Politécnica de Madrid el día.........de. ..de 2017

\title{
Tribunal
}

Presidente :
Secretario :
Vocal :
Vocal :
Vocal :
Suplente :
Suplente :

Realizado el acto de lectura y defensa de la tesis el día de de 2017.

calificación de la Tesis.

El Presidente:

Los Vocales:

El Secretario: 

A mis padres Domingo y Ana,

A mi novia Lola,

A Domingo e Irene y Agustín, Al resto de mi familia,

$Y$ a mis amigos,

Que me han ayudado a descubrir el valor de continuar explorando y la satisfacción que proporciona enfrentarse a nuevos desafios.

Jesús Pestana Puerta 



\section{Acknowledgements}

I am delighted to express my gratitude for those who have enabled the realization of this $\mathrm{PhD}$ thesis. First of all, I am profoundly thankful to my supervisors, professor Pascual Campoy Cervera for his direction, scientific insights, innovative ideas and tireless support, and professor Sergio Domínguez Cabrerizo for his advice and guidance along the years.

I am grateful to the past and current members of the Computer Vision and Aerial Robotics (CVAR) group, previously Computer Vision Group (CVG), of the Technical University of Madrid (UPM), and its related professors Martin Molina and José María Sebastian; and specifically to my colleagues: Ignacio Mellado, José Luis Sánchez López, Dr. Paloma de la Puente, Adrian Carrio, Ramón Suarez, Carlos Sampedro, Changhong Fu, Michele Pratusevich, Dr. Iván Fernando Mondragón Bernal, Dr. Jean-François Collumeau, Dr. Carol Martínez, Dr. Aneesh Chauhan, David Galindo, Juan Carlos Garcia Gordo, Dr. Miguel Olivares and Hriday Bavle among others.

I would like to thank all the members of the Centre for Automation and Robotics (CAR, UPM-CSIC), specially the professors from whom I had the opportunity to learn during my Master's degree; and the members, researchers and colleagues of the former Institute of Industrial Automation (IAI, CSIC) with whom I had the pleasure to work.

It is my pleasure to thank the Autonomous Systems Technologies Research \& Integration Laboratory (ASTRIL) for hosting my research stay at the Arizona State University (ASU) and specially to professor Srikanth Saripalli for his supervision and innovative scientific vision; and my colleagues: Patrick McGarey, Yucong Lin, Aravindhan K. Krishnan and Ben Stinnett.

I am profoundly thankful to the Institute of Computer Graphics and Vision (ICG) of the Technical University of Graz (TU Graz) and its members for hosting my research stay and following employment for over two years. I would like to thank specifically professors Horst Bischof for providing me with this opportunity and letting me be part of his scientific endeavors, and Friedrich Fraundorfer for his supervision, scientific insights, deep research discussions and support. I would also like to thank specifically my colleagues: Michael Maurer, Dr. Daniel Muschick, Alexander Isop, Thomas Holzmann, Christian Mostegel, Dr. Manuel Hofer, Markus Rumpler, Dr. Gabriele Ermacora, Rudolf Prettenthaler, Horst Possegger, Felix Egger and Devesh Adlakha.

It is my pleasure to thank all the institutions that have provided funding for the realization of this thesis and its related works: the Spanish National Research Council (CSIC) for the pre-doctoral scholarship through the "Junta de Ampliación de Estudios" (JAE, 2010) programme; the UECIMUAVS Project (PIRSES-GA-2010) included in the Marie Curie Program; the Spanish Ministry of Science MICYT DPI2010-20751-C02-01 for project funding; the Austrian Science Fund (FWF) for funding under the projects V-MAV (I-1537), UFO - Semi-Autonomous Aerial Vehicles for Augmented Reality (I-153644); and the Austrian Research Promotion Agency (FFG) and OMICRON electronics GmbH for funding through the "FreeLine" project (Bridge1/843450).

I am very grateful with my family and close friends for their unconditional support, specially to my parents, my girlfriend and my brothers and their partners.

I also thank all the persons that, while not specifically mentioned, have supported the realization of this $\mathrm{PhD}$ thesis. 


\section{Resumen}

El objetivo de esta tesis es conseguir una navegación basada en visión fiable y explorar el potencial de los algoritmos del estado del arte moderno en visión por computador para la robótica aérea, y especialmente aplicados a drones de tipo multirotor. Dado que las cámaras digitales pueden proveer potencialmente de una cantidad enorme de información al robot, que son menos costosas que otras alternativas de sensado y que son muy ligeras; los métodos de estimación basados en visión son muy prometedores para el desarrollo de aplicaciones civiles con drones. La parte principal de esta tesis consiste en el diseño, la implementación y la evaluación de tres módulos nóveles de propósito general para multirotores que utilizan métodos basados en visión para tomar decisiones de navegación.

El primer módulo propuesto es un controlador de navegación para multirotores que fue diseñado para conseguir un vuelo fiable en entornos sin disponibilidad de señal GPS por medio del uso de la visión por computador. Con este objetivo en mente, la estimación de velocidad basada en el flujo óptico del suelo fue seleccionada para explorar las implicaciones de la utilización de métodos basados en la visión como principal sistema de medida para la retroalimentación del controlador. Fijar y respetar una limitación en la velocidad de navegación fue identificada como una manera fiable de asegurar que la estimación de velocidad basada en flujo óptico no falle. El controlador propuesto tiene en cuenta las limitaciones de velocidad establecidas por el uso de un método de estimación de la posición basado en odometría visual. Esta capacidad del controlador de respetar una velocidad máxima preconfigurada ha sido utilizada con éxito para realizar investigación en la navegación descentralizada simultánea de múltiples drones.

Una capacidad comúnmente buscada en drones, es la realización de un seguimiento visual y la estabilización del vuelo mientras se adquieren imágenes de un objeto de interés. El segundo módulo propuesto en esta tesis es una arquitectura para el seguimiento en vuelo de objetos. Esta arquitectura fue desarrollada con el propósito de explorar el potencial de los métodos modernos de seguimiento visual basados en aprendizaje de máquina, o "machine learning" en inglés. Este tipo de algoritmos tienen como objeto determinar la posición de un objeto en el flujo de imágenes. Los métodos de seguimiento visual tradicionales funcionan correctamente en las condiciones para las cuales fueron originalmente diseñados, pero a menudo fallan en situaciones reales. La solución propuesta ha demostrado que los métodos actuales de seguimiento visual basados en aprendizaje de máquina permiten conseguir un seguimiento en vuelo fiable de una gran variedad de objetos.

El despliegue rápido de drones autónomos en entornos desconocidos es todavía un problema actual en investigación. Uno de los principales desafíos es el cálculo de trayectorias que permitan una navegación rápida en dichos entornos. Con este problema en mente, el tercer módulo propuesto permite la generación automática de trayectorias seguras en entornos abarrotados de obstáculos. Este módulo ha sido probado utilizando mapas adquiridos en tiempo real a bordo del dron mediante el uso de algoritmos de fotogrametría basados en la visión por computador. Aun usando navegación basada en GPS, mediante la realización de experimentos se ha mostrado que el despliegue rápido de drones es factible incluso utilizando solo el ordenador y los sensores a bordo del dron.

Parte de los módulos desarrollados han sido liberados en código abierto, contribuyendo al entorno de desarrollo "Aerostack" de código abierto del CVAR, previamente CVG, (UPM). Los módulos propuestos en esta tesis han sido repetidamente probados con éxito en experimentos fuera del laboratorio y en eventos públicos, demostrando su fiabilidad y potencial, y promoviendo su evaluación en diferentes escenarios. 



\section{Abstract}

The aim of this thesis is to attain reliable vision-based navigation and explore the potential of state of the art Computer Vision algorithms in Aerial Robotics, and specifically for multirotor drones. Since digital cameras can potentially provide a vast amount of information to the robot, are less expensive than their alternatives and are very lightweight, vision-based estimation methods are very promising for the development of civilian applications for drones. The main part of this thesis consists on the design, implementation and evaluation of three novel general purpose modules for multirotors that utilize visionbased methods to make navigation decisions.

The first proposed module is a novel multirotor navigation controller that was designed to achieve reliable flight in GPS-denied environments by using Computer Vision. With this objective in mind, ground optical flow speed estimation was selected to explore the implications of using vision-based methods as the main measurement feedback for the controller. Setting and enforcing a speed limitation during navigation was identified as a reliable way to ensure that the optical flow speed estimation method does not malfunction. The proposed controller takes into account the speed limitations established by the vision-based odometry estimation method. In addition, by leveraging this capability of the controller to ensure navigation respecting the preconfigured maximum speed, it has also been successfully utilized to research on decentralized multi-drone navigation.

A common objective for drones is to be able to fly and stabilize looking at and close-by to an object of interest. The second module proposed in this thesis is a novel vision-based object tracking architecture for multirotor drones, which was designed to explore the reliability of machine learning based visual trackers to provide feedback to an object following controller. Visual trackers have the purpose to determine the position of an object in an image stream and are, therefore, good candidates to achieve this objective. However traditional visual tracking methods, though very effective in the specific conditions for which they were designed, have the drawback of not generalizing well to the myriad of different visual appearances that real objects exhibit. The proposed architecture has demonstrated that current machine learning based visual tracking algorithms can reliably provide feedback to a visual servoing controller.

The fast deployment of autonomous drones in unknown environments is still an ongoing research problem. One of the main challenges to achieve it is the calculation of trajectories that allow fast navigation in such areas. With this problem in mind, the third proposed module is a trajectory planner that delivers smooth safe trajectories in relatively cluttered environments. The algorithm has been tested experimentally on maps obtained using an on-board real-time capable Computer Vision mapping method. Although other modules would be needed to decide the exact navigation objectives of the drone, using this planner we have shown that it is feasible to deploy drones in unknown outdoors environments, by leveraging the good qualities of maps obtained using state of the art photogrammetry methods.

Part of these modules have been released as open-source, contributing to the CVAR's, previously CVG, (UPM) Open-Source Aerial Robotics Framework "Aerostack", which main purpose is enabling drone civilian applications. The proposed modules have been repeatedly evaluated with successful results in several out-of-the-lab demonstrations, therefore showing their potential and good qualities. The utilization of the proposed modules in public events has promoted their further testing in different settings and applications, e. g. indoors and outdoors flight, narrow corridors, passage through windows and landing in different conditions. 



\section{Acronyms}

Following is a description of most commonly acronyms referenced on this work:

API: Application Programming Interface

AR: Augmented Reality

AUVSI: Association for Unmanned Vehicles Systems International

BA: Bundle Adjustment

CEA: Spanish Committee of Automation

CVAR: Computer Vision and Aerial Robotics group (UPM), previously CVG

CVG: Computer Vision Group (UPM)

EKF: Extended Kalman Filter

ERSG: European RPAS Steering Group (ERSG)

EuRoC or EuRoC2014: European Robotics Challenge (2014)

FSM: Finite State-Machine

GCS: Ground Control Station

GPS: Global Positioning System

GS: Ground Station

IBVS: Image Based Visual Servoing

IMAV: International Micro Air Vehicle Conference and Flight Competition

IMU: Inertial Measurement Unit

KF: Kalman Filter

MAV: Micro-Aerial Vehicles

MAVWork: CVG Framework for interfacing with MAVs (2011)

MCL: Monte Carlo Localization

mUAV: Micro Unmanned Aerial Vehicle

NAAs: National Aviation Authorities

LRF: Laser Range Finder

LQR: Linear Quadratic Regulator 
PF: Particle Filter

PID: : Proportional-Integral-Derivative controller

QP: Quadratic Programming minimization problem

RC: Radio Controlled or Radio Controller

ROS: Robot Operating System

RPM: Revolutions per Minute

RPA: Remotely Piloted Aircraft

RPAS: Remotely Piloted Aircraft System

R\&D: Research and Development

SDK: Software Development Kit

UA: Unmanned Aircraft

UAS: Unmanned Aircraft System

UAV: Unmanned Aerial Vehicle

UVS International: Association for Unmanned Vehicles Systems International

VTOL: Vertical Take-Off and Landing 


\section{Contents}

$\begin{array}{ll}\text { Thesis Cover } & 1\end{array}$

Dedication i

\begin{tabular}{ll}
\hline Acknowledgements & iii
\end{tabular}

Resumen v v

Abstract vii vi v

\begin{tabular}{ll}
\hline Acronyms & ix
\end{tabular}

Contents $\quad$ xi

$\begin{array}{ll}\text { List of Figures } & \text { xv }\end{array}$

\begin{tabular}{lll}
\hline List of Tables & xix \\
\hline
\end{tabular}

\begin{tabular}{|l|c|}
\hline List of Algorithms & xxi \\
\hline
\end{tabular}

1 Introduction 1

1.1 Introduction to UAVs and Multirotors . . . . . . . . . . . . . . . . . . . . . 1

1.2 Problem Statement . . . . . . . . . . . . . . . . . . . . 2

1.3 Thesis proposal and objectives $\ldots \ldots \ldots \ldots \ldots \ldots \ldots$. . . . . . . . . . . 4

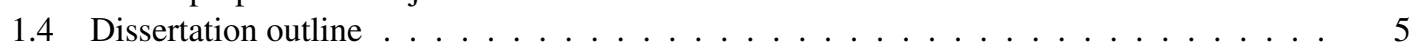

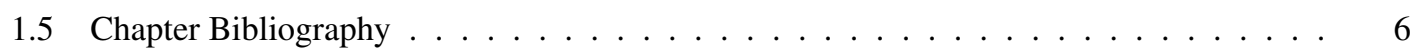

$2 \quad$ A General Purpose Configurable Controller for Multirotor MAVs 9

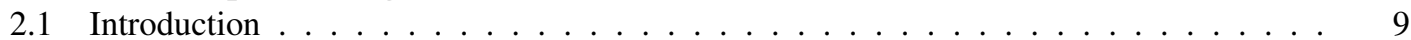

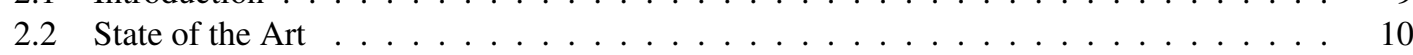

2.3 Controller Algorithm . . . . . . . . . . . . . . . . . . . . . . . . . 12

$2.3 .1 \quad$ Control Architecture . . . . . . . . . . . . . . . . . . . . . . . . . . . . 13

2.3 .2 Multirotor Point Mass Kinematic Model . . . . . . . . . . . . . . . . . . . . 14

2.3 .3 Middle-Level Controller . . . . . . . . . . . . . . . . . . . . . . . . . . 16

2.3 .4 High-Level Controller - State-Machine . . . . . . . . . . . . . . . . . . . 17

2.3 .5 Speed Planner Algorithm $\ldots \ldots \ldots \ldots$

$2.3 .6 \quad$ Configurable Parameters Set $\ldots \ldots \ldots \ldots \ldots$ 


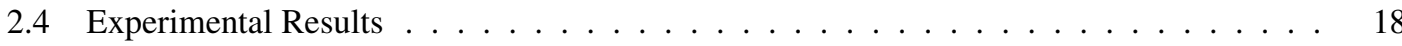

$2.4 .1 \quad$ Videos of Experiments . . . . . . . . . . . . . . . . . . . . . 19

$2.4 .2 \quad$ Experimental Results - Indoors Flight . . . . . . . . . . . . . . . . . . . . . 19

$2.4 .3 \quad$ Experimental Results - Outdoors Flight . . . . . . . . . . . . . . . . . . . 22

2.4 .4 Experimental Results - Flights in IMAV2013 Replica . . . . . . . . . . . . . . . 27

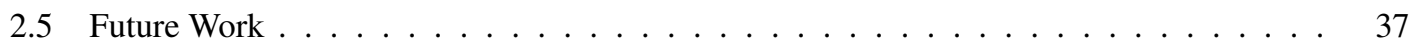

2.6 Conclusions . . . . . . . . . . . . . . . . . . . . . . . 37

2.7 Chapter Bibliography $\ldots \ldots \ldots \ldots \ldots \ldots \ldots$

3 Visual Servoing of Arbitrary Objects for Multirotors 4

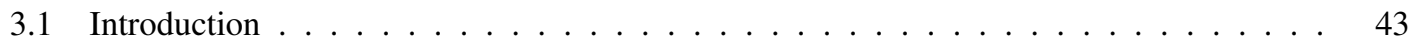

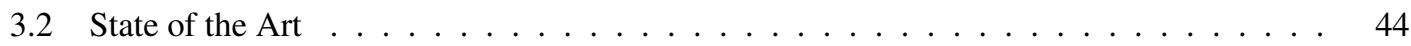

3.3 System Description . . . . . . . . . . . . . . . . . . . . . . . . . . . . . . 46

$3.3 .1 \quad$ Image Based Visual Servoing (IBVS) Controller . . . . . . . . . . . . . . . 47

3.3 .2 IBVS PD Controller Parameters Breakdown . . . . . . . . . . . . . . . . . . 48

3.4 Experiments and Results . . . . . . . . . . . . . . . . . . . . 50

$3.4 .1 \quad$ Videos and Dataset of Flight Experiments . . . . . . . . . . . . . 51

3.4.2 Qualitative performance - navigation following suburban house elements . . . . 53

3.4.3 Qualitative performance - navigation along a street in a suburban area . . . . . . 55

3.4.4 Quantitative performance - person following without decoupling control heuristics 57

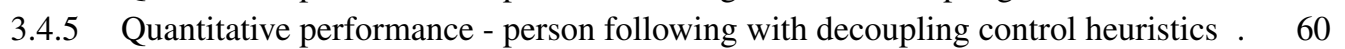

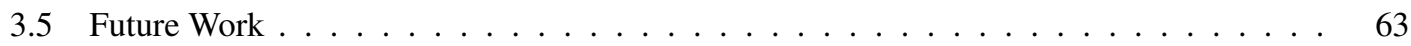

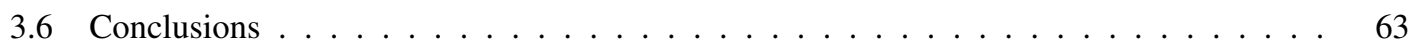

3.7 Chapter Bibliography $\ldots \ldots \ldots \ldots \ldots$. . . . . . . . . . . . . . . . 66

4 Smooth Trajectory Planning for Multirotors $\quad 69$

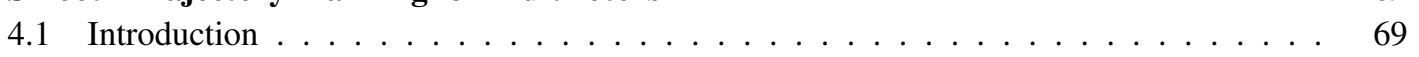

4.2 State of the Art . . . . . . . . . . . . . . . . . . . . . . . . 70

4.3 Developed Trajectory Planner - Summary . . . . . . . . . . . . . . . . . . 71

$4.3 .1 \quad$ Online Structure-from-Motion (SfM) - Real-Time Mapping . . . . . . . . . . . 72

4.4 Trajectory Planning - Theoretical Background . . . . . . . . . . . . . . . . . . 74

$4.4 .1 \quad$ OctoMap - Probabilistic 3D Mapping Framework . . . . . . . . . . . . . . . . . 75

4.4 .2 Dynamic Efficient Obstacle Distance Calculation . . . . . . . . . . . . . . . . 76

$4.4 .3 \quad$ Probabilistic RoadMap - Planning Algorithm . . . . . . . . . . . . . . . . . . 76

$4.4 .4 \quad$ Rapidly-Exploring Random Trees - Planning Algorithm . . . . . . . . . . . . . 77

$4.4 .5 \quad$ Utilized open-source libraries $\ldots \ldots \ldots \ldots$. . . . . . . . . . . . . . . . . . . . . . . .

4.5 Obstacle-free Smooth Trajectory Planning . . . . . . . . . . . . . . . . . . . . . . . 79

$4.5 .1 \quad$ Planner initialization $\ldots \ldots \ldots \ldots \ldots$

4.5 .2 Obstacle-aware Geometric Trajectory Planning . . . . . . . . . . . . . . . . . . 79

4.5 .3 Obstacle-aware Trajectory Shortening and Smoothing . . . . . . . . . . . . . 80

$4.5 .4 \quad$ Speed, Acceleration and Time-of-passage Planning . . . . . . . . . . . . . . . . 80

4.5 .5 Communication with other modules $\ldots \ldots \ldots$. . . . . . . . . . . . 86

4.5 .6 Configuration Parameters . . . . . . . . . . . . . . . . . . . . . . . . . . . . . . . . . . . 87

4.6 Geometric Speed Planner . . . . . . . . . . . . . . . . . . . . . . . . . . . . 87

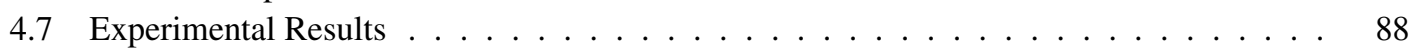

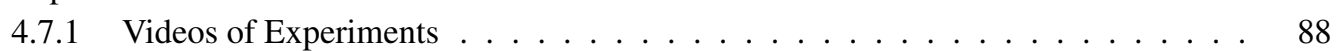

$4.7 .2 \quad$ Experimental Setup . . . . . . . . . . . . . . . . . . . . . . . 88

4.7 .3 Results in EuRoC 2014 - Challenge 3 - Simulation Contest . . . . . . . . . . . . 89

4.7 .4 Results in 2016 DJI Developer Challenge - Experimental Flights . . . . . . . . . 103

4.8 Future Work . . . . . . . . . . . . . . . . . . . . . . 108

4.9 Conclusions . . . . . . . . . . . . . . . . . . . . . . . . . . 108

4.10 Chapter Bibliography $\ldots \ldots \ldots \ldots \ldots \ldots$ 
5 Conclusions and Future Work 113

5.1 Thesis Contributions _. . . . . . . . . . . . . . . . . . . . . . . . . . 113

5.2 Conclusions . . . . . . . . . . . . . . . . . . . . . . . . . . . . . 118

5.3 Future Work . . . . . . . . . . . . . . . . . . . . . . . . . . . . . . . . . . . . . . . 119

\begin{tabular}{ll}
\hline Appendices & 121
\end{tabular}

\begin{tabular}{|lll}
\hline A Scientific Dissemination & 123 \\
\hline
\end{tabular}

A.1 Journals . . . . . . . . . . . . . . . . . . . . . . . . . . . . . . . 123

A.2 Publications in peer-reviewed conferences . . . . . . . . . . . . . . . . . . . . . . 124

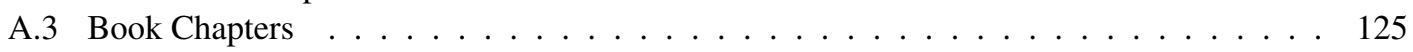

A.4 Participation in Conference Workshops . . . . . . . . . . . . . . . . . . . 125

\begin{tabular}{|lr|r|}
\hline$B$ UAVs and Multicopters & 127
\end{tabular}

B.1 Appendix Bibliography . . . . . . . . . . . . . . . . . . . . . . . . 130

\begin{tabular}{ll}
\hline C Multirotor Software Interface & 131
\end{tabular}

C.1 Introduction . . . . . . . . . . . . . . . . . . . . . . . . . . . . 131

C.2 Robotics Middleware . . . . . . . . . . . . . . . . . . . . . . . . . . . . 131

C.3 Multirotor Driver . . . . . . . . . . . . . . . . . . . . . . . . 132

C.3.1 Notes on the adaptation of the Asctec Pelican to the Multirotor Driver . . . . . . 133

C.4 Example Software Architecture (IMAV2012) $\ldots \ldots \ldots \ldots$. . . . . . . . . . . . . . 134

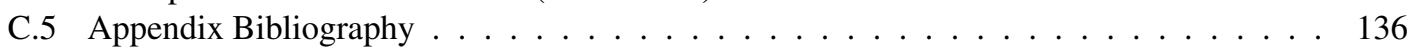

\begin{tabular}{|lr}
\hline D Multirotor dynamics modeling & 137
\end{tabular}

D.1 Introduction . . . . . . . . . . . . . . . . . . . . . . . . . . . . 137

D.2 Model based on rigid body dynamics . . . . . . . . . . . . . . . . . . . . . 137

D.2.1 $\quad$ Brief explanation of the Low-level and Mid-level control of a quadrotor . . . . . 138

D.2.2 Shortcomings of the model based on rigid body dynamics $\ldots \ldots$. . . . . . . . 139

D.3 Model based on rigid body dynamics with autopilot . . . . . . . . . . . . . . . . 140

D.4 Simplified model with altitude and yaw hold . . . . . . . . . . . . . . . . . . . . . . . 142

D.5 Complete multirotor simplified model $\ldots \ldots \ldots$. . . . . . . . . . . . . . . . . . . 143

D.5.1 Identification method for this model . . . . . . . . . . . . . . . . . . 145

D.5.2 Identification example: Parrot AR Drone with indoors hull . . . . . . . . . . . . 146

D.5.3 4 AR Drone model, comparison with experimental data. . . . . . . . . . . . . . . 149

D.5.4 Identification example: Asctec Pelican . . . . . . . . . . . . . . . . . . . . 153

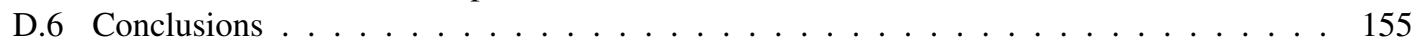

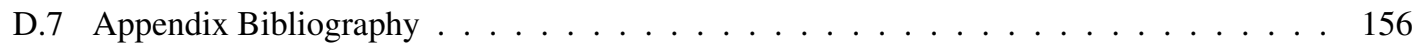

\begin{tabular}{lll}
\hline E & An Extended Kalman Filter for Multirotors with Ground Speed Sensing & 157
\end{tabular}

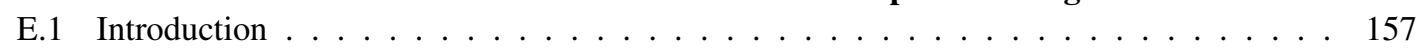

E.2 State of the Art . . . . . . . . . . . . . . . . . . . . 158

E.3 Dynamic systems and filtering algorithms $\ldots \ldots \ldots \ldots$. . . . . . . . . . . 163

E.3.1 Filtering in State-Observation models . . . . . . . . . . . . . . . . . 164

E.4 Kalman filter . . . . . . . . . . . . . . . . . . . . . . . . . . . . . 165

E.4.1 The Kalman Filter algorithm . . . . . . . . . . . . . . . . . . . . . 166

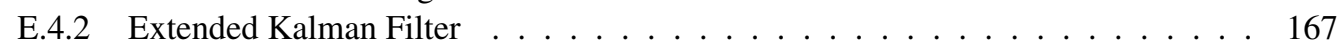

E.5 Complete simplified multirotor model, state equations . . . . . . . . . . . . . . . . . . 168

E.6 $\quad$ Testing the EKF on experimental data $\ldots \ldots \ldots$

E.6.1 EKF with odometry measurements . . . . . . . . . . . . . . . . . . 170

E.6.2 $\quad$ EKF with position measurements $\ldots \ldots \ldots \ldots \ldots$

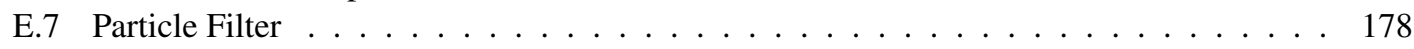

E.7.1 The PF algorithm . . . . . . . . . . . . . . . . . . . . . . . 178

E.7.2 Testing the PF on experimental data . . . . . . . . . . . . . . . . . . . . 179

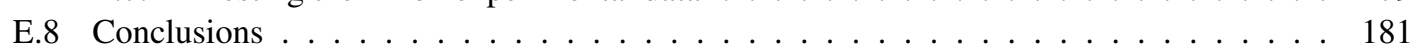

E.9 $\quad$ Appendix Bibliography . . . . . . . . . . . . . . . . . . . . . . . . . . . . . 182 
\begin{tabular}{|ll|l|l|}
\hline F & Proportional-Integral-Derivative (PID) controllers & 187
\end{tabular}

G Contributions to the Open-Source Aerial Robotics Framework Aerostack 189

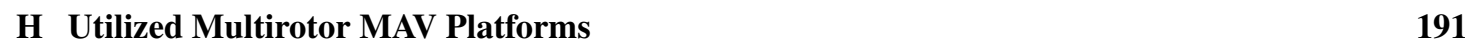

H.1 AscTec Pelican from Ascending Technologies . . . . . . . . . . . . . . . . . . . . . . . 192

H.1.1 Asctec Pelican Multirotor Experimental Setup - IMAV 2012 . . . . . . . . . . . 193

H.1.2 Asctec Pelican Multirotor Experimental Setup - IARC 2014 . . . . . . . . . . . 193

H.2 Parrot AR Drone 1\&2 . . . . . . . . . . . . . . . . . . . . . . . . . . . . . . . . . 194

H.3 DJI M100 Quadcopter . . . . . . . . . . . . . . . . . . . . . . . . . . . . . . . 196

H.3.1 DJI M100 Multirotor Experimental Setup - DJIC 2016 . . . . . . . . . . . . . . 196

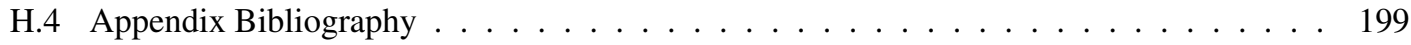

\begin{tabular}{lll}
\hline I International Micro Aerial Vehicle Competitions & 201
\end{tabular}

I.1 2016 DJI Developer Challenge - DJIC 2016 . . . . . . . . . . . . . . . . . . . 202

$[.1 .1$ External Links . . . . . . . . . . . . . . . . . . . . . . . . 202

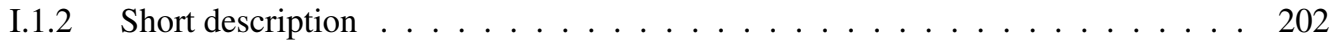

I.1.3 Achieved Results . . . . . . . . . . . . . . . . . . . . . . . . . . . 202

I.2 International Micro Air Vehicle Conference and Flight Competition 2016 - IMAV 2016 . 203

I.2.1 External Links $\ldots \ldots \ldots \ldots$. . . . . . . . . . . . . . . . . 203

I.2.2 Short description . . . . . . . . . . . . . . . . . . . . . . . . 203

I.2.3 Achieved Results . . . . . . . . . . . . . . . . . . . . . . . . . . . . . . . . . 203

I.3 European Robotics Challenges 2014 - Simulation Challenge 3 - EuRoC 2014 . . . . . . 204

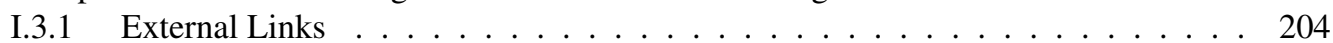

I.3.2 Short description . . . . . . . . . . . . . . . . . . . . . . . . . . . 204

I.3.3 Achieved Results . . . . . . . . . . . . . . . . . . . . . . . . . . . . . . . . . 204

I.4 International Aerial Robotics Competition 2014 - Mission 7 - IARC 2014 . . . . . . . . 205

$\left[\begin{array}{l}I .4 .1 \\ \text { External Links } \ldots \ldots \ldots \ldots\end{array}\right.$

I.4.2 Short description . . . . . . . . . . . . . . . . . . . . . . . . . . . 205

I.4.3 Achieved Results . . . . . . . . . . . . . . . . . . . . . . . . . 205

I.5 International Micro Air Vehicle Conference and Flight Competition 2013 - IMAV 2013 . 206

I.5.1 External Links . . . . . . . . . . . . . . . . . . . . . . . . . . . . . 206

$[.5 .2 \quad$ Short description $\ldots \ldots \ldots \ldots \ldots \ldots$

I.5.3 Achieved Results . . . . . . . . . . . . . . . . . . . . . . . . 206

I.6 International Micro Air Vehicle Conference and Flight Competition 2012 - IMAV 2012 . 207

$\left[\begin{array}{ll}{[.6 .1} & \text { External Links }\end{array} \ldots \ldots \ldots \ldots \ldots\right.$

I.6.2 Short description . . . . . . . . . . . . . . . . . . . . . . . . . . . . . 207

I.6.3 Achieved Results . . . . . . . . . . . . . . . . . . . . . . . . . . . . . . . . . 207

I.7 Contest on Control Engineering 2012 - CEA $2012 \ldots \ldots \ldots \ldots \ldots \ldots$

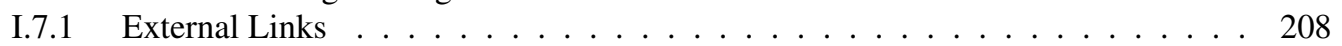

I.7.2 Short description . . . . . . . . . . . . . . . . . . . . . . . . . . . . 208

I.7.3 Achieved Results . . . . . . . . . . . . . . . . . . . . . . . . . . . . . . . 208

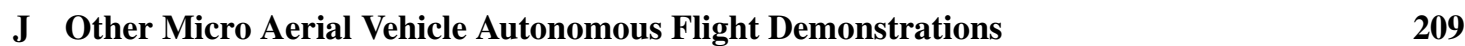

J.1 Using Trajectory Controller and/or State Estimator _ . . . . . . . . . . . . . . . . . . . 210

J.2 Using Visual Servoing Architecture . . . . . . . . . . . . . . . . . . . . . . . 211

J.3 Using Trajectory and Speed Planner $\ldots \ldots \ldots$. . . . . . . . . . . . . . . . . . 212

\begin{tabular}{ll}
\hline Bibliography & 213
\end{tabular} 


\section{List of Figures}

$2.1 \quad$ Asctec Pelican quadrotor in the IMAV2012 . . . . . . . . . . . . . . . . . . . . 10

2.2 General architecture of the controller . . . . . . . . . . . . . . . . . . . 13

2.3 Free body diagram of a quadrotor $\ldots \ldots \ldots \ldots \ldots \ldots$

$2.4 \quad$ Mid-level controller architecture, IMAV 2012 . . . . . . . . . . . . . . . . . . . 16

2.5 High-level control finite state machine . . . . . . . . . . . . . . . . . . . . 17

2.6 Controller, experimental test, vertical eight-shape trajectory . . . . . . . . . . . . 20

2.7 Controller, experimental test, horizontal square trajectory . . . . . . . . . . . . . 20

2.8 Controller, experimental test, horizontal eight-shape trajectory . . . . . . . . . . . . . 21

2.9 Controller, experimental test, horizontal eight-shape trajectory . . . . . . . . . . . . 21

2.10 Controller, outdoors test, pseudo-square trajectory . . . . . . . . . . . . . . . . . 24

2.11 Controller, outdoors test, pseudo-hexagon trajectory . . . . . . . . . . . . . . . . 25

2.12 Controller, outdoors test, pseudo-octagon trajectory . . . . . . . . . . . . . . . . . 26

2.13 Flights in IMAV2013 Replica - Summary figure . . . . . . . . . . . . . . . . . . 27

2.14 Flights in IMAV2013 Replica - IMAV2013 Coordinate Frames . . . . . . . . . . . . . . 28

2.15 IMAV2013 Replica Experimental Flight - Overview . . . . . . . . . . . . . . . . . . . . . . 31

2.16 IMAV2013 Replica Experimental Flight - Highlights . . . . . . . . . . . . . . . . . . . . 32

2.17 IMAV2013 Replica Experimental Flight - Drift correction . . . . . . . . . . . . . . . . . 33

2.18 IMAV2013 Replica Experimental Flight - Drone 1 . . . . . . . . . . . . . . . . . . . . . . . . . . 34

2.19 IMAV2013 Replica Experimental Flight - Drone 2 . . . . . . . . . . . . . . . . . . 35

2.20 IMAV2013 Replica Experimental Flight - Drone 3 . . . . . . . . . . . . . . . . . 36

$3.1 \quad$ Visual Servoing Summary with AR Drone . . . . . . . . . . . . . . . . . . . . . . . . 44 44

3.2 Visual Servoing, main reference frames . . . . . . . . . . . . . . . . . . . . . . . . . . . . . . . . . . . .

3.3 Visual Servoing, system overview f . . . . . . . . . . . . . . . . . . . . . 47

3.4 Diagram of the Image Based Visual Servoing (IBVS) Controller . . . . . . . . . . . . . 48

3.5 Visual Servoing, Controller Gains Breakdown . . . . . . . . . . . . . . . . . . . . . 49

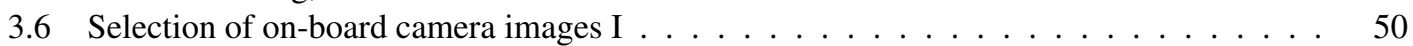

3.7 Selection of on-board camera images II $\ldots \ldots \ldots \ldots$. . . . . . . . . . . 50

3.8 Selection of on-board camera images - navigation following suburban house elements . . 54

3.9 Selection of on-board camera images - navigation along a street in a suburban area . . . 55

3.10 Selection of images - navigation along a street in a suburban area II . . . . . . . . . . 56

3.11 Selection of on-board camera images III $\ldots \ldots \ldots \ldots \ldots$

3.12 Visual Servoing, test 1, measured and reference values . . . . . . . . . . . . . . . 58

3.13 Visual Servoing, test 1 , controller commands . . . . . . . . . . . . . . . . . . . . 59

3.14 Selection of on-board camera images IV . . . . . . . . . . . . . . . . . . 60 
3.15 Visual Servoing, test 2, measured and reference values . . . . . . . . . . . . . . 61

3.16 Visual Servoing, test 2, controller commands . . . . . . . . . . . . . . . 62

4.1 Simulated outdoor static environment of EuRoC 2014. . . . . . . . . . . . . . 71

4.2 Outdoor map generated using the SfM "Off-board Approach". . . . . . . . . . . . . . . 72

4.3 Outdoor map from SfM "Real-Time On-board Approach". . . . . . . . . . . . . . . . . 73

4.4 OcTree, data-structure schematic representation . . . . . . . . . . . . . . . . . 75

4.5 Probabilistic roadmap (PRM), motion planning algorithm . . . . . . . . . . . . . . . 77

4.6 Rapidly-exploring random tree (RRT), motion planning algorithm . . . . . . . . . . . 78

$4.7 \quad$ Velocity Smoothing - EuRoC2014 - Example Trajectory 1 - Plots . . . . . . . . . . . . . 91

4.8 Velocity Smoothing - EuRoC2014 - Example Trajectory 7 - Plots . . . . . . . . . . . . . 92

4.9 Velocity Smoothing - Number of passes - Example Trajectory 7 . . . . . . . . . . . 93

4.10 Trajectory Planner - EuRoC2014 - Example Trajectory 1 - RGB . . . . . . . . . . . . . 94

4.11 Trajectory Planner - EuRoC2014 - Example Trajectory 1 - Plots . . . . . . . . . . . . . 94

4.12 Trajectory Planner - EuRoC2014 - Example Trajectory 2 - RGB . . . . . . . . . . . . . 95

4.13 Trajectory Planner - EuRoC2014 - Example Trajectory 2 - Plots . . . . . . . . . . . . . 95

4.14 Trajectory Planner - EuRoC2014 - Example Trajectory 3 - RGB . . . . . . . . . . . . . 96

4.15 Trajectory Planner - EuRoC2014 - Example Trajectory 3 - Plots . . . . . . . . . . . . . 96

4.16 Trajectory Planner - EuRoC2014 - Example Trajectory 4 - RGB . . . . . . . . . . . . . 97

4.17 Trajectory Planner - EuRoC2014 - Example Trajectory 4 - Plots . . . . . . . . . . . . . 97

4.18 Trajectory Planner - EuRoC2014 - Example Trajectory 5 - RGB . . . . . . . . . . . . . 98

4.19 Trajectory Planner - EuRoC2014 - Example Trajectory 5 - Plots . . . . . . . . . . . . . 98

4.20 Trajectory Planner - EuRoC2014 - Example Trajectory 6 - RGB . . . . . . . . . . . . . 99

4.21 Trajectory Planner - EuRoC2014 - Example Trajectory 6 - Plots . . . . . . . . . . . . . 99

4.22 Trajectory Planner - EuRoC2014 - Example Trajectory 7 - RGB . . . . . . . . . . . . 100

4.23 Trajectory Planner - EuRoC2014 - Example Trajectory 7 - Plots . . . . . . . . . . . . . 100

4.24 Trajectory Planner - EuRoC2014 - Example Trajectory 8 - RGB . . . . . . . . . . . . . 101

4.25 Trajectory Planner - EuRoC2014 - Example Trajectory 8 - Plots . . . . . . . . . . . . . 101

4.26 Trajectory Planner - EuRoC2014 - Example Trajectory 9 - RGB . . . . . . . . . . . . . 102

4.27 Trajectory Planner - EuRoC2014 - Example Trajectory 9 - Plots . . . . . . . . . . . . . 102

4.28 Trajectory Planner - DJIC2016 - Example Trajectory 1 - RGB . . . . . . . . . . . . . . 104

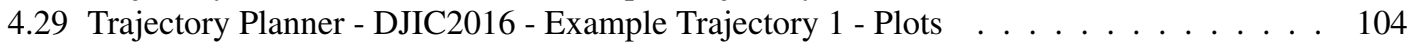

4.30 Trajectory Planner - DJIC2016 - Example Trajectory 2 - RGB . . . . . . . . . . . . 105

4.31 Trajectory Planner - DJIC2016 - Example Trajectory 2 - Plots . . . . . . . . . . . . . . 105

4.32 Trajectory Planner - DJIC2016 - Example Trajectory 3 - RGB . . . . . . . . . . . . . . 106

4.33 Trajectory Planner - DJIC2016 - Example Trajectory 3 - Plots . . . . . . . . . . . . 106

4.34 Trajectory Planner - DJIC2016 - Example Trajectory 4 - RGB ． . . . . . . . . . . . . . 107

4.35 Trajectory Planner - DJIC2016 - Example Trajectory 4 - Plots $\ldots$. . . . . . . . . . . . . 107

B.1 Example of fixed-wing UAV. . . . . . . . . . . . . . . . . . . . . . . . . . . 128

B.2 Example of multirotor drone. . . . . . . . . . . . . . . . . . . . . . . . . . 128

C.1 Abstract multirotor representation or Multirotor Driver . . . . . . . . . . . . . . . . . . 132

C.2 IMAV2012 software architecture overview. . . . . . . . . . . . . . . . . . . . . . . . 134

D.1 Free body diagram of a quadrotor . . . . . . . . . . . . . . . . . . . . . . . 138

D.2 Rigid body model with autopilot . . . . . . . . . . . . . . . . . . . . . . 141

D.3 AR Drone black-box model . . . . . . . . . . . . . . . . . . . . . . . . . . . 141

D.4 Pelican black-box model as equipped by the CVG_UPM team for the IMAV2012 . . . . 142

D.5 Quadrotor 2D simplified model . . . . . . . . . . . . . . . . . . . . 143

D.6 Complete multirotor simplified model . . . . . . . . . . . . . . . . . . . . . 143

D.7 Experimental identification of LTI blocks for the AR Drone, multirotor simplified model 146

D.8 $\quad$ Experimental aerodynamic friction data, AR Drone identification . . . . . . . . . . . . 147

D.9 Experimental aerodynamic friction data (2), AR Drone identification . . . . . . . . . . . 147

D.10 Experimental aerodynamic friction data (3), AR Drone identification . . . . . . . . . . . 148

D.11 Comparison of AR Drone identified model to experimental data, X-Y trajectory . . . . . 149

D.12 Comparison of AR Drone identified model to experimental data, speed in body frame . . 150 
D.13 Comparison of AR Drone identified model to experimental data, position coordinates . . 151

D.14 Comparison of the AR Drone identified model to experimental data, attitude angles . . . 152

D.15 Experimentally identified LTI blocks for Asctec Pelican, multirotor simplified model . . 153

E.1 Multirotor complete simplified model f . . . . . . . . . . . . . . . . . 168

E.2 EKF performance using odometry data - X-Y trajectory . . . . . . . . . . . . . . . . . 170

E.3 EKF performance using odometry data - speed in the body frame . . . . . . . . . . . . 171

E.4 EKF performance using odometry data - position coordinates . . . . . . . . . . . . . . 172

E.5 EKF performance using odometry data - attitude angle . . . . . . . . . . . . . . . 173

E.6 EKF performance using odometry and Vicon data - X-Y trajectory . . . . . . . . . . . . 174

E.7 $\quad$ EKF performance using odometry and Vicon data - speed in the body frame . . . . . . . 175

E.8 $\quad$ EKF performance using odometry and Vicon data - position coordinates . . . . . . . . 176

E.9 $\quad$ EKF performance using odometry and Vicon data - attitude angles . . . . . . . . . . . . 177

E.10 Particle Filter on a real replica of the IMAV 2012 house, autonomy challenge . . . . . . 179

E.11 Particle Filter on a real replica of the IMAV 2012 house, autonomy challenge (2) . . . . 180

F.1 Proportional-Integral-Derivative (PID) controller block diagram . . . . . . . . . . . . 187

H.1 Asctec Pelican quadrotor - CVG-UPM at IARC2014 . . . . . . . . . . . . . . . . . . 192

H.2 Pelican on CVG Framework for interfacing with MAVs . . . . . . . . . . . . . . . . . 193

H.3 Parrot AR Drone 2.0 quadcopter . . . . . . . . . . . . . . . . . . . . . . . . . . . . . 194

H.4 Fully equipped DJI M100 quadcopter . . . . . . . . . . . . . . . . . . . . . . . . . . . 196

H.5 Our fully equipped DJI M100 Quadrotor MAV _ . . . . . . . . . . . . . . . . . . . . . 197

H.6 MAV Hardware - Onboard Computer, Autopilot and GPS Module . . . . . . . . . . . . 197

H.7 MAV Hardware - Guidance Sensing System . . . . . . . . . . . . . . . . . . . . . . 198

H.8 MAV Hardware - Gimbal Camera and Guidance Sensing System . . . . . . . . . . . . . 198 



\section{List of Tables}

$2.1 \quad$ Controller, results summary of experimental tests (IMAV2012) . . . . . . . . . . . . . 22

2.2 Controller, results summary of experimental tests (IMAV2012) . . . . . . . . . . . . . . 22

2.3 IMAV2013 Replica Experimental Flight - Performance parameters . . . . . . . . . . . . 30

$4.1 \quad$ Trajectory planning and control performance - EuRoC2014 . . . . . . . . . . . . 9 90

4.2 Trajectory planning and control performance - DJIC2016 . . . . . . . . . . . . 103

H.1 Asctec Pelican characteristics, from Asctec Pelican website . . . . . . . . . . . . . . . . 192

H.2 Parrot AR Drone characteristics . . . . . . . . . . . . . . . . . . . . . . . . . 194 



\section{List of Algorithms}

$1 \quad$ Velocity_Plan_Sweep_Double_Pass $\left(\mathbf{s}, \mathbf{r},\left\{\mathbf{t}_{i}^{*}\right\}_{i}, \mathbf{v}_{\text {desired }}\right.$, config $) \ldots \ldots . \ldots$. . . . 82

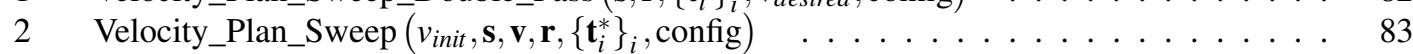

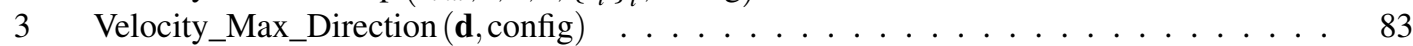

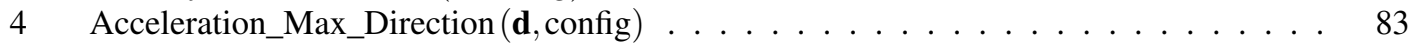

$5 \quad$ Velocity_Smoothing $\left(\mathbf{s}, \mathbf{r},\left\{\mathbf{t}_{i}^{*}\right\}_{i}, \mathbf{v}_{\text {plan }}, \Delta \mathbf{t}_{\text {plan }}, \mathbf{r}\right) \ldots \ldots \ldots \ldots \ldots$. . . . . . . . 87

6 The Monte Carlo Localization algorithm for a robot using a LRF sensor . . . . . . . . . 179 



\section{1}

\section{Introduction}

\subsection{Introduction to UAVs and Multirotors}

The Research and Development (R\&D) in the field of Unmanned Aerial Systems is experiencing an increasing quantity of investment all around the world. Small UAVs are often used as experimental platforms to research on state estimation, control, robotic collaboration, etc; since their dynamic maneuvering and natural instability pose important challenges that are not present with other robotic platforms. In addition, the maneuverability of UAVs is combined with a low payload capability, which in turn requires the development of algorithms with constrained computation requirements. This fact has resulted in many research focused on testing and demonstrating the possibilities of many different sensors on these platforms. It is specially remarkable the usage of cameras (Campoy et al., 2009; Y.-C. Liu et al., 2010; P. Liu et al., 2014), since they are very lightweight and consume little power they are a potentially adequate sensor for UAVs. And consequently, small UAVs have been the target platform for the development and demonstration of very competitive Computer Vision algorithms.

Many of the R\&D is being performed by Universities and other Research institutions, but in the past few years there has been a growing number of companies selling UAV platforms, and offering civilian application services such as $3 \mathrm{D}$ reconstruction based mapping and surveying, visual inspection, etc. The paper (Pajares, 2015) presents an overview on remote sensing technologies utilized on small UAVS for surveying. Since there exists a wide range of possible civilian applications to UAVs, there are many studies that can be consulted. It is interesting to compare and see the evolution of the usage of UAVs over the years. In the late 90s, the usage of UAVs was still too expensive to allow their commercial development, since manned flights were more cost-effective. The only exception was the development of Japanese UAVs for agriculture applications (Aerosystems, 1999; Van Blyenburgh, 1999). The progressive miniaturization of computers, inertial measurements units and the extended use of GPS have made possible the manufacturing of small drones. A very good example is the toy AR Drone quadrotor, which demonstrates automatic take-off, hovering and landing capabilities (Bristeau et al., 2011). These factors and others have allowed the wider set of civilian applications that are possible to perform nowadays (Pajares, 2015), driving many countries around the world to almost simultaneously conceive a legal regulation framework for these platforms. The regulations are also aiming to address the growing privacy concerns of the general 
public, and some research also focuses on how the current legal framework affects UAVs and proposes solutions (Finn et al., 2012). The general time schedule for the deployment of these frameworks can be consulted in the following document (ERSG, 2013), which mainly concerns the European Union (EU). The United States of America, Canada, Australia, among many other countries; are advancing the implementation of their legal regulation in a similar pace as the EU. With the advent of these necessary legal frameworks, it is expected that UASs will generate a wide range of new commercial applications. The interest on these applications is directing part of the research effort to the necessary safety measures to be required on commercial UAVs such as obstacle detection and avoidance, visual detectability of UAS, safe communication data-links and related human factors (ERSG, 2013).

The work in this thesis is mainly concerned with multirotors. Although this work may be applicable to various UAV sizes, the conducted experimental works have been performed with multirotors on the $0.350 \mathrm{~kg}$ to $4.00 \mathrm{~kg}$ weight range and with horizontal sizes of approximately $0.450 \mathrm{~m}$ to $1.20 \mathrm{~m}$. Based on these weight and size characteristics, these aircrafts can be currently classified as multirotors of the Micro Aerial Vehicle (MAV) category. In general, throughout this text the term MAV can be considered to be referred to a multirotor drone or UAV.

Considering only civilian applications, UAVs and their related usages are very active fields of research and development. Considering only small ones, MAVs are aerial data acquisition platforms that can be utilized at a fraction of the cost of any manned counterpart. Furthermore, this reduced cost allows the development of aerial data gathering solutions for sectors that could not possibly afford to use a manned helicopter; effectively opening untapped commercial markets. For instance, this is the case of periodical surveying of agriculture crops.

In addition, UAVs, as any other robotic platforms are suitable to be used in tasks termed as "3D", Dull, Dangerous or Dirty (Welch et al., 2005); or other inaccessible tasks. UAVs can fly for very extended periods of time, longer than any human pilot could possible withstand. Additionally, they can be put at risk, without endangering a human pilot. These are the reasons why they are used as communication relays in remote locations, for border patrol and control and surveillance applications. UAVs can be equipped with adequate payloads and be converted into efficient aerial sensing platforms, making them suitable not only for aerial photography, but for wildlife monitoring, detection of illegal hunting or general (land, air and some sea) surveying applications. The only factors currently impeding the expansion of commercial applications based on these platforms are the ongoing development of appropriate regulations, and the still open robotic technical challenges.

\subsection{Problem Statement}

During the last few years the ongoing trend towards the integration of robotic systems into existing industrial applications and their utilization in new innovative and well-suited operations is intensifying. Therefore, there exists a big effort towards the vision-based realization of fully autonomous tasks using lightweight Unmanned Aerial Systems (UAS). The increasing computational power of small computers and the advent of lightweight general-purpose and application-specific sensors and sensing systems is accelerating the design of lightweight UAS for civilian applications. In order to put this trend into context, the reader may consider the several new products related to the development of lightweight UAS that have been released in the last 4 years. Regarding small computing platforms consider, for instance, the power and weight of the following on-board computers: the Snapdragon Flight (Qualcomm Technologies, Inc., 2015), the Intel Aero (Intel Corporation, 2016a) and the Nvidia Jetson TK1 (Nvidia Corporation, 2014). There exist now several developer drones which ease the design and implementation of new applications using lightweight multirotors. Some examples of them are: the Intel Aero development drone (Intel Corporation, 2016b), the DJI Matrice (DJI - Da-Jiang Innovations Science and Technology Co., Ltd, 2015) multirotor series and the Parrot Bebop 2 (Parrot SA, 2014). The following modern vision-based sensing systems are examples of specialized lightweight sensors for UAVs. The extremely lightweight depth-sensor RealSense (Intel Corporation, 2014). And the sensing systems that are able to estimate their pose and perform obstacle sensing using vision-based depth estimation: the DJI Guidance (Zhou et al., 2015) and the Parrot SLAM dunk (Parrot SA, 2016).

Given the increasing interest in utilizing drones for civilian applications were people may be around, there is an interest in achieving drones lightweight enough for them be harmless. In this sense, the legislation is heavily rewarding lightweight UAVs, thus motivating the research in vision-based navigation 
and control, since digital cameras are lightweight and feature a low power consumption. Stabilizing and controlling the drone by means of vision-based feedback, odometry (Fraundorfer and Scaramuzza, 2012; Scaramuzza et al., 2011) and localization are important steps in this direction. Vision-based navigation may enable the drone to navigate in GPS-denied and indoor environments, for instance (Bachrach et al., 2011). The control and pose estimation problems are not independent, since aggressive maneuvers result in more rapidly changing image streams from the on-board cameras, therefore they can potentially cause the drone to fail to estimate its motion properly. The controller, thus, needs to account for the sensor limitations and be able to perform smooth navigation at optimal speeds for the on-board sensors to function properly. Thus, the development of novel odometry algorithms that can process very high frame-rate streams (Forster et al., 2014) is important for UAS to be able to navigate at high speeds in indoor environments. There are still many challenges for drones to perform vision-based navigate indoors, like badly lit areas, reflective surfaces, moving objects and self-projected shadows and obstacle-avoidance in highly cluttered environments. Another issue are the presence of disturbances such as wind drafts which may be addressed by means of a better dynamics modeling of the multirotor, specifically identifying the major causes contributing to aerodynamic friction in drones (Leishman et al., 2014). In some civilian applications, for drones to be cost effective, it will be necessary to increase the number of drones per operator, and consider multi-drone systems (Ars Electronica Linz Gmbh, 2012; Hörtner et al., 2012), that can be successfully flown while performing a task for interest.

A common objective for drones is to be able to fly and stabilize looking at and close-by to an object of interest. This kind of application is defined as visual servoing (Corke, 2011), or object following, and it is of great interest for the visual inspection of infrastructure. Drones have a lot of economic potential for this purpose, as they promise to lower the cost of such necessary operations or even change their implications entirely by allowing to inspect big infrastructure, e. g. bridges, without stopping its normal operation. A closely related task is the realization of person following, enabling a drone to take pictures and video of a person from interesting vantage points, for instance, while practicing sports or while doing tourism. In order to perform the visual servoing operation, the usage of vision-based methods is very promising since images contain a lot of information. Algorithms that are able to locate an object in a sequential image stream are defined as visual trackers. There exists a diversity of such methods depending on their working principle: based on features, direct methods, based on statistical properties of the tracked object such as color or shape and others (Baker et al., 2004). Some recent research have worked in the improvement of traditional methods (Possegger et al., 2015), achieving very competitive results. However, setting aside such important works, traditional methods, though very effective in the specific conditions for which they were designed, have the drawback of not generalizing well to the myriad of different visual appearances that real objects exhibit. A very important trend in modern visual tracking research focuses on tackling the problem through data driven approaches and machine learning (Kalal et al., 2012). These methods learn a model for the object of interest on-the-fly, thus they can potentially create a model tailored for the object of interest and its surrounding environment and arguably that better discriminates between both, thus achieving more reliable tracking.

The autonomous navigation in real-world environments, for the realization of civilian applications in general situations, requires the drone to map the environment and specifically to be able to detect static and moving obstacles and avoid collisions with them. Since assuming that the drone knows its surrounding environment is a big limitation, a common task that needs to be performed by the drone, while realizing a task of interest, is the exploration (Fraundorfer, Heng, et al., 2012) of the environment. During this operation, it is required to incrementally build a map, usually named SLAM (Davison et al., 2007), and decide which parts of the space are occupied by obstacles using statistical methods and memory and computationally efficient map representations (Hornung et al., 2013). Navigation through cluttered environments can be performed reactively (Ross et al., 2013) or by calculating collision-free paths based on the currently mapped obstacles, a problem which is generally defined as trajectory planning (LaValle et al., 2001). The navigation control performs better when following smooth trajectories, allowing faster motion, and it benefits from faster trajectory generation algorithms, allowing the drone to make fast decisions while navigating in cluttered environments; therefore giving rise to different research trends in trajectory planning. Since robots can move and affect which data is going to be gathered for the mapping of the environment, another innovative research trend is acknowledging the inter-dependence in robotics of exploration, obstacle mapping and trajectory planning.

The scientific community is starting to acknowledge the importance of experiment reproducibility. In Computer Science related fields, the best way to accomplish it is by releasing the source-code as 
open-source and sharing the experimental data, so that other researchers can individually confirm the results of scientific publications and investigate the capabilities of published methods. In robotics this kind of reproducibility can be achieved at the level of task-specific libraries and modules or at the bigger scale of general-purpose robotics architectures. Since modularization can ease this process greatly, researchers favor the adoption of common robotics middle-ware frameworks that provide inter-module communication and other features, among which the Robot Operating System (ROS) has achieved the biggest acceptance (Willow Garage, 2007). Additionally, in the case of drones there exists a number of open-hardware and open-source autopilot boards, one particularly favored board is the Pixhawk autopilot (Meier et al., 2015). At a bigger scale the Dronecode Project (Linux Foundation, 2014), supported by the Linux Foundation, seeks to develop an open-source UAV platform for civilian applications.

The necessity of implementing novel and reliable vision-based navigation methods, exploring the capabilities of state of the art Computer Vision methods and their applicability to Robotics defines the research framework from which this thesis has been developed. This thesis has a strong experimental basis and the main research problems tackled during its realization have been: vision-based navigation, vision-based object tracking and following, smooth trajectory and speed planning for drones, and the utilization of vision-based acquired maps for the calculation of obstacle-free paths.

\subsection{Thesis proposal and objectives}

The main goal of the Computer Vision Group (CVG-UPM) (CVG-UPM, 2012) in the field of aerial robotics is the use of Unmanned Aerial Vehicles guided and controlled by a computer to perform applications in non-segregated civilian spaces in an autonomous or semi-autonomous fashion; or to develop post-processing algorithms to increase the degree of automation of the subsequent tasks that are to be conducted after inspection works. This $\mathrm{PhD}$ thesis aims to improve the state of the art in this growing field by making contributions to this general proposal considering the following objectives.

- The development of a novel middle-level controller for multirotors. Its main specification is to consider possible speed limitations established by the sensing capabilities of the on-board sensors. This main objective is further split into the following requirements:

- The controller will have as output a common interface specification for multirotors. This module should enable the MAV to autonomously track position or speed control references.

- The controller needs to be extended to be able to follow a trajectory and be thoroughly debugged and tested, so that it can be used in subsequent projects.

- It is also required for the controller to have several configurable parameters so as to be adjustable to work with different multirotors, sensors and odometry estimation algorithms.

- Building up on the prior requirement, some odometry estimation algorithms may fail to deliver estimates when navigating at inadequate velocities. As an example, in vision-based odometry estimation it becomes increasingly difficult to match image patches or features in subsequent images, due to a lack of overlap between them, thus, in practice, setting a maximum navigation velocity. Therefore, the controller, under normal working conditions, should be able to ensure that the trajectory will be executed at the optimum speed for the on-board sensors to work properly.

- The development of a novel visual servoing control architecture for arbitrary object following. The main objective is attaining stable flight against various objects, and to explore the possibility of tracking objects determined by the user on-the-fly. The development of this architecture will allow to understand the feasibility of safely performing the following of arbitrary objects that are specified sequentially by an user during flight.

- The development of a novel trajectory planner for multirotors to fly in outdoors cluttered environments. This main objective is further split into the following requirements:

- The algorithm needs to be lightweight enough to be executed on the on-board computer of the multirotor. 
- The algorithm has to be tested experimentally on maps obtained using real-time capable Computer Vision methods.

- It has to work using a commonly accepted map representation in robotics, calculate obstaclefree smooth trajectories when possible at a safe distance from obstacles and provide a speed, acceleration and time-of-passage plans along with the trajectory path.

- It has to be possible to set the maximum desired speed and acceleration for the trajectory calculation.

- The speed plan should bound the jerk, i. e. the derivative of the acceleration, of the trajectory so that the multirotor can fly through it using smooth rotations.

- Providing contributions, in the form of tested and working modules, to the general software architecture for UAVs, and specifically for multirotors, named "Aerostack" that is being continuously developed and improved by the CVAR, previously CVG, (UPM). Aerostack is a multipurpose software framework that aims to enable MAVs to perform civilian applications. The author has to actively participate in the publication of open-source software for this framework. The contributed modules to Aerostack need to be, therefore, reusable by others and reconfigurable so that they can be utilized in tasks related to the main purpose of the module.

In order to ease the diffusion of results, once the previously listed objectives were achieved and tested, the author has actively participated in public flight demonstrations, such as international MAV competitions and other events. The participation in such events also showcases the performance and capabilities of Aerostack, fosters the research collaboration among team members and colleagues and promotes the further testing of the developed modules in different settings and applications, e. g. indoors and outdoors flight, narrow corridors, passage through windows, landing in different conditions and so on.

\subsection{Dissertation outline}

The chapters of this $\mathrm{PhD}$ thesis are dedicated to the main contributions of the author to the state of the art. A general purpose controller for multirotor MAVs, that has been showcased in many public international MAV competitions, is presented in Chap. 2. An innovative visual servoing architecture for multirotor MAVs that enables them to follow arbitrarily selected objects is presented in Chap. 3 A trajectory and speed planner developed to be compatible with the reknown robotics mapping representation Octomap and that has been shown to work using vision-based reconstructed geo-referenced 3D models is presented in Chap. 4 The multirotor drones utilized in the realization of experiments during the course of this thesis are described in Appx. $\mathrm{H}$. The main body of this $\mathrm{PhD}$ thesis ends with Chap. 5 that discusses the thesis contributions, conclusions and future work.

The software modules, developed during this thesis, that have been released as open-source as part of "Aerostack", a multipurpose software framework for MAVs developed mainly at the CVAR, or CVG, (UPM), are enumerated in Appx. G. They include the controller architectures discussed in Chap. 2 \& 3 , and a simple kinematics simulator and a state estimator, presented in Appx. E that are based on the model presented in Appx.D.

During the course of this $\mathrm{PhD}$ thesis and as part of the collaboration on the development of "Aerostack", the robotics modules developed by the author have been utilized for the participation in the International MAV competitions described, and listed with their corresponding achieved results, in Appx.[1 These modules have been also showcased in several public events, which are listed in Appx. J] 


\subsection{Chapter Bibliography}

Aerosystems, B. (1999). "Civilian applications: the challenges facing the UAV industry". Air \& Space Europe (cit. on p. 1).

Ars Electronica Linz Gmbh (2012). SPAXELS: DRONE SHOWS SINCE 2012. https : / / www . spaxels.at/. Accessed: 2017-03-19 (cit. on p. 3).

Bachrach, A., Prentice, S., He, R., and Roy, N. (2011). "RANGE-Robust autonomous navigation in GPSdenied environments". Journal of Field Robotics 28.5, pp. 644-666 (cit. on p. 3).

Baker, S. and Matthews, I. (2004). "Lucas-kanade 20 years on: A unifying framework". International journal of computer vision 56.3, pp. 221-255 (cit. on p. 3).

Bristeau, P.-J., Callou, F., Vissiere, D., Petit, N., et al. (2011). "The navigation and control technology inside the ar. drone micro uav". In: 18th IFAC World Congress. Vol. 18, pp. 1477-1484 (cit. on p. 1).

Campoy, P., Correa, J. F., Mondragón, I., Martínez, C., Olivares, M., Mejías, L., and Artieda, J. (2009). "Computer Vision Onboard UAVs for Civilian Tasks". Journal of Intelligent and Robotic Systems 54.1-3, pp. 105-135 (cit. on p. 1).

Corke, P. (2011). Robotics, vision and control: fundamental algorithms in MATLAB. Vol. 73. Springer (cit. on p. 3).

Davison, A. J., Reid, I. D., Molton, N. D., and Stasse, O. (2007). "MonoSLAM: Real-time single camera SLAM". IEEE transactions on pattern analysis and machine intelligence 29.6 (cit. on p. 3).

DJI - Da-Jiang Innovations Science and Technology Co., Ltd (2015). DJI Matrice developer drone line. https://www.dji.com/matrice100. Accessed: 2017-03-18 (cit. on p.2).

ERSG (2013). Roadmap for the integration of civil Remotely - Piloted Aircraft Systems into the European Aviation System. Tech. rep. European RPAS Steering Group (cit. on p.2).

Finn, R. L. and Wright, D. (2012). "Unmanned aircraft systems: Surveillance, ethics and privacy in civil applications". Computer Law \& Security Review 28.2, pp. 184-194 (cit. on p.2).

Forster, C., Pizzoli, M., and Scaramuzza, D. (2014). "SVO: Fast Semi-Direct Monocular Visual Odometry". In: IEEE International Conference on Robotics and Automation (ICRA) (cit. on p. 3 .

Fraundorfer, F., Heng, L., Honegger, D., Lee, G. H., Meier, L., Tanskanen, P., and Pollefeys, M. (2012). "Vision-based autonomous mapping and exploration using a quadrotor MAV". In: Intelligent Robots and Systems (IROS), 2012 IEEE/RSJ International Conference on. IEEE, pp. 4557-4564 (cit. on p.3).

Fraundorfer, F. and Scaramuzza, D. (2012). "Visual odometry: Part II: Matching, robustness, optimization, and applications". IEEE Robotics \& Automation Magazine 19.2, pp. 78-90 (cit. on p. 3).

Hornung, A., Wurm, K. M., Bennewitz, M., Stachniss, C., and Burgard, W. (2013). "OctoMap: An efficient probabilistic 3D mapping framework based on octrees". Autonomous Robots 34.3, pp. 189-206 (cit. on p. 3).

Hörtner, H., Gardiner, M., Haring, R., Lindinger, C., and Berger, F. (2012). "Spaxels, Pixels in Space-A Novel Mode of Spatial Display.” In: International Conference on Signal Processing and Multimedia Applications, SIGMAP, pp. 19-24 (cit. on p. 3).

Intel Corporation (2014). Intel RealSense Developer Kits. https : / / click . intel . com / realsense.html. Accessed: 2017-03-18 (cit. on p. 2).

Intel Corporation (2016a). Intel Aero Compute Board. https : / / click . intel . com / intelaero-platform-for-uavs-compute-board.html. Accessed: 2017-03-18 (cit. on p.2).

Intel Corporation (2016b). Intel Aero Ready to Fly Drone. https : / / click . intel . com/intelaero-ready-to-fly-drone.html. Accessed: 2017-03-18 (cit. on p.2).

Kalal, Z., Mikolajczyk, K., and Matas, J. (2012). "Tracking-Learning-Detection”. IEEE Transactions on Pattern Analysis and Machine Intelligence 34.7, pp. 1409-1422 (cit. on p.3).

LaValle, S. M. and Kuffner, J. J. (2001). "Randomized kinodynamic planning”. The International Journal of Robotics Research 20.5, pp. 378-400 (cit. on p. 3).

Leishman, R. C., Macdonald, J., Beard, R. W., and McLain, T. W. (2014). "Quadrotors and accelerometers: State estimation with an improved dynamic model". Control Systems, IEEE 34.1, pp. 28-41 (cit. on p. 3).

Linux Foundation (2014). Dronecode Project. https : / / www . dronecode . org / about/ Accessed: 2017-03-18 (cit. on p.4).

Liu, Y.-C. and Dai, Q.-h. (2010). “A survey of computer vision applied in aerial robotic vehicles”. In: Optics Photonics and Energy Engineering (OPEE), 2010 International Conference on. Vol. 1. IEEE, pp. 277-280 (cit. on p. 11). 
Liu, P., Chen, A. Y., Huang, Y.-N., Han, J.-Y., Lai, J.-S., Kang, S.-C., Wu, T.-H., Wen, M.-C., and Tsai, M.-H. (2014). “A review of rotorcraft Unmanned Aerial Vehicle (UAV) developments and applications in civil engineering”. SMART STRUCTURES AND SYSTEMS 13.6, pp. 1065-1094 (cit. on p.1).

Meier, L., Honegger, D., and Pollefeys, M. (2015). "PX4: A node-based multithreaded open source robotics framework for deeply embedded platforms". In: Robotics and Automation (ICRA), 2015 IEEE International Conference on. IEEE, pp. 6235-6240 (cit. on p. 4).

Nvidia Corporation (2014). NVIDIA Jetson TK1 developer kit. https : / / wWw . nvidia . com / object/ jetson-tk1-embedded-dev-kit.html Accessed: 2017-03-18 (cit. on p.2).

Pajares, G. (2015). "Overview and Current Status of Remote Sensing Applications Based on Unmanned Aerial Vehicles (UAVs)". Photogrammetric Engineering \& Remote Sensing 81.4, pp. 281-330 (cit. on p. 1).

Parrot SA (2014). Parrot BEBOP 2. https: / / www . parrot . com/us / Drones / Parrot-bebop2. Accessed: 2017-03-18 (cit. on p.2).

Parrot SA (2016). Parrot S.L.A.M. dunk. https : / / www . parrot . com / us / business solutions/parrot-slamdunk. Accessed: 2017-03-14 (cit. on p.2).

Possegger, H., Mauthner, T., and Bischof, H. (2015). "In defense of color-based model-free tracking”. In: Proceedings of the IEEE Conference on Computer Vision and Pattern Recognition, pp. 2113-2120 (cit. on p. 3).

Qualcomm Technologies, Inc. (2015). Snapdragon Flight Kit. https : / developer. qualcomm . com/hardware/snapdragon-flight. Accessed: 2017-03-18 (cit. on p.2).

Willow Garage, S. A. I. L. (2007). Robot Operating System (ROS). http : / / www . ros . org/wiki/ (cit. on p. 4).

Ross, S., Melik-Barkhudarov, N., Shankar, K. S., Wendel, A., Dey, D., Bagnell, J. A., and Hebert, M. (2013). "Learning monocular reactive uav control in cluttered natural environments". In: Robotics and Automation (ICRA), 2013 IEEE International Conference on. IEEE, pp. 1765-1772 (cit. on p. 3).

Scaramuzza, D. and Fraundorfer, F. (2011). "Visual odometry [tutorial]". IEEE robotics \& automation magazine 18.4, pp. 80-92 (cit. on p. 3).

Van Blyenburgh, P. (1999). "UAVs: an overview". Air \& Space Europe 1.5, pp. $43-47$ (cit. on p. 1).

CVG-UPM (2012). Universidad Politécnica de Madrid. Computer Vision Group. Vision for UAV Project. http: //www.vision4uav.com (cit. on p. 4).

Welch, G. and Bishop, G. (2005). European Civil Unmanned Air Vehicle Roadmap, Volume 3 Strategic Research Agenda. Tech. rep. European Civil UAV FP5 R\&D Program Members (cit. on p. 2).

Zhou, G., Fang, L., Tang, K., Zhang, H., Wang, K., and Yang, K. (2015). "Guidance: A visual sensing platform for robotic applications". In: IEEE Conference on Computer Vision and Pattern Recognition Workshops, 2015 IEEE CVPR Workshop, pp. 9-14 (cit. on p.2). 



\section{Chapter}

\section{A General Purpose Configurable Controller for Multirotor MAVs}

\subsection{Introduction}

The final objective of robot applications is for the robot to perform some useful task. The problem of making an autonomous or semi-autonomous robot perform a task is hard and is realized by a combination of control, odometry, state estimation and planning algorithms. Planning algorithms have to decide a succession of steps that leads to the accomplishment of the required objectives. They require to approach the problem in a friendly set of coordinates, usually related to position coordinates. They may also work with speed coordinates, but only to optimize certain specifications of the task, and the planning problem usually does not output actuator/engine commands. For instance, if an application requires a quadrotor to approach multiple points of interest to send video and images to a ground station, the common solution is to approach the problem in position coordinates where static obstacles are defined. Another aspect is that the task planner has to assume that the multirotor will be stabilized automatically, and that it will follow commands in position coordinates.

These and other issues are solved by adding interface layers between the multirotor and the task planner. In the case of multirotors, the main two problems that have to be addressed are:

- The estimation problem: both the planning algorithm and the controller require an estimation of the state of the quadrotor. For example the task scheduler, that ensures that the steps specified by the planning algorithm are being accomplished, will need feedback to determine the accomplishment of the successive mission objectives. The estimation problem has been described and discussed in Appx. E

- The stabilization of the vehicle and the capacity to follow paths and position commands are solved using 'controllers'. Controllers are automation algorithms that ultimately generate the commands for the engines of the robot, in real-time using feedback from sensors or from an estimator such as an Extended Kalman Filter (EKF). In the case of multirotors the controller should offer an interface to specify a trajectory, position or speed commands; thus, simplifying the planning problem. 


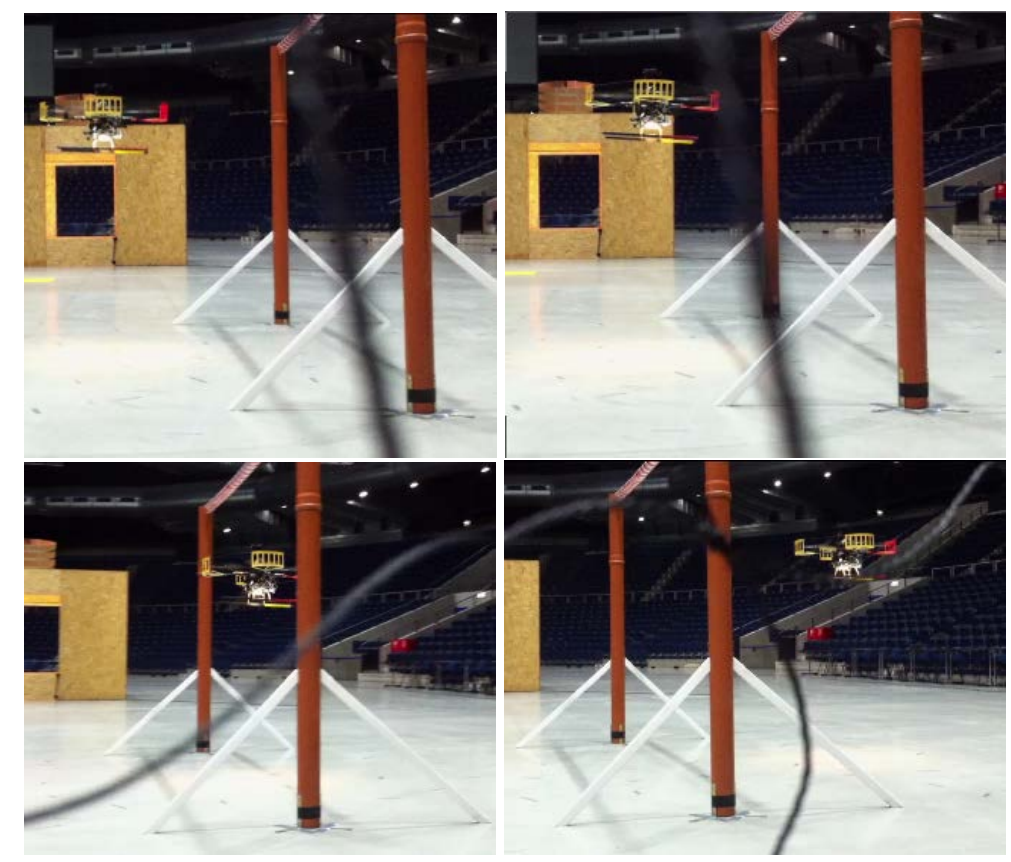

Figure 2.1: The Asctec Pelican quadrotor, equipped as explained in Sec. H.1.1, is shown flying in autonomous mode on the IMAV 2012 competition with an implementation of the controller presented in this paper. An EKF performs state estimation on the odometry measurements, and its estimation is fused with the position estimations from a Montecarlo Localization algorithm that is processing the Laser Range Finder readings. The quadrotor also had to be able to fly safely on areas where no obstacles were in range of the laser sensor. In this image sequence the quadrotor passes through a pair of poles using the laser sensor to estimate its position with respect to the poles. This flight gained the two IMAV 2012 awards stated in Appx. I. see http://vision4uav.com/?q=IMAV12 . The full video of the flight is available online on http://vision4uav.com/?q=node/323 .

This chapter is dedicated to the development of a general-purpose controller for drones. The objective is developing a control layer for the multirotor that offers an appropriate interface to the task-level supervisor, such as GPS checkpoint navigation or position checkpoints for indoors navigation. For a detailed state of the art the reader is referred to Sec. 2.2. The presented controller was first developed for the participation in the IMAV2012 competition, see Fig. 2.1, where an Asctec Pelican quadrotor equipped with additional sensors was used, and is explained in Sec.2.3, including: the speed, position and trajectory controllers presented in Sec. 2.3.3, and an state machine, see Sec. 2.3.4 that defines the commands to reach a sequence of waypoints along the lines that join them. This controller has been used by the CVG_UPM to participate in the following competitions: IMAV2012, IMAV2013, IARC2014 and IMAV2016 (see Appx. II). The experimental results obtained with this controller flying the Asctec Pelican, a single and multiple AR Drones are presented in Secs. 2.4.2 \& 2.4.3\& 2.4.4

\subsection{State of the Art}

The following cited work has shown that the navigation control loops must be designed taking into account the non-linear dynamics of the multirotor, so that the control actions are approximately decoupled. Many successful results can be obtained by linearizing the dynamics of the multirotor around the hovering state. However, as it will be later discussed, more recent work has shown much better results using various non-linear approaches to controller design. If the control laws are well designed the multirotor can perform smooth trajectories in position coordinates, while orientating its yaw heading in any required direction.

Since 2010 and onwards, some research projects have demonstrated, using motion capture systems, like for instance a Vicon system, that multirotors can be controlled successfully when accurate position measurements are available. The researchers at the GRASP Laboratory of the University of Pennsylvania 
have been able to execute very precise trajectory following, using cascade control laws with non-linear decoupling functions and precise feedforward command laws (Mellinger et al., 2010; B. Y. N. Michael et al., 2010). They have also demonstrated excellent performance in aggressive maneuvering with small Asctec Hummingbird quadrotors, such as multi-flips, perching in vertical landing platforms and precise maneuvers synchronized with environment events. Several experimental tests have been carried out involving $4+$ quadrotors in collaborative tasks, examples of such experiments are quadrotor swarming 1 (Kushleyev et al., 2012), a swarming artistic quadrotor show 2 , with lighting effects and movement, that took place on Cannes Lions Festival of Creativity 2012; and the construction of small-sized buildings using toy construction sets 3

In a similar research line, also making use of a motion capture system, the ETH - IDSC - Flying Machine Arena project at the Eidgenössische Technische Hochschule Zürich (ETHZ), have also demonstrated impressive quadrotor flight performance on aggressive maneuvering (S. et al., 2010), and synchronizing with music (Schölling et al., 2010). The synchronization with music has been shown playing the piand 4 and performing diverse navigation maneuvers following the rhythm of musis 5 . The multirotor platforms used in this lab are Asctec Hummingbird quadrotors. The synchronization between multiple sUAS platforms is useful for swarming and the exploration of indoors buildings, so that quadrotors can fly together precisely maneuvering without crashing with each other. The environmental music is used to create human emotional appeal to the experiments.

The main issue with these works is the requirement of a structured environment, where a motion capture system has to be in place. Their research work has been extremely fruitful demonstrating what many different control approaches can attain. In principle these controllers should perform equally well outside these environments, if they are fed with a good enough state estimate. However, it is important to know what is possible for a quadrotor to do outside such structured environments. By that time, there were research groups that had shown already good autonomous multirotor navigation capabilities using only GPS positioning data, or performing (SLAM) during building exploration.

The STARMAC project 6 , from the Stanford University shows competitive outdoors performance in trajectory tracking (Hoffmann, Gabriel M et al., 2008) relying only on GPS. Unlike both prior research labs, the STARMAC (, 2012) is a bigger multirotor, about $80 \times 80 \mathrm{~cm}$ large; and it is also designed by the Stanford and Berkeley Universities. Prior work for post-disaster assessment was presented on (Hoffmann, Waslander, et al., 2006). Their work has also been focused on the study of the aerodynamic disturbances that are present during quadrotor navigation, for example in the stall turn maneuver (Hoffmann, G.M. et al., 2009), where the multirotor has to change its navigation direction to move backwards. An interesting study, from both the theoretical and experimental point of view, was done in the work (Gillula et al., 2010), where the STARMAC platform was able to make single-flip maneuvers in an outdoors environment The calculations performed in this study, would allow a quadrotor to determine if a maneuver can be performed successfully, and how near would it be to instability. The control laws used in the STARMAC projects remained relying on cascade control laws around the hovering linearized state and that took into account the non-linear decoupling, using the yaw, of the pitch and roll commands.

Around that time other impressive work, not involving the usage of tracking systems, had been performed. The project on collaborative and swarming of multirotor platforms, like the Collaborative Microdrones project (Quaritsch et al., 2008), works at the GRASP Lab (N. Michael et al., 2012) and the sFly project 8 (Scaramuzza et al.,2013). In terms of control, one of the biggest contributions of the sFly project was to show that their visual-inertial based architecture could function quite well even with a gap of 1 second between image measurements (Weiss et al.,2012), which were simulated as pose measurements. This paper really showed what a well integrated IMU sensor can attain, in terms of adding robustness to the whole architecture. In terms of autonomous exploration using quadrotors, some works also have shown that it was possible for a quadrotor to explore a man-made environments using different sensors: liked LIDAR (A. G. Bachrach, 2009, Shen, N. Michael, et al., 2011), LIDAR and Stereo Cameras (Achtelik et al., 2009, A. Bachrach et al., 2011), Stereo cameras only (Heng et al., 2014) and Kinect sensors (A. S.

\footnotetext{
1 https://www $\cdot$ youtube.com/watch?v=YQIMGV5vtd4, 2012

2 http://www.uasvision.com/2012/07/06/a-flying-quadrotor-light-show-spectacular/, 2012

3 http://www.youtube.com/watch?v=W18Z3UnnS_0, 2011

4 http://www.youtube.com/watch?v=ToACDIXTzo0,, 2010

5 http://www.youtube.com/watch?v=7r281vgfotg, 2011

(http://www. youtube.com/watch?v=rJ9r2orcaYo, 2008

http://www.youtube.com/watch?v=iD3QgGpzzIM, 2009

8 http://WwW.sfly.org/
} 
Huang et al., 2011). Other groups have increased the situational awareness of the mUAVs using optical flow sensors and cooperative robots. For instance, a ground vehicle can estimate the position and attitude of the mUAV (Rudol et al., 2008). These two research works (Lippiello et al., 2011; Zingg et al., 2010) are focused on navigation in corridors and obstacle collision avoidance strategies using multiple optical flow sensors. In outdoors navigation, optical flow sensors were used in a fixed-wing mUAV in (Zufferey et al., 2010) to avoid the collision with trees and other obstacles in a GPS waypoint trajectory.

Currently, among the most aggressive maneuvering multirotor research projects performed in unstructured environments are probably the following. Fast dynamic trajectory maneuvering has been achieved in indoors flight using LIDAR and IMU integration, but for a previously mapped environment, by Richter et al. (Richter, Charles et al., 2013). And using a mixture of monocular and stereo vision, Shen et al. (Shen, Shaojie et al., 2013, Shen, Mulgaonkar, et al., 2013) have achieved similar capabilities in both indoors and outdoors environments with a limited short-time visual mapping capability. However this last UAS was still not able to track trajectories as fast as in Richter et al.'s approach.

Next the related research work will be summarized in terms of the control laws themselves, whether they can be considered as linear control laws or non-linear ones. The discussion is first done with controllers that are linear or designed by linearizing the quadrotor dynamics. Most of these works explicitly linearize the multirotor dynamics around the hovering position, and then develop the control law for the linearized system. Then the control problem is subdivided into the attitude stabilization problem, which is then used as the inner control loop of a nested control architecture, and into the position control problem (Bouabdallah, Murrieri, et al., 2005, Hoffmann, H. Huang, et al., 2007, B. Y. N. Michael et al., 2010). Some of these works still take into account part of the nonlinear characteristics of the system to improve the general performances of the controller due to varying thrust requirements when tilted and to the rotor drag (Hoffmann, G.M. et al., 2009); or use different operating modes for trajectory following and hovering (Hoffmann, Gabriel M et al., 2008; Mellinger et al., 2010). The performances of quadrotors using LQR control allows around the hovering positions are well studied in the following articles (Cowling et al., 2010; Nice, 2004; Valenti et al., 2006). However, as shown in (Castillo, Lozano, et al., 2005) the stability may be lost when the flight develops far from the linearized hovering state. This same work also shows how a non-linear controller may keep the stability in these cases.

Now, turning the discussion into research on non-linear controllers. Some of them still rely on the linearized model around the hovering state (Castillo, Lozano, et al., 2005), and saturating or limiting the values of interim control commands, effectively saturating the commanded attitude values (Guenard et al., 2005). Very promising results were shown in the following work that applied backstepping (Bouabdallah and Siegwart, 2005, Madani et al., 2007), sliding mode control techniques to multirotors (Bouabdallah and Siegwart, 2005, D. Lee et al., 2009), and research that separate the attitude control problem from the translation one (Kendoul et al., 2010; Raffo et al., 2010), feedback linearization control (Driessen et al., 2004; Fritsch et al., 2012, D. Lee et al., 2009, Mistler et al., 2001) and other techniques (Castillo, Dzul, et al., 2004; Guenard et al., 2005, Hua et al., 2009).

A very influencing work by Taeyoung Lee et al. (T. Lee et al., 2010) was done based on the Geometric Control Theory (Agrachev et al., 2004; Jurdjevic, 1997). Although this work only tested the developed control laws in simulation, it demonstrated the stability qualities of the proposed controller, of exponential attractiveness for attitude errors of up to $90^{\circ}$, and almost exponential attractiveness for errors of up to $180^{\circ}$; and also was formulated in a way that there were no singularities in terms of the attitude representation.

Another influencing work by Oliver Fritsch et al. (Fritsch et al., 2012) performed quasi-static feedback linearization for the translation dynamics using a quaternion formulation for the attitude of the multirotor. First the altitude control loop and the heading are controlled using feedback linearization and then in a second step the lateral translation is also feedback linearized. This control law can be considered to be built on the differential flatness property of the dynamics of the multirotor.

\subsection{Controller Algorithm}

In this chapter a navigation controller that uses a set of configurable parameters to be adaptable to different quadrotors and sensing options is proposed. It is inspired on previous work from other research centers (Hoffmann, Gabriel M et al., 2008; B. Y. N. Michael et al., 2010) and by the STARMAC project (Hoffmann, Waslander, et al., 2006). The proposed controller consists of a main control architecture and a state machine that provides trajectory following commands to the main control architecture. 
Both subsystems are designed to provide control commands that are consistent with a configurable maximum speed parameter.

First, the overall control architecture is introduced in Sec.2.3.1. Then a simple multirotor point mass kinematic model is described in Sec. 2.3.2. Then the main control architecture, named the middle-level controller, is introduced in Sec. 22.3.3 Then the high level controller state-machine is introduced with the description of the configurable parameters that are derived from the modelin Secs. 2.3.4 \& 2.3.5 \& 2.3.6. Then final part of the chapter introduces the experimental results in Sec 2.4 and the conclusions in Sec. 2.6.

\subsubsection{Control Architecture}

The goal for the controller design task is to make the multirotor follow a path specified by a sequence of checkpoints. The general controller architecture, shown in Fig. 2.2. consists of: a mid-level controller, that calculates the actual commands that will be sent to the quadrotor; and a high-level controller that tracks the quadrotor position relative to the desired path and calculates the speed and position references for the mid-level controller. The state estimator that was utilized in the experiments and its related model are explained in Appx.E\&Drespectively.

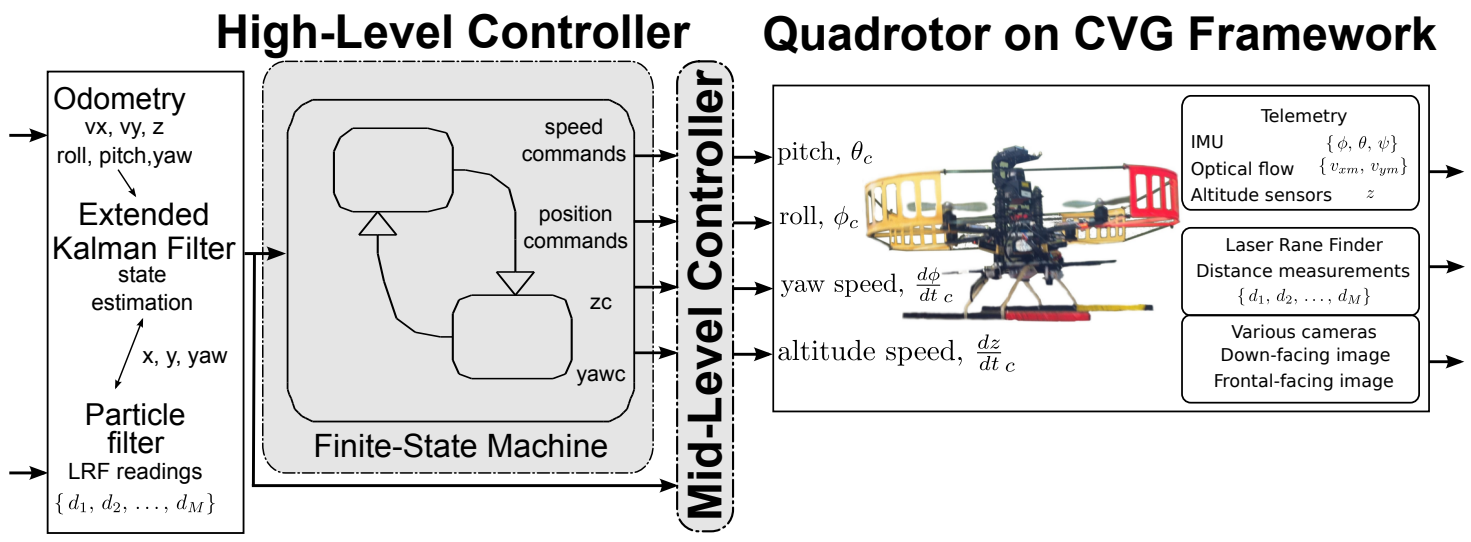

Figure 2.2: General architecture of the controller. The measurements are processed by an estimator that includes an EKF and a Particle Filter, which are discussed in Appx. E Then, in order to minimize the route time, the controller includes a speed planner in a state machine that sends optimized reachable speed commands to the mid-level controller. The state estimation is used by the high-level controller to determine optimized reachable speed commands to the mid-level controller, which sends commands to the drone through the CVG Framework (Mellado Bataller, I., 2012, Mellado-Bataller, Mejias, et al., 2012; Mellado-Bataller, Pestana, et al.,2013) or its pseudo-equivalent ROS node.

The multirotor is stabilized using odometry-based position estimates and for that reason the boundaries where the quality of these estimates is lost must be determined. For instance, the optical flow algorithm that is calculated on the down-facing images is affected by the texture and reflectiveness of the floor surface, the size of the floor texture details, the lighting conditions of the environment and the computing power of the on-board computer. This characteristics set a maximum speed boundary on the speed estimation over which the estimates do not provide meaningful information and may cause the malfunction of the estimation and control architecture.

For instance, the maximum speed that could be reliably estimated at low altitude by our optical flow algorithm in the indoors playground where most of the tests where carried out was about $0.5-0.75 \mathrm{~m} / \mathrm{s}$, and so, this boundary was used in our indoors tests. Based on our experience, the AR Drone at the same altitude provides speed estimation feedback even navigating at high speeds, up to $3 \mathrm{~m} / \mathrm{s}$. The direction of the speed estimation maintains a good accuracy at all working conditions if the yaw rotations are kept low. On the other hand, the absolute value of the speed can be affected by several conditions. For example, when the drone switches from ultrasound sensor based to pressure-only based altitude estimation, the accuracy of the speed estimates is degraded. Also the speed measurement at speed in the $2-3 \mathrm{~m} / \mathrm{s}$ speed range is not accurate, but its enough to keep our controller architecture working. 


\subsubsection{Multirotor Point Mass Kinematic Model}

A multirotor is a flying vehicle that propels itself by pointing the thrust force in the required direction and thus can be simplified as a point mass moving in the 3D space. The thrust force is perpendicular the propeller's rotating surface, as shown in Fig. 2.3 The vehicle tilts to exert force in the horizontal plane, and changes the average propeller speeds to control the amount of thrust force. A maximum speed is set in practice by various reasons, for instance, due to the aerodynamic friction of the movement through the air. It is also important to take into account that the multirotor is commanded using feedback loop control laws that produce a response that can be characterized by a set of parameters. The speed planner can take advantage of a simple kinematic model built upon these facts in order to take decisions.

The body frame, $\left\{X_{m}, Y_{m}, Z_{m}\right\}$, of the vehicle, shown in Fig. 2.3, is described as follows. The $\left\{X_{m}, Y_{m}\right\}$ axis are horizontal and the $Z_{m}$ axis points down to the floor. The $X_{m}$ axis points to the front of the vehicle and the $Y_{m}$ axis points to the right obtaining a orthonormal right-handed reference frame. The euler angles are denoted $\{\phi$, roll $\},\{\theta$, pitch $\}$ and $\{\psi$, yaw $\}$, and they define the rotation of the the body frame, $\left\{X_{m}, Y_{m}, Z_{m}\right\}$, with respect to the world frame, $\{X, Y, Z\}$.

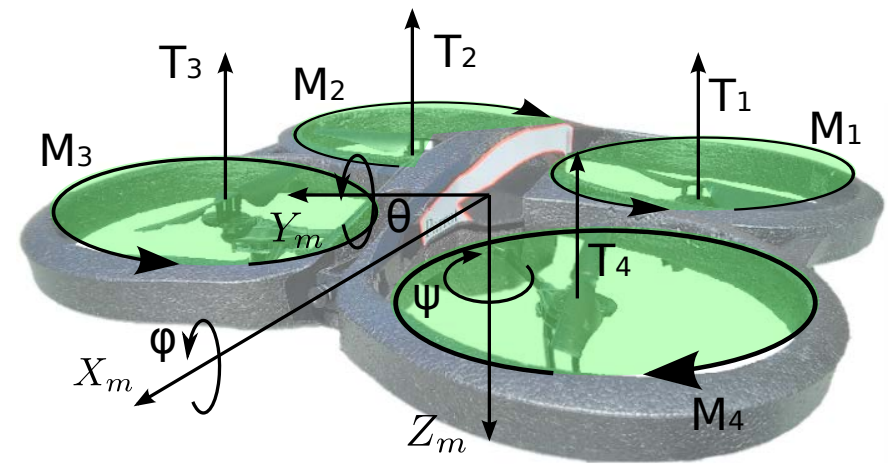

Figure 2.3: Free body diagram of a quadrotor. The four propellers, and each of their performed thrust $T_{i}$ and torque $M_{i}$ are labeled 1-4. The euler angles are denoted $\{\phi, \operatorname{roll}\},\{\theta, \operatorname{pitch}\}$ and $\{\psi$, yaw $\}$

The quadrotor rigid body dynamics model, see Eq. 2.1 and Fig. 2.3 is infered from physical laws as explained in Appx. D.

$$
\begin{aligned}
I_{x} \ddot{\phi} & =\dot{\psi} \dot{\theta}\left(I_{y}-I_{z}\right)+l\left(T_{1}+T_{2}-T_{3}-T_{4}\right) \\
I_{y} \ddot{\theta} & =\dot{\phi} \dot{\psi}\left(I_{z}-I_{x}\right)+l\left(T_{1}+T_{4}-T_{2}-T_{3}\right) \\
I_{z} \ddot{\psi} & =\dot{\theta} \dot{\phi}\left(I_{x}-I_{y}\right)+\sum_{i=1}^{4} M_{i} \\
m \ddot{x} & =(-\sin \phi \sin \psi-\cos \phi \sin \theta \cos \psi) \sum_{i=1}^{4} T_{i}-K_{f r} \dot{x}^{2} \\
m \ddot{y} & =(-\cos \phi \sin \theta \sin \psi+\sin \phi \cos \psi) \sum_{i=1}^{4} T_{i}-K_{f r} \dot{y}^{2} \\
m \ddot{z} & =m g-\cos \theta \cos \phi \sum_{i=1}^{4} T_{i}-K_{f r} \dot{z}^{2}
\end{aligned}
$$

Where the following variables have been introduced: the position of the quadrotor with respect to the world frame is denoted by the $\{x, y, z\}$ coordinates, the attitude of the vehicle is represented by the yaw $\psi$, pitch $\theta$ and roll $\phi$ euler angles; the quadrotor rigid body is characterized by its mass $m$ and its three principal mass moments of inertia $\left\{I_{x}, I_{y}, I_{z}\right\}$, each propeller $i$ generates a thrust $T_{i}$ and a heading torque $M_{i}, K_{f r}$ is an aerodynamic friction constant and the $l$ constant is the arm between each pair of thrusts, in this case the distance between the propellers 1 and 2, as denoted in Fig. 2.3 .

The following control loops are usually implemented inside the autopilot board of the multirotor: the attitude (roll, $\phi$, pitch, $\theta$, and yaw, $\psi$ ) control loops and altitude, $z$, control loop. The autopilot boards usually accept roll, pitch, yaw speed and altitude speed references. So that the saturation bounds for these variables is set by the autopilot board and drone capabilities. Thus, it is only required to further develop the equations of movement in the horizontal plane.

If the flight is performed at constant altitude then, $\sum_{i=1}^{4} T_{i} \approx m g$, and taking the approximation to low roll and pitch angles, then the equations that define the mouvement in the horizontal plane are derived 
from Eqs. 2.1

$$
\begin{aligned}
& m \ddot{x}=(-\theta \cos \psi-\phi \sin \psi) m g-K_{f r} \dot{x}^{2} \\
& m \ddot{y}=(-\theta \sin \psi+\phi \cos \psi) m g-K_{f r} \dot{y}^{2}
\end{aligned}
$$

Two additional attitude variables are defined $\left\{\phi_{v}\right.$, virtual roll $\},\left\{\theta_{v}\right.$, virtual pitch $\}$, which control de position of the vehicle in the horizontal $\{X, Y\}$ plane. They are related to the actual $\{\phi, \theta\}$ through the yaw $(\psi)$ angle as follows:

$$
\left[\begin{array}{l}
\theta_{v} \\
\phi_{v}
\end{array}\right]=\left[\begin{array}{cc}
\cos (\psi) & \sin (\psi) \\
-\sin (\psi) & \cos (\psi)
\end{array}\right]\left[\begin{array}{l}
\theta \\
\phi
\end{array}\right]
$$

The attitude controller, with a response time of $t_{r} \approx 200-300 \mathrm{~ms}$, is significantly faster than the horizontal speed loop, that has a response time of $\left(t_{r} \approx 1-2 \mathrm{~s}\right)$. This difference in their response speeds allows to correct the coupling introduced by the yaw commands in the horizontal speed loops using the decoupling law alone, defined by Eq. 2.3. The reference frame change defined by Eq. 2.3 is included in the controller, so that the $\left\{\phi_{v}, \theta_{v}\right\}$ can be used for the controller design.

$$
\begin{aligned}
& m \ddot{x}=-\theta_{v} m g-K_{f r} \dot{x}^{2} \\
& m \ddot{y}=\phi_{v} m g-K_{f r} \dot{y}^{2}
\end{aligned}
$$

The dynamic model of the multirotor is much simpler when expressed using the virtual angles and a maximum value for the quadrotor acceleration saturation can be infered from Eq. 2.4 as follows:

$$
\begin{aligned}
& \ddot{x} \approx-g \theta_{v}-\left(K_{f r} / m\right) \dot{x}^{2} \rightarrow|\ddot{x}| \leq g\left|\theta_{v \max }\right|-\left(K_{f r} / m\right) \dot{x}^{2} \\
& \ddot{y} \approx g \phi_{v}-\left(K_{f r} / m\right) \dot{y}^{2} \rightarrow|\ddot{y}| \leq g\left|\phi_{v \max }\right|-\left(K_{f r} / m\right) \dot{y}^{2}
\end{aligned}
$$

These equations show that the maximum horizontal speed of the vehicle, $v_{x y \max }$, is physically limited by the aerodynamic friction. Thus, the maximum quadrotor speed is expressed by Eq. 2.8 and the following model:

$$
\left(\dot{x}^{2}+\dot{y}^{2}\right) \leq v_{x y \max }^{2}
$$

The altitude acceleration and speed can be modeled similarly, but since many autopilots accept altitude speed commands we constrain the altitude speed only and not its acceleration:

$$
|\dot{z}| \leq{\frac{d z}{d t_{\text {max }}}}
$$

The point mass model has to take into account that the vehicle is being commanded using feedback control laws, and that the controller will not be able to push the vehicle capabilities to the physical limits stated above. Thus the following set of parameters is selected based on this fact and on the previous considerations:

- The combination of Eqs. $2.8 \& 2.7$ set a maximum speed on any direction.

- The speed response of the vehicle can be characterized by the response time of the speed control loop $t_{\text {rvmax }}$

- The maximum acceleration is physically limited by Eqs. 2.5 \& 2.6 and can be further determined by experimental tests. But the actual accelerations obtained during trajectotry control depend on the performances of the controller and on the smoothness of the speed references set to the speed control loop. Thus, the maximum acceleration parameters in the horizontal plane are derived $\left\{a_{x \max }, a_{y \max }\right\}$.

- The trajectory around checkpoints can be approximately modeled by a sector of circumference thus requiring a centripetal acceleration that is limited by

$$
a_{c x y} \leq g\left\{\theta_{v \max }, \phi_{v \max }\right\}=\frac{v_{t}^{2}}{R_{c}}
$$

where $v_{t}$ and $R_{c}$ are the average speed and curve radius during the turn. 


\subsubsection{Middle-Level Controller}

The mid-level controller is shown in Fig 2.4 and it is inpired on previous research from other groups (Hoffmann, Gabriel M et al., 2008; Mellinger et al., 2010, B. Y. N. Michael et al., 2010). Its general architecture is a cascade controller consisting of an inner speed loop and an outer position loop. But it also includes control laws to take into account the overall system capabilities.

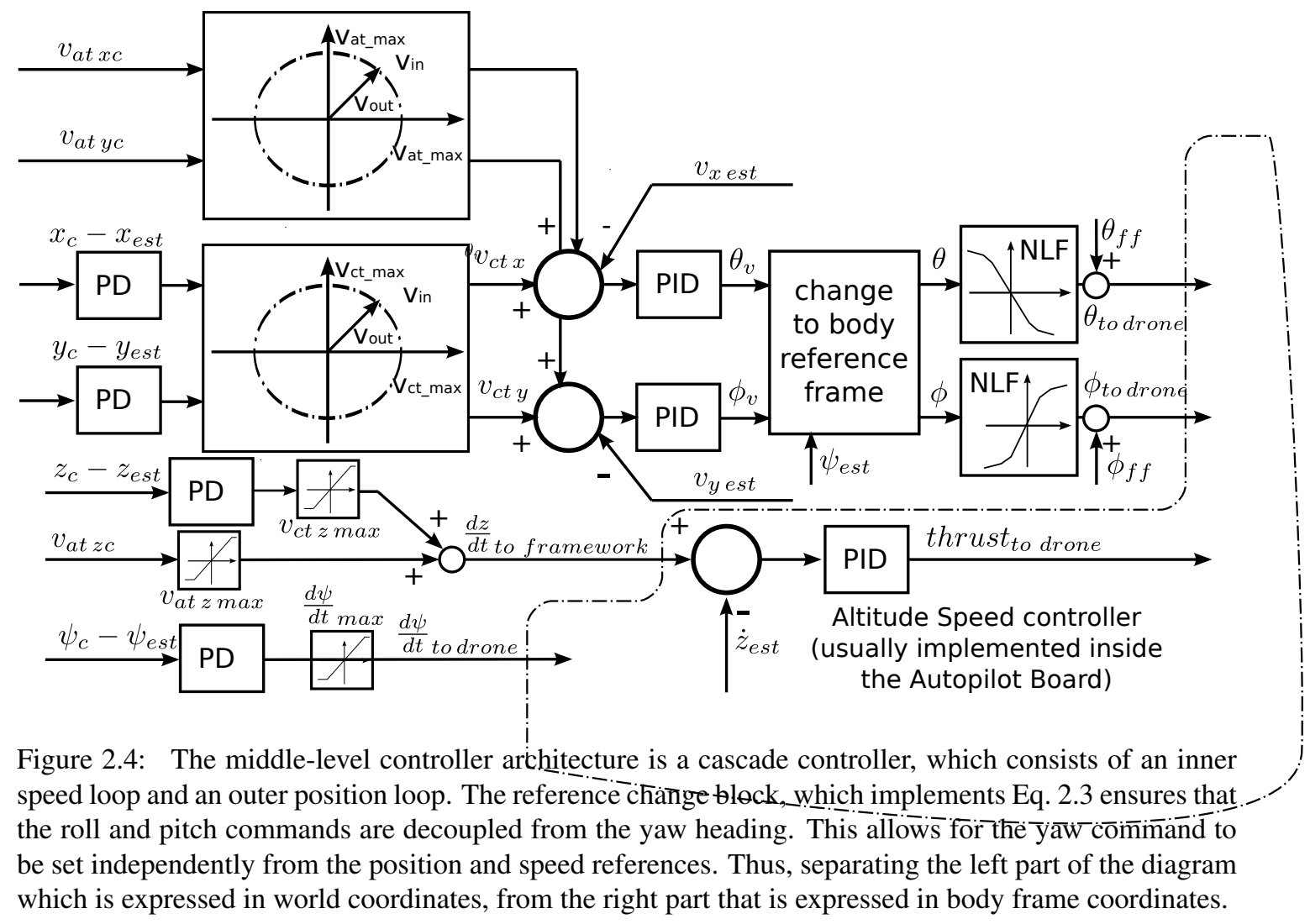

Some characteristics of the control architecture decipted in Fig. 2.4 are:

- The position controller loop only commands achievable speeds to the speed controller loop, see left block in Fig. 2.4. The reference (feedforward) speed along the trajectory is saturated to $v_{a t \max }$ and $v_{\text {at zmax }}$; and the cross-track reference speed is saturated to $v_{c t \max }$ and $v_{c t z \max }$. Both speed limits are set so that $v_{\text {at } \max }+v_{\text {ctmax }} \leq v_{x y \max }, v_{\text {at zmax }}+v_{\text {ctzmax }} \leq v_{z \max }$.

- The planned along-track velocity $\left\{v_{a t x c}, v_{a t y c}, v_{a t z c}\right\}$ is lower than the maximum velocity, thus, giving the relative position controller a speed margin to work on.

- The reference change block, which implements Eq. 2.3 ensures that the roll and pitch commands are decoupled from the yaw heading.

- The aerodynamic friction is partially linearized, in the NLF blocks, using the inverse of the identified aerodynamic friction.

- In the PID modules the derivative action is filtered to limit noise amplification and the integral action features anti-windup saturation.

- The variables $\left\{\theta_{f f}, \psi_{f f}\right\}$ are feed-forward commands that are calculated by the state machine using the planned acceleration and Eqs. $2.5 \& 2.6$ without considering the aerodynamic friction. 


\subsubsection{High-Level Controller - State-Machine}

The HL controller is implemented using a Finite State Machine (FSM), Fig. 2.5, which uses the state estimation from the EKF to calculate the mid-level controller references. The FSM has three states corresponding to three control strategies: hover in a desired position, follow a straight segment of the trajectory and turn to head to the next trajectory segment.

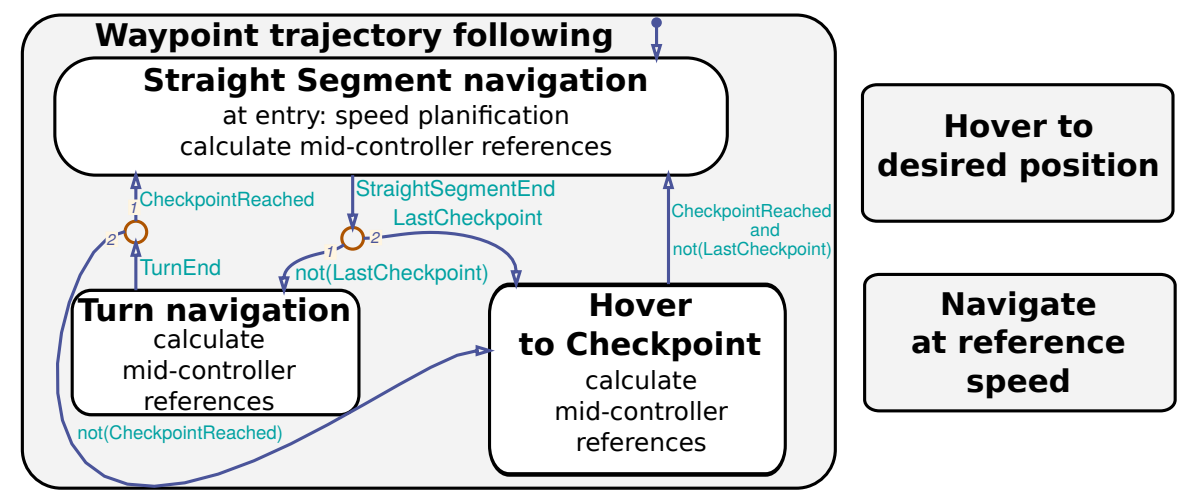

Figure 2.5: High-level control finite state machine. The state machine accepts three flying modes: 'waypoint trajectory following', 'hover to reference position' and 'navigate at reference speed'. The states correspond to navigation control strategies. The state transitions are activated depending on the position of the drone relative to the current trajectory.

The main purpose of the FSM is to navigate along a waypoint trajectory, and therefore its main actions, during trajectory tracking, can be summarized as the repetition of the following steps:

1. Follow a straight segment, accelerating at first, and slowing down before reaching the next turn,

2. Around the mid-waypoints of the trajectory, perform a turn controlling the speed direction to achieve a soft alignment with the next straight segment.

The FSM has also two additional operation modes where the quadrotor is controlled either only in position control, or in speed control. These can be used by the task scheduler to perform certain tasks. During trajectory tracking, every time the controller output is calculated the FSM calculates the planned speed at the current position $v_{\text {plan }}(s)$, where $s$ is a coordinate along the trajectory:

1. The speed, $\left\{V_{\text {turni }}\right\}$, inner turn angle, $\left\{\alpha_{i}\right\}$, and radius, $\left\{R_{\text {turn } i}\right\}$, at checkpoint $i$ during each turn are precalculated each time the reference trajectory is changed, for instance when a waypoint is added. The radius is calculated so that the trajectory passes at a distance $R_{\text {conf }} / 2$ from the checkpoint, where $R_{\text {conf }}$ is the maximum distance at which the checkpoint is considered to be reached. The planned speed for each turn is selected either according to Eq. 2.9 either considering that the turn requires a stall-turn or turn-around maneuver, $v_{\text {stall turn }}$, which corresponds to a planned speed near zero.

2. The algorithm explained in Sec. 2.3.5 is used to calculate the planned speed, $v_{\text {plan }}(s)$, at the current position.

The middle-level (ML) controller, see Fig. 2.2 receives position, $\left\{x_{c}, y_{c}, z_{c}\right\}$, yaw heading, $\psi_{c}$, speed, $\left\{v_{a t x c}, v_{a t y c}, v_{a t z c}\right\}$, and tilt feed-forward, $\left\{\boldsymbol{\theta}_{f f}, \psi_{f f}\right\}$, commands from the FSM, as shown in Fig. 2.4. The presented controller allows to use a single mid-level architecture for the three control modes: trajectory, position and speed modes. These references to the ML controller are calculated by the FSM in such a way that the relative position and speed commands are orthogonal:

- The position references $\left[x_{c}, y_{c}, z_{c}\right]$ are caltulated projecting the estimated position $\left[x_{e s t}, y_{e s t}, z_{e s t}\right]$ onto the reference trajectory.

- The along-track speed commands, $\left[v_{a t x c}, v_{a t y c}, v_{a t z c}\right]$, are parallel to the trajectory $v_{\text {plan }}(s) \vec{u}_{\text {tangent }}$. 
- The along-track speed commands, $\left[v_{a t x c}, v_{a t y c}, v_{a t z c}\right]$, are derivated and smoothed using a low-pass filter to obtain an acceleration command based on Eqs. 2.5 \& 2.6, so that the feed-forward attitude references, $\left\{\boldsymbol{\theta}_{f f}, \phi_{f f}\right\}$, are calculated as follows:

$$
\left\{\theta_{f f}, \phi_{f f}\right\}=\left\{-\operatorname{asin}\left(\frac{\ddot{x}_{m}}{g}\right), \operatorname{asin}\left(\frac{\ddot{y}_{m}}{g}\right)\right\}
$$

\subsubsection{Speed Planner Algorithm}

The planned speed depends on the current speed and position of the multirotor, and on the previous and subsequent trajectory waypoints. Similarly to Hoffman et al. (Hoffmann, Gabriel M et al.,, 2008), the speed planner uses a uniform acceleration motion model to calculate both: the acceleration profile from the previous trajectory waypoints, and the deceleration profile to perform the following turns succesfully. But, in addition to this model, the speed loop response is also modeled by taking into account its response time.

The algorithm calculates the following distances for each of the neighbouring waypoints:

1. $\Delta d_{c h k i}$, the distance to waypoint $i$ from the current estimated quadrotor position.

2. $\Delta d_{\text {turn } i}=\left(R_{\text {turn } i}+\frac{R_{\text {conf }}}{2}\right) \sin \left(\alpha_{i} / 2\right)$, the distance to waypoint $i$ when the turn is to be started; where $R_{\text {conf }}$ is the waypoint maximum clearance distance, $\left\{R_{\text {turni }}\right\}$ is the planned turn radius at waypoint $i$ and $\left\{\alpha_{i}\right\}$ is the inner turn angle at waypoint $i$.

3. $\Delta d_{t r v i}=t_{r v \max } \sqrt{v_{x e s t}^{2}+v_{y e s t}^{2}}$, the distance required by the speed loop to slow down, where $\left\{v_{\text {xest }}, v_{\text {yest }}\right\}$ is the current estimated velocity and $t_{\text {rvmax }}$ is the speed loop response time.

The optimal speed, $v_{\text {plani }}$, for the neighboring checkpoints is calculated. For each waypoint $i, v_{p_{\text {plan }}}$ depends on the sign of $\Delta d$, with $\Delta d=\left(\Delta d_{\text {chki }}-\Delta d_{t u r n i}-\Delta d_{t r v i}\right)$. If $\Delta d \geq 0$ then $v_{\text {plan }_{i}}=\sqrt{V_{\text {turni }}^{2}+2 a_{\text {xymax }} \Delta d}$, else if $\Delta d<0$ then $v_{\text {plan }}=V_{\text {turni }}$. Where $\left\{V_{\text {turn }}\right\}$ is the planned speed at turn $i$.

The planned speed at the current position, $v_{\text {plan }}(s)$, is calculated as the minimum of the precaltulated optimal speeds $v_{\text {plan }}(s)=\arg _{\min }\left(v_{\text {plan }}\right)$. The coordinate $s$ denotes the position of the quadrotor along the trajectory, and the expression $v_{\text {plan }}(s)$ highlights that the planned speed depends only on $s$ and the set of configurable parameters listed in Sec.2.3.6.

\subsubsection{Configurable Parameters Set}

From the prior explanation, the set of configurable parameters of the proposed controller architecture is:

- $\left\{v_{a t \max }, v_{c t \max }, v_{a t z \max }, v_{c t z \max }\right\}$, are the saturation speed limits used inside the middle level controller that are imposed by either the kinematic capabilities of the vehicle, or the measurement range of the onboard sensors or the precision requirements of the task at hand.

- $\left\{R_{\text {conf }}, v_{\text {stallturn }}, t_{r v \max }, a_{x y \max }\right\}$, are the parameters that during trajectory tracking are used to determine the planned speed at each turn, $\left\{V_{t u r n i}\right\}$, and to calculate the planned speed, $v_{\text {plan }}(s)$, at every controller step iteration.

\subsection{Experimental Results}

Several experimental results obtained using the presented control architecture are detailed in this section. The experiments and flying platform are the following:

- Videos of many experiments performed with the proposed controller are listed in Sec.2.4.1

- Indoor experiments, Sec. 2.4.2 four flights performed using an Asctec Pelican quadrotor, equipped as described in Appx. H.1.1 This experiment was published in (Pestana, Mellado-Bataller, Fu, et al., 2013). 
- Outdoor experiments, Sec. 2.4.3 three flights performed using an AR Drone 2.0 quadrotor, described in Appx. H.2 This experiment was published in (Pestana, Mellado-Bataller, SanchezLopez, et al., 2014).

- Indoor experiments in a replica of the IMAV 2013 competition environment, Sec. 2.4.4. one flight with three AR Drone 2.0 quadrotors flying simultaneously in the same area. This experiment was published in (Pestana, Sanchez-Lopez, et al., 2015).

\subsubsection{Videos of Experiments}

Several playlists are uploaded in YouTube related to experiments and flights by the CVAR/CVG (UPM) that have been performed using the proposed controller. The main ones are related to the participation of our research group in international MAV competitions, for details on this events with their corresponding achieved results the reader is refered to Appx. I. Therefore the following list is given:

- International Micro Air Vehicle Conference and Flight Competition 2016 (IMAV 2016), video playlist: https://goo.gl/tdZyGu.

- Asia Venue of the AUVSI International Aerial Robotics Competition 2014 (IARC 2014), video playlist: https://goo.gl/bEvK3F.

- International Micro Air Vehicle Conference and Flight Competition 2013 (IMAV 2013), video playlist: https://goo.gl/KosWKH.

- International Micro Air Vehicle Conference and Flight Competition 2012, (IMAV 2012), video playlist: https://goo.gl/PRns2D.

\subsubsection{Experimental Results - Indoors Flight}

This section describes four separate experimental flights that show the navigation capabilities of the presented controller architecture. These flights were performed using an Asctec Pelican quadrotor, equipped as described in Sec. H.1.1. These flights were originally performed as part of the publication (Pestana, Mellado-Bataller, Fu, et al., 2013). All the flights are trajectory tracking tests in autonomous navigation carried out in an indoor espacious room, simulating a GPS-denied situation. Thus, the GPS signal is not used in the estimation algorithms. One of the tests consists on the Pelican following a trajectory in a vertical $X Z$ plane, see Fig. 2.6. There is a second test performed by this quadrotor following a square horizontal trajectory shown on Fig. 2.7. The last two tests were done using the AR Drone following the horizontal eight-shaped trajectory shown in Figs. 2.8 \& 2.9. A summary of the controller performances in these tests is shown in Table 2.1 .

The Pelican was configured with the following parameters values. $\left\{v_{a t \max }, v_{c t \max }, v_{\text {at zmax }}, v_{c t z \max }\right\}$ where fixed to $0.5 \mathrm{~m} / \mathrm{s}$. and the horizontal speed was constrained with an additional saturation to $0.70 \mathrm{~m} / \mathrm{s}$. This limitation ensured that the speed reference for the speed control loop was inside the reliable measurement range of our optical flow implementation. The FSM parameters were: $R_{\text {conf }}=0.30 \mathrm{~m}$, $v_{\text {stall turn }}=0.30 \mathrm{~m} / \mathrm{s}, t_{\text {rvmax }}$ was set to $0 \mathrm{~s}$ because the planned speed was always lower than $0.7 \mathrm{~m} / \mathrm{s}$ (this parameter does not give an advantage in this situation), and the maximum acceleration $a_{x y \max }$ was set to $0.5 \mathrm{~m} / \mathrm{s}^{2}$.

The vertical flight of the Pelican on the $X Z$ plane shown in Fig. 2.6 consisted on two laps. The mean and maximum tracking errors during the test were $0.15 \mathrm{~m}$ and $0.38 \mathrm{~m}$ correspondingly. The vehicle navigated at a mean and maximum speeds of $0.42 \mathrm{~m} / \mathrm{s}$ and $0.7 \mathrm{~m} / \mathrm{s}$. During half of the flight the vehicle was commanded to change its yaw heading, without affecting the trajectory tracking objectives.

The horizontal flight of the Pelican shown in Fig. 2.7 was carried out setting a constant yaw heading reference so that the results could be compared with those of the previous test, shown on Fig. 2.6. The mean and maximum tracking errors during the test were $0.09 \mathrm{~m}$ and $0.27 \mathrm{~m}$ correspondingly. The Pelican navigated at a mean and maximum speeds were $0.51 \mathrm{~m} / \mathrm{s}$ and $0.60 \mathrm{~m} / \mathrm{s}$. The trajectory controller features similar performances on both types of trajectory. 


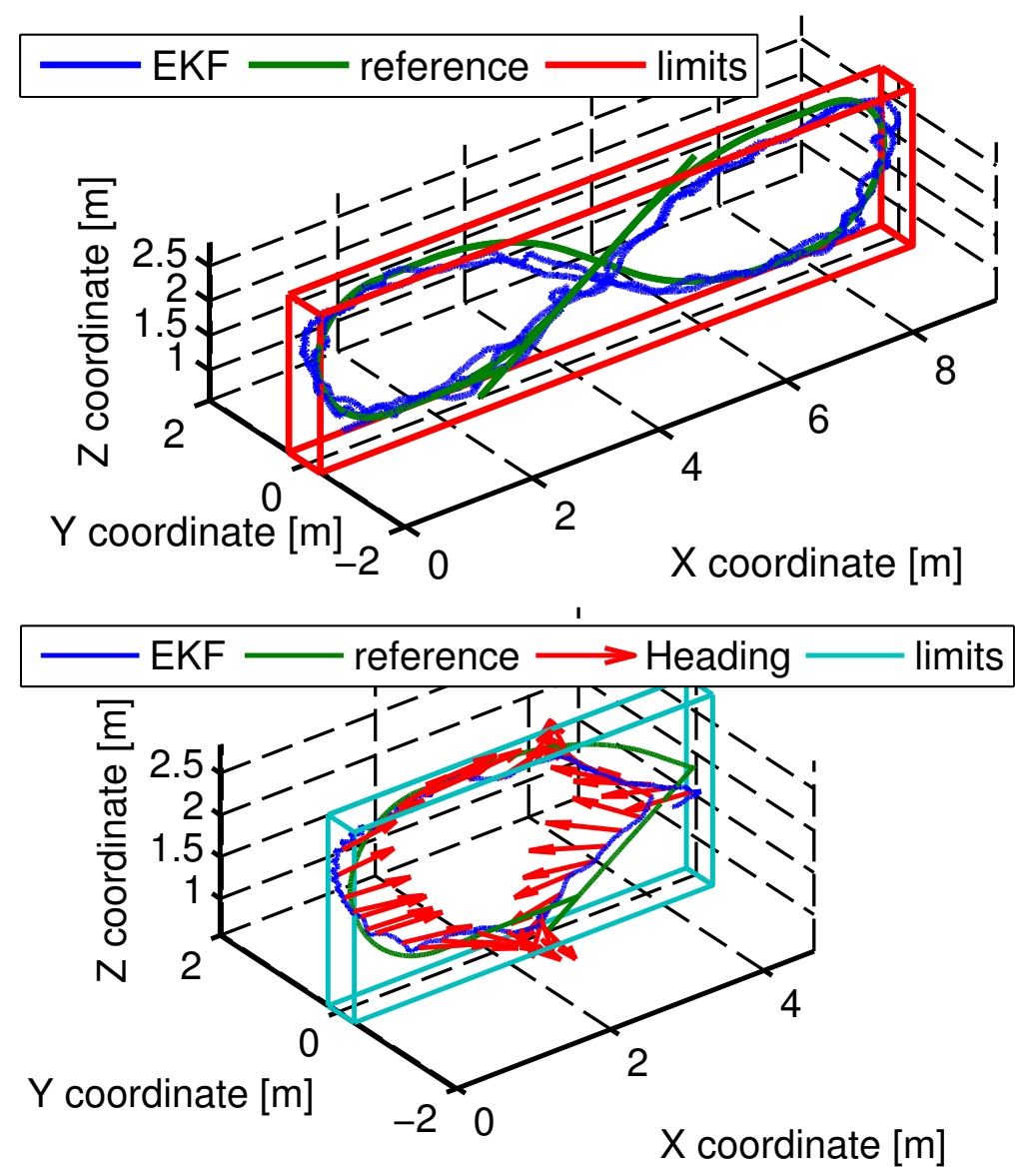

Figure 2.6: Asctec Pelican test on a vertical eight-shape trajectory, where two laps were completed. The graph above shows the trajectory in the XYZ space, and the bounding box graph ('limits' on the legend) shows that the $Y$ error was kept low. A video of the test is available on http://vision4uav.com/?q=node/338 . During the second lap the yaw reference was changed repeatedly without affecting the trajectory tracking.

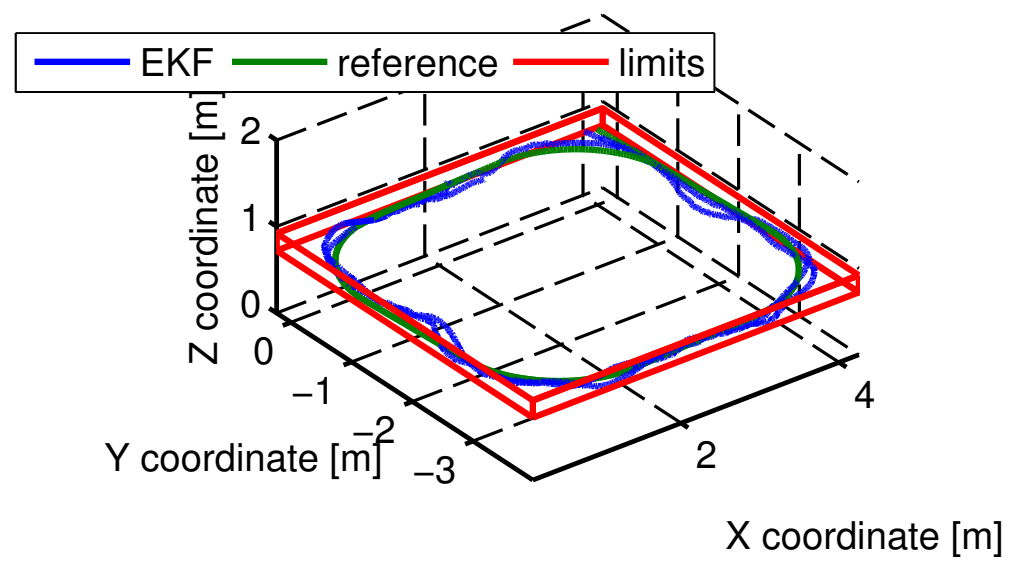

Figure 2.7: Asctec Pelican test on horizontal square trajectory. The quadrotor performed two turns carrying out the trajectory tracking task. The altitude hold worked successfully during this test, and the yaw heading reference was kept constant. 

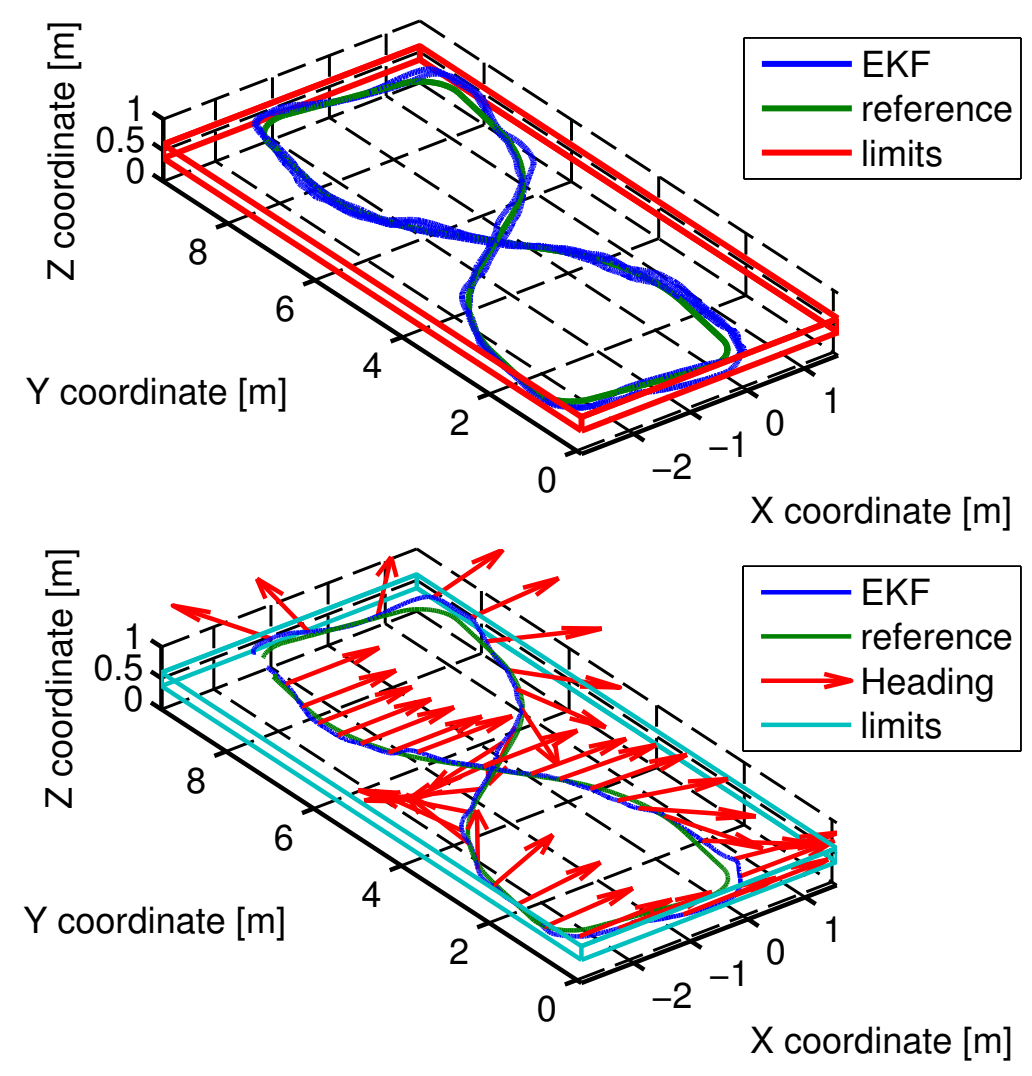

Figure 2.8: AR Drone following a horizontal eight-shape trajectory. The first graph shows the whole flight consisting of two and a half laps. In the second graph only the lap where yaw heading reference was changed is shown. This rotation does not affect much the trajectory tracking, however, small oscillations around the trajectory reference can be observed. A video of the test is available in http://vision4uav.com/?q=node/338 .

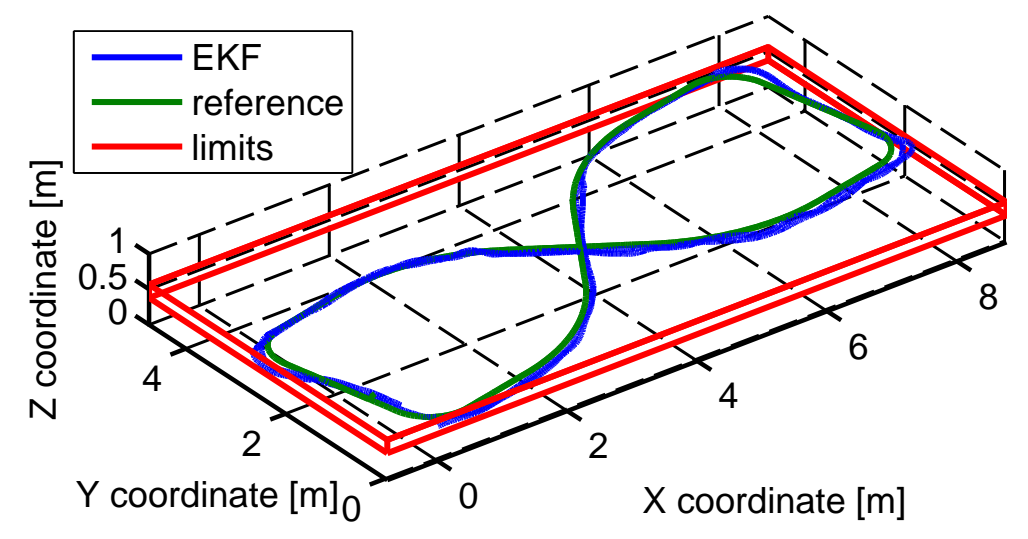

Figure 2.9: AR Drone following a horizontal eight-shape trajectory, the yaw heading is kept constant. The quadrotor performs the tracking task smoothly. The maximum speed is reached on the middle of the eight-shape. 
The AR Drone was configured with the following parameters values. The maximum horizontal speed was set to $2 \mathrm{~m} / \mathrm{s}$, where $v_{\text {at } \max }=1.4 \mathrm{~m} / \mathrm{s}, v_{\text {ct } \max }=0.8 \mathrm{~m} / \mathrm{s}$. The FSM parameters were set to $R_{\text {conf }}=0.50 \mathrm{~m}, v_{\text {stall turn }}=0.25 \mathrm{~m} / \mathrm{s}, t_{\text {rvmax }}=1.7 \mathrm{~s}$, and the maximum acceleration to $a_{x y \max }=0.4 \mathrm{~m} / \mathrm{s}^{2}$. The configuration parameters were selected to work on trajectories made up of short segments, with the objective of optimizing speed while keeping low tracking error.

\begin{tabular}{c|cc|cc} 
Fig. & (Pel.) 2.6 & (Pel.) 2.7 & (ARD.) 2.8 & (ARD.) 2.9 \\
\hline$d_{\text {mean }}[\mathrm{m}]$ & 0.15 & 0.09 & 0.10 & 0.09 \\
$d_{\text {max }}[\mathrm{m}]$ & 0.38 & 0.27 & 0.29 & 0.28 \\
\hline$v_{\text {mean }}[\mathrm{m} / \mathrm{s}]$ & 0.42 & 0.51 & 0.61 & 0.61 \\
$v_{\text {max }}[\mathrm{m} / \mathrm{s}]$ & 0.70 & 0.60 & 1.10 & 1.10
\end{tabular}

Table 2.1: Summary of results of the experimental tests: average, $d_{\text {mean }}$, and maximum, $d_{\text {max }}$, tracking error; average, $v_{\text {mean }}$, and maximum, $v_{\max }$, speed during the test

Both flights shown in Figs. 2.8 \& 2.9, were performed following the same eight-shape trajectory to check whether the trajectory control actions are decoupled from the yaw reference. The obtained results were a mean and maximum tracking errors of $0.09 \mathrm{~m}$ and $0.28 \mathrm{~m}$ correspondingly. The vehicle navigated at a mean and maximum speeds of $0.61 \mathrm{~m} / \mathrm{s}$ and $1.10 \mathrm{~m} / \mathrm{s}$. The similarity between both tests demonstrates that the yaw heading is approximately decoupled from the trajectory tracking.

The flights of both quadrotors were mostly unaffected during yaw heading rotation. In the flights exposed in this section the yaw rotation speed was about $25-30 \%$. The experimental data clarify, however, that both vehicles tend to oscillate around the reference trajectory during the yaw rotation, a comparison between Figs. $2.8 \& 2.9$ shows this fact. High yaw rotation speeds do affect the position control, but this is not an interesting action for many applications.

\subsubsection{Experimental Results - Outdoors Flight}

This section describes three separate experimental flights that show the navigation capabilities of the presented controller architecture. These flights were performed using an AR Drone 2.0 quadrotor. All the flights are trajectory tracking tests in autonomous navigation carried out in an outdoors environment, simulating a GPS-denied situation. Thus, GPS was unavailable and unused during the tests. All the flights were performed with the AR Drone 2.0 with the outdoors hull. These flights were originally performed as part of the publication (Pestana, Mellado-Bataller, Sanchez-Lopez, et al.,2014). The flight trajectories are shown in Figs. 2.10 \& 2.11 \& 2.12. The system has been tested following regular polygon trajectories on the XY plane, with varying altitude on each waypoint, of values of 0-3 m higher than the first checkpoint's altitude. A summary of the controller performances in these tests is shown in Table 2.2

\begin{tabular}{c||c|c||c|c||c|c}
$\begin{array}{c}\text { Parameter } \\
\text { units }\end{array}$ & $\begin{array}{c}d_{\text {mean }} \\
{[\mathrm{m}]}\end{array}$ & $\begin{array}{c}d_{\text {max }} \\
{[\mathrm{m}]}\end{array}$ & $\begin{array}{c}v_{\text {mean }} \\
{[\mathrm{m} / \mathrm{s}]}\end{array}$ & $\begin{array}{c}v_{\text {max }} \\
{[\mathrm{m} / \mathrm{s}]}\end{array}$ & $\begin{array}{c}\Delta L_{\text {mean }} \\
{[\mathrm{m}]}\end{array}$ & $\begin{array}{c}\Delta t_{\text {test }} \\
{[\mathrm{s}]}\end{array}$ \\
\hline Square, Fig. 2.10 & 0.17 & 0.70 & 0.92 & 1.90 & 7.72 & 123.0 \\
Hexagon, Fig. 2.11 & 0.31 & 1.71 & 1.09 & 1.95 & 7.75 & 123.0 \\
Octagon, Fig. 2.12 & 0.32 & 2.90 & 1.17 & 2.10 & 7.75 & 95.0
\end{tabular}

Table 2.2: Summary of results of the experimental tests obtained for the trajectories shown in Figs. $2.10 \& 2.11 \& 2.12$. The specified parameters are: average, $d_{\text {mean }}$, and maximum, $d_{\text {max }}$, tracking error; average, $v_{\text {mean }}$, and maximum, $v_{\text {max }}$, speed along-trajectory; $\Delta L_{\text {mean }}$, mean distance between waypoints and $\Delta t_{\text {test }}$, duration of the test. The tested trajectories and poligons on the XY plane, with varying values of altitude on each waypoint. The tracking error is calculated from the odometry based position estimates. The mean and maximum speed are calculated taking into account the component along the trajectory, where the number of laps on each test was $2-3$ laps.

The AR Drone 2.0 was configured with the following parameters values. The maximum horizontal speed was set to $2.75 \mathrm{~m} / \mathrm{s}$, where $v_{\text {at } \max }=2.0 \mathrm{~m} / \mathrm{s}, v_{\text {ctmax }}=0.75 \mathrm{~m} / \mathrm{s}$. The FSM parameters were set to $R_{\text {conf }}=0.25 \mathrm{~m}, v_{\text {stall turn }}=0.25 \mathrm{~m} / \mathrm{s}, t_{\text {rvmax }}=1.20 \mathrm{~s}$, and the maximum acceleration to $a_{x y \max }=0.70 \mathrm{~m} / \mathrm{s}^{2}$. 
The configuration parameters were selected to obtain high speeds on the straight segments, with the objective of studying the controller performance on this configuration on outdoors environments.

Each experiment is shown in one figure: the first experiment consisted of a pseudo-square trajectory shown on Fig. 2.10, the second one was a pseudo-hexagon shown on Fig. 2.11, and the last one was a pseudo octagon shown on Fig.2.12. As shown the drone follows a trajectory made out of straight segments and circle segments around the corners. The first graphs $\{(\mathrm{a})$, (c) and (e) $\}$ show only the trajectory reference and the estimated position, and the second ones $\{(\mathrm{b})$, (d) and (f) $\}$ show also the yaw heading during one of the laps. As can be appreciated from the graphs; the yaw control can be handled separately from the position control.

The trajectory tracking position error is measured against the odometry based position estimation. As expected this estimation is not accurate, and accumulates error over time. A ground truth to calculate an accurate tracking error is difficult to obtain without a motion capture system, which is the reason why it is not calculated. The drift depends on many circumstances that affect the quality of the optical flow speed estimation: texture of the floor, size of identifiable features in floor texture, illumination, altitude of the flight, yaw rotation speed, etc. To keep a good quality on the position measurements the yaw rotation speed commands are saturated at $50 \%$, and are usually below $30 \%$. The flights were mostly anaffected during yaw heading rotation. In the flights exposed in this section the yaw rotation speed attained values of about $30-50^{\circ} / \mathrm{s}$. The experimental data clarify, however, that the vehicle tends to oscillate around the reference trajectory during the yaw rotation. High yaw rotation speeds do affect the position control, but $50 \%$ is an acceptable value for many applications.

The altitude on each experiment was $2.5-5.5 \mathrm{~m}, 5.5-8.5 \mathrm{~m}$ and $3.0-6.0 \mathrm{~m}$ respectively. The AR Drone 2.0 can measure altitude precisely of up to $6 \mathrm{~m}$ (Bristeau et al., 2011) using the ultrasound sensor. For higher altitudes the drone has to switch to using the pressure sensor. In practice, the drone telemetry will show a good tracking on the altitude coordinate, while on reality the vehicle is correcting the altitude internally moving upwards or downwards. This was specially appreciable during some of the experiments, but is not visible on the plots because the sensor reading is misleading.

The selected tests show how our architecture is able to deal with moderate winds and how it takes into account automatically the curve inner angles of the trajectory at each waypoint. The trajectories have a varying number of waypoints to provide a varying inside angle of the trajectory at their waypoints. Since they are all regular polygons on the XY plane, then the higher the number of waypoints the bigger the inner angles of the trajectory will be, allowing for faster passes through them. This is appreciated by the mean speed during trajectory tracking, see $v_{\text {mean }}$ in Table 2.1. The other factor that affects the maximum and mean speeds, $v_{\max }$ and $v_{\text {mean }}$ respectively, is the distance between waypoints; but all the trajectories where performed with similar distances. Longer distances between waypoints allow the multirotor to accelerate to and decelerate from higher speeds.

In some occasions, the drone drifts in the curves of the trajectory. But this behavior is not deterministic and the same error is not committed in every corner and is not even repeated on the next lap. However, it could be avoided lowering the $a_{x y \max }$ and $t_{\text {rvmax }}$ to more restrictive values. It would also be convenient to have separate values of acceleration for the curves and the straight lines, as the controller architecture does not perform equally well on straight trajectory segments than in the curves. Then the solutions are improving the controller, or adding parameters to the speed planning algorithm to take into account this issue. Another occasional behavior is that the odometry error may increase substantially and the drone might perform a 20-50\% bigger trajectory than commanded, due to odometry estimation errors; or subsequently accumulate a constant drift per lap on a certain direction. This behavior might be triggered by the AR Drone's altitude sensor switching solution, but we do not have experimental evidence to ascertain the source of this occasional problem. 


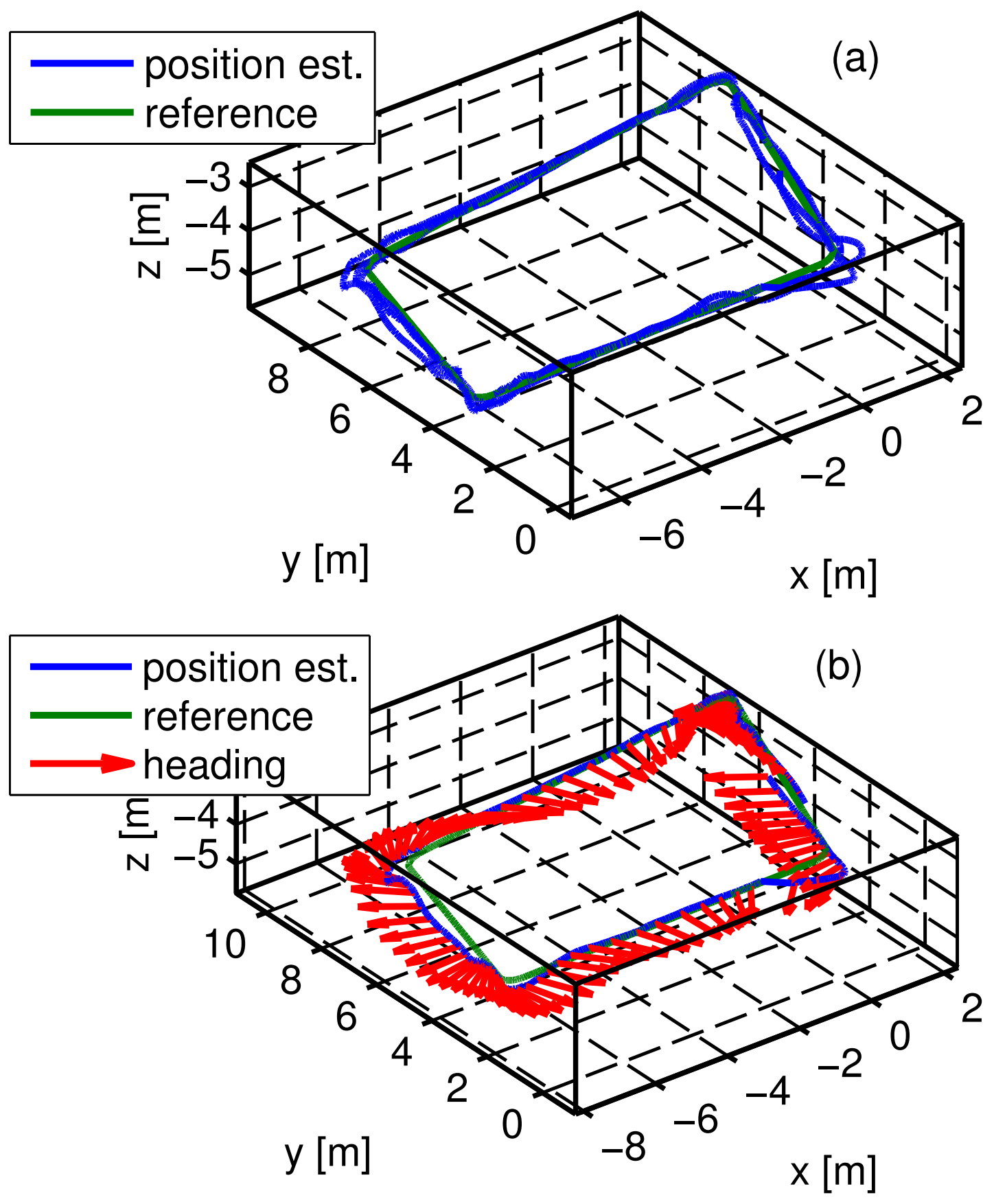

Figure 2.10: Pseudo-square trajectory following experimental test on an outdoors environment performed using our achitecture and an AR Drone 2.0. The (a) graph shows the referece and followed trajectory in the XYZ space, and the (b) graph additionally shows the vehicle heading during the second lap of the experiment were the yaw controller interaction with the trajectory following task was tested. The trajectory is a square on the XY plane with varying values of altitude, on the 2.5-5.5 m range, at each checkpoint. The test consisted on three laps, where the yaw heading control was tested on the second lap, which is plotted on graph (b). Some performance parameters obtained from this test are shown in Table 2.1 

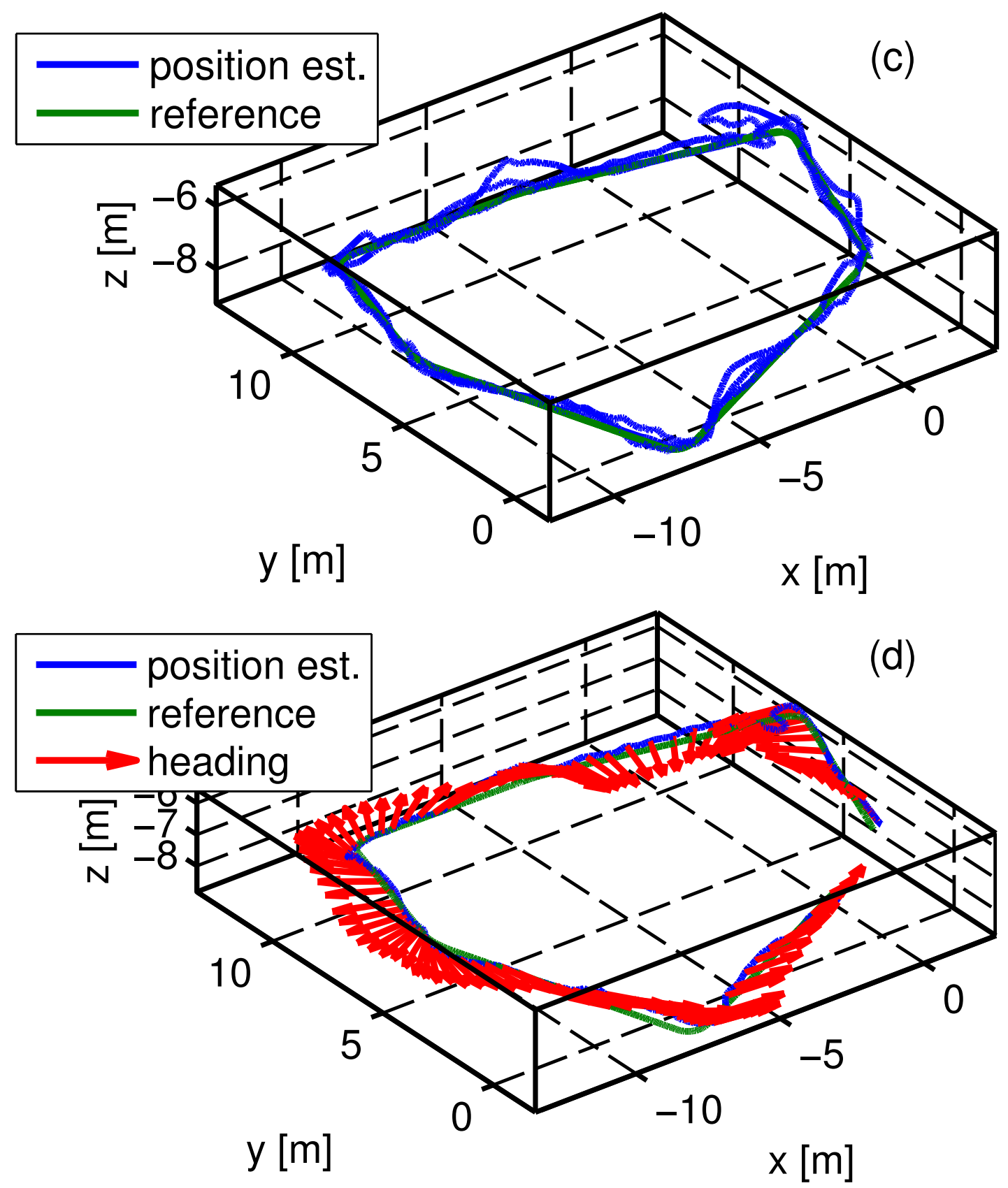

Figure 2.11: Pseudo-hexagon trajectory following experimental test on an outdoors environment performed using our achitecture and an AR Drone 2.0. The (c) graph shows the referece and followed trajectory in the XYZ space, and the (d) graph additionally shows the vehicle heading during the second lap of the experiment were the yaw controller interaction with the trajectory following task was tested. The trajectory is an hexagon on the XY plane with varying values of altitude, on the 5.5-8.5 m range, at each checkpoint. The test consisted on three laps, where the yaw heading control was tested on the second lap, which is plotted on graph (d). Some performance parameters obtained from this test are shown in Table 2.1. 


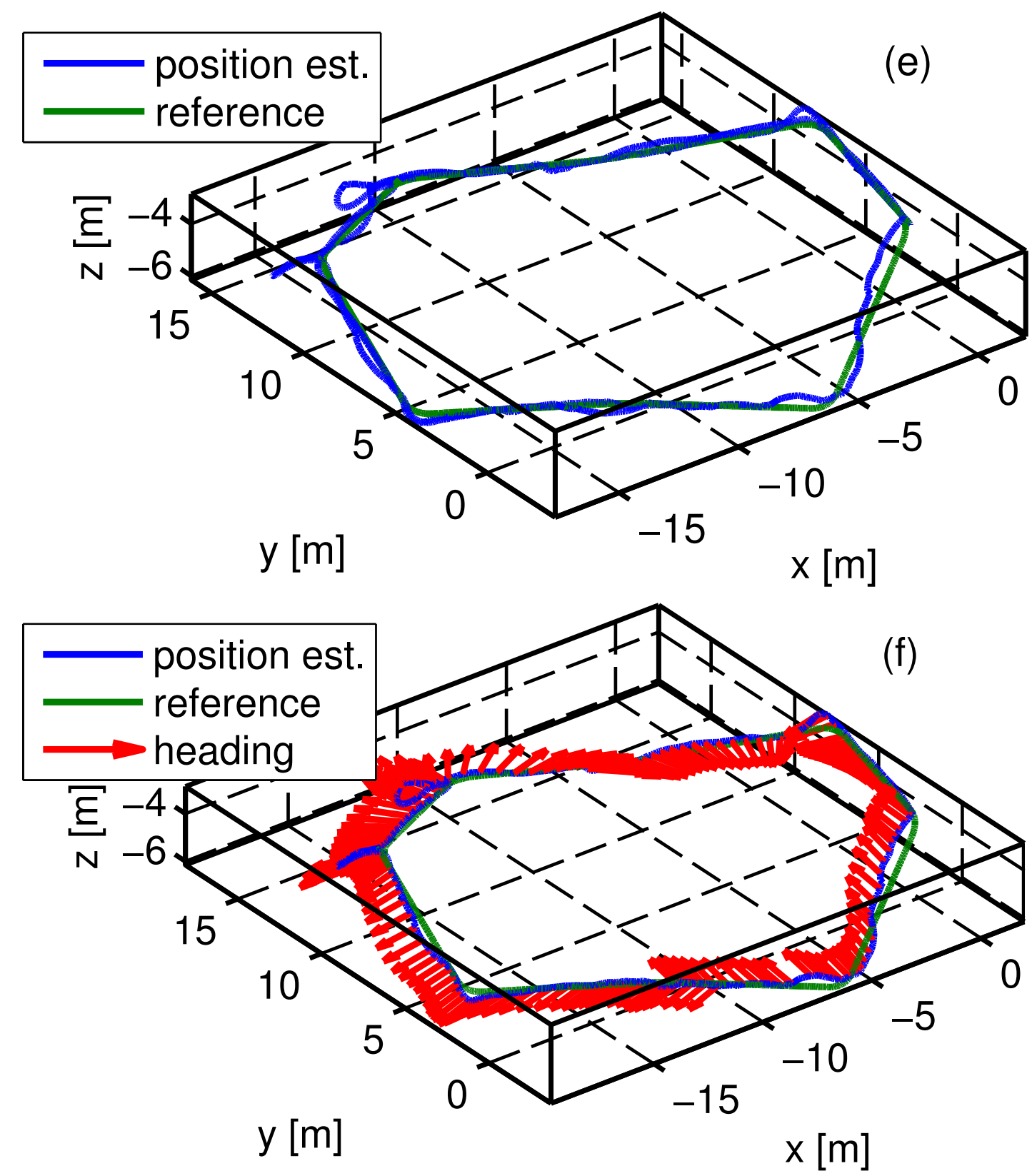

Figure 2.12: Pseudo-octagon trajectory following experimental test on an outdoors environment performed using our achitecture and an AR Drone 2.0. The (e) graph shows the referece and followed trajectory in the XYZ space, and the (f) graph additionally shows the vehicle heading during the second lap of the experiment were the yaw controller interaction with the trajectory following task was tested. The trajectory is an octagon on the XY plane with varying values of altitude, on the 3.0-6.0 m range, at each checkpoint. The test consisted on two laps, where the yaw heading control was tested on the second lap, which is plotted on graph (f). The test was interrupted at the end of the second lap due to poor WiFi performance. Some performance parameters obtained from this test are shown in Table 2.1 . 


\subsubsection{Experimental Results - Flights in IMAV2013 Replica}

In this section one experimental test with three AR Drone 2.0 quadrotors flying simultaneously is described. This experiment was published in (Pestana, Sanchez-Lopez, et al., 2015). This test shows the performance of our architecture in a replica of the IMAV 2013 competition environment.

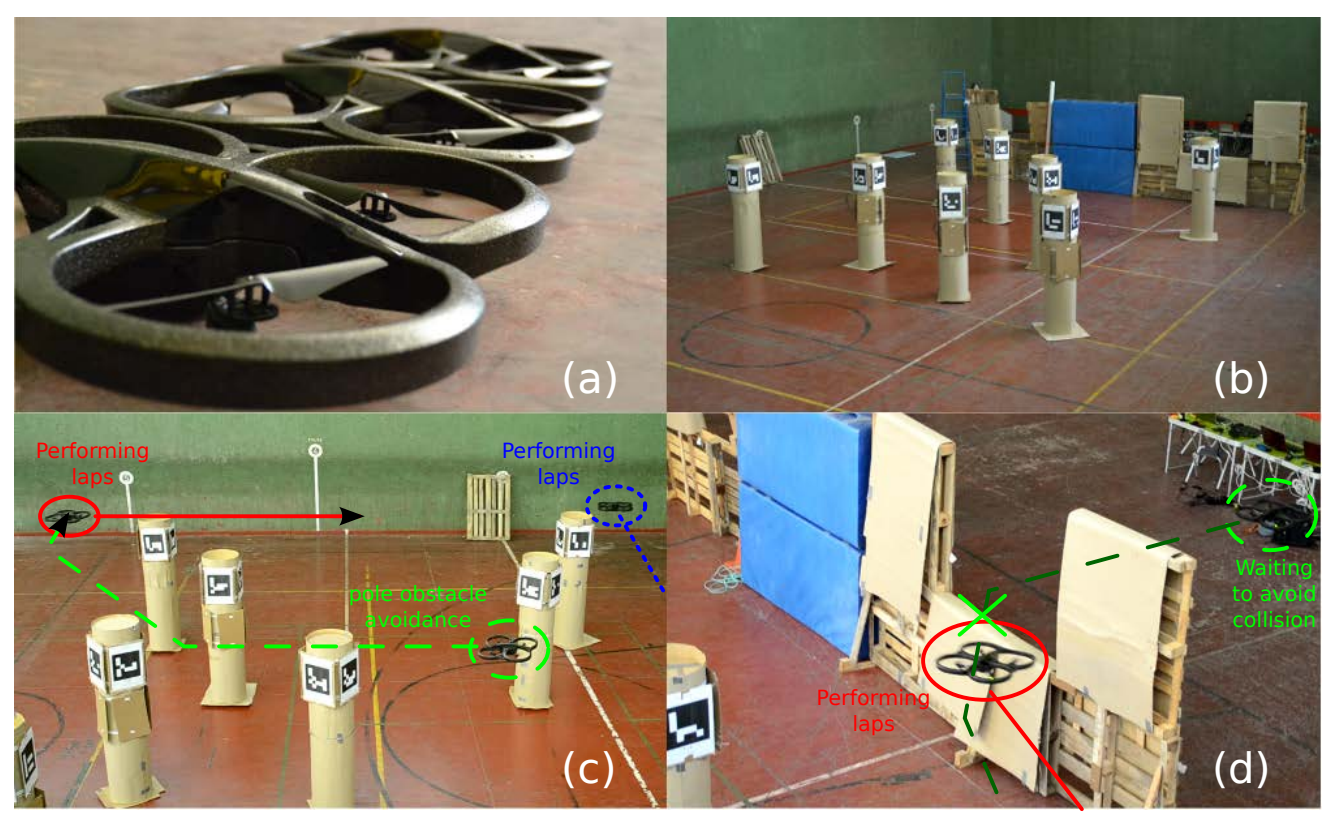

Figure 2.13: A navigation architecture has been designed for AR Drone 2.0 platforms. Figure (a) shows a picture of three of these quadrotors. The experiments are performed in a replica of the IMAV 2013 Indoor Challenge environment, shown in figure (b). This environment consists of a wall with two windows and eight poles. The position of the wall is previously known but not the location of the windows along it. The wall and the poles represent obstacles that must be avoided by the drones while flying. Only the position of the four corner poles is previously known. All the poles and the windows are stamped using ArUco visual markers (Garrido-Jurado et al., 2014), see (b) and (c). Figure (c) shows an experimental flight at the moment when the quadrotors are crossing the unknown poles area. The unknown poles are robustly located in previous laps, when the drone performs laps around the known poles, ensuring a good estimation of their positions. The collision avoidance with other agents is solved at the trajectory planning step. For instance in (d), a drone has to wait until the path to cross the window is free. The flight shown in (c) is described and analyzed in Sec. 2.4.4.3. Videos of this and other flights are available at the website http://www.vision4uav.com/?q=node/386. 


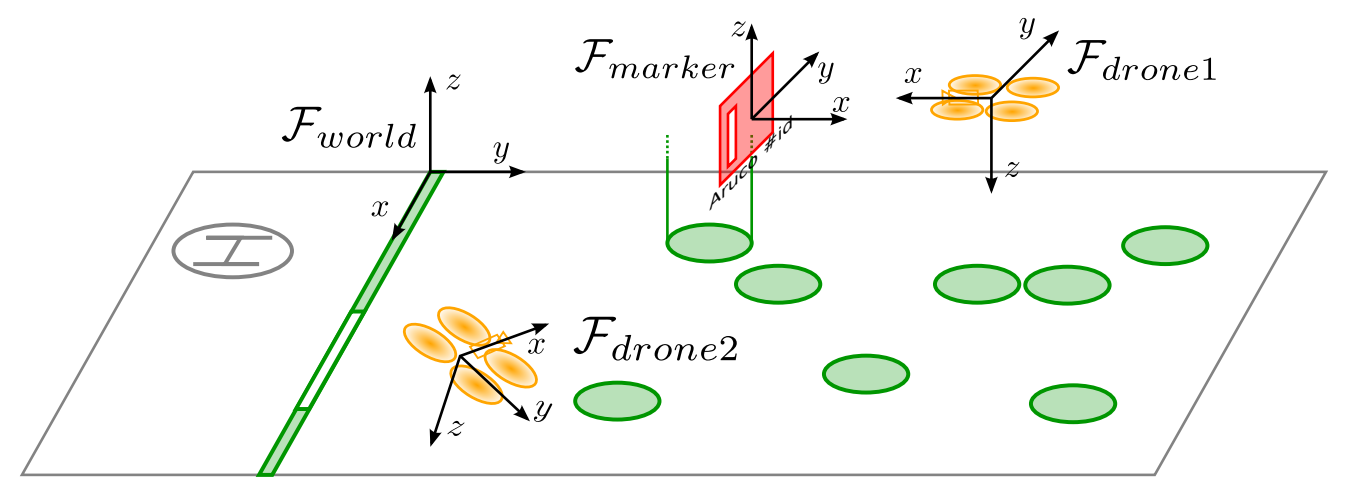

Figure 2.14: Relevant coordinate frames of our proposed solution. The figure shows a simplified depiction of the IMAV2013 environment: the initial take-off position is shown in gray; the static obstacles, the wall and columns, in green; two drones in green and a marker in red. The world coordinate frame, $\mathscr{F}_{\text {world }}$, is located in one of the corners of the wall. The frames of the drones, $\mathscr{F}$ drone 1 and $\mathscr{F}$ drone 2 ; and the marker, $\mathscr{F}_{\text {marker }}$, are also shown. Only the position of the windows and the four inner columns are not known.

\subsubsection{Multi-Agent-Level Mission Coordination}

For the realization of a multi-robot flight in the IMAV2013 competition, a trajectory planner was utilized. Visual markers were positioned in the flying field, so that all obstacles were tagged, and the drones could periodically locate themselves in global coordinates against them. The position in global coordinates was known only for part of the visual markers. The trajectory planner module had a map of the competition environment which was precise except for the position of the windows and part of the obstacles. The map is shown in Fig. 2.14. The trajectory planner also knew the position of all flying drones. Therefore this module had the capability to deliver obstacle-free trajectories during the flight. The drones performed only navigation related missions. The overall expected behavior of the agents during mission execution is:

- Each agent follows a sequence of waypoints given by its mission specification. The mission scheduler executes it sequentially.

- During execution, the trajectory planner will attempt to find trajectories that are collision-free. If it fails, the quadrotor will be controlled to stay in the current position.

- The other drones are considered obstacles only when they are inside a 4 m radius from the agents' position. When other drones are nearby, the expected behavior is the following:

- If other robotic agents enter the current trajectory, the trajectory planner will detect it and it will command the trajectory controller, and thus also the quadrotor, to stay in its current position while attempting to find a collision-free path to the current waypoint.

- If another robotic agent is on or enters the current waypoint, then the trajectory planner handles the situation in the same manner. The trajectory controller is commanded to stay in the current position. A new collision-free path will be calculated when the current waypoint is free again.

One interesting characteristic of this multi-robot system is that there is no central mission supervisor. Rather, the synchronization of the drone navigation mission is achieved by the set of rules and conventions specified in the Trajectory Planner and the Trajectory Controller, of which one instance is running on each agent. Arguably, this intelligence does not allow to solve every possible navigation conflict between the robotic agents. However, if the mission is specified correctly unsolvable conflicts will not occur.

The number of drones that can be flown using our architecture is limited by the WiFi bandwidth requirements of the AR Drones. Experimental testing has shown us, that by limiting the quality of the video stream sent by each drone, it is possible to fly up to 9 drones, which share the three available independent WiFi channels in groups of 3. The problem is the requirement of the AR Drone 2.0 for visual-based navigation of sending the imagery to the ground station. Otherwise, using drones with an on-board computer running an instance of our agent-level architecture would allow for higher numbers of drones to fly simultaneously. 


\subsubsection{Mission specification - Flights in IMAV2013 Replica}

The mission specification is identical for all drones flying and corresponds to the following sequence of tasks, see Fig.2.14.

1. Start the agent flight architecture (autopilot interface, controller, state estimators, visual marker detector, trajectory planner, mission scheduler and agent monitor) and take-off,

2. Move in front of the window and then move to one corner of the poles area (thus, crossing the big window). During this task, the quadrotor is controlled to look at the poles area perpendicularly to the wall, which results in a constant yaw reference,

3. Perform laps around the poles area for 3 minutes,

4. Cross the poles area moving towards the upper right corner of the map,

5. Navigate to the final scoring location and perform the landing, hovering and landing task specified in the IMAV 2013 rules. During this task, the quadrotor is controlled to look at the poles area perpendicularly to the wall, which results in a constant yaw reference.

The laps and the crossing through the unknown poles area tasks are specified so that all the drones will probably navigate in the same direction. This mission specification requirement minimizes the occurrence of navigation conflicts. During tasks 3 and 4, the quadrotor is controlled to look at a specific corner column. The laps around the poles area are specified by waypoints in the corners and in the middle points of the square lap. The drone switches to looking at the next column in these middle points.

Considering the size of the map, $10 \times 15 \mathrm{~m}$, and the size of the AR Drones, around $52 \times 52 \mathrm{~cm}$, the competition environment gets relatively crowded when more than 5 drones are navigating around the columns' area. In our experiments, the main factor, more important than localization and control inaccuracies, that affects the selected safety distance between quadrotors is the necessity to avoid interference among the ultrasound altimeters of the AR Drones. Additionally to the altimeter problems, in real flights, WiFi bandwidth limitations may also limit the maximum number of drones to around six, and in our experience maximally nine, drones flying simultaneously.

\subsubsection{Experimental Results - Flights in IMAV2013 Replica}

The mission specification was described in Sec.2.4.4.1. Subfig. 2.13(c) shows the drones attempting to cross the unknown pole area during this flight. And the figures 2.15 and 2.16 summarize the experimental flight. In these figures, the obstacles, the wall with two windows and the eight poles, are shown in black. The trajectories executed by the drones, as estimated by the localization module, are shown with lines. The agents know the position of the four corner poles and the wall, and the size of the windows; but need to estimate the position of the four unknown poles and the windows.

A 2D overview plot of the flight is shown in Fig. 2.15 As shown in the videos, the quadrotors are commanded to look at specific known poles to control the odometry position error. At the middle of each side the agents are commanded to look at the next corner pole. At this moment the position estimate might change. These events increase the localization error in specific points of the mission execution and trigger the planning of new trajectories. In this flight they occur repeatedly in the points $[-0.5,7.75]$ and $[5.5,10.5]$, see Fig. 2.15

The explained emergent synchronized behavior of the drones is shown in Fig. 2.16 The mission is specified to minimize navigation conflicts and maximize the final achieved competition score. For example all the agents perform laps in the same direction.

In Fig. 2.16 the current estimated position and orientation of each quadrotor is shown with a marker and an arrow, the last 30 seconds of the executed trajectory are shown by a line and the currently planned trajectory is shown by a segmented line with markers. Subfigs. 2.16(a)-(d) show the startup sequence, where the drones cross the big window and the automatic synchronization is achieved. Subfigs. 2.16(e)(f) show the final steps of the mission execution, where the drones cross the unknown pole area and land in the final position. More details of the execution of this mission can be read in the caption of the experiment figures 2.15 and 2.16

Additionally, Fig. 2.17] shows, for drone1 only, plots of the drift-free (green) and the odometry-based (blue) estimates. The known environment elements are shown in black and the progressively mapped 
unknown elements are shown in red. This figure shows how the Localization module is able to calculate a drift-free pose from the odometry-based estimate.

The performance in terms of trajectory following capabilities, and tracking error of each drone are shown in Figs. 2.18, 2.19 and 2.20 In each of the figures the control references and the estimated positions, output of the localization module, are shown. There is no ground truth available, so these estimates are utilized to measure the performance of the system. $\{X, Y, Z, y a w\}$ are expressed in the same reference system as Fig. 2.15 $e_{\text {cross track }}$ is the trajectory following position error, including horizontal and vertical error. In these figures it can be appreciated that the tracking error is less than $0.5 \mathrm{~m}$ most of the time and that yaw is commanded slowly to limit the blur in the acquired video stream, so as to improve the performance of the marker detector module.

\begin{tabular}{|c|c|c|c|}
\hline & drone1 & drone2 & drone3 \\
\hline$e_{c t}[\mathrm{~m}]$ & 0.1971 & 0.1986 & 0.1290 \\
\hline hori $_{c t}[\mathrm{~m}]$ & 0.1723 & 0.1678 & 0.1166 \\
\hline vert $_{c t}[\mathrm{~m}]$ & 0.0957 & 0.1063 & 0.0551 \\
\hline yaw $_{\text {error }}[\mathrm{deg}]$ & 26.97 & 26.40 & 24.37 \\
\hline
\end{tabular}

Table 2.3: Flight performance of the drones in the experimental flight shown in Figs. 2.15 and 2.16 The values are the RMS error over the whole mission execution shown in Figs. 2.18, 2.19 and 2.20 $e_{c t}$, horiz $_{c t}$, vert $c t$ are the total, horizontal and vertical position errors with respect to the trajectory reference respectively. The yawerror is the error with respect to the commanded yaw, which corresponds to looking to one of the map columns or in an specific direction (which happens only in the first and last tasks).

The overall performance of the navigation tasks is summarized by the RMS error of $\{X, Y, Z$, yaw $\}$ during trajectory following. These values are shown in Table 2.3. The RMS horizontal tracking error is around $10-15 \mathrm{~cm}$, which is between a fourth and a third of the size of the drone. The RMS horizontal tracking error is around $10-15 \mathrm{~cm}$, which is between a fourth and a third of the size of the drone. The RMS yaw error is between $2 \mathrm{deg}$ and $25 \mathrm{deg}$ depending on the current mission task that the agent is performing. The lowest value corresponds to a constant yaw reference, during the first task before crossing the window, and the highest values correspond to the maximum yaw reference variations, during the navigation task around the columns. The reason for this surprisingly high RMS yaw error is that its controller is tuned with a very low proportional gain, so that the blurring on the acquired images is minimized. This was required to accommodate the image acquisition for the lowest light conditions that could occur in the indoor environment of the competition. 


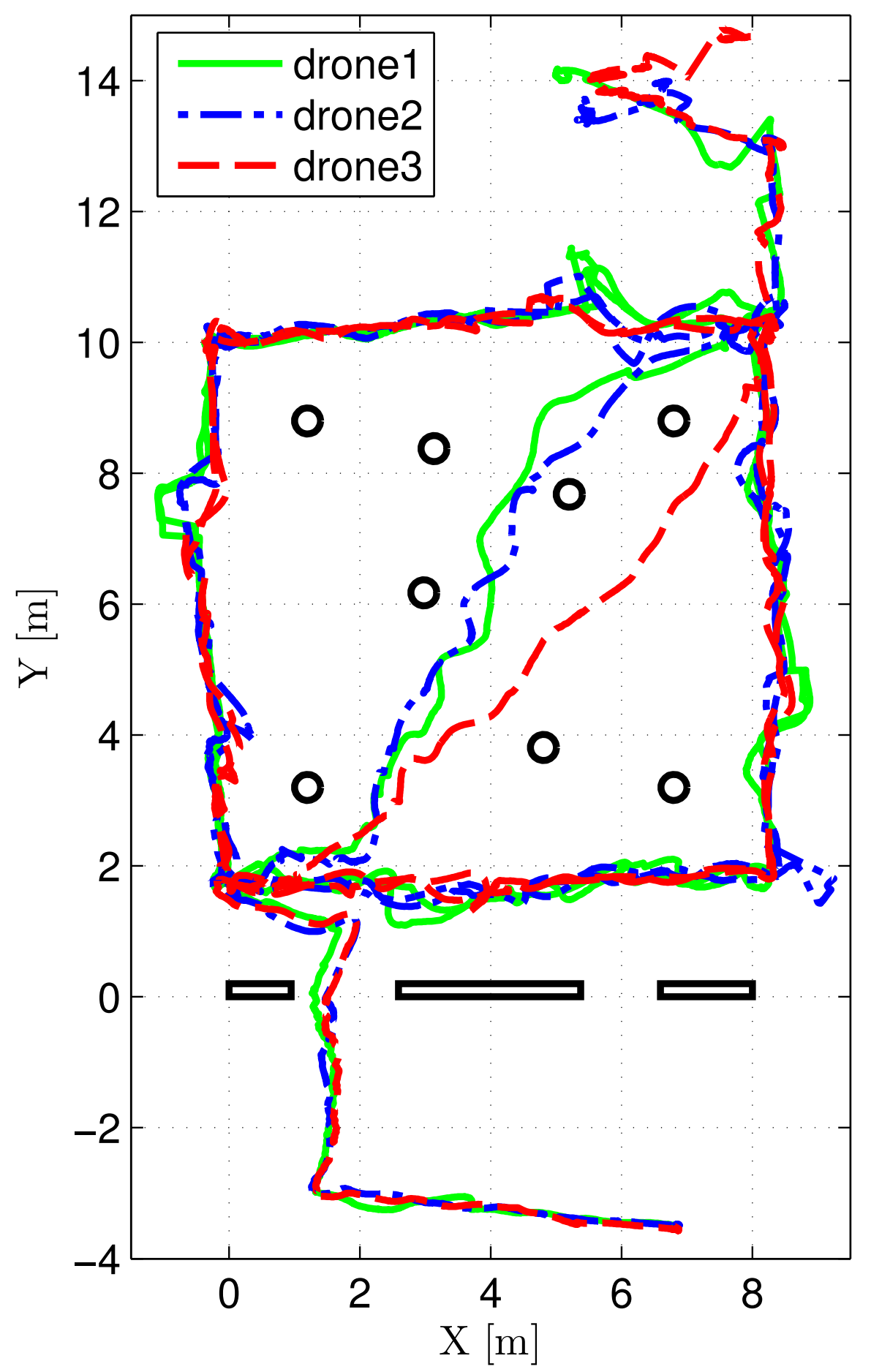

Figure 2.15: Experimental flight, three drones fly simultaneously performing navigation tasks. The environment is a replica of the IMAV 2013 Indoor Challenge environment. Figs. 2.15, 2.16, 2.18, 2.19 and 2.20 correspond to this experimental flight. The inner unknown pole locations are the estimates of drone1. The flight through this area is usually successful even when the position of the unknown poles is not precisely known, because the agents localize themselves with respect to these poles when they see them, thus planning obstacle-free trajectories with respect to their position estimate. The execution of this flight was successful, with all agents accomplishing their missions. A video of this test is available at the website: http://www.vision4uav.com/?q=node/386. 

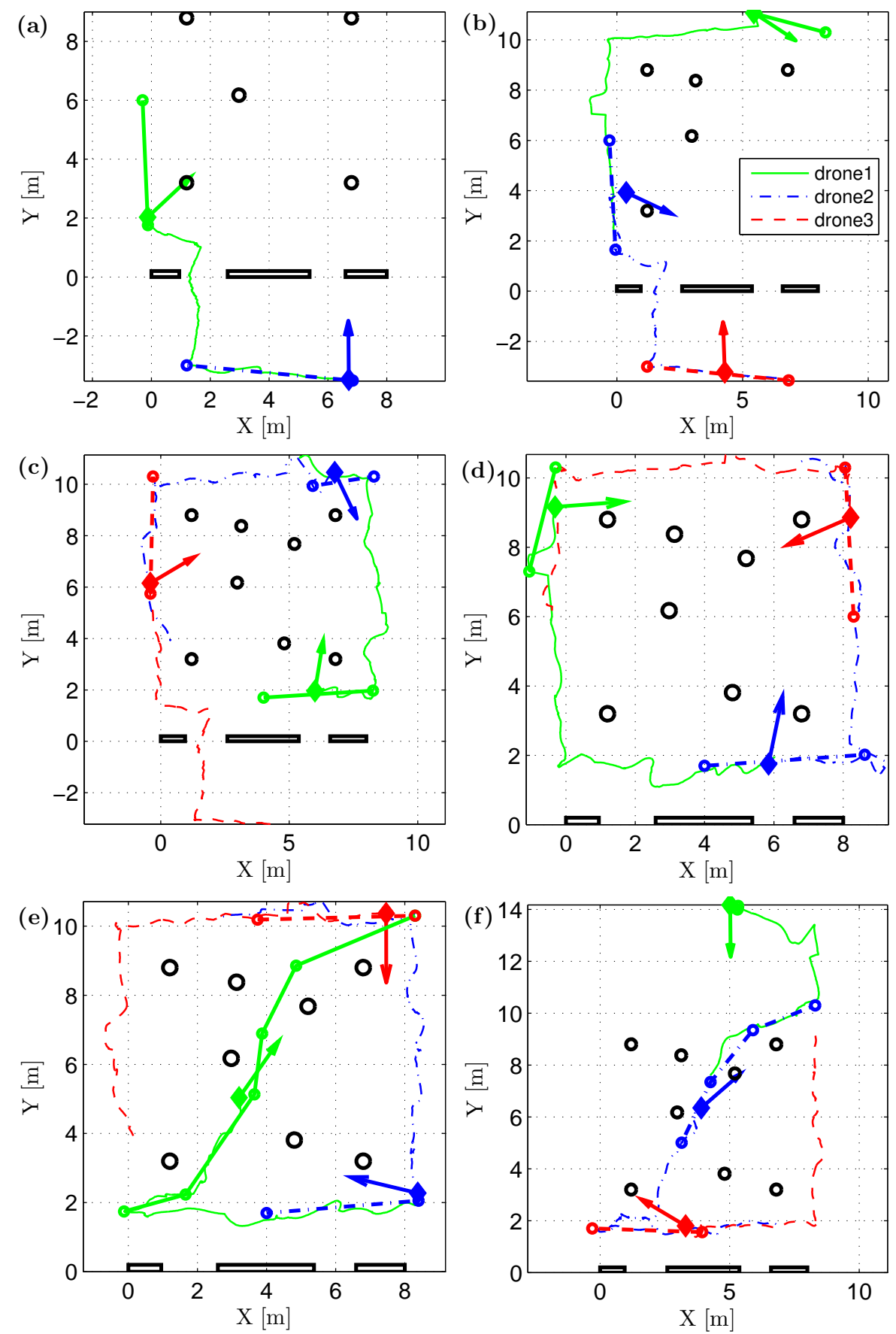

Figure 2.16: Same experimental flight as shown in Fig. 2.15 These figures, labeled a-f, are ordered in time, and each agent's plots have a different color and line style. The drones ordered by their launch times are plotted with: (drone1) green-solid lines, (drone2) blue-dash dotted lines and (drone3) red-dashed lines. The planned trajectory is shown with a thicker width than the actual executed trajectory. The dotted lines are the current trajectory references at the end of the plotted time period. The arrows indicate the direction at which the quadrotor is estimated to be looking in the plotted position. As shown, the quadrotors are commanded to look at one of the four known corner columns of the map. The unknown poles localized by drone 1 are shown in these figures. (a), (b), (c) and (d) show consecutive flight intervals of 30 seconds showing the startup sequence of the multi-robot system. In this period, dronel crosses the window and performs 1.25 laps. (e) and (f) show consecutive flight intervals of 30 second of the final stages of the mission. The drones cross the unknown poles area by planning and executing obstacle-free trajectories. 

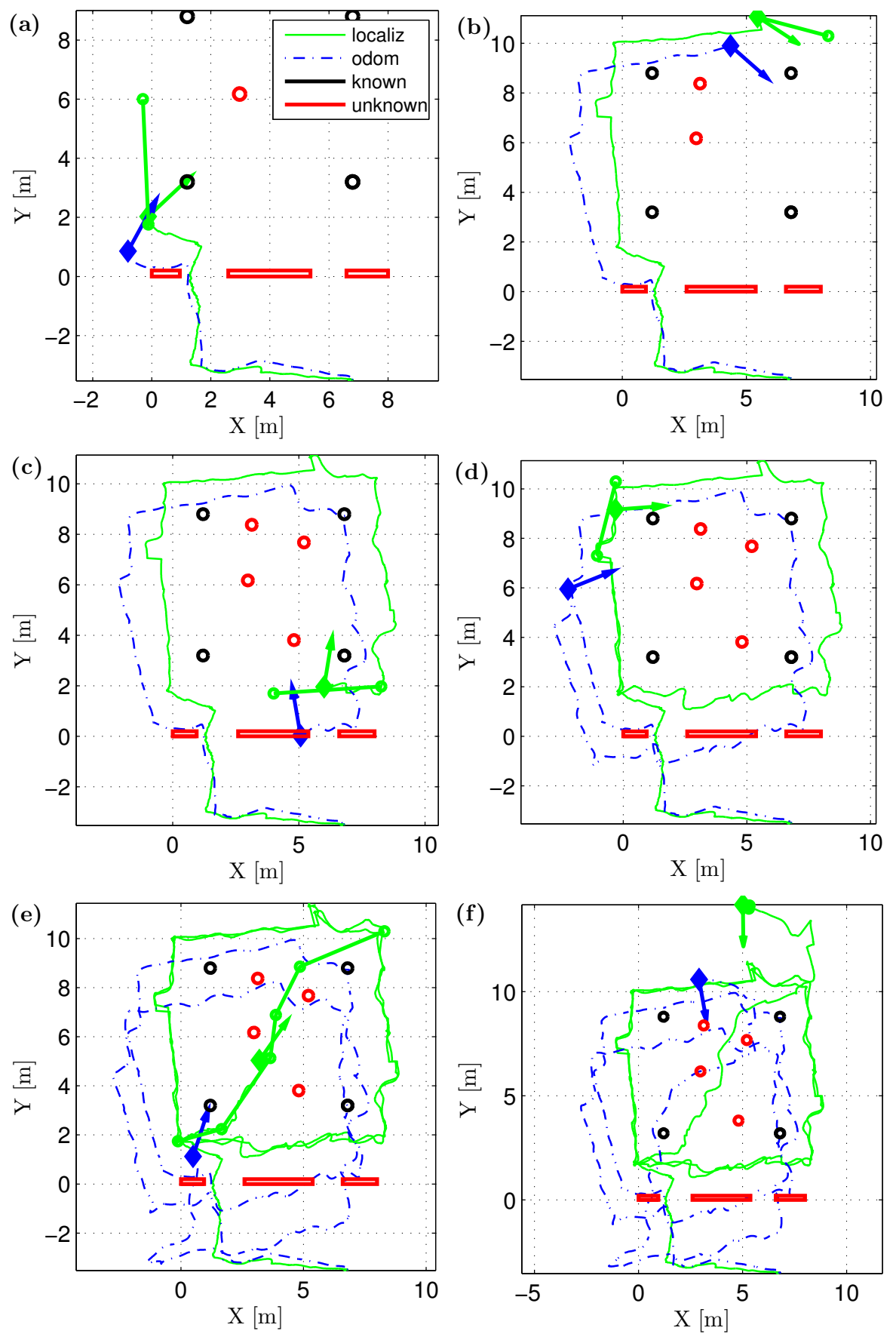

Figure 2.17: Same experimental flight as shown in Fig. 2.15 These figures, labeled a-f, are ordered in time, all the plotted elements are from drone1. The timestamps at which these plots were generated are the same as in Fig. 2.16(a-f). The Localization module drift-free estimate from dronel is shown with thin green-solid lines, the odometry-based estimate from the Pose Estimator module is shown with bluedash dotted lines, the known environment elements are shown with thick black lines and the unknown environment elements are shown with thick red lines. The drone estimated positions are shown with diamonds and the yaw estimates with an arrow of the same color. The thick green line plot, shows the currently commanded trajectory. These figures show how the Localization module is able to calculate a drift-free pose from the odometry-based estimate. The corrections are specially visible after the yaw turns, when the drone can localize with respect to the next corner column. It is noted that in this experiment some known markers were placed on the wall in the taking-off side, but that the windows were localized as unknown elements. 

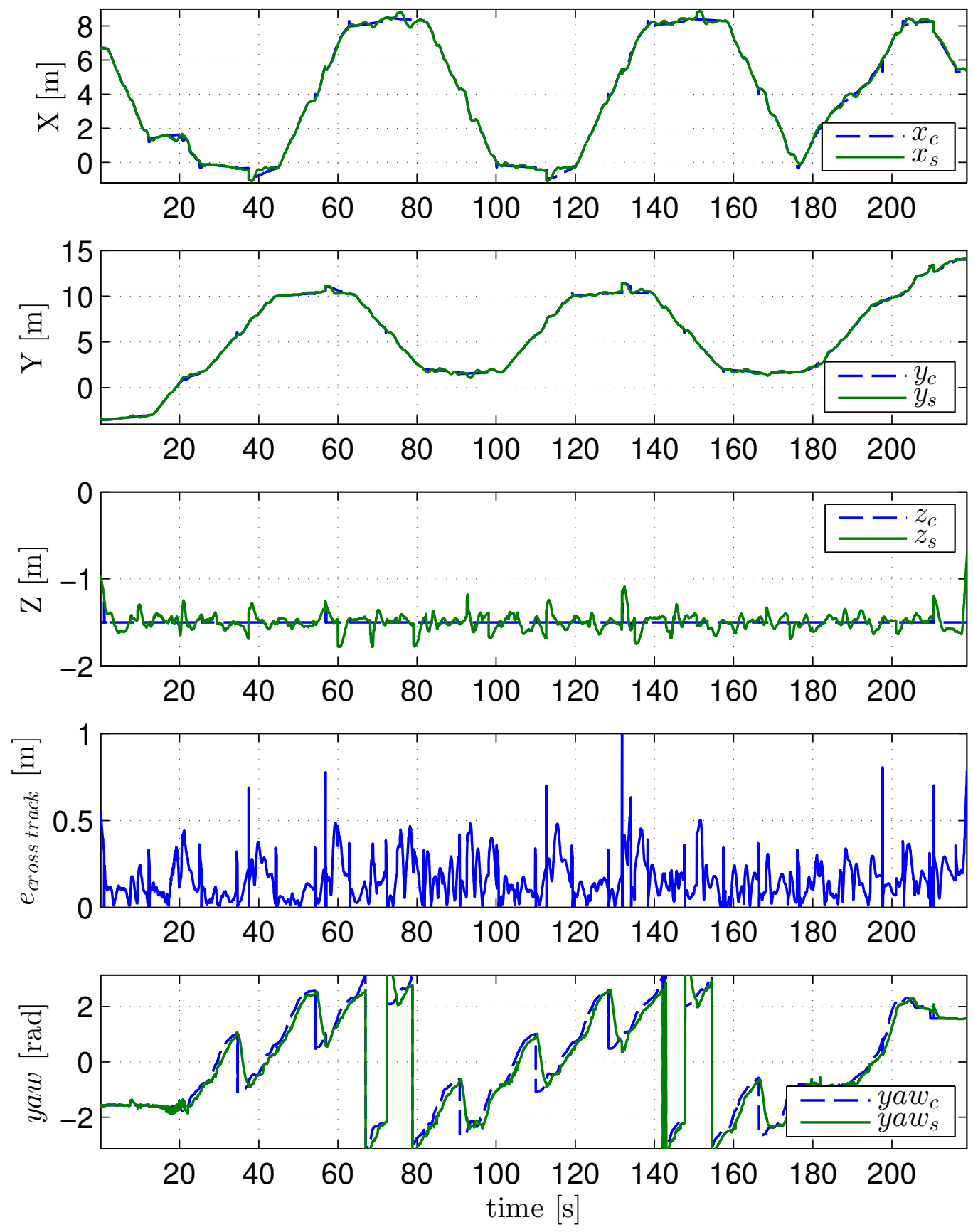

Figure 2.18: Flight performance of drone1, same experimental flight as shown in Figs. 2.15 and 2.16 The plots show the estimated position and yaw of the quadrotor and the inputs given to the position and speed control loops inside the trajectory controller module. The $e_{\text {crosstrack }}$ plot shows the absolute position control error based on these commands and the estimated position, as calculated by the localization module. The yaw command only changes sharply when the drone is commanded to look at a different column. The rest of the time when a sharp change is shown it has just evolved to the opposite side of the $[-\pi,+\pi]$ interval. 

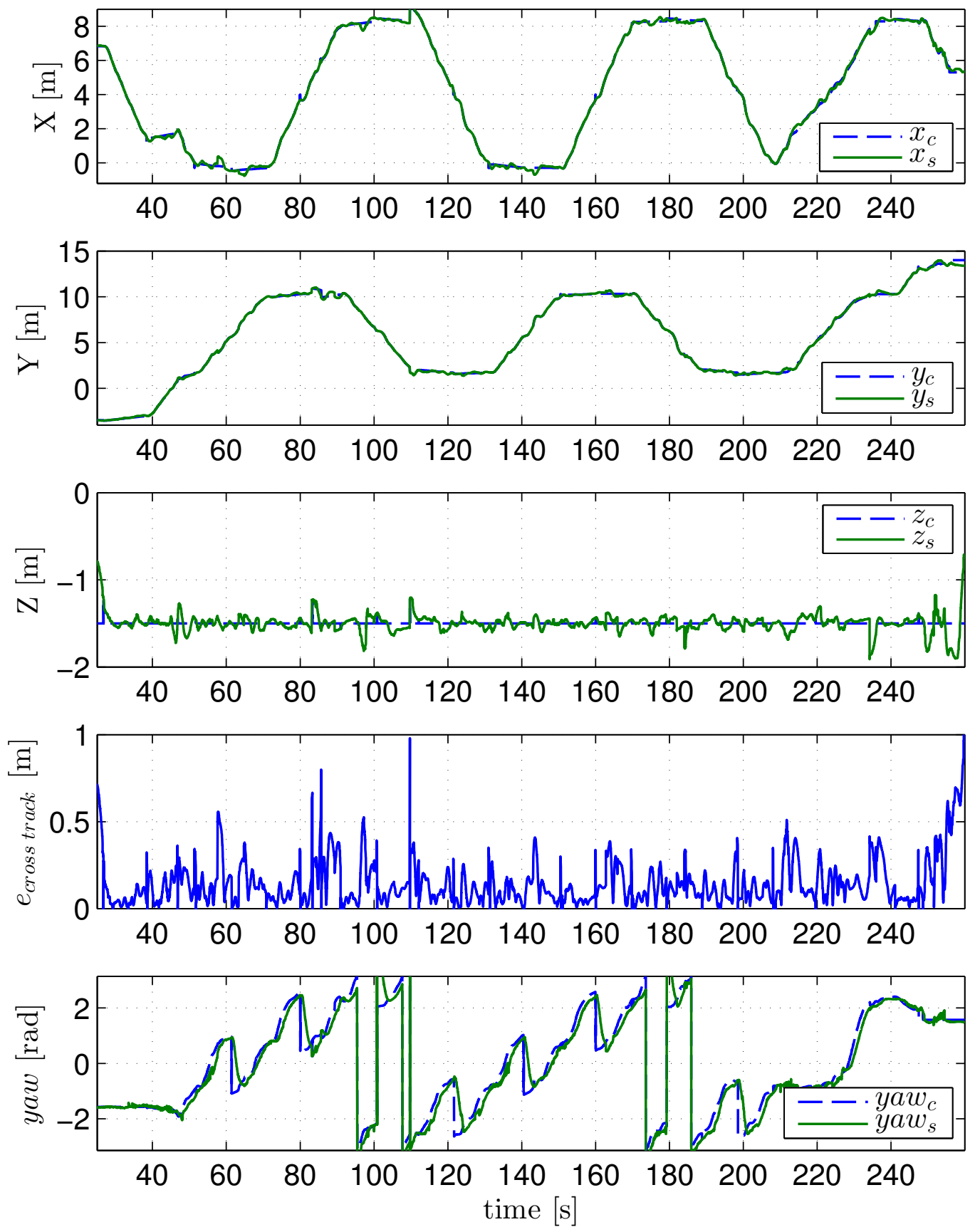

Figure 2.19: Flight performance of drone2, same experimental flight as shown in Figs. 2.15 and 2.16. The interpretation of this figure is similar to that of Fig. 2.18 . 

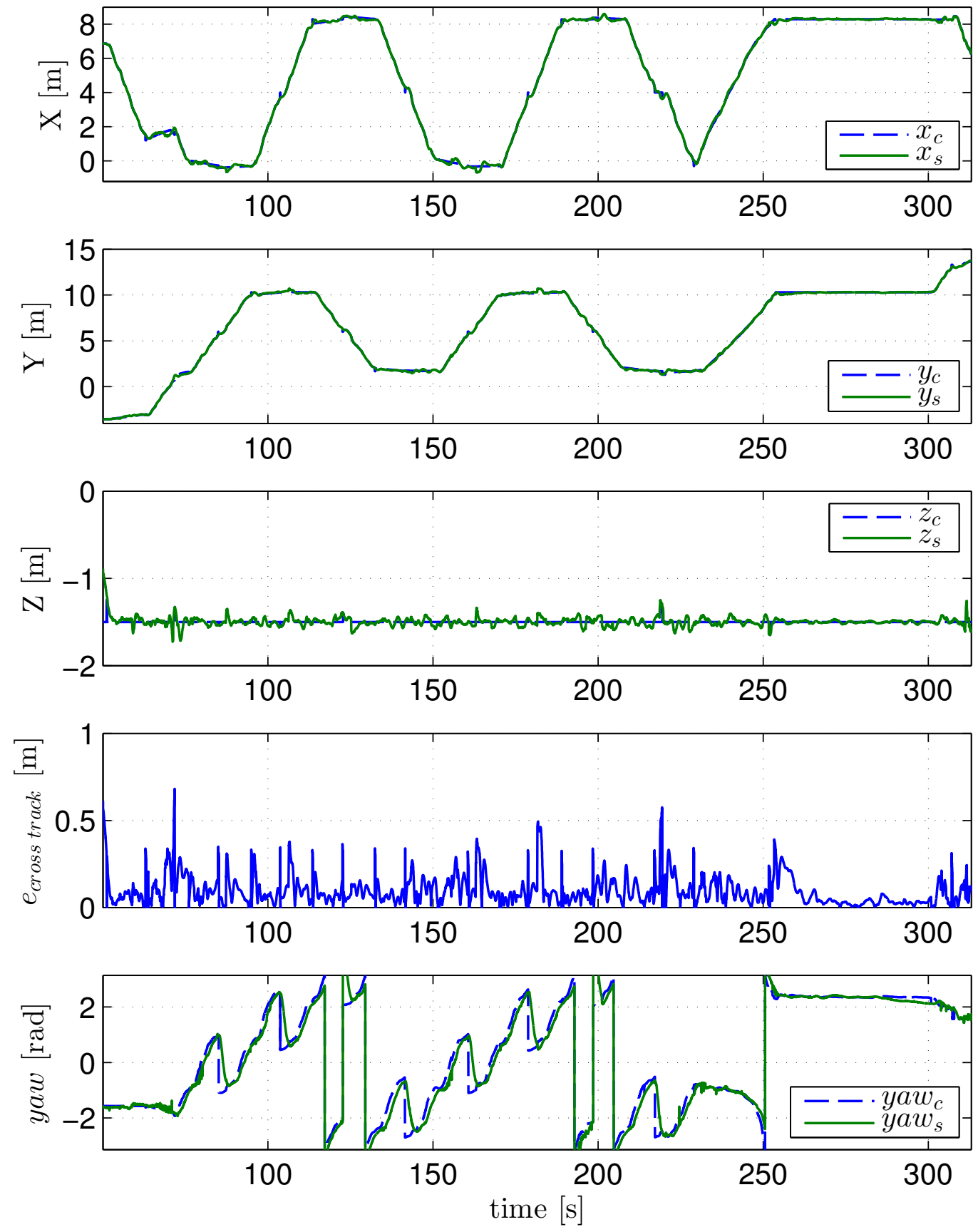

Figure 2.20: Flight performance of drone3, same experimental flight as shown in Figs. 2.15 and 2.16. The interpretation of this figure is similar to that of Fig. 2.18. 


\subsection{Future Work}

In the area of control, estate estimation and dynamics modeling of drones, there are promising works that show that better models allow a much more precise estimation of the wind speed, or, in the absence of wind, of the speed of the drone itself, see Sec. 3.2 and Appx.E.2. In addition to providing more precise state estimates, the research in this direction may lead to safer flight maneuvering in environments that become very challenging under the presence of wind disturbances such as urban corridors and indoor hallways. Another interesting area of research is the development of state estimators that detect flight issues. Consider, for instance, soft collisions of the drone protective hull with walls. Detecting such events may result in interesting behaviors such as control techniques that avoid the worsening of the flight situation, after the event is detected by the state estimator, like avoiding a full crash against the said wall. Other physical contact events that are important to be detected are the approach to the ground, the ceiling or walls, based on how these events affect the flight of the drone; and determining the landing event more precisely, that may enable faster landing maneuvers on moving landing platforms. In the area of state estimation, many autopilots have a very big dependence on the magnetometer sensor for the drone heading estimation. There currently exist UAVs that can operate under magnetic interference by using multiple GPS sensors. In GPS-denied environments, for indoor navigation and flying near ferromagnetic infrastructure, it is required to use other sensor data for heading estimation, so as to decrease the dependence on the magnetometer sensor.

\subsection{Conclusions}

The main section of this chapter describes a novel general purpose controller for multirotors, Sec. 2.3 . This controller was inspired by prior work by Hoffmann et al. (Hoffmann, Gabriel M et al., 2008) and Michael et al. (B. Y. N. Michael et al., 2010):

- The main difference of our controller when compared to these two prior works is the addition of several saturation laws that can intrinsically take into account speed limitations established by the on-board sensors. This feature was tested during our participation in the international MAV competition IMAV2012, where our MAV had to deal with low frame-rate optical-flow measurements (Pestana, Mellado-Bataller, Fu, et al., 2013). These saturations are used to set a desired maximum navigation speed for the multirotor and they affect the velocity planning during trajectory tracking and the execution of the controller itself. In addition to this feature:

- In comparison to the work by Michael et al. (B. Y. N. Michael et al., 2010), our experiments are performed without reliance of GPS or a motion capture system, e. g. a Vicon system. We utilize instead the EKF discussed in Annex. Eduring the experimental validation of the controller.

- In comparison to the work by Hoffmann et al. (Hoffmann, Gabriel M et al., 2008), our experiments were carried out in indoor environments and using different sensing solutions: ground optical-flow and LIDAR (Pestana, Mellado-Bataller, Fu, et al., 2013), and ground optical-flow and fiducial markers (Pestana, Sanchez-Lopez, et al., 2015).

- This feature of the presented controller is quite advantageous, considering that some odometry estimation algorithms may fail to deliver estimates when navigating at inadequate velocities. For instance, in vision-based odometry estimation it becomes increasingly difficult to match image patches or features in subsequent images, due to a lack of overlap between them, thus, in practice, setting a maximum navigation velocity. The controller architecture proposed in this chapter under normal working conditions, e.g. within the sensors and actuators limits and in absence of strong enough wind and wind-gusts, is able to ensure that the trajectory is executed at an optimum speed for the on-board sensors to work properly. The actual acceptable wind speed depends on the maximum speed that can be sensed by the utilized sensing algorithm. By adopting a conservative approach, in practice, the acceptable wind speed is set lower than the sensor limits.

- An additional usage of the speed saturations proposed in the controller architecture and in the speed planning step is to simplify the flight rules of decentralized multi-robot navigation, as showcased 
in Sec. 2.4.4 This particular use of the speed saturations was utilized by the CVG_UPM during its participation in the IMAV 2013 competition.

- The presented controller has been successfully utilized as part of an autonomous flight architecture in several international MAV competitions, showcasing its tested reliable implementation, and its code has been released as open-source in github.

The main publications related to this chapter are:

- J. Pestana, J. L. Sanchez-Lopez, P. de la Puente, A. Carrio, P. Campoy. "A Vision-based Quadrotor Multi-robot Solution for the Indoor Autonomy Challenge of the 2013 International Micro Air Vehicle Competition”. In Journal of Intelligent and Robotic Systems, JINT2015. Springer Netherlands.

- J. Pestana, I. Mellado-Bataller, C. Fu, J. L. Sanchez-Lopez, I. F. Mondragon, P. Campoy. “A General Purpose Configurable Controller for Indoors and Outdoors GPS-Denied Navigation for Multirotor Unmanned Aerial Vehicles". In JINT2013.

- J. L. Sanchez-Lopez, J. Pestana, P. de la Puente, P. Campoy. “A reliable open-source system architecture for the fast designing and prototyping of autonomous multi-uav systems: Simulation and experimentation”. In JINT2015.

- I. Mellado-Bataller, J. Pestana, M. A. Olivares-Mendez, P. Campoy, and L. Mejias, book chapter, "MAVwork: a Framework for Unified Interfacing between Micro Aerial Vehicles and Visual Controllers", in book "Frontiers of Intelligent Autonomous Systems", serie "Studies in Computational Intelligence Series”, Springer, 2013.

- J. Pestana, I. Mellado-Bataller, C. Fu, J. L. Sanchez-Lopez, I. F. Mondragon, P. Campoy. “A general purpose configurable navigation controller for micro aerial multirotor vehicles”, In 2013 International Conference on Unmanned Aircraft Systems (ICUAS), 2013.

This general purpose controller for multirotors has been used by the CVG in its participation in five international MAV competitions:

- International Micro Air Vehicle Conference and Flight Competition 2016 (IMAV 2016) $\sqrt{9}$ where the CVG team was able to solve multiple tasks in fully autonomous mode and was the team to achieve the highest number of tasks, although in separate flights, in fully autonomous mode.

Despite its outstanding performance, the CVG team earned the $4^{\text {th }}$ position due to the scoring system leaning very heavily towards the usage of very small MAVs.

- Asia Venue of the AUVSI International Aerial Robotics Competition 2014 (IARC 2014) ${ }^{10}$ same as other teams, we were still far from achieving the interaction with the ground robots. Therefore, the CVG_UPM team showed similar capabilities to other contestants. Our level of development was good as a first step towards continuing our participation the next year for IARC 2015.

- International Micro Air Vehicle Conference and Flight Competition 2013 (IMAV 2013 11 where the team that represented the UPM and the Computer Vision Group (CVG, UPM) was awarded with: first place in the "Indoor Autonomy - IMAV 2013".

- International Micro Air Vehicle Conference and Flight Competition 2012, (IMAV 2012) 12 , The team that represented the CAR and the Computer Vision Group (CVG) was twice awarded with:

- the special award “Best Automatic Performance - IMAV 2012”, as part of the overall results of the competitions, and

- second place in the "Indoor Flight Dynamics - Rotary Wing MAV" challenge, where the first place was obtained by a remotely piloted multirotor.

\footnotetext{
${ }^{9}$ IMAV 2016 video playlist: https: / / goo.gl/tdZyGu

${ }^{10}$ IARC 2014 video playlist: https://goo.gl/bEvK3F

${ }^{11}$ IMAV 2013 video playlist: https://goo.gl/KosWKH

${ }^{12}$ IMAV 2012 video playlist: https://goo.gl/PRns2D
} 
- Contest on Control Engineering 2012, (IC CEA 2012), organized by the Spanish Committee of Automation. Two teams participated representing the Universidad Politécnica de Madrid (UPM) and the Computer Vision Group (CVG_UPM), and were awarded with:

- Undergraduate students' of the ETSII-UPM engineering school using their own developed software supervised by J. Pestana: the first (phase 1 - simulation) and third places (final phase - autonomous flight event)

- CVG PhD students' team, using the presented controller: the second (phase 1 - simulation) and fifth places (final phase - autonomous flight event). In the final phase of the competition our team achieved the fifth place, due to our controller, when compared to other teams, leaning towards low tracking error with higher round-trip times.

The source of this controller has been released as open-source as part of the stack for the control of multirotor MAVs from the CVG, first integrated to MAVwork $\left.{ }^{13}\right|^{4}$ and currently as part of Aerostack $\left.k^{15}\right|^{6}$

This controller has been showcased in several live demos by the CVG (UPM):

- CivilDRON 2017 congres ${ }^{17}$ Madrid (Spain)

Simplified version of IMAV 2016 competition mission

January 2017

- CivilDRON 2016 congres ${ }^{18}$ Madrid (Spain)

Multi-robot mission and vision-based person following

May 2016

- Semana de la Ciencia 2015 - Science Week 201519/20 Madrid (Spain)

Multi-robot mission and vision-based person following

November 2-15, 2015

- Noche Europea de los investigadores - European Researchers' Night 201521

Multi-robot mission, vision-based person following and a simple search and rescue mission, which included the usage of a multi-modal human-machine interface with the MAV.

Madrid (Spain), September 2015

- Semana de la Ciencia 2014 - Science Week 201422, Madrid (Spain)

Multi-robot mission and vision-based person following

November 2014

- Semana de la Ciencia 2013 - Science Week 201323, Madrid (Spain)

Multi-robot mission and vision-based person following

November 4-17, 2013

\footnotetext{
${ }^{13}$ Git repository: https://github.com/uavster/mavwork

${ }^{14}$ Git repository: https://github.com/jespestana/MultirotorController4mavwork

${ }^{15}$ Git repository: https://github.com/Vision4UAV/cvg_quadrotor_swarm

${ }^{16}$ Git repository: https://github.com/Vision4UAV/Aerostack

${ }^{17}$ CivilDRON 2017: http://wwW.civildron.com/

${ }^{18}$ CivilDRON 2016: http://www. aerpas.es/wp-content/uploads/2015/04/CivilDRON16_v2.pdf

${ }^{19}$ Semana de la Ciencia 2015 - Website http://bit.ly/21N9RNg

${ }^{20}$ Semana de la Ciencia 2015 - Results Report http://bit.ly/2muELHG

${ }^{21}$ European Researchers' Night 2015 - Website: http://bit.1y/2mM6a9Q

${ }^{22}$ Semana de la Ciencia 2014 - Results Report http://bit.ly/21Ng1NA

${ }^{23}$ Semana de la Ciencia 2013 - Results Report http://bit.ly/2npz2lF
} 


\subsection{Chapter Bibliography}

Achtelik, M., Bachrach, A., He, R., Prentice, S., and Roy, N. (2009). "Stereo vision and laser odometry for autonomous helicopters in GPS-denied indoor environments". In: SPIE Defense, Security, and Sensing. International Society for Optics and Photonics, pp. $733219-733219$ (cit. on p. 11).

Agrachev, A. A. and Sachkov, Y. (2004). Control theory from the geometric viewpoint. Vol. 2. Springer Science \& Business Media (cit. on p. 12).

Bachrach, A. G. (2009). "Autonomous flight in unstructured and unknown indoor environments". MA thesis. Massachusetts Institute of Technology (cit. on p. 11).

Bachrach, A., Prentice, S., He, R., and Roy, N. (2011). "RANGE-Robust autonomous navigation in GPSdenied environments". Journal of Field Robotics 28.5, pp. 644-666 (cit. on p. 11).

Bouabdallah, S., Murrieri, P., and Siegwart, R. (2005). "Towards autonomous indoor micro VTOL". Autonomous Robots 18.2, pp. 171-183 (cit. on p.112).

Bouabdallah, S. and Siegwart, R. (2005). "Backstepping and sliding-mode techniques applied to an indoor micro quadrotor". In: Robotics and Automation, 2005. ICRA 2005. Proceedings of the 2005 IEEE International Conference on. IEEE, pp. 2247-2252 (cit. on p. 12).

Bristeau, P.-J., Callou, F., Vissiere, D., Petit, N., et al. (2011). "The navigation and control technology inside the ar. drone micro uav". In: 18th IFAC World Congress. Vol. 18, pp. 1477-1484 (cit. on p. 23).

Castillo, P., Dzul, A., and Lozano, R. (2004). "Real-time stabilization and tracking of a four-rotor mini rotorcraft”. Control Systems Technology, IEEE Transactions on 12.4, pp. 510-516 (cit. on p. 12).

Castillo, P., Lozano, R., and Dzul, A. (2005). "Stabilization of a mini rotorcraft with four rotors". IEEE Control Systems Magazine 25.6, pp. 45-55 (cit. on p. 12).

Cowling, I. D., Yakimenko, O. A., Whidborne, J. F., and Cooke, A. K. (2010). "Direct method based control system for an autonomous quadrotor". Journal of Intelligent \& Robotic Systems 60.2, pp. 285 316 (cit. on p. 12).

Mellado Bataller, I. (2012). A new framework for interfacing with MAVs. https : / / github . com / uavster/mavwork (cit. on p. 13).

Driessen, B. J. and Robin, A. L. (2004). “A globally convergent tracking controller for the X4 flyer rotor craft for reference trajectories with positive thrust". Robotica 22.04, pp. 375-388 (cit. on p. 12).

Fritsch, O., De Monte, P., Buhl, M., and Lohmann, B. (2012). "Quasi-static feedback linearization for the translational dynamics of a quadrotor helicopter". In: American Control Conference (ACC), 2012. IEEE, pp. 125-130 (cit. on p. 12).

Garrido-Jurado, S., Muñoz-Salinas, R., Madrid-Cuevas, F. J., and Marín-Jiménez, M. J. (2014). “Automatic generation and detection of highly reliable fiducial markers under occlusion". Pattern Recognition 47.6, pp. 2280-2292 (cit. on p. 27).

Gillula, J. H., Huang, H., Vitus, M. P., and Tomlin, C. J. (2010). "Design of Guaranteed Safe Maneuvers Using Reachable Sets : Autonomous Quadrotor Aerobatics in Theory and Practice". IEEE Electrical Engineering (2010), pp. 1649-1654 (cit. on p. 11).

Guenard, N., Hamel, T., and Moreau, V. (2005). "Dynamic modeling and intuitive control strategy for an" X4-flyer"”. In: Control and Automation, 2005. ICCA'05. International Conference on. Vol. 1. IEEE, pp. 141-146 (cit. on p.12).

Heng, L., Honegger, D., Lee, G. H., Meier, L., Tanskanen, P., Fraundorfer, F., and Pollefeys, M. (2014). "Autonomous visual mapping and exploration with a micro aerial vehicle". Journal of Field Robotics 31.4, pp. 654-675 (cit. on p. 11).

Hoffmann, Gabriel M, Waslander, Steven L, and Tomlin, Claire J (2008). "Quadrotor Helicopter Trajectory Tracking Control”. Electrical Engineering (2008), pp. 1-14 (cit. on pp. 11, 12, 16, 18, 37).

Hoffmann, G.M., Waslander, S.L., and Tomlin, C.J. (2009). "Aerodynamics and control of autonomous quadrotor helicopters in aggressive maneuvering". In: 2009 IEEE International Conference on Robotics and Automation. Ieee, pp. 3277-3282 (cit. on pp.11,12).

Hoffmann, G. M., Huang, H., Waslander, S. L., and Tomlin, C. J. (2007). "Quadrotor helicopter flight dynamics and control: Theory and experiment". In: Proc. of the AIAA Guidance, Navigation, and Control Conference. Vol. 2 (cit. on p. 12).

Hoffmann, G. M., Waslander, S. L., and Tomlin, C. J. (2006). "Distributed Cooperative Search using Information-Theoretic Costs for Particle Filters, with Quadrotor Applications". Electrical Engineering, pp. 1-21 (cit. on pp. 11, 12). 
Hua, M.-D., Hamel, T., Morin, P., and Samson, C. (2009). "A control approach for thrust-propelled underactuated vehicles and its application to VTOL drones". Automatic Control, IEEE Transactions on 54.8, pp. 1837-1853 (cit. on p. 12).

Huang, A. S., Bachrach, A., Henry, P., Krainin, M., Fox, D., and Roy, N. (2011). "Visual Odometry and Mapping for Autonomous Flight Using an RGB-D Camera". Camera (cit. on p. 11).

Jurdjevic, V. (1997). Geometric control theory. Cambridge university press (cit. on p. 12).

Kendoul, F., Yu, Z., and Nonami, K. (2010). "Guidance and nonlinear control system for autonomous flight of minirotorcraft unmanned aerial vehicles". Journal of Field Robotics 27.3, pp. 311-334 (cit. on p. 12.

Kushleyev, A., Kumar, V., and Mellinger, D. (2012). “Towards A Swarm of Agile Micro Quadrotors”. In: Proceedings of Robotics: Science and Systems. Sydney, Australia (cit. on p.11).

Lee, D., Kim, H. J., and Sastry, S. (2009). "Feedback linearization vs. adaptive sliding mode control for a quadrotor helicopter". International Journal of control, Automation and systems 7.3, pp. 419-428 (cit. on p.12).

Lee, T., Leoky, M., and McClamroch, N. H. (2010). "Geometric tracking control of a quadrotor UAV on SE (3)". In: Decision and Control (CDC), 2010 49th IEEE Conference on. IEEE, pp. 5420-5425 (cit. on p.12).

Lippiello, V., Loianno, G., and Siciliano, B. (2011). "MAV indoor navigation based on a closed-form solution for absolute scale velocity estimation using Optical Flow and inertial data". In: Decision and Control and European Control Conference (CDC-ECC), 2011 50th IEEE Conference on, pp. 35663571 (cit. on p. 12).

Madani, T. and Benallegue, A. (2007). "Backstepping control with exact 2-sliding mode estimation for a quadrotor unmanned aerial vehicle”. In: Intelligent Robots and Systems, 2007. IROS 2007. IEEE/RSJ International Conference on. IEEE, pp. 141-146 (cit. on p.12).

Mellado-Bataller, I., Mejias, L., Campoy, P., and Olivares-Mendez, M. A. (2012). "Rapid prototyping framework for visual control of autonomous micro aerial vehicles". In: 12th International Conference on Intelligent Autonomous System (IAS-12). Jeju Island, Korea (cit. on p. 13).

Mellado-Bataller, I., Pestana, J., Olivares-Mendez, M. A., Campoy, P., and Mejias, L. (2013). “MAVwork: a framework for unified interfacing between micro aerial vehicles and visual controllers". In: Frontiers of Intelligent Autonomous Systems. Springer, pp. 165-179 (cit. on p. 13).

Mellinger, D., Michael, N., and Kumar, V. (2010). "Trajectory Generation and Control for Precise Aggressive Maneuvers with Quadrotors". In: Int Symposium on Experimental Robotics (2010) (cit. on pp. $11,12,16$.

Michael, B. Y. N., Mellinger, D., and Lindsey, Q. (2010). "The GRASP Multiple Micro UAV Testbed". IEEE Robotics \& Automation Magazine, pp. 56-65 (cit. on pp. 11, 12, 16, 37).

Michael, N., Shen, S., Mohta, K., Mulgaonkar, Y., Kumar, V., Nagatani, K., Okada, Y., Kiribayashi, S., Otake, K., Yoshida, K., et al. (2012). "Collaborative mapping of an earthquake-damaged building via ground and aerial robots". Journal of Field Robotics 29.5, pp. 832-841 (cit. on p. 11).

Mistler, V., Benallegue, A., and M'sirdi, N. (2001). "Exact linearization and noninteracting control of a 4 rotors helicopter via dynamic feedback”. In: Robot and Human Interactive Communication, 2001. Proceedings. 10th IEEE International Workshop on. IEEE, pp. 586-593 (cit. on p. 12).

Nice, E. B. (2004). "Design of a four rotor hovering vehicle". PhD thesis. Cornell University (cit. on p. 12,

Pestana, J., Mellado-Bataller, I., Fu, C., Sanchez-Lopez, J. L., Mondragon, I. F., and Campoy, P. (2013). "A general purpose configurable navigation controller for micro aerial multirotor vehicles". In: Unmanned Aircraft Systems (ICUAS), 2013 International Conference on. IEEE, pp. 557-564 (cit. on pp. 18, 19 37).

Pestana, J., Mellado-Bataller, I., Sanchez-Lopez, J. L., Fu, C., Mondragón, I. F., and Campoy, P. (2014). "A general purpose configurable controller for indoors and outdoors gps-denied navigation for multirotor unmanned aerial vehicles”. Journal of Intelligent \& Robotic Systems 73.1-4, pp. 387-400 (cit. on pp. 19 22.

Pestana, J., Sanchez-Lopez, J. L., Puente, P. de la, Carrio, A., and Campoy, P. (2015). “A Vision-based Quadrotor Multi-robot Solution for the Indoor Autonomy Challenge of the 2013 International Micro Air Vehicle Competition”. Journal of Intelligent \& Robotic Systems, pp. 1-20 (cit. on pp. 19, 27, 37).

Quaritsch, M., Stojanovski, E., Bettstetter, C., Friedrich, G., Hellwagner, H., Rinner, B., Hofbaur, M., and Shah, M. (2008). “Collaborative Microdrones: Applications and Research Challenges”. In: Pro- 
ceedings of the Second International ICST Conference on Autonomic Computing and Communication Systems. Gent, BELGIUM: Icst (cit. on p. 11).

Raffo, G. V., Ortega, M. G., and Rubio, F. R. (2010). "An integral predictive/nonlinear Hinf control structure for a quadrotor helicopter”. Automatica 46.1, pp. 29-39 (cit. on p. 12).

Richter, Charles, Bry, Adam, and Roy, Nicholas (2013). "Polynomial trajectory planning for aggressive quadrotor flight in dense indoor environments". In: Proceedings of the International Symposium on Robotics Research (ISRR) (cit. on p. 12).

Rudol, P., Wzorek, M., Conte, G., and Doherty, P. (2008). "Micro unmanned aerial vehicle visual servoing for cooperative indoor exploration". In: Aerospace, 2008 IEEE Conference on (cit. on p. 12).

S., L., A., S., M, S., and D’Andrea, R. (2010). “A Simple Learning Strategy for High-Speed Quadrocopter Multi-Flips". In: 2010 IEEE International Conference on Robotics and Automation (ICRA 2010), pp. 1642-1648 (cit. on p. 11).

Scaramuzza, D., Achtelik, M., Doitsidis, L., Fraundorfer, F., Kosmatopoulos, E., Martinelli, A., et al. (2013). "Vision-controlled micro flying robots: from system design to autonomous navigation and mapping in gps-denied environments". IEEE Robotics and Automation Magazine (cit. on p. 11).

Schölling, A., Augugliaro, F., Lupashin, S., and D'Andrea, R. (2010). "Synchronizing the Motion of a Quadrocopter to Music". In: IEEE International Conference on Robotics and Automation ICRA, pp. 3355-3360 (cit. on p.11).

Shen, Shaojie, Mulgaonkar, Yash, Michael, Nathan, and Kumar, Vijay (2013). "Vision-based state estimation for autonomous rotorcraft mavs in complex environments". In: Robotics and Automation (ICRA), 2013 IEEE International Conference on. IEEE, pp. 1758-1764 (cit. on p. 12).

Shen, S., Michael, N., and Kumar, V. (2011). "Autonomous multi-floor indoor navigation with a computationally constrained MAV". Robotics and Automation (ICRA), 2011 IEEE International Conference on, pp. 20-25 (cit. on p.11).

Shen, S., Mulgaonkar, Y., Michael, N., and Kumar, V. (2013). "Vision-Based State Estimation and Trajectory Control Towards High-Speed Flight with a Quadrotor." In: Robotics: Science and Systems. Citeseer (cit. on p. 12).

(2012). The Stanford/Berkeley Testbed of Autonomous Rotorcraft for Multi-Agent Control (STARMAC) project. http://hybrid.eecs.berkeley.edu/starmac/ (cit. on p. 11).

Valenti, M., Bethke, B., Fiore, G., How, J. P., and Feron, E. (2006). "Indoor multi-vehicle flight testbed for fault detection, isolation, and recovery". In: Proceedings of the AIAA Guidance, Navigation, and Control Conference and Exhibit, Keystone, CO. Vol. 63, p. 64 (cit. on p. 12).

Weiss, S., Achtelik, M. W., Chli, M., and Siegwart, R. (2012). "Versatile distributed pose estimation and sensor self-calibration for an autonomous MAV". In: Robotics and Automation (ICRA), 2012 IEEE International Conference on. IEEE, pp. 31-38 (cit. on p.11).

Zingg, S., Scaramuzza, D., Weiss, S., and Siegwart, R. (2010). "MAV Navigation through Indoor Corridors Using Optical Flow”. In: Robotics and Automation (ICRA), 2010 IEEE International Conference on (cit. on p. 12).

Zufferey, J.-C., Beyeler, A., and Floreano, D. (2010). "Autonomous flight at low altitude with visionbased collision avoidance and GPS-based path following". In: Proceedings of the IEEE International Conference on Robotics and Automation (ICRA). Anchorage, Alaska: IEEE (cit. on p. 12). 


\section{3}

\section{Visual Servoing of Arbitrary Objects for Multirotors}

\subsection{Introduction}

This research showcases visual object tracking for Unmanned Air Vehicles (UAV) to perform GPSdenied object following tasks, on a big variety of objects in outdoors suburban environments. Navigation is more challenging for a flying robot than for ground robots because it requires feedback to stabilize itself. This fact provides a second objective, which is to show that visual servoing or visual based object following is possible and reliable for a great variety of objects. The capability of autonomous tracking and following of arbitrary objects is interesting by itself; because it can be directly applied to visual inspection among other civilian tasks. So far, this work has been a feasibility project to showcase the possibilities of visual servoing to operate in relatively spacious unknown outdoors environments in suburban areas. The proposed architecture addresses these objectives through the knowledgeable choice of robust reliable components, namely: the open-source object tracker OpenTLD (Kalal, 2011, Kalal et al., 2012), and the AR Drone 2.0 (see Fig. 3.1 left).

The Vertical Take-Off and Landing (VTOL) UAV platform used in the experimental work is the multirotor Parrot AR.Drone 2.0, shown in Figs. 3.1 \& 3.2 Recent research has demonstrated that the AR Drone is a realiable platform for VTOL UAV vision based navigation algorithm prototyping. For instance, the AR Drone has been used on the following research: autonomous navigation of hallways and stairs (Bills et al., 2011), visual SLAM based navigation (Engel et al., 2012) and reactive obstacle avoidance in natural environments (Ross et al., 2013).

The AR Drone 2 platform was selected for experimental testing of the presented work for multiple reasons: it is practically harmless to people and third-party objects, its ease of use thanks to its built-in flying modes, its low price, and its robustness to crashes. Since one of the main objectives of the presented research work was to perform person following from close distances, the chosen multirotor platform had to be safe to fly close to people.

This work is a continuation of previous work by the CVG group (Mondragoón et al., 2011) where 


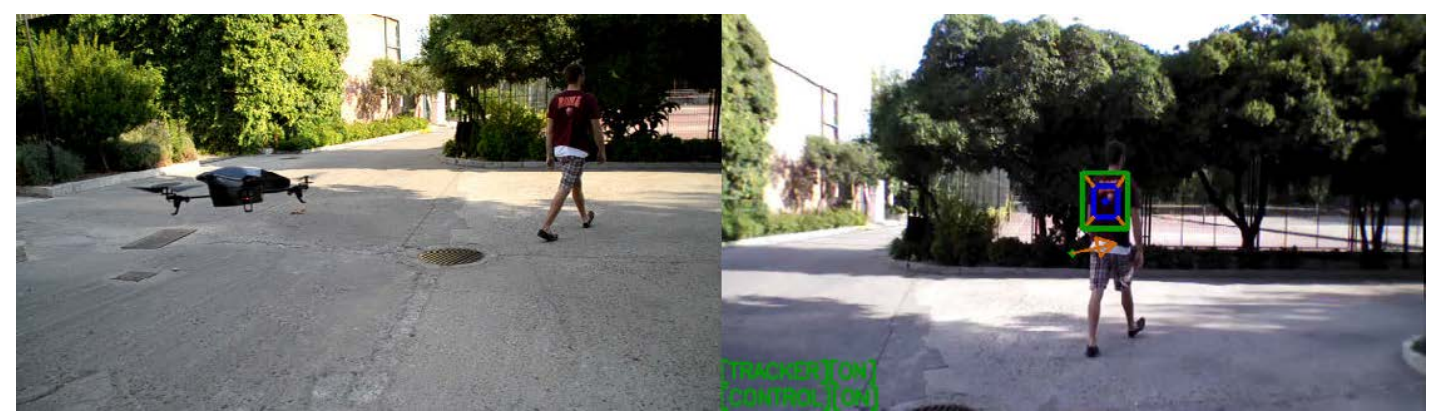

Figure 3.1: (left) Image of the AR Drone 2.0 during one of the object tracking and following experiments. The drone is equipped with the outdoors hull to improve the system's wind disturbance rejection. (right) Modified front image of the drone to show the controller (green) depth reference, (blue) feedback, and (orange) the altitude and lateral movement control error. The drone is controlled from an off-board computer through a WiFi link, and the target object is selected by the experimenter. The utilized tracker features on-line learning which causes problems if the target's tracked part includes background. This is the reason why, for person following tasks, a logo on the person's outfit is selected as the tracker's target.

visual based GPS-dependent object following was achieved using a more expensive multirotor. In addition to performing GPS-denied visual servoing the system presented in this chapter can follow a larger variety of objects.

\subsection{State of the Art}

Visual Servoing or Visual Servo Control is a group of techniques that allow accurate robot motion using a mixture of Computer Vision and Control Theory (Hutchinson, S et al., 2006, 2007). This means that vision data is acquired and processed to compute the commands executed by the robot. These visual data may be acquired from a camera on-board the robot, in the so called eye-in-hand setup, or from cameras fixed to the environment where the robot is operating and observing its movements, in the so called eye-to-hand configuration. These names are derived from the traditional visual servoing research, where manipulator robots were used as experimental platforms (P. I. Corke et al., 1996). For instance, the control of a multirotor by means of the data gathered by on-board cameras is a case of an eye-in-hand visual servoing configuration. Related to this topic, specific research is focused on "eye-in-hand" systems where the camera is fixed to a rigid body with actuated dynamics (Hamel et al., 2002a), which is very related to the multirotor visual servoing problem.

Works on Image Based Visual Servoing (IBVS) track visual features in the camera image, and pose the control problem determining the necessary camera motion to achieve a desired movement or position of the visual features. For instance, this image features may be the parallel lines of a road or specific points and texture on a followed object. Corke et al. (Mahony, R. et al., 2002) have shown that the selection of the tracked image features can affect the overall behavior of the robotic system, specially when several attitude and position variables are to be controlled, and that a knowledgeable combination of image features (Tahri et al., 2010) allows to attain decoupled image-based visual servoing. For instance, this is important for the motion along the depth axis that mainly controls the distance to a followed object. In this context, the desired behavior of a decoupled image-based visual servoing approach is to control the distance to the target without causing unnecessary movements in other directions. An appropriate set of image features can be selected in various manners, the following works show choices that have already been explored: image points can be used but the jacobian formulation must be selected with care (Chaumette, 1998), based on image moments on a group of points located on a planar surface of the target (Tahri and Chaumette, 2005a), the utilization of such image moments was studied by Tahri et al. with respect to their decoupling and linearizing capabilities (Tahri, 2004; Tahri and Chaumette, 2005b), distance between two points of a planar object and the orientation of the line that connects them (Feddema et al., 1989), the centroid of the followed target to control only the translational DOFs of a simulated helicopter (Hamel et al., 2002b), a comparison of the target following performance in the depth axis based on different image features (Mahony, P. Corke, et al., 2002), the usage of a spherical camera model (Fom- 
ena et al., 2007), projecting image features into the unit sphere and using them in the control law (Tahri, Chaumette, and Mezouar, 2008), and also even projecting the triangle surfaces between image features into the unit sphere (Tahri et al., 2009). Interestingly, recent research has included non-overlapping multicamera robotic systems (Comport et al., 2011).

The visual servoing strategy con be modified to avoid following non-straight or far-away image feature position references, by defining desired feature-paths using different techniques: including the nonlinear minimization methods to select the desired image feature path on the image plane based on a Hessian calculation (Lapresté et al., 2004, Malis, 2004, Tahri and Mezouar, 2010); a combination of path planning and visual servoing allows to perform longer camera displacements around the target while keeping the target in the field of view of the camera and respecting the joint limits of the robot (Chesi and Vicino, 2004; Mezouar et al., 2002), including big orientation reference changes (Schramm et al., 2007), and also to avoid obstacles during object tracking (Chesi, 2009).

Different research work has been done where a vision system with range sensors was used for Object Following tasks. On (Argyros et al., 2002) an omnidirectional visual system was used as a bearing sensor, while the distance to the target is measured using a range sensor, for control a robotic wheelchair on indoors. Others systems have been proposed only based on visual information for cooperative robotics (Korodi et al., 2008).

Visual information have been exploited on many projects involving UAVs, for instance to allow: fixed wing UAVs to fly at a constant altitude following a moving object on a ground planar surface (Fahd Rafi et al., 2006, Savkin et al., 2008), rotary wing UAVs to follow a terrestrial target (Deng et al., 2009, Lin et al., 2010), pose-based methods for autonomous landing on moving objects (Saripalli et al., 2007), and GPS dependent visual servoing for low altitude moving targets (Mondragoón et al., 2011). Also, recent work on visual based navigation using monocular SLAM methods for indoors environment has been achieved with promising results (Achtelik et al., 2011; Engel et al., 2012).

Recent research on Visual Tracking has been focused on online learning and detection algorithms (Babenko et al., 2009, Kalal et al., 2012). Such trackers have demonstrated to be able to track a wide variety of targets in real-time. When compared to prior research, the main advantage of the UAV Visual Servoing system proposed in this Thesis is that we use a tracker, OpenTLD, which allows to perform visual servoing with a large number of different targets. Our proposed architecture addresses these objectives through the knowlegeable choice of robust reliable components, namely: the open-source object tracker OpenTLD (Kalal, 2011; Kalal et al., 2012), and the AR Drone 2.0. Following arbitrarily chosen targets is a big improvement compared to following targets marked with blobs of different sizes, which is a commonly used target to measure the performance of visual servoing controllers, see for instance (Bourquardez et al., 2009) or (Espiau et al., 1992), or when compared to following balloons using color based tracking (Mondragoón et al., 2011; Zhang et al., 1999). However, our architecture is not able to estimate the depth at which the target is located as in (Mahony, Brasch, et al., 2005), or the relative attitude of the target with respect to the drone, as in (Martínez et al., 2013).

Other works have focused on developing image trackers designed with UAV object following in mind. Teuliere et al. (Teuliere et al., 2011) propose using a color based tracker using a multi-part representation of the followed target using particle filter based estimation. Danelljan et al. (Danelljan, Khan, et al., 2014; Danelljan, Shahbaz Khan, et al., 2014) used a novel color based tracking method and a combination of state of the art filtering and Artificial Intelligence (AI) algorithms to achieve person following and multiperson visual tracking. Haag. et al. (Haag et al., 2015) proposed a novel visual-based tracker to follow a person and tested it on various following scenarios, like walking at different speeds or riding a bike.

Road following and corridor navigation tasks by UAVs have also received attention. A commonly referenced work for including computer vision directly in a control loop where only visual features are used as measurements and control references was done by Espiau et al. (Espiau et al., 1992), which included experiments on target and road following. A road following task by an airship which follows the horizon line and a vanishing point on the image plane was proposed by Rives et al. (Rives et al., 2004). Bills et al. (Bills et al., 2011) proposed using a CV classifier to determine the type of indoor environment where an quadrotor is navigating, and then used this information to control the UAV based on perspective cues specifically tailor to the detected environment such as the perspective vanishing point. Ross et al. (Ross et al., 2013) used visual cues for achieving relatively safe UAV flight in forests by avoiding trees on the direction of navigation using a reactive control law. 


\subsection{System Description}

Our system consists of several modules which communicate with each other under the Robot Operating System (ROS) framework (Quigley et al., 2009). The main modules of our system are an object tracker and an Image Based Visual Servoing IBVS controller. As shown in Fig. 3.3, the AR.Drone 2.0 is commanded from a computer via WiFi link using the ardrone_autonomy ROS package (Mani Monajjemi, 2012).

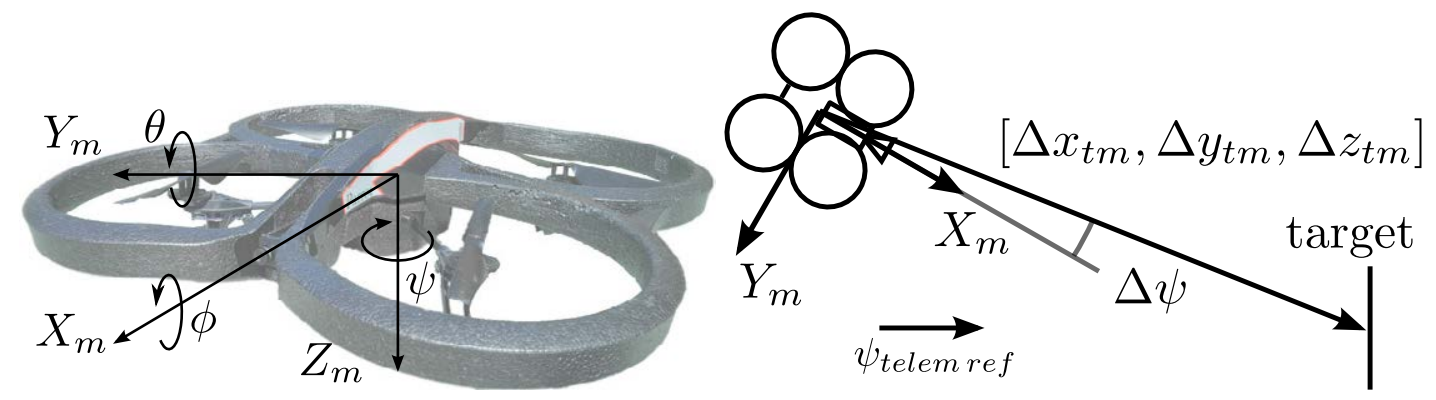

Figure 3.2: (left) Parrot AR.Drone 2.0 and its body reference frame, $\left\{X_{m}, Y_{m}, Z_{m}\right\} . X_{m}$ points towards the front of the vehicle, $Y_{m}$ points towards the right and $Z_{m}$ points downwards, obtaining an orthonormal right-handed reference frame. The attitude is defined using Euler angles, which are denoted $\{\phi$, roll $\}$, $\{\theta$, pitch $\}$ and $\{\psi$, yaw $\}$.

(right) While performing the visual servoing tasks the drone tracks the target from a constant distance. The target's relative position, $\left[\Delta x_{t m}, \Delta y_{t m}, \Delta z_{t m}\right]$, is estimated from the image feedback using an expected target's size value, $A_{\text {exp }}$. $\psi_{\text {telemref }}$ is a variable internal yaw reference which specifies the prefered relative tracking direction.

The following is a brief description of each of the modules:

1. Object tracker: our software is currently using a $\mathrm{C}++$ open-source implementation of the OpenTLD tracker (Nebehay, 2012). The OpenTLD tracker was originally developed by Z. Kalal at the University of Surrey during his PhD Thesis (Kalal, 2011; Kalal et al., 2012). The repositories related to this library can be found in (Kalal, Z, 2011; Nebehay, G, 2012; Chauvin, R, 2012).

OpenTLD can robustly track objects in the drone's video stream without any previous knowledge of the target. The tracker feeds back a bounding box (location, height and width) around the tracked object along with a confidence ratio. OpenTLD was tested during flight with a great variety of objects, see the objects shown in Figs. 3.6 \& 3.7 \& 3.11 \& 3.14. The only constraints that were determined to be important to get high repeatability during testing were that: the tracker's learning feature has to be switched off to enable the target occlusion capability, and small targets require the bounding box not to include any background.

2. IBVS controller: the controller closes four feedback loops based on three image features, which are the target's bounding box centroid and size, see Fig. 3.4 The references to the controller are its desired values. The resulting system's behavior is that the drone will turn to look at the target and approximately control its relative position with regards to it.

As a result of the above mentioned system architecture, the sensor information required during the experiments is, see Fig. 3.4.

1. During successful object tracking: the built-in operation of the drone requires, at all times, to use the IMU and the ultrasound altitude sensor. Additionally, our off-board software uses the front camera image and part of the IMU telemetry data. Since the AR Drone is executing its internal "flying mode", it does not use optical flow based speed estimation during the execution of this operation mode of our architecture. 


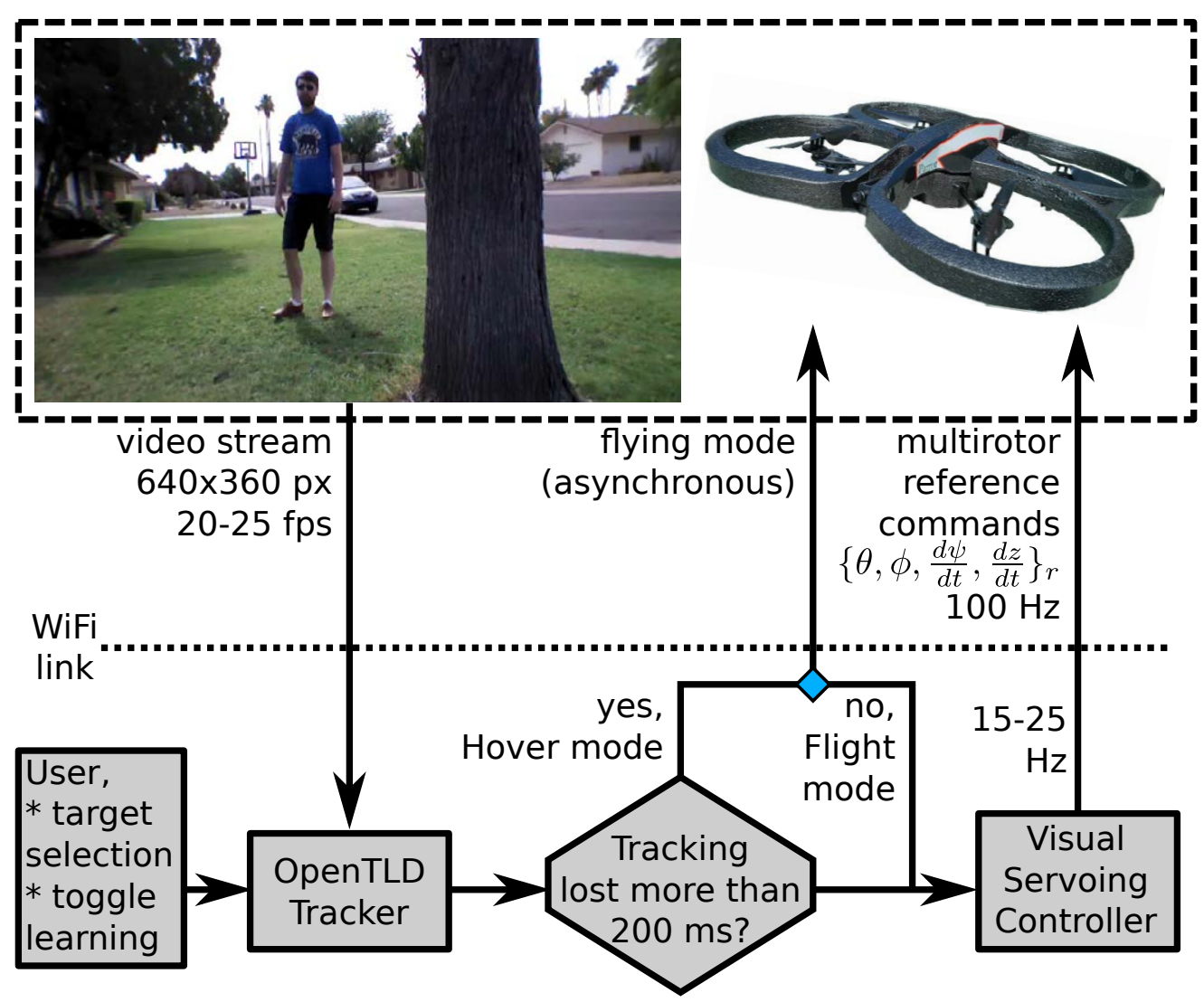

Figure 3.3: System overview, the AR.Drone 2.0 is commanded from a computer over a WiFi link. The main components of the system are: the drone, the drone driver (ardrone_autonomy ROS package), the image rectification stage, the object tracker (OpenTLD tracker) and the controller.

2. Whenever the object tracking is lost or when the object is out of the image frame: the AR Drone 2.0 is automatically commanded to enter the internal "hovering mode". As a result, the AR Drone uses the on-board optical flow speed estimation to self-stabilize.

\subsubsection{Image Based Visual Servoing (IBVS) Controller}

An overview of the system focused on the description of the visual servoing controller is shown in Fig. 3.4 The tracker provides the horizontal, $x_{b b}$, and vertical, $y_{b b}$, location of the upper-left corner, and the width, $w_{b b}$, and height, $h_{b b}$, of the target on the image plane. The image features that are provided as feedback to the controller are calculated as follows, see Eq.3.1. and Figs. 3.2 \& 3.4.

$$
\begin{aligned}
f_{u} & =\frac{x_{b b}+\left(w_{b b} / 2\right)}{w_{i m}} \\
f_{v} & =\frac{y_{b b}+\left(h_{b b} / 2\right)}{h_{i m}} \\
f_{\Delta} & =\sqrt{\frac{w_{i m} \cdot h_{i m}}{w_{b b} \cdot h_{b b}}} \propto x_{t m}
\end{aligned}
$$

Note that the image feature $f_{\Delta}$ is approximately proportional to $x_{t m}$, the frontal distance from drone to target, which results in better visual servoing performance (Tahri et al., 2010).

The utilization of a fixed camera on a visual servoing multirotor system naturally couples the controlled variables through the target's movement on the image plane, which has required the design of 


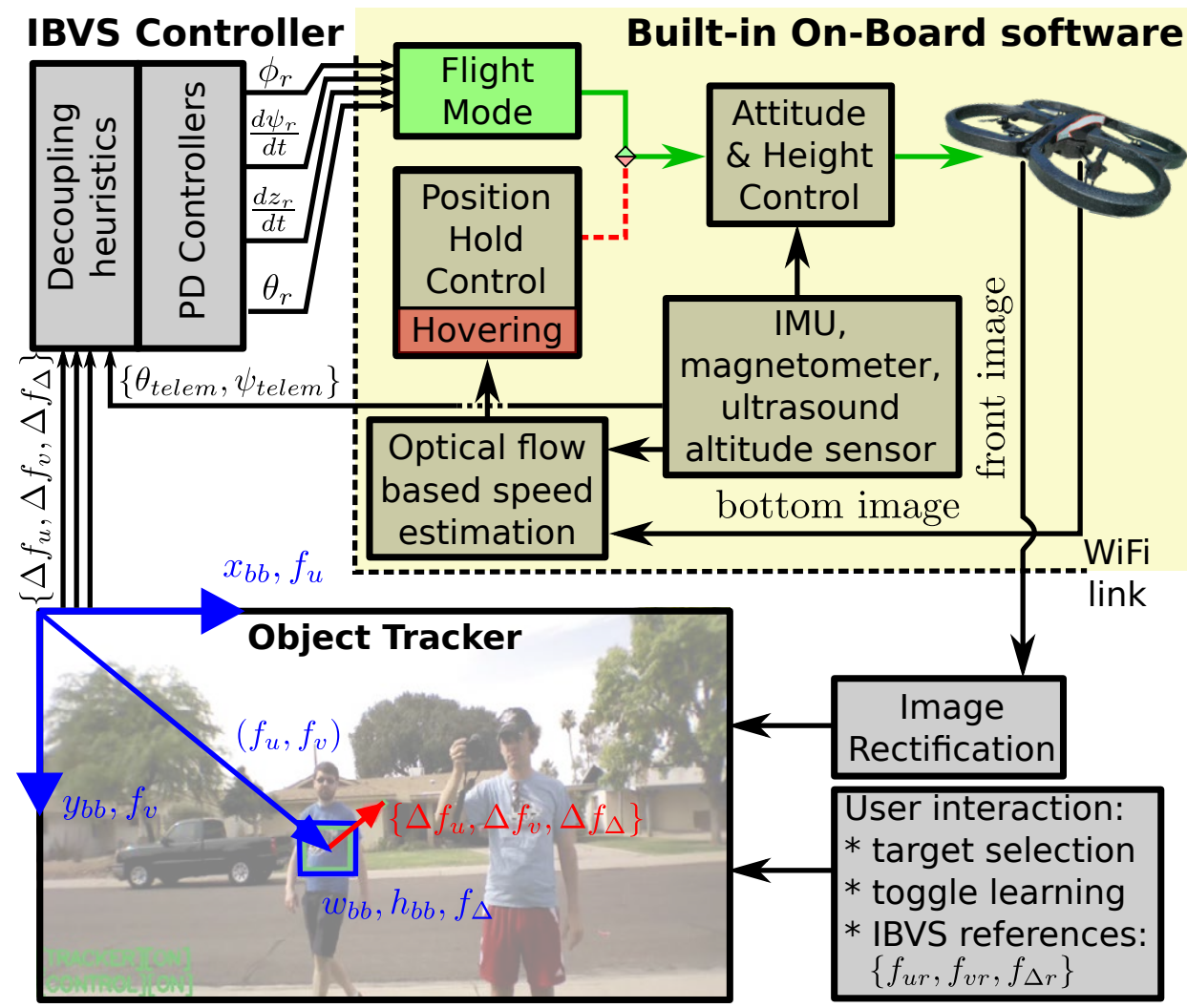

Figure 3.4: Diagram of the Image Based Visual Servoing (IBVS) Controller. Since the tracker is working properly at this moment, the drone is operating under the flight mode and following the drone reference commands that the off-board computer is sending via WiFi. During flight mode, the optical flow speed estimation is unused. The figure shows the image features utilized by the controller, which are: $\left\{f_{u}, f_{v} \in[0,1]\right\}$ related to the centroid position and $\left\{f_{\Delta}>0\right\}$ to the size of the bounding box.

decoupling control heuristics for the altitude and lateral movement degrees of freedom. Thus, the correction shown on Eq. 3.2 on the tracking error is calculated, which greatly decreases the mentioned DOF couplings. The terms related to the decoupling control heuristics for the image features and the DOFs of the quadrotor are the two terms specified on the right side of Eq. 3.2 .

$$
\begin{aligned}
& \Delta f_{u \psi}=\Delta f_{u} \\
& \Delta f_{u y}=\Delta f_{u}-\frac{\psi_{\text {telemref }}-\psi_{\text {telem }}}{F O V_{u}} \\
& \Delta f_{v z}=\Delta f_{v}-\frac{\theta_{\text {centroidref }}-\theta_{\text {telem }}}{F O V_{v}} \\
& \Delta f_{\Delta x}=\Delta f_{\Delta}
\end{aligned}
$$

$\theta_{\text {telem }}$ and $\psi_{\text {telem }}$ are the pitch and yaw angles of the vehicle respectively, as shown in Fig. 3.2, which are obtained from the AR Drone telemetry data. $F O V_{u}$ and $F O V_{v}$ denote the horizontal and vertical field of view of the camera. $\psi_{\text {telem ref }}$ is a yaw heading reference which is updated to $\psi_{\text {telem }}$ everytime that the drone is commanded to enter the Flight Mode or whenever $\left|\psi_{\text {telemref }}-\psi_{\text {telem }}\right|>25^{\circ} . \theta_{\text {centroidref }}$ is always 0.0 , and $\theta_{\text {telem }}$ is obtained from the drone's telemetry data.

\subsubsection{IBVS PD Controller Parameters Breakdown}

In this subsection the controller gains are decomposed, see Fig. 3.5. in parameters related to various system components: process of image formation on the camera, a previously tunned position controller; 


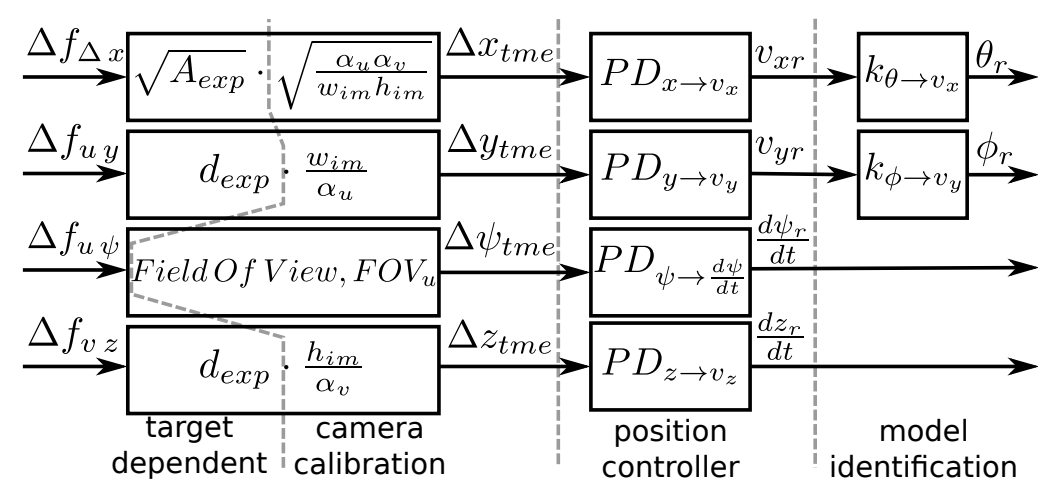

Figure 3.5: IBVS Controller Breakdown, the PD controllers shown in Fig. 3.4 can be decomposed to show how they relate to a regular position controller. The shown parameters come from: the camera parameters, the expected target size $A_{\exp }$ and distance to the target $d_{\text {exp }}$, the position controller gains and the aerodynamic friction of the vehicle. The obtained PD gains are: $\left\{P D_{f_{u} \rightarrow y_{t m}}, K p=-0.298, K d=-0.145\right\},\left\{P D_{f_{u} \rightarrow \Delta \psi}, K p=-0.990, K d=-0.119\right\}$, $\left\{P D_{f_{v} \rightarrow z_{t m}}, K p=1.430, K d=0.371\right\},\left\{P D_{f_{\Delta x} \rightarrow x_{t m}}, K p=0.0254, K d=0.0124\right\}$, for the following AR Drone command gains configuration: $\left\{\theta_{r}=1 \rightarrow 12^{\circ}\right\},\left\{\phi_{r}=1 \rightarrow 12^{\circ}\right\},\left\{\frac{d \psi_{r}}{d t}=1 \rightarrow 100 \frac{\mathrm{deg}}{\mathrm{s}}\right\},\left\{\frac{d z}{d t}=1 \rightarrow\right.$ $\left.1 \frac{\mathrm{m}}{\mathrm{s}}\right\}$.

and the stationary relationship between speeds and drone reference commands. This decomposition allows to understand the effect that each parameter can have on the overall behaviour of the system during the Visual Servoing task.

The meaning of the the constants in Fig. 3.5 is:

- The target dependent parameters are the size of the tracked object's target surface, $A_{\text {exp }}=40 \times 30 \mathrm{~cm}$; and the expected distance to the target $d_{\exp }=3 \mathrm{~m}$.

- The camera dependent parameters are: the image resolution along width $w_{i m}$ and height $h_{i m}$; the focal length measured in pixels $\alpha_{u}, \alpha_{v}$; and the horizontal field of view of the camera, $F O V_{u}$, obtained from the rectified image projection matrix $P$, see Eq. 3.3

$$
P=\left[\begin{array}{cccc}
\alpha_{u} & 0 & u_{0} & 0 \\
0 & \alpha_{v} & v_{0} & 0 \\
0 & 0 & 1 & 0
\end{array}\right]
$$

- $\left\{\Delta x_{t m e}, \Delta y_{t m e}, \Delta z_{t m e}, \Delta \psi_{t m e}\right\}$ is the estimated relative position of the target with respect to the drone, expressed in the drone's reference frame, as shown in Fig. 3.2 $\left\{\Delta v_{x m r}, \Delta v_{y m r}\right\}$ are speed commands in the same reference frame.

- $P D_{\text {var }_{1} \rightarrow v a r_{2}}$ are the PD controllers of an already tunned position controller which outputs speed commands. For the experimental work the position controller presented in (Pestana, MelladoBataller, et al., 2013), and Sec.2, was used.

- $\left\{k_{\theta \rightarrow v_{x}}, k_{\phi \rightarrow v_{y}}\right\}$, are the static relationship between the multirotor tilt and the attained horizontal steady-state speed. In multirotors this constant is related to the aerodynamic profile of the vehicle. Using the methodology presented in (Pestana, 2012), and Appx. D for the AR Drone with the outdoors hull, they were estimated to be $k_{t i l t \rightarrow v_{x y}} \approx 5-7 \frac{\mathrm{m} / \mathrm{s}}{24^{\circ}}$ for angles lower than $12^{\circ}$.

- $\left\{\theta_{r}, \phi_{r}, \frac{d \psi_{r}}{d t}, \frac{d z}{d t}\right\}$ are the reference commands, Fig. 3.4 


\subsection{Experiments and Results}

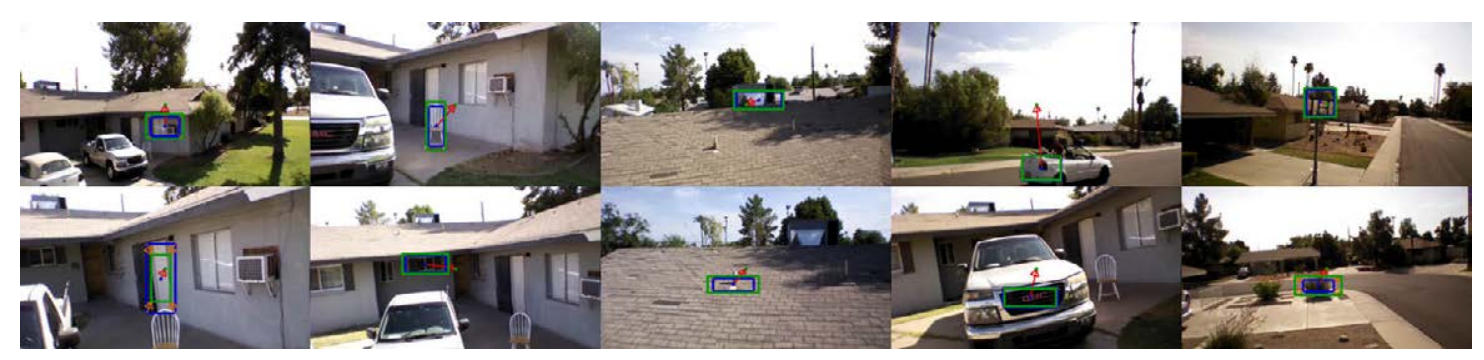

Figure 3.6: Selection of on-board camera images showing targets upon which our system was tested: (house elements) a window with an AC machine, a chair, a door and a small window; (roof elements) AC machinery on the roof and a roof part; (car elements) a moving car and a car logo; (street elements) a basketball basket and a plant. The red arrow shows the target centroid tracking error on the image plane.

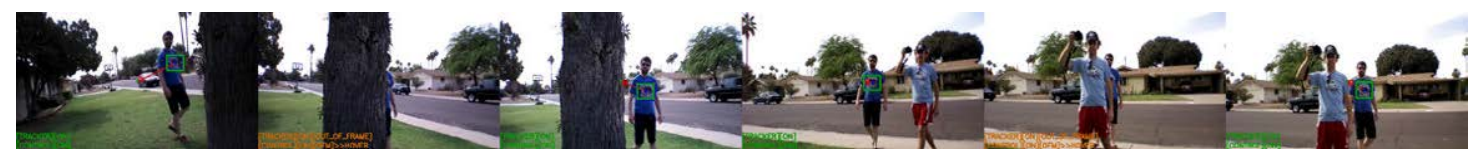

Figure 3.7: Selection of on-board camera images, showing a person following test and how the system handles target occlusion. When target occlusion occurs the AR Drone 2.0 starts hovering until the target is detected again, and then it proceeds with the visual servoing task. The first three images show target occlusion by a tree, and the second three images show occlusion by another person. The learning feature of the tracker is used at the beginning of every experiment and then it is switched off. Otherwise the tracker may not be able to recognize the occlusion event appropriately.

The main focus of the experimental work was to demonstrate that our system performs the object following task, as described in Sec. 3.3. successfully. Note that the presented architecture does not rely on GPS at any level. A series of real-world experiments were conducted on suburban areas, of which experimental flight videos can be watched online at the ASTRIL lab website: http://robotics.asu.edu/ardrone2_ibvs/1 The available videos show: two tests where the target matches the $A_{\text {exp }}$ and $d_{\text {exp }}$ parameters used to calculate the controller gains (Fig. 3.5); testing against various objects present on suburban areas; a car and a person following tests along suburban area streets; two tests where people were followed from close distances showing occlusion handling; the videos in the section 3.3 of the website show people following tests where the indoors hull was used and without using the decoupling heuristics including the test presented in Sec. 3.4.4, the videos in the section 3.5 of the website show people following tests where the outdoors hull and the decoupling heuristics were utilized. including the test presented in Sec. 3.4 .5 .

Various tests were performed to ascertain what kind of objects could be tracked visually. A selection of images acquired during test flights is shown in Fig. 3.6. The selected targets ranged from a quarter of the tunned size to more than ten times the tunned target surface, $A_{\text {exp }}$. The drone was able to visually track all these targets even when the objects were at a distance, relatively far from the preconfigured expected visual tracking distance, $d_{\text {exp }}$.

A second battery of tests was performed to showcase moving object following, mainly including people following and some car following tests. For small moving targets, such as logos on people's tshirts, the best performance was achieved when no background is included in the tracked object bounding box. However, for big moving targets, the bounding box can be chosen including some background and the tracker and system will still work successfully. The reason for this is that big targets tend to evolve slowly in the image plane, which accounts for the tracker's better performance.

\footnotetext{
${ }^{1}$ Otherwise, https: / / www $\cdot$ youtube.com/playlist? list=PLdjvAEULWOEHq_jwxQkd5RXC4qvGMLRQb
} 
People following was highly successful. As shown in Fig. 3.7, our solution can handle occlusion by objects such as trees and also by other people. In this kind of situation, the system will automatically switch to hovering mode until the target is detected again; and then it will proceed with the visual servoing task. On these experiments, the learning feature of the tracker was switched off after a 10-30 seconds period of learning. Occlusion handling was highly degraded if the tracker still had the learning feature enabled.

The flight time, when including target loss, second detection, etc; can reach battery depletion provided that the outdoors environment is spacious.

\subsubsection{Videos and Dataset of Flight Experiments}

The videos of the experiments can be watched online at the ASTRIL lab website http://robotics.asu.edu/ardrone2_ibvs/; or directly in YouTube and Vimeo in the following links:

- Playlist with all the videos:

- https://wWw.youtube.com/playlist?list=PLdjvAEULWOEHq_jwXQkd5R XC 4 qVGMLRQb

- Overview video showing how the drone can follow different targets:

- https://youtu.be/ruboUgHM-PQ

- https://vimeo.com/71961365

- the videos in the section 3.5 of the website show people following tests where the outdoors hull and the decoupling heuristics were utilized, including the test presented in Sec. 3.4.5.

- https://youtu.be/67RXf5P7bfg

- https://youtu.be/MkQOlZdQn0Q

- https://vimeo.com/75887484

- https://vimeo.com/76358967

- two tests where people were followed from close distances showing occlusion handling:

- https://youtu.be/aKIdJLnJK_A

- https://youtu.be/JcEXlozOzbI

- https://youtu.be/9uzCmyVJzow

- https://youtu.be/G-95g_5EUB0

- https://vimeo.com/72177117

- https://vimeo.com/72177116

- https://vimeo.com/72177118

- https://vimeo.com/72177115

- a car and a person following tests along suburban area streets:

- https://youtu.be/I-Y0yisBI4Y

- https://youtu.be/PKs5f3taLW0

- https://youtu.be/c9oIdGefyVU

- https://youtu.be/Ansxfhvs12g

- https://vimeo.com/71953201

- https://vimeo.com/71960163

- https://vimeo.com/71960162

- https://vimeo.com/71960164 
- testing against various objects present on suburban areas:

- https://youtu.be/JbvaEupa_jA

- https://youtu.be/JKsHZSP2ntw

- https://vimeo.com/71953202

- https://vimeo.com/71959032

- two tests where the target matches the $A_{\exp }$ and $d_{\exp }$ parameters used to calculate the controller gains (Fig. 3.5):

- https://youtu.be/K9uKbp_KEbs

- https://youtu.be/FTUMDGZYm4E

- https://youtu.be/jE5_AMT3BIo

- https://vimeo.com/71952027

- https://vimeo.com/71952028

- https://vimeo.com/71887823

- Other videos:

- Flight with Asctec Pelican quadrotor: https://youtu.be/24x20ed91Ps

- In the Spanish Antena 3 news: https://youtu.be/0Y8Ikv6bor4

The images and telemetry data from these experiments are available, for research purposes, here:

- In the CVG-UPM ftp (user:publicftp , pass:usuarioftp): ftp://138.100.76.91/CVG_PUBLIC/DataSets/IBVS_ardrone2_datasets/

- In google drive: http://bit.ly/1sp3qxG 


\subsubsection{Qualitative performance evaluating navigation following suburban house elements - without decoupling control heuristics}

In this experiment we show how the proposed visual servoing architecture is reliable enough to follow multiple static targets in the front of a suburban house. A sequence of on-board images of this experiment is shown in Fig. 3.8. In this case, since the targets are static, the background changes slower than in other experiments allowing to keep the learning of the OpenTLD tracker activated during the experiment. The drone flew quite stable while switching from one object to the next in the sequence. Only in some parts of the flight the coupling between pitch and vertical movement caused the drone to oscillate, but never resulting in a crash. This is partly thanks to the experience of the experimenter, who switches to hovering in such cases. The conclusions about this experiment and the next one are given in Sec. 3.4.3. 

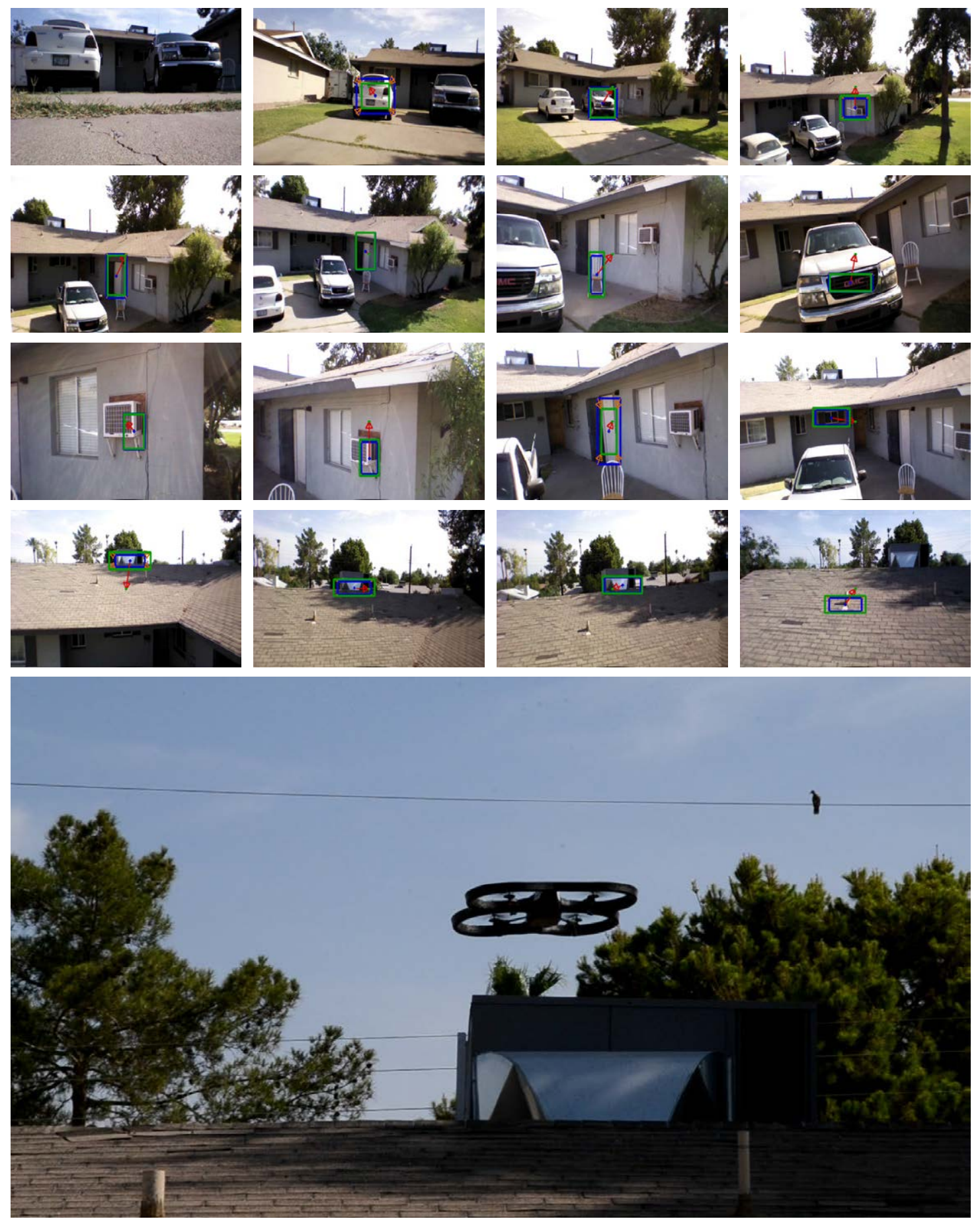

Figure 3.8: Selection of on-board camera images from an experiment testing the presented architecture for the navigation following suburban house elements. The followed objects are: a car, a second car, a window with an AC system on the side, a door, a chair, a car brand symbol, an AC system, a door, a window, a second $\mathrm{AC}$ system and a roof element. Afterwards the landing was performed by commanding the drone manually using a computer interface. Video: https://youtu.be/JbvaEupa_jA. 


\subsubsection{Qualitative performance evaluating navigation along a street in a suburban area - without decoupling control heuristics}

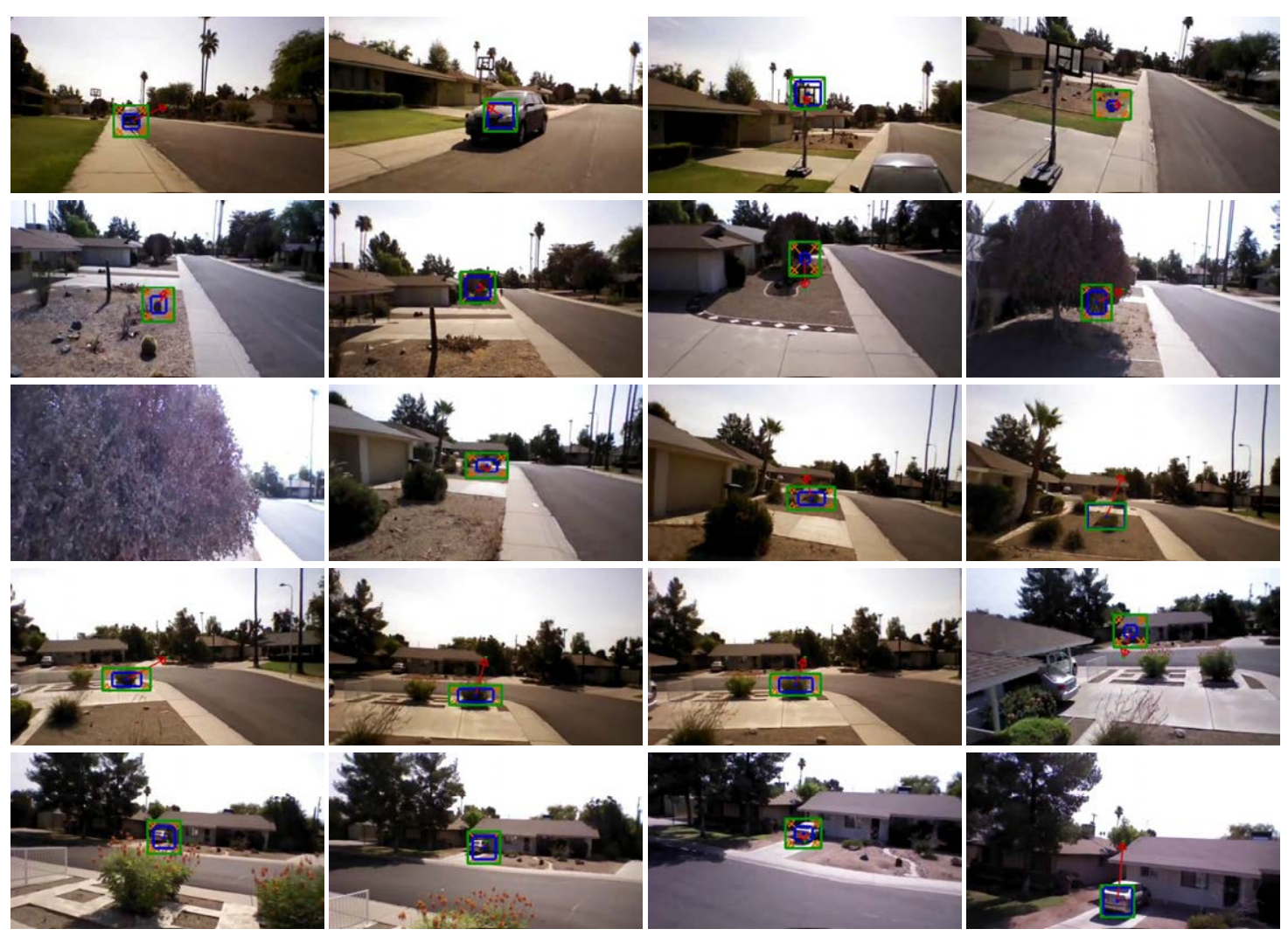

Figure 3.9: Selection of on-board camera images from an experiment testing the presented architecture for the traversal of suburban streets by following subsequent targets. The followed objects are: a car, a basketball basket, a plant, a tree, three more plants and a car. After approaching the tree the user needed to command the drone to move right, to get the tree out of sight because it was blocking most of the camera view. Otherwise the flight was performed by commanding the drone to follow one target after the other. The flown distance in this experiment is in the 150-200 $\mathrm{m}$ range.

Video: https: //youtu.be/JKsHZSP2ntw.

In this experiment we show how the proposed visual servoing architecture is reliable enough to follow multiple static targets along a street. The drone moved along the street for around 150-200 $\mathrm{m}$, while following a sequence of user defined targets, listed in the caption of Fig. 3.9 On-board images together with pictures of the drone during flight are shown in Fig. 3.10 In this case, since the targets are static, the background changes slower than in other experiments allowing to keep the learning of the OpenTLD tracker activated during the experiment. The drone flew quite stable while switching from one object to the next in the sequence. Only in some parts of the flight the coupling between pitch and vertical movement caused the drone to oscillate, but never resulting in a crash. This is partly thanks to the experience of the experimenter, who switches to hovering in such cases. Otherwise, the only other user interaction with the system was required after approaching a tree, where the user commanded the control to move right to get the tree out of sight because it was blocking most of the camera view.

Studying the results from this experiment, Sec. 3.4.3, and the prior one, Sec. 3.4.2, we showed how the proposed system as a whole is robust to temporary losses of the visual tracking. This fact is provided by the flying mode switching strategy and by the reliability of the AR Drone 2.0 hovering mode. The OpenTLD algorithm has shown to be very reliable for target tracking and detection of many objects that were found in front of a suburban house and along a suburban street. The drone, using the proposed visual servoing architecture, was able to traverse the street successfully. 

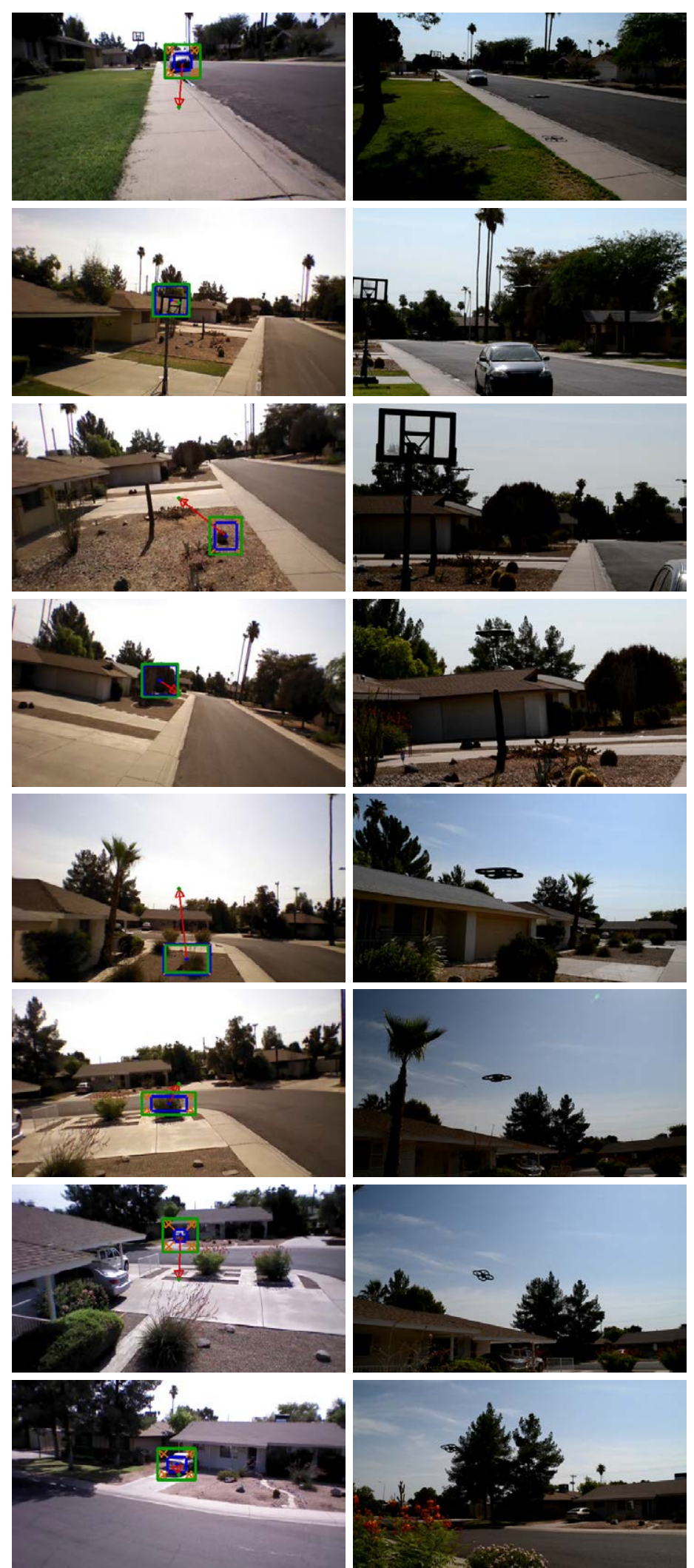

Figure 3.10: Selection of on-board camera images from an experiment testing the presented architecture for the traversal of suburban streets by following subsequent targets. (left) On-board image (right) Approximately corresponding image of the drone during the flight experiment.

Video: https://youtu.be/JKsHzSP2ntw. 


\subsubsection{Quantitative performance during a person following task - without decou- pling control heuristics}

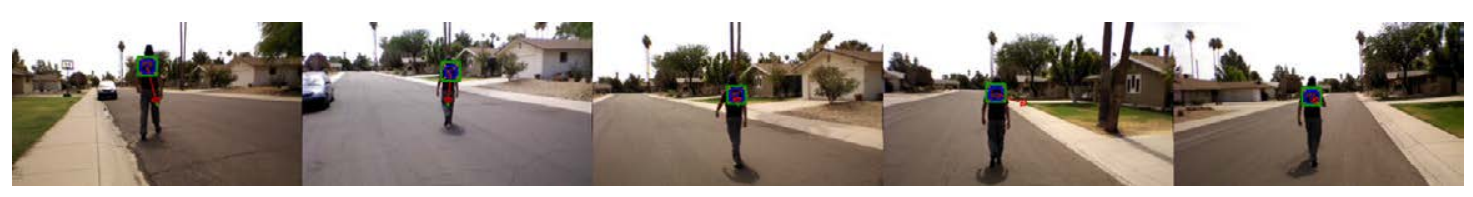

Figure 3.11: Selection of on-board camera images from another person following test, which is explained in subsection 3.4.4 The drone follows a person along a street in a suburban area, the experiment lasted 1.5 minutes and the tracking had to be recovered by hand only once.

Video: https: / /youtu.be/PKs5f3taLW0.

The experimental work presented in this subsection was published in (Pestana, Sanchez-Lopez, Campoy, et al., 2013). The experimental test corresponding to the images shown in Fig. 3.11 was selected to showcase the performance of our Visual Servoing controller. The test lasted for about 1 minute and 30 seconds, where the drone followed a person in a suburban area. The main variables of the controller are plotted on Figs. $3.12 \& 3.13$.

Figs. 3.12 \& 3.13 show the main variables of the controlled system and show an overall succesful behaviour. There is no noise in the image features, the drone commands or the attitude and altitude of the drone. The coupling between pitch and altitude through the $f_{y}$ image feature is noticeable. The reason why there are big variations on $f_{y}$ is that this image feature is tightly coupled to the vehicle's pitch, because the camera is fixed to the vehicle's body and the pitch commands are required to follow the moving target. $f_{y}$ variations are then introduced in the altitude speed command through the calculations of the PD altitude controller. The yaw commands dominate $f_{x}$, as demonstrated by the fact that the roll commands are near zero during all the 30 seconds, see Fig. 3.16 The tracking is lost for a short moment at about $t=24 \mathrm{~s}$, and the visual servoing task resumes immediately.

The size of the target was about $22 \times 25 \mathrm{~cm}$, which is 2.2 times smaller than expected $A_{\text {exp }}$. As shown in Fig. 3.5, this affects the estimate of the distance to the target. So a more correct estimate, on plot Fig. 3.15. $f_{\Delta}$, would be that the target stayed $\operatorname{sqrt}(2.2)=1.47$ closer than shown; at about 3.4-5 m.

This flight, shown in Figs. $3.11 \& 3.12 \& 3.13$ lasted one more minute. After that point the tracking was lost due to the tracker increasingly learning the background and finally loosing the target. After this event, the main target was reentered by hand again and the vehicle successfully followed the target along the street for another 30 seconds. The total flight distance was about 90-110 m, with no user interaction other than target selection. These values allow us to estimate that the drone had to move at an average forward velocity of $1.5 \mathrm{~m} / \mathrm{s}$.

As discussed on this chapter and supported by the experimental videos, the system as a whole has demonstrated to be robust to temporary loss of the visual tracking. This fact is provided by the flying mode switching strategy and by the reliability of the AR Drone 2.0 hovering mode. The system has also been shown to be able to perform visual servoing on a great variety of targets, with robustness to low winds and no dependence on GPS. 

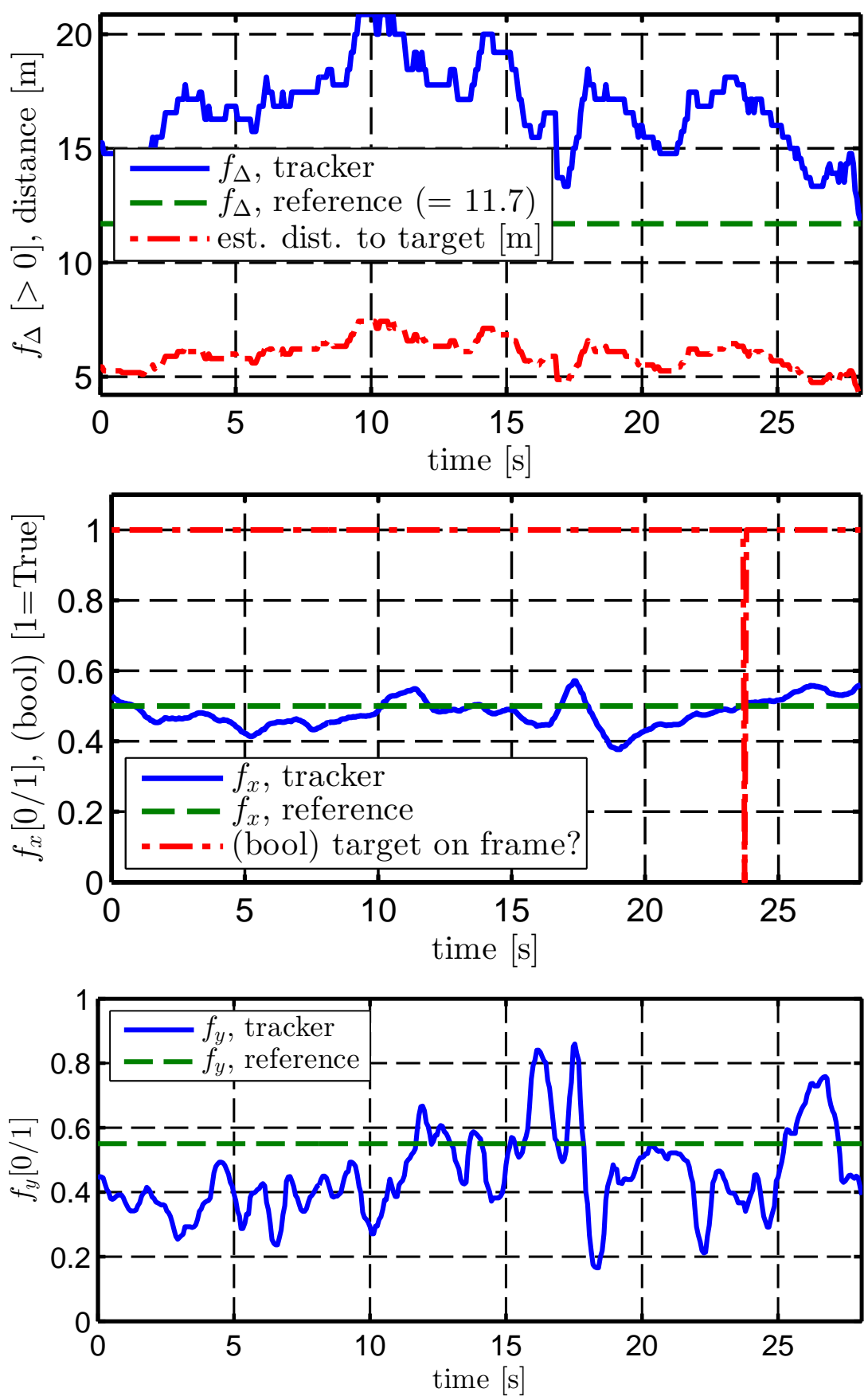

Figure 3.12: Measured and reference values of the image features during a 30 seconds period of the experiment shown in Fig. 3.11 The $f_{\Delta}$ plot shows that the distance to the target varies slowly and it was estimated to be on the 5-7.5 m range, due to the target being 2.2 times smaller than expected; the real distance was about sqrt (2.2) smaller, thus, in the 3.4-5 m range. Note that the object tracker does not estimate $f_{\Delta}$, the size of the target's bounding box, smoothly. $f_{x}$ has been well controlled around the reference value; its graph also shows (red, dash-dot line) that the tracking was lost for a very small period of time once. The reason why there are big variations on $f_{y}$ is that this image feature is tightly coupled to the vehicle's pitch, because the camera is fixed to the vehicle's body and the pitch commands are required to follow the moving target. 

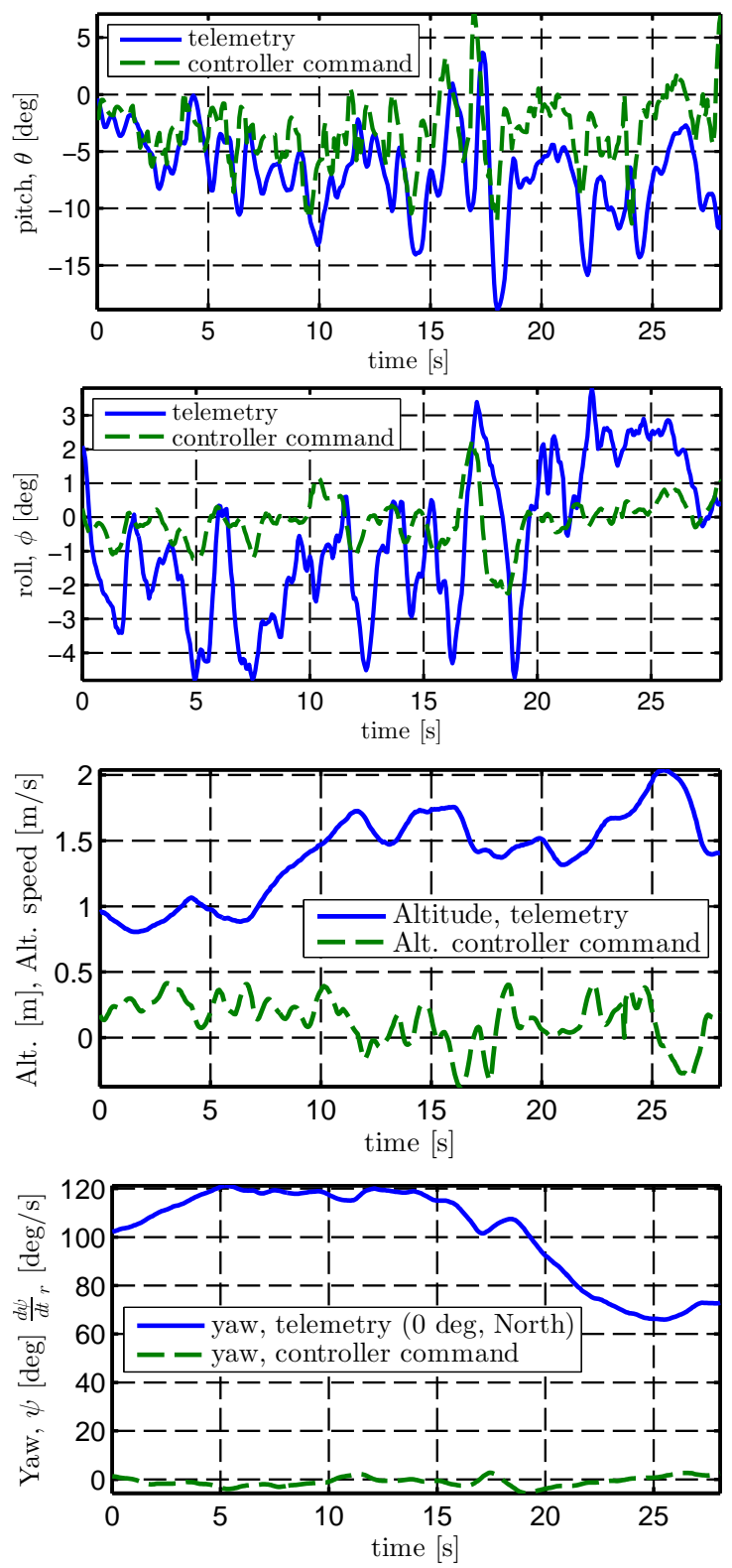

Figure 3.13: Controller commands during a 30 seconds period of the experiment shown in Fig. 3.11 As shown, none of the commands have a noticeable level of noise. The pitch command has a negative average because the drone is following a person and it has to move forward. The roll command is approximately zero, as explained on the article the roll command is used just to stabilize the vehicle laterally. The drone stayed at an altitude of 1 to $2.0 \mathrm{~m}$ during the experiment, as measured by the ultrasound altitude sensor. The altitude speed command suffer from the influence of $f_{y}$ on the pitch commands, because $f_{y}$ is the image feature used to feedback the altitude controller. The yaw plot shows a constant heading while crossing the street and then it changes when the drone moves parallel to the street. 


\subsubsection{Quantitative performance during a person following task - with decoupling control heuristics}

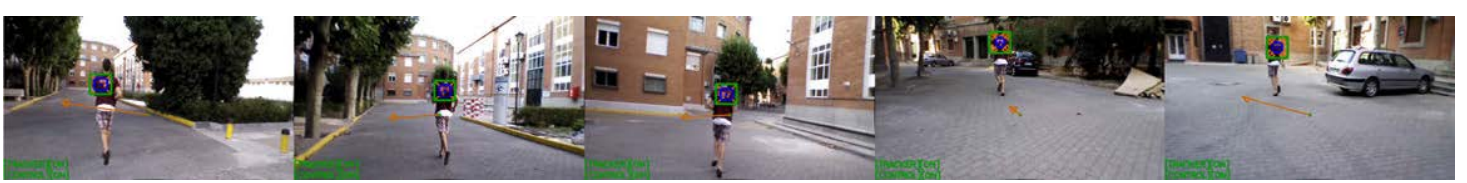

Figure 3.14: Selection of on-board camera images from another person following test, which is explained in subsection 3.4.5. The robot tracks and follows a person along a street corridor in a suburban area. The experiment lasted 45 seconds where the AR Drone 2.0 covered a distance of about $120-140 \mathrm{~m}$, thus navigating at an average speed of 2.65-3.10 m/s. The orange arrow shows the decoupled altitude and lateral movement control actions. Video: https://youtu.be/67RXf5P7bfg.

The experimental work presented in this subsection was published in (Pestana, Sanchez-Lopez, Saripalli, et al., 2014). The experimental test corresponding to the images shown in Fig. 3.14 was selected to showcase the performance of our Visual Servoing controller. First, the tracker was trained to learn the target. Then, the learning feature of the tracker was switched off before the experimenter started running. The run lasted 45 seconds where the AR Drone 2.0 covered a distance of about $120-140 \mathrm{~m}$. Thus, the drone navigated at an average speed of 2.65-3.10 m/s. Thus far, these values show the peak performance of our IBVS controller.

The Figs. 3.15 \& 3.16 show the main variables of the controlled system during the experiment. There is low noise in the controlled image features, the drone commands and the attitude and altitude of the drone. In spite of the decoupling heuristics, the coupling between pitch and altitude through the $f_{v}$ image feature near the end of the test is still noticeable. The reason why there are big variations in $f_{v}$ is that this image feature is tightly coupled to the vehicle's pitch, because the camera is fixed to the vehicle's body and the pitch commands are required to follow the moving target. $f_{v}$ variations are then introduced in the altitude speed command through the calculations of the PD altitude controller. Another improvement that was introduced lately in our controller was the utilization of the outdoors hull and the yaw-lateral movement decoupling heuristic. Again, it is difficult to quantify the wind disturbance rejection improvement in any other way than investigating the external videos of the tests, which in our opinion show a clear improvement.

As discussed in this chapter and supported by the experimental videos, the system as a whole has demonstrated to be robust to temporary loss of the visual tracking. This fact is provided by the flying mode switching strategy and by the reliability of the AR Drone 2.0 hovering mode. The OpenTLD algorithm, to the extent of our limited group of experiments, has shown to be very reliable for target tracking and detection and it has only rarely detected a wrong object. 

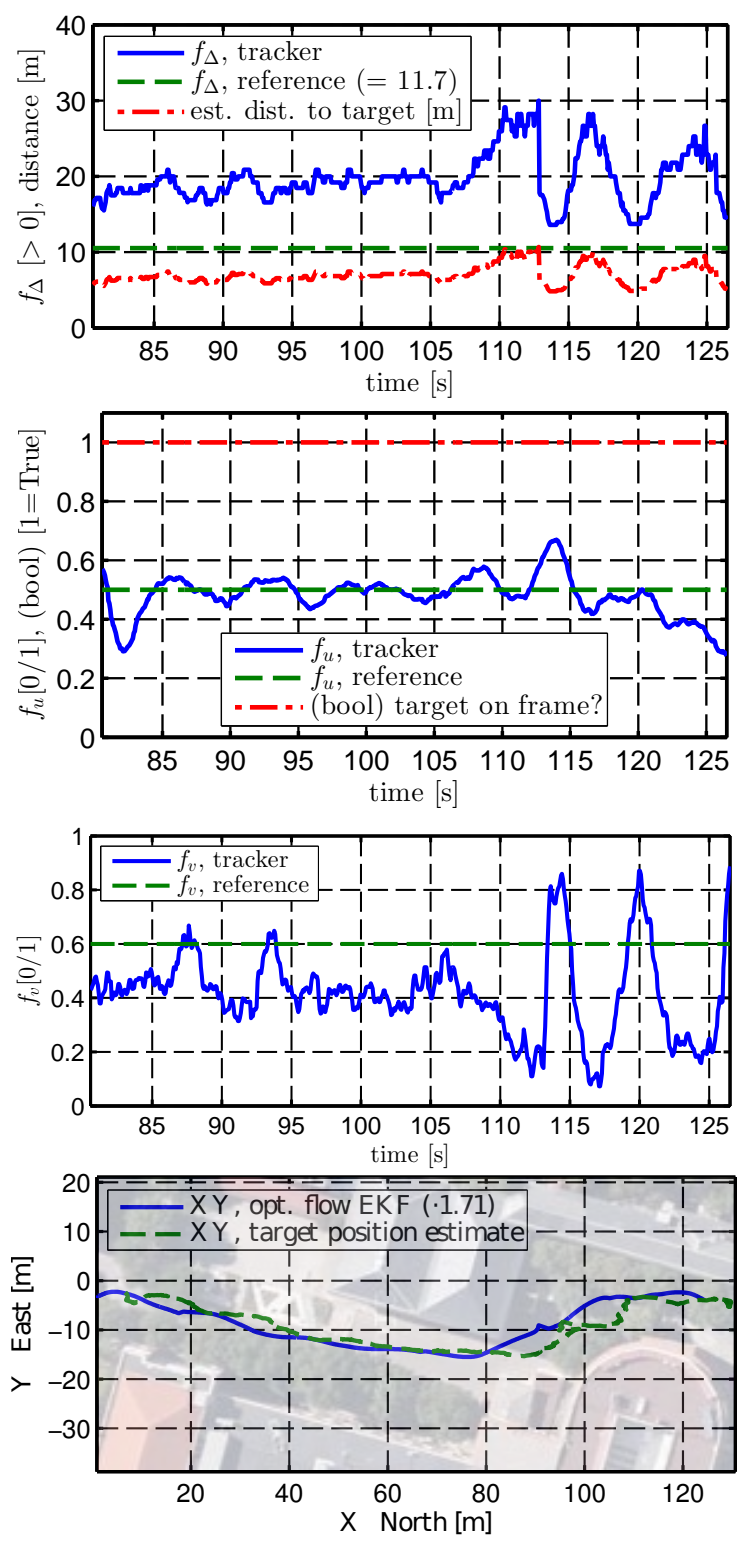

Figure 3.15: Measured and reference values of the image features during the experiment shown in Fig. 3.14. The $f_{\Delta}$ plot shows that the distance to the target varies slowly and it was estimated to be on the 5-10.5 m range. Due to the target being 2.2 times smaller than expected; the real distance was about sqrt (2.2) smaller, thus, in the 3.5-7 m range. We note that the object tracker does not estimate $f_{\Delta}$, the size of the target's bounding box, smoothly all the time; the target bounding box sometimes jumps to another estimate on the image plane. $f_{u}$ has been well controlled around the reference value; it's graph also shows (red, dash-dot line) that the tracking was not lost during this test. There are big variations in $f_{v}$ near the end of test which might be due to this feature being coupled to the vehicle's pitch. The navigation speed of the vehicle or an estimation error on the vehicle's pitch might be the cause of the decoupling's heuristic degradation on that part of the test. The last plot shows an estimate of the vehicle's trajectory based on the vehicle's telemetry using the EKF presented on (Pestana, 2012). The target's position estimate is obtained adding the estimate $\left\{\Delta x_{\text {tme }}, \Delta y_{\text {tme }}, \Delta z_{\text {tme }}\right\}$, shown in Fig. 3.5 , to the EKF's drone's position estimate. 

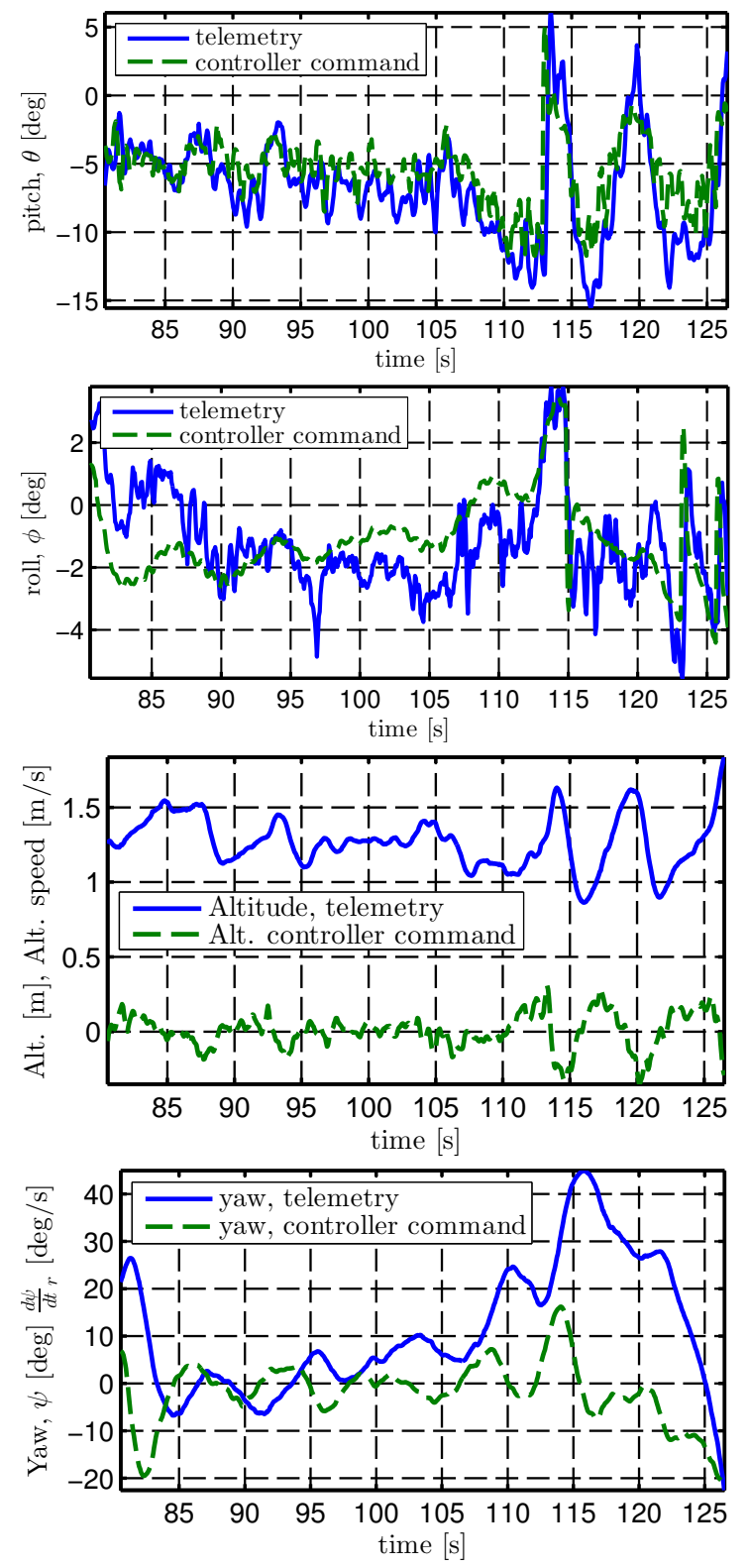

Figure 3.16: Controller commands during the experiment shown in Fig. 3.14. As shown, none of the commands present a high level of noise. The pitch command has a negative average because the drone is following a person who is running and it has to move forward to keep up. The roll command is low and only used during lateral movement or wind disturbance rejection to stabilize the vehicle's lateral movement. The drone stayed at an altitude of 1 to $1.5 \mathrm{~m}$ during most of the experiment, as measured by the ultrasound altitude sensor. Near the end of the test, the altitude speed command is coupled with the pitch command, which is caused by the coupling of the $f_{v}$ image feature with the pitch command. The yaw plot shows a constant heading during the first half of the experiment and then it shows the turn on the last part of the street corridor covered during the experiment, see the last plot of Fig. 3.15 . As explained before, the yaw heading is controlled directly to keep the target in the middle of the image which is important to avoid tracking loss events. The effect of the internal yaw reference $\psi_{\text {telem ref }}$ is that the vehicle tends to look at the target from the same direction. 


\subsection{Future Work}

The first line is related with the tracking algorithm. Since the OpenTLD is one of the best tracking algorithms which includes not only the tracking step, but also the detection one. However, it only provides translation, and scaling to specify the real movement of the followed object in the image. But, this assumption is restrictive. As future work, our aim is to use other trackers, like (Martínez et al., 2013), which provide the projective transformation in the image plane.

The second proposed improvement to the system is related with the reliability of the architecture. Now, when the tracked object leaves the image, the quadrotor starts to hover and it is not able to recover it unless the object appears again in the image. One option could be to switch from the IBVS controller to a PBVS controller when the object is lost. An EKF would be estimating the quadrotor's and also the target's 3D pose while the IBVS controller is working. Then when the target is lost, the PBVS controller is enabled based on the EKF prediction of the followed object's pose.

In the area of visual servoing there is an strong inter-dependence between the performance of the visual tracker and the successful realization of an object following task by a controller. Therefore, new methods could combine specific characteristics of the tracked objects and the surrounding environment into a better control strategy. For instance, it may be beneficial to select positions relative to the followed object that result in viewing angles without light glare reflections or strong shadows. In the same direction, the development of visual trackers that allow for object model introspection should be preferred. For instance, consider models that include the 3D structure of the object or the reflective properties of the different surfaces of the object. A very interesting ongoing challenge that may require model introspection is the International Aerial Robotics Competition (IARC) Mission 7, see Sec. I.4, where the drone needs to physically interact with ground robots that exhibit pseudo-random navigation behavior. From a navigation stand point, obstacle avoidance needs to be incorporated more strongly into the visual servoing task to enable following an object through a cluttered environment, like a sportsman through a forest. In this sense, obstacles that are difficult to map, such as trees, could be avoided considering their visual appearance rather than their difficult to reconstruct 3D structure.

\subsection{Conclusions}

In this chapter, a novel visual based object tracking and following architecture for multirotor vehicles is presented. The contributions of this work are two-fold:

- First, it has been demonstrated that current tracking algorithms, such as OpenTLD, can reliably work on a fixed camera multirotor vehicle to feedback an Image Based Visual Servoing (IBVS) controller, even during high velocity autonomous navigation.

- Second, our architecture has been able to follow a large variety of unmarked targets of different sizes and from a wide range of distances. Moreover, the algorithm is validated using a low-cost platform, the Parrot AR Drone 2.0, in outdoor conditions while tracking and following people. The system has successfully and repeatedly handled occlusion events and tracked fast moving targets, such as a person running, showing the robustness of our system against wind disturbances and illumination changes.

The main features of our architecture that made it unique at the time of the publication of our works are:

- Our system is able to follow and stabilize itself and it is able to track a large variety of different objects.

- Additionally, safety is assured even when the wireless connection is suddenly degraded, the tracking is lost or the target is occluded. This is achieved by using a multirotor platform that can attain onboard autonomous hovering by means of floor optical flow based odometry. The experimental work was performed using an AR Drone 2.0, and the algorithms where run on an off-board laptop computer via a WiFi link.

- Our system has been able to repeatedly perform object following tasks for long periods of time of up to 7 minutes (see the related videos), and fully depleting the UAV battery, after the user is experienced enough to understand the capabilities of our object following solution. 
- Our system has been able to perform visual servoing with targets of varying size, from a quarter to more than ten times the tunned target size, at varying distances from 1-2 m to 10-15 $\mathrm{m}$ of distance from the target, and it has has achieved person following at speeds up to $2.5-3.0 \mathrm{~m} / \mathrm{s}$ for a period of 45 seconds.

- The reliability of our system has been demonstrated in live demos: several performed by the CVG, at the SSRR2013 conference and with the ICG - TU Graz.

The main publications related to this chapter are:

- J. Pestana, J. L. Sanchez-Lopez, S. Saripalli, P. Campoy. “Computer Vision Based General Object Following for GPS-denied Multirotor Unmanned Vehicles”. In 2014 American Control Conference (ACC 2014). Portland, Oregon (United States). June 4th - 6th 2014

- J. Pestana, J. L. Sanchez-Lopez, S. Saripalli, P. Campoy. "Vision based GPS-denied Object tracking and Following for Unmanned Aerial Vehicles". Best Paper Award Finalist in 2013 The 11th IEEE International Symposium on Safety, Security, and Rescue Robotics (SSRR 2013). Linköping (Sweden). Oct 21-26, 2013.

The source of this aerial robotics visual servoing architecture has been released as open-source $\mathrm{2}^{2}$, and it has been integrated by my colleagues as part of the stack for the control of multirotor MAVs from the CVAR/CVG (UPM) Aerostack ${ }^{3}$. The architecture has been successfully tested using an Asctec Pelican and a powerful on-board computer, the Asctec Mastermind. Therefore, flights have been performed not requiring an external ground station.

This aerial robotics visual servoing architecture has been showcased in several live demos:

- CivilDRON 2016 congress 4 Madrid (Spain)

Multi-robot mission and vision-based person following

May 2016

- Semana de la Ciencia 2015 - Science Week 2015 5 . Madrid (Spain)

Multi-robot mission and vision-based person following

November 2-15, 2015

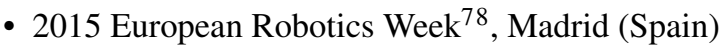

Vision-based person following

September 2015

- Noche Europea de los investigadores - European Researchers' Night 20159

Multi-robot mission, vision-based person following and a simple search and rescue mission

Madrid (Spain), September 2015

- 2014 European Robotics ${ }^{1911}$, Madrid (Spain)

Vision-based person following

November 2014

- Semana de la Ciencia 2014 - Science Week 2014 12 , Madrid (Spain)

Multi-robot mission and vision-based person following

November 2014

${ }^{2}$ GitHub Repository: https://github.com/Vision4UAV/cvg_ardrone2 ibvs

${ }^{3}$ GitHub Repository: https://github.com/Vision4UAV/Aerostack

${ }^{4}$ CivilDRON 2016: http: //www. aerpas.es/wp-content/uploads/2015/04/CivilDRON16_v2.pdf

${ }^{5}$ Semana de la Ciencia 2015 - Website http: / / bit.ly/2lN9RNg

${ }^{6}$ Semana de la Ciencia 2015 - Results Report http://bit.ly/2muELHG

${ }^{7} 2015$ European Robotics Week - Website: http://bit.1y/2mcMglt

${ }^{8}$ European Robotics Week - Website: http://bit.ly/2mT12Bh

${ }^{9}$ European Researchers' Night 2015 - Website: http://bit.1y/2mM6a9Q

${ }^{10} 2014$ European Robotics Week - Website: http://bit.ly/2mMaTIV

${ }^{11}$ European Robotics Week - Website: http://bit.ly/2mT12Bh

${ }^{12}$ Semana de la Ciencia 2014 - Results Report http://bit.ly/21Ng1NA 
- OpenLabNight 2014 13 , ICG - TU Graz (Austria)

Vision-based person following

October 9, 2014

- Semana de la Ciencia 2013 - Science Week 2013 ${ }^{14}$, Madrid (Spain)

Multi-robot mission and vision-based person following

November 4-17, 2013

- The 11th IEEE International Symposium on Safety, Security, and Rescue Robotics (SSRR 2013) Vision-based person following

Linköping (Sweden), October 21-26, 2013

For a full list of videos showing the performance of the presented visual servoing architecture the reader is referred to $\mathrm{Sec}$. 3.4.1.

\footnotetext{
${ }^{13}$ OpenLabNight 2014, ICG - TU Graz, Website: http: / / bit. ly/2mwigU4

${ }^{14}$ Semana de la Ciencia 2013 - Results Report http://bit.ly/2npz2lF

${ }^{15}$ SSRR 2013 Demos Website http://www.ssrr-conference.org/2013/Demos.html
} 


\subsection{Chapter Bibliography}

Achtelik, M., Achtelik, M., Weiss, S., and Siegwart, R. (2011). "Onboard IMU and monocular vision based control for MAVs in unknown in- and outdoor environments". In: Robotics and Automation (ICRA), 2011 IEEE International Conference on, pp. 3056-3063 (cit. on p. 45.

Mani Monajjemi (2012). ardrone_autonomy : A ROS Driver for ARDrone 1.0 \& 2.0. https : / / github . com / AutonomyLab/ardrone_autonomy, Autonomy Lab, Simon Fraser University (cit. on p. 46).

Argyros, A., Georgiadis, P., Trahanias, P., and Tsakiris, D. (2002). "Semi-autonomous Navigation of a Robotic Wheelchair". Journal of Intelligent and Robotic Systems 34 (3), pp. 315-329 (cit. on p. 45).

Babenko, B., Yang, M.-H., and Belongie, S. (2009). "Visual tracking with online Multiple Instance Learning”. In: Computer Vision and Pattern Recognition, 2009. CVPR 2009. IEEE Conference on, pp. $983-$ 990 (cit. on p. 45).

Bills, C., Chen, J., and Saxena, A. (2011). “Autonomous MAV flight in indoor environments using single image perspective cues”. In: Robotics and Automation (ICRA), 2011 IEEE International Conference on, pp. 5776-5783 (cit. on pp. 43, 45).

Bourquardez, O., Mahony, R., Guenard, N., Chaumette, F., Hamel, T., and Eck, L. (2009). “Image-Based Visual Servo Control of the Translation Kinematics of a Quadrotor Aerial Vehicle". Robotics, IEEE Transactions on 25.3 , pp. 743-749 (cit. on p. 45.

Chaumette, F. (1998). "Potential problems of stability and convergence in image-based and position-based visual servoing". In: The confluence of vision and control. Springer, pp. 66-78 (cit. on p. 44).

Chesi, G. (2009). "Visual servoing path planning via homogeneous forms and LMI optimizations". Robotics, IEEE Transactions on 25.2, pp. 281-291 (cit. on p. 45.

Chesi, G. and Vicino, A. (2004). "Visual servoing for large camera displacements". Robotics, IEEE Transactions on 20.4 , pp. 724-735 (cit. on p. 45).

Comport, A., Mahony, R., and Spindler, F. (2011). “A visual servoing model for generalised cameras: Case study of non-overlapping cameras". In: Robotics and Automation (ICRA), 2011 IEEE International Conference on, pp. 5683-5688 (cit. on p. 45).

Corke, P. I. et al. (1996). Visual Control of Robots: high-performance visual servoing. Research Studies Press Taunton, UK (cit. on p. 44).

Danelljan, M., Khan, F. S., Felsberg, M., Granström, K., Heintz, F., Rudol, P., Wzorek, M., Kvarnström, J., and Doherty, P. (2014). "A low-level active vision framework for collaborative unmanned aircraft systems". In: Workshop at the European Conference on Computer Vision. Springer, pp. 223-237 (cit. on p. 45.

Danelljan, M., Shahbaz Khan, F., Felsberg, M., and Van de Weijer, J. (2014). “Adaptive color attributes for real-time visual tracking". In: Proceedings of the IEEE Conference on Computer Vision and Pattern Recognition, pp. 1090-1097 (cit. on p.45).

Deng, H., Zhao, X., and How, Z. (2009). "A Vision-Based Ground Target Tracking System for a SmallScale Autonomous Helicopter". Image and Graphics, International Conference on, pp. 739-743 (cit. on p. 45.

Engel, J., Sturm, J., and Cremers, D. (2012). "Camera-Based Navigation of a Low-Cost Quadrocopter". In: Proc. of the International Conference on Intelligent Robot Systems (IROS) (cit. on pp. 43, 45).

Espiau, B., Chaumette, F., and Rives, P. (1992). "A new approach to visual servoing in robotics". ieee Transactions on Robotics and Automation 8.3, pp. 313-326 (cit. on p. 45).

Fahd Rafi Saad Khan, K. S. and Shah, M. (2006). "Autonomous target following by unmanned aerial vehicles”. In: Proc. SPIE. Orlando (Kissimmee), FL, USA (cit. on p.45).

Feddema, J. T. and Mitchell, O. R. (1989). "Vision-guided servoing with feature-based trajectory generation [for robots]". Robotics and Automation, IEEE Transactions on 5.5, pp. 691-700 (cit. on p. 44).

Fomena, R. T. and Chaumette, F. (2007). "Visual servoing from spheres using a spherical projection model". In: Robotics and Automation, 2007 IEEE International Conference on. IEEE, pp. 2080-2085 (cit. on p.44).

Haag, K., Dotenco, S., and Gallwitz, F. (2015). "Correlation filter based visual trackers for person pursuit using a low-cost Quadrotor”. In: Innovations for Community Services (I4CS), 2015 15th International Conference on. IEEE, pp. 1-8 (cit. on p.45). 
Hamel, T. and Mahony, R. (2002a). "Visual servoing of an under-actuated dynamic rigid-body system: an image-based approach". Robotics and Automation, IEEE Transactions on 18.2, pp. 187-198 (cit. on p. 44).

Hamel, T. and Mahony, R. (2002b). "Visual servoing of an under-actuated dynamic rigid-body system: an image-based approach". Robotics and Automation, IEEE Transactions on 18.2, pp. 187-198 (cit. on p. 44).

Hutchinson, S and Chaumette, F (2006). "Visual servo control, Part I: Basic approaches". IEEE Robotics and Automation Magazine 13.4, pp. 82-90 (cit. on p. 44).

Hutchinson, S and Chaumette, F (2007). "Visual servo control, Part II: Advanced approaches". IEEE Robotics and Automation Magazine 14.1, pp. 109-118 (cit. on p.44).

Kalal, Z. (2011). “Tracking Learning Detection". PhD thesis. University of Surrey (cit. on pp. 43, $45,46$.

Kalal, Z., Mikolajczyk, K., and Matas, J. (2012). “Tracking-Learning-Detection”. IEEE Transactions on Pattern Analysis and Machine Intelligence 34.7, pp. 1409-1422 (cit. on pp. 43, 45, 46).

Korodi, A., Codrean, A., Banita, L., and Volosencu, C. (2008). "Aspects regarding the object following control procedure for wheeled mobile robots”. WSEAS Trans. Sys. Ctrl. 3 (6), pp. 537-546 (cit. on p. 45.

Lapresté, J.-T. and Mezouar, Y. (2004). "A Hessian approach to visual servoing”. In: Intelligent Robots and Systems, 2004.(IROS 2004). Proceedings. 2004 IEEE/RSJ International Conference on. Vol. 1. IEEE, pp. 998-1003 (cit. on p. 45).

Lin, F., Chen, B., Lum, K. Y., and Lee, T. (2010). "A robust vision system on an unmanned helicopter for ground target seeking and following". In: Intelligent Control and Automation (WCICA), 2010 8th World Congress on, pp. 276-281 (cit. on p. 45).

Mahony, R., Corke, P., and Chaumette, F. (2002). "Choice of image features for depth-axis control in image based visual servo control”. In: Intelligent Robots and Systems, 2002. IEEE/RSJ International Conference on. Vol. 1, 390-395 vol.1 (cit. on p.44).

Mahony, R., Brasch, A., Corke, P., and Hamel, T. (2005). "Adaptive depth estimation in image based visual servo control of dynamic systems." In: Decision and Control, 2005 and 2005 European Control Conference. CDC-ECC '05. 44th IEEE Conference on, pp. 5372-5378 (cit. on p. 45).

Mahony, R., Corke, P., and Chaumette, F. (2002). "Choice of image features for depth-axis control in image based visual servo control”. In: Intelligent Robots and Systems, 2002. IEEE/RSJ International Conference on. Vol. 1. IEEE, pp. 390-395 (cit. on p. 44).

Malis, E. (2004). "Improving vision-based control using efficient second-order minimization techniques". In: Robotics and Automation, 2004. Proceedings. ICRA'04. 2004 IEEE International Conference on. Vol. 2. IEEE, pp. 1843-1848 (cit. on p. 45).

Martínez, C., Mondragón, I. F., Campoy, P., Sánchez-López, J. L., and Olivares-Méndez, M. A. (2013). “A Hierarchical Tracking Strategy for Vision-Based Applications On-Board UAVs”. Journal of Intelligent \& Robotic Systems, pp. 1-23 (cit. on pp. 45, 63).

Mezouar, Y. and Chaumette, F. (2002). "Path planning for robust image-based control". Robotics and Automation, IEEE Transactions On 18.4, pp. 534-549 (cit. on p. 45.

Mondragoón, I., Campoy, P., Olivares-Mendez, M., and Martinez, C. (2011). “3D object following based on visual information for Unmanned Aerial Vehicles”. In: Robotics Symposium, 2011 IEEE IX Latin American and IEEE Colombian Conference on Automatic Control and Industry Applications (LARC), pp. 1-7 (cit. on pp. 43, 45.

Nebehay, G. (2012). "Robust Object Tracking Based on Tracking-Learning-Detection". MA thesis. Austria: Vienna University of Technology (cit. on p. 46).

Kalal, Z (2011). OpenTLD object image tracker source repositories: (2011) Kalal, Z (PhD Thesis); Matlab OpenTLD implementation https://github.com/zk00006/OpenTLD (cit. on p.46).

Nebehay, G (2012). OpenTLD object image tracker source repositories: (2012) Nebehay, G(Msc. Thesis); C++ OpenTLD implementation https://github.com/gnebehay/OpenTLD (cit. on p. 46).

Chauvin, R (2012). OpenTLD object image tracker source repositories: (2013) Chauvin, $R$; $C++R O S$ OpenTLD wrapper https: / /github.com/Ronan0912/ros_opent Id (cit. on p. 46.

Pestana, J. (2012). “On-board control algorithms for Quadrotors and indoors navigation". MA thesis. Spain: Universidad Politécnica de Madrid (cit. on pp. 49,61).

Pestana, J., Mellado-Bataller, I., Fu, C., Sanchez-Lopez, J. L., Mondragon, I. F., and Campoy, P. (2013). "A general purpose configurable navigation controller for micro aerial multirotor vehicles". In: Un- 
manned Aircraft Systems (ICUAS), 2013 International Conference on. IEEE, pp. 557-564 (cit. on p. 49.).

Pestana, J., Sanchez-Lopez, J. L., Campoy, P., and Saripalli, S. (2013). "Vision based gps-denied object tracking and following for unmanned aerial vehicles". In: 2013 IEEE International Symposium on Safety, Security, and Rescue Robotics (SSRR). IEEE, pp. 1-6 (cit. on p. 57).

Pestana, J., Sanchez-Lopez, J. L., Saripalli, S., and Campoy, P. (2014). "Computer vision based general object following for GPS-denied multirotor unmanned vehicles". In: 2014 American Control Conference. IEEE, pp. 1886-1891 (cit. on p.60).

Quigley, M., Gerkey, B., Conley, K., Faust, J., Foote, T., Leibs, J., Berger, E., Wheeler, R., and Ng, A. (2009). "ROS : an open-source Robot Operating System". In: IEEE International Conference on Robotics and Automation (ICRA 2009) (cit. on p.46).

Rives, P. and Azinheira, J. R. (2004). "Linear structures following by an airship using vanishing point and horizon line in a visual servoing scheme". In: Robotics and Automation, 2004. Proceedings. ICRA'04. 2004 IEEE International Conference on. Vol. 1. IEEE, pp. 255-260 (cit. on p. 45).

Ross, S., Melik-Barkhudarov, N., Shankar, K. S., Wendel, A., Dey, D., Bagnell, J. A., and Hebert, M. (2013). "Learning monocular reactive uav control in cluttered natural environments". In: Robotics and Automation (ICRA), 2013 IEEE International Conference on. IEEE, pp. 1765-1772 (cit. on pp. 43. 45.

Saripalli, S. and Sukhatme, G. S. (2007). "Landing a Helicopter on a Moving Target". In: Proceedings of IEEE International Conference on Robotics and Automation. Rome, Italy, pp. 2030-2035 (cit. on p. 45.).

Savkin, A. and Teimoori, H. (2008). "Bearings-only guidance of an autonomous vehicle following a moving target with a smaller minimum turning radius". In: Decision and Control, 2008. CDC 2008. 47th IEEE Conference on, pp. 4239-4243 (cit. on p. 45).

Schramm, F., Geffard, F., Morel, G., and Micaelli, A. (2007). "Calibration free image point path planning simultaneously ensuring visibility and controlling camera path”. In: Robotics and Automation, 2007 IEEE International Conference on. IEEE, pp. 2074-2079 (cit. on p. 45).

Tahri, O. and Chaumette, F. (2005a). "Point-based and region-based image moments for visual servoing of planar objects". Robotics, IEEE Transactions on 21.6, pp. 1116-1127 (cit. on p. 44).

Tahri, O., Mezouar, Y., Chaumette, F., and Corke, P. (2010). "Decoupled Image-Based Visual Servoing for Cameras Obeying the Unified Projection Model”. Robotics, IEEE Transactions on 26.4, pp. 684-697 (cit. on pp. 44, 47).

Tahri, O. (2004). "Utilisation des moments en asservissement visuel et en calcul de pose". PhD thesis. Rennes 1 (cit. on p.44).

Tahri, O. and Chaumette, F. (2005b). "Point-based and region-based image moments for visual servoing of planar objects". Robotics, IEEE Transactions on 21.6, pp. 1116-1127 (cit. on p. 44).

Tahri, O., Chaumette, F., and Mezouar, Y. (2008). "New decoupled visual servoing scheme based on invariants from projection onto a sphere". In: Robotics and Automation, 2008. ICRA 2008. IEEE International Conference on. IEEE, pp. 3238-3243 (cit. on p. 45).

Tahri, O. and Mezouar, Y. (2010). "On visual servoing based on efficient second order minimization". Robotics and Autonomous Systems 58.5, pp. 712-719 (cit. on p. 45).

Tahri, O., Mezouar, Y., Chaumette, F., and Corke, P. (2009). "Generic decoupled image-based visual servoing for cameras obeying the unified projection model". In: Robotics and Automation, 2009. ICRA'09. IEEE International Conference on. IEEE, pp. 1116-1121 (cit. on p. 45).

Teuliere, C., Eck, L., and Marchand, E. (2011). "Chasing a moving target from a flying UAV". In: 2011 IEEE/RSJ International Conference on Intelligent Robots and Systems. IEEE, pp. 4929-4934 (cit. on p. 45).

Zhang, H. and Ostrowski, J. (1999). "Visual servoing with dynamics: control of an unmanned blimp". In: Robotics and Automation, 1999. Proceedings. 1999 IEEE International Conference on. Vol. 1, 618623 vol.1 (cit. on p. 45, 


\section{Chapter}

\section{Smooth Trajectory Planning for Multirotors}

\subsection{Introduction}

This chapter describes the development of a trajectory planner that generates smooth sampled obstacle-free trajectories and accompanying speed and acceleration plans for multirotors. This trajectory planner was developed to participate in the Simulation Contest of the European Robotics Challenge (EuRoC 2014) - Challenge 3, which focus was on drones applied to plant servicing and inspection. The planner was later improved, verified experimentally and executed on-board a quadrotor while participating in the 2016 DJI Developer Challenge. The main purpose of this module is to generate paths that can be traversed smoothly and fast by the quadrotor, while remaining at a safe distance from nearby obstacles.

A state-of-the-art planner is used to obtain a path that minimizes a weighted length. Each stretch of path is weighted depending on various factors, e. g. proximity to obstacles. A fixed amount of time is alotted for this task, and the best solution found so far is used for the rest of the calculations. With these objectives in mind:

- The planner calculates obstacle-free trajectories for multirotor drones. Given that these platforms are omnidirectional, they can move at any point in any direction, the trajectory planning step is performed in 3D space; no attitude angles, only position.

- A map of the environment must be provided as an octomap representation. The map does not need to be static, this planner was extended to work in dynamic maps/environments while participating in the 2016 DJI Developer Challenge.

- The generated paths stay away from obstacles by associating, inside an optimization formulation, an increased cost to navigating near them. Similarly a higher cost is associated to trajectories that realize unnecessary changes in altitude.

- The trajectories are smoothed in order to avoid sharp angles in curves, so that the multirotor can follow them smoothly without decelerating strongly on every curve. The smoothing operation is 
obstacle-aware, which ensures that the smoothed trajectory is approximately as far from obstacles as the originally planned raw trajectory.

- The trajectories are finely sampled to allow for the estimation of the radius of curvature at every waypoint of the trajectory. Its calculation is required to compute a time of passage through every waypoint, a speed and an acceleration plan.

- In addition to the positions, the sets of values for the time of passage, speed and acceleration are used by a controller to calculate interpolating splines of third order (or higher, depending on the utilized data) over the waypoints of the path. The resulting spline is later used during trajectory execution by the controller to perform navigation.

- For our work, it is important to use algorithms that can provide a feasible trajectory in a reasonable amount of time, for path lengths of 30-150 m, so that they can be used in real-time operation in the on-board computer of the multirotor. Consider as an application scenario the plant servicing and inspection in industrial environments.

The layout of this chapter is the following. First, the state of the art and a summary of the developed trajectory planning approach is presented. Then, the theoretical background related to the developed trajectory planner is introduced. It includes a description of the probabilistic map representation Octomap (Hornung et al., 2013), an efficient calculation of the distance to obstacles (Lau et al., 2013) using the said map representation, the two tested state-of-the-art trajectory planning algorithms Probabilistic RoadMap Star (PRM*) (Karaman et al., 2011; Kavraki et al., 1996) and Rapidly-exploring Random Tree Star $\left(\right.$ RRT $\left.^{*}\right)$ (LaValle, 1998); and the open-source libraries utilized during implementation (Şucan et al., 2012). Then, the detailed description of the developed planner is introduced; including the related utilization of the said open-source libraries. Afterwards, the results in two international competitions are discussed, including a description of the utilized experimental platform. The chapter finishes with the conclusions and the objectives achieved along with the utilization of the developed trajectory planner.

\subsection{State of the Art}

Modern efficient trajectory planning approaches for multirotors are based on them being differentially flat dynamic systems. For instance, the control law by Fritsch et al. (Fritsch et al., 2012) can be considered to be built on the differential flatness property of the dynamics of the multirotor (Fliess et al., 1995; Murray et al., 1995), which is well explained in (Mellinger et al., 2011). A system is differentially flat if there exists a set of outputs from it which fully determine the state of the system given the values of the flat outputs and their derivatives (to a given order). When a non-linear system is differentially flat, this characteristic can be used for both: control and trajectory generation (Dunbar et al., 2003, Van Nieuwstadt et al., 1998). Some of the first works to exploit the differential flatness of multirotors to perform trajectory planning and generation was performed by (Cowling et al., 2010). In this work, many different approaches to parametrize the trajectory were proposed: Chebyshev polynomials, Laguerre polynomials, trigonometric functions and piecewise polynomials among others; however results were only shown for a single polynomial per coordinate.

Probably, one of the most known works that makes use of the differential flatness to perform trajectory generation and tracking with quadrotors was carried out by Daniel Mellinger et al. (Mellinger et al., 2011) from the GRASP Laboratory. In this work the trajectory was divided in segments were a high-degree polynomial is fit, and the polynomials were fitted optimizing a Quadratic Programming (QP) problem that minimize the snap of the trajectory and includes corridor constraints, that allow to traverse tight passages demonstrated by making the quadrotor go through static and moving hula hoops. In this work, the trajectory could also be scaled in time to perform either faster tracking or a slower more stable flight.

However, it was not yet clearly developed how this trajectory generation methods can be combined with traditional trajectory planning techniques, such as Probabilistic RoadMaps (PRMs) or Rapidly exploring Random Tree (RRTs). The work by Charles Richter et al. (Richter, Charles et al., 2013; Richter et al., 2013) from the MIT, showed how to input a trajectory calculated by an RRT* planning algorithm into a Quadratic Programming (QP) to calculate an optimal flight trajectory using splines. Their novel approach optimizes the trajectory in terms of traversal time and aggressiveness, so that it can be used to 
calculate smoother but slower trajectories when required. In addition, the flights were performed in an unstructured environmen 1 using a Hokuyo LIDAR and a Microstrain IMU as sensors.

\subsection{Developed Trajectory Planner - Summary}
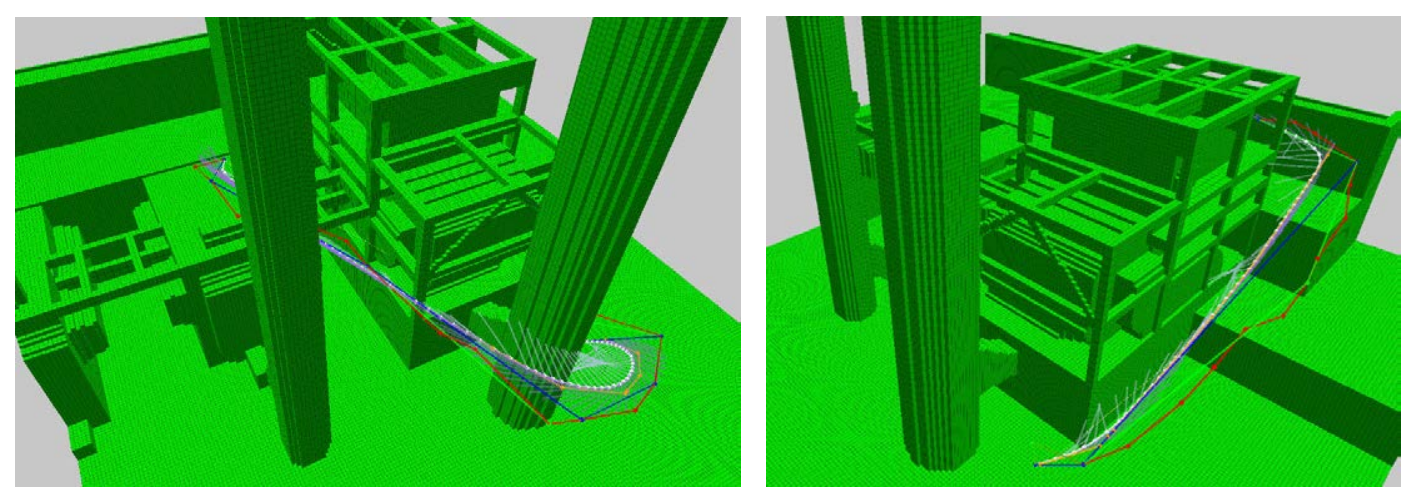

Figure 4.1: Simulated outdoor static environment of size $50 \mathrm{~m} \times 50 \mathrm{~m} \times 40 \mathrm{~m}$ from the Simulation Contest of the European Robotics Challenge (EuRoC 2014) - Challenge 3. The shown environment representation (green) is the multi-resolution octree-based method Octomap. A typically planned trajectory obtained by using our approach, 35-60m long, is shown: The output of the $P R M^{*}$ algorithm (red) is consecutively shortened (green-blue-orange-white). The resulting smoothed trajectory is shown in white, which is at a safe distance from nearby obstacles.

Whenever a new goal position is received, a new path is delivered to the controller. In order to allow fast and safe navigation, the calculated path should stay away from obstacles and be smooth. Our approach is similar to the approach proposed by Richter et al. (Richter et al., 2013), where a trajectory is planned in 3D space based on a traditional planning algorithm and, then, used to fit a high order polynomial.

Our method proceeds as follows: First, the path that minimizes a cost function is planned, which penalizes proximity to obstacles, length and unnecessary changes in altitude. Limiting the cost increase, the raw output path from the planning algorithm (shown in red in Fig. 4.1) is shortened (white). Finally, a speed plan is calculated based on the path curvature. The resulting path and timing information are used to fit a spline used as reference by the controller for feedback and feedforward control. In EuRoC2014 a quintic spline was used, and in the 2016 DJI Developer Challenge a third order spline was utilized.

In the EuRoC2014 Challenge (Fig.4.1), as input we get a static map provided as an Octomap (Hornung et al., 2013). To take advantage of the environment's staticity, we selected a Probabilistic Roadmap (PRM) based algorithm (Kavraki et al., 1996). As implementation, we used PRM* (PRMStar) from the OMPL library (Şucan et al., 2012). The roadmap and an obstacle proximity map are calculated prior to the mission. For the latter the dynamicEDT3D library (Lau et al., 2013) is used.

In the 2016 DJI Developer Challenge (Fig. 4.2), as input we calculated a first version of the map using an incremental Structure from Motion approach (see Sec.4.3.1, which we convert to an Octomap representation (Hornung et al., 2013). This map needs to be updated during flight, as dynamic obstacles and unmapped obstacles have to be avoided during flight. Since the map is not static and may change between trajectory queries, we used a fast single-query planning approach. We selected a Rapidly exploring Random Tree (RRT) based algorithm (LaValle, 1998). As implementation, we used RRT* (RRTStar) from the $O M P L$ library (Sucan et al., 2012). The obstacle map and the obstacle proximity map are updated on-board in real-time during the mission. For the latter the dynamicEDT3D library (Lau et al., 2013) is used.

[http://groups.csail.mit.edu/rrg/quad_polynomial_trajectory_planning, 2013 

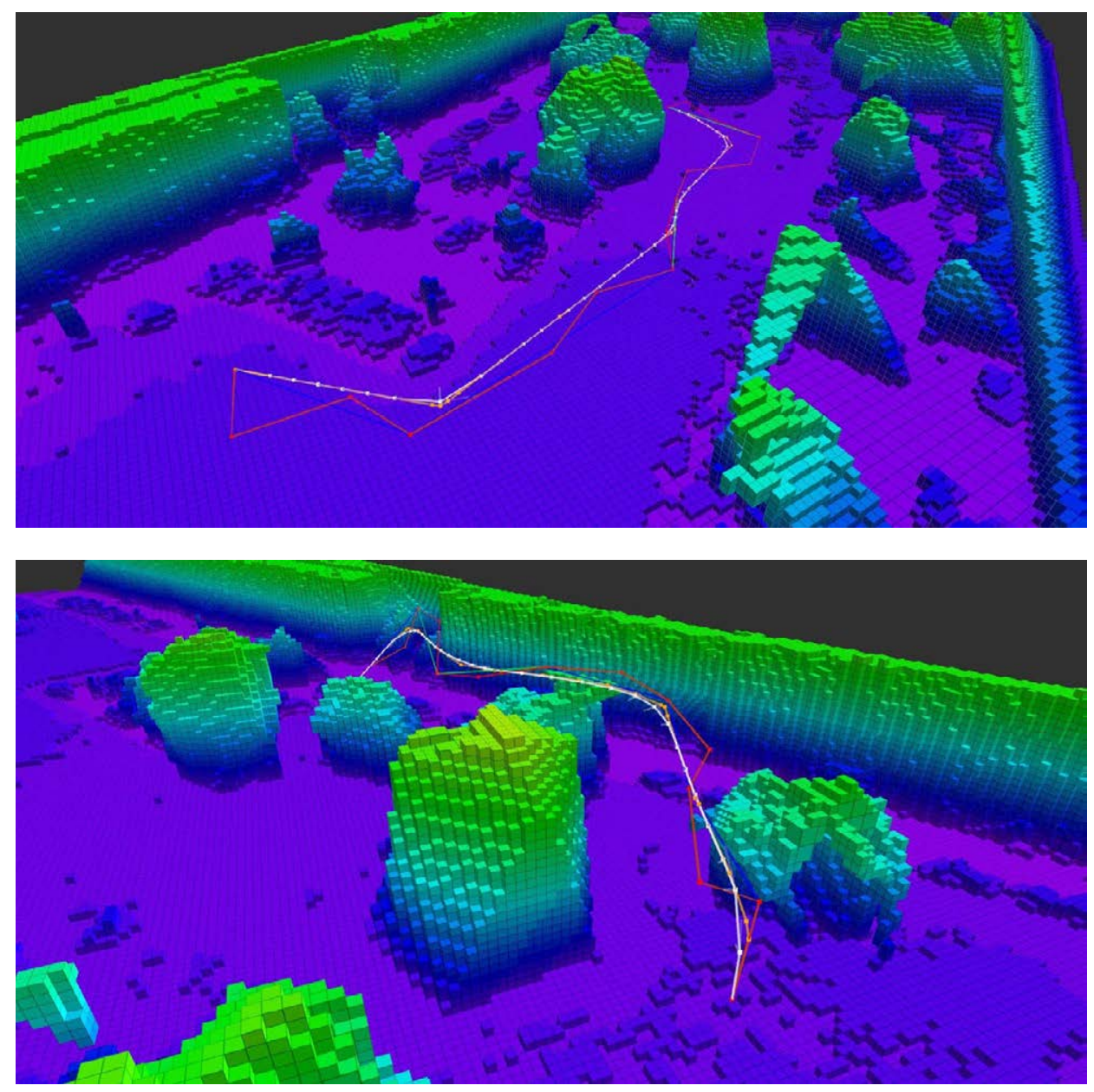

Figure 4.2: Outdoor map environment of size $55 \mathrm{~m} \times 170 \mathrm{~m} \times 40 \mathrm{~m}$ generated using the "Off-board Approach" explained in Sec. 4.3.1. The shown environment representation is the Octomap obstacle map. Typical planned trajectories, up to around $100 \mathrm{~m}$ long, are shown: The output of the $R R T^{*}$ algorithm (red) is consecutively shortened (green-blue-orange-white). The resulting smoothed trajectory is shown in white, which is at a safe distance from nearby obstacles. Our DJI M100 quadrotor flew such trajectories as part of our second deliverable for the 2016 DJI Developer Challenge, see video: http://bit.ly/2fauble.

\subsubsection{Online Structure-from-Motion (SfM) - Rescue Area Real-Time Mapping - 2016 DJI Developer Challenge}

We use an in-house 3D Reconstruction and Structure from Motion library developed over the years by the Institute of Computer Graphics of the TU Graz 2 . We use the real-time (on-line) Structure-from-Motion (SfM) pipeline which was first proposed and developed by Hoppe et al. (Hoppe, Klopschitz, Rumpler, et al., 2012). This Online SfM framework (or pipeline) is very runtime efficient. It uses SIFT features (Lowe, 2004). The model is initialized from the first two images for which a valid relative pose estimate can be computed. It iteratively utilizes the fast absolute pose estimation developed by Kneip et al. (Kneip et al., $2011)$ to align the latest acquired high-resolution image to the current sparse 3D model in real-time. Using an incremental and real-time version of Line3D++ (Hofer et al., 2016), a set of 3D lines is also calculated from the aligned images. Thus, the model consists of a sparse point cloud, a set of line segments and the camera poses related to each acquired image. The set of 3D lines helps to visually interpret the sparse 3D map, specially in the case of man-made environments (and other line-rich environments).

Utilizing the GPS measurements at the acquisition time of the images, the sparse 3D map is correctly

2 http://icg.tugraz.at/ 

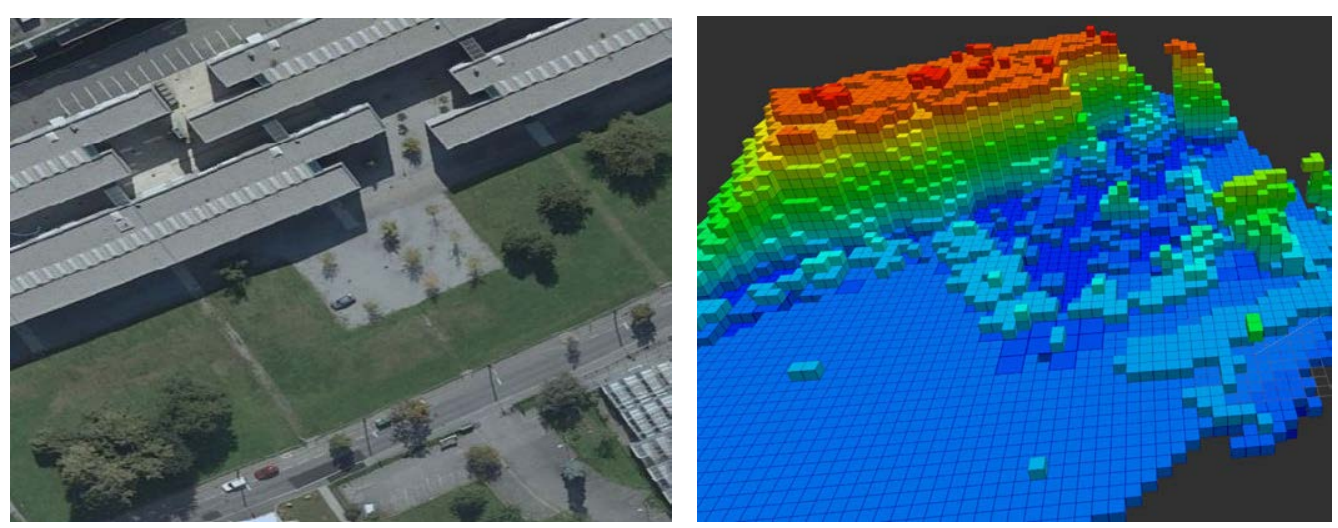

Figure 4.3: Outdoor map environment of size $60 \mathrm{~m} \times 95 \mathrm{~m}$ generated using the "Real-Time On-board Approach" explained in Sec.4.3.1. The left image is an overview of the mapped area, the target $50 \mathrm{~m} \times$ $50 \mathrm{~m}$ region is around the parking lot. Man-made obstacles, such as the buildings and cars, and big trees are quite well acquired as obstacles. However, smaller tries are often not correctly mapped and need to be sensed during nearby flight at low altitude. Our DJI M100 quadrotor is equipped with several stereo heads (the DJI Guidance System) that correctly acquires point-clouds of trees; which we use to update the map with dynamic and unmapped obstacles during flight. The trajectory planner, then, uses the updated map to calculate obstacle free smooth paths in the area.

scaled and geo-referenced. The on-board implementation matches the GPS measurements to the camera locations of the model by means of a robust RANSAC-based minimum least squares minimization of the distance between both sets of locations. This effectively provides the correct scale, rotation and relative translation for the sparse 3D map. In addition, a better geo-referenciation can be obtained by placing geo-referenced fiducial markers on site (Rumpler et al., 2016), but this approach was not possible in the Search and Rescue scenario proposed in the 2016 DJI Developer Challenge.

In addition to the sparse 3D map, the pipeline also creates a surface model on-the-fly (Hoppe, Klopschitz, Donoser, et al., 2013). The current implementation uses the approach by Labatut et al. (Labatut et al., 2007), rather than the approach outlined in Hoppe's work (Hoppe, Klopschitz, Donoser, et al., 2013). This model can then directly be converted to an Octomap (Hornung et al.,2013) by direct point-sampling of the triangle-mesh. The obtained Octomap can be updated in real-time and utilized by the presented trajectory planner during flight.

All these map representations of the environment are geo-referenced, based on the GPS data from the acquired images. This allows to locate the drone, a DJI M100 quadrotor, with respect to this map by simply using its GPS measurements. Other advantages of the geo-referencing of the map are that: no-fly zones can be added, and that the survivors can be located on the acquired map and referred to corresponding images (and pixel locations) of the mapping dataset.

To generate the map, the multirotor is first flown autonomously over the area to be mapped at a safe altitude (e. g. 34m). A set of top down images is used to generate a sparse 3D map on-the-fly, as explained above. Simultaneously a 3D mesh is fitted to the sparse model, which is finally used to obtain the Octomap representation of the environment, the obstacle map, by fine sampling of the said 3D mesh.

[Off-board Approach] During our first experiments, the Online SfM framework was used to process an image stream acquired by flying above the Rescue Area from a safe height. The images from the Zenmuse X3 Gimbal (resolution of 1280x720 pixels) were processed on a laptop during the acquisition flight. Our Online SfM runs faster than real-time on a 4 fps image stream.

[Real-Time On-board Approach] Currently the image stream from the Zenmuse X3 Gimbal (resolution of 1280x720 pixels) is fully processed on-board our DJI M100 quadrotor. This is made possible by leveraging the GPU of the on-board computer, the DJI Manifold (basically a Nvidia Tegra K1 development board). The Online SfM pipeline is able to process 1 image every 3.0s during flight (or 1 image every $1.5 \mathrm{~s}$ at the office). An area of $50 \times 50 \mathrm{~m}$ with a final map size of up to around $65 \times 105 \mathrm{~m}$ (for level-ground) is mapped on-board the DJI M100 in $2.5 \mathrm{~min}$. This time includes the overview-flight, acquisition of images, the generation of the sparse 3D model, the meshing, the geo-referenciation an the conversion to the Octomap obstacle map representation. One of the seven 3D reconstructions (Oc- 
tomap model), processed on-board during the 2016 DJI Developer Challenge is shown in the see video: http://bit.ly/2faublQ, and another map obtained in the same fashion is shown in Fig. 4.3

\subsection{Trajectory Planning - Theoretical Background}

For a comprehensive description of trajectory planning algorithms, the reader is referred to the freely available book from S.M. LaValle (LaValle and Kuffner, 2001). In the context of this chapter, the objective of the trajectory planning task is to calculate a smooth path to the next target point from the current position of the multirotor, while remaining reasonably away from obstacles and resulting in a reasonably short path. Staying away from obstacles and calculating the shortest path are often rather opposite and competing objectives that need to be combined in a common weighting scheme. The path that achieves both objectives "reasonably" is preferred over the shortest path or over the one that stays away from obstacles as much as possible.

The reason why the planning problem is focused on the positions only is the following. Common multirotors can theoretically follow trajectories defined in position as long as they are continuous up to the fourth derivative, with the heading angle being continuous up to the second derivative (Fritsch et al., 2012; Mellinger et al., 2011). The heading can otherwise be set to look in any desired direction during the trajectory. This very interesting property of the quadrotor dynamics model is exploited in the works by Mellinger et al. (Mellinger et al., 2011) and Fritsch et al. (Fritsch et al., 2012); and it can be understood as the quadrotor dynamics model being differentially flat with respect to the trajectory path and heading. This property allows to solve the trajectory planning problem including the dynamics of the quadrotor intrinsically considering a position-only path, by just enforcing the continuity of the path up to the said derivatives. This is a very advantageous approach, because it allows to use simpler path planning algorithms with a faster computation time than general-purpose dynamics-aware trajectory planners.

A map representation is needed to describe the environment and divide it between the free-space and the obstacle-space. For a given robot, this allows to determine which positions of the vehicle do not collide with obstacles and are thus permissible. In order to achieve real-time execution time, an efficient map representation able to represent general-shaped obstacles is used, the Octomap map representation (Hornung et al., 2013). This map representation has the additional advantage to be able to encode mapping uncertainty, by encoding the probability of a point in space to be occupied by an obstacle. A related open-source algorithm specifically designed for regular grid-based representations of 3D space, including Octomap, is available to efficiently determine the distance of every point in the free-space to the closest obstacle (Lau et al.,2013).

In order to achieve a real-time competitive execution time while calculating an optimal path, samplingbased trajectory planning algorithms were selected. The reason for selecting sampling-based methods is that they have proofed to work very well in practice. More specific reasons for this decision are:

- the speed with which the solution is obtained will not depend on the sampling resolution of the Octomap (as it would happen with a grid-based planning approach).

- that the planning time should scale better with the complexity and the size of the map than gridbased approaches.

Specifically, our planner has been tested using the Probabilistic RoadMap Star (PRM*)(Karaman et al., 2011, Kavraki et al., 1996) and Rapidly-exploring Random Tree Star (RRT*)(LaValle, 1998) planning algorithms. As stated above, the path is optimal using a combined path weight for: path-length, proximityto-obstacles and height changes. The main difference between both is that PRM* is a multiple-query algorithm, allowing to retrieve multiple trajectories faster when the map is known and static; and RRT*, which is single-query, requires to calculate the path from scratch for every trajectory query, but that is straight-forward to use in situations where the obstacle map needs to be updated during execution, e. g. when updating an uncertain map, or in the presence of dynamic obstacles. These algorithms do not consider positions of the robot uniformly on the environment, but sample them randomly. The use of this approaches result in a number of advantages over grid-based methods, a discussion about this topic can be found in (LaValle and Kuffner, 2001).

The rest of this section (4.4) is dedicated to describing the main works, that were introduced in this section, leveraged to achieve the implementation of our trajectory planner. 


\subsubsection{OctoMap - Probabilistic 3D Mapping Framework}

OctoMap is a volumetric map representation. It serves the purpose of specifying which parts of the surroundings of the robot are occupied by obstacles, which are free-space and which parts have not yet been explored. OctoMap was developed by Hornung et al. (Hornung et al., 2013) in 2012 and has since received a lot of attention, being used in many research works in Robotics. One of the main motivations of the development of OctoMap was to provide a tested, efficient and reusable mapping framework software for researchers and robotics applications, which its authors pursued by making their implementation opensource ${ }^{3}$ and available to be easily used with $\operatorname{ROS}^{4}$.

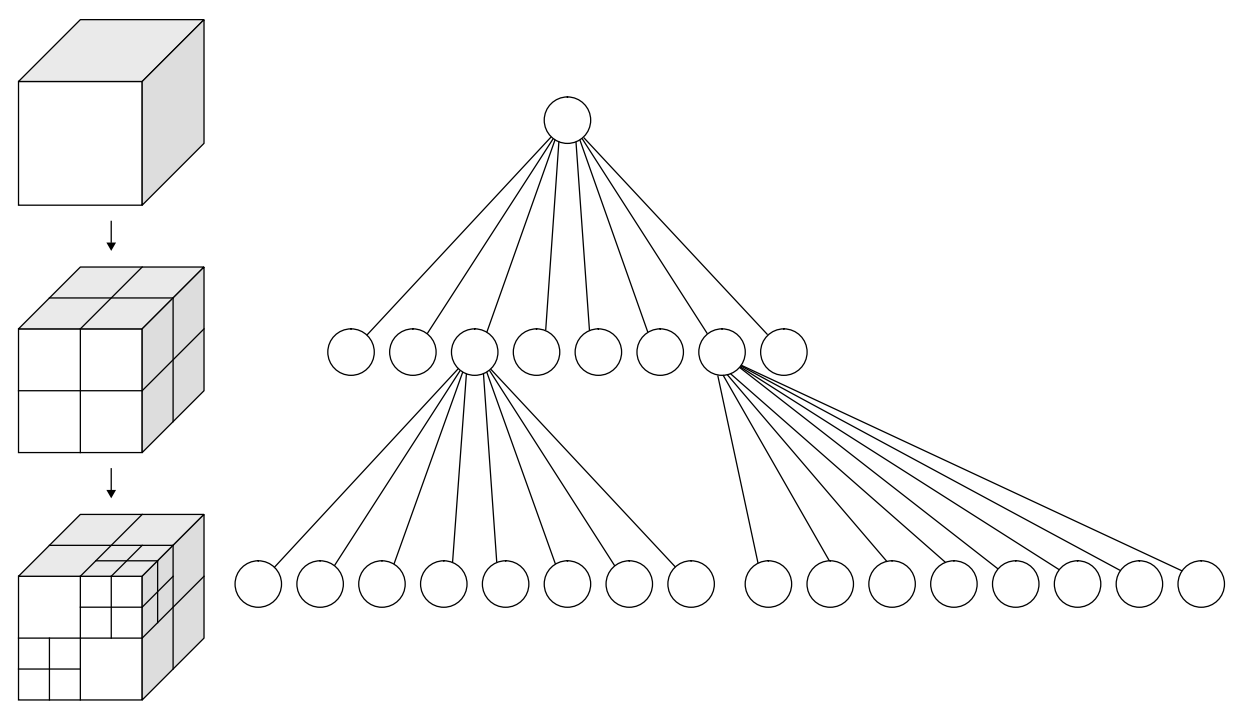

Figure 4.4: OcTree, data-structure schematic representation. On the left, a cubic region of space is iteratively subdivided into octants, eight smaller cubes at a time. On the right, a convenient data-structure to store information about each cube is shown, a tree data-structure. Thus the name OcTree. Original image author: WhiteTimberwolf - Wikipedia 6 , Creative Commons license.

The data-structure supporting this map representation is an Octree, see Fig. 4.4. This structure subdivides a cubic region in 3D space iteratively in octants, eight smaller cubes at a time. A convenient data-structure to store information about these subregions is a tree, where each node correspond to one of the cubes. OctoMap by default stores the state of each node: unexplored, free or occupied; in addition to the probability of the corresponding region of space of being occupied. This last data is quite an important feature in robotics, as it allows to trade off false observations of obstacles and free space by the robot (to account for sensor noise and the stochastic nature of the position estimates of the robot) with the necessity to sample obstacle more times by using a probabilistic mapping approach. The OcTree structure confers OctomMap with the capability of being a multi-resolution map representation; allowing to represent different structures or objects in the environment with varying degrees of precision, for instance allowing to use smaller cubes for objects of interest. In addition, the implementation of OctoMap effectively uses object-oriented programming and allows to extend the quantity of data stored per node, allowing to additionally store for instance the color information related to each node.

Another interesting feature of OctoMap is its efficiency in terms of memory consumption and access time. The map may occupy a big space in memory when including the probability occupancy values. However, these values can be dropped and unnecessary parts of the tree structure can be pruned resulting in a compressed map representation. The OcTree can be simplified without loosing any information when all the octants of a cube have the same value of their internal variables. This operation is usually refered as pruning. The compressed version of the map can be stored or serialized and shared by

\footnotetext{
${ }^{3}$ https://github.com/OctoMap/octomap

${ }^{4}$ http://wiki.ros.org/octomap_mapping

${ }^{6}$ https://en.wikipedia.org/wiki/File:Octree2.svg
} 
multiple autonomous agents, resulting in a reduced utilization of communication bandwidth. The OctoMap implementation provides a serialization interface that allows to send the compressed map so that it can be shared between robots over bandwidth constrained networks, such as wireless over long distances (Yanmaz et al., 2014). For more details, the reader is refered to the publication about OctoMap by Hornung et al. (Hornung et al., 2013).

Various OctoMap representations have been shown already on this chapter: the simulation environment of the EuRoC2014 autonomous flight challenge, see Fig. 4.1. and two maps obtained by using a full vision-based Structure from Motion pipeline, see Sec. 4.3.1, that were utilized in the participation in the 2016 DJI Developer Challenge, see Figs. $4.2 \& 4.3$.

\subsubsection{Dynamic Efficient Obstacle Distance Calculation}

As shown in Fig. 4.4, the OcTree representation of the environment subdivides the space in cubic regions. A grid representation of the OcTree at a fixed resolution can be extracted, to which it is possible to apply a distance transform resulting in a distance map to obstacles. The distance map is a matrix, from which each value corresponds to a small cube in space at the mentioned fixed resolution. The value stores the distance to the closest mapped obstacle in 3D space.

An implementation for Octomap of such an approach was developed by Lau et. al. (Lau et al., 2013) and released as open-source as a library named "DynamicEDT3D: A library for Incrementally Updatable Euclidean distance transforms in 3D". The code is available inside the OctoMap source-code repository. This distance map implementation is well tested and still supported in 2016. Its main characteristics are being compatible with 2D and 3D maps, time efficiency and being compatible with OctoMap.

This type of distance map is grown from the obstacle-space towards the interior of the free-space, up to a maximum desired distance (for computational efficiency reasons). Points further away from obstacles are all filled in to be at this said maximum distance. The distance map can be updated incrementally when new obstacles are located in the environment. Such incremental modifications to the distance map are directly performed after updates to the OctoMap representation.

Since the internal data-structure storing the distance map is basically an array, its access time is extremely fast. This means that a node distance lookup requires constant time: checking whether a point in space is occupied, collision checks, calculating the clearance of a patch from obstacles and the calculation of obstacle-free paths can be integrated very efficiently inside the motion planning algorithm.

In the present chapter, the distance map has been utilized to speed the calculation of obstacle-free paths for quadrotor drones in relatively open outdoors environments. For more details about the DynamicEDT3D distance map implementation, the reader is refered to the publication (Lau et al., 2013).

\subsubsection{Probabilistic RoadMap - Planning Algorithm}

This motion planning method solves the problem of finding an obstacle-free path between an starting position and a goal position. The Probabilistic RoadMap (PRM) algorithm was proposed by Kavraki et. al. (Kavraki et al., 1996). It is a sampling-based motion planning algorithm that works in the configuration space of the robot, where each point corresponds to a fully defined position of the robot in $3 \mathrm{D}$ space.

The trajectory planning problem is separated in two phases: the roadmap construction phase and the query phase. These two phases are depicted in Fig. 4.5 The roadmap construction is shown in the first five drawings of the figure, followed by an example of a query phase in the last drawing. The roadmap is basically a graph where the nodes are feasible robot configurations and the edges represent simple motion primitives that are only added when they are tested to be obstacle-free.

The PRM constitutes a multiple-query planning algorithm, i. e. it allows to solve multiple motion planning queries potentially making the planning queries less computationally demanding as the roadmap grows and becomes more complete. The queries are handled quite fast by first trying to connect the starting and goal points to the roadmap, and then solving for the shortest path by using the A* Dijkstra's algorithm (Dijkstra, 1959). If the starting or goal points cannot be added to the roadmap by simple motion primitives, then the roadmap is grown with the objective of connecting these two points to it. The downside, is that the roadmap needs to be remade or at least updated after every update to the obstacle

\footnotetext{
${ }^{8}$ https://commons.wikimedia.org/wiki/File:Motion_planning_configuration_space_road_map_path.svg
} 

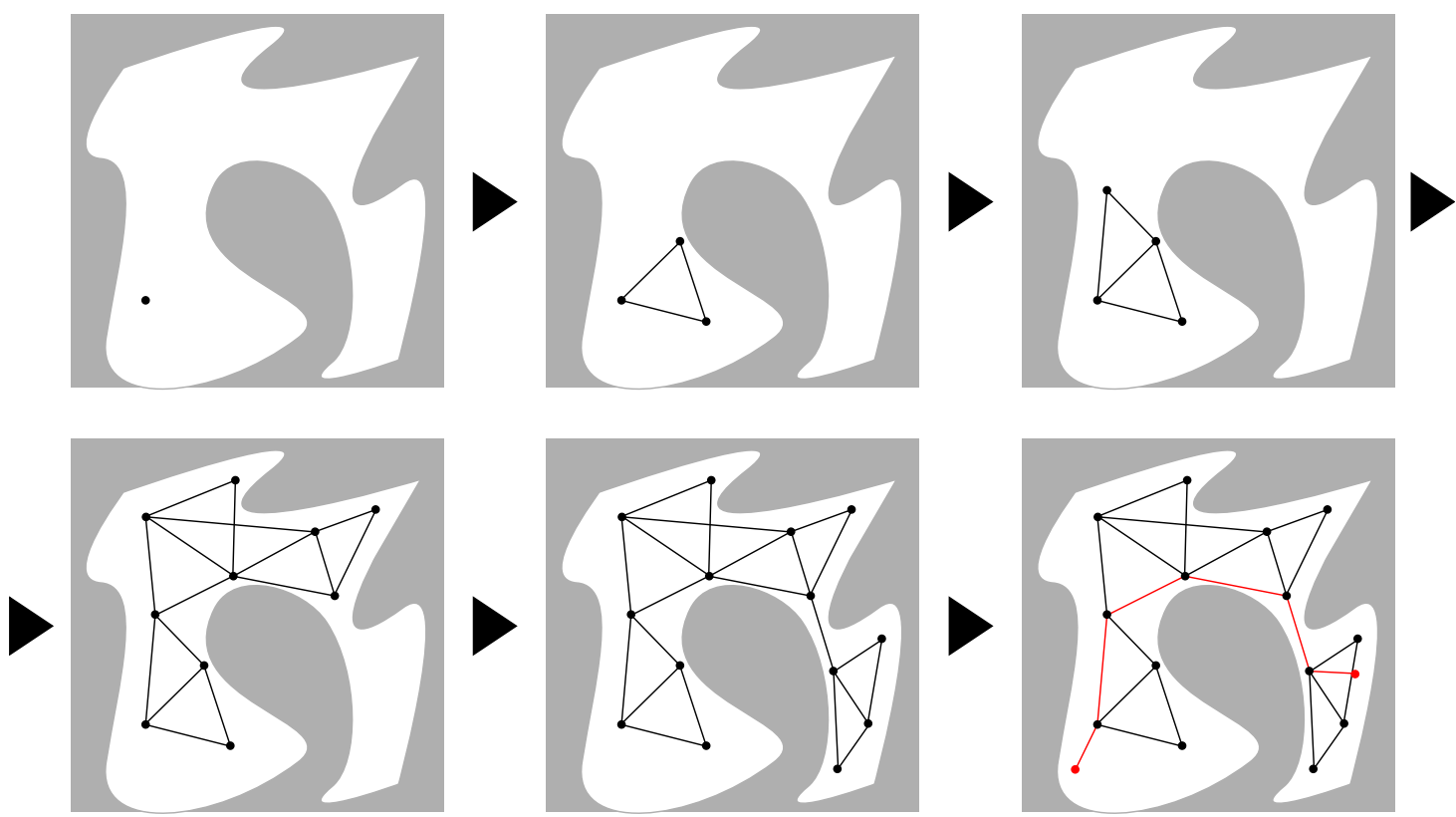

Figure 4.5: Probabilistic roadmap (PRM), motion planning algorithm. The main insight of this algorithm is to construct a graph of connected points in the configuration space of the robot. The gray region shows the obstacle-space, and the white the free-space. This graph sown by the nodes and edges in the figure is what is called the roadmap. Every edge represents obstacle-free paths. The graph is constructed by iteratively randomly sampling points in the configuration space of the robot, and trying to connect to nearby points and to the current roadmap by simple motion primitives. The first five drawings depict the roadmap construction. The last drawing shows how the roadmap can be used to quite fast answer to new motion queries by using the A* Dijkstra's shortest path algorithm (Dijkstra, 1959). Original image author: Simeon87 - Wikipedia 8 Creative Commons license.

map, as priorly obstacle-free edges and feasible nodes of the graph might become occluded by newly located obstacles. This fact limits the advantages of the multiple-query capability in applications where the obstacle map needs to be explored in real-time or in presence of dynamic obstacles.

The PRM sampling-based motion planning algorithm is proven to have very good statistical properties with regards to the motion planning objective. The PRM algorithm is probabilistically complete, meaning that given that there is a solution path for the current motion planning query, then the PRM method ensures that given enough time to perform the calculation it can find a feasible solution path. In addition, if the points in the configuration space have a good visibility, meaning that there not many obstacles with small bottlenecks, then a solution to the motion planning query will be found in a relatively short time.

The algorithm utilized in our work is the $P R M^{*}$ (Karaman et al., 2011), which is an improvement on the PRM. It allows not only to find a solution path to the motion planning query, but it also converges to the minimum cost optimal solution. We utilized the open-source implementation from the Open Motion Planning Library (OMPL) (Şucan et al., 2012).

\subsubsection{Rapidly-Exploring Random Trees - Planning Algorithm}

This motion planning method solves the problem of finding an obstacle-free path between an starting position and a goal position. The Rapidly-Exploring Random Trees (RRT) algorithm was proposed by Lavalle in 1998 et. al. (LaValle, 1998). It is a sampling-based motion planning algorithm that works in the configuration space of the robot, where each point corresponds to a fully defined position of the robot in 3D space.

The main insight to this algorithm lies in the creation of a tree shaped graph that tends to fill in all the free-space available to the robot for navigation. The interpretation of the graph is similar to that of the

\footnotetext{
${ }^{10}$ https://commons.wikimedia.org/wiki/File:Rapidly-exploring_Random_Tree_(RRT)_500x373.gif
} 


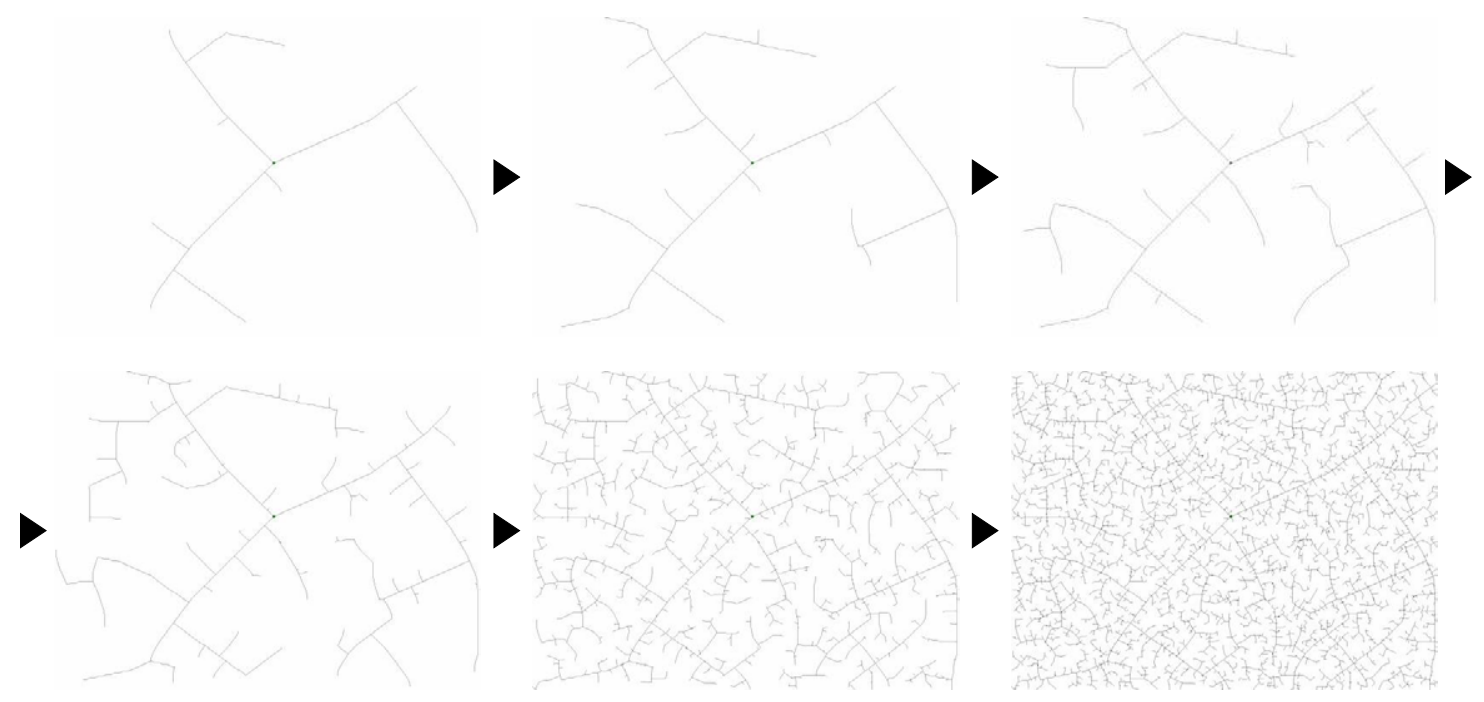

Figure 4.6: Rapidly-exploring random tree (RRT), motion planning algorithm. These drawings show how this planning algorithm grows a tree-shaped connected graph of feasible states by means of obstacle-free paths. In this case there are no obstacles. The purpose of the figure is to show how the nodes are sampled, and how they tend to explore the whole free-space. The RRT is a single-query algorithm. Original image author: Javed Hossain - Wikipedia ${ }^{10}$ Creative Commons license.

roadmap in the PRM. Nodes correspond to feasible positions and edges to obstacle-free simple motion primitives between nodes. The tree is grown by randomly sampling points in the configuration space of the robot. The algorithm tries to connect each new sample to the current RRT tree. The growth of an RRT tree in a region with no obstacles is depicted in Fig. 4.6 The tree is grown from the starting position of the motion planning query, and every certain number of samples the algorithm will try to connect the goal point to the tree. At this point, a feasible path is returned as solution solution to the query. This methods is a single-query algorithm, and it is very well suited to applications where the obstacle map is updated in real-time.

The RRT sampling-based motion planning algorithm, similarly to the PRM, is also proven to have very good statistical properties with regards to the motion planning objective. For instance the RRT is proven to be probabilistically complete.

The algorithm utilized in our work is the $R R T^{*}$ (Karaman et al., 2011), which is an improvement on the RRT. It allows not only to find a solution path to the motion planning query, but it also converges to the minimum cost optimal solution. We utilized the open-source implementation from the Open Motion Planning Library (OMPL) (Şucan et al., 2012).

\subsubsection{Utilized open-source libraries}

These are the utilized external software libraries:

- Octomap: map representation, see Sec.4.4.1

- ROS octomap_mapping package ${ }^{11}$, visualization in Rviz.

- DynamicEDT3DOctomap: this library delivers a clearance distance map for every cell in the map, see Sec.4.4.2

- OMPL - Open Motion Planning Library: $P R M^{*}$ and $R R T^{*}$ planning algorithms, see Secs. 4.4.3 \& 4.4.4. This library was selected because it is used in the MoveIt community in ROS, and also because it is under active development.

- Eigen3 library 12 for matrix operations.

\footnotetext{
${ }^{11}$ https://github.com/OctoMap/octomap_mapping

${ }^{12} \mathrm{http}$ ://eigen.tuxfamily.org/
} 


\subsection{Obstacle-free Smooth Trajectory Planning}

The main calculations realized during the operation of the trajectory planner are the following:

1. Planer initialization.

2. Answering to planning queries.

(a) Geometric Trajectory Planning.

(b) Geometric Trajectory Shortening and Smoothing.

(c) Speed planning along the trajectory.

The following information is also provided in this section:

- Communication with other modules

- Configuration Parameters

\subsubsection{Planner initialization}

1. Open the file that contains the octomap representation of the static map/environment.

2. The map is stored as a octomap octree (low memory consumption) and as a DynamicEDT3DOctomap distance map (high memory consumption). Every cell of the distance map contains the location of the nearest obstacle and the distance to it (or clearance).

3. Trajectory planner object initialization, the type depends on the kind of environment under consideration:

(a) Static: the $P R M^{*}$ planning algorithm is used. The RoadMap of the planner is initialized with a prefixed number of nodes.

(b) Dynamic: the $R R T^{*}$ planning algorithm is used. The trajectories are calculated in a singlequery fashion using the latest version of the Octomap, which is updated with the latest obstacle data, for instance using depth maps acquired with a stereo camera.

\subsubsection{Obstacle-aware Geometric Trajectory Planning}

The trajectory planning is performed in 3D space (no attitude angles, only position), assuming that the quadrotor is omnidirectional (it can move at any point in any direction) using the $P R M^{*}$ or the $R R T^{*}$ algorithms. In this step a cost function is evaluated over candidate paths and the delivered trajectory is of minimum cost. The cost penalizes length, proximity to obstacles and changes in the altitude of the trajectory. Ideally the trajectory is preferred to, in this order, not have unnecessary changes in altitude, not be unnecessarily near obstacles and be as short as possible.

The trajectory queries are from the current pose estimate as starting point to the goal position. The collision check is performed using the DynamicEDT3D distance map, which was is very fast in run-time. The collision check is done recursively from bigger to smaller parts of the quadrotor bounding box. If the nearest obstacle is nearer than the size of the currently evaluated bounding box, then the 8 neighbouring sub-bouding boxes are checked and so on.

A cost function is used to assign a cost to the obstacle-free trajectories that are a valid solution to the planning query. The cost function evaluates:

- Changes in altitude between consecutive waypoints: a more horizontal trajectory is preferred.

- Clearance to nearby obstacles : higher clearance is preferred.

- Path length : shorter paths are preferred. 
The trajectory is a sequence of states, $s_{i} \in S$, joined by the straight path segments, $l_{i} \in L$, in 3D space. The path cost function, $c_{\text {path }}$, is the sum of state costs, $c_{\text {state }}\left(s_{i}\right)$, and segment costs, $c_{\text {segment }}\left(l_{i}\right)$. The segment cost is evaluated as the curvilinear integral of a cost per unit length function. This cost is evaluated along the segment by sampling points over it, $\mathbf{x} \in \mathbb{R}^{3}$. This means that the path cost function, $c_{\text {path }}$, is the following:

$$
\begin{gathered}
c_{\text {path }}=\sum_{s_{i} \in S} c_{\text {state }}\left(s_{i}\right)+\sum_{l_{i} \in L} c_{\text {segment }}\left(l_{i}\right) \\
c_{\text {state }}\left(s_{i}\right)=c_{\text {state }}(\mathbf{x})=0 \\
c_{\text {segment }}\left(l_{i}\right)=\int_{l_{i}}\left\{K_{c} c_{\text {clearance }}(\mathbf{x})+K_{a} c_{\text {altitude }}(\mathbf{x})+K_{l} c_{\text {length }}(\mathbf{x})\right\}|d l| \\
c_{\text {clearance }}(\mathbf{x})=\left\{1+K_{c 2}\left(1-d_{\text {clearance }}(\mathbf{x}) / d_{\text {max }}\right)\right\}^{4} \\
c_{\text {altitude }}(\mathbf{x})=\left|u_{z}(\mathbf{x})\right| \\
c_{\text {length }}(\mathbf{x})=1
\end{gathered}
$$

where $K_{c}, K_{c 2}, K_{a}$ and $K_{l}$ are tuning constants to set the relative strength of each cost contribution, $d_{\text {max }}$ is the maximum clearance distance that saturates the distance map, $d_{\text {clearance }}(\mathbf{x})$ is the value of the clearance provided by the distance map at $\mathbf{x}, u_{z}(\mathbf{x})$ is the $z$ component of the unitary vector along the path at $\mathbf{x}$. Note that $X$ and $Y$ world axis are horizontal and the $Z$ axis is vertical and pointing upwards.

The output of the trajectory planner, the $P R M^{*}$ or the $R R T^{*}$ algorithm, is a feasible trajectory that is optimal with respect to the defined path cost. However, the resulting trajectory is usually not smooth and presents sharp angles at many checkpoints.

\subsubsection{Obstacle-aware Trajectory Shortening and Smoothing}

In this step the trajectory is shortened and smoothed. The blind application of these operations would result in a path which would pass near obstacles, and thus of high cost. In order to avoid this, some increase in the cost of the trajectory is allowed; but the cost increase is constrained to a fraction of to the original trajectory cost. This way the above mentioned qualities of the trajectory are kept in the smoothed trajectory. The shortening and smoothing operations are the result of applying small sub-operations iteratively, for which a cost increase can be individually calculated. The simplifications performed to the path are all cost-aware, meaning that a shortening or smoothing sub-operation is only accepted when its related cost increase, measured by means of Eq. 4.1, is below a threshold.

The shortening and smoothing operations are the following in the specified order:

1. Reduce the number of vertices that are present in the current path: interim waypoints are removed if the trajectory is still collision-free and until the total cost does not increase more than $10 \%$.

2. Collapse waypoints that are too near each other (allowed cost increase: 10\%) and reduce vertices again (allowed cost increase: $10 \%$ )

3. Shortcut the path twice (each allowed cost increase: 10\%): not only waypoints are considered for the path length reduction, but also points in the segments of the path themselves.

4. Smooth the path using the BSpline algorithm (allowed cost increase: 15\%): new wapoints are sampled making the path rounder near the corners.

5. New waypoints are sampled in the current trajectory segments and others are reduced to get a minimum distance between waypoints but not too small (allowed cost increase: 10\%).

6. New waypoints are sampled near the first and last waypoints to have a different minimum length of the first and last path segments.

\subsubsection{Speed, Acceleration and Time-of-passage Planning}

In the last step, a speed plan for the checkpoints in the trajectory is delivered. The speed plan is calculated so that maximum acceleration constraints can be enforced, the algorithm is inspired by the work 
of Hoffman. et al. (Hoffmann, Gabriel M et al., 2008), but with improvements. A speed plan is calculated iteratively to obtain an acceleration plan whose derivative is bounded. The speed plan calculation is performed by the following steps, in the specified order:

1. Geometric calculations are performed to estimate the tangent, normal and binormal vectors at each waypoint.

2. Then, the radius of curvature in each waypoint is calculated:

- A system of equations to determine the circumference that approximates each waypoint[i] and its neighbours is calculated.

- For each point[i], its neighbours are projected into the plane formed by the normal and tangent vectors estimated at point[i].

- The radius of curvature in each waypoint is calculated and the tangent and normal are corrected based on the solution of the system of equations.

Steps $1 \& 2$ amount to the following calculations. For each point, $\mathbf{p}_{i}$. First, $N_{n c}$ neighboring points, $\mathbf{p}_{\mathbf{n}_{i}}$, are sampled around, $\mathbf{p}_{i}$, each one separated by a predefined distance $d_{n c}$ to $\mathbf{p}_{i}$ or to another neighbor, $\mathbf{p}_{\mathbf{n}_{i} j}$. By this definition, $\mathbf{p}_{\mathbf{n}_{i} 0}=\mathbf{p}_{i}$ and for $j<0(j>0)$ the point $\mathbf{p}_{\mathbf{n}_{i}}$ is before (after) $\mathbf{p}_{i}$ in the trajectory path. Second, see Eqs. $4.7 \& 4.8 \& 4.9$ the tangent, $\mathbf{t}_{i}$, binormal, $\mathbf{b}_{i}$, and normal, $\mathbf{n}_{i}$, of the trajectory at point $\mathbf{p}_{i}$ are estimated by calculating mean values using the $N_{n c}$ neighbors $\mathbf{p}_{\mathbf{n}_{i} j}$ around the point $i, \mathbf{p}_{i}$ :

$$
\begin{aligned}
& \mathbf{t}_{i j}=\frac{\mathbf{p}_{\mathbf{n}_{i j}+1}-\mathbf{p}_{\mathbf{n}_{i j}}}{\left|\mathbf{p}_{\mathbf{n}_{i j}+1}-\mathbf{p}_{\mathbf{n}_{i} j}\right|} \quad \text { with } j=\left\{-\frac{N_{n c}}{2},-\frac{N_{n c}}{2}+1, \ldots, \frac{N_{n c}}{2}-1\right\} \\
& \mathbf{t}_{i}=\frac{\sum_{j} \mathbf{t}_{i j}}{N_{n c}} ; \quad \mathbf{t}_{i}=\frac{\mathbf{t}_{i}}{\left|\mathbf{t}_{i}\right|} \quad \text { with } j=\left\{-\frac{N_{n c}}{2},-\frac{N_{n c}}{2}+1, \ldots, \frac{N_{n c}}{2}-2\right\} \\
& \mathbf{b}_{i j}=\mathbf{t}_{i j} \times \mathbf{t}_{i(j+1)} \quad \text { with } j=\left\{-\frac{N_{n c}}{2},-\frac{N_{n c}}{2}+1, \ldots, \frac{N_{n c}}{2}-2\right\} \\
& \mathbf{b}_{i}=\frac{\sum_{j} \mathbf{b}_{i j}}{N_{n c}-1} ; \quad \mathbf{b}_{i}=\frac{\mathbf{b}_{i}}{\left|\mathbf{b}_{i}\right|} \\
& \mathbf{n}_{i j}=\mathbf{b}_{i j} \times \mathbf{t}_{i j} \\
& \mathbf{n}_{i}=\frac{\sum_{j} \mathbf{n}_{i j}}{N_{n c}-1} ; \quad \mathbf{n}_{i}=\frac{\mathbf{n}_{i}}{\left|\mathbf{n}_{i}\right|}
\end{aligned}
$$

And third, the radius of curvature $r_{i}$ at the point $\mathbf{p}_{i}$ is calculated. In order to perform the estimation of this value, the $N_{n c}$ neighbors are projected onto the plane defined by the tangent, $\mathbf{t}_{i}$, and normal, $\mathbf{n}_{i}$, vectors of the trajectory at the point $\mathbf{p}_{i}$. Then, see Eqs. 4.10\&4.11 \&4.12, their 2D coordinates $\left\{x_{i j}, y_{i j}\right\}$ on this plane are used to solve the following system of equations. It provides an estimate of the radius of curvature $r_{i}$, and the corresponding center of curvature, $\mathbf{c}_{i}$ or $\left\{c_{x i}, c_{y i}\right\}$, at the point $\mathbf{p}_{i}$. This is also used to obtain a better estimate of the normal and tangent vectors, $\mathbf{n}_{i}^{*}$ and $\mathbf{t}_{i}^{*}$, which are used in the rest of the calculations.

$$
\begin{aligned}
& x_{i j}=\left(\mathbf{p}_{\mathbf{n}_{i} j}-\mathbf{p}_{i}\right) \cdot \mathbf{t}_{i} \quad y_{i j}=\left(\mathbf{p}_{\mathbf{n}_{i} j}-\mathbf{p}_{i}\right) \cdot \mathbf{n}_{i} \\
& {\left[\begin{array}{ccc}
x_{i j} & y_{i j} & 1 \\
\vdots & \vdots & \vdots
\end{array}\right]\left[\begin{array}{c}
-2 c_{x i} \\
-2 c_{y i} \\
c_{x i}^{2}+c_{y i}^{2}-r_{i}^{2}
\end{array}\right]=\left[\begin{array}{c}
-x_{i j}^{2}-y_{i j}^{2} \\
\vdots
\end{array}\right]} \\
& \text { with } j=\left\{-\frac{N_{n c}}{2},-\frac{N_{n c}}{2}+1, \ldots, \frac{+N_{n c}}{2}\right\} \\
& \mathbf{n}_{i}^{*}=\frac{c_{x i} \mathbf{t}_{i}+c_{y i} \mathbf{n}_{i}}{\left|c_{x i} \mathbf{t}_{i}+c_{y i} \mathbf{n}_{i}\right|} \quad \mathbf{t}_{i}^{*}=\mathbf{n}_{i}^{*} \times \mathbf{b}_{i}
\end{aligned}
$$


Configuration values for this calculation that have worked well in our experiments are $N_{n c}=8$ and $d_{n c}=0.50 \mathrm{~m}$. Predefined result values are used for special cases. For instance, when all the $N_{n c}$ neighboring points around waypoint $j$ are aligned in a straight line, then its radius of curvature is set to a high value for the rest of the algorithm.

3. The main configuration of the algorithm consists of the maximum upwards, downwards and horizontal velocities and accelerations. These values are used to calculate the maximum velocity and acceleration as a function of a unitary direction vector, see Algs. 3 \& 4 . Note that in Algs. 3 \& 4 the maximum velocity and acceleration constraints are set by an ellipsoid so as to make the output of these functions continuous with respect to the trajectory direction.

4. The desired speed for the trajectory is set at every point to the minimum of the following two values: (1) the maximum configured velocity Velocity_Max_Direction, see Alg. 3, and (2) the maximum attainable velocity set by the radius of curvature $v_{\max , i}=\sqrt{a_{\max , n} \cdot r_{i}}$ where $a_{\text {max }, n}=$ Acceleration_Max_Direction $\left(\mathbf{n}_{i}^{*}\right.$, config $)$, see Alg. 4 Then, the desired initial and final velocities are set, which are usually both set to zero.

5. A first speed plan is calculated that complies with the acceleration constraints. This algorithm, that we are going to call velocity_plan_sweep_double_pass, see Alg. 1. was implemented following the publication (Hoffmann, Gabriel M et al., 2008) with only minor modifications. Note that the equations relating path trajectory lengths, velocities and accelerations correspond to those of linear uniformly accelerated motion (for every section of the trajectory). The modifications to this algorithm are: (1) see line 3 of Alg. 2 and (2) Algs. 3 \& 4. Later on, a velocity smoothing step is proposed that provides a much more continuous and feasible acceleration plan than proposed by Hoffman et al. in (Hoffmann, Gabriel M et al., 2008). For a comparison between smoothed and non-smoothed speed-plans, the reader is referred to Sec. 4.7.3.1.

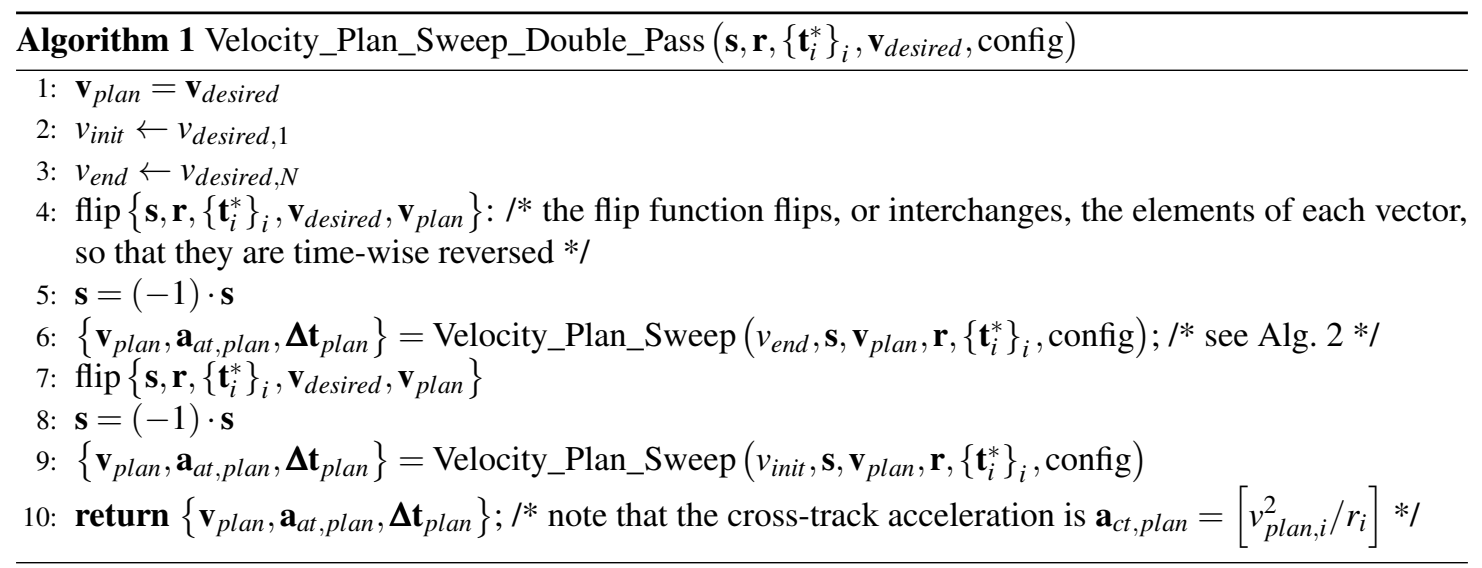



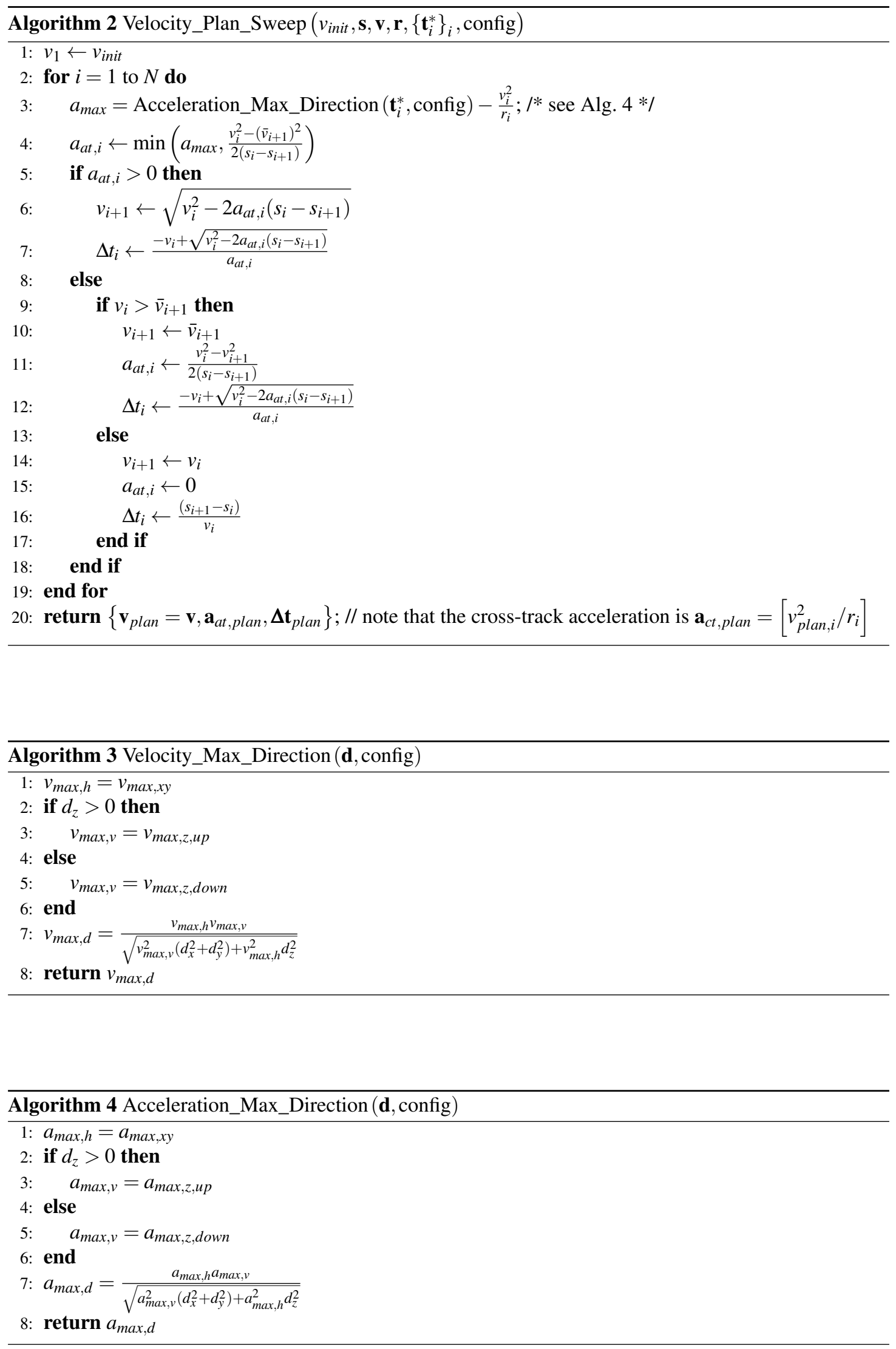
6. The output speed plan, $\mathbf{v}_{\text {plan }}$, of the velocity_plan_sweep_double_pass, Alg. 1, complies with the configured velocity and acceleration constraints, considering both normal and along-track accelerations. The only drawback is that it provides a bang-bang solution, with the acceleration usually at maximum values changing signs at certain points of the trajectory. For this reason, the speed plan is smoothed iteratively to obtain a more continuous acceleration plan whose derivative does not contain values that are too high:

(a) Using the current velocity plan as a data term a new velocity plan is calculated applying a smoothing spline type optimization, see related chapter of the book (James et al., 2013). This corresponds to minimizing the following cost-function by using Gauss-Newton Least Squares minimization (Triggs et al., 1999) (note that 1 and $N$ are the indexes of the initial and end velocities):

$$
\begin{aligned}
& f(\mathbf{v})=\lambda_{1}\left[\sum_{i=2}^{i=N-1}\left(v_{i}-v_{i, \text { old }}\right)^{2}\right]+\lambda_{2}\left[\int_{t_{1}}^{t_{N}}\left(\frac{d^{2} v}{d t^{2}}\right)^{2} d t\right]+\lambda_{3}\left[\int_{t_{1}}^{t_{N}}\left(\frac{d^{3} v}{d t^{3}}\right)^{2} d t\right] \\
& =\lambda_{1}\left[\sum_{i=2}^{i=N-1}\left(v_{i}-v_{i, o l d}\right)^{2}\right]+ \\
& \lambda_{2}\left[\sum_{i=1}^{i=N-2}\left\{\left(\frac{\left(a_{a t, i+1}-a_{a t, i}\right)}{\frac{\Delta t_{i+1}+\Delta t_{i}}{2}}\right)^{2} \frac{\Delta t_{i+1}+\Delta t_{i}}{2}\right\}\right]+ \\
& \lambda_{3}\left[\sum_{i=1}^{i=N-3}\left\{\left(\frac{T_{a t, i+1}-T_{a t, i}}{\frac{\Delta t_{i+2}+2 \Delta t_{i+1}+\Delta t_{i}}{4}}\right)^{2} \frac{\Delta t_{i+2}+2 \Delta t_{i+1}+\Delta t_{i}}{4}\right\}\right] \\
& =\lambda_{1}\left[\sum_{i=2}^{i=N-1}\left(v_{i}-v_{i, \text { old }}\right)^{2}\right]+ \\
& \lambda_{2}\left[\sum_{i=1}^{i=N-2}\left\{\left(\frac{\left(\frac{v_{i+2}-v_{i+1}}{\Delta t_{i+1}}-\frac{v_{i+1}-v_{i}}{\Delta t_{i}}\right)}{\frac{\Delta t_{i+1}+\Delta t_{i}}{2}}\right)^{2} \frac{\Delta t_{i+1}+\Delta t_{i}}{2}\right\}\right]+ \\
& \lambda_{3}\left[\sum_{i=1}^{i=N-3}\left\{\left(\frac{\frac{\left(\frac{v_{i+3}-v_{i+2}}{\Delta t_{i+2}}-\frac{v_{i+2}-v_{i+1}}{\Delta t_{i+1}}\right)}{\frac{\Delta t_{i+2}+\Delta t_{i+1}}{2}}-\frac{\left(\frac{v_{i+2}-v_{i+1}}{\Delta t_{i+1}}-\frac{v_{i+1}-v_{i}}{\Delta t_{i}}\right)}{\frac{\Delta t_{i+1}+\Delta t_{i}}{2}}}{\frac{\Delta t_{i+2}+2 \Delta t_{i+1}+\Delta t_{i}}{4}}\right) \frac{\Delta t_{i+2}+2 \Delta t_{i+1}+\Delta t_{i}}{4}\right\}\right]
\end{aligned}
$$

In these equations:

i. $a_{a t, i}$ is the along-track acceleration at waypoint $i, a_{a t, i}=\frac{v_{i+1}-v_{i}}{\Delta t_{i}}$

ii. $T_{a t, i}$ is the derivative of the along-track acceleration at waypoint $i$, $T_{a t, i}=\frac{\left(a_{a t, i+1}-a_{a t, i}\right)}{\frac{\Delta t_{i+1}+\Delta t_{i}}{2}}=\frac{\left(\frac{v_{i+2}-v_{i+1}}{\Delta t_{i+1}}-\frac{v_{i+1}-v_{i}}{\Delta t_{i}}\right)}{\frac{\Delta t_{i+1}+\Delta t_{i}}{2}}$.

The similarity of the smoothing cost function to the one minimized by a smoothing spline is shown in Eq. 4.13, see related chapter of the book (James et al., 2013). The expression is first developed using the intermediary variables $a_{a t, i}$ and $T_{a t, i}$ resulting in Eq. 4.14; and finally developed as a function of the velocity plan $\mathbf{v}$ and related times of passage in Eq. 4.15. The data term, the old velocity plan $\mathbf{v}_{\text {old }}$, and the time intervals between waypoints, $\Delta \mathbf{t}_{\text {plan }}$, are held constant during the optimization. The optimization is performed using Gauss-Newton Least Squares, where only the current velocity plan is considered as optimization variable $\mathbf{v}$. The initial, $v_{1}$, and end, $v_{N}$, velocities are kept constant, and therefore not optimized. The parameters $\left\{\lambda_{1}, \lambda_{2}, \lambda_{3}\right\}$ are weights adjusting the strength of each type optimization residuals. $\lambda_{1}$ regulates the strength of the data term. $\lambda_{2}$ regulates the strength of the smoothing for the acceleration. $\lambda_{3}$ regulates the strength of the smoothing for the derivative of the acceleration. 
A set of parameters weights that has provided good results and that was used to obtain the values shown in the simulation and experimental tests at the end of the chapter are the following: $\lambda_{1}=300, \lambda_{2}=1.12$ and $\lambda_{3}=0.08$.

Considering the notation from the review paper (Triggs et al., 1999), the previously defined cost function, Eq. 4.15, can be rewritten using residuals as follows, see Eq.4.16. In these equations $W$ is the weight matrix and $\Delta \mathbf{z}$ is the residual vector. The residual vector is subdivided in the three types of terms shown before $\Delta \mathbf{z}_{v_{0}} \Delta \mathbf{z}_{a} \Delta \mathbf{z}_{T} . \Delta z_{v_{0}, i}$ is defined for $i=2, \ldots, N-1$. $\Delta z_{a, i}$ is defined for $i=1, \ldots, N-2 . \Delta z_{T, i}$ is defined for $i=1, \ldots, N-3$.

$$
\begin{aligned}
& f(\mathbf{v})=\frac{1}{2} \Delta \mathbf{z}^{\top} W \Delta \mathbf{z}=\frac{1}{2}\left[\Delta \mathbf{z}_{v_{0}}, \Delta \mathbf{z}_{a}, \Delta \mathbf{z}_{T}\right] W\left[\Delta \mathbf{z}_{v_{0}}, \Delta \mathbf{z}_{a}, \Delta \mathbf{z}_{T}\right]^{\top} \\
& W=\operatorname{diag}\left(\lambda_{1} I_{N-2}, \lambda_{2} I_{N-2}, \lambda_{3} I_{N-3}\right) \\
& \Delta z_{v_{0}, i}=\left(v_{i}-v_{i, o l d}\right) \\
& \Delta z_{a, i}=T_{a t, i} \sqrt{\Delta t_{T_{a t, i}}}=\left(\frac{\left(a_{a t, i+1}-a_{a t, i}\right)}{\Delta t_{T_{a t, i}}}\right) \sqrt{\Delta t_{T_{a t, i}}} \\
& =\left(\frac{\left(\frac{v_{i+2}-v_{i+1}}{\Delta t_{i+1}}-\frac{v_{i+1}-v_{i}}{\Delta t_{i}}\right)}{\frac{\Delta t_{i+1}+\Delta t_{i}}{2}}\right) \sqrt{\frac{\Delta t_{i+1}+\Delta t_{i}}{2}} \\
& \Delta z_{T, i}=\left(\frac{T_{a t, i+1}-T_{a t, i}}{\Delta t_{\Delta T}}\right) \sqrt{\Delta t_{\Delta T}} \\
& =\left(\frac{\frac{\left(\frac{v_{i+3}-v_{i+2}}{\Delta t_{i+2}}-\frac{v_{i+2}-v_{i+1}}{\Delta t_{i+1}}\right)}{\frac{\Delta t_{i+2}+\Delta t_{i+1}}{2}}-\frac{\left(\frac{v_{i+2}-v_{i+1}}{\Delta t_{i+1}}-\frac{v_{i+1}-v_{i}}{\Delta t_{i}}\right)}{\frac{\Delta t_{i+1}+\Delta t_{i}}{2}}}{\frac{\Delta t_{i+2}+2 \Delta t_{i+1}+\Delta t_{i}}{4}}\right) \sqrt{\frac{\Delta t_{i+2}+2 \Delta t_{i+1}+\Delta t_{i}}{4}}
\end{aligned}
$$

Using this notation, the increment to the speed plan, $\Delta \mathbf{v}_{c}$, at each iteration is calculated from the following set of equations $\left(J^{\top} W J\right) \Delta \mathbf{v}_{c}=-J^{\top} W \Delta \mathbf{z}$, where $\mathrm{J}$ is the jacobian matrix of $\Delta \mathbf{z}$. The jacobian components of these residuals are:

- $\Delta z_{v_{0}, i}$

$$
J_{\Delta z_{v_{0}, i}, v_{i}}=1.0
$$

- $\Delta z_{a, i}$

$$
\begin{aligned}
& J_{\Delta z_{a, i}, v_{i}}=\frac{1}{\Delta \mathrm{t}_{\mathrm{i}} \sqrt{\frac{\Delta \mathrm{t}_{\mathrm{i}}}{2}+\frac{\Delta \mathrm{t}_{\mathrm{i}+1}}{2}}} \\
& J_{\Delta z_{a, i}, v_{i+1}}=-\frac{\frac{1}{\Delta \mathrm{t}_{\mathrm{i}}}+\frac{1}{\Delta \mathrm{t}_{\mathrm{i}+1}}}{\sqrt{\frac{\Delta \mathrm{t}_{\mathrm{i}}}{2}+\frac{\Delta \mathrm{t}_{\mathrm{i}+1}}{2}}} \\
& J_{\Delta z_{a, i}, v_{i+2}}=\frac{1}{\Delta \mathrm{t}_{\mathrm{i}+1} \sqrt{\frac{\Delta \mathrm{t}_{\mathrm{i}}}{2}+\frac{\Delta \mathrm{t}_{\mathrm{i}+1}}{2}}}
\end{aligned}
$$




$$
\begin{aligned}
& J_{\Delta z_{T, i}, v_{i}}=-\frac{1}{\Delta \mathrm{t}_{\mathrm{i}}\left(\frac{\Delta \mathrm{t}_{\mathrm{i}}}{2}+\frac{\Delta \mathrm{t}_{\mathrm{i}+1}}{2}\right) \sqrt{\frac{\Delta \mathrm{t}_{\mathrm{i}}}{4}+\frac{\Delta \mathrm{t}_{\mathrm{i}+1}}{2}+\frac{\Delta \mathrm{t}_{\mathrm{i}+2}}{4}}} \\
& J_{\Delta z T, i}, v_{i+1}=\frac{\frac{1}{\Delta \mathrm{t}_{\mathrm{i}+1}\left(\frac{\Delta \mathrm{t}_{\mathrm{i}+1}}{2}+\frac{\Delta \mathrm{t}_{\mathrm{i}+2}}{2}\right)}+\frac{\frac{1}{\Delta \mathrm{t}_{\mathrm{i}}}+\frac{1}{\Delta \mathrm{t}_{\mathrm{i}+1}}}{\frac{\Delta \mathrm{t}_{\mathrm{i}}}{2}+\frac{\Delta \mathrm{t}_{\mathrm{i}+1}}{2}}}{\sqrt{\frac{\Delta \mathrm{t}_{\mathrm{i}}}{4}+\frac{\Delta \mathrm{t}_{\mathrm{i}+1}}{2}+\frac{\Delta \mathrm{t}_{\mathrm{i}+2}}{4}}} \\
& J_{\Delta z T, i}, v_{i+2}=-\frac{\frac{1}{\Delta \mathrm{t}_{\mathrm{i}+1}\left(\frac{\Delta \mathrm{t}_{\mathrm{i}}}{2}+\frac{\Delta \mathrm{t}_{\mathrm{i}+1}}{2}\right)}+\frac{\frac{1}{\Delta \mathrm{t}_{\mathrm{i}+1}}+\frac{1}{\Delta \mathrm{t}_{\mathrm{i}+2}}}{\frac{\Delta \mathrm{t}_{\mathrm{i}+1}}{2}+\frac{\Delta \mathrm{t}_{\mathrm{i}+2}}{2}}}{\sqrt{\frac{\Delta \mathrm{t}_{\mathrm{i}}}{4}+\frac{\Delta \mathrm{t}_{\mathrm{i}+1}}{2}+\frac{\Delta \mathrm{t}_{\mathrm{i}+2}}{4}}} \\
& J_{\Delta z_{T, i}, v_{i+3}}=\frac{1}{\Delta \mathrm{t}_{\mathrm{i}+2}\left(\frac{\Delta \mathrm{t}_{\mathrm{i}+1}}{2}+\frac{\Delta \mathrm{t}_{\mathrm{i}+2}}{2}\right) \sqrt{\frac{\Delta \mathrm{t}_{\mathrm{i}}}{4}+\frac{\Delta \mathrm{t}_{\mathrm{i}+1}}{2}+\frac{\Delta \mathrm{t}_{\mathrm{i}+2}}{4}}}
\end{aligned}
$$

(b) After every iteration of the smoothing spline optimization the resulting speed plan is not selfconsistent. That means that the values $\left\{\mathbf{v}_{\text {plan }}, \mathbf{a}_{\text {at, plan }}, \Delta \mathbf{t}_{\text {plan }}\right\}$ do not verify the equations of an uniformly accelerated motion for all segments of the trajectory. This is because the smoothing procedure does not enforce them. For this reason, after every iteration of the smoothing spline optimization a new pass of velocity_plan_sweep_double_pass is performed, so that a new set of self-consistent values for $\left\{\mathbf{v}_{\text {plan }}, \mathbf{a}_{\text {at }}\right.$ plan,$\left.\Delta \mathbf{t}_{\text {plan }}\right\}$ is obtained. This amounts to the following function call:

$\left[\mathbf{v}_{\text {plan }}, \mathbf{a}_{\text {at }, \text { plan }}, \Delta \mathbf{t}_{\text {plan }}\right]=$ Velocity_Plan_Sweep_Double_Pass $\left(\mathbf{s}, \mathbf{r},\left\{\mathbf{t}_{i}^{*}\right\}_{i}, \mathbf{v}_{\text {newmax }}\right.$, config $)$

Where $\mathbf{v}_{\text {newmax }}$ is defined for every component as the minimum of $\mathbf{v}_{\text {plan }}+\Delta \mathbf{v}_{c}$ and $\mathbf{v}_{\text {desired }}$, that is: $\mathbf{v}_{\text {new max }}=\left\{v_{\text {newmax }, i}=\min \left(v_{\text {plan }, i}+\Delta v_{c, i}, v_{\text {desired }, i}\right)\right\}$.

The Gauss-Newton Least Squares optimization, that encodes velocity smoothing, is stopped: when (1) the norm of the jacobian of the cost function, $f(\mathbf{v})$ increased in the last iteration or (2) when a preset number of iterations, 10 provides consistently good results, is reached.

(c) Rerunning the smoothing spline optimization exchanging the data term by the last smoothed velocity plan allows the algorithm to gradually forget the strong initial bang-bang velocity plan. For a given set of the smoothing strength parameters $\left\{\lambda_{1}, \lambda_{2}, \lambda_{3}\right\}$, increasing the number of reruns of the smoothing spline optimization results in smoother speed plans, with lower maximum values for the acceleration derivative. This effect is demonstrated in the results section, see Sec.4.7.3.2. This fact allows to calculate a speed plan with an acceleration derivative bounded by a certain value. In practice, in order to obtain efficient computation times, the smoothing spline optimization is repeated a fixed number of times. In our experiments 10 provides consistently good results, see Secs. 4.7.3.3 \& 4.7.4.1.

The velocity smoothing operation is summarized in Alg. 5 .

7. The calculated trajectory message for the trajectory controller is filled-in including the speed and acceleration plans. This is the final trajectory which is to be executed by the trajectory controller. Take into account that the trajectory controller, used in both in EuRoC and the DJI Challenge, calculates a smoother trajectory by fitting a spline to the provided trajectory, times-of-passage, speed and acceleration plans.

\subsubsection{Communication with other modules}

Our trajectory planner ROS node communicates using the following topics:

- Subscription to the goal position.

- Subscription to the current pose estimate.

- Subscription to the depth maps of the stereo cameras on the drone. The depth data is used to update the planner's internal map representation. 


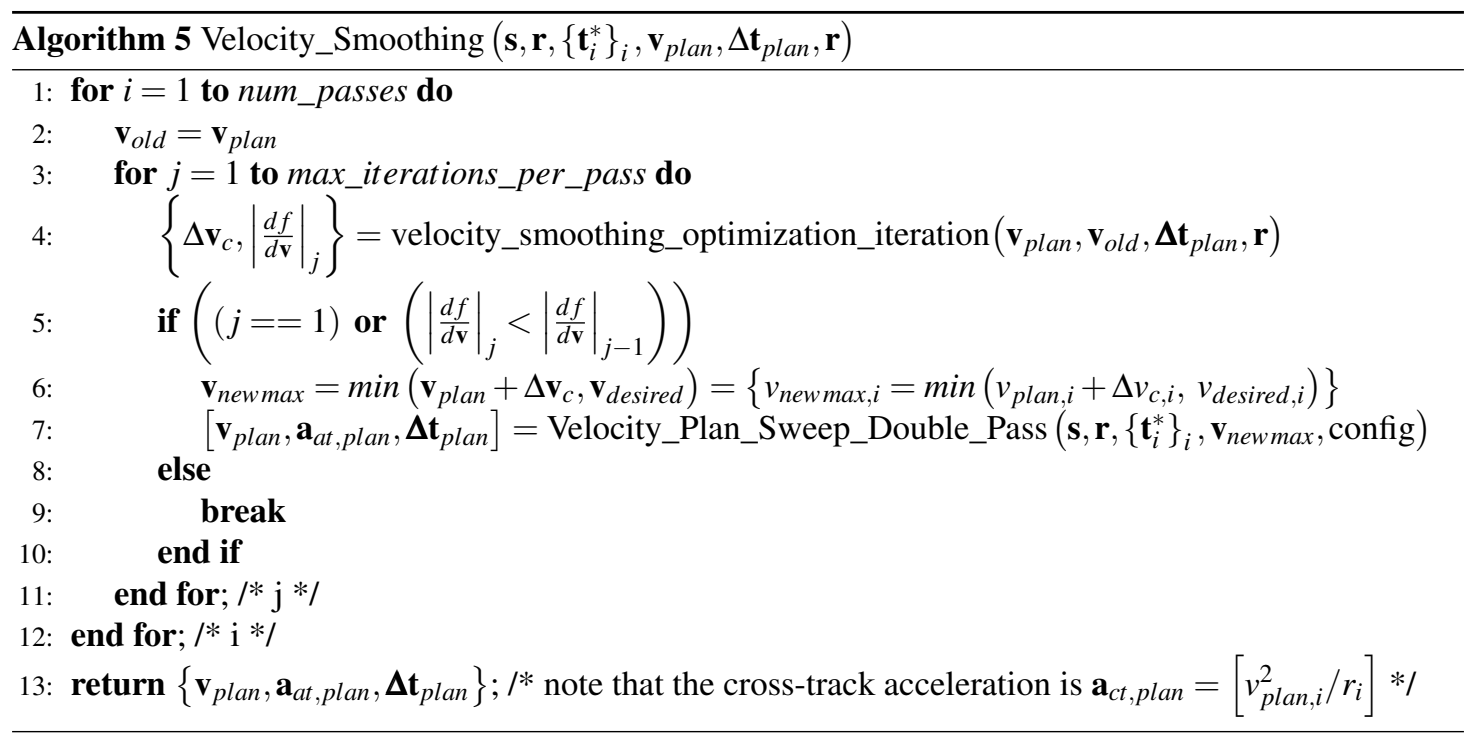

- Publication of the calculated trajectory, which includes the waypoints and the corresponding timesof-passage, acceleration and speed plans.

\subsubsection{Configuration Parameters}

The most important parameters are the following:

- Desired maximum velocity over the trajectory (vertical upwards and downwards; and horizontal).

- Desired maximum acceleration over the trajectory (vertical upwards and downwards; and horizontal).

- Maximum clearance distance, $d_{\max }$, calculated by the DynamicEDT3D library. Points at further distances are simply set to this maximum value, therefore reducing the computation requirements of the distance map calculation.

- The multirotor's horizontal and vertical sizes in $\mathrm{m}$.

- The maximum planning time in seconds allocated for the calculation of an obstacle free trajectory by the OMPL library.

- Maximum distance between waypoints in the output trajectory.

- For the $P R M^{*}$ algorithm, the minimum number of nodes calculated to populate the free space during the planner initialization.

- The parameters $\left\{\lambda_{1}, \lambda_{2}, \lambda_{3}\right\}$ that adjust the weights of each type of optimization residuals of the velocity smoothing operation.

\subsection{Geometric Speed Planner}

The previous section, Sec. 4.5 "Obstacle-free Smooth Trajectory Planning”, described a planning approach which divides the problem in separate subproblems: obstacle-free path planning, obstacle-aware trajectory shortening and smoothing and speed planning. The presented speed planning approach can also be used when the drone needs to fly in obstacle-free areas over paths defined by waypoints. For this purpose, a module named "Geometric Speed Planner" was developed. In the obstacle-free scenario, this module serves as an interface to the controller which is expecting as trajectory a path supplemented with a speed, an acceleration and a time-of-passage plans. The "Geometric Speed Planner" implements only the following two subproblems: trajectory smoothing and speed planning. These steps correspond to the following: 
1. Trajectory smoothing with no cost-checking:

1. Add waypoints in the current path until the path segments are all smaller than a given small distance, for instance $1 \mathrm{~m}$. Then, reduce consecutive waypoints that are too near each other at less distance than a given value, for instance $7 \mathrm{~m}$. This has the effect of dividing sharp trajectory corners into two or more softer corners, which helps in the next step.

2. Smooth the path using the BSpline algorithm (for instance 6 passes): new wapoints are sampled making the path rounder near the corners.

3. Reduce consecutive waypoints that are too near each other at less distance than a given value, for instance $1.5 \mathrm{~m}$. This reduces the number of waypoints that are sampled very close to each other.

4. Reduce consecutive waypoints that are in a straight line and too near each other at less distance than a given value, for instance $5.0 \mathrm{~m}$. This is important to reduce the number of points over which the speed plan is calculated, and also the time that the controller needs to calculate the interpolating spline over the trajectory.

5. The first and last trajectory segments are subdivided two times to increase the number of waypoints for the initial and final accelerations, which is helpful for the subsequent speed planning approach.

2. Speed planning: the algorithm explained in Sec.4.5.4 is used.

\subsection{Experimental Results}

\subsubsection{Videos of Experiments}

The following videos show a multirotor in real and simulated flight executing trajectories delivered by the presented trajectory planner:

- 2016 DJI Developer Challenge (DJIC 2016), http: / / bit. ly/2faublQ: this video summarizes the participation of the "Graz Griffins" team in the challenge. Starting at 2:38, first, some planned trajectories and, second, the experimental autonomous flight where the trajectories plotted in Sec.4.7.4 were executed are shown.

- An experimental fully autonomous package delivery flight demonstration made by the ICG - TU Graz, https://youtu.be/bxM6dls2wuo:this demo was made in collaboration with the company SFL Technologies as part of the roll-out of their brand new electric vehicle, the ELI. The roll-out of this vehicle and the autonomous flight demo have been described by two Austrian newspapers: Mein Bezirk ${ }^{13}$ and Kleine Zeitung ${ }^{14}$

- European Robotics Challenge (EuRoC 2014), https : / bit. ly/2cQnO6I): this video shows the performance of a simulated Asctec Firefly hexacopter MAV executing trajectories produced by the presented planner by means of a controller developed by D. Muschick, a colleague from the Institute of Automation and Control - TU Graz. Both algorithms where succinctly described in the paper (Pestana et al.,2015).

\subsubsection{Experimental Setup}

\subsubsection{Flights in a Simulation Environment}

The trajectory planner was first tested using the octomap map provided by the EuRoC2014 organizers. The Simulation Contest of EuRoC Challenge 3 takes places in a simulated outdoors industry environment of size 50mx50mx40m, see Fig. 4.1, which is described using an Octomap with a leaf size of $0.25 \mathrm{~m}$. The quadrotor has a diameter of (actually less than) $1.00 \mathrm{~m}$ and a height of (actually less than) $0.50 \mathrm{~m}$. For task 4.3 of the simulation contest, the trajectories had to be calculated rather fast and executed at average

\footnotetext{
${ }^{13}$ ELI roll-out - Mein Bezirk - http://bit. ly/2hott $8 t$

${ }^{14}$ ELI roll-out - Kleine Zeitung - http://bit.ly/2it6N2P
} 
speeds of around $3.5 \mathrm{~m} / \mathrm{s}$ to attain the best score in the simulation contest. The overall trajectory planning and execution had to be done in less than 10 seconds.

The feasibility of the calculated speed and acceleration plans were then tested in simulation, on the accompanying simulator. A publication explaining the implementation, capabilities, and configuration of this simulator, the RotorS simulator framework (Furrer et al., 2016 ${ }^{15}$ has been published in 2016. The main feature of the RotorS simulator are that it utilizes the Gazebo physics engine, that it can be easily run and interfaced using ROS, that it provides a controller and a state estimator to help the user getting started, and that it provides an already implemented evaluation framework. For instance, the propellers are modeled separately, so that multirotors with different number of propellers can be meaningfully simulated. There are currently a few MAVs available in the simulator: the AR Drone; the Asctec Firefly, Pelican and Hummingbird and the 3D Robotics Iris.

It is noted here that there are other open-source multirotor drone simulators, among others: the hector_quadrotor (Meyer et al., 2012 [16 hardware and software in the loop simulators for the Pixhawk autopilo ${ }^{11}{ }^{18}$ and the AirSim simulato: ${ }^{19}$ (Shah et al., 2017) from Microsoft Research.

\subsubsection{Experimental Flights with a Multirotor}

The experimental flights were done as part of the participation on the 2016 DJI Developer Challenge, and they have been performed using a DJI M100 quadrotor drone. Our fully equipped DJI M100 quadrotor includes: the DJI Manifold onboard computer, the DJI Guidance sensing system and the DJI Zenmuse X3 gimbal camera. For more details on the used setup the reader is referred to Appx. H.3.1.

\subsubsection{Results in EuRoC 2014 - Challenge 3 - Simulation Contest}

The trajectory planner used in the participation of the "Graz Griffins" to the EuRoC 2014 challenge utilized the $P R M^{*}$ planning algorithm. The performance characteristics of the planner are the following:

- Selected planning algorithm: $P R M^{*}$.

- Memory usage:

$-300 \mathrm{MB}=200 \mathrm{MB}$ map representations $+100 \mathrm{MB}[20.000$ nodes $]$ in planner data

$-633 \mathrm{MB}=200 \mathrm{MB}$ map representations $+433 \mathrm{MB}[100.000$ nodes $]$ in planner data

- 20.000 nodes were used for the final submission. New nodes are created with each trajectory planning task. When there are too many nodes in the data structure the planner becomes slower in answering individual queries.

- The loading time of the planner in the laptop Intel Core i7-4710HQ processor @ $2.5 \mathrm{GHz}$ for 100k nodes is around 50-70seconds.

- The planner is set to execute as a low priority process.

- The total computation time is around $0.7-1.2 \mathrm{~s}$. This time is used as follows: $0.5 \mathrm{~s}$ for planning, $0.1 \mathrm{~s}$ for trajectory simplification and $0.3 \mathrm{~s}$ for speed planning; resulting in a mean planning time of around $0.9 \mathrm{~s}$.

- Main configuration parameters:

- $d_{\max }$ approximately set a desired safety distance to be kept between the MAV and the environment. A wall can be approached as much as needed (for instance if the goal target is near obstacles). However, the planner won't do this unless needed and will stay away from obstacles otherwise. This parameter was set to $4.0 \mathrm{~m}$.

\footnotetext{
15 https://github.com/ethz-asl/rotors_simulator

16 http://wiki.ros.org/hector_quadrotor

17 http://ardupilot.org/dev/docs/sitl-simulator-software-in-the-loop.html

18 https://pixhawk.org/users/hil

19 https://github.com/Microsoft/Airsim
} 
- One of the last trajectory simplification steps resamples the trajectory before performing the speed planning and delivering both to the Trajectory Controller. It sets the minimum (desired) distance between trajectory waypoints. This parameters was set to $1.0 \mathrm{~m}$.

The evaluation of the EuRoC2014 organizers showed that the control framework developed by D. Muschick (Holzmann et al., 2015, Pestana et al., 2015) for the "Graz Griffins" team achieved a position RMS error of $0.055 \mathrm{~m}$ and an angular velocity RMS error of $0.087 \mathrm{rad} / \mathrm{s}$ in stationary hovering. For the evaluation they used the RoToRs simulator, briefly described above. The simulated sensor uncertainties were typical of a multirotor such as the Asctec Firefly. The controlled drone was able to reject constant and variable wind disturbances. The effect of a constant lateral wind is rejected in $3.6 \mathrm{~s}$.

\begin{tabular}{||c|ccc|c||}
\hline path length [m] & time plan & execution & total $[\mathrm{s}]$ & $\max$ speed[m/s] \\
\hline \hline 33.70 & 0.77 & $7.55 / 8.60$ & $8.32 / 9.37$ & 7.72 \\
20.25 & 0.56 & $6.00 / 6.33$ & $6.56 / 6.89$ & 5.50 \\
25.17 & 0.57 & $6.00 / 7.13$ & $6.62 / 7.70$ & 6.18 \\
37.60 & 0.72 & $8.79 / 9.22$ & $9.57 / 9.94$ & 7.66 \\
29.18 & 0.65 & $7.08 / 7.83$ & $7.76 / 8.47$ & « averages \\
\hline
\end{tabular}

Table 4.1: Trajectory planning and control performance - EuRoC2014. Results for the goal positions sequence utilized be the challenge organizers for evaluation. The shown values are a combination of evaluation made on my own computer and the feedback from the organizers after their evaluation of all teams' submissions. The planning times are different because different processors were used: (ours) Intel Core i7-4710HQ and (eval) Intel® CoreTM2 6700. The path length, planning time and max speed are for the evaluation on our computer. The execution and total time are shown as (ours) / (eval). This shows that similar results are obtained using both computers. The last row shows average values. The average achieved navigation speed is: [(ours) / (eval)] $4.12 / 3.73 \mathrm{~m} / \mathrm{s}$. The planning time corresponds to the whole trajectory generation time. The execution time to the time that it takes for the controller to perform the commanded trajectory. And the total time is the sum of both.

The trajectory controller achieved the performance detailed in table 4.1 in combination with the mentioned controller. The EuRoC2014 organizers executed our architecture in a Intel® CoreTM2 6700 processor@ $2.66 \mathrm{Ghz}$, which can be mounted in a Asctec Firefly quadrotor. The performance of the trajectory following is such that paths of $35 \mathrm{~m}$ are planned in $0.75 \mathrm{~s}$ and can be safely executed in $7.55 \mathrm{~s}$ to $8.8 \mathrm{~s}$ with average speeds of $4.2 \mathrm{~m} / \mathrm{s}$ and peak speeds of $7.70 \mathrm{~m} / \mathrm{s}$.

In the next subsection trajectories resulting from the presented trajectory planning and subsequent speed and acceleration planning algorithms are shown. They are calculated using the EuRoC2014 - Challenge 3 octomap. These plots show the following:

- The calculated obstacle-free smooth trajectories

- The calculated speed and acceleration plans, and the corresponding times-of-passage plan.

- The estimated radius of curvature at each point of the trajectory path. Note how the radius of curvature affects the maximum velocity of the speed plan before, inside and after curves.

- Note how the acceleration and speed plans are correctly limited to the desired values, and how the derivative of the acceleration remains reasonable. 


\subsubsection{Comparison - with and without velocity smoothing - speed and acceleration plans}
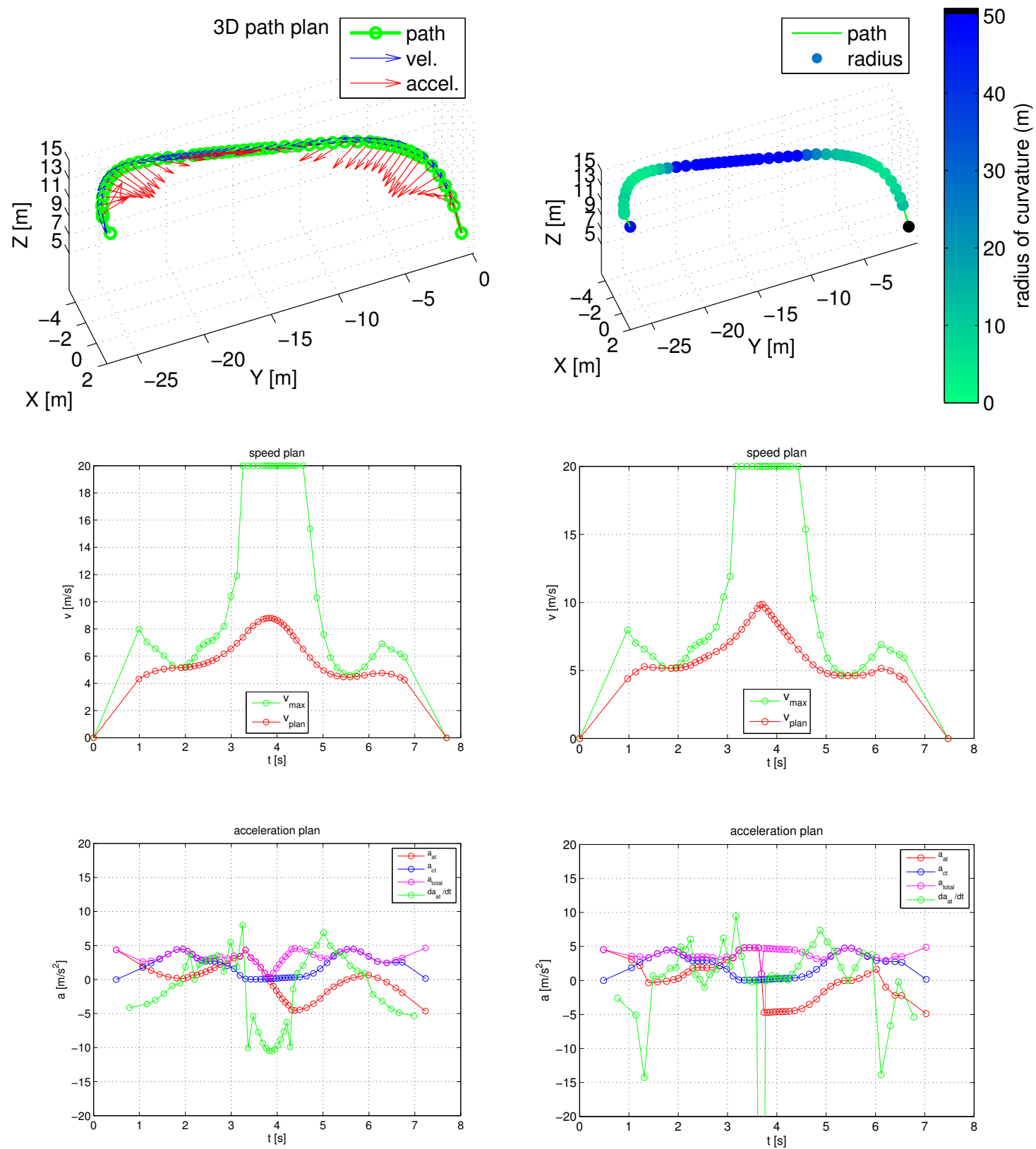

Figure 4.7: Velocity Smoothing - EuRoC2014 - Example Trajectory 1 - Plots. See Figs. 4.10 for a visualization the corresponding planned trajectory. The top left plot shows the trajectory path in 3D (green) along with the speed (blue) and acceleration (red) plans. The top right plot shows the trajectory path color coded with the radius of curvature, black color denote parts of the trajectory estimated to be straight. For the center and bottom plots, (left) is the result of applying the velocity smoothing optimization and (right) the result of applying only the velocity_plan_sweep_double_pass. The center plots show the final along-track speed plan (red) with the maximum desired velocity (green) affected and limited by the radius of curvature. The bottom plots show the along-track (red), cross-track (blue) and total (magenta) acceleration, along with the derivative of the acceleration (green). As shown, the velocity smoothing ends up with a very similar final trajectory execution time, but with a much smoother and feasible velocity and acceleration plan. 

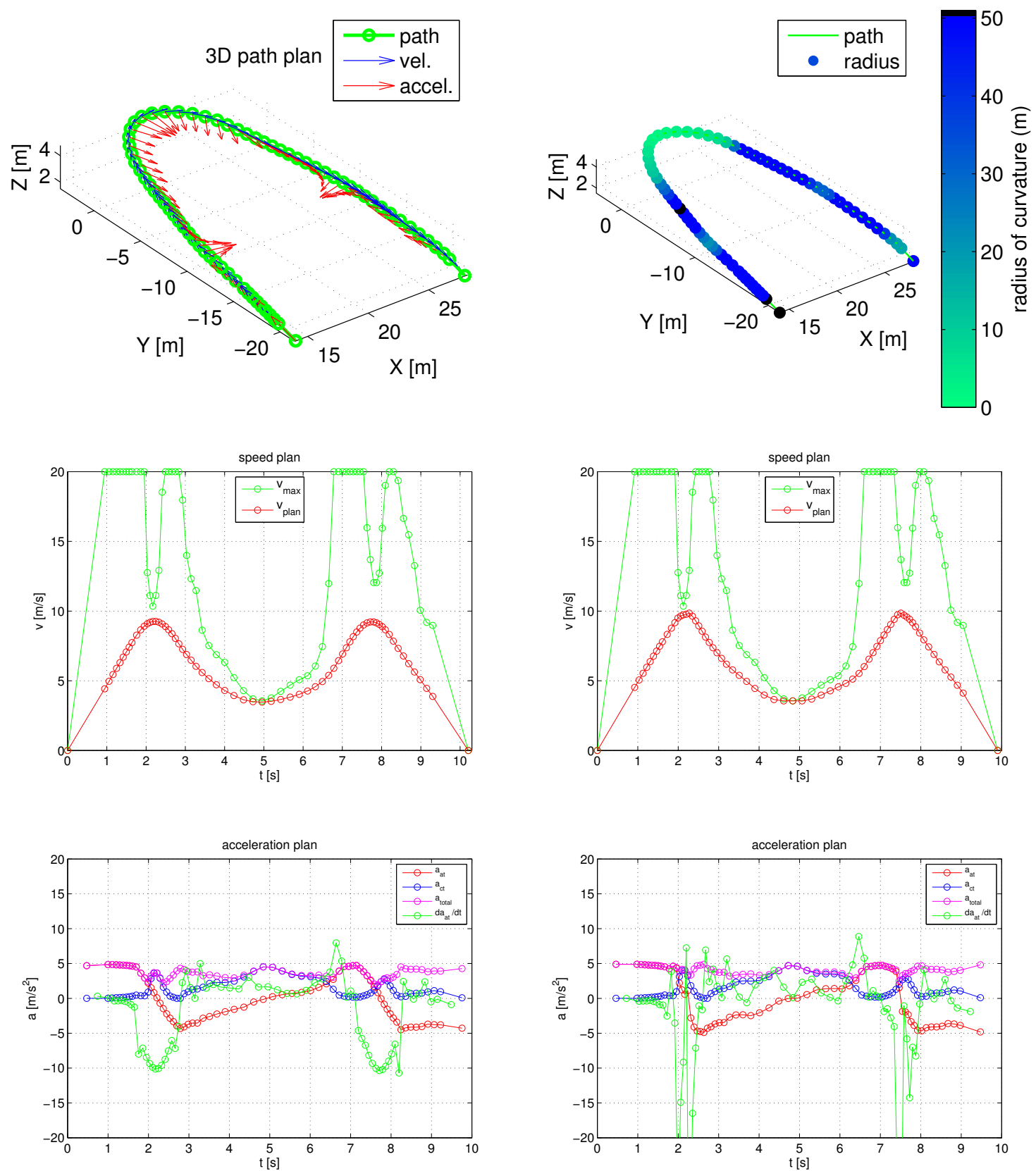

Figure 4.8: Velocity Smoothing - EuRoC2014 - Example Trajectory 7 - Plots. See Figs. 4.22 for a visualization the corresponding planned trajectory. The top left plot shows the trajectory path in 3D (green) along with the speed (blue) and acceleration (red) plans. The top right plot shows the trajectory path color coded with the radius of curvature, black color denote parts of the trajectory estimated to be straight. For the center and bottom plots, (left) is the result of applying the velocity smoothing optimization and (right) the result of applying only the velocity_plan_sweep_double_pass. The center plots show the final along-track speed plan (red) with the maximum desired velocity (green) affected and limited by the radius of curvature. The bottom plots show the along-track (red), cross-track (blue) and total (magenta) acceleration, along with the derivative of the acceleration (green). As shown, the velocity smoothing ends up with a very similar final trajectory execution time, but with a much smoother and feasible velocity and acceleration plan. 


\subsubsection{Comparison - Number of velocity smoothing passes - speed and acceleration plans}
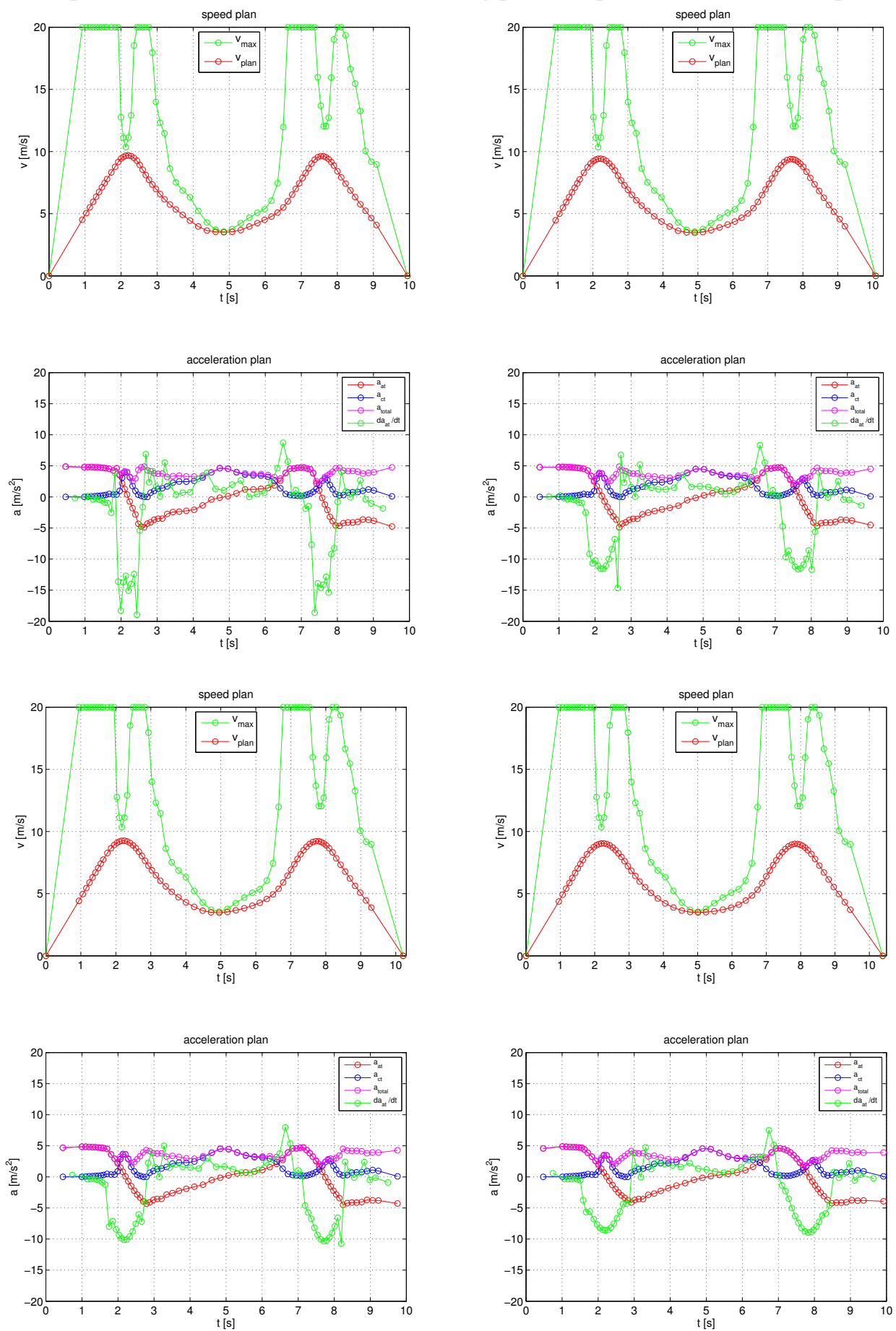

Figure 4.9: Velocity Smoothing - EuRoC2014 - Example Trajectory 7 - Plots. See Figs. 4.22 for a visualization the corresponding planned trajectory. Each pair of plots shows a different number of velocity smoothing passes and maximum acceleration derivative, (upper left) $\left[1 ; 19 \frac{\mathrm{m}}{\mathrm{s}^{3}}\right]$ (upper right) $\left[5 ; 15 \frac{\mathrm{m}}{\mathrm{s}^{3}}\right]$ (down left) $\left[10 ; 11 \frac{\mathrm{m}}{\mathrm{s}^{3}}\right]$ (down right) $\left[20 ; 9 \frac{\mathrm{m}}{\mathrm{s}^{3}}\right]$. The top plots show the final along-track speed plan (red) with the maximum desired velocity (green) affected and limited by the radius of curvature. The bottom plots show the along-track (red), cross-track (blue) and total (magenta) acceleration, along with the derivative of the acceleration (green). As shown, for a given set of the smoothing strength parameters $\left\{\lambda_{1}, \lambda_{2}, \lambda_{3}\right\}$, increasing the number of reruns of the smoothing spline optimization results in smoother speed plans, with lower maximum values for the acceleration derivative. 


\subsubsection{Plots of trajectories, speed and acceleration plans}

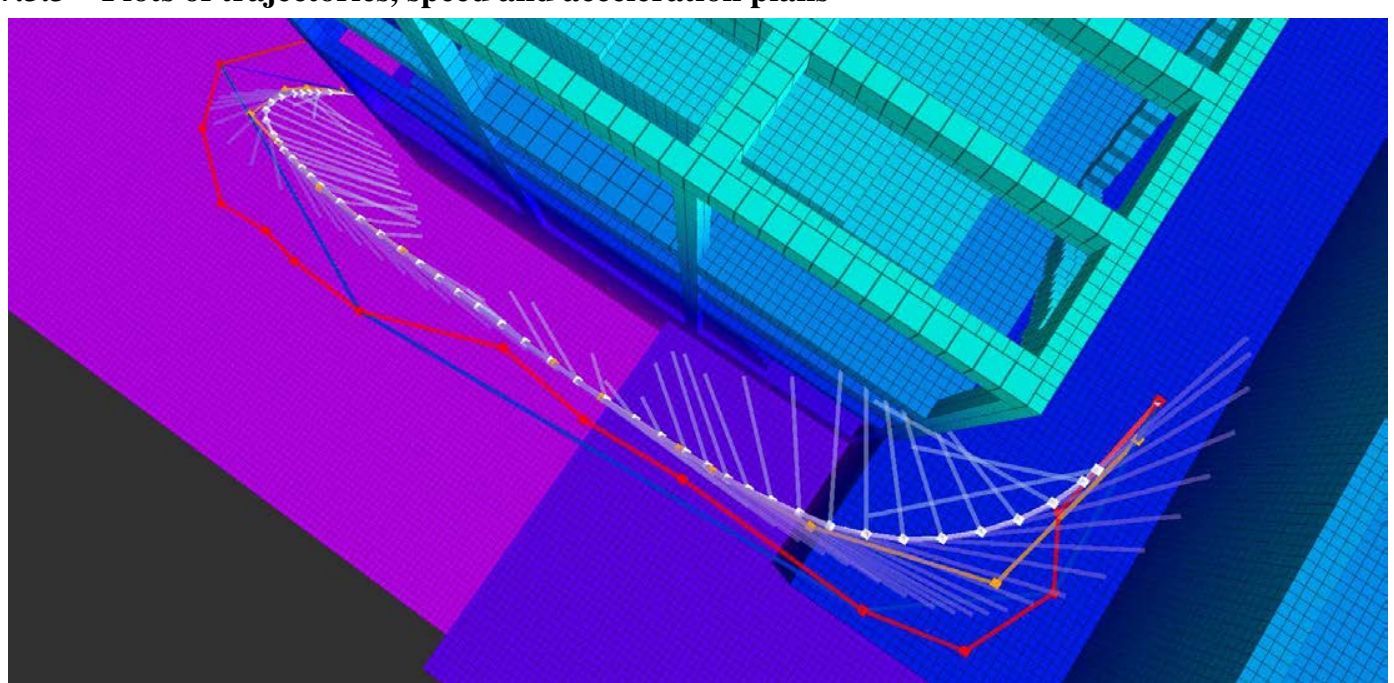

Figure 4.10: Trajectory Planner - EuRoC2014 - Example Trajectory 1 - RGB. The octomap is colorcoded from low altitude (pink) to high altitude (clear blue). A typical planned trajectory is shown: The output of the PRMStar algorithm (red) is consecutively shortened (green-blue-orange-white). The semi transparent lines show the velocity and acceleration plans.
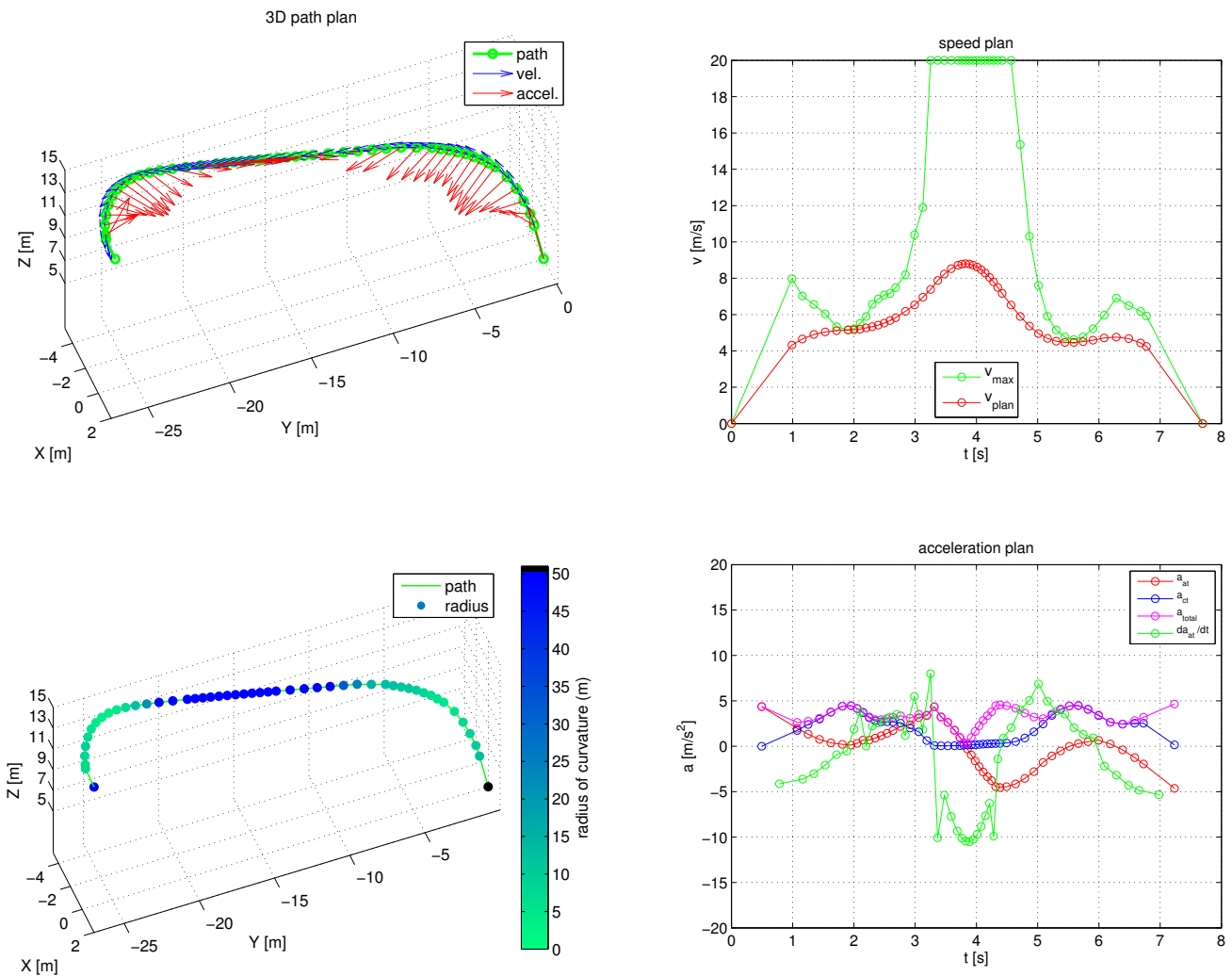

Figure 4.11: Trajectory Planner - EuRoC2014 - Example Trajectory 1 - Plots. The top left plot shows the trajectory path in 3D (green) along with the speed (blue) and acceleration (red) plans. The bottom left plot shows the trajectory path color coded with the radius of curvature, black color denote parts of the trajectory estimated to be straight. The right top plot shows the final along-track speed plan (red) with the maximum desired velocity (green) affected and limited by the radius of curvature. The right bottom plot shows the along-track (red), cross-track (blue) and total (magenta) acceleration, along with the derivative of the acceleration (green). 


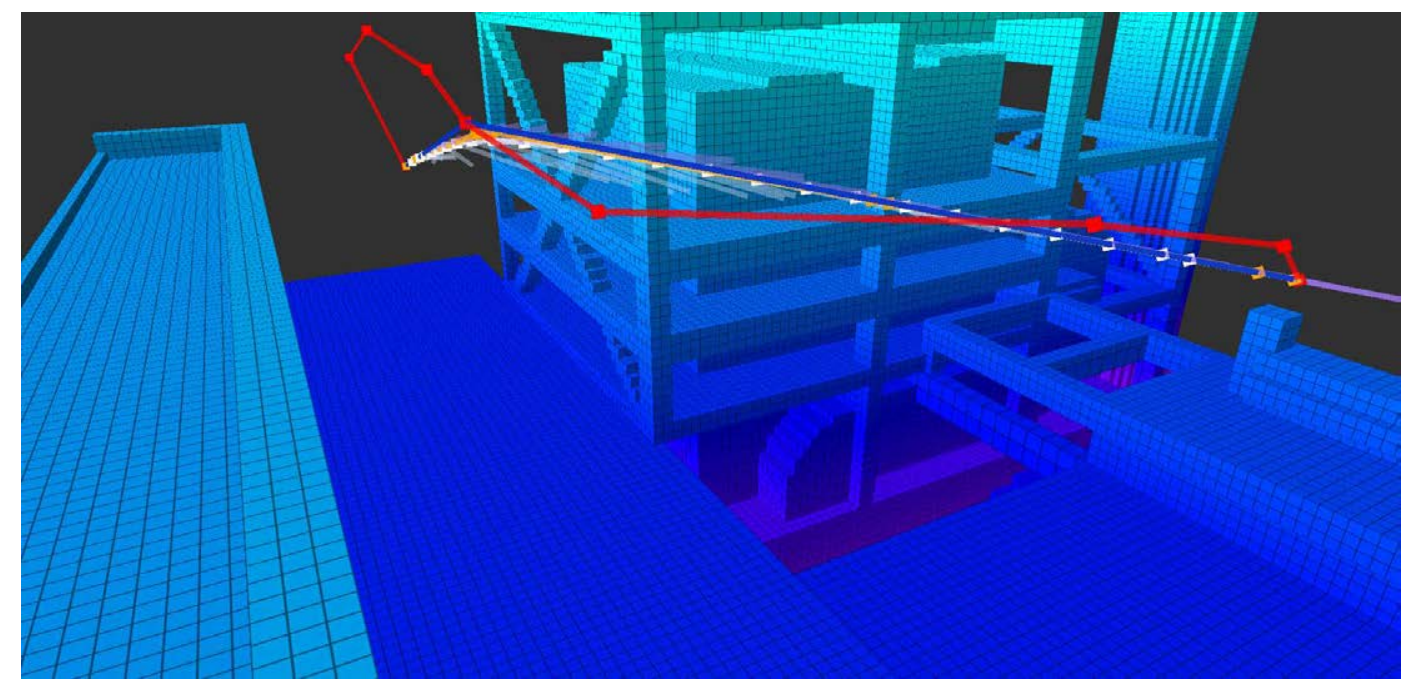

Figure 4.12: Trajectory Planner - EuRoC2014 - Example Trajectory 2 - RGB. The octomap is colorcoded from low altitude (pink) to high altitude (clear blue). A typical planned trajectory is shown: The output of the PRMStar algorithm (red) is consecutively shortened (green-blue-orange-white). The semi transparent lines show the velocity and acceleration plans.
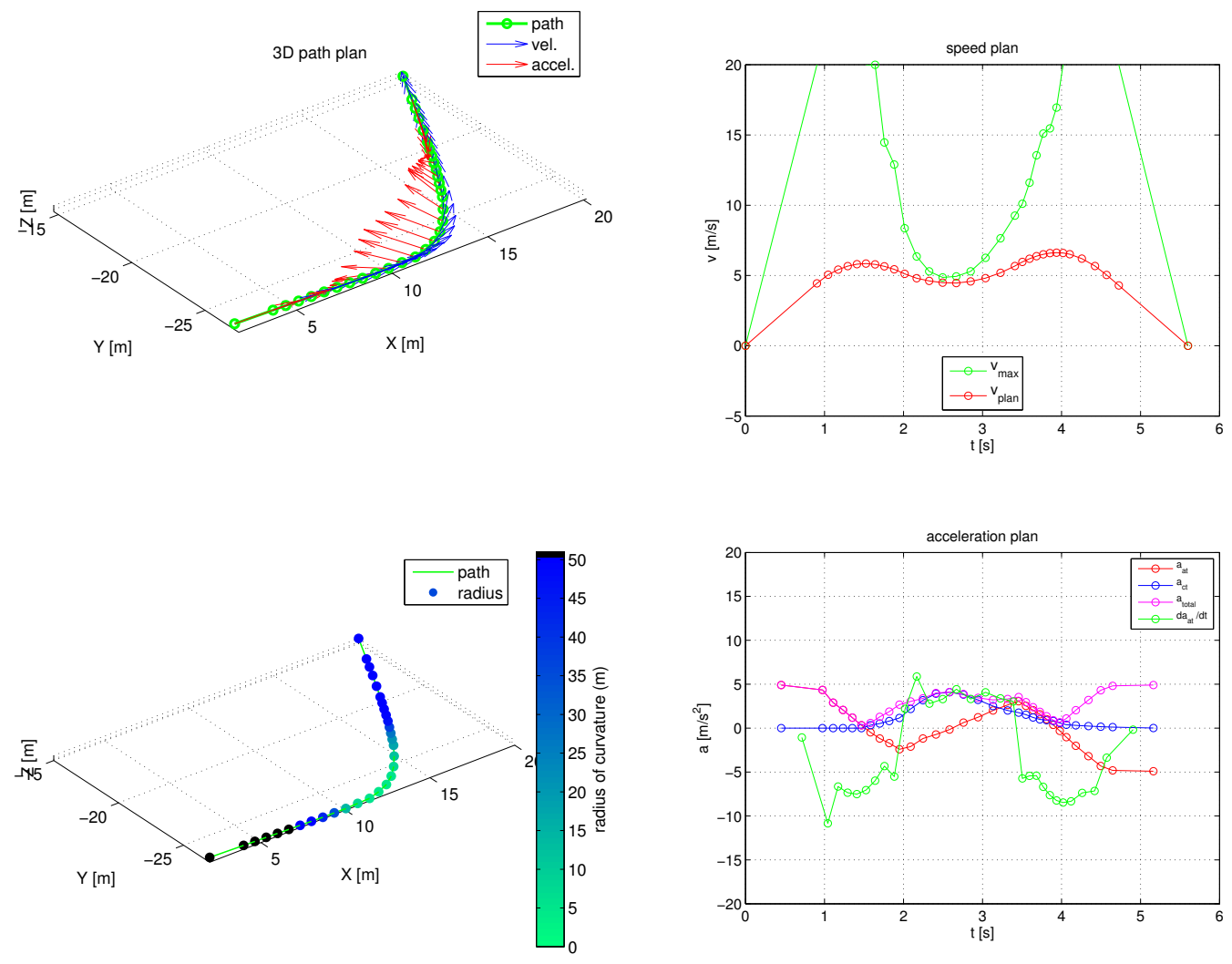

Figure 4.13: Trajectory Planner - EuRoC2014 - Example Trajectory 2 - Plots. The top left plot shows the trajectory path in 3D (green) along with the speed (blue) and acceleration (red) plans. The bottom left plot shows the trajectory path color coded with the radius of curvature, black color denote parts of the trajectory estimated to be straight. The right top plot shows the final along-track speed plan (red) with the maximum desired velocity (green) affected and limited by the radius of curvature. The right bottom plot shows the along-track (red), cross-track (blue) and total (magenta) acceleration, along with the derivative of the acceleration (green). 


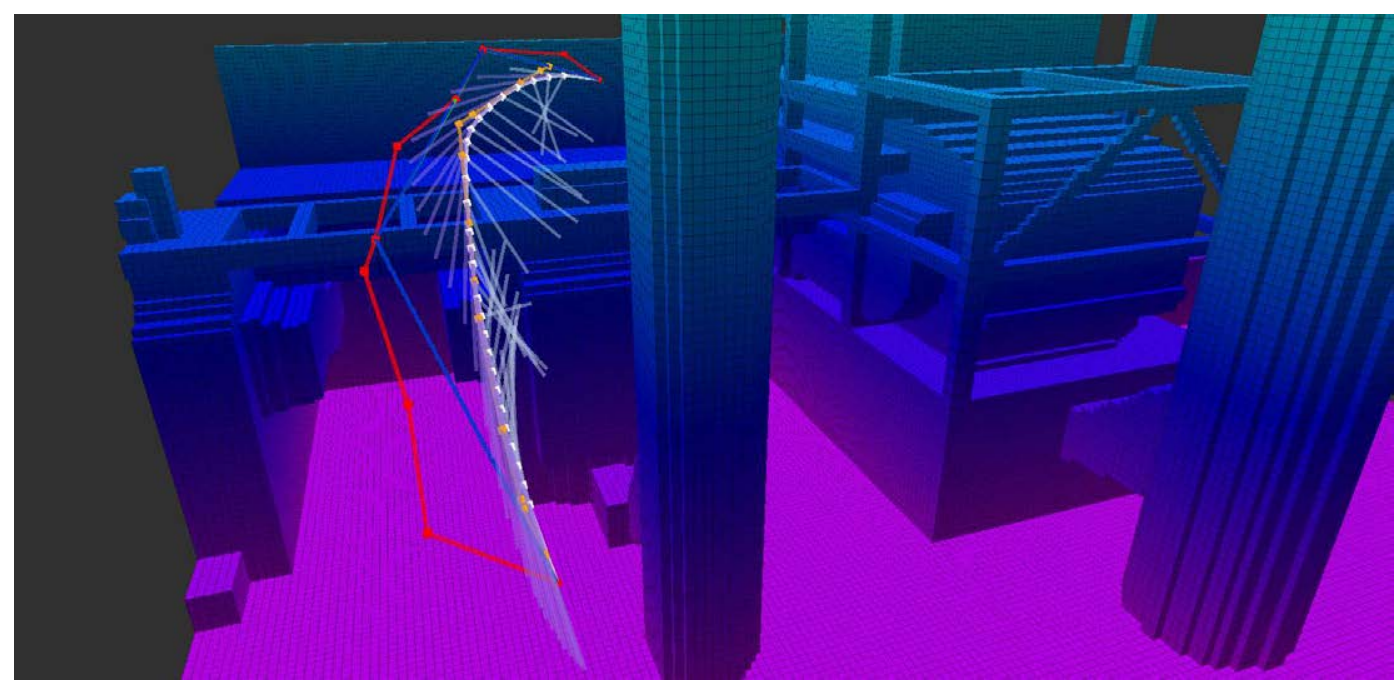

Figure 4.14: Trajectory Planner - EuRoC2014 - Example Trajectory 3 - RGB. The octomap is colorcoded from low altitude (pink) to high altitude (clear blue). A typical planned trajectory is shown: The output of the PRMStar algorithm (red) is consecutively shortened (green-blue-orange-white). The semi transparent lines show the velocity and acceleration plans.
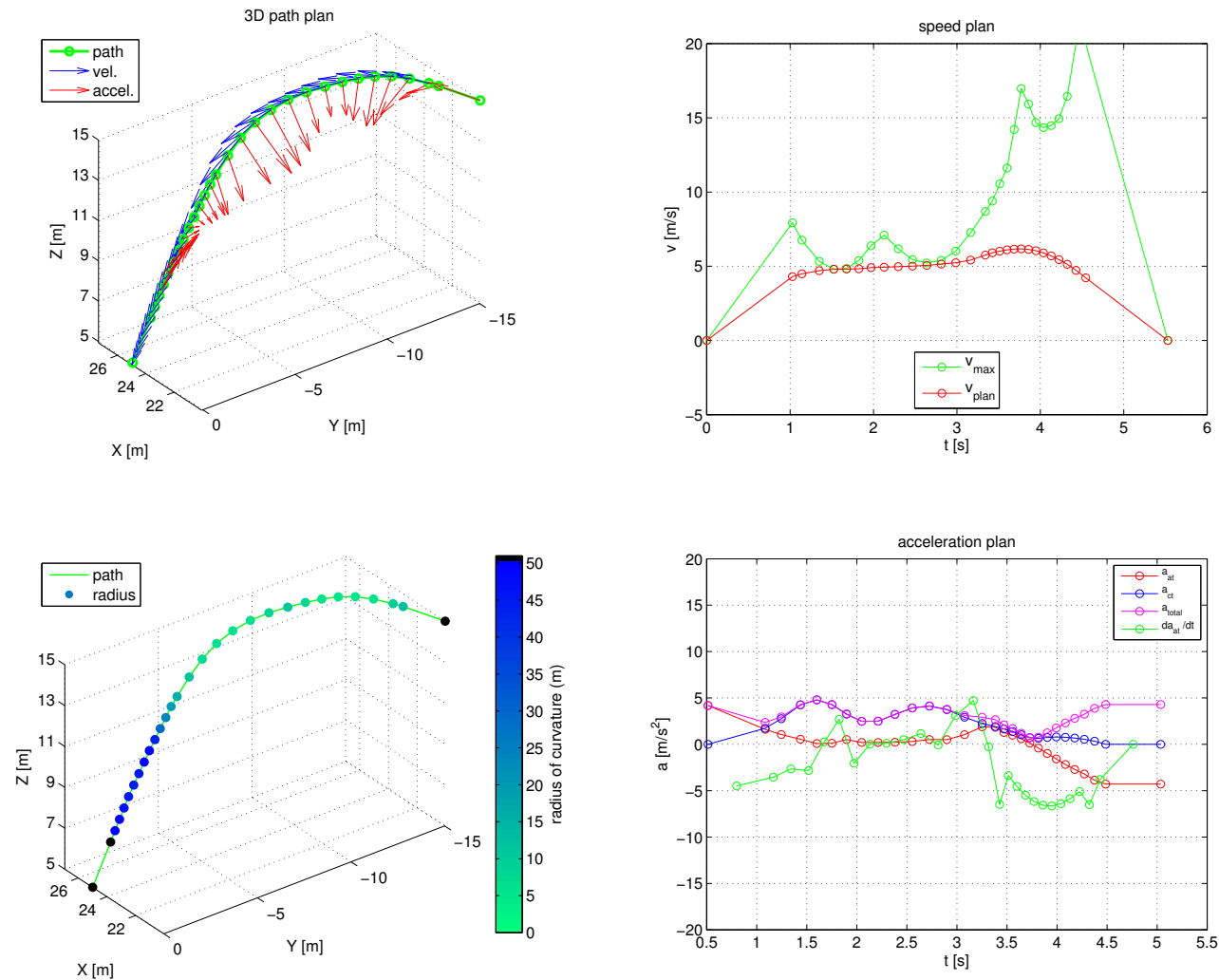

Figure 4.15: Trajectory Planner - EuRoC2014 - Example Trajectory 3 - Plots. The top left plot shows the trajectory path in 3D (green) along with the speed (blue) and acceleration (red) plans. The bottom left plot shows the trajectory path color coded with the radius of curvature, black color denote parts of the trajectory estimated to be straight. The right top plot shows the final along-track speed plan (red) with the maximum desired velocity (green) affected and limited by the radius of curvature. The right bottom plot shows the along-track (red), cross-track (blue) and total (magenta) acceleration, along with the derivative of the acceleration (green). 


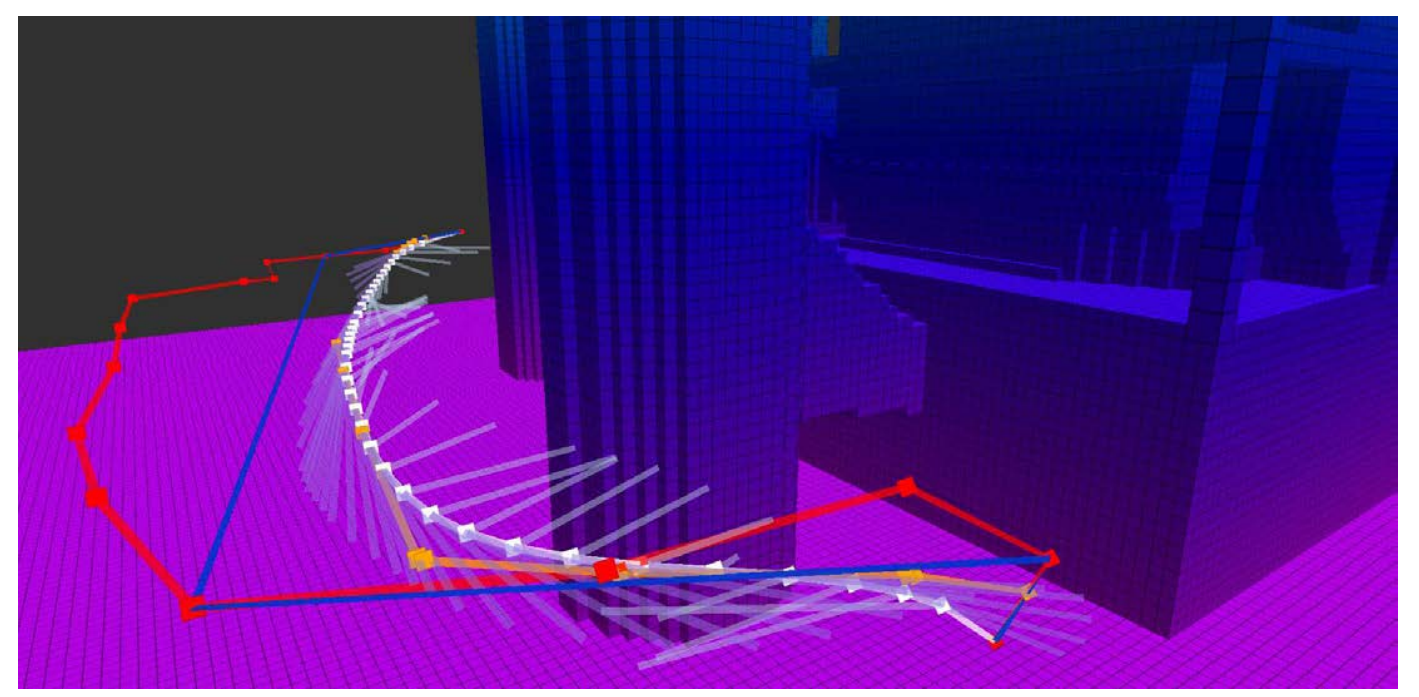

Figure 4.16: Trajectory Planner - EuRoC2014 - Example Trajectory 4 - RGB. The octomap is colorcoded from low altitude (pink) to high altitude (clear blue). A typical planned trajectory is shown: The output of the PRMStar algorithm (red) is consecutively shortened (green-blue-orange-white). The semi transparent lines show the velocity and acceleration plans.
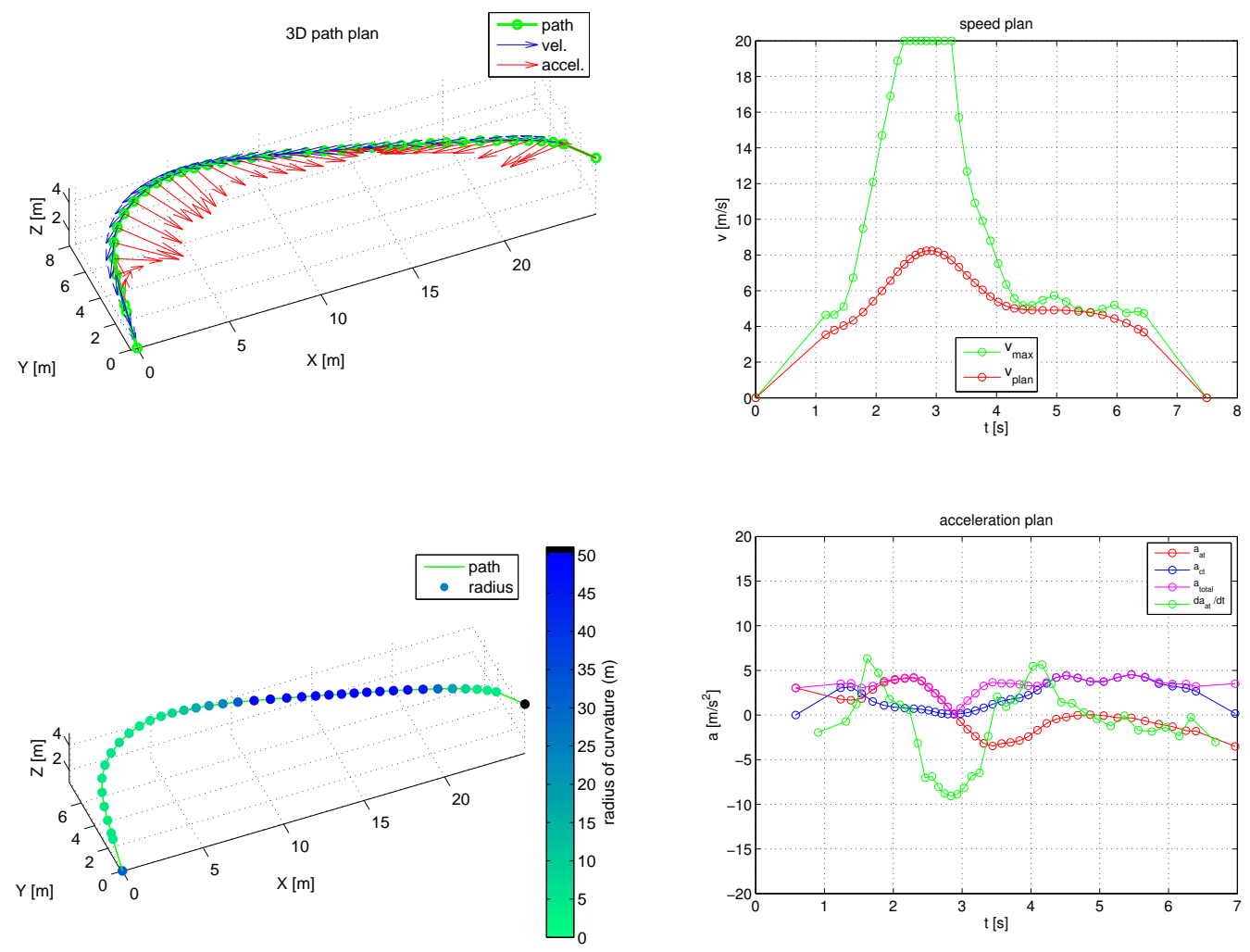

Figure 4.17: Trajectory Planner - EuRoC2014 - Example Trajectory 4 - Plots. The top left plot shows the trajectory path in $3 \mathrm{D}$ (green) along with the speed (blue) and acceleration (red) plans. The bottom left plot shows the trajectory path color coded with the radius of curvature, black color denote parts of the trajectory estimated to be straight. The right top plot shows the final along-track speed plan (red) with the maximum desired velocity (green) affected and limited by the radius of curvature. The right bottom plot shows the along-track (red), cross-track (blue) and total (magenta) acceleration, along with the derivative of the acceleration (green). 


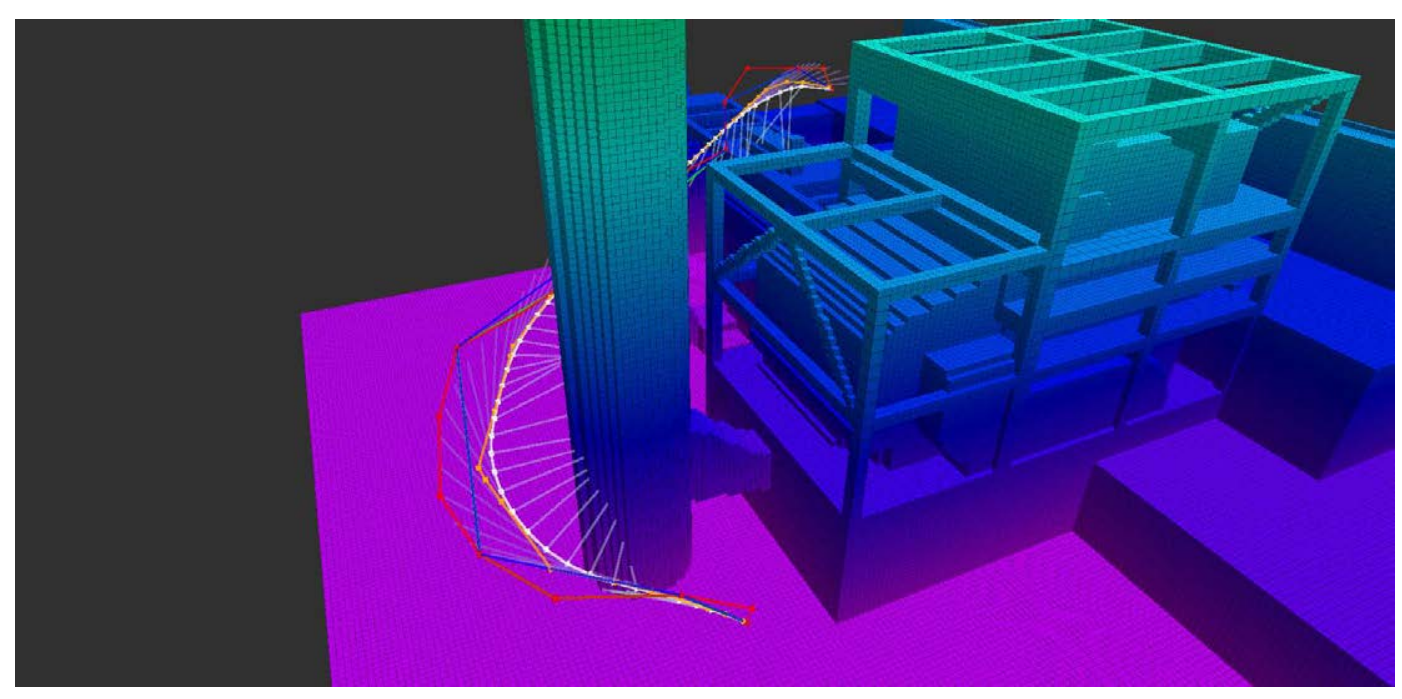

Figure 4.18: Trajectory Planner - EuRoC2014 - Example Trajectory 5 - RGB. The octomap is colorcoded from low altitude (pink) to high altitude (clear blue). A typical planned trajectory is shown: The output of the PRMStar algorithm (red) is consecutively shortened (green-blue-orange-white). The semi transparent lines show the velocity and acceleration plans.
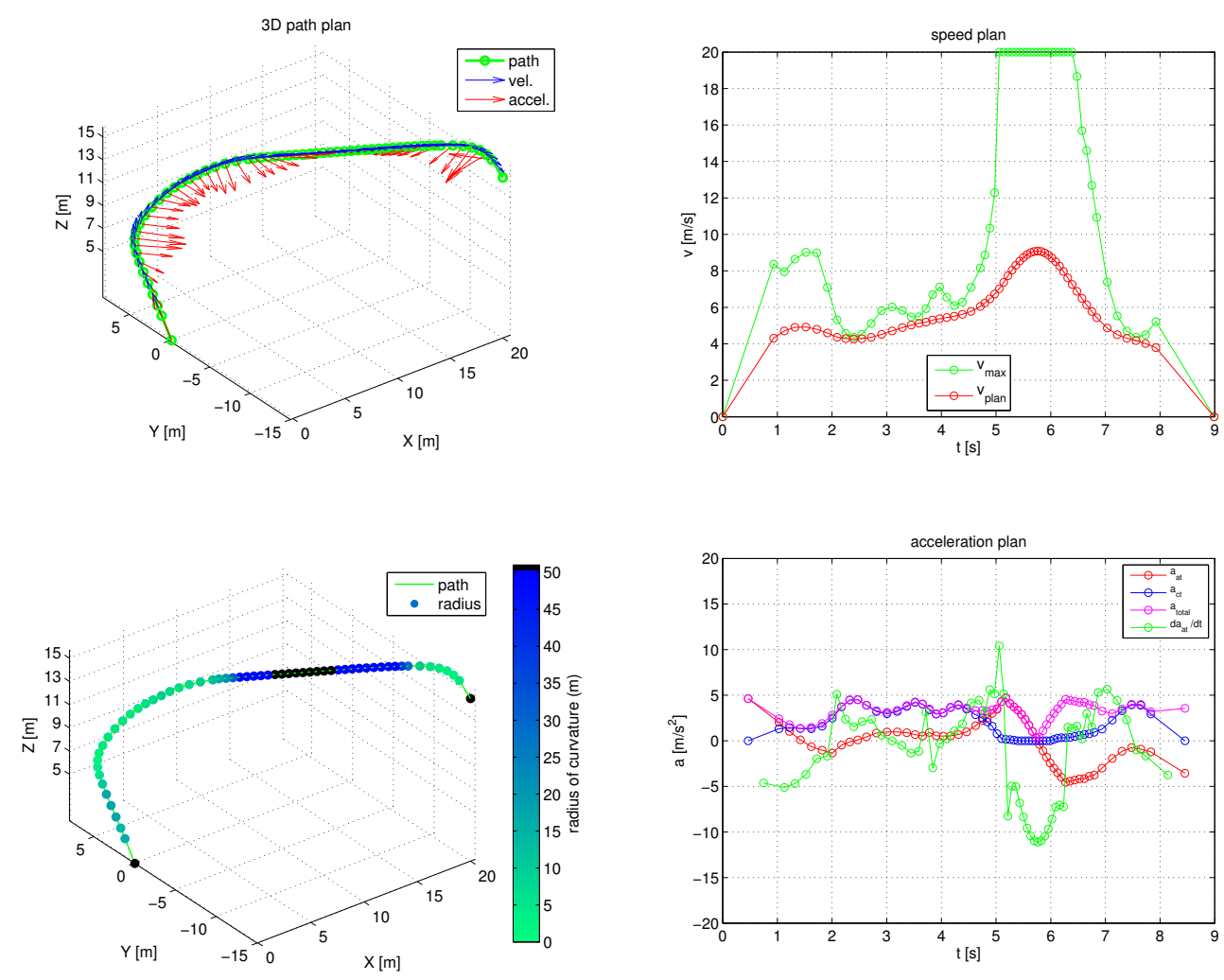

Figure 4.19: Trajectory Planner - EuRoC2014 - Example Trajectory 5 - Plots. The top left plot shows the trajectory path in 3D (green) along with the speed (blue) and acceleration (red) plans. The bottom left plot shows the trajectory path color coded with the radius of curvature, black color denote parts of the trajectory estimated to be straight. The right top plot shows the final along-track speed plan (red) with the maximum desired velocity (green) affected and limited by the radius of curvature. The right bottom plot shows the along-track (red), cross-track (blue) and total (magenta) acceleration, along with the derivative of the acceleration (green). 


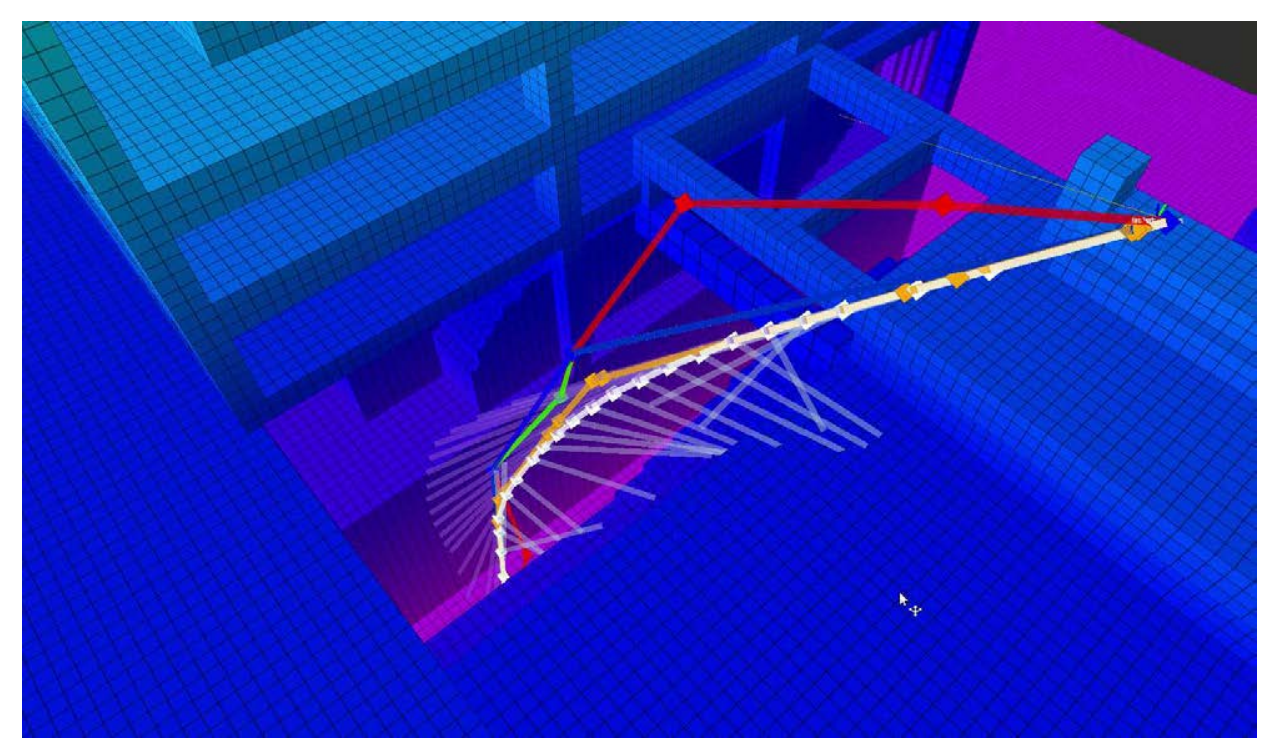

Figure 4.20: Trajectory Planner - EuRoC2014 - Example Trajectory 6 - RGB. The octomap is colorcoded from low altitude (pink) to high altitude (clear blue). A typical planned trajectory is shown: The output of the PRMStar algorithm (red) is consecutively shortened (green-blue-orange-white). The semi transparent lines show the velocity and acceleration plans.
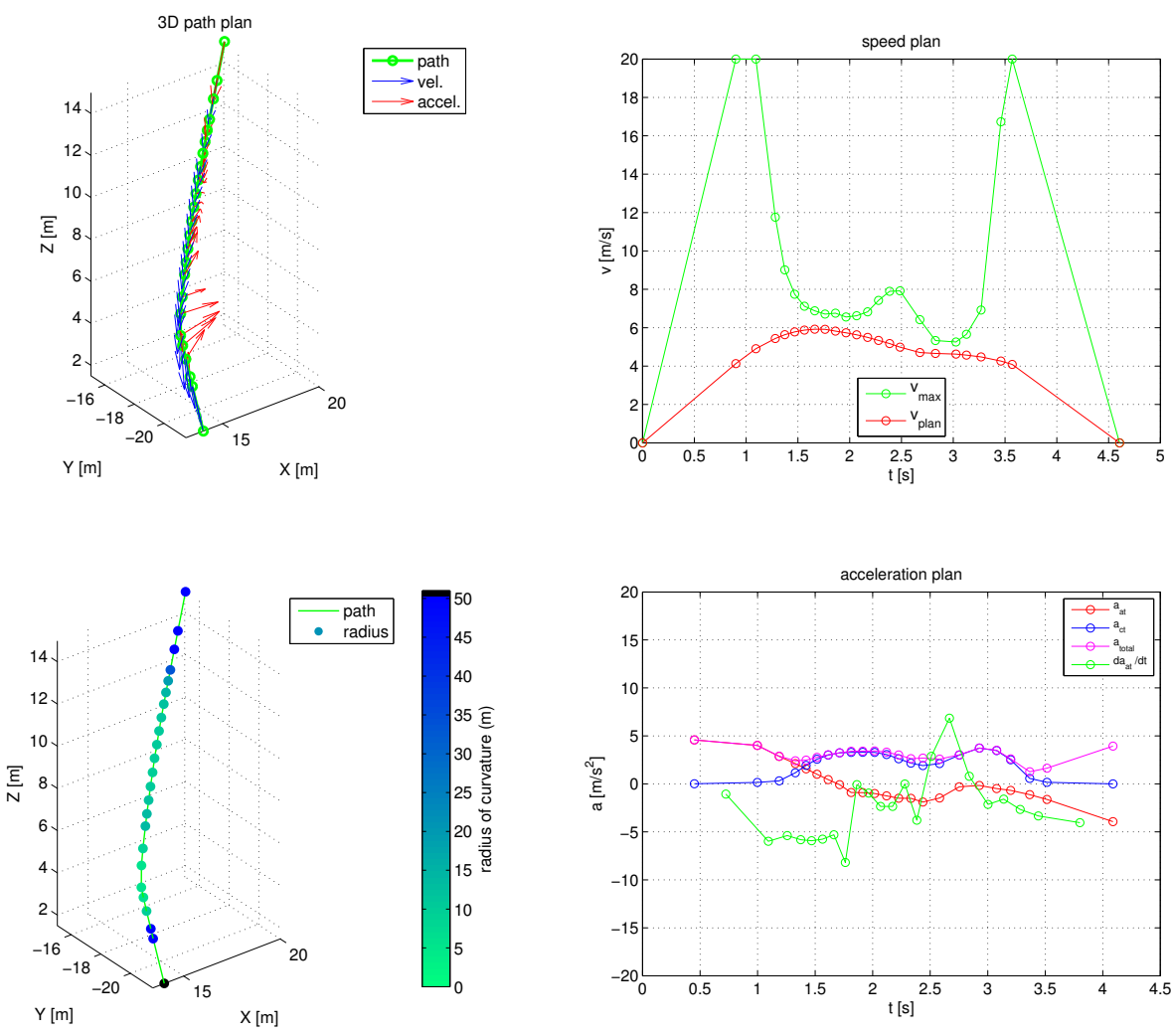

Figure 4.21: Trajectory Planner - EuRoC2014 - Example Trajectory 6 - Plots. The top left plot shows the trajectory path in 3D (green) along with the speed (blue) and acceleration (red) plans. The bottom left plot shows the trajectory path color coded with the radius of curvature, black color denote parts of the trajectory estimated to be straight. The right top plot shows the final along-track speed plan (red) with the maximum desired velocity (green) affected and limited by the radius of curvature. The right bottom plot shows the along-track (red), cross-track (blue) and total (magenta) acceleration, along with the derivative of the acceleration (green). 


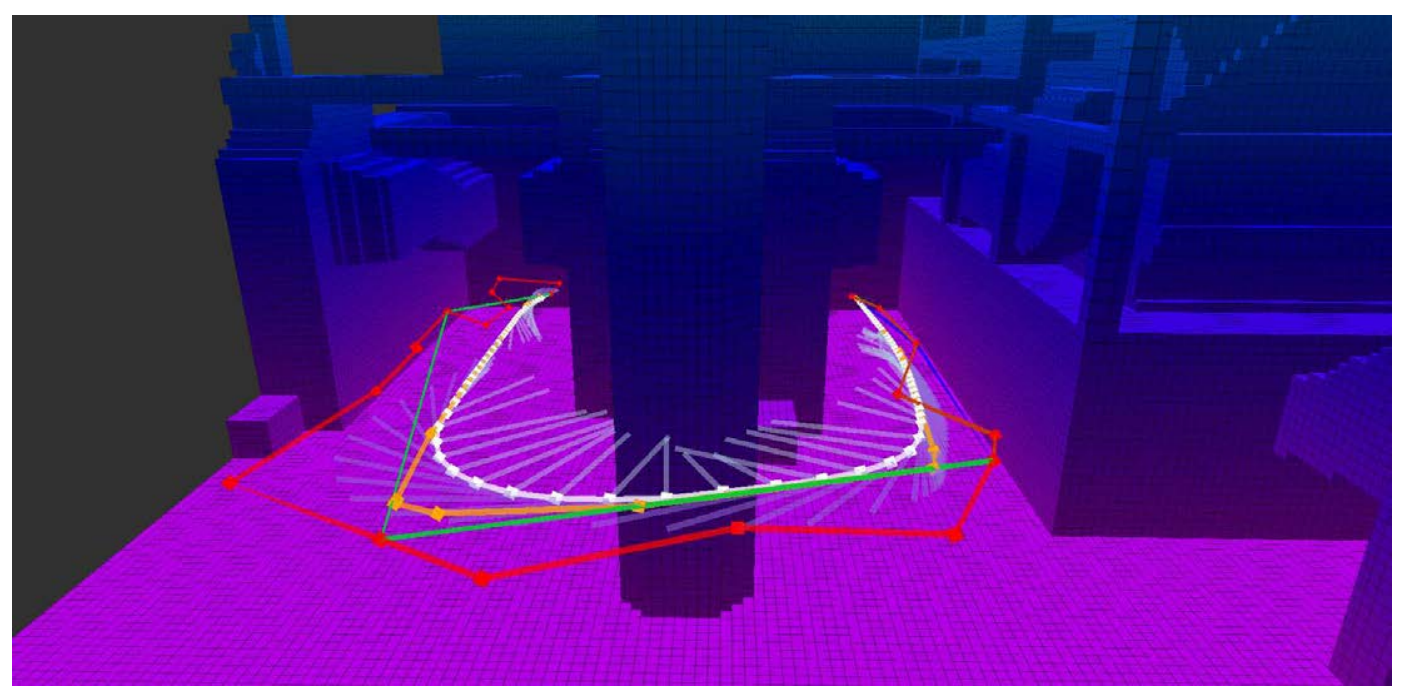

Figure 4.22: Trajectory Planner - EuRoC2014 - Example Trajectory 7 - RGB. The octomap is colorcoded from low altitude (pink) to high altitude (clear blue). A typical planned trajectory is shown: The output of the PRMStar algorithm (red) is consecutively shortened (green-blue-orange-white). The semi transparent lines show the velocity and acceleration plans.
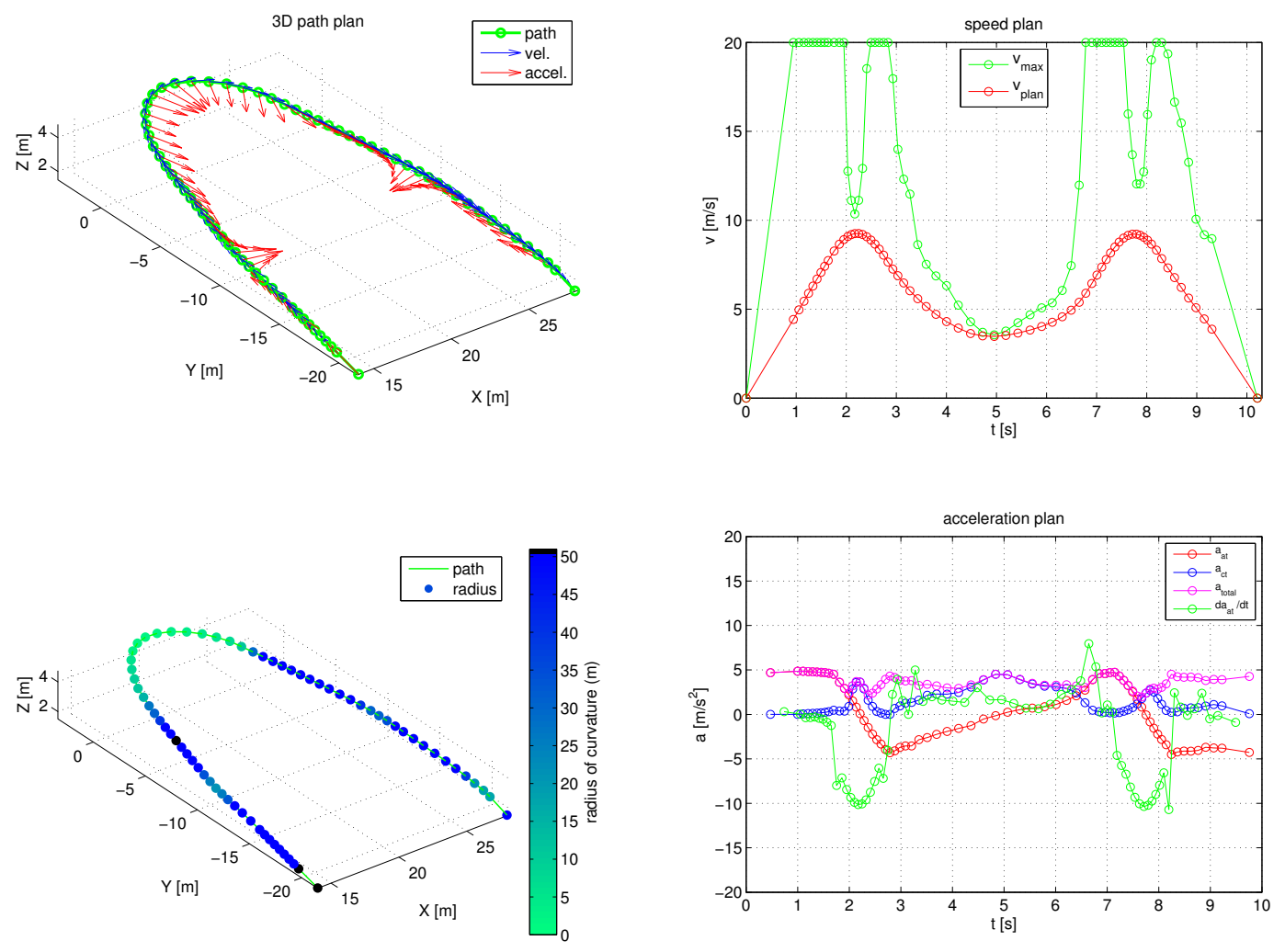

Figure 4.23: Trajectory Planner - EuRoC2014 - Example Trajectory 7 - Plots. The top left plot shows the trajectory path in 3D (green) along with the speed (blue) and acceleration (red) plans. The bottom left plot shows the trajectory path color coded with the radius of curvature, black color denote parts of the trajectory estimated to be straight. The right top plot shows the final along-track speed plan (red) with the maximum desired velocity (green) affected and limited by the radius of curvature. The right bottom plot shows the along-track (red), cross-track (blue) and total (magenta) acceleration, along with the derivative of the acceleration (green). 


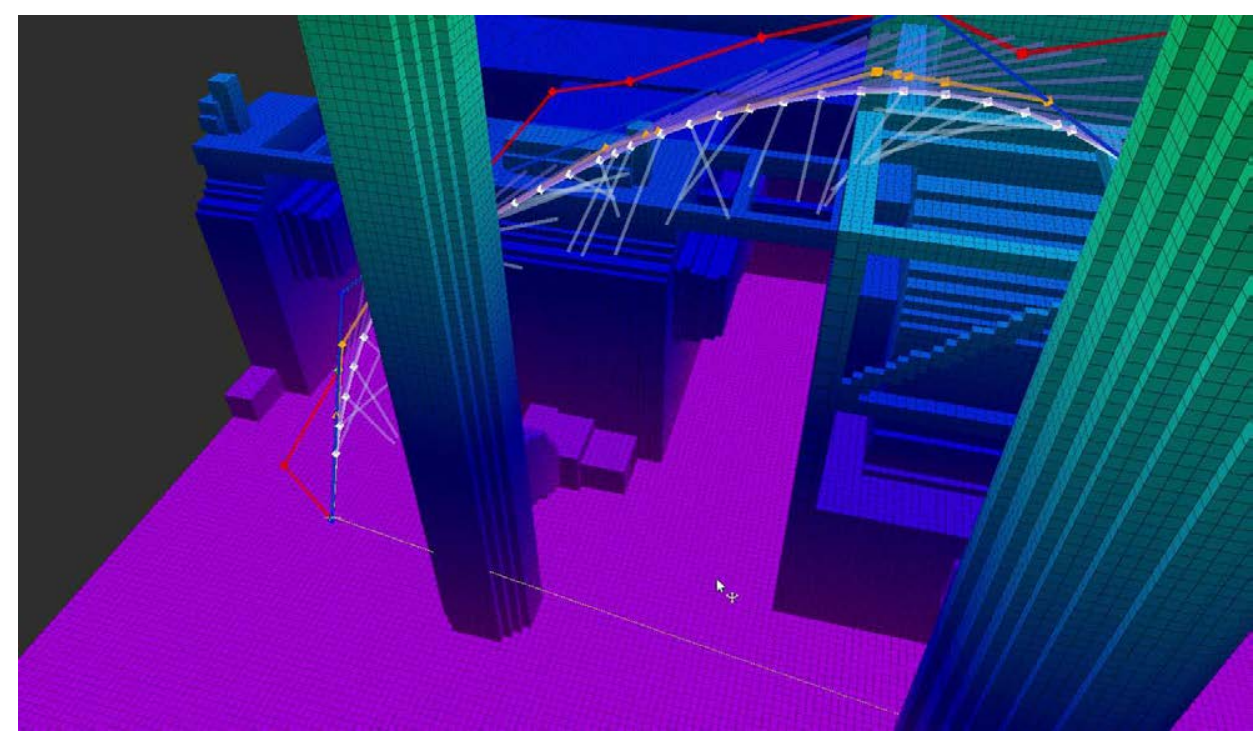

Figure 4.24: Trajectory Planner - EuRoC2014 - Example Trajectory 8 - RGB. The octomap is colorcoded from low altitude (pink) to high altitude (clear blue). A typical planned trajectory is shown: The output of the PRMStar algorithm (red) is consecutively shortened (green-blue-orange-white). The semi transparent lines show the velocity and acceleration plans.
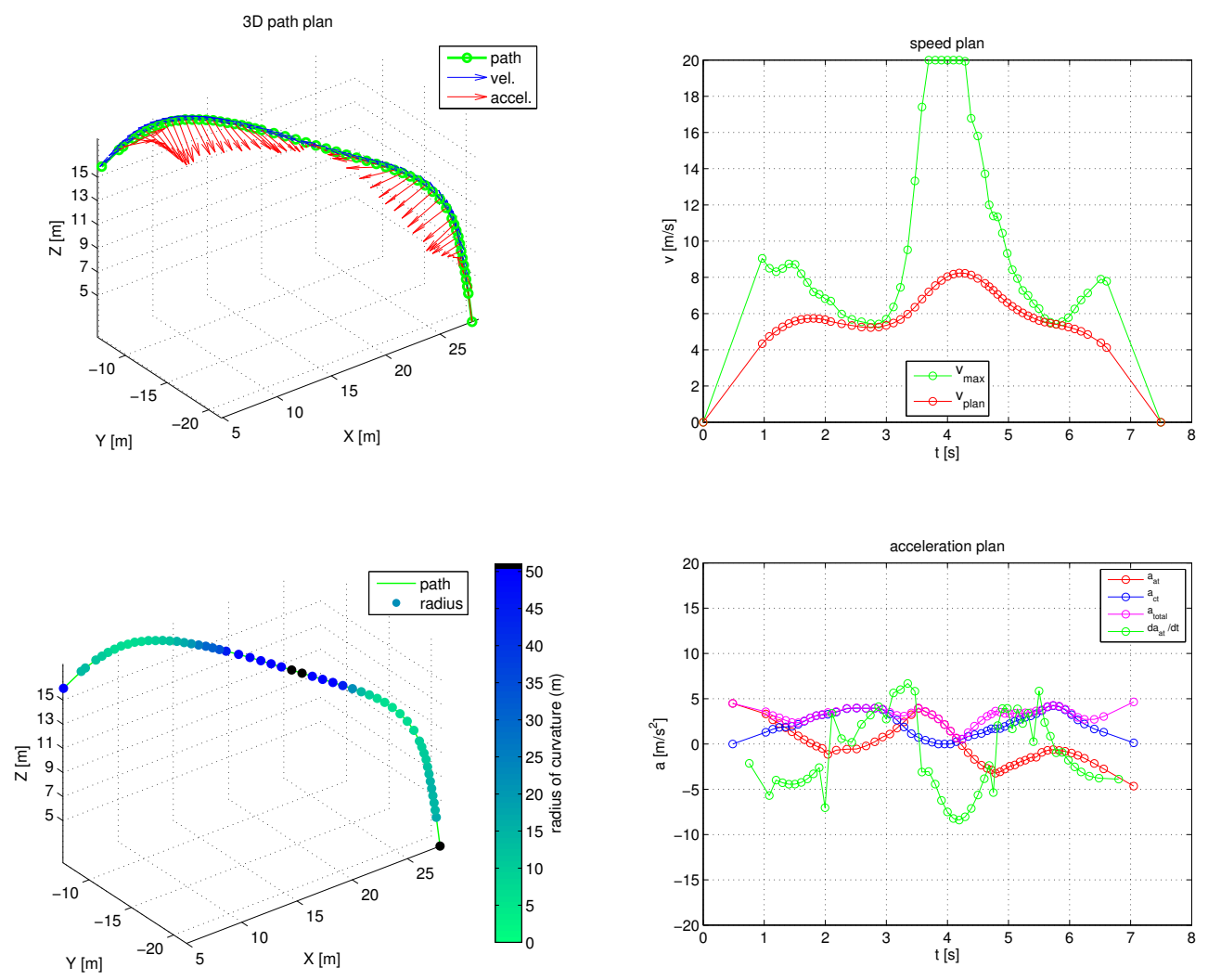

Figure 4.25: Trajectory Planner - EuRoC2014 - Example Trajectory 8 - Plots. The top left plot shows the trajectory path in 3D (green) along with the speed (blue) and acceleration (red) plans. The bottom left plot shows the trajectory path color coded with the radius of curvature, black color denote parts of the trajectory estimated to be straight. The right top plot shows the final along-track speed plan (red) with the maximum desired velocity (green) affected and limited by the radius of curvature. The right bottom plot shows the along-track (red), cross-track (blue) and total (magenta) acceleration, along with the derivative of the acceleration (green). 


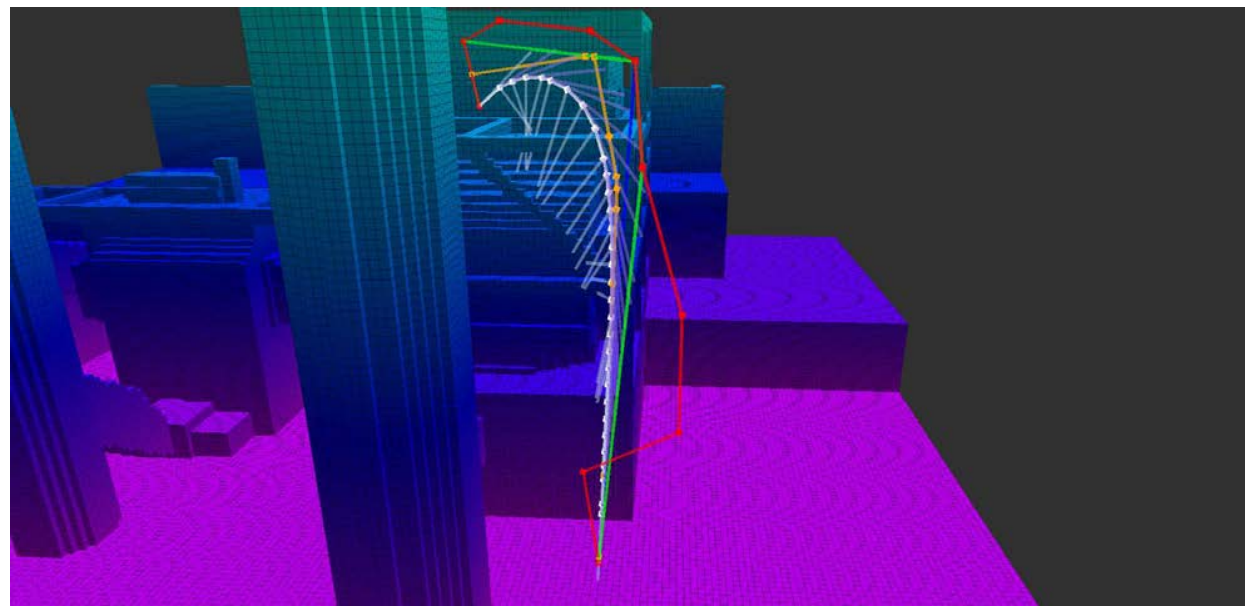

Figure 4.26: Trajectory Planner - EuRoC2014 - Example Trajectory 9 - RGB. The octomap is colorcoded from low altitude (pink) to high altitude (clear blue). A typical planned trajectory is shown: The output of the PRMStar algorithm (red) is consecutively shortened (green-blue-orange-white). The semi transparent lines show the velocity and acceleration plans.
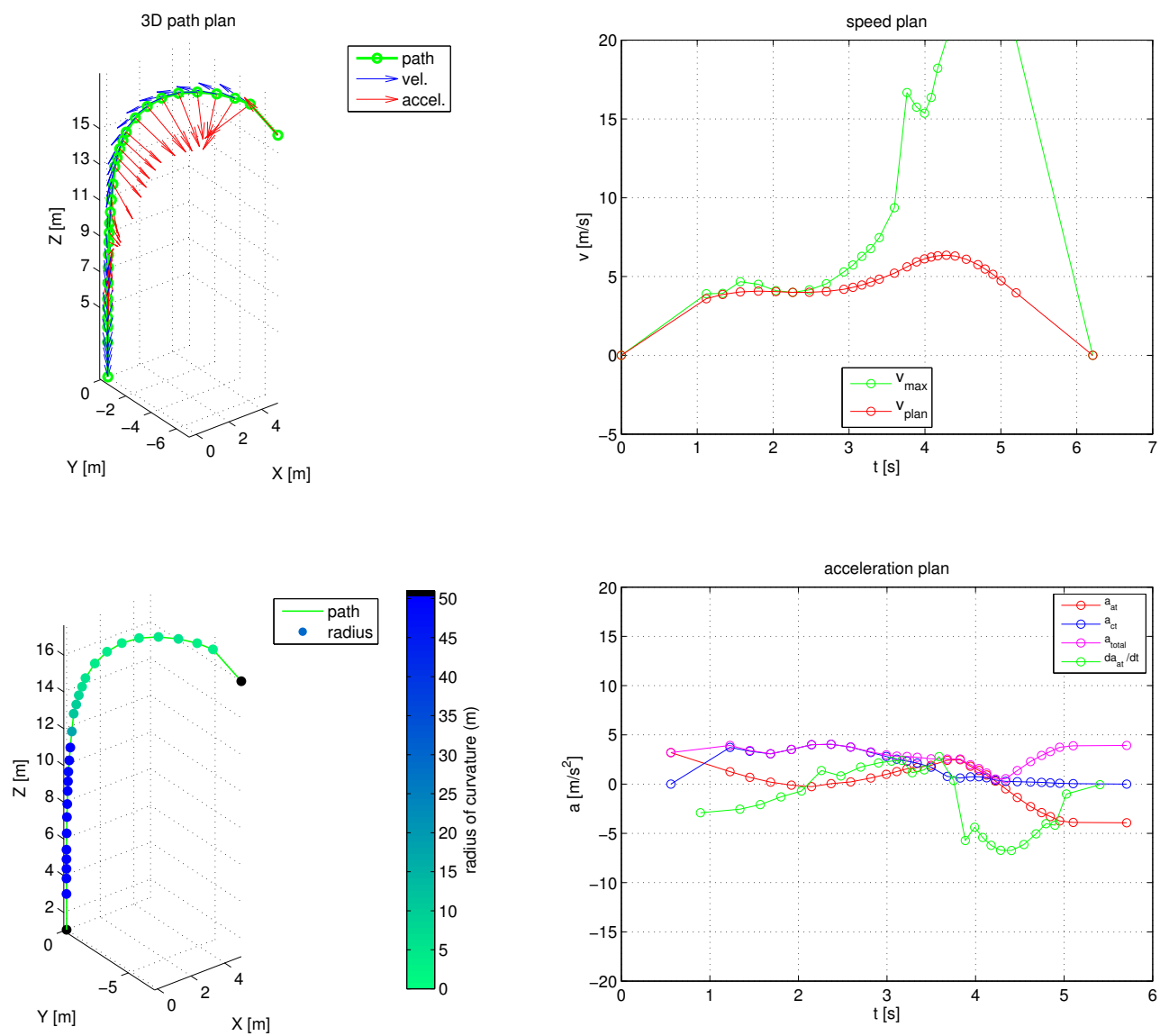

Figure 4.27: Trajectory Planner - EuRoC2014 - Example Trajectory 9 - Plots. The top left plot shows the trajectory path in 3D (green) along with the speed (blue) and acceleration (red) plans. The bottom left plot shows the trajectory path color coded with the radius of curvature, black color denote parts of the trajectory estimated to be straight. The right top plot shows the final along-track speed plan (red) with the maximum desired velocity (green) affected and limited by the radius of curvature. The right bottom plot shows the along-track (red), cross-track (blue) and total (magenta) acceleration, along with the derivative of the acceleration (green). 


\subsubsection{Results in 2016 DJI Developer Challenge - Experimental Flights}

The trajectory planner used in the participation of the "Graz Griffins" to the 2016 DJI Developer Challenge utilized the $P R M^{*}$ for the second deliverable and the $R R T^{*}$ later on. The main difference between using one or the other in terms of usability is that using the $R R T^{*}$ allows to work with dynamic environments. The trajectories shown in this section where generated using the $P R M^{*}$ algorithm.

The controller utilized in this challenge was inspired by the one used by the team in the EuRoC2014 and it was also developed by D. Muschick for the "Graz Griffins" team. In this case, a third order spline is fitted to the provided trajectory path and times of passage. The following performance parameters, see Table 4.2, correspond to the video uploaded here ${ }^{20}$ In this experiment the octomap size is around $200 \times 55 \mathrm{~m}$, and the octomap resolution used by the planner is $1 \mathrm{~m}$ (which does not match what is shown in the figures of the next subsection, that show an octomap of resolution $0.50 \mathrm{~m}$ ). For the trajectory planner, the maximum speed was set to $4.0 \mathrm{~m} / \mathrm{s}$ and the acceleration to $0.15 \mathrm{~g}=1.47 \mathrm{~m} / \mathrm{s}^{2}$ All the control architecture is executed on-board the drone except for a user interface to command the drone on where to fly next.

\begin{tabular}{||c|ccc|c||}
\hline path length [m] & UI+plan & execution & total time [s] & max speed[m/s] \\
\hline \hline 77.24 & 2.52 & $26.57 / 25.47$ & $29.09 / 27.99$ & 4.00 \\
43.53 & 1.66 & $15.67 / 14.93$ & $17.33 / 16.59$ & 4.00 \\
44.96 & 2.09 & $15.58 / 14.89$ & $17.67 / 16.98$ & 4.00 \\
80.52 & 2.88 & $24.68 / 23.78$ & $27.56 / 26.66$ & 4.00 \\
61.56 & 2.29 & $20.63 / 19.77$ & $22.91 / 22.06$ & « averages \\
\hline
\end{tabular}

Table 4.2: Trajectory planning and control performance - 2016 DJI Developer Challenge. All the values shown in the table are derived from a datalog of the flight, considering the times at which ROS messages were published. All the control architecture is executed on-board the drone except for a user interface to command the drone on where to fly next. The UI+plan time corresponds to the whole trajectory generation time plus the time that it took between clicking on the next target point on the User Interface (ROS Rviz) to receiving the goal point in the drone through WiFi. The execution time is the time that it took the controller to perform the commanded trajectory, where the left column shows the actual execution time and the right expected or planned execution time. And the total time is the sum of both. The average achieved navigation speed was: [(real) / (planned) $2.98 / 3.11 \mathrm{~m} / \mathrm{s}$.

In the next subsection trajectories resulting from the presented trajectory planning and subsequent speed and acceleration planning algorithms are shown. The trajectories are the ones utilized during the execution of the same vided 20. These plots show the following:

- The calculated obstacle-free smooth trajectories

- The calculated speed and acceleration plans, and the corresponding times-of-passage plan.

- The estimated radius of curvature at each point of the trajectory path. Note how the radius of curvature affects the maximum velocity of the speed plan before, inside and after curves.

- Note how the acceleration and speed plans are correctly limited to the desired values, and how the derivative of the acceleration remains reasonable.

20 https://youtu.be/9DXzIGKKqbu 


\subsubsection{Plots of trajectories, speed and acceleration plans}

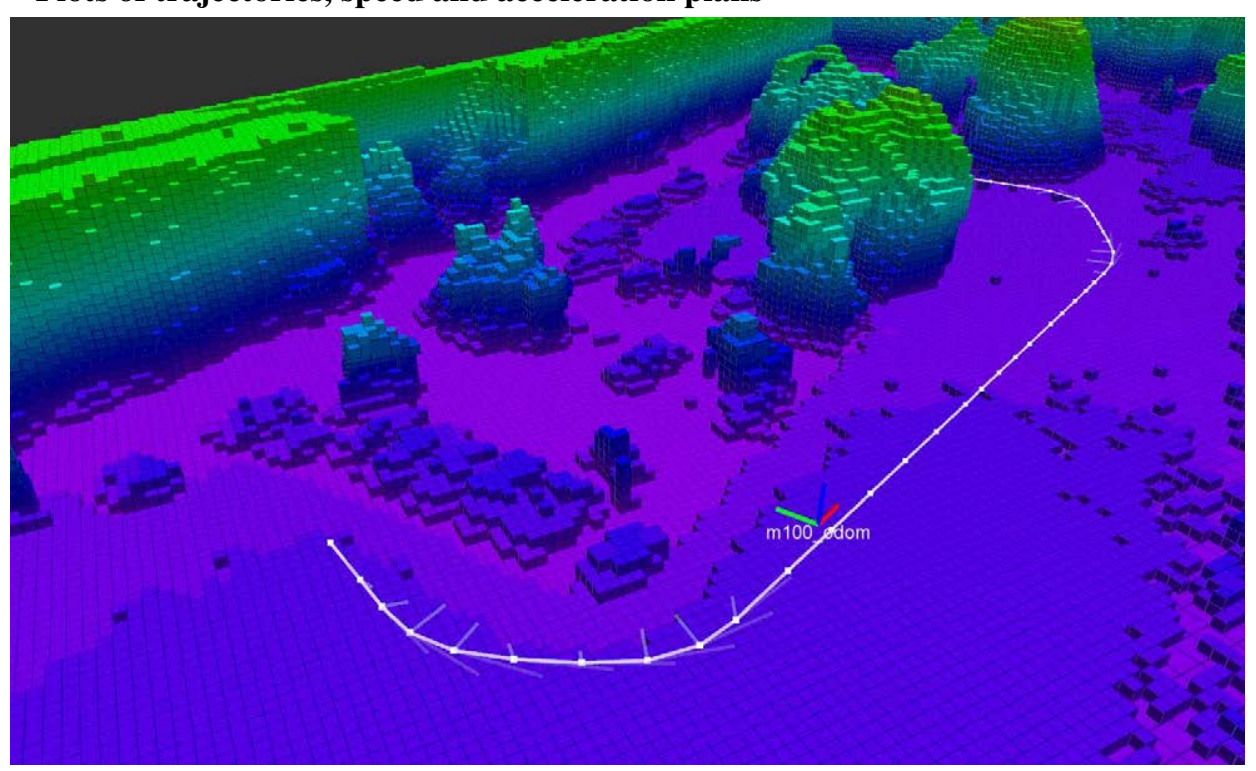

Figure 4.28: Trajectory Planner - 2016 DJI Developer Challenge - Example Trajectory 1 - RGB. The octomap is color-coded from low altitude (pink) to high altitude (green). A typical planned trajectory is shown in white, and the semi transparent lines show the velocity and acceleration plans.
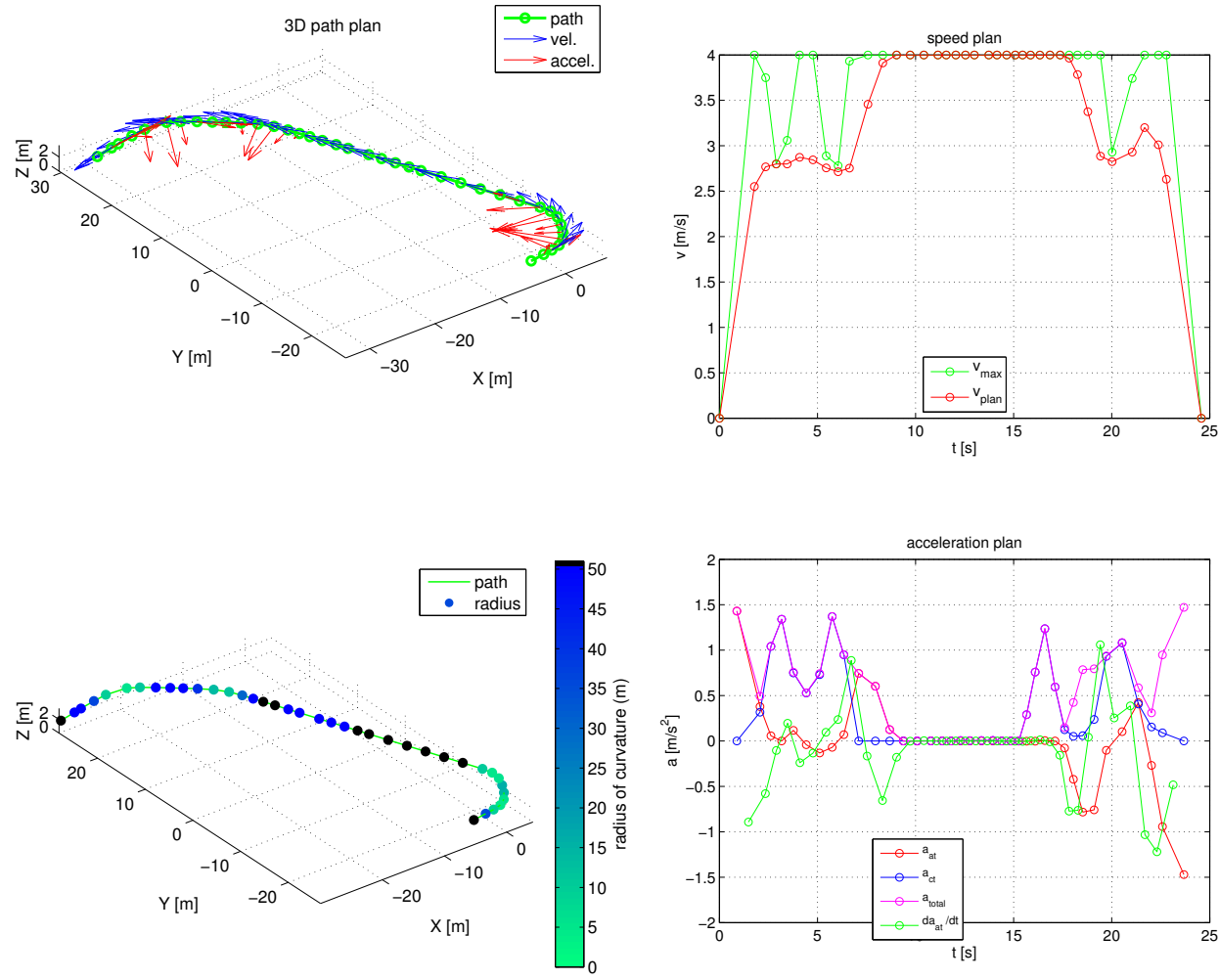

Figure 4.29: Trajectory Planner - 2016 DJI Developer Challenge - Example Trajectory 1 - Plots. The top left plot shows the trajectory path in 3D (green) along with the speed (blue) and acceleration (red) plans. The bottom left plot shows the trajectory path color coded with the radius of curvature, black color denote parts of the trajectory estimated to be straight. The right top plot shows the final along-track speed plan (red) with the maximum desired velocity (green) affected and limited by the radius of curvature. The right bottom plot shows the along-track (red), cross-track (blue) and total (magenta) acceleration, along with the derivative of the acceleration (green). 


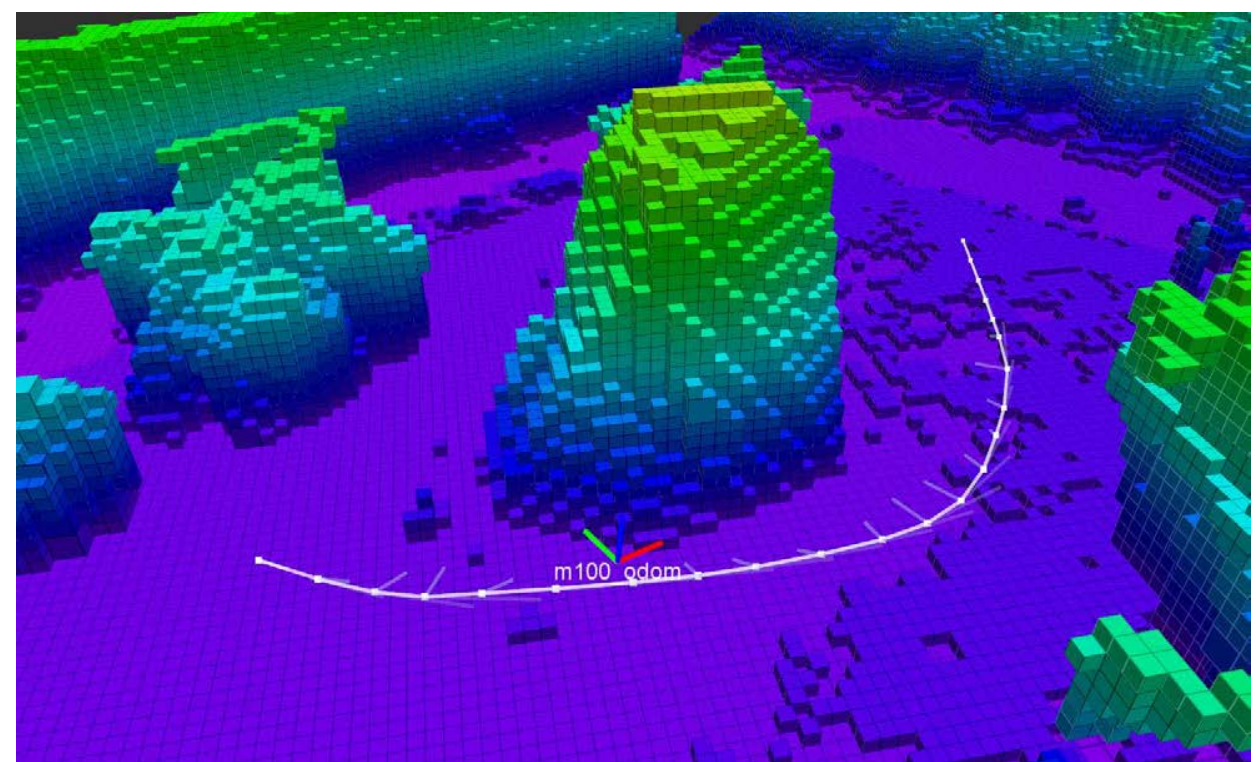

Figure 4.30: Trajectory Planner - 2016 DJI Developer Challenge - Example Trajectory 2 - RGB. The octomap is color-coded from low altitude (pink) to high altitude (green). A typical planned trajectory is shown in white, and the semi transparent lines show the velocity and acceleration plans.
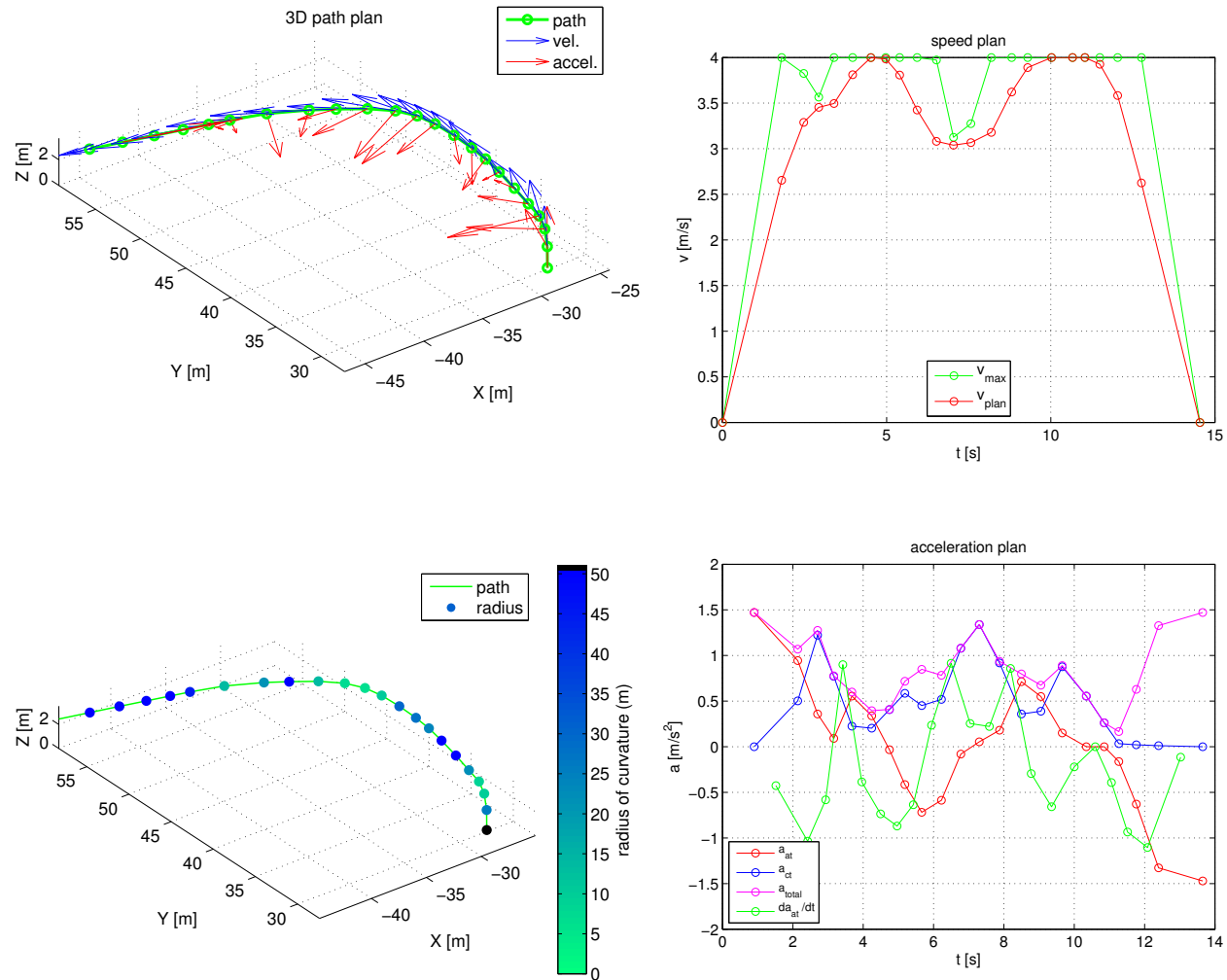

Figure 4.31: Trajectory Planner - 2016 DJI Developer Challenge - Example Trajectory 2 - Plots. The top left plot shows the trajectory path in 3D (green) along with the speed (blue) and acceleration (red) plans. The bottom left plot shows the trajectory path color coded with the radius of curvature, black color denote parts of the trajectory estimated to be straight. The right top plot shows the final along-track speed plan (red) with the maximum desired velocity (green) affected and limited by the radius of curvature. The right bottom plot shows the along-track (red), cross-track (blue) and total (magenta) acceleration, along with the derivative of the acceleration (green). 


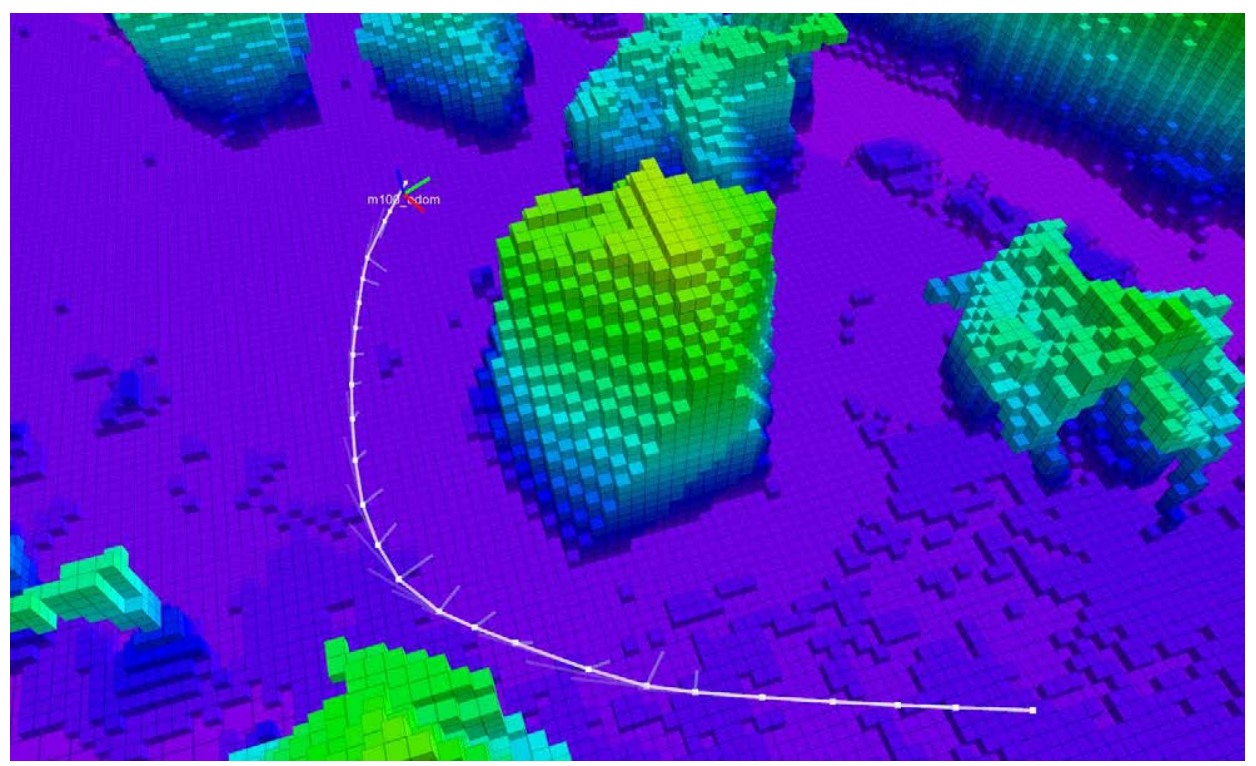

Figure 4.32: Trajectory Planner - 2016 DJI Developer Challenge - Example Trajectory 3 - RGB. The octomap is color-coded from low altitude (pink) to high altitude (green). A typical planned trajectory is shown in white, and the semi transparent lines show the velocity and acceleration plans.
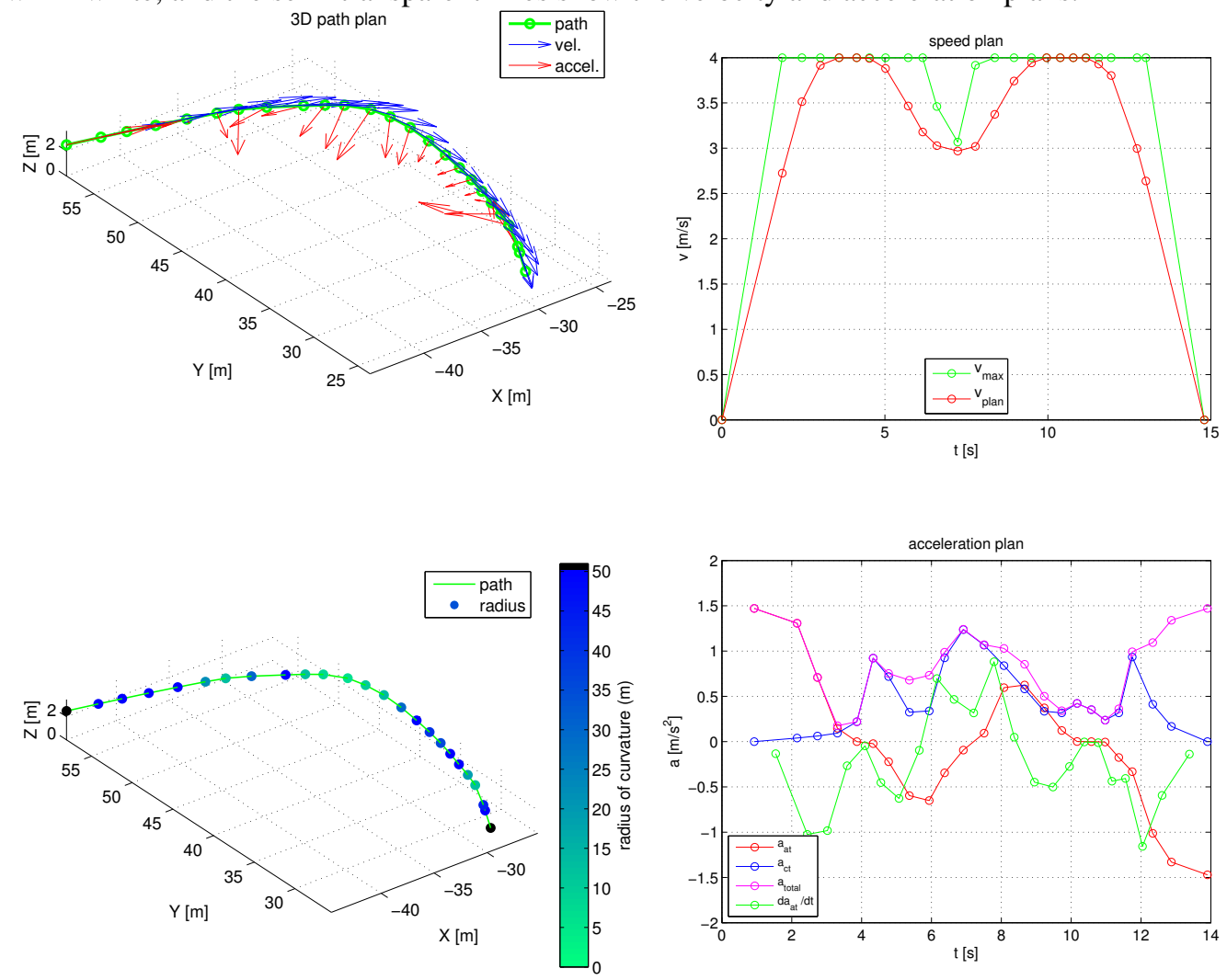

Figure 4.33: Trajectory Planner - 2016 DJI Developer Challenge - Example Trajectory 3 - Plots. The top left plot shows the trajectory path in 3D (green) along with the speed (blue) and acceleration (red) plans. The bottom left plot shows the trajectory path color coded with the radius of curvature, black color denote parts of the trajectory estimated to be straight. The right top plot shows the final along-track speed plan (red) with the maximum desired velocity (green) affected and limited by the radius of curvature. The right bottom plot shows the along-track (red), cross-track (blue) and total (magenta) acceleration, along with the derivative of the acceleration (green). 


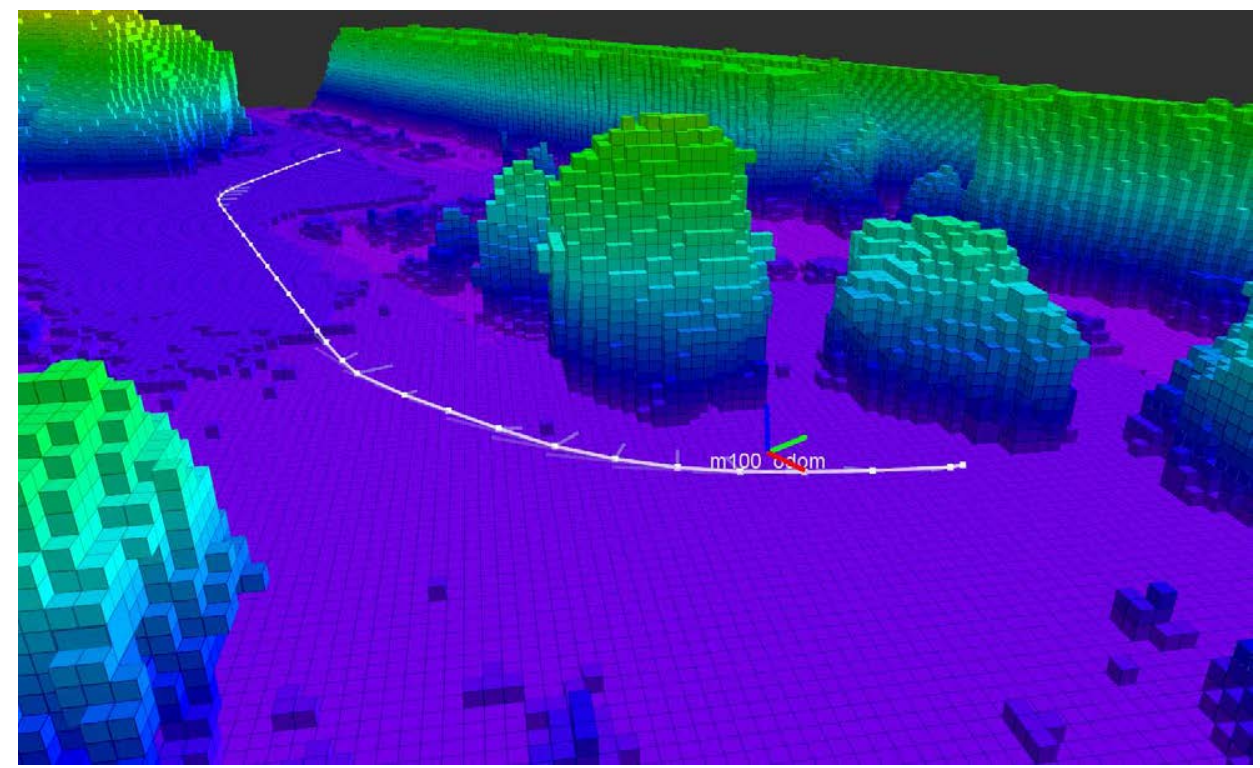

Figure 4.34: Trajectory Planner - 2016 DJI Developer Challenge - Example Trajectory 4 - RGB. The octomap is color-coded from low altitude (pink) to high altitude (green). A typical planned trajectory is shown in white, and the semi transparent lines show the velocity and acceleration plans.
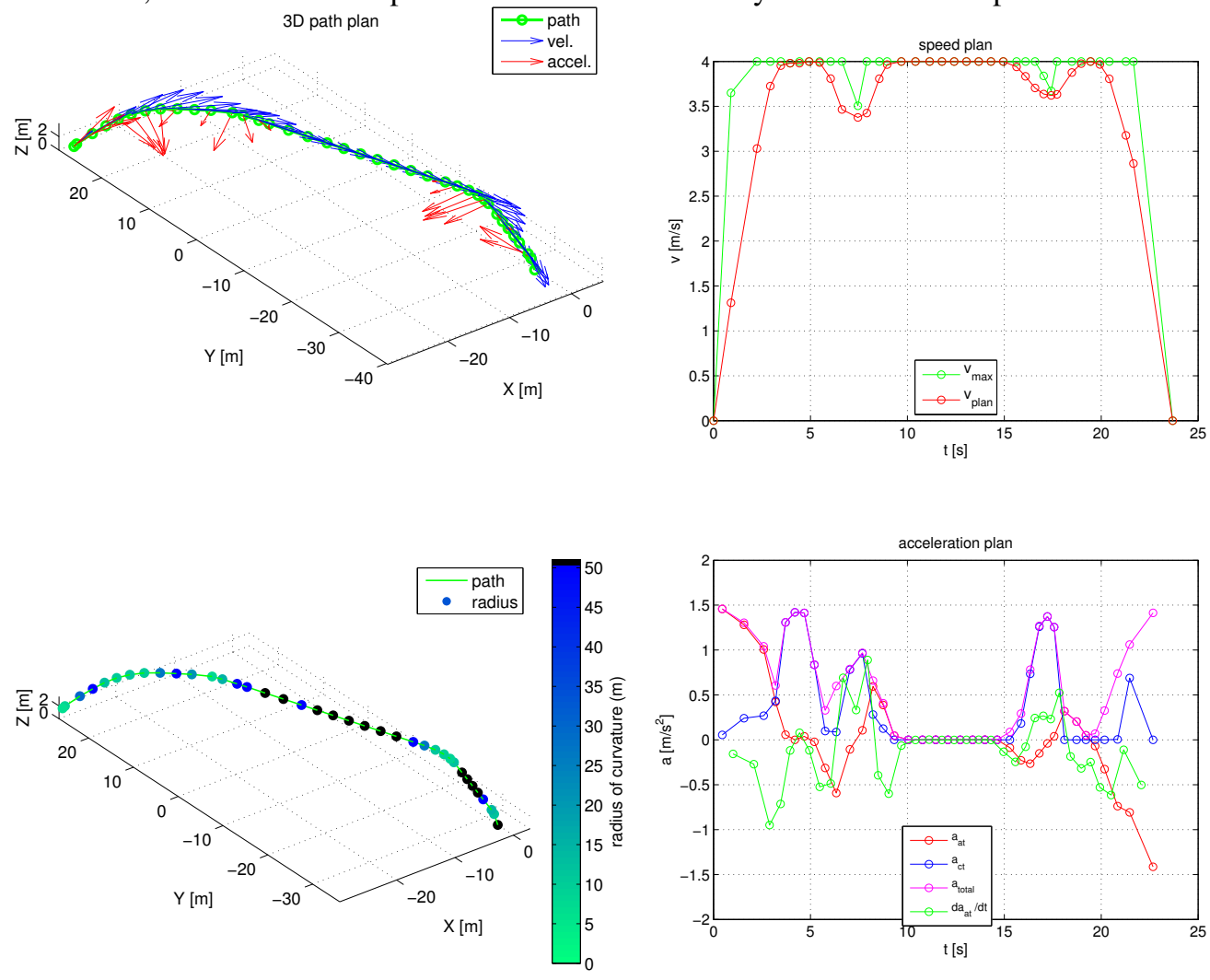

Figure 4.35: Trajectory Planner - 2016 DJI Developer Challenge - Example Trajectory 4 - Plots. The top left plot shows the trajectory path in 3D (green) along with the speed (blue) and acceleration (red) plans. The bottom left plot shows the trajectory path color coded with the radius of curvature, black color denote parts of the trajectory estimated to be straight. The right top plot shows the final along-track speed plan (red) with the maximum desired velocity (green) affected and limited by the radius of curvature. The right bottom plot shows the along-track (red), cross-track (blue) and total (magenta) acceleration, along with the derivative of the acceleration (green). 


\subsection{Future Work}

In the area of trajectory planning, the navigation control performs better when following smooth trajectories, allowing faster motion. Additionally, less computer intensive trajectory generation algorithms allow the drone to make rapid decisions while navigating in cluttered environments or while performing time critical operations. Since robots can move and affect which data is going to be gathered for the mapping of the environment, another innovative research trend is acknowledging the inter-dependence in robotics of exploration, obstacle mapping and trajectory planning. For instance, the development of trajectory planning strategies that utilize obstacle maps that encode uncertainty. There is also a trend to combine image processing and motion planning where these two tasks are not separated in modules but rather solved together by means of deep learning. Some works have already shown promising results in object grasping and door opening by robot arm manipulators, but similar techniques are still to be applied to drones.

\subsection{Conclusions}

In this chapter, a novel trajectory planner for multirotors has been presented. The main purpose of this planner is to enable outdoors flight in relatively cluttered environments. As shown in the results the planner is able to deliver smooth safe trajectories when flying near buildings and other obstacles. The following features of the planner have been demonstrated:

- The algorithm is lightweight enough that it can be executed on the on-board computer of the drone.

- The algorithm has been tested experimentally on maps obtained using an on-board real-time capable Computer Vision photogrammetry method.

- It utilizes the Octomap obstacle map representation, which is commonly used in robotics. The utilization of a distance map, to all present obstacles, is leveraged for the calculation of obstaclefree smooth trajectories.

- A feasible speed, acceleration and time-of-passage plans are delivered along with the trajectory path. The algorithm enforces the set maximum desired speed and acceleration. The speed plan is the result of a refinement to bound the jerk, i. e. the derivative of the acceleration, of the trajectory so that the drone can fly through it using smoother rotations. The speed plan refinement has been shown to improve the result in terms of lower acceleration derivative values and of smoother speed and acceleration plans.

- Although other modules would be needed to decide the exact navigation objectives of the drone, using this planner we have shown that it is feasible to deploy drones in unknown outdoors environments, by leveraging the good qualities of maps obtained using state of the art photogrammetry mapping methods.

The main publications related to this chapter are:

- J. Pestana, M. Maurer, D. Muschick, D. Adlakha, H. Bischof, F. Fraundorfer, "Package Delivery Experiments with a Camera Drone”. In Austrian Robotics Workshop 2017, OAGM/AAPR2017 joint Workshop. To be published.

- J. Pestana and F. Fraundorfer, "Vision based safe navigation for search and rescue drones" in the IEEE/RSJ IROS2016 Workshop "Vision-Based High Speed Autonomous Navigation of UAVs". [No Proceedings] https://www. seas . upenn.edu/ loiannog/workshopIRos2016uav/

- J. Pestana, R. Prettenthaler, T. Holzmann, D. Muschick, C. Mostegel, F. Fraundorfer, H. Bischof “Graz Griffins' Solution to the European Robotics Challenges 2014”. In Austrian Robotics Workshop 2015, ARW2015.

- T Holzmann, R Prettenthaler, J. Pestana, D Muschick, G Graber, C Mostegel, F Fraundorfer, H Bischof "Performance Evaluation of Vision-Based Algorithms for MAVs". In Austrian Association for Pattern Recognition Conference 2015, OAGM2015. 
- The author of this thesis is currently working in two publications based on works related to either the presented trajectory planner or the 2016 DJI Developer Challenge.

The presented trajectory planner and related speed planning algorithm has been used by the ICG - TU Graz in its participation in two international MAV competitions as the team named the "Graz Griffins":

- 2016 DJI Developer Challenge (DJIC 2016 ${ }^{21}$, the competition recreated a drone Search and Rescue scenario. The "Graz Griffins" team qualified through successive rounds, from the initial $\approx 140$ teams, to be part of the last 10 teams showing their solution experimentally in the finals. The team showed a very innovative solution using on-board mapping based on photogrammetry algorithms. The on-board calculated map is converted to an obstacle-map that can be used by the presented planner to deliver trajectories that are then executed by the controller, all in the same flight and without requiring any off-board computation.

- European Robotics Challenge (EuRoC 2014 ${ }^{22}$ The "Graz Griffins" took part in the Simulation Contest of the European Robotics Challenge (EuRoC) - Challenge 3. The developed fast real-time trajectory and speed planner enabled reliable obstacle avoidance and very fast trajectory execution by the simulated quadrotor that replicated the capabilities of an Asctec Firefly MAV. The developed planner contributed to the $6^{\text {th }}$ position of the "Graz Griffins" out of 21 teams, which included world renowned European universities and laboratories. This fact enabled the team to present a proposal in collaboration with a company to the next stage of the challenge.

The works related to the 2016 DJI Developer Challenge have been presented or showcased in two public events so far:

- An experimental fully autonomous package delivery flight demonstration made by the ICG - TU Graz ${ }^{23}$ in collaboration with the company SFL Technologies as part of the roll-out of their brand new electric vehicle, the ELI. The roll-out of this vehicle and the autonomous flight demo have been described by two Austrian newspapers: Mein Bezirk ${ }^{24}$ and Kleine Zeitung 25 November 2016

- A public presentation, with no experimental flights, named Intelligent Search and Rescue Drone in the world renowned Ars Electronica Festival 2016 - DroneLab - FutureLab 26 This festival shows modern artwork that utilizes technology as a medium of expression and tries to deliver an outstanding and open view of current technology developments to its public.

September 8-12, 2016

\footnotetext{
${ }^{21}$ DJIC 2016 video playlist: http: / / bit. ly/2 fauble

${ }^{22}$ EuRoC 2014 trajectory flight video: http://bit.1y/2cQno6I

${ }^{23}$ Fully autonomous package delivery flight demonstration: https://youtu . be/bxM6dls 2wuo

${ }^{24}$ ELI roll-out - Mein Bezirk - http://bit. ly/2hott8t

${ }^{25}$ ELI roll-out - Kleine Zeitung - http://bit.ly/2it6N2P

${ }^{26}$ Ars Electronica Festival 2016 - DroneLab: http://www.aec.at/radicalatoms/en/ars-electronica-dronelab/
} 


\subsection{Chapter Bibliography}

Cowling, I. D., Yakimenko, O. A., Whidborne, J. F., and Cooke, A. K. (2010). "Direct method based control system for an autonomous quadrotor". Journal of Intelligent \& Robotic Systems 60.2, pp. 285316 (cit. on p. 70).

Dijkstra, E. W. (1959). "A note on two problems in connexion with graphs". Numerische mathematik 1.1, pp. 269-271 (cit. on pp.76, 77).

Dunbar, N. and Franz, R. (2003). "Online control customization via optimization, based control". Software-Enabled Control: Information Technology for Dynamical Systems, p. 149 (cit. on p.70).

Fliess, M., Lévine, J., Martin, P., and Rouchon, P. (1995). "Flatness and defect of non-linear systems: introductory theory and examples". International journal of control 61.6, pp. 1327-1361 (cit. on p. 70).

Fritsch, O., De Monte, P., Buhl, M., and Lohmann, B. (2012). "Quasi-static feedback linearization for the translational dynamics of a quadrotor helicopter". In: American Control Conference (ACC), 2012. IEEE, pp. 125-130 (cit. on pp.70,74).

Furrer, F., Burri, M., Achtelik, M., and Siegwart, R. (2016). "RotorS-A Modular Gazebo MAV Simulator Framework". In: Robot Operating System (ROS). Springer, pp. 595-625 (cit. on p. 89.

Hofer, M., Maurer, M., and Bischof, H. (2016). "Efficient 3D scene abstraction using line segments". Computer Vision and Image Understanding (cit. on p. 72).

Hoffmann, Gabriel M, Waslander, Steven L, and Tomlin, Claire J (2008). "Quadrotor Helicopter Trajectory Tracking Control”. Electrical Engineering (2008), pp. 1-14 (cit. on pp. 81, 82).

Holzmann, T., Prettenthaler, R., Pestana, J., Muschick, D., Graber, G., Mostegel, C., Fraundorfer, F., and Bischof, H. (2015). "Performance Evaluation of Vision-Based Algorithms for MAVs". arXiv preprint arXiv:1505.02247 (cit. on p.90).

Hoppe, C., Klopschitz, M., Donoser, M., and Bischof, H. (2013). "Incremental Surface Extraction from Sparse Structure-from-Motion Point Clouds.” In: BMVC, pp. 94-1 (cit. on p. 73).

Hoppe, C., Klopschitz, M., Rumpler, M., Wendel, A., Kluckner, S., Bischof, H., and Reitmayr, G. (2012). "Online Feedback for Structure-from-Motion Image Acquisition.” In: BMVC. Vol. 2, p. 6 (cit. on p.72,

Hornung, A., Wurm, K. M., Bennewitz, M., Stachniss, C., and Burgard, W. (2013). "OctoMap: An efficient probabilistic 3D mapping framework based on octrees". Autonomous Robots 34.3, pp. 189-206 (cit. on pp.70,71,73,76).

James, G., Witten, D., Hastie, T., and Tibshirani, R. (2013). An introduction to statistical learning. Vol. 6. Springer (cit. on p. 84).

Karaman, S. and Frazzoli, E. (2011). "Sampling-based algorithms for optimal motion planning". The international journal of robotics research 30.7, pp. 846-894 (cit. on pp. 70, 74, 77, 78,.

Kavraki, L. E., Svestka, P., Latombe, J.-C., and Overmars, M. H. (1996). "Probabilistic roadmaps for path planning in high-dimensional configuration spaces". IEEE transactions on Robotics and Automation 12.4, pp. 566-580 (cit. on pp.70,71,74, 76,.

Kneip, L., Scaramuzza, D., and Siegwart, R. (2011). “A novel parametrization of the perspective-threepoint problem for a direct computation of absolute camera position and orientation". In: Computer Vision and Pattern Recognition (CVPR), 2011 IEEE Conference on. IEEE, pp. 2969-2976 (cit. on p.72).

Labatut, P., Pons, J.-P., and Keriven, R. (2007). "Efficient multi-view reconstruction of large-scale scenes using interest points, delaunay triangulation and graph cuts". In: 2007 IEEE 11th international conference on computer vision. IEEE, pp. 1-8 (cit. on p. 73).

Lau, B., Sprunk, C., and Burgard, W. (2013). "Efficient grid-based spatial representations for robot navigation in dynamic environments". Elsevier RAS2013 (cit. on pp. 70, 71, 74, 76,.

LaValle, S. M. (1998). "Rapidly-Exploring Random Trees: A New Tool for Path Planning” (cit. on pp.70. 71, 74, 77,.

LaValle, S. M. and Kuffner, J. J. (2001). "Randomized kinodynamic planning”. The International Journal of Robotics Research 20.5, pp. 378-400 (cit. on p. 74).

Lowe, D. G. (2004). "Distinctive image features from scale-invariant keypoints". International journal of computer vision 60.2, pp. 91-110 (cit. on p.72).

Mellinger, D. and Kumar, V. (2011). "Minimum snap trajectory generation and control for quadrotors". In: Robotics and Automation (ICRA), 2011 IEEE International Conference on. IEEE, pp. 2520-2525 (cit. on pp.70, 74). 
Meyer, J., Sendobry, A., Kohlbrecher, S., Klingauf, U., and Stryk, O. von (2012). "Comprehensive simulation of quadrotor uavs using ros and gazebo". In: Simulation, Modeling, and Programming for Autonomous Robots. Springer, pp. 400-411 (cit. on p. 89).

Murray, R. M., Rathinam, M., and Sluis, W. (1995). "Differential flatness of mechanical control systems: A catalog of prototype systems". In: ASME International Mechanical Engineering Congress and Exposition. Citeseer (cit. on p.70).

Pestana, J., Prettenthaler, R., Holzmann, T., Muschick, D., Mostengel, C., Fraundorfer, F., and Bischof, H. (2015). “Graz griffins' solution to the european robotics challenges 2014”. In: Proceedings of Austrian Robotics Workshop 2015, pp. 11-12 (cit. on pp. 88, 90).

Richter, Charles, Bry, Adam, and Roy, Nicholas (2013). "Polynomial trajectory planning for aggressive quadrotor flight in dense indoor environments". In: Proceedings of the International Symposium on Robotics Research (ISRR) (cit. on p. 70).

Richter, C., Bry, A., and Roy, N. (2013). "Polynomial trajectory planning for quadrotor flight". In: International Conference on Robotics and Automation (cit. on pp. 70,71).

Rumpler, M., Tscharf, A., Mostegel, C., Daftry, S., Hoppe, C., Prettenthaler, R., Fraundorfer, F., Mayer, G., and Bischof, H. (2016). "Evaluations on multi-scale camera networks for precise and geo-accurate reconstructions from aerial and terrestrial images with user guidance". Computer Vision and Image Understanding (cit. on p.73,.

Shah, S., Dey, D., Lovett, C., and Kapoor, A. (2017). Aerial Informatics and Robotics Platform. Tech. rep. MSR-TR-2017-9. Microsoft Research (cit. on p. 89).

Şucan, I. A., Moll, M., and Kavraki, L. E. (2012). "The Open Motion Planning Library". IEEE Robotics $\&$ Automation Magazine (cit. on pp. 70, 71, 77, 78,

Triggs, B., McLauchlan, P. F., Hartley, R. I., and Fitzgibbon, A. W. (1999). "Bundle adjustment - a modern synthesis”. In: International workshop on vision algorithms. Springer, pp. 298-372 (cit. on pp. 84, 85).

Van Nieuwstadt, M. J. and Murray, R. M. (1998). "Real-time trajectory generation for differentially flat systems". International Journal of Robust and Nonlinear Control 8.11, pp. 995-1020 (cit. on p. 70).

Yanmaz, E., Hayat, S., Scherer, J., and Bettstetter, C. (2014). "Experimental performance analysis of twohop aerial 802.11 networks". In: 2014 IEEE Wireless Communications and Networking Conference (WCNC). IEEE, pp. 3118-3123 (cit. on p.76). 



\section{5}

\section{Conclusions and Future Work}

A series of works and contributions to the field of vision-based control and trajectory planning have been presented in this $\mathrm{PhD}$ thesis. This chapter presents the thesis contributions, see Sec 5.1, conclusions, see $\operatorname{Sec} 5.2$, and future work, see $\operatorname{Sec} 5.3$ The proposed methods have been successfully utilized in the participation in MAV competitions, achieving the results presented in Appx. I]

\subsection{Thesis Contributions}

\section{Main contributions}

The main contributions presented in this dissertation are the following:

\section{- A General Purpose Configurable Controller for Multirotor MAVs:}

The controller proposed in Chap. 2 was inspired by prior work by Hoffmann et al. (Hoffmann, Gabriel M et al., 2008) and Michael et al. (B. Y. N. Michael et al., 2010):

- The main difference of our controller when compared to these two prior works is the addition of several saturation laws that can intrinsically take into account speed limitations established by the on-board sensors. This feature was tested during our participation in the international MAV competition IMAV2012, where our MAV had to deal with low frame-rate optical-flow measurements (Pestana et al., 2013). These saturations are used to set a desired maximum navigation speed for the multirotor and they affect the velocity planning during trajectory tracking and the execution of the controller itself. In addition to this feature:

* In comparison to the work by Michael et al. (B. Y. N. Michael et al., 2010), our experiments are performed without reliance of GPS or a motion capture system, e. g. a Vicon system. We utilize instead the EKF discussed in Annex. Eduring the experimental validation of the controller.

* In comparison to the work by Hoffmann et al. (Hoffmann, Gabriel M et al., 2008), our experiments were carried out in indoor environments and using different sensing solutions: ground optical-flow and LIDAR (Pestana et al., 2013), and ground optical-flow and fiducial markers (Pestana, Sanchez-Lopez, Puente, et al., 2015). 
- This feature of the presented controller is quite advantageous, considering that some odometry estimation algorithms may fail to deliver estimates when navigating at inadequate velocities. For instance, in vision-based odometry estimation it becomes increasingly difficult to match image patches or features in subsequent images, due to a lack of overlap between them, thus, in practice, setting a maximum navigation velocity. The controller architecture proposed in this chapter under normal working conditions, e.g. within the sensors and actuators limits and in absence of strong enough wind and wind-gusts, is able to ensure that the trajectory is executed at an optimum speed for the on-board sensors to work properly. The actual acceptable wind speed depends on the maximum speed that can be sensed by the utilized sensing algorithm. By adopting a conservative approach, in practice, the acceptable wind speed is set lower than the sensor limits.

- An additional usage of the speed saturations proposed in the controller architecture and in the speed planning step is to simplify the flight rules of decentralized multi-robot navigation, as showcased in Sec. 2.4.4. This particular use of the speed saturations was utilized by the CVG_UPM during its participation in the IMAV 2013 competition.

- The presented controller has been successfully utilized as part of an autonomous flight architecture in several international MAV competitions, showcasing its tested reliable implementation, and its code has been released as open-source in github.

\section{- Visual Servoing of Arbitrary Objects for Multirotors:}

In Chap. 3, a novel visual based object tracking and following architecture for multirotor vehicles is presented. The contributions of this work are two-fold:

- First, it has been demonstrated that current tracking algorithms, such as OpenTLD, can reliably work on a fixed camera multirotor vehicle to feedback an Image Based Visual Servoing (IBVS) controller, even during high velocity autonomous navigation.

- Second, our architecture has been able to follow a large variety of unmarked targets of different sizes and from a wide range of distances. Moreover, the algorithm is validated using a lowcost platform, the Parrot AR Drone 2.0, in outdoor conditions while tracking and following people. The system has successfully and repeatedly handled occlusion events and tracked fast moving targets, such as a person running, showing the robustness of our system against wind disturbances and illumination changes.

The main features of our architecture that made it unique at the time of the publication of our works are:

- Our system is able to follow and stabilize itself and it is able to track a large variety of different objects.

- Additionally, safety is assured even when the wireless connection is suddenly degraded, the tracking is lost or the target is occluded. This is achieved by using a multirotor platform that can attain on-board autonomous hovering by means of floor optical flow based odometry. The experimental work was performed using an AR Drone 2.0, and the algorithms where run on an off-board laptop computer via a WiFi link.

- Our system has been able to repeatedly perform object following tasks for long periods of time of up to 7 minutes (see the related videos), and fully depleting the UAV battery, after the user is experienced enough to understand the capabilities of our object following solution.

- Our system has been able to perform visual servoing with targets of varying size, from a quarter to more than ten times the tunned target size, at varying distances from 1-2 $\mathrm{m}$ to 10$15 \mathrm{~m}$ of distance from the target, and it has has achieved person following at speeds up to 2.5-3.0 m/s for a period of 45 seconds.

- The reliability of our system has been demonstrated in live demos: several performed by the CVG, at the SSRR2013 conference and with the ICG - TU Graz.

\section{- Smooth Trajectory Planning for Multirotors:}

In Chap. 4, a novel trajectory planner for multirotor drones has been presented. The main purpose of this planner is to enable outdoors flight in relatively cluttered environments. As shown in the 
results the planner is able to deliver smooth safe trajectories when flying near buildings and other obstacles. The following features of the planner have been demonstrated:

- The algorithm is lightweight enough that it can be executed on the on-board computer of the drone.

- The algorithm has been tested experimentally on maps obtained using an on-board real-time capable Computer Vision photogrammetry method.

- It utilizes the Octomap obstacle map representation, which is commonly used in robotics. The utilization of a distance map, to all present obstacles, is leveraged for the calculation of obstacle-free smooth trajectories.

- A feasible speed, acceleration and time-of-passage plans are delivered along with the trajectory path. The algorithm enforces the set maximum desired speed and acceleration. The speed plan is the result of a refinement to bound the jerk, i. e. the derivative of the acceleration, of the trajectory so that the drone can fly through it using smoother rotations. The speed plan refinement has been shown to improve the result in terms of lower acceleration derivative values and of smoother speed and acceleration plans.

- Although other modules would be needed to decide the exact navigation objectives of the drone, using this planner we have shown that it is feasible to deploy drones in unknown outdoors environments, by leveraging the good qualities of maps obtained using state of the art photogrammetry mapping methods.

Main scientific publications:

The main scientific publications derived from the work realized during the realization of this PhD Thesis are the following:

\section{- A General Purpose Configurable Controller for Multirotor MAVs}

The main publications related to control architecture proposed in Chap. 2 are:

- J. Pestana, J. L. Sanchez-Lopez, P. de la Puente, A. Carrio, P. Campoy. "A Vision-based Quadrotor Multi-robot Solution for the Indoor Autonomy Challenge of the 2013 International Micro Air Vehicle Competition”. In Journal of Intelligent and Robotic Systems, JINT2015. Springer Netherlands.

- J. Pestana, I. Mellado-Bataller, C. Fu, J. L. Sanchez-Lopez, I. F. Mondragon, P. Campoy. “A General Purpose Configurable Controller for Indoors and Outdoors GPS-Denied Navigation for Multirotor Unmanned Aerial Vehicles”. In JINT2013.

- J. L. Sanchez-Lopez, J. Pestana, P. de la Puente, P. Campoy. “A reliable open-source system architecture for the fast designing and prototyping of autonomous multi-uav systems: Simulation and experimentation”. In JINT2015.

- I. Mellado-Bataller, J. Pestana, M. A. Olivares-Mendez , P. Campoy, and L. Mejias, book chapter, "MAVwork: a Framework for Unified Interfacing between Micro Aerial Vehicles and Visual Controllers", in book "Frontiers of Intelligent Autonomous Systems", serie "Studies in Computational Intelligence Series", Springer, 2013.

- J. Pestana, I. Mellado-Bataller, C. Fu, J. L. Sanchez-Lopez, I. F. Mondragon, P. Campoy. “A general purpose configurable navigation controller for micro aerial multirotor vehicles", In 2013 International Conference on Unmanned Aircraft Systems (ICUAS), 2013.

\section{- Visual Servoing of Arbitrary Objects for Multirotors}

The main publications related to visual servoing architecture proposed in Chap. 3 are:

- J. Pestana, J. L. Sanchez-Lopez, S. Saripalli, P. Campoy. “Computer Vision Based General Object Following for GPS-denied Multirotor Unmanned Vehicles”. In 2014 American Control Conference (ACC 2014). Portland, Oregon (United States). June 4th - 6th 2014

- J. Pestana, J. L. Sanchez-Lopez, S. Saripalli, P. Campoy. "Vision based GPS-denied Object tracking and Following for Unmanned Aerial Vehicles”. Best Paper Award Finalist in 2013 The 11th IEEE International Symposium on Safety, Security, and Rescue Robotics (SSRR 2013). Linköping (Sweden). Oct 21-26, 2013. 


\section{- Smooth Trajectory Planning for Multirotors}

The main publications related to the trajectory planning approach presented in Chap. 4 are:

- J. Pestana, M. Maurer, D. Muschick, D. Adlakha, H. Bischof, F. Fraundorfer, "Package Delivery Experiments with a Camera Drone”. In Austrian Robotics Workshop 2017, OAGM/AAPR2017 joint Workshop. To be published.

- J. Pestana and F. Fraundorfer, "Vision based safe navigation for search and rescue drones" in the IEEE/RSJ IROS2016 Workshop "Vision-Based High Speed Autonomous Navigation of UAVs". [No Proceedings] https://www. seas.upenn.edu/ loiannog/workshopIRos2016uav/

- J. Pestana, R. Prettenthaler, T. Holzmann, D. Muschick, C. Mostegel, F. Fraundorfer, H. Bischof "Graz Griffins' Solution to the European Robotics Challenges 2014”. In Austrian Robotics Workshop 2015, ARW2015.

- T Holzmann, R Prettenthaler, J. Pestana, D Muschick, G Graber, C Mostegel, F Fraundorfer, $\mathrm{H}$ Bischof "Performance Evaluation of Vision-Based Algorithms for MAVs". In Austrian Association for Pattern Recognition Conference 2015, OAGM2015.

- The author of this thesis is currently working in two publications based on works related to either the presented trajectory planner or the 2016 DJI Developer Challenge.

Contributions to the Open-Source Aerial Robotics Framework Aerostack:

Aerostack I s an Open-Source Aerial Robotics Framework developed mainly at the CVG (UPM), see Appx. G Its main purpose is enabling MAV civilian applications. The tasks that have been performed with Aerostack are: single-MAV navigation, multi-MAV navigation, object tracking and following, person tracking and following, human-machine interaction with the drone, entering buildings through windows or doors and pick and place operations with objects. As a reference of the types of missions achieved using Aerostack, this framework was used to participate in IMAV 2013, IARC 2014 and IMAV 2016, see Appx. I. This appendix also explains which was the role and contribution of the author to the participation on each of the listed international MAV competitions.

The contributions of the author of this dissertation to the Aerostack framework are:

- Flight controller module presented in Ch. 2

- Aerial robotics visual servoing module presented in Chap. 3

- State estimator module presented in Appx. E

- Simple kinematics simulator module based on the model presented in Appx.D.

The author has actively participated in the release of open-source software for the Aerostack framework. The contributed modules to Aerostack are reusable by others and reconfigurable so that they can be utilized in tasks related to the main purpose of the module. The author has actively participated in public flight demonstrations, such as international MAV competitions and other events, with the objective of publicly showcasing the performance of Aerostack. The participation in such events has resulted in the further testing of the developed modules in different settings and applications, e. g. indoors and outdoors flight, narrow corridors, passage through windows, landing in different conditions and so on.

\section{Participation in international MAV competitions and other public events:}

In order to ease the diffusion of results, the author has actively participated in public flight demonstrations, such as international MAV competitions, see Appx. I and other events, see Appx. J Appx. I also explains which was the role and contribution of the author to the participation on each of the listed international MAV competitions. The participation in such events has showcased the performance and increasing capabilities of Aerostack and it has promoted the further testing of the developed modules in different settings and applications, e. g. indoors and outdoors flight, narrow corridors, passage through windows, landing in different conditions and so on. The achieved results by the teams of which the author has been part, or by colleagues using modules developed by the author, in international aerial robotics competitions are the following:

- 2016 DJI Developer Challenge - DJIC 2016:

The competition recreated a drone Search and Rescue scenario. The "Graz Griffins" team qualified

${ }^{1}$ Aerostack website: https://github.com/Vision4UAV/Aerostack/wiki
${ }^{2}$ Github repository https://github.com/Vision4UAV/Aerostack 
through successive rounds, from the initial $\approx 140$ teams, to be part of the last 10 teams showing their solution experimentally in the finals.

Although the team was unable to integrate all prepared tasks in a single flight for the competition event, overall the team showed a very innovative solution using on-board mapping based on photogrammetry algorithm $s^{3}$ The on-board calculated map is converted to an obstacle-map that can be used by the presented planner to deliver trajectories that are then executed by the controller, all in the same flight and without requiring any off-board computation.

- International Micro Air Vehicle Conference and Flight Competition 2016 - IMAV 2016:

The only contribution of the author to the results was the utilization of controller module presented in Ch.2. The CVG_UPM team was able to solve multiple tasks in fully autonomous mode and was the team to achieve the highest number of tasks, although in separate flights, in fully autonomous mode $\epsilon^{4}$ Despite its outstanding performance, the CVG team earned the $4^{\text {th }}$ position due to the scoring system leaning very heavily towards the usage of very small MAVs.

- European Robotics Challenges 2014 - Simulation Challenge 3 - EuRoC 2014:

The developed fast real-time trajectory and speed planner ${ }^{5}$ enabled reliable obstacle avoidance and very fast trajectory execution by the simulated quadrotor that replicated the capabilities of an Asctec Firefly. The "Graz Griffins" team achieved the $6^{\text {th }}$ position out of 21 teams, which included world renowned European universities and laboratories, in the EuRoC 2014 - Challenge 3 Simulation Competition. This fact enabled the team to present a proposal in collaboration with a company to the next stage of EuRoC.

- International Aerial Robotics Competition 2014 - Mission 7 - IARC 2014:

Same as other teams, we were still far from achieving the interaction with the ground robots. Therefore, the CVG_UPM ${ }^{6}$ team showed similar capabilities to other contestants. Our level of development was good as a first step towards continuing our participation the next year for IARC 2015.

- International Micro Air Vehicle Conference and Flight Competition 2013 - IMAV 2013:

The CVG_UPM showed an outstanding multi-robot autonomous solution to IMAV 2013 7 The team that represented the UPM and the Computer Vision Group (CVG, UPM) was awarded with: first place in "Indoor Autonomy - IMAV 2013".

- International Micro Air Vehicle Conference and Flight Competition 2012 - IMAV 2012:

The CVG_UPM was the only team to show a fully autonomous solution to the IMAV 2012 Indoor Dynamics Competition 8 . The team that represented the UPM and the Computer Vision Group (CVG) was twice awarded with:

- the special award "Best Automatic Performance - IMAV 2012", as part of the overall results of the competitions, and

- second place in the "Indoor Flight Dynamics - Rotary Wing MAV" challenge, where the first place was obtained by a remotely piloted multirotor.

- Contest on Control Engineering 2012 - CEA 2012:

Two teams participated representing the Universidad Politécnica de Madrid (UPM) and the Computer Vision Group (CVG_UPM), and were awarded with:

- Undergraduate students' team of the ETSII-UPM engineering school using their own developed software supervised by J. Pestana: the first (phase 1 - simulation) and third places (final phase - autonomous flight event)

- CVG_UPM PhD students' team: the second (phase 1 - simulation) and fifth places (final phase - autonomous flight event). In the final phase of the competition our team achieved the fifth place, due to our controller, when compared to other teams, leaning towards low tracking error with higher round-trip times.

\footnotetext{
${ }^{3}$ DJIC 2016 video playlist: http: / / bit. ly/2 faublQ

${ }^{4}$ IMAV 2016 video playlist: https://goo.gl/tdZyGu

${ }^{5}$ EuRoC 2014 trajectory flight video: http://bit.1y/2cono6 I

${ }^{6}$ IARC 2014 video playlist: https://goo.gl/bEvK3F

${ }^{7}$ IMAV 2013 video playlist: https://goo.gl/KosWKH

${ }^{8}$ IMAV 2012 video playlist: https://goo.gl/PRns2D
} 


\subsection{Conclusions}

The necessity of implementing novel and reliable vision-based navigation methods, exploring the capabilities of state of the art Computer Vision methods and their applicability to Robotics have defined the research framework from which this thesis has been developed. The principal contributions derived from this $\mathrm{PhD}$ thesis are summarized in the following:

\section{- A General Purpose Configurable Controller for Multirotor MAVs:}

A novel multirotor navigation controller has been designed, implemented and experimentally evaluated. The main difference of our controller when compared to prior works is the addition of several saturation laws that can intrinsically take into account speed limitations established by the on-board sensors. This feature of the presented controller is quite advantageous, considering that some odometry estimation algorithms may fail to deliver estimates when navigating at inadequate velocities. An additional usage of the speed saturations proposed in the controller architecture and in the speed planning step is to simplify the flight rules of decentralized multi-robot navigation that was utilized by the CVG_UPM during its participation in the IMAV 2013 international competition.

\section{- Visual Servoing of Arbitrary Objects for Multirotors:}

A novel vision-based object tracking and following architecture for multirotor drones has been designed, implemented and experimentally evaluated. This architecture has demonstrated that current visual tracking algorithms, such as OpenTLD, can reliably work on a fixed camera multirotor vehicle to feedback an Image Based Visual Servoing (IBVS) controller. Our architecture has been able to follow a large variety of unmarked targets of different sizes and from a wide range of distances. Moreover, the algorithm was validated using a low-cost platform, the Parrot AR Drone 2.0, in outdoor conditions while tracking and following people. The system has successfully and repeatedly handled occlusion events and tracked fast moving targets, such as a person running, showing the potential capabilities of the combination of modern machine learning based visual tracking methods for the vision-based object following task.

\section{- Smooth Trajectory Planning for Multirotors:}

A novel trajectory planner for multirotor drones has been designed, implemented and experimentally evaluated. The algorithm has been tested experimentally on maps obtained using an on-board real-time capable Computer Vision mapping method. Although other modules would be needed to decide the exact navigation objectives of the drones, using this planner we have shown that the fast deployment of MAVs to perform a mission in an unknown outdoors environment using only on-board computation is possible, by leveraging the good qualities of maps obtained using state of the art photogrammetry methods. The planner utilizes the Octomap obstacle map representation, which is commonly used in robotics, and its related distance map, to all present obstacles, resulting in the fast calculation of obstacle-free smooth trajectories. A feasible speed, acceleration and time-of-passage plans, configurable by maximum speed and acceleration parameters, are delivered along with the trajectory path. The speed plan is refined to bound the jerk, i. e. the derivative of the acceleration, of the trajectory so that the drone can fly through it using smoother rotations.

\section{- Contributions to the Open-Source Aerial Robotics Framework Aerostack:}

Aerostack ${ }^{9}$ s an Open-Source Aerial Robotics Framework developed mainly at the CVG (UPM), see Appx. G, which main purpose is enabling MAV civilian applications. The tasks that have been performed with Aerostack are: single-MAV navigation, multi-MAV navigation, object tracking and following, person tracking and following, human-machine interaction with the drone, entering buildings through windows or doors and pick and place operations with objects. As a reference of the types of missions achieved using Aerostack, this framework was used to participate in IMAV 2013, IARC 2014 and IMAV 2016, see Appx. I. This appendix also explains which was the role and contribution of the author to the participation on each of the listed international MAV competitions. The author has actively participated in the release of open-source software for the Aerostack framework, his contributions have included: the flight controller module presented in

\footnotetext{
${ }^{9}$ Aerostack website: https://github.com/Vision4UAV/Aerostack/wiki

${ }^{10}$ Github repository https://github.com/Vision4UAV/Aerostack
} 
Ch. 2. the aerial robotics visual servoing module presented in Chap. 3, the state estimator module presented in Appx. Eland the simple kinematics simulator module based on the model presented in Appx.D

\section{- Participation in international MAV competitions and other public events:}

In order to ease the diffusion of results, the author has actively participated in public flight demonstrations, such as international MAV competitions, see Appx. I and other events, see Appx. J. Appx. [1 also explains which was the role and contribution of the author to the participation on each of the listed international MAV competitions. The participation in such events has showcased the performance and increasing capabilities of Aerostack and it has promoted the further testing of the developed modules in different settings and applications, e. g. indoors and outdoors flight, narrow corridors, passage through windows, landing in different conditions and so on. Teams of which the author has been part or in which colleagues have used modules explained in this dissertation and developed by author have participated in the following international aerial robotics competitions: 2016 DJI Developer Challenge - DJIC 2016, International Micro Air Vehicle Conference and Flight Competition 2016 - IMAV 2016, European Robotics Challenges 2014 - Simulation Challenge 3 - EuRoC 2014, International Aerial Robotics Competition 2014 - Mission 7 - IARC 2014, International Micro Air Vehicle Conference and Flight Competition 2013 - IMAV 2013, International Micro Air Vehicle Conference and Flight Competition 2012 - IMAV 2012 and the Concurso en Ingeniería de Control 2012 - CEA 2012.

\subsection{Future Work}

The strong experimental basis of this work and the main research problems tackled during its realization point towards the following areas for potential future work:

- In the area of control, estate estimation and dynamics modeling of drones, there are promising works that show that better models allow a much more precise estimation of the wind speed, or, in the absence of wind, of the speed of the drone itself, see Sec.3.2 and Appx.E.2. In addition to providing more precise state estimates, the research in this direction may lead to safer flight maneuvering in environments that become very challenging under the presence of wind disturbances such as urban corridors and indoor hallways. Another interesting area of research is the development of state estimators that detect flight issues. Consider, for instance, soft collisions of the drone protective hull with walls. Detecting such events may result in interesting behaviors such as control techniques that avoid the worsening of the flight situation, after the event is detected by the state estimator, like avoiding a full crash against the said wall. Other physical contact events that are important to be detected are the approach to the ground, the ceiling or walls, based on how these events affect the flight of the drone; and determining the landing event more precisely, that may enable faster landing maneuvers on moving landing platforms. In the area of state estimation, many autopilots have a very big dependence on the magnetometer sensor for the drone heading estimation. There currently exist UAVs that can operate under magnetic interference by using multiple GPS sensors. In GPS-denied environments, for indoor navigation and flying near ferromagnetic infrastructure, it is required to use other sensor data for heading estimation, so as to decrease the dependence on the magnetometer sensor.

- In the area of visual servoing there is an strong inter-dependence between the performance of the visual tracker and the successful realization of an object following task by a controller. Therefore, new methods could combine specific characteristics of the tracked objects and the surrounding environment into a better control strategy. For instance, it may be beneficial to select positions relative to the followed object that result in viewing angles without light glare reflections or strong shadows. In the same direction, the development of visual trackers that allow for object model introspection should be preferred. For instance, consider models that include the 3D structure of the object or the reflective properties of the different surfaces of the object. A very interesting ongoing challenge that may require model introspection is the International Aerial Robotics Competition (IARC) Mission 7, see Sec. [I.4, where the drone needs to physically interact with ground robots that exhibit pseudo-random navigation behavior. From a navigation stand point, obstacle avoidance 
needs to be incorporated more strongly into the visual servoing task to enable following an object through a cluttered environment, like a sportsman through a forest. In this sense, obstacles that are difficult to map, such as trees, could be avoided considering their visual appearance rather than their difficult to reconstruct $3 \mathrm{D}$ structure.

- In the area of trajectory planning, the navigation control performs better when following smooth trajectories, allowing faster motion. Additionally, less computer intensive trajectory generation algorithms allow the drone to make rapid decisions while navigating in cluttered environments or while performing time critical operations. Since robots can move and affect which data is going to be gathered for the mapping of the environment, another innovative research trend is acknowledging the inter-dependence in robotics of exploration, obstacle mapping and trajectory planning. For instance, the development of trajectory planning strategies that utilize obstacle maps that encode uncertainty. There is also a trend to combine image processing and motion planning where these two tasks are not separated in modules but rather solved together by means of deep learning. Some works have already shown promising results in object grasping and door opening by robot arm manipulators, but similar techniques are still to be applied to drones.

- In the area of vision-based navigation, it would be important to increase the effort on the development of algorithms that can use a minimal setting of sensors and low power on-board computers. The research on novel vision-based and low computationally intensive pose estimation techniques is of great importance for the development of autonomous very lightweight UAVs. These platforms can be rather harmless and may enable the use of drones around people in civilian applications. 
Appendices 



\section{Appendix}

\section{Scientific Dissemination}

\section{A.1 Journals}

J. Pestana, J. L. Sanchez-Lopez, P. de la Puente, A. Carrio, P. Campoy. "A Vision-based Quadrotor Multi-robot Solution for the Indoor Autonomy Challenge of the 2013 International Micro Air Vehicle Competition". In Journal of Intelligent and Robotic Systems. 2015. Springer Netherlands. Print ISSN: 0921-0296. Online ISSN: 1573-0409.

J. L. Sanchez-Lopez, J. Pestana, P. de la Puente, P. Campoy. “A reliable open-source system architecture for the fast designing and prototyping of autonomous multi-uav systems: Simulation and experimentation". In Journal of Intelligent and Robotic Systems. 2015. Springer Netherlands. Print ISSN: 0921-0296. Online ISSN: 1573-0409.

J. Pestana, I. Mellado-Bataller, C. Fu, J. L. Sanchez-Lopez, I. F. Mondragon, P. Campoy. “A General Purpose Configurable Controller for Indoors and Outdoors GPS-Denied Navigation for Multirotor Unmanned Aerial Vehicles". In Journal of Intelligent and Robotic Systems. 2013. Springer Netherlands. Print ISSN: 0921-0296. Online ISSN: 1573-0409.

J. L. Sanchez-Lopez, J. Pestana, S. Saripalli, P. Campoy. “An Approach Towards Visual Autonomous ship board landing of a VTOL UAV”. In Journal of Intelligent and Robotic Systems. 2013. Springer Netherlands. Print ISSN: 0921-0296. Online ISSN: 1573-0409.

L. Hernández Hernández, J. Pestana, D. Casares Palomeque, P. Campoy, J. L. Sanchez-Lopez “Identificación y control en cascada mediante inversión de las no linealidades del cuatrirrotor del concurso de control inteligente CEA IFAC 2012”, in Revista Iberoamericana de automática e informática industrial, July-September 2013. Volume 10, Issue 3, pp. 356-367. ISSN: 1697-7912. ISSN: 1697-7920. 


\section{A.2 Publications in peer-reviewed conferences}

J. Pestana, M. Maurer, D. Muschick, D. Adlakha, H. Bischof, F. Fraundorfer, "Package Delivery Experiments with a Camera Drone”. In Austrian Robotics Workshop 2017, OAGM/AAPR2017 joint Workshop.

J. L. Sanchez-Lopez, J. Pestana, P. Campoy. "A Robust Real Time Path Planner for the Collision Free Navigation of Multirotor Aerial Robots in Dynamic Environments”. 2017 International Conference on Unmanned Aircraft Systems (ICUAS).

W. A. Isop, J. Pestana, G. Ermacora, F. Fraundorfer, D. Schmalstieg “Micro Aerial Projector - Stabilizing Projected Images Of An Airborne Robotics Projection Platform”. In IEEE/RSJ International Conference on Intelligent Robots and Systems, IROS2016

J. L. Sanchez-Lopez, R. A. Suárez Fernández, H. Bavle, C. Sampedro, M. Molina, J. Pestana, P. Campoy “AEROSTACK: An Architecture and Open-Source Software Framework for Aerial Robotics”. In 2016 International Conference on Unmanned Aircraft Systems, ICUAS16

J. Pestana, R Prettenthaler, T Holzmann, D Muschick, C Mostegel, F Fraundorfer, H Bischof "Graz Griffins' Solution to the European Robotics Challenges 2014”. In Austrian Robotics Workshop 2015, ARW2015

T Holzmann, R Prettenthaler, J. Pestana, D Muschick, G Graber, C Mostegel, F Fraundorfer, H Bischof "Performance Evaluation of Vision-Based Algorithms for MAVs". In Austrian Association for Pattern Recognition Conference 2015, OAGM2015

J. L. Sanchez-Lopez, J. Pestana, JF Collumeau, Ramon Suarez-Fernandez, P. Campoy, M. Molina " $A$ vision based aerial robot solution for the Mission 7 of the International Aerial Robotics Competition". In 2015 International Conference on Unmanned Aircraft Systems, ICUAS15

J. Pestana, J. L. Sanchez-Lopez, R. Suarez-Fernandez, J. F. Collumeau, P. Campoy, J. MartinCristobal, M. Molina, J. De Lope, and D. Maravall. "A Vision Based Aerial Robot solution for the IARC 2014 by the Technical University of Madrid". In AUVSI International Aerial Robotics Competition 2014

J. Pestana, J. L. Sanchez-Lopez, P. de la Puente, A. Carrio, P. Campoy. "A Vision-based Quadrotor Swarm for the participation in the 2013 International Micro Air Vehicle Competition”. In 2014 International Conference on Unmanned Aircraft Systems, ICUAS14

J. L. Sanchez-Lopez, J. Pestana, P. de la Puente, R. Suarez-Fernandez, P. Campoy. “A System for the Design and Development of Vision-based Multi-robot Quadrotor Swarms". In 2014 International Conference on Unmanned Aircraft Systems, ICUAS14

A. Carrio, C. Fu, J. Pestana, P. Campoy. “A Ground-Truth Video Dataset for the Development and Evaluation of Vision-based Sense-and-Avoid systems”. In 2014 International Conference on Unmanned Aircraft Systems, ICUAS14

J. Pestana, J. L. Sanchez-Lopez, S. Saripalli, P. Campoy. “Computer Vision Based General Object Following for GPS-denied Multirotor Unmanned Vehicles”. In 2014 American Control Conference (ACC 2014). Portland, Oregon (United States). June 4th - 6th 2014

J. Pestana, J. L. Sanchez-Lopez, S. Saripalli, P. Campoy. “Vision based GPS-denied Object tracking and Following for Unmanned Aerial Vehicles". Best Paper Award Finalist in 2013 The 11th IEEE International Symposium on Safety, Security, and Rescue Robotics (SSRR 2013). Linköping (Sweden). Oct 21-26, 2013. 
J. Pestana, I. Mellado-Bataller, C. Fu, J. L. Sanchez-Lopez, I. F. Mondragon, P. Campoy. “ $A$ general purpose configurable navigation controller for micro aerial multirotor vehicles", In 2013 International Conference on Unmanned Aircraft Systems (ICUAS), pp. 557-564, 28-31 May 2013 doi: 10.1109/ICUAS.2013.6564733

J. L. Sanchez-Lopez, S. Saripalli, P. Campoy, J. Pestana, C. Fu. "Visual Autonomous Ship Board Landing of a VTOL UAV”. In 2013 International Conference on Unmanned Aircraft Systems (ICUAS'13). Atlanta, Georgia (USA). May 28-31, 2013.

J. Pestana, I. Mellado-Bataller, J. L. Sanchez-Lopez, C. Fu, I. F. Mondragon, P. Campoy. “Floor Optical Flow Based Navigation Controller for Multirotor Aerial Vehicles”. Robot 2013: First Iberian Robotics Conference (ROBOT 2013).

J. L. Sanchez-Lopez, J. Pestana, P. de la Puente, A. Carrio, P. Campoy. "Visual Quadrotor Swarm for IMAV 2013 Indoor Competition”. Robot 2013: First Iberian Robotics Conference (ROBOT 2013).

Jesús Pestana, Ignacio Mellado-Bataller, Fu Changhong, Jose Luis Sanchez-Lopez, Iván F. Mondragón, and Pascual Campoy "A Visual Guided Quadrotor for IMAV 2012 Indoor Autonomy Competition and Visual Control of a Quadrotor for the IMAV 2012 Indoor Dynamics Competition”, at International Micro Air Vehicle Conference and Flight Competition, IMAV 2012, July 2012

Changhong Fu, Jesús Pestana, Ignacio Mellado-Bataller, Jose Luis Sanchez-Lopez, and Pascual Campoy "Visual Identification and Tracking for Vertical and Horizontal Targets in Unknown Indoor Environment”, at International Micro Air Vehicle Conference and Flight Competition, IMAV 2012, July 2012

J. Pestana, J. L. Sánchez-López, I. Mellado, F. Changhong, P. Campoy, “AR Drone Identification and Navigation Control at CVG-UPM”, in XXXIII Jornadas de Automática, September 2012

J. Pestana, R. Bombín, E. Garcia, "Characterization of Magnetic Shape Memory Alloys (MSMA) Oriented to Periodic Actuation”, in 12th International Conference on New Actuators/6th International Exhibition on Smart Actuators and Drive Systems, Bremen, Germany, 2010

J. Pestana, R.Bombín, J. C. Arevalo and E. Garcia, "Characterization of emerging actuators for empowering legged robots", in 13th International Conference on Climbing and Walking Robots, CLAWAR, Nagoya, Japan, 2010

J. C. Arevalo, J. Pestana, F. Sanchez, J. F. Sarria and E. Garcia, "Impedance control of an agilelocomotion robotic leg”, in 13th International Conference on Climbing and Walking Robots, CLAWAR, Nagoya, Japan, 2010

\section{A.3 Book Chapters}

I. Mellado-Bataller, J. Pestana, M. A. Olivares-Mendez, P. Campoy, and L. Mejias, book chapter, "MAVwork: a Framework for Unified Interfacing between Micro Aerial Vehicles and Visual Controllers", in book "Frontiers of Intelligent Autonomous Systems", serie "Studies in Computational Intelligence Series”, Springer, 2013, Volume 466, ISBN 978-3-642-35484-7, pp 165-179

\section{A.4 Participation in Conference Workshops}

Oct. 14, 2016: IEEE/RSJ International Conference IROS2016

Daejeon, Korea Workshop on Vision-Based High Speed Autonomous Navigation of UAVs, presented "Vision based safe navigation for search and rescue drones" based on participation on the 2016 DJI Developer Challenge. 



\section{B}

\section{UAVs and Multicopters}

An Unmanned Aircraft (UA) or Unmanned Aerial Vehicle (UAV) is an aircraft without a human pilot on-board. These aircrafts are either piloted remotely by a human operator or autonomous, in which case the UAV does not depend on a human operator to fly safely. The degree of autonomy varies from navigation-only capabilities, to performing the decision-making necessary to perform a task or mission automatically. Certainly, as it is common with current robotic technology, these systems cannot yet accomplish general complex tasks by themselves; and full autonomy is still limited to specific use cases, such as automated data gathering for surveying purposes. However, autonomy does divide UAVs in the two major types specified above: Remotely Piloted Aircrafts (RPA), which require a human pilot, and Autonomous UAVs, which navigate themselves. It is remarked that RPA do have an on-board control system that performs lower-level operations, such as tracking the attitude of the vehicle and adjusting it to follow the pilot's commands. The main difference between both types is, thus, in the capability to navigate themselves without direct human interaction.

A second term has been adopted to refer, not only to the aircraft, but to all the associated equipment. This term is Unmanned Aerial System (UAS), and includes the Ground Control Station (GCS), the communications, data links, telemetry and all the other equipment necessary to operate the UAV. The term UAS was initially used only for large sized systems, but it is currently more and more commonly used for small ones too. Both terms are correct, but one or the other should be utilized depending on whether only the aircraft or the whole system is alluded. In the same manner the term Remotely Piloted Aircraft (RPA) refers to the flying platform and the term Remotely Piloted Aircraft System (RPAS) refers also to the rest of the equipment and instruments. Note that all RPAS are UAS, but not all UAS, for instance fully autonomous ones, are RPAS.

UAVs can be classified by various characteristics, mainly weight, endurance and propulsion principle. The most common types, based on the latter, are fixed and rotary UAVs. Fixed wing aircraft produce lift force by means of their forward speed and the shape of their wings, an example of them are airplanes. Various fixed-wing UAVs are shown in Fig. B.2. In rotary wing aircraft, or rotorcraft, the wings revolve around a mast, and a common example are helicopters. There are other types of propulsion used in UAVs, for instance flapping wing aircraft move their wings as a bird to produce lift force. This thesis is focused 


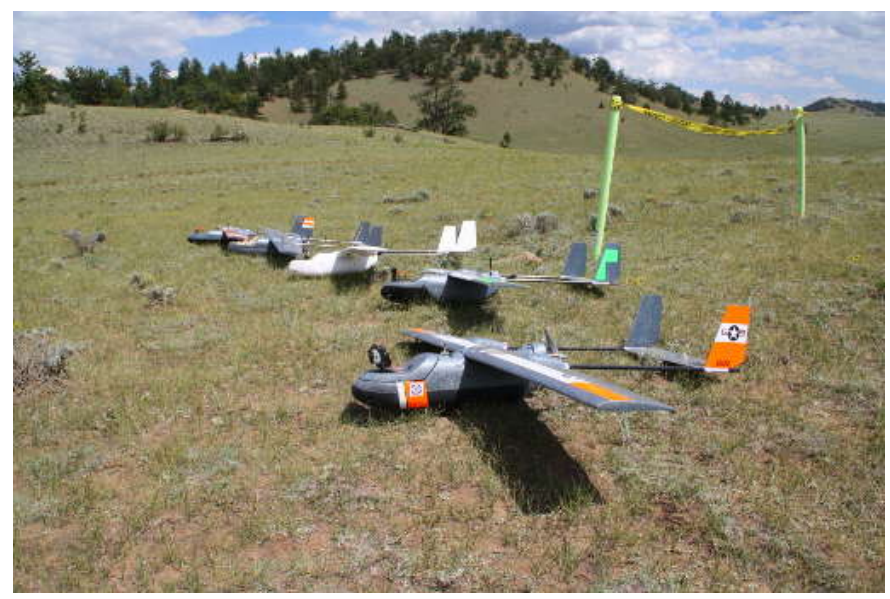

Figure B.1: Various fixed-wing micro UAVs (MAVs) of "Sky Hunter" at a meetup in Colorado (in (July 2013), photography by Patrick McKay - Wikimedia - Creative Commons Attribution-Share Alike 3.0 Unported license.

on multirotors which will be now presented.

A multirotor is a rotary wing aircraft with more than two rotors, see Fig. B.1. In most cases, and in contrast with helicopters, their rotors are equipped with propellers of fixed-pitch blades. This fact introduces mechanical differences that make multirotors mechanically much simpler and cheaper to maintain than helicopters. In addition, their propellers are smaller and more lightweight, making them less dangerous. In a multicopter, the aircraft motion is controlled by varying the relative speed of the rotors, which effectively determines the thrust and torques acting on the aircraft body. In RPA multirotors, the commanded rotor speeds are calculated by an on-board flight controller called autopilot based on the thrust and body attitude commands specified by the pilot. Multicopters are Vertical Take-Off and Landing (VTOL) aircraft because they can hover, take off, and land vertically. Their capability to hover and navigate at low speeds makes them adequate to perform tasks in cluttered environments. Overall, these platforms are very versatile and cost-effective and they are being adopted as the preferred solution for many surveying civilian applications.

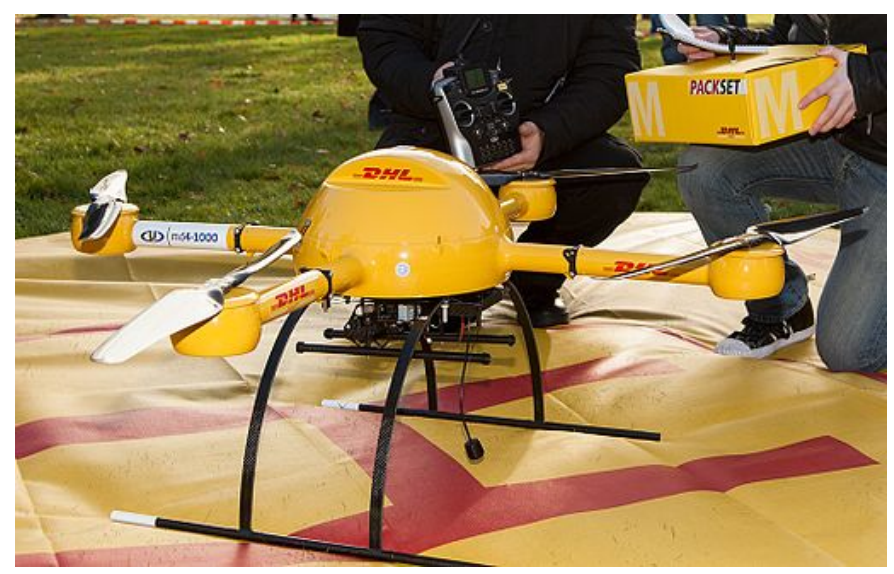

Figure B.2: A Microdrones MD4-1000 quadrotor tested for medicine delivery in collaboration with DHL (2013), photography by Frankhöffner - Wikimedia - Creative Commons Attribution-Share Alike 3.0 Unported license.

The classification of UAVs by propulsion principle is clear. In terms of the weight and size of the aircraft, however, there does not exist a general accepted standard to classify them. For instance, the civil regulations that concern UAVs are usually categorized based on the weight of the aircraft, but specific 
category thresholds differ between countries. There does not exist a common regulation framework for all countries, and each National Aviation Authority (NAA) has a similar but different set of rules concerning UAVs. A reliable perspective of how the European regulations will evolve to allow autonomous UAVs to fly in the common airspace is provided by the report (ERSG, 2013): "Roadmap for the integration of civil Remotely - Piloted Aircraft Systems into the European Aviation System", written by the European RPAS Steering Group (June 2013).

A comprehensive classification of UAVs, which is still applicable nowadays, was proposed by Blyenburgh (Blyenburgh, 2006) and is backed by the Association for Unmanned Vehicles Systems International (UVS International). Two scholar articles that can also be consulted to understand this classification are (Bento, 2008), which focuses on military UAVs, and (Watts et al., 2012) that describes also UAS used for civilian applications. 


\section{B.1 Appendix Bibliography}

Bento, M. (2008). “Unmanned aerial vehicles: an overview”. Inside GNSS 3.1, pp. 54-61 (cit. on p. 129). Blyenburgh, P. v. (2006). "UAV Systems : Global Review”. In: Avionics'06 Conference. Amsterdam, The Netherlands (cit. on p. 129).

ERSG (2013). Roadmap for the integration of civil Remotely - Piloted Aircraft Systems into the European Aviation System. Tech. rep. European RPAS Steering Group (cit. on p. 129).

Watts, A. C., Ambrosia, V. G., and Hinkley, E. A. (2012). "Unmanned aircraft systems in remote sensing and scientific research: Classification and considerations of use". Remote Sensing 4.6, pp. 1671-1692 (cit. on p.129. 


\section{C}

\section{Multirotor Software Interface}

\section{C.1 Introduction}

In order to convert a commercial multirotor into an autonomous aerial robot several modules need to be developed and integrated together. The development of software modules is eased by the utilization of frameworks that manage the data communication between them. To aid the development of the various processing modules, the multirotor is adapted to provide a minimal set of functionalities and to communicate using common interface commands and sensing data. This chapter describes the communication framework, the abstract interface for the multirotor and an example of a software architecture used during the course of this thesis. The purpose of the main processing modules related to the works realized in this thesis are introduced along with an example software architecture.

First the Robotics Middleware frameworks utilized for communicating data between processes are shortly introduced in Sec. C.2. Then, the abstract interface of the multirotor is described in Sec. C.3. Finally the software modules and architecture utilized to participate in the IMAV2012 competition are shortly discussed on Sec. C.4

\section{C.2 Robotics Middleware}

There are many ways of putting the software of a robot together. Nowadays, there are various frameworks that allow each module to be developed separately; and that offer an easy way to communicate each module with each other. The main goal of this approach is to free the developer of the burden of putting together a complex communication system. Every member of the team can focus on their part of the project, without having to work on the underlying system architecture that takes care of communicating the data. In robotics, this type of software is commonly known as middleware. Using popular robotics middleware is very advantageous for open-source projects because it increases the shareability of the code. Any developer that is familiar with the utilized middleware knows how the communication interface works.

For instance, the Robot Operating System (ROS) (Quigley et al., 2009, Willow Garage, 2007) offers 
the possibility to easily communicate processing modules and computers over a network. ROS allows the developer to visualize the software architecture as a graph where the processing takes place in nodes and the edges of the graph represent information channels. It offers different types of communication modalities, so that common requirements and functionalities can be addressed with them. ROS has become a standard to share open-source code in robotics and it is currently widely utilized in this community.

Until 2013, the CVG-UPM was developing and using their own middleware, MAVwork (Mellado Bataller, I., 2012; Mellado-Bataller et al., 2013) which was used successfully to participate in the IMAV2012 competition. The main drawback of this middleware is that it requires to use a multithreaded approach where each processing module is a thread, which makes the addition and removal of modules programmatically cumbersome when compared to ROS.

One of the drawbacks of ROS at the time was that it was not efficient for communicating images between processes, which could pose a problem in vehicles with limited onboard computing capabilities. However, this was solved with the introduction of ROS "nodelets", which allow to use a shared memory between a sensor driver and its processing module. This fact and the increasing popularity of ROS in the robotics community are the main reasons why the CVG (UPM) decided in 2013 to switch to using ROS as communication middleware for its projects.

\section{C.3 Multirotor Driver}

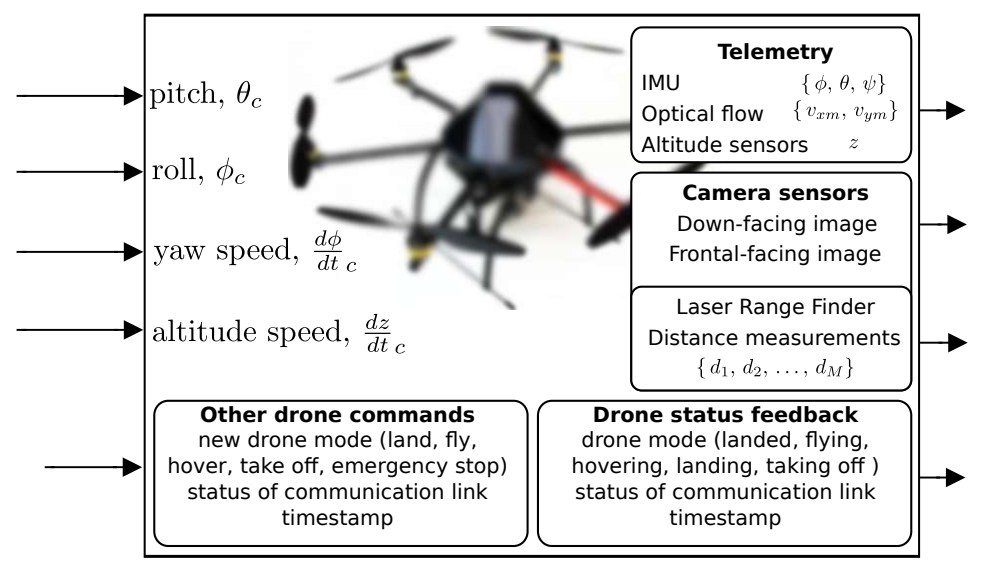

Figure C.1: The abstract multirotor representation or Multirotor Driver offers two main feedback channels: the telemetry package with IMU attitude, body horizontal speed and altitude data and the drone status feedback channel. Other sensors, such as a laser range finder or cameras, can be added to the multirotor but they are not mandatory. The drone modes can be sent to command autonomous take-off, landing and hovering or to accept control commands from an external controller. In order to provide these functionalities, a sensor or module estimating the horizontal velocity and the altitude of the multirotor has to be used and integrated.

A general representation for a drone was designed and developed as part of MAVwork (Mellado Bataller, I., 2012; Mellado-Bataller et al., 2013) and it is hereon described from the point of view of the author of this dissertation. It offers a basic drone representation where interfacing functions are already defined. This representation defines a common interface to be used with different multirotors which offers basic functionalities such as autonomous take-off, landing and hovering. The multirotor representation was inspired by functionalities available in commercial drones but that are not standard. It eases the work of the developers, because it allows them to start the experiments from a hovering position and to enter a safe hovering mode. This abstract multirotor representation was later on adapted and ported to ROS. The actual implementation of this representation is the ROS module that is termed "Multirotor Driver" and it is part of the ROS-based open-source code of the CVG (UPM). The Fig. C.1 summarizes the interface that the multirotor representation offers to the developer, and that is further explained in the following:

- A function to send roll, pitch, yaw speed and altitude speed commands to the drone autopilot. The 
commands can be sent in an asynchronous fashion, at the pace defined by the user,

- Several drone modes can be commanded, offering autonomous take off and landing. During flight the drone can be commanded to enter position hold mode (hover mode), where the drone approximately stays in position by using the speed estimates. The 'flying' mode obeys the attitude and altitude speed commands sent by the user application,

- Timestamps and the status of the communication link are exchanged between the drone and the application with no intervention from the client application,

- The telemetry feedback channel sends back IMU attitude, body horizontal speed an altitude data,

- The actual drone mode is sent back to the client application inside the telemetry package.

- Other sensors, such as a laser range finder or cameras, can be added to the multirotor but they are not mandatory,

- From the user point of view, the feedback channels are independent threads that are woken up when a new measurement is available. And the commands are transfered to the multirotor autopilot as soon as they are received.

In order to provide the take-off, landing and hovering functionalities, a sensor or module estimating the horizontal velocity and the altitude of the multirotor has to be integrated with the Multirotor Driver. This data is readily available in the AR Drone, where it is obtained using an ultrasound altimeter and a ground optical flow speed sensor. Other drones have to be adapted by using appropriate sensors depending on the application.

\section{C.3.1 Notes on the adaptation of the Asctec Pelican to the Multirotor Driver}

The Pelican does not natively provide support for the described MAVwork framework. In order to add it, by the members of the CVG (UPM) as part of prior works and for the participation in IMAV2012, a serial communication link was first implemented between the high-level autopilot board and the onboard Intel Atom running Linux, which is used as main processing platform. In this way, a framework-compliant proxy could be implemented on the Atom onboard computer, using the WiFi interface for networking. For the IMAV2012, the Pelican proxy was interfaced with a Hokuyo range finder, whose readings were sent to the control application through a camera channel.

The Pelican autopilot does not naturally support the MAV1 protocol, used by MAVwork (Mellado Bataller, I., 2012; Mellado-Bataller et al., 2013). It neither interprets commands in the required format nor gives navigation information as expected by the protocol. Therefore, some software adaptations involving reference frame changes and conversion of units were carried out on the already available commands and feedback to suit the MAV1 specification. However, some measures, like the drone speed estimate or the altitude, required also the addition of new hardware and special processing. For the altitude measurements, a couple of ultrasound sensors were added. When they point to the ground they can give a much better estimate than the Pelican internal altitude sensor, which is based on air pressure measurements. The drone speed is estimated with a camera, also pointing to the ground. At every frame, the optical flow is computed with the OpenCV implementation of the pyramidal Lucas-Kanade algorithm for some image features selected by the Shi-Tomasi method.

The ROS version of the Multirotor Driver for the Asctec Pelican was integrated with the PX4Flow sensor (Honegger et al., 2013), which provides altitude and horizontal speed measurements in a similar manner to the AR Drone. This version of the driver was first used to participate in the IARC2014 competition.

By using the Multirotor Driver, the Pelican and the AR Drone offer a common interface and their behaviors are similar. In 2012, a control application for them was implemented using MAVwork (Mellado Bataller, I., 2012), which later came to be adapted to the common Multirotor Driver interface in ROS. MAVwork exposes an Application Programming Interface (API), independent of the drone type, through which visual control applications can communicate with MAVs without worrying about their hardware specifics. Deeper explanations of how the framework is implemented can be found on (Mellado Bataller, I., 2012, Mellado-Bataller et al., 2013, Pestana et al., 2012). Thanks to this approach, the trajectory controller used in the IMAV2012 dynamics challenge was first tested and debugged on a Parrot AR Drone, 
which we often use as prototyping platform. Once tests showed that the software was bug-free and robust, the controller was run and fine-tuned on the Pelican without changing the application structure; only the parameters of the dynamic controllers were modified to suit the Pelican flight dynamics.

\section{C.4 Example Software Architecture (IMAV2012)}

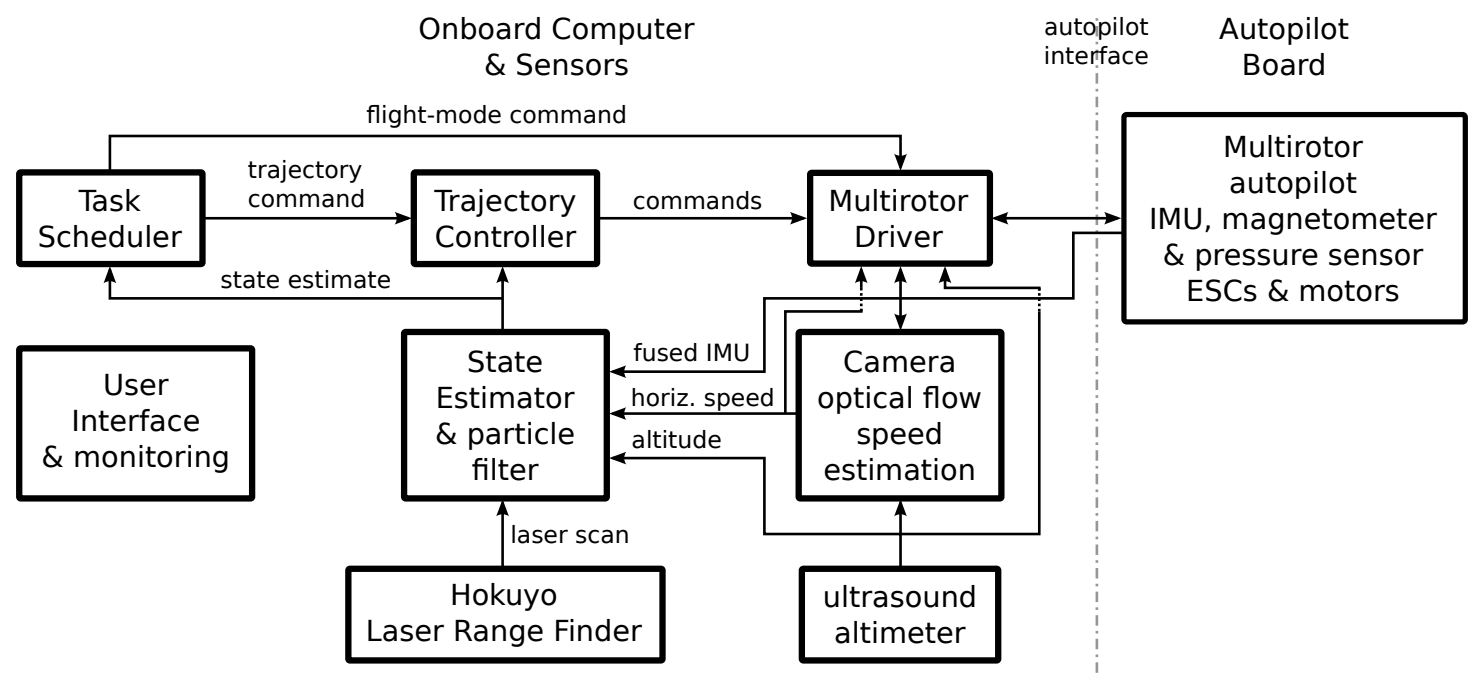

Figure C.2: Overview of the software architecture utilized to participate in the IMAV2012 competition. The autopilot takes care of the data acquisition and low-level control tasks of the multirotor. The onboard computer acquires information from additional sensors, a camera, an ultrasound altimeter and a Hokuyo laser range finder, and uses it to enable the multirotor to perform the autonomous flight in the competition. The Multirotor Driver centralizes the communications with the autopilot and offers the practical flightmode commands interface by taking care of middle-level tasks such as take-off, land and hover. The state estimator assesses the current position and speed of the multirotor based on the sensor data. It also feeds its estimate to the particle filter that can calculate a drift-free position estimate by comparing the laser scans to the known obstacle map. The controller calculates attitude and altitude speeds commands for stabilizing the drone and follow trajectory commands. The competition dynamics challenge is achieved autonomously by using a rigid task scheduler that leverages the discussed software architecture. It monitors the state of the drone and its battery status and issues a series of flight-mode and trajectory commands as the realization of the navigation tasks proceeds and are accomplished.

The development of an autonomous robot requires engineering many different software modules. For the participation in the IMAV2012, the software architecture was implemented using a single program in a multi-threaded approach based on MAVwork (Mellado Bataller, I., 2012, Mellado-Bataller et al., 2013; Pestana et al., 2012). The following list of modules and Fig. C.2 describe the software that was running during the competition, which allowed to follow trajectories and perform the dynamics challenge autonomously in the environment described by the competition rules:

- Low-level sensing, control and hardware interfaces: many multirotors provide these functionalities out of the box, these tasks being accomplished by a board called autopilot. However, many commercial drones do not provide a convenient way to command the drone from an onboard computer, and are only designed to be flown by human pilots. The information from several sensors is acquired to determine the attitude of the aerial robot and, usually using GPS, its position. These information together with the flight commands is used to stabilize the flight of the multirotor.

- Interfacing the onboard computer with the autopilot and various sensors: each sensor module is interfaced using the libraries provided by the manufacturer. A multi-threaded approach or separate programs that communicate through a robotics middleware are used to obtain the sensor data as soon as it is available. These interfaces have to be reliable and thus require debugging and testing. 
- A state estimation module provides a real-time assessment of the position, attitude and velocity of the flying robot, based on the information available in the sensor measurements.

- A controller module which principal objective is to allow other modules to move the vehicle with a more friendly set of commands. In the case of multirotors a position controller allows commanding the multirotor directly in the $\{x, y, z, \psi\}$ coordinates, which represent its position and heading. Another options which was used in the IMAV2012, is to make a controller that can follow trajectory commands. The control module has to be able to safely communicate with the estimation module in a bidirectional fashion. The estimation algorithm can benefit from knowing which commands the controller is sending to the multirotor autopilot, and the controller needs the estimation of the position and velocities of the robot to calculate the commands.

- A task scheduler, which is in charge of chaining a set of steps that accomplish the mission objectives. The task scheduler requires to communicate with the priorly explained modules: the Multirotor Driver, the Trajectory Controller and the State Estimator. The communications with the estimation module and additional sensors are used to update the status of accomplishment of the mission objectives; and the scheduler uses the controller to command the robot where to move next.

- A user interface, either graphical or through a simple console interface: the user needs to be able to monitor what the robot is doing and if necessary to take command over the robot, basically deactivating the task scheduler and/or controller; and sending commands himself. 


\section{C.5 Appendix Bibliography}

Mellado Bataller, I. (2012). A new framework for interfacing with MAVs. https: / / github . com/ uavster/mavwork (cit. on pp. 132, 134).

Honegger, D., Meier, L., Tanskanen, P., and Pollefeys, M. (2013). “An open source and open hardware embedded metric optical flow cmos camera for indoor and outdoor applications". In: Robotics and Automation (ICRA), 2013 IEEE International Conference on. IEEE, pp. 1736-1741 (cit. on p. 133).

Mellado-Bataller, I., Pestana, J., Olivares-Mendez, M. A., Campoy, P., and Mejias, L. (2013). "MAVwork: a framework for unified interfacing between micro aerial vehicles and visual controllers". In: Frontiers of Intelligent Autonomous Systems. Springer, pp. 165-179 (cit. on pp.132 134.

Pestana, J., Mellado-Bataller, I., Fu, C., Sanchez-Lopez, J. L., Mondragon, I. F., and Campoy, P. (2012). "A Visual Guided Quadrotor for IMAV 2012 Indoor Autonomy Competition and Visual Control of a Quadrotor for the IMAV 2012 Indoor Dynamics Competition”. International Micro Air Vehicle (IMAV), 2012 Conference and Flight Competition (cit. on pp. 133, 134).

Quigley, M., Gerkey, B., Conley, K., Faust, J., Foote, T., Leibs, J., Berger, E., Wheeler, R., and Ng, A. (2009). "ROS : an open-source Robot Operating System". In: IEEE International Conference on Robotics and Automation (ICRA 2009) (cit. on p. 131).

Willow Garage, S. A. I. L. (2007). Robot Operating System (ROS). http: / /wWw . ros . org/wiki/ (cit. on p.131). 


\section{D}

\section{Multirotor dynamics modeling}

\section{D.1 Introduction}

The purpose of this appendix is to present the multirotor models that have been used during the course of this PhD Thesis. Sec. D.2, describes the rigid body dynamics of the multirotor. This model is useful to understand the attitude controller which in the multirotors utilized in this thesis has always been executed inside the autopilot, which motivates the next section. For medium-level controllers the rigid body model, Sec. D.3, is augmented to simulate a multirotor with an autopilot board that accepts the usual multirotor attitude and thrust or altitude velocity command set. For the design of the Extended Kalman Filter (EKF) and the controllers designed and implemented as part of this $\mathrm{PhD}$ Thesis simplified models were considered, so that only the main characteristics of the multirotor dynamics system are represented. These simplified models are presented in the rest of this chapter. Sec. D.4 introduces a simplification to 2 degrees of freedom of the multirotor model, where the simulated vehicle flies in a horizontal plane and with a constant heading. A complete simplified model for the multirotor vehicle is proposed in Sec. D.5. which is inspired in the previously discussed models from Secs. D.3 \& D.4 The last section, Sec. D.6. summarizes the uses of these models during the progress of this PhD Thesis.

\section{D.2 Model based on rigid body dynamics}

The Fig. D.1 shows the free body diagram of a quadrotor. The principal magnitudes that affect the dynamic movement of this aircraft are: the mass properties of the vehicle body, and the forces and torques generated by the propellers. The following symbols describe them and are used in later equations and discussion:

- the position of the quadrotor is denoted by the $\{x, y, z\}$ coordinates,

- the attitude of the vehicle is represented by the yaw, pitch and roll Euler angles. Which are denoted: $\{\phi$, roll $\},\{\theta$, pitch $\}$ and $\{\psi$, yaw $\}$, 


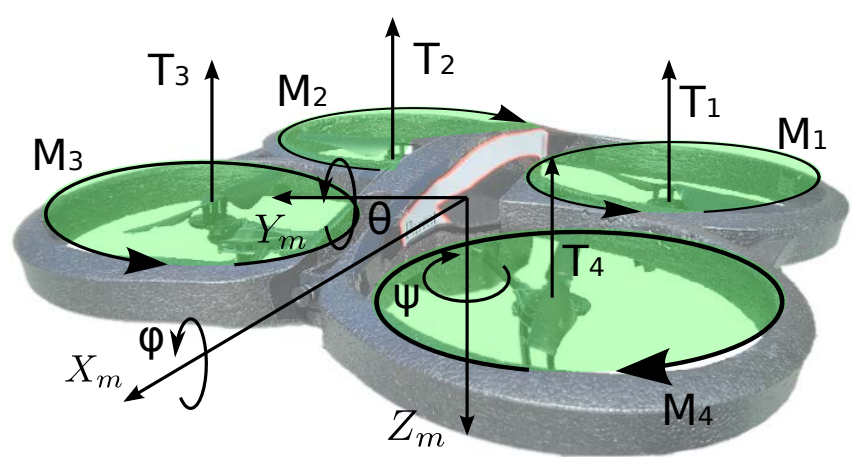

Figure D.1: Free body diagram of a quadrotor. The four propellers, and each of their performed thrust $T_{i}$ and torque $M_{i}$ are labeled 1-4. The euler angles are denoted $\{\phi, \operatorname{roll}\},\{\theta, \operatorname{pitch}\}$ and $\{\psi$, yaw $\}$

- the quadrotor rigid body is characterized by its mass $m$ and its three principal mass moments of inertia $\left\{I_{x}, I_{y}, I_{z}\right\}$,

- each propeller $i$ generates a thrust $T_{i}$ and a heading torque $M_{i}$,

- the $l$ constant is the distance between the centers of each pair of opposite propellers, such us propellers 1 and 3 in Fig. D.1.

- each propeller rotates at speed $\omega_{i}$; and its thrust causes a torque with respect to the center of mass of the quadrotor that can tip the aircraft body frontward, backward or sideways.

The quadrotor rigid body dynamics model, see Eq. D.1 and Fig. D.1] is inferred by considering the effects of the forces described above and is explained in multiple articles (Hoffmann, Gabriel M et al., 2008; Michael et al., 2010).

$$
\left\{\begin{array}{l}
I_{x} \ddot{\phi}=\dot{\psi} \dot{\theta}\left(I_{y}-I_{z}\right)+(l / \sqrt{2})\left(+T_{1}-T_{2}-T_{3}+T_{4}\right) \\
I_{y} \ddot{\theta}=\dot{\phi} \dot{\psi}\left(I_{z}-I_{x}\right)+(l / \sqrt{2})\left(-T_{1}-T_{2}+T_{3}+T_{4}\right) \\
I_{z} \ddot{\psi}=\dot{\theta} \dot{\phi}\left(I_{x}-I_{z}\right)+\left(-M_{1}+M_{2}-M_{3}+M_{4}\right) \\
m \ddot{x}=(-s \phi s \psi-c \phi s \theta c \psi) \sum_{i=1}^{4} T_{i} \\
m \ddot{y}=(-c \phi s \theta s \psi+s \phi c \psi) \sum_{i=1}^{4} T_{i} \\
m \ddot{z}=m g-c \theta c \phi \sum_{i=1}^{4} T_{i}
\end{array}\right.
$$

It is generally accepted to approximate $T_{i}$ and $M_{i}$ as $T_{i}=k_{T} \cdot \omega^{2}$ and $M_{i}=k_{M} \cdot \omega^{2}$, where $\omega, k_{M}$ and $k_{T}$ are the propeller speed and two propeller characteristic constants. The differences between these theoric equations and the reality are dealt with by the attitude controller implemented in the autopilot. The attitude controllers can be PIDs, and still provide good results (Hoffmann, Gabriel M et al., 2008; Michael et al., 2010). However, this is suboptimal because the attitude of the quadrotor determines its linear acceleration. Thus the derivatives of the acceleration are determined by the rotation velocity of the quadrotor body. Nonlinear controllers based on this observation outperform its PID counterparts (Fritsch et al., 2012), but require the usage of more complex state estimators that estimate the correspondingly augmented state of the aircraft, which needs to estimate the derivatives of the acceleration.

Using the above mentioned relationships $T_{i}=k_{T} \cdot \omega^{2}$ and $M_{i}=k_{M} \cdot \omega^{2}$, the input to this model is the speed of each propeller, and the output would be the position and attitude of the quadrotor. These inputs correspond to the autopilot outputs, because the vehicle has interface boards, called Electronic Speed Controllers (ESC), that receive the autopilot commands and approximately control the speed of the propeller rotors.

\section{D.2.1 Brief explanation of the Low-level and Mid-level control of a quadrotor}

The terms of the Eq. D.1 are explained here, and then their relations to the low-level and mid-level control laws are clarified. This point of view of the problem helps to better understand the quadrotor rigid body model, and how a cascade of PID controllers can be used to command the aircraft. The terms in Eq. D.1 can be understood as follows: 
- $\sum_{i=1}^{4} T_{i}$ is the total thrust developed by the four propellers, and is perpendicular to the vehicle body. Thus, if the attitude of the vehicle can be controlled, the direction of the total thrust can be used to control the position of the vehicle. The three projections with respect to the world reference of the total thrust in world coordinates are:

- $c \theta c \phi \sum_{i=1}^{4} T_{i}$ which compensates the vehicle weight and is used to move the vehicle up and down,

- $(s \phi s \psi+c \phi s \theta c \psi) \sum_{i=1}^{4} T_{i}$ and $(c \phi s \theta s \psi-s \phi c \psi) \sum_{i=1}^{4} T_{i}$, are the horizontal proyections and are used to move the vehicle in the horizontal plane.

- $\left(-M_{1}+M_{2}-M_{3}+M_{4}\right)$ is the total heading torque which is used to control the vehicle's yaw,

- $(l / \sqrt{2})\left(+T_{1}-T_{2}-T_{3}+T_{4}\right)$ and $(l / \sqrt{2})\left(-T_{1}-T_{2}+T_{3}+T_{4}\right)$ are the torques used to control the roll and pitch angles respectively,

- $\dot{\psi} \dot{\theta}\left(I_{y}-I_{z}\right), \dot{\phi} \dot{\psi}\left(I_{z}-I_{x}\right), \dot{\theta} \dot{\phi}\left(I_{x}-I_{z}\right)$ are inertia torque disturbances related to an uneven distribution of the vehicle mass. This disturbances tend to be low because the mass is concentrated in the main body of the vehicle.

As explained, linear combinations of the propeller mechanical efforts can be used to control the altitude and attitude of the vehicle. The understanding of the terms in Eq.D.1 lead to the following decoupling law for the speed commands of the quadrotor, as described in Eq. D.2 In this equation $\omega_{h}$ is the rotor speed that applied to all propellers, compensates the weight of the vehicle. Then $\Delta \omega_{F}$ is the new rotor speed command for the altitude coordinate. And $\Delta \omega_{\phi}, \Delta \omega_{\theta}$ and $\Delta \omega_{\psi}$ are the new rotor speed commands for roll, pitch and yaw respectively.

$$
\left[\begin{array}{c}
\omega_{h}+\Delta \omega_{F} \\
\Delta \omega_{\phi} \\
\Delta \omega_{\theta} \\
\Delta \omega_{\psi}
\end{array}\right]=\left[\begin{array}{cccc}
1 & 1 & 1 & 1 \\
1 & -1 & -1 & 1 \\
-1 & -1 & 1 & 1 \\
-1 & 1 & -1 & 1
\end{array}\right]\left[\begin{array}{c}
\omega_{1}^{\text {des }} \\
\omega_{2}^{\text {des }} \\
\omega_{3}^{\text {des }} \\
\omega_{4}^{\text {des }}
\end{array}\right]
$$

These subdivision of the problem leads to two control laws:

- Low-level attitude control: the new speed commands $\Delta \omega_{\phi}, \Delta \omega_{\theta}$ and $\Delta \omega_{\psi}$ are decoupled commands that control the roll, pitch, and yaw respectively. Decoupled means that one command affects theoretically only one of the outputs. Then roll, pitch and yaw are regulated using SISO feedback controllers, for example PIDs.

- Mid-level speed or position control: the usual mid-level control approach involves using (Hoffmann, Gabriel $\mathrm{M}$ et al., 2008; Michael et al., 2010) the average thrust $\sum_{i=1}^{4} T_{i}$ as control variable for the altitude coordinate. The low-level attitude control is commanded by roll and pitch reference angles. The vehicle and its overall thrust are tilted to obtain two linear acceleration commands in the horizontal plane. Which leads to a speed or position controller in the position coordinates $\{x, y, z\}$. If the controller laws take into account the yaw angle, then the heading of the quadrotor can be commanded independently from the position control loop.

The result is that the four degrees of freedom that the vehicle had initially $\left\{\omega_{1}^{\text {des }}, \omega_{2}^{\text {des }}, \omega_{3}^{\text {des }}, \omega_{4}^{\text {des }}\right\}$ are now $\{x, y, z, \psi\}$, which continues to be a four DOF system; but much better fitted for higherlevel control and task design.

\section{D.2.2 Shortcomings of the model based on rigid body dynamics}

Some of the reasons listed below are also accounted for in the articles (Gillula et al., 2010; Michael et al., 2010):

- The propeller dynamics: the propellers take some time to respond. In other words, the rotor speed does not change instanteously when its reference is set by the autopilot. The ESC boards take some time to attain the commanded rotor speed. This presents an actuation limit to the attitude control problem, and is important for low-level controller design. 
- Aerodynamic disturbances: aside from wind disturbances, there are aerodynamic disturbances that do not manifest in normal navigation, and appear for example during a stall turn maneuver (Hoffmann, G.M. et al., 2009).

- The communication delay between the ESCs and the controller which might be implemented on the autopilot, an onboard computer or a groundstation,

- The damping coefficients in the horizontal plane, that model the aerodynamic friction, serve to partly explain the response time of the linear speed dynamics of the quadrotor and to have a good steady-state model fixing the correspondence between roll and pitch values to steady-state horizontal speed values. It is also important to model the maximum speed of the vehicle, because it is a limiting factor for the velocity controller design. For example, based on our experiments, when using the indoors hull the Parrot AR Drone 1 has a maximum speed of about $2.5 \mathrm{~m} / \mathrm{s}$, even tilting the vehicle further will not achieve faster speeds.

- Regarding the aerodynamic friction, recent studies (Abeywardena et al., 2013, Leishman et al., 2014) have identified the horizontal aerodynamic friction of the propellers as a mayor force contributing to the dynamics of the quadrotor that affects the readings of the accelerometers in such vehicles. These frictions are explained as blade flapping, profile and induced drag forces on the propellers.

Some reasons for not using the rigid body model are:

- A multirotor with a closed autopilot software, such as the AR Drone does not accept rotor speed commands, but roll, pitch and yaw speed commands; for the altitude the are two possibilites either an altitude speed or a thrust command.

- Most closed autopilots that are intended for private or public developers give at least attitude measurements. But in order to use the acceleration measurements from the IMU the bias affecting them needs to be identified in real-time, for example using a Kalman Filter formulation.

- For the linear speed or position controller design of a quadrotor with an embedded attitude controller, there are other parameters that need to be identified. All saturations that affect the model and the response time of the variables that are to be controlled have to be. The following are list of such parameters that are considered in the identification process: the maximum speeds that the vehicle can reach, the response time of the attitude angles (as controlled by the autopilot), the response time of the horizontal speed after step commands in pitch and roll; the response time of the altitude speed to a step command; and similarly for the yaw speed. A methodology to obtain these data will also be explained in later sections; it basically requires to make judicious experimental tests.

To solve the first problem, in the next section, Sec. D.3 an attitude controller is added to the model. For the last issue some simplified models have been developed in Sec. D.4\&D.5.

\section{D.3 Model based on rigid body dynamics with autopilot}

The difference between this model and the rigid body model is on the inputs and outputs. The inputs are not rotor speed comands, but roll, pitch and yaw speed commands; and for the altitude there are two possibilities either an altitude speed or a thrust command. These corresponds to the usual inputs of a multirotor autopilot. Similarly, the outputs are selected to be the same as the autopilot available measurements. This would result in a model with the block diagram shown in Fig. D.2.

The idea is to imitate the response of a multirotor that has already a low-level controller implemented in the autopilot board. A way of obtaining such a model is the following, see Fig. D.2

- Identify the rigid body model, as explained in Sec.D.2

- Perform experimental tests to identify the saturation limits of the system: $\phi_{\max }, \theta_{\max }, \dot{\psi}_{\max }, \dot{z}_{\max }$ and $v_{x y \max } . v_{x y \max }$ is the maximum horizontal speed of the multirotor. It is also worthwhile to determine the maximum speed that the speed measurement method can safely estimate. 


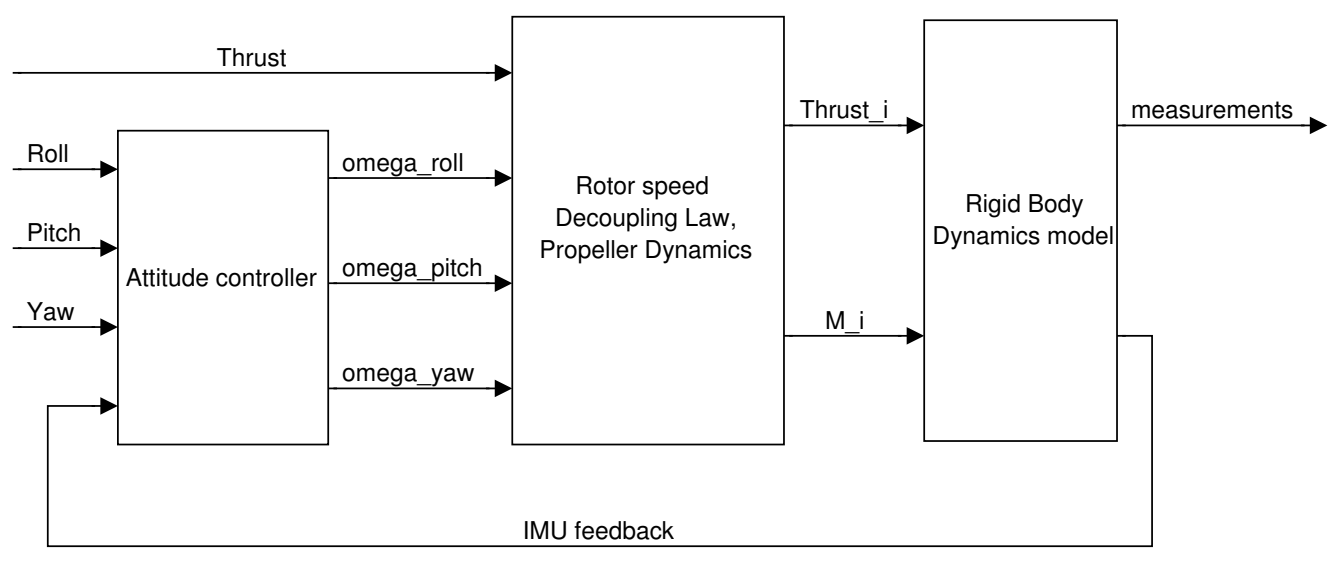

Figure D.2: Rigid body model with autopilot. The altitude control is added if it is present on the autopilot. The most important thing to do, is to have a model that has the same inputs and outputs as the actual multirotor. And that both have the same saturation limits and response times, among other characteristics, as the real system.

- Perform experimental tests to identify the critical response times of the multirotor:

- Response times on $\phi, \theta, \dot{\psi}, \dot{z}$.

- Estimate the response time of the horizontal speed. This can be done measuring the speed after a roll or a pitch step command.

- Implement the "Rotor speed Decoupling Law, and Propeller Dynamics" box: the thrust command must be converted to $\Delta \omega_{F}$ using the relation Thrust $=k_{T}\left(\omega_{h}+\Delta \omega_{F}\right)^{2}$. Then the decoupling law specified in Eq. D.2 must be implemented to pass from $\Delta \omega_{i}$ commands to $\omega_{i}$ references for the ESC boards. Then the propellers can be modeled as a first order system with an appropiate response time. The output of these blocks would be the simulated rotor speeds $\omega_{i}$. For example in (Michael et al., 2010) a first order system with response time of $50 \mathrm{~ms}$ was used for the propeller dynamics, it is probable that the response time is bigger for quadrotors of bigger size such as the Asctec Pelican, Sec. H.1. And finally the rotor speeds are converted to thrusts and heading torques using the relations $T_{i}=k_{T} \cdot \omega^{2}$ and $M_{i}=k_{M} \cdot \omega^{2}$.

- The attitude controller and, if necessary, the altitude controller have to be implemented, for instance PID controllers can be used. The most important thing is that the simulated system must present response times similar to the experimental data from the identification tests and to the multirotor itself.

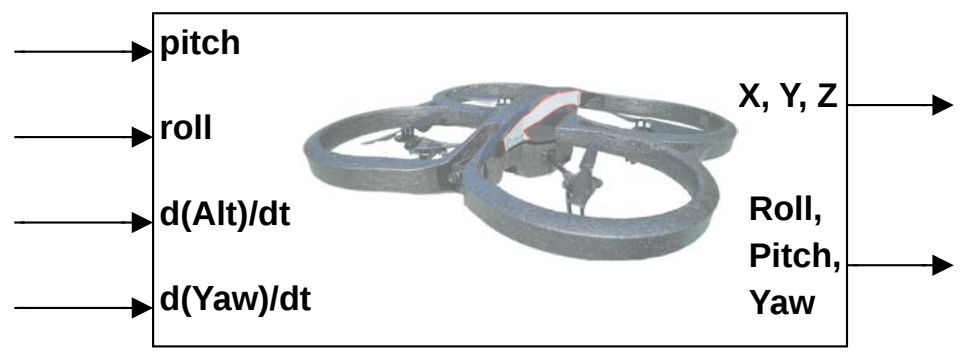

Figure D.3: AR Drone black-box model. The specified inputs match the real system's. The Euler angles and the altitude outputs are available from the AR Drone telemetry data. To the contrary, the only available data from the drone about the $X$ and $Y$ directions are speed estimates. These estimates are obtained by means of a down-facing camera and optical flow algorithms. 
A good example of a multirotor that can be represented by the rigid body model with autopilot is the Parrot AR Drone. The input commands of the drone are roll, pitch, yaw speed and altitude speed commands, as illustrated in Fig. D.3. The software of the autopilot is totally closed, and the measurements are communicated to the host computer via WiFi every 60-70 ms. The available measurements from the drone are attitude and altitude measurements, and about the $X$ and $Y$ coordinates only speed measurements are available; the parrot does not have a GPS receiver. The horizontal speed measurements are obtained from the down-facing camera image by means of visual odometry algorithms (Bristeau et al., 2011).

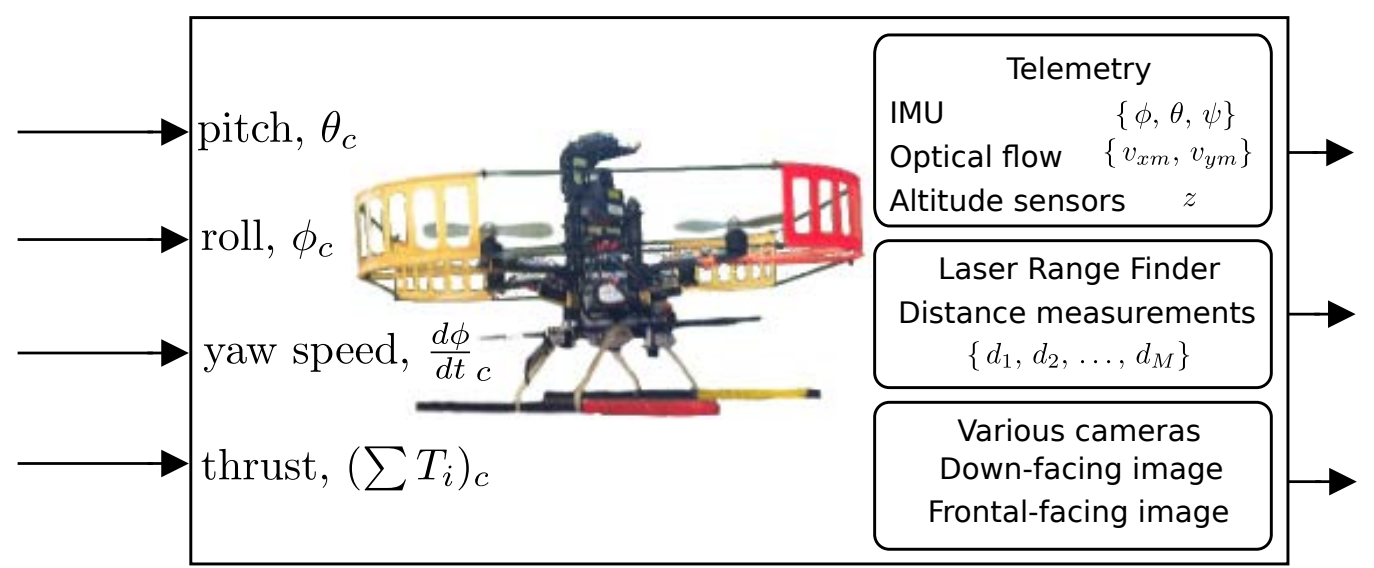

Figure D.4: Pelican black-box model as equipped by the CVG_UPM team for the IMAV2012. The specified inputs match the real system's. The Euler angles are available from the Pelican telemetry data. Two sonar altitude sensors were added using a USB mini acquisition board, "PhidgetInterfaceKit 2/2/2", to obtain an altitude measurement. The available data from the quadrotor about the $X$ and $Y$ directions are speed measurements obtained by means of an optical flow algorithm applied to down-facing images; and the measurement from a Hokuyo Scanning Laser Range Finder that was added to perform localization.

Another good example of a multirotor that can be represented by the rigid body model with autopilot is the Asctec Pelican. The vehicle was used as illustrated in Fig. D.4 to participate on the IMAV2012 international competition. The autopilot was configured to accept attitude and thrust commands. The Asctec Pelican has a closed embedded autopilot that performs the attitude control, and that provides measurements regarding the low-level state of the vehicle. Two sonar sensors where added to obtain altitude measurements. A down-facing camera is used to have an image of the ground, the optical flow processing on its images combined with the altitude measurements results on a speed estimate. Finally a Hokuyo Scanning Laser Range Finder was added to perform localization.

\section{D.4 Simplified model with altitude and yaw hold}

This model was developed to participate in the international competition "Concurso de Ingeniería de Control 2012, Control Autónomo del seguimiento de trayectorias de un vehículo cuatrirrotor”, which final phase was held in Vigo, Galicia; in September 2012. This competition will be called CEA 2012 competition for the rest of this appendix. The model is easier to identify and control, because it has only 2 inputs and 2 outputs. The altitude and yaw heading are not modeled. Only the roll and pitch commands are available; and the outputs of the model are only related to the $X$ and $Y$ coordinates.

To simplify the identification and control problems, the organizers of the CEA 2012 competition had decided to constrain the quadrotor movement to the horizontal plane and also to fix the yaw heading. Thus, leaving roll and pitch as the only command variables. An inspection of the underlying dynamic model, from Eq. D.1, reveals that the pitch angle controls the speed in the $X$ direction, and the roll angle controls the speed in the $Y$ direction.

Taking into account these contraints the simplified model depicted in Fig. D.5 is proposed: 


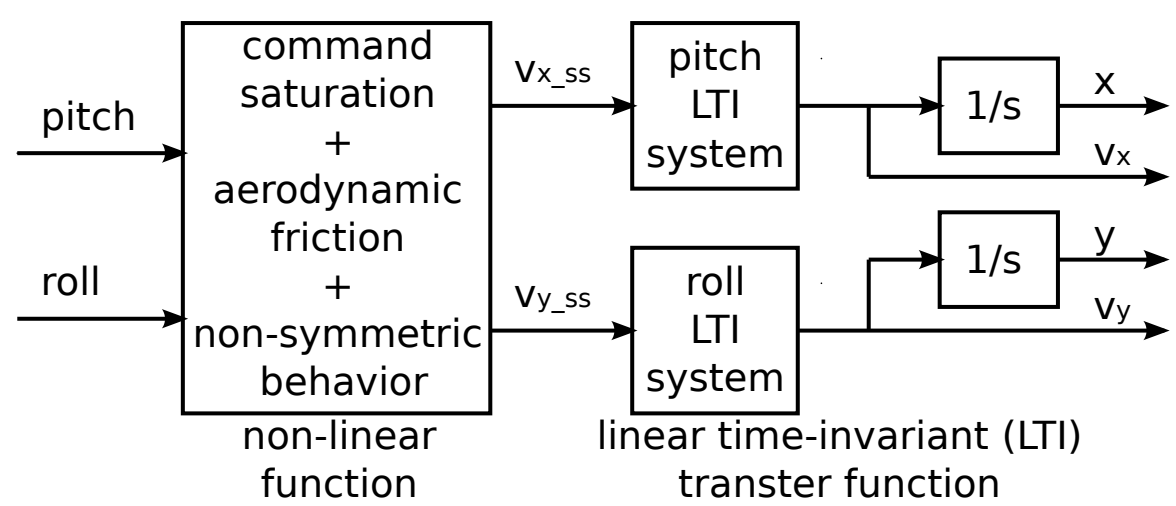

Figure D.5: Quadrotor simplified model, only the pitch and roll inputs are kept, along with the main resulting quadrotor behavior.

- A non-linear function models and fixes the steady-state correspondance between $\{\phi, \theta\}$ and $\left\{v_{x}, v_{y}\right\}$. The quadrotor characteristics modeled by this non-linear function are:

- aerodynamic friction: it can be expressed as a correspondance between constant input values and steady-state speed values $\left\{v_{x_{-} s s}, v_{y_{-} s s}\right\}$. This relationship can be non-symmetric, $\left[v_{x_{-} s s}, v_{y_{-} s s}\right]=\mathbf{f}(\theta, \phi)$, i. e. presenting different maximum speed values for each direction.

- maximum tilt angle: expressed by a $2 \mathrm{D}$ command saturation $\left(\phi / \phi_{\max }\right)^{2}+\left(\theta / \theta_{\max }\right)^{2} \leq 1$

- The dynamics of the quadrotor are modeled by a linear time invariant (LTI) system, i. e. a transfer function. The LTI systems model: the roll and pitch closed loop response, and the resultant damped speed response. Instead of including all the high frequency poles and the whole physical behavior of the drone, the actual transfer function can be simplified and just include the slowest poles and dynamics.

\section{D.5 Complete multirotor simplified model}

The model presented in this subsection retains the simplicity of the previous model in the sense that the identification experimental tests are easy to perform. The quadrotor movement in the horizontal plane is strongly determined by the aerodynamic friction and the attitude offset of the IMU. It is really important that the friction stays opposite to the actual speed of the quadrotor in the world frame, being independent of the yaw orientation of the vehicle.

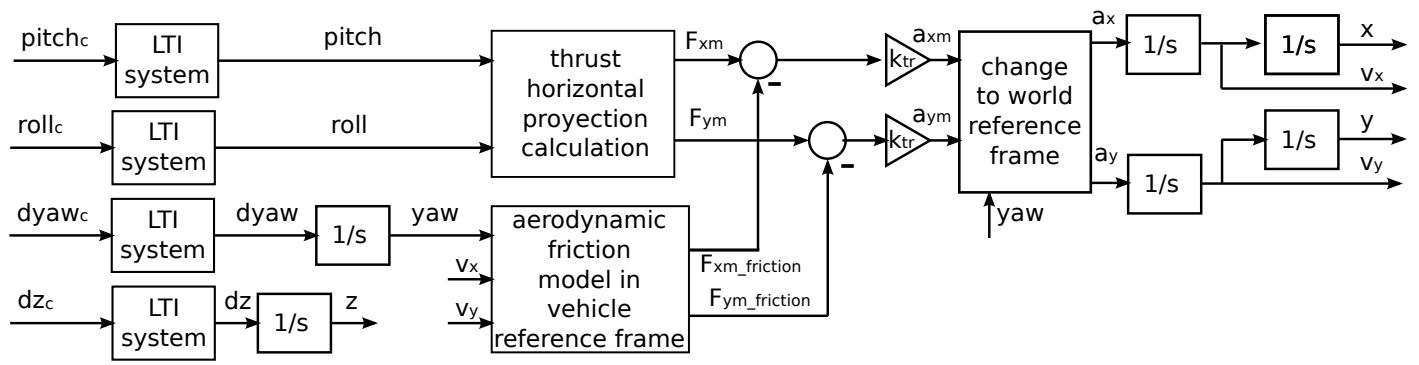

Figure D.6: Complete multirotor simplified model, all inputs are taken into account. The altitude and yaw loops are supposed to have a speed controller, so that the model roughly identifies the closed loop behavior.

Taking into account these constraints the simplified model depicted in Fig. D.6 is proposed: 
- A non-linear function models the aerodynamic friction, which can be specified in the vehicle reference frame. In order to obtain a friction force opposite to the speed in the world frame the model includes a change of the reference coordinates from the world to the vehicle frame:

- The roll and pitch angles $\{\phi, \theta\}$ produce a force specified in the vehicle frame: $\left\{F_{x}=\right.$ $\left.-m g \sin (\theta), F_{y}=m g \sin (\phi)\right\}$. This force is a gross magnitude, from which the friction must be substracted.

- Aerodynamic friction: the friction is specified by a non-linear function that depends on the orientation of the horizontal speed, $\alpha$, in the vehicle frame and on the modulus of the speed. Experimental data is being fitted to the following model: $F_{\text {friction }}=c_{1}(\alpha)\left(\left|v_{m}\right|+c_{2}(\alpha)\right) v_{m}$. The vehicle speed is changed from the world frame to the body frame, using the yaw angle. And then the aerodynamic friction equation is used. The result is the friction force in the body frame coordinates, that can now be subsctracted from the gross horizontal thrust force.

- The aerodynamic friction is substracted to the horizontal component of the quadrotor's thrust, obtaining a resulting pseudo-acceleration. The acceleration that will be applied to calculate the speed is proportional to the one calculated before.

- Horizontal speed response time: the pseudo-acceleration is multiplied by a positive gain, to tune the response time to experimental data. Then an artificial gain $k_{t r}$ is added to the model, so that the response time of the model can be tuned. The relation between this constant and the approximate obtained response time is: $t_{r}=\frac{3 k_{t r}}{c_{1}\left(2\left|v_{m}\right|+c_{2}\right)}$. The obtained response is similar to a first-order model response.

- The other dynamics of the quadrotor are modeled by several linear time invariant (LTI) systems, i. e. transfer functions.

- The fastest multirotor dynamics that are modeled explicitly by the model are the pitch and roll angles. In the AR Drone the response time of these variables is about 150-300 ms; and the response time of the yaw speed command and altitude speed is about $500 \mathrm{~ms}$. In the rigid body model, Secs. D.2 \& D.3 the fastest response time is the propeller response, which will respond in about $50 \mathrm{~ms}$. This fact, makes that the system can be simulated at slower sampling times, and also that there are less state variables; which may be useful for certain purposes.

An advantage of the model is that several saturation limits of the system can be directly specified:

- maximum roll, pitch, altitude speed and yaw speed can be fixed putting saturation limits at the input of the model.

- the aerodynamic friction together with the roll and pitch limits fix the maximum horizontal speed of the multirotor model.

- disturbances can be added to the model, for example, pitch and roll disturbances. This would make the quadrotor not hover in position with zero pitch and roll commands.

Another convenience of this model is that the response times of the principal variables can be fixed easily in the model:

- the roll, pitch, altitude speed and yaw speed response times are fixed by their respective (LTI) systems.

- the response time of the horizontal speed is fixed by $k_{t r}$.

Some other interesting facts about the model are:

- The multirotor model can perform a horizontal circle with similar inputs as the real multirotor. No tests have been done checking actual values of both inputs. The meaning of this is that with a constant command of roll, pitch and yaw speed, a multirotor will perform horizontal circle trajectories of varying radius. 
- The aerodynamic friction is always parallel to the current speed of the multirotor. For instance, if the vehicle has a certain velocity direction and then the pitch and roll are set to zero, and then a yaw turn is performed; the model will continue moving in the same direction as before, but slowing down due to aerodynamic friction. This was one of the characteristics that were sought in the design of this multirotor model.

- the model is simple and has parameters to adjust many important aspects of the multirotor dynamics.

It is important to keep in mind that this is an approximate model, and that there are characteristics of the multirotor behavior that are not be explained by the model.

\section{D.5.1 Identification method for this model}

A list of steps to perform identification on the "Complete simplified model", Sec D.5 will be enumerated. This identification process does not require to have expensive sensors such us a Vicon motion capture system. The tests require to have a sports playground such as a basketball, or a volleyball field with some space around it. It is important that the test software has some kind of safe stop switch. For example, the AR Drone quadrotor can enter hover mode, sending just one command to it. And the Asctec Pelican can be completely passed to manual mode just with one switch, passing the control to the RC pilot. The test to be performed on the system are step commands of different values on the various inputs.

The identification process requires to perform the tests explained later in this section. It is highly advised to perform first the tests that the experimenter estimate the least dangerous. It is also important to make the tests in a closed room with no other people than the two experimenters; one that will be in charge of the user interface of the test software and the other that will be the pilot and that must take control of the vehicle if necessary. The steps explained here are specific to multirotors with inputs similar to the AR Drone's, see Fig. D.3. The reader should compare the following steps with Fig. D.6 The steps of the identification process are:

1. Test the safe stop switch command, and make several training flights with the pilot and the experimenter. Multirotors are easy to fly so that an expert pilot is not required for these tests as long as there is good communication between both persons of the team. When the test gets dangerous for the vehicle it is important that both team members now how to react and are able to do it fast enough.

2. Perform separate step commands for each input: roll, pitch, yaw speed and altitude speed. With a step time of two seconds the system should be able to reach the steady state response. These tests can be used to identify the LTI blocks at the input of the model.

3. Obtain the saturation limits of the inputs. These corresponds to performing tests to obtain the maximum roll, pitch, and yaw speed that the multirotor can reach. Note that the yaw speed tests are dangerous because they can make the pilot lose the reference of the front part of the vehicle. It is also necessary to obtain the maximum altitude speed (going upwards only), this test can be really dangerous for the vehicle. Actually it is enough to try to reach a maximum desired speed. For example, the team may decide that they need to climb at most at $1 \mathrm{~m} / \mathrm{s}$.

4. The rest of the flights are pitch and roll step tests, to identify the horizontal speed behavior of the multirotor:

- It is important to perform the tests in a place where some distances are known. As an example, suppose a basketball field is available; the length of it can be measured and it can be used to calculate average speed during the tests.

- The multirotor is placed so that when it is tilted by the pitch or roll command it will move along the basketball field. The step commands are initiated approximately from hover position in one side of the field, and they are finished when the vehicle arrives to the other side.

- If possible the vehicle speed is acquired and saved for later analysis.

- It is important to test increasing values of the pitch (roll) steps to obtain a correspondence between pitch (roll) step commands and the speed values, $v_{x}\left(v_{y}\right)$. 
- The $v_{x}$ (or $v_{y}$ ) response time is measured from the data when the speed curves are analyzed.

- Other directions, not only front and sideways, can be tested using combined pitch and roll steps. From hover position, the vehicle will move along the maximum-tilt direction.

- The aerodynamic friction law is then fitted to the data. In the authors experience, multirotors have a really similar behavior in all directions so that a fit to the simpler friction law $F_{\text {friction }}=c_{1}\left(\left|v_{m}\right|+c_{2}\right) v_{m}$ can be used. If a maximum speed is intended to be enforced by the model, a good option is to saturate the pitch and roll inputs to the corresponding speed value in the obtained friction law.

- $k_{t r}$ is selected to obtain a judicious response time with respect to the results of the experiments. The response time is different depending on the amplitude of the step command, but it does not seem to vary more than by a factor of 2 .

- It is important to carefully analyze the data, specially the calculation of the speed limits $v_{x \max }$ and $v_{y \max }$. In a later chapter, Chap. 2, a controller law will be presented. The proposed controller law uses an inner speed control loop, for $v_{x}$ and $v_{y}$. It is important that the command reference of the loop does not surpass the maximum speed that the multirotor can reach, so that the controller does not saturate. Another issue is the maximum speed that the sensors can measure, it places a limit to the speeds that can be attained by the speed loop.

These tests can be performed without expensive motion capture systems, and yield an acceptable model. The models obtained using this model were utilized to design controllers for two multirotors. The obtained controllers required to decrease the obtained gains for some PIDs by a factor of 2 to 5 , $K_{\text {real }}=0.2-0.5 \cdot K_{\text {simulation }}$. The reason is that the speed measurements were much more noisy in reality than in simulation, but the result stills demonstrates that such a model is useful for the controller design.

\section{D.5.2 Identification example: Parrot AR Drone with indoors hull}

First the LTI blocks are identified, see Fig. D.7, using the Matlab System Identification Toolbox. Note that the pitch and roll LTI blocks have a gain of about 2.35. It seems that the parrot configuration by our software of the maximum pitch and roll commands may have a bug. The measurements from the AR Drone correspond to the commands with a factor of about 2.35 , this is the reason why the LTI blocks do not present unit gain.

$$
\begin{gathered}
G(s)=\frac{K}{\left(\frac{s}{w_{n}}\right)^{2}+\left(\frac{2 \xi}{w_{n}}\right) s+1} \\
G(s)=\frac{K}{\frac{s}{w_{1}}+1}
\end{gathered}
$$

\begin{tabular}{c|c|c} 
& $\dot{\psi}_{c}, \dot{\psi}$ & $\dot{z}_{c}, \dot{z}$ \\
\hline \hline$K$ & .930 & .972 \\
$\xi$ & .754 & .440 \\
$w_{n}$ & 8.50 & 3.82
\end{tabular}

(a) yaw speed and altitude speed, Eq.D.3

\begin{tabular}{c|c|c} 
& $\phi_{c}, \phi$ & $\theta_{c}, \theta$ \\
\hline \hline$K$ & 2.52 & 2.37 \\
$w_{1}$ & 15.4 & 24.4
\end{tabular}

(b) roll and pitch, model Eq.D.4

Figure D.7: Experimentally identified LTI blocks for the Parrot AR Drone using the complete simplified model, Fig. D.6. These LTI blocks approximately model the response of the attitude and altitude variables that area already controlled with a feedback loop in the autopilot. The autopilot is closed, and identifying its closed-loop response is a first approximation to the system identification of this drone. fixed to:

Then the input saturation limits in the AR Drone can be configured using the official API, and are

- Roll and pitch input values must be, $-1 \leq \phi \leq+1$ and $-1 \leq \theta \leq+1$. The AR Drone can be configured to set what is the physical reference for the value +1 , the usual used configuration during this work has been $24^{\circ}$. 
- The yaw speed is configured to $u_{\psi}=+1$ command $=100 \%=1.745 \mathrm{rad} / \mathrm{s}$

- The altitude speed is configured to $u_{z}=+1$ command $=1.0 \mathrm{~m} / \mathrm{s}$

Then several tests where performed in order to obtain the aerodynamic friction model. It is important to note that all tests where done with the protective hull of the AR Drone. These tests were performed in a volleyball field that has a length of $18 \mathrm{~m}$. The aerodynamic friction law was fitted using the Matlab Curve Fitting Toolbox, see Figs. D.8 \& D.9.

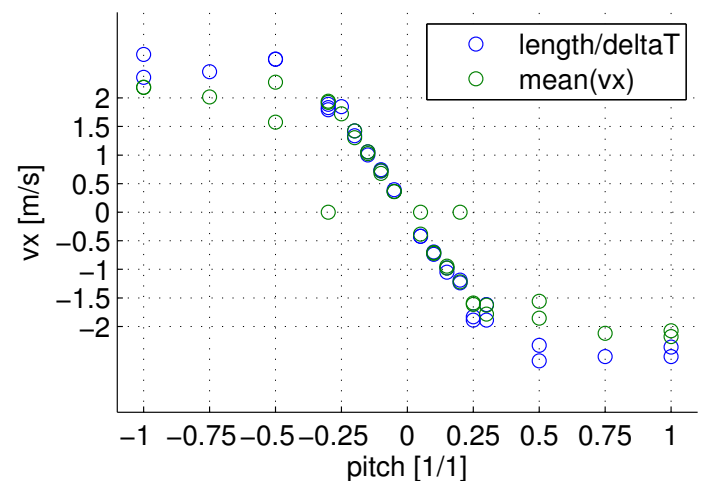

(a) Pitch vs $v_{x}$

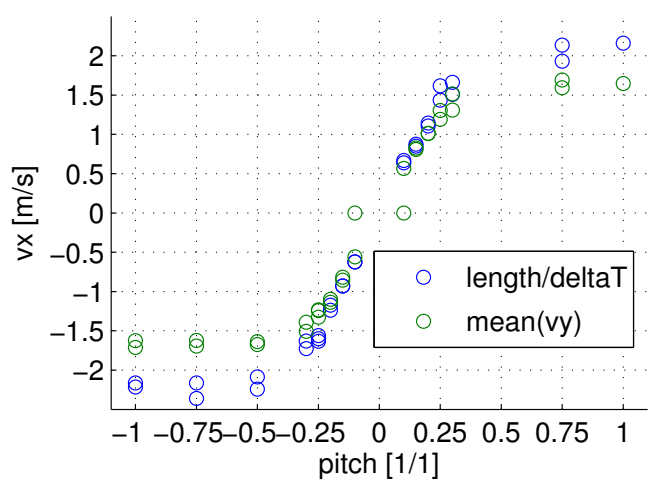

(b) Roll vs $v_{y}$

Figure D.8: The figures show the average values of speed obtained in the aerodyncamic friction tests for the Parrot AR Drone. Each graph correspondes to a different direction of movement: front movement using step commands in pitch, and lateral movement using step commands in roll. The maximum speed obtained during tests was about $2.25 \mathrm{~m} / \mathrm{s}$, and for tilt values higher that $12^{\circ}$ the speed was approximately the same and around $2 \mathrm{~m} / \mathrm{s}$.

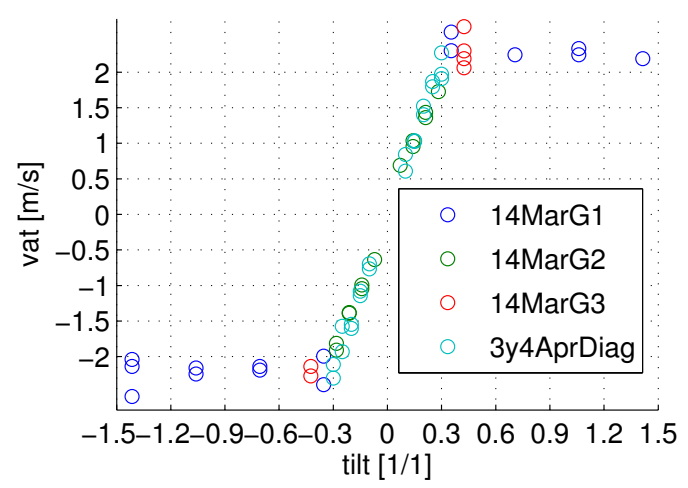

Figure D.9: The figures show the average values of speed obtained in the aerodyncamic friction tests for the Parrot AR Drone. Several datasets on the diagonal movement of the drone using step commands with equal commands, $\phi=\theta$. The maximum speed obtained during tests was about $2.25 \mathrm{~m} / \mathrm{s}$, and for tilt values higher than $12^{\circ}$ the speed was approximately the same around $2 \mathrm{~m} / \mathrm{s}$. The commands with tilt $\geq+1$ were sent with the API, but probably the drone saturates this commands to +1 .

The results for pitch step commands are shown in Fig. D.8(a) corresponding to frontal movement. The first thing that stands out is that there is a part of the data that is linear, until pitch $\approx 0.5$; but the rest of the graph shows that the steady-state speed is constant.

A possible explanation for this behavior is that for small angles the vehicle speed increases as the pitch increases. If the data is analyzed carefully the relationship is less linear as the angle increases, this is due to the increasing surface area that the vehicle is showing in the direction of movement. This behavior 
continues until at a specific angle, pitch, $\phi \approx 12^{\circ}$. A possible explanation is that at this point the flow around the vehicle changes; causing that there is a maximum speed of about $2.25 \mathrm{~m} / \mathrm{s}$.

Data curves with similar characteristics where obtained for two more test directions: lateral and $45^{\circ}$ diagonal directions. These curves are shown in Figs. D.8(b) \& D.9.

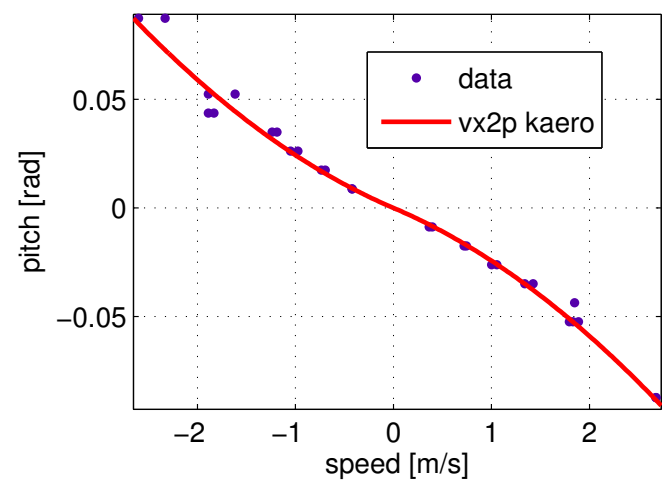

(a) front direction

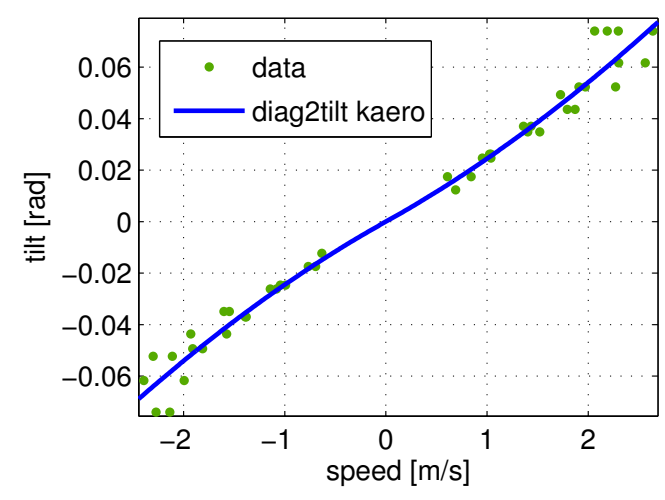

(c) diagonal direction

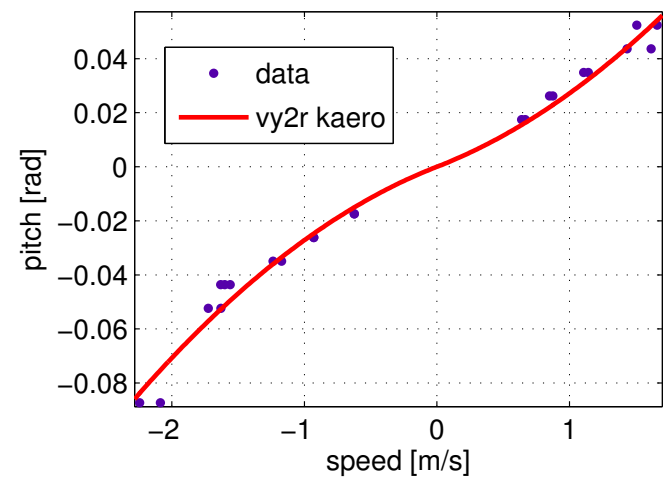

(b) laterial direction

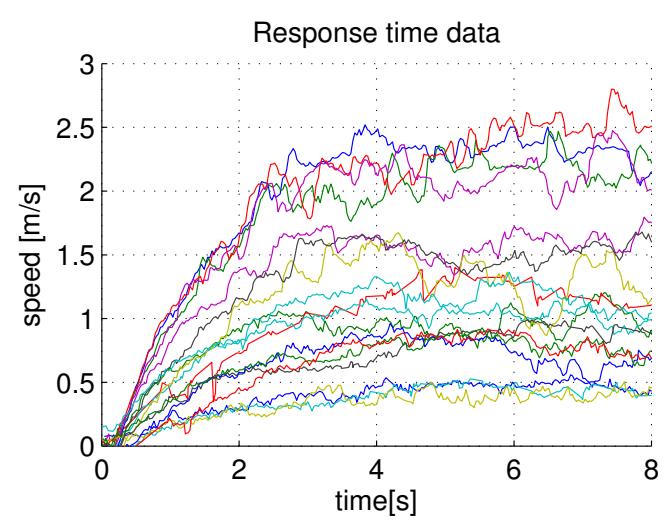

(d) speed response for different step values

Figure D.10: Experimental identification of the aerodynamic friction law. The subfigures D.10(a) \& D.10(b) \& D.10(c) show the same data points as shown in Figs. D.8 \& D.9. and also the curve that was obtained using data regression techniques with a funcion model: $F_{\text {friction }}=c_{1}\left(\left|v_{m}\right|+c_{2}\right) v_{m}$. The subfigure D.10(d) shows the acquired speed measurements over time for several of the step commands with different step values. A careful analysis of the curves in subfigure D.10(d) shows that the response time is about 3-4 s.

The subfigures D.10(a) \& D.10(b) \& D.10(c), shown in Fig. D.10, show the regression curves that where used to fit the aerodynamic friction test data to the model $F_{\text {friction }}=c_{1}\left(\left|v_{m}\right|+c_{2}\right) v_{m}$. The Matlab Curve Fitting Toolbox was used to solve the regression problem. The values that were obtained were $c_{1}=0.0496 \mathrm{rad} /(\mathrm{m} / \mathrm{s})$ and $c_{2}=4.0 \mathrm{~m} / \mathrm{s}$, where $c_{2}$ was fixed around the values obtained for three datasets (one for each test direction). In the actual code, it is important to scale $c_{1}$ by the gain of the pitch and roll LTI blocks, and $c_{1}=0.0496 \cdot 2.35 \mathrm{rad} /(\mathrm{m} / \mathrm{s})$.

The last thing that was obtained from these tests was the response time of the horizontal speed in step commands tests. The obtained speed curves are shown in Fig. D.10(d), where several tests are shown. There are tests with different step values shown in the graph, but the response times remain approximately 3-4 s, which corresponds to $k_{t r} \approx 2.0$ (adim). 


\section{D.5.3 AR Drone model, comparison with experimental data}

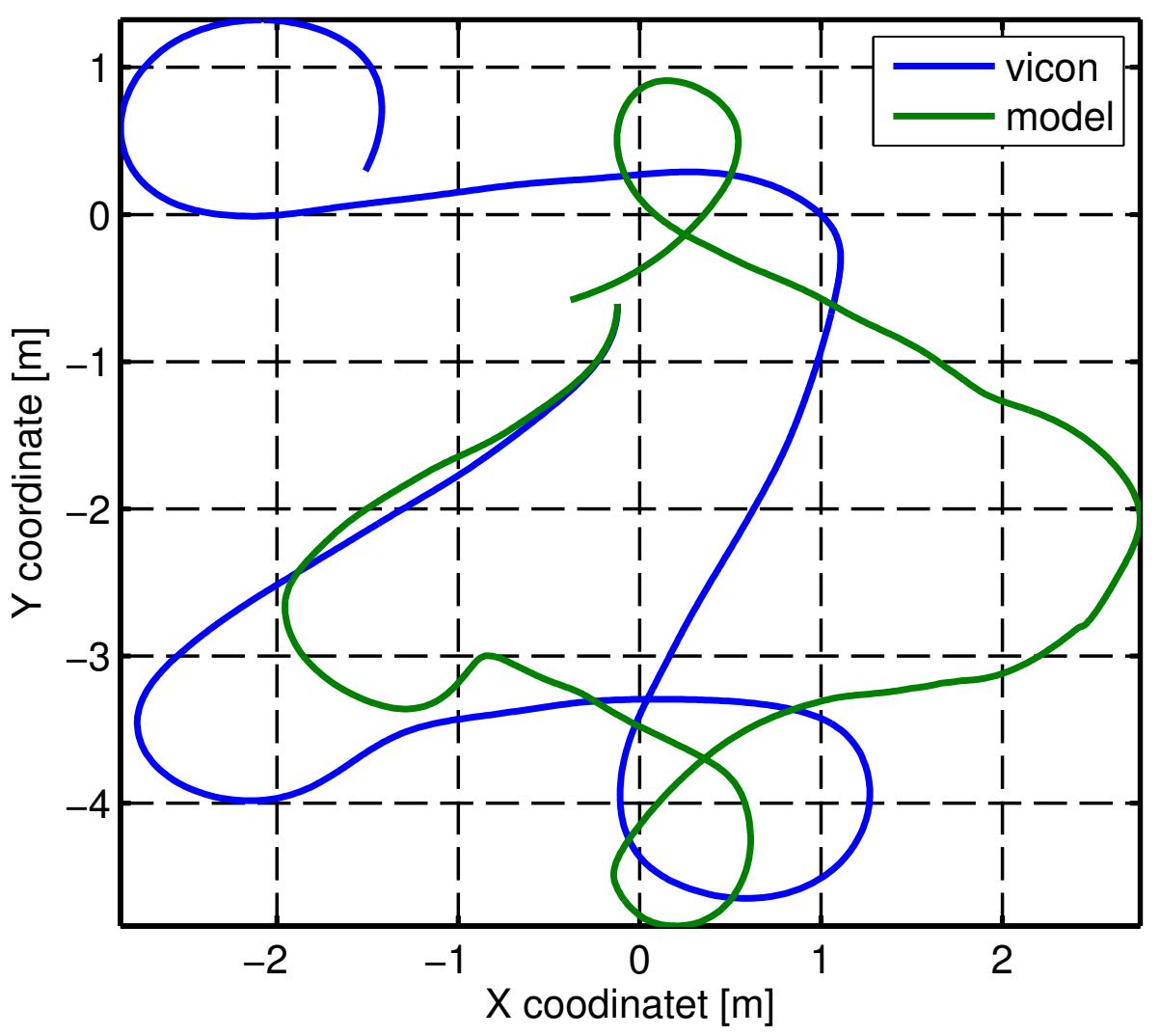

Figure D.11: Comparison of AR Drone identified model to experimental data, X-Y trajectory. The shape of the model's trajectory is similar to the ground truth, the model estimates the loops in the waypoints of the trajectory. On the other hand, a lot of error is accumulated as the time passes.

It is important to compare the identified model with reality. As part of the development of the CVG Framework for interfacing with MAVs (Mellado Bataller, I., 2012; Mellado-Bataller et al., 2013), the author of the framework performed a test showing the viability of of the framework with a trajectory controller. The AR Drone control problem was simplified using feedback information from a Vicon motion capture system, thus isolating the navigation problem from the localization/estimation problem. The video recorded during the test is available online in the website https://www.youtube.com/watch?v=MFxfaHb7jT0. The data from the test offers: experimental input, telemetry and ground truth data (from the Vicon system). The availability of ground truth data for this test was the main reason to use it as a comparison with the model implementation. The control during this test was based on four PIDs: forward speed, lateral speed, yaw and altitude, which were adjusted from a model estimation. The controller is a trajectory controller that follows a trajectory defined by waypoints. The feedback information required for navigation was obtained from a Vicon motion capture system.

The measurements are available with a common timescale and the data is divided in three sets:

1. Input data: $\{\phi$ (roll), $\theta$ (pitch), $\dot{\psi}$ (yaw speed), $-\dot{z}$ (altitude speed) $\}$

2. Telemetry data: the data that the AR Drone sends to the ground station which are the $\{\phi$ roll, $\theta$ pitch, $\psi$ yaw $\}$ coordinates, the altitude (from the sonar) and the $\left\{v_{x m}, v_{y m}\right\}$ speed estimations. The speed estimations are given in body frame coordinates ( $x$, frontal direction, and $y$, lateral direction).

3. Ground truth localization dataset (Vicon): position and attitude data. The $\{x, y, \psi$ (yaw) $\}$ coordinates with respect to the world frame are compared with the model estimation. 
In the following, several graphs are shown where the model data is either compared with the vicon data or the telemetry data. It is important to note that the model is only using the input dataset, no measurements are being used to increase the estimation quality. The take-off was eliminated from the estimation process, because the model did not perform well in that part; it estimated much higher speeds than compared to reality. It was decided not to consider that part of the data for the analysis, for three reasons:

- the take-off mode of the AR Drone ignores the roll and pitch commands until the maneuver is finished,

- it helps in the analysis task to work on data that corresponds to the conditions that the identification process was aimed for,

- the take-off situation differs from the normal flying conditions and should be analyzed separately.

In general terms, the model behaves similarly to the system: the shape of the trajectory and the general appearance of the curves in terms of rapidity/response time are comparable. However, it is clear from the horizontal $X-Y$ coordinates graph, Fig. D.11, that the position error increases gradually over time. This is expected as errors in the estimated velocities are integrated, resulting in the gradual increase of the position error.

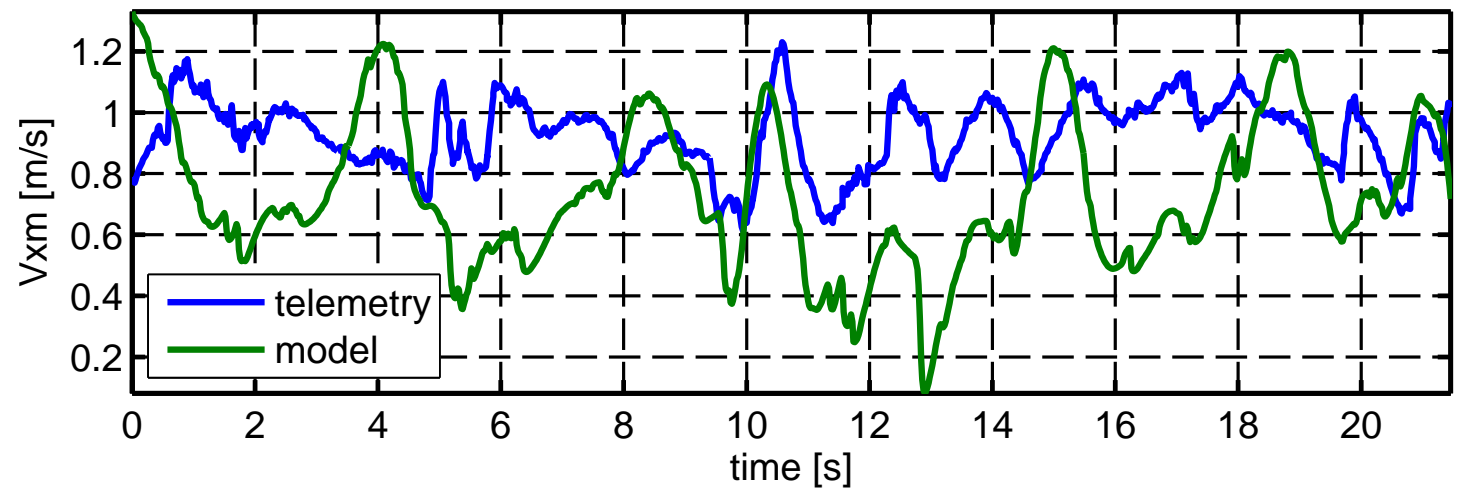

(a) Speed in body frame, frontal speed $v_{x m}$

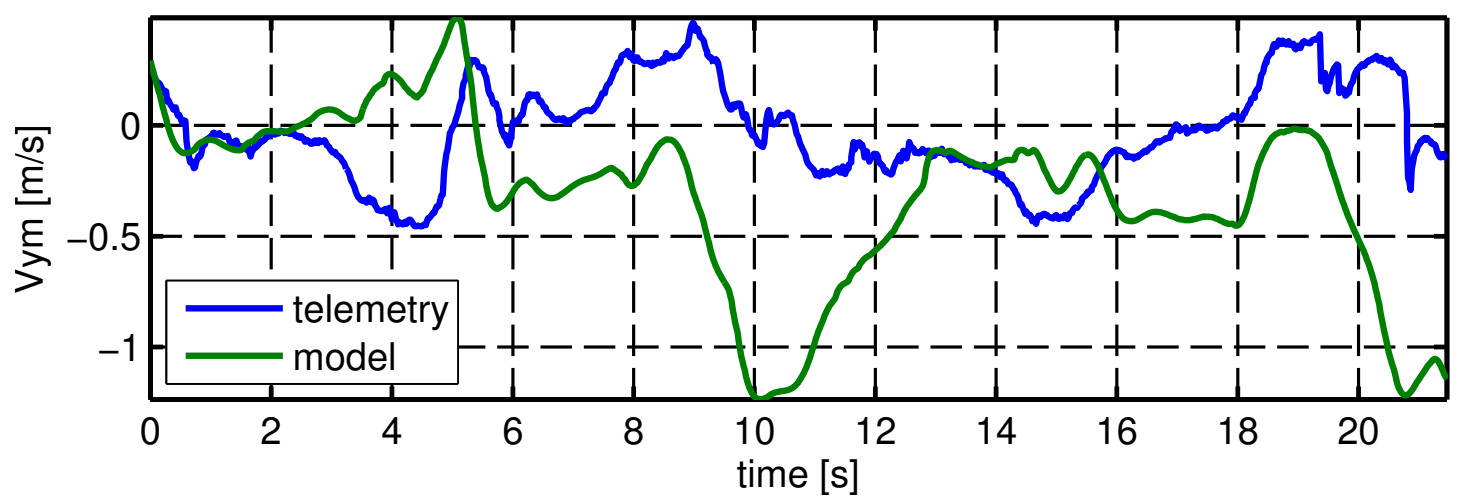

(b) Speed in body frame, laterial speed $v_{y m}$

Figure D.12: Comparison of AR Drone identified model to experimental data, speed in body frame. The frontal speed estimation, $v_{x m}$, is much more acceptable than the lateral speed, $v_{y m}$. The error in the lateral speed estimations makes the estimated trajectory shown in Fig. D.11] deviate to one side of the ground truth. Both curves, estimated and telemetry, evolve similarly, with comparable response times. The take-off part of the simulation was not taken into account for the analysis and is not shown in the graphs. 


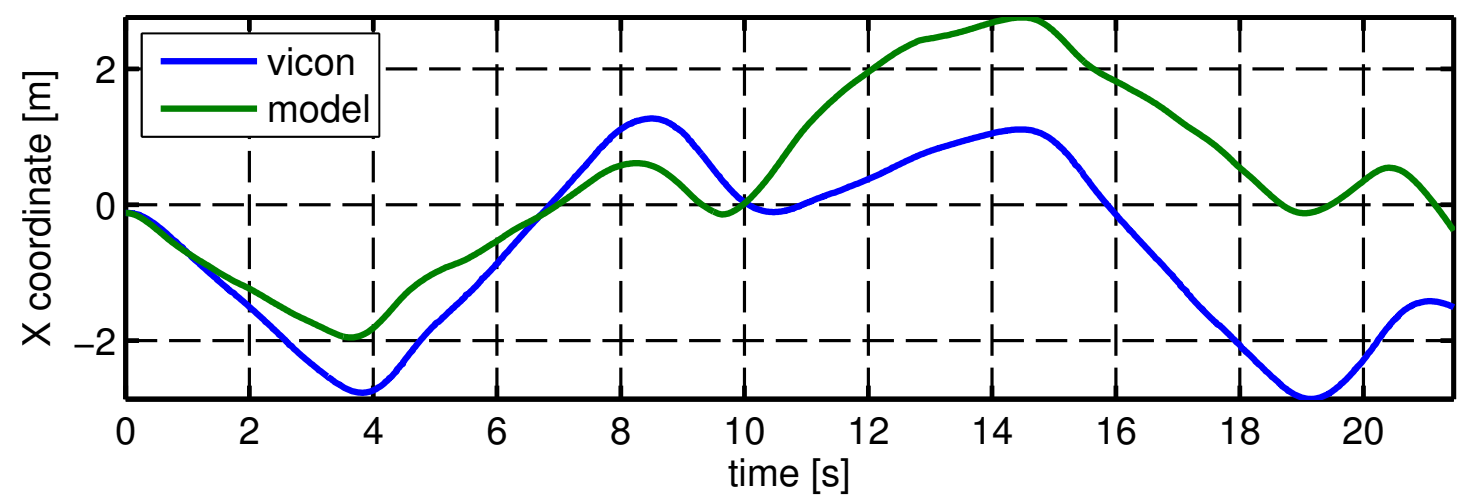

(a) X coordinate, in the world frame

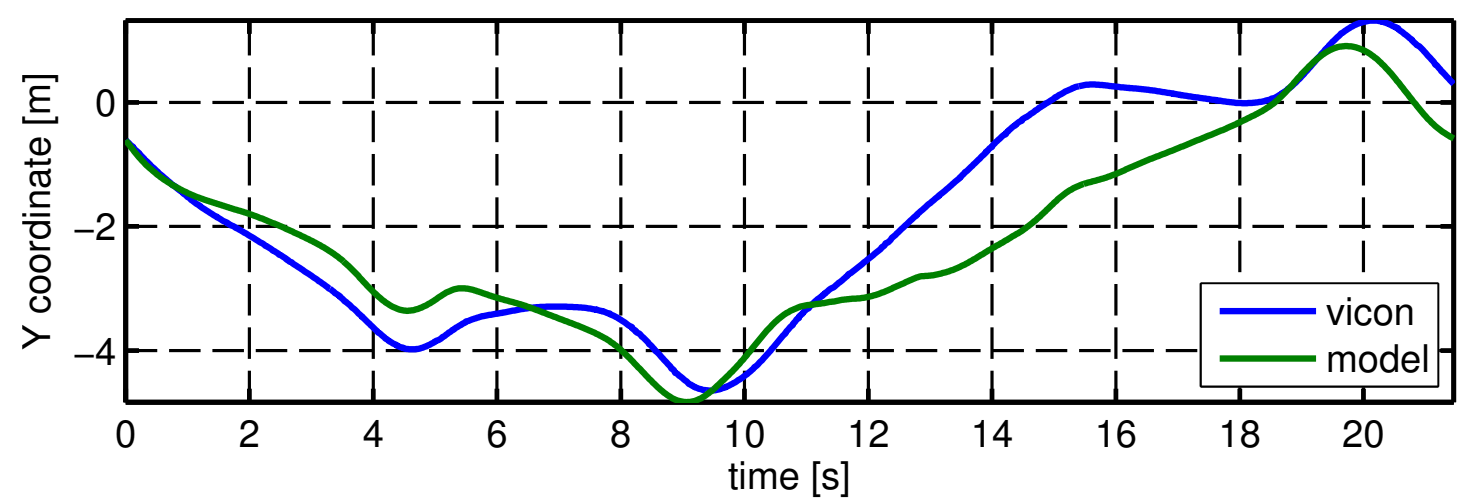

(b) Y coordinate, in the world frame

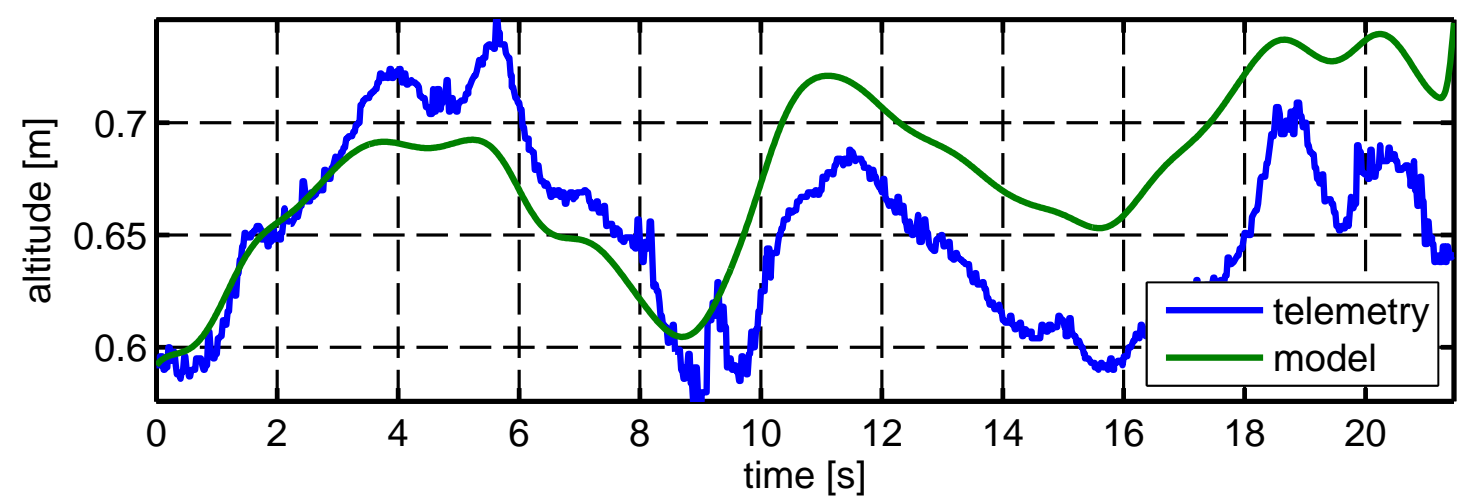

(c) Altitude

Figure D.13: Comparison of AR Drone identified model to experimental data, position coordinates. The general shape of the $X$ and $Y$ estimations is comparable to the ground truth, but a lot of error is accumulated over time. The take-off part of the simulation was not taken into account for the analysis and is not shown in the graphs. 


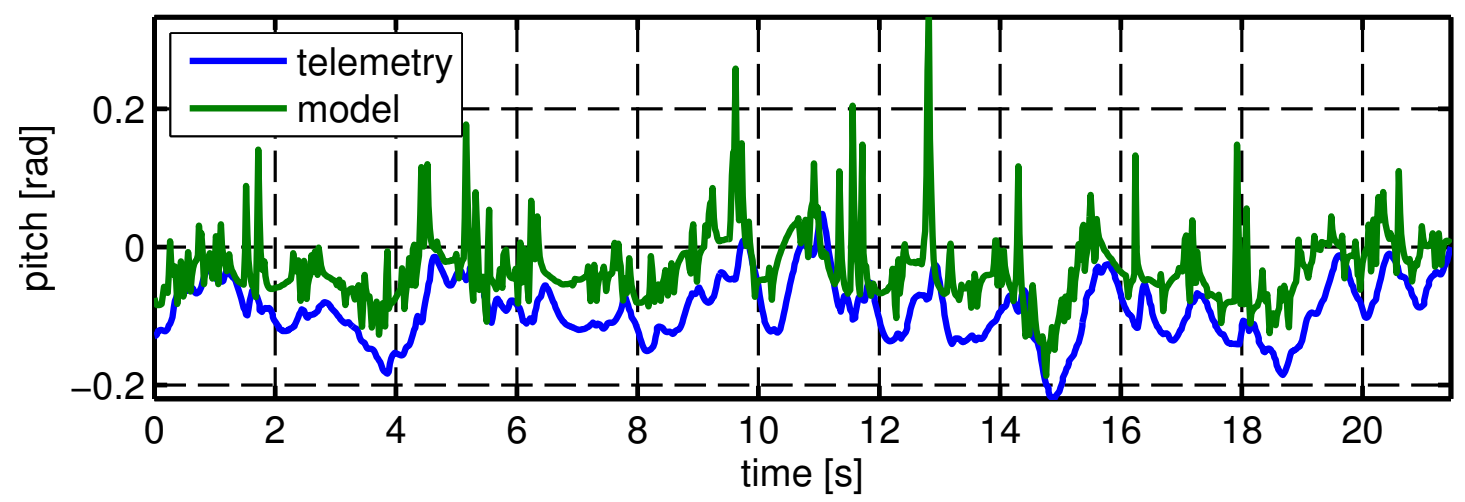

(a) Pitch angle

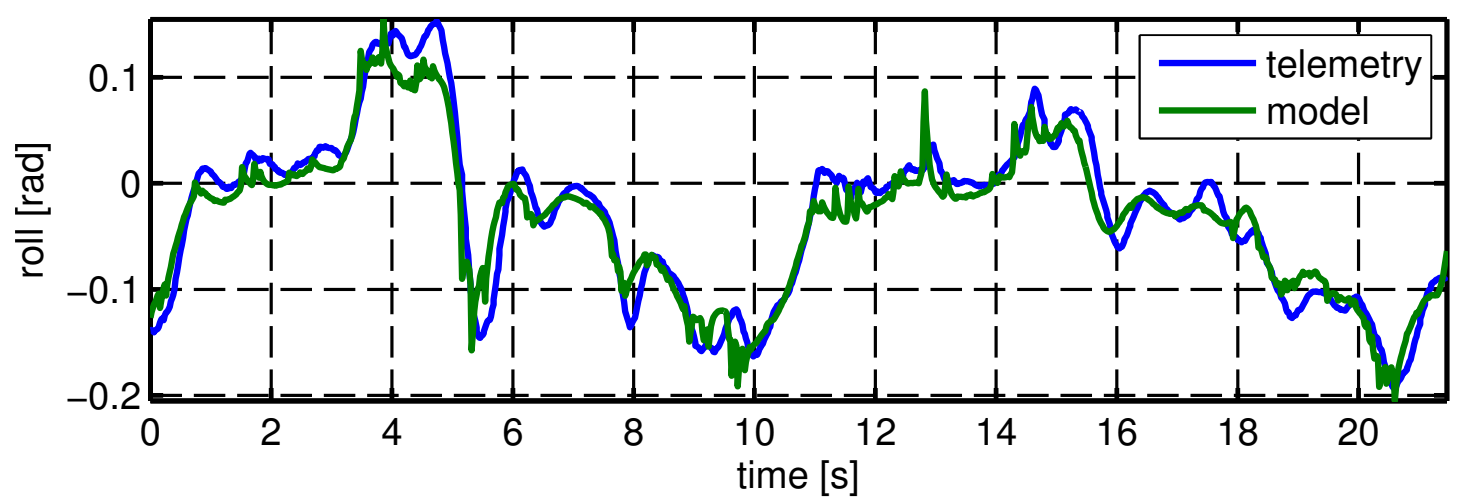

(b) Roll angle

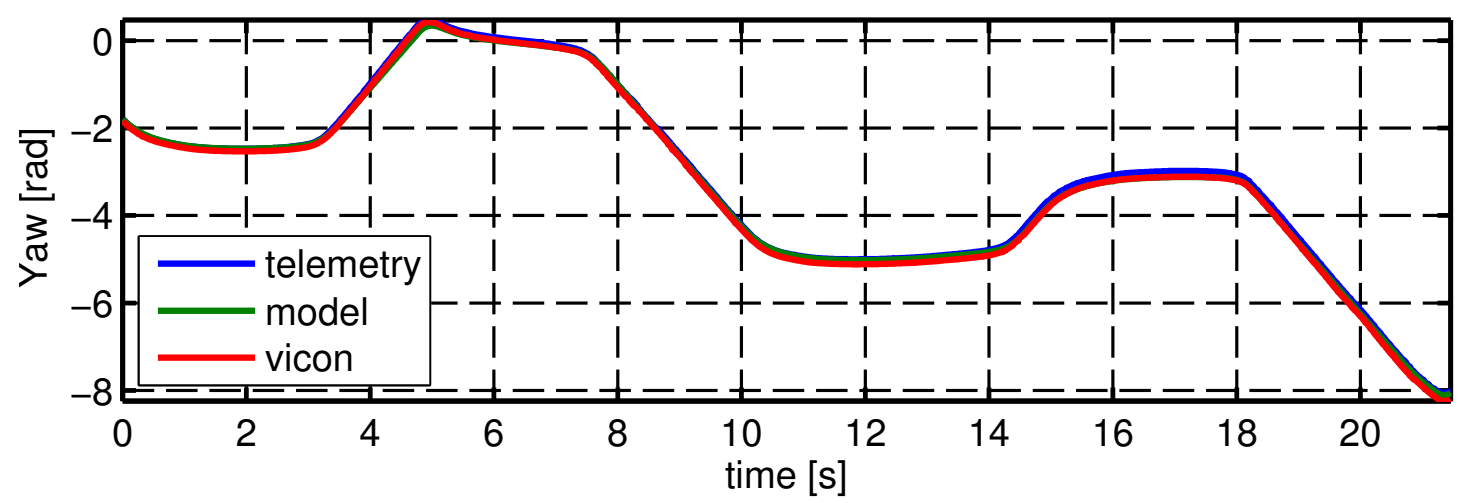

(c) Yaw angle

Figure D.14: Comparison of the AR Drone identified model to experimental data, attitude angles. The pitch estimation has an offset and it is noisy. The noise in the estimation is due to the control input, which was noisy for some reason (for instance, it could be a problem in the derivative part of the utilized PID controller). The roll and the yaw estimation show good results. The take-off part of the simulation was not taken into account for the analysis and is not shown in the graphs. 
The speed estimation shows better results, Fig. D.12. The frontal speed, $v_{x m}$, of the model has similar average value compared to the telemetry data. The laterial speed, $v_{x m}$, is also similar, but the error is much bigger. A really good characteristic of the model is that the curves in this figure have a similar appereance. Both curves seem to change smoothly, and present similar response times. The constant offset between the commanded pitch and roll and its real values, see Fig. D.14 causes part of the estimation error in velocity.

The position coordinates $\{x, y\}$ (with respect to the world frame) and the altitude are shown in Fig D.13. It can be seen again the similarity in the appereance of the curves. The estimation error is big as commented before.

Finally, the attitude angles are shown in Fig. D.14 The pitch is much noisier than the roll estimation. This is probably due to differences in the frontal speed controller, and the lateral position controller; leading to the pitch command to be noisy. The roll estimation is good, and the pitch estimation presents an offset with respect to the telemetry data. The yaw estimation is good and has little error.

It is interesting that during all the trajectory, the model estimation is oriented a little bit to the right, compared to the ground truth. This can be appreciated in the $X-Y$, Fig. D.11 The trajectory can be divided in four segments, and comparing the corresponding segments between the estimation and the ground truth; in three of them the model segment is deviated to the right with respect to the ground truth. The AR Drone was always oriented along the trajectory; with the heading parallel to the trajectory. It is possible that the estimation error is due to an internal estimation offset in the roll and pitch angles.

\section{D.5.4 Identification example: Asctec Pelican}

\begin{tabular}{c|c|c} 
& $\dot{\psi}_{c}, \dot{\psi}$ & $z_{c}, z$ \\
\hline \hline$K$ & 1.164 & 1.097 \\
$\xi$ & 1.398 & .636 \\
$w_{n}$ & 16.99 & 3.33
\end{tabular}

(a) yaw speed and altitude speed, Eq. D.3

\begin{tabular}{c|c|c} 
& $\phi_{c}, \phi$ & $\theta_{c}, \theta$ \\
\hline \hline$K$ & 0.962 & 0.934 \\
$w_{1}$ & 10.48 & 11.76
\end{tabular}

(b) roll and pitch, model Eq. D.4

Figure D.15: Experimentally identified LTI blocks for the Asctec Pelican using the complete simplified model, Fig. D.6. These LTI blocks approximately model the response of the attitude variables that area already controlled with a feedback loop in the autopilot. The altitude controller is implemented in the MAVwork framework.

First the LTI blocks are identified, see Fig. D.7, using the Matlab System Identification Toolbox. Then the input saturation limits in the Asctec Pelican can be configured using the CVG Framework for interfacing with MAVs (Mellado Bataller, I., 2012; Mellado-Bataller et al., 2013). The values that were used as saturation limits are:

- Roll and pitch input values are limited to $10^{\circ}$.

- The yaw speed is configured for a maximum speed of $100 \%$ s.

- The altitude speed is configured to obtain a maximum of $1.0 \mathrm{~m} / \mathrm{s}$.

The aerodynamic friction tests where not performed for several reasons:

- A the moment our speed measurement system is a down-facing camera which images are processed using optical flow algorithms. The optical flow is computed with the OpenCV implementation of the pyramidal Lucas-Kanade algorithm for some image features selected by the Shi-Tomasi method. The maximum speed that can be measured reliably at low altitude with this system was around $v_{\text {measurement, } \max }=0.75 \mathrm{~m} / \mathrm{s}$.

- The pelican was not well balanced at the moment, and it was difficult to stabilize it in hovering position. Many tests ended because the vehicle was not moving in the correct direction but sideways, probably due to an offset in the commanded attitude angles internally controlled by the Asctec Autopilot. 
- A the moment the developer team had the priority to participate in the IMAV 2012 competition.

As a summary, some tests were done and the following parameters were selected:

- For the aerodynamic friction law $F_{\text {friction }}=c_{1}\left(\left|v_{m}\right|+c_{2}\right) v_{m}, c_{1}=0.1139 \mathrm{rad} /(\mathrm{m} / \mathrm{s})$ and $c_{2}=$ $3.0 \mathrm{~m} / \mathrm{s}$

- The response time was about 4-6 s, and a value of 5 seconds was kept as response time. Then a value of $k_{t r}=1.317$ (adim) was used.

The Asctec Pelican model has no been compared to ground thruth data, because no such data was available. 


\section{D.6 Conclusions}

More details on the identification methods and evaluations on real data of the described models can be found in the MSc. thesis of the author Pestana, 2012, where these models were first presented. The models presented on this appendix have been used during the course of this PhD Thesis for the design of:

1. A speed, position and trajectory controller and an EKF for multirotors with an attitude controller on their Autopilot board. The EKF is used to estimate the position and velocity of the multirotor. The combined architecture has repeatedly allowed stable and precise quadrotor flight and our research group has successfully used it and been awarded in its participation on various International Micro Aerial Vehicle competitions, see Appx. [1]

2. The design of an image based visual servoing controller, showcasing the capability of following many different targets using the low-cost AR Drone platform, see Chap. 3

The main purpose of the discussed models was the design of reliable controllers for out of the lab autonomous flight, which was successfully achieved as demonstrated by the participation of the CVG_UPM in international MAV competitions and by the reliability of the developed visual-based object following architecture.

However, more precise non-linear models have been identified successfully by other researchers during the course of this thesis. For the identification of the parameters of the rigid body dynamics based model for multirotors the work by Achtelik et al. (Achtelik et al., 2013) is recommended. Also recent work by other authors (Abeywardena et al., 2013; Leishman et al., 2014) have identified the horizontal aerodynamic friction on the propellers as a mayor force contributing to the dynamics of the multirotor and have used this fact to derive improved estimation algorithms of the multirotor state. 


\section{D.7 Appendix Bibliography}

Abeywardena, D., Kodagoda, S., Dissanayake, G., and Munasinghe, R. (2013). "Improved State Estimation in Quadrotor MAVs: A Novel Drift-Free Velocity Estimator". IEEE Robotics \& Automation Magazine 20.4, pp. 32-39 (cit. on pp. 140, 155).

Achtelik, M. W., Lynen, S., Chli, M., and Siegwart, R. (2013). "Inversion based direct position control and trajectory following for micro aerial vehicles". In: Intelligent Robots and Systems (IROS), 2013 IEEE/RSJ International Conference on. IEEE, pp. 2933-2939 (cit. on p. 155).

Bristeau, P.-J., Callou, F., Vissiere, D., Petit, N., et al. (2011). "The navigation and control technology inside the ar. drone micro uav". In: 18th IFAC World Congress. Vol. 18, pp. 1477-1484 (cit. on p. 142).

Mellado Bataller, I. (2012). A new framework for interfacing with MAVs. https : / / github . com / uavster/mavwork (cit. on pp.149.153).

Fritsch, O., De Monte, P., Buhl, M., and Lohmann, B. (2012). "Quasi-static feedback linearization for the translational dynamics of a quadrotor helicopter". In: American Control Conference (ACC), 2012. IEEE, pp. 125-130 (cit. on p. 138).

Gillula, J. H., Huang, H., Vitus, M. P., and Tomlin, C. J. (2010). "Design of Guaranteed Safe Maneuvers Using Reachable Sets : Autonomous Quadrotor Aerobatics in Theory and Practice". IEEE Electrical Engineering (2010), pp. 1649-1654 (cit. on p. 139).

Hoffmann, Gabriel M, Waslander, Steven L, and Tomlin, Claire J (2008). "Quadrotor Helicopter Trajectory Tracking Control”. Electrical Engineering (2008), pp. 1-14 (cit. on pp.138,139).

Hoffmann, G.M., Waslander, S.L., and Tomlin, C.J. (2009). "Aerodynamics and control of autonomous quadrotor helicopters in aggressive maneuvering". In: 2009 IEEE International Conference on Robotics and Automation. Ieee, pp. 3277-3282 (cit. on p.140).

Leishman, R. C., Macdonald, J., Beard, R. W., and McLain, T. W. (2014). "Quadrotors and accelerometers: State estimation with an improved dynamic model". Control Systems, IEEE 34.1, pp. 28-41 (cit. on pp. 140,155.

Mellado-Bataller, I., Pestana, J., Olivares-Mendez, M. A., Campoy, P., and Mejias, L. (2013). "MAVwork: a framework for unified interfacing between micro aerial vehicles and visual controllers". In: Frontiers of Intelligent Autonomous Systems. Springer, pp. 165-179 (cit. on pp. 149, 153.

Michael, B. Y. N., Mellinger, D., and Lindsey, Q. (2010). "The GRASP Multiple Micro UAV Testbed". IEEE Robotics \& Automation Magazine, pp. 56-65 (cit. on pp. 138, 139, 141.

Pestana, J. (2012). "On-board control algorithms for Quadrotors and indoors navigation". MA thesis. Spain: Universidad Politécnica de Madrid (cit. on p. 155. 


\section{Appendix}

\section{An Extended Kalman Filter for Multirotors with Ground Speed Sensing}

\section{E.1 Introduction}

For a mobile robot to navigate safely in any environment, it requires to have feedback about its own motion and about its localization. But multirotors and other rotary wing flying vehicles require information about their own speed to attain stabilization. This is an important difference between multirotors and other ground robots. A ground robot has some certainty about how to be in a stable position, and measuring the speed of its wheels gives a reliable speed measurement for this purpose. The direction of the movement can be commanded with a good level of certainty, and together with the speed estimation gives a somewhat reliable source of information. Its control algorithms can be based in the relative certainty that is obtained from the speed measurements and of the effects of the commands on the movement of the robot to navigate. The robot still needs to localize to perform missions; but at least the robot can navigate slowly or stop moving when it is too uncertain of its localization or about nearby obstacles.

To the contrary, a multirotor has some uncertainty about its attitude. The problem is that little angle offsets in the pitch or roll estimates, can lead to motion with a relatively high speed; easily of $0.5 \mathrm{~m} / \mathrm{s}$ after some seconds. This means that if a speed controller with no reliable feedback has as objective to attain hover conditions, it can fail if it does not have reliable speed measurements. And more often than not, the vehicle is going to move at relatively high speeds. The attitude measurements alone are not enough to stabilize a multirotor. Multirotors can be in this situation for several reasons, for instance, if the weight is not well balanced the angle offset is aggravated. The consequence is that multirotors require good self-motion feedback to attain reliable stabilization, in addition to the localization problem.

In multirotors the high-level (mission planning and supervision) and mid-level (speed, position and trajectory control) control laws require a certain amount of accurate information about the quadrotor speed and position relative to the environment. This problem is not fully solved, but specific approaches give 
an acceptable estimation for simplified environment models. For a detailed state of the art the reader is referred to Sec. E.2.

The estimation and localization problem is really complex, some relevant bibliography about the problem is (Koller et al., 2009; Rudy, 2003, Russell et al., 2003; Thrun, 2002). The measurements that are available for a robot can be classified as one of the following:

- The robot may have access to an a-priori known map describing the environment.

- The robot can use local navigation and measurements information to obtain his relative position from a certain starting point. This gives rise to relative position measurements that are usually classified as:

- Inertial: it involves using rotation speed and acceleration measurements from gyroscopes and accelerometers. The IMU from a quadrotor uses both types of sensors to estimate the attitude of the multirotor. However, this information is not enough to stabilize a multirotor autonomously.

- Odometry: essentially these are measurements related to the speed of the robot; with no additional localization information. The odometry measurements are integrated over time to obtain a relative position. The localization obtained has a good short-term accuracy, but the position uncertainty increases over time. As explained before a multirotor requires odometry or relatively frequent position measurements to stabilize.

- Absolute position measurements supply information about the location of the robot with regards to the environment and nearby obstacles; the location is not derived from integrating a sequence of measurements, but directly from measurements that contain information about the robots relative position to nearby objects or from signals from beacons.

The methods to obtain absolute measurements can be divided into methods based on the use of landmarks or beacons and methods based on the use of maps. Landmarks can be passive or active, depending on wether they actively send information to the robot or not. And maps can be geometric or topological: geometric maps describe the environment in metric terms, and topological maps describe the environment in terms of characteristic features at specific locations and ways to get from one location to another. Both kind of maps can be known a priori by the robot, thus only having to match sensor reading to the known map; or learnt while navigating through the environment. The map learning task is known as SLAM (Thrun, 2002), Simultaneous Localization and Mapping, and it constitutes a very important and widely studied research topic.

This chapter is dedicated to explaining the filtering algorithms that have been implemented and successfully utilized during the course of this work. Sec. E.3, explains the filtering problem from a statistical point of view introducing the state-observation model and the assumptions used by recursive filters, then, the Sec. E.3.1 is dedicated to general aspects of recursive filters. Sec. E.4, is dedicated to the Kalman Filter, with and explanation of the algorithm on Sec. E.4.1, and a brief explanation of the Extended Kalman Filter on Sec. E.4.2. The equations of the complete multirotor simplified model are stated on Sec. E.5. which was introduced in Appx. D.5. The experimental tests using the Kalman filter are shown in Secs. E.6 \& E.6.1 \& E.6.2 The Monte Carlo Localization is introduced in Sec. E.7, then the algorithm is explained in Sec. E.7.1, and a test of the implemented filter on experimental data is discussed in Sec. E.7.2

\section{E.2 State of the Art}

In general terms, state estimation refers to the problem of calculating an estimate of the internal state of the robot. It is interesting to understand this problem from a theoretical point of view, for instance as it is explained in the following two books: Artificial Intelligence: A Modern Approach (Russell et al., 2003), and Probabilistic Graphical Models: Principles and Techniques (Koller et al., 2009); and with a more experimental approach in the Msc. Thesis by Negenborn R., which gives a good insight into Kalman filters and other estimation algorithms applied to ground mobile robots (Rudy, 2003).

In order for a multicopter to, first, fly autonomously and, second, to take decisions, the robotic platform needs an accurate estimate of its position with regards to the environment. For the stabilization of 
the platform in the air, the mid-level control laws are provided with speed and position measurements, then enabling the position hold capacity to the multirotor. This basic operation is, for instance, interesting in maintenance operations of communication towers where the vehicle provides a close view of the components of the tower.

The state estimation problem for multirotors remains an active research field, because dynamic maneuvers require fast and reliable estimates in the limited on-board hardware of the platform. However, specific approaches using Laser Range Finders (LRFs or lidars) give successful results for simplified environment models. In recent years, vision-based approaches are showing drastically improved results.

The environment model utilized by a robot limits the hazardous situations to which it can react successfully, thus limiting the commercial applications of mobile robots in unstructured environments. For instance, the ability to avoid an obstacle is limited by the type of obstacles that the robot can detect. Regarding the problem of indoors obstacle avoidance many previous work used a small number of ultrasound or infrared sensors to attain basic obstacle avoidance in indoor environments (Bouabdallah, Samir, Murrieri, Pierpaolo, et al., 2005; Matsue et al., 2005; Roberts et al., 2007). Moving to the field of Computer Vision (CV), a camera can be used to classify the type of indoor environment where the MAV is in, and use this information to control the UAV based on perspective cues to estimate the desired direction to fly (Bills et al., 2011). However, more generally applicable solutions are required, such as real-time Simultaneous Localization and Mapping (SLAM).

Inside buildings, a popular approach is the usage of laser range finders (LRFs) (such as the Hokuyo URG-04LX-UG01) and lightweight implementations of SLAM algorithms. The following survey by (Thrun, 2002) by Thrun S., gives an early introduction to the robotics SLAM problem. The CSAIL MIT laboratory has used an Asctec Pelican quadrotor platform to explore unknown areas successfully (A. G. Bachrach, 2009, A. Bachrach et al., 2011). This areas would be similar to human-made environments, were self-localization with respect to the lateral walls gives a good idea of the flyable obstacle-free space. The UAV used an stereo camera rig and an LRF to obtain the speed and position measurements (A. Bachrach et al., 2011), and it was able to win the 2009 International Aerial Robotics competition (IARC2009), hosted by the Association of Unmanned Vehicle Systems Inter-national (AUVSI); where the vehicle had to explore an environment made of hallways and rooms with little clutter, and locate a blinking device on a wall.

The GRASP laboratory of the Univesity of Pennsylvania has desmonstrated similar exploring capabilities ${ }^{1}$ using a similar arrange of sensors with the Asctec Pelican (Shen, Michael, and Kumar, 2011). The vehicle was able to explore an unknown building with multiple floors, joining perfectly the obtained map whenever the vehicle came back to the same place through a new path. Later on (Shen and Michael, 2013), the setup was improved to allow the UAV to fly changing from indoors to outdoors environments. The localization successfully switched to add GPS measurements to the state estimate 2 In a similar line collaborative mapping was also investigated, using a mobile robot along with the quadrotor (Michael et al., 2012).

There are many industrial sites that are difficult to reach or hazardous for humans to enter, but they still require inspection from time to time for routine or emergency reasons. An application research project aimed to work on these conditions using an autonomous hexarotor was carried out at the Warwick Mobile Robotics group (Abdul Shukor, 2013, Winkvist, 2013, Winkvist et al., 2013). As a motivation to the possibilities of UAVs equipped with a LRF, the following are two demonstration videos of this project where the UAV performs mapping during the a semi-autonomous flight: mapping of nuclear sites ${ }^{3}$ and a flight demonstration 4

The recent proliferation of cameras that provide depth along with the image, such as the Microsoft Kinect 5 , has moved forward the field of processing point clouds in real-time. The Kinect and other related sensors, use a hardware setup similar to an stereo camera, where one of the cameras is substituted by an infrared projector that projects a pattern on the environment, which allows to perform triangulation with the partner camera. A collaboration between the CSAIL lab of the MIT, and the Robotics and State Estimation Lab (RSE-Lab) of the University of Washington gave as a result an Asctec Pelican quadrotor that performed SLAM using a Kinect sensor. The so called RGB-D SLAM algorithm creates a 3D colored

\footnotetext{
1 http://www $\cdot$ youtube. com/watch?v=IMSozUpFFkU, 2010

2 https://www.youtube.com/watch?v=G_vtm46eGtU, 2012

3 http://www.youtube.com/watch?v=Mz5z_xaB9i8, 2011

4 http://www.youtube.com/watch?v=w5tQ7iVJ4p8 , 2011

5 http://en.wikipedia.org/wiki/Kinect, 2010
} 
map of the environment (A. S. Huang et al., 2011). The paper from the RSE-Lab regarding this work is the following (Henry et al., 2010).

In the last years there has been an increasing potential of computer vision based algorithms. The following study, on the performances of vision based point cloud algorithms versus their LIDAR (LRF) based counterparts (Leberl et al., 2010), shows that computer vision bundle adjustment methods are, since 2010 , cost-effective and obtain better results. The study is based in two practical examples comparing point clouds created from LIDAR systems and from images: aerial surface mapping and street mapping. In general terms the image based methods have an accuracy comparable to LRFs, but they offer a higher pixel density, meaning that more points are reconstructed per unit surface. Regarding this topic, the reference book to introduce the relationships between image points on images of the same scene is the book Multiple View Geometry in Computer Vision, (Hartley et al., 2004).

One of the first works that achieved a real-time SLAM algorithm based on vision, and that was able to perform real-time robot exploration was the thesis by Andrew Davidson (A. J. Davison, 1998), which was demonstrated using a ground robot. The approach uses an algorithm that is able to recognize new features of the scene that can be located repeatedly from different viewpoints. An EKF is used to filter the data and obtain the camera position with respect to the environment, the estimated position of the features is added as part of the state representation of the filter. This work gave way to more improvements (Molton et al., 2004) and applications, for example the development of a wearable camera that interacts with the user to highlight objects in the scene (Mayol et al., 2004).

In recent research, new approaches to the visual SLAM problem showed much better results than the filtering or EKF approach, both in terms of computation efficiency and accuracy. George Klein et al. designed a new localization algorithm (Klein et al., 2007) called Parallel Tracking and Mapping (PTAM) for Augmented Reality applications. The algorithm first required the computing power of a laptop, but two years later was able to make it work on an iPhone (Klein et al., 2009). Further works by the group led by Davidson studied the advantages of PTAM over the former filtering-based algorithms (Strasdat et al., 2010), explaining the reasons for its better performance. An improved version of PTAM was designed by this group, and was called Live Dense Reconstruction (DTAM) (R. A. N. Davison et al., 2010). This new algorithm uses not only salient points and image features, but also the texture of potentially all points in the image, to perform the localization calculations. DTAM has been shown to be stable and very precise in more difficult conditions: fast camera movements and camera defocusing among others 6 The approach was to include more pixel information on the map by combining the current camera position estimations with dense optical flow techniques. However, PTAM has been preferred in the UAV community, first because is available as open-source software for research, and second because it has less computing requirements, which allows to run it on-board an UAV.

The PTAM algorithm was widely used in the sFly project 7 . First results, showed how to fuse the measurements from the IMU with the output of the PTAM algorithm (Nützi et al., 2011), based on the usage of an Extended Kalman Filter (EKF). Later results, showed autonomous flight performance in outdoors environments and scale recovery of the PTAM reconstructed map (M. W. Achtelik, Lynen, S. Weiss, et al., 2012; S. Weiss, M. W. Achtelik, Lynen, et al., 2012). The following paper summarizes the research contribution of the sFly project, in terms 3D Mapping, collaborative mapping and of visualinertial fusion for state estimation and control of multirotors (Scaramuzza et al.,2013). The latter has been partially released as open-source software 8 , which can work in a low-end on-board computer. Related to this works, there are two other software repositories which are designed to perform a visual-inertial based state estimation for multicopters by fusing the output of PTAM, IMU measurements, and other sensors. They are the result of multiple consecutive works (Lynen et al., 2013, S. M. Weiss, 2012, S. Weiss, M. W. Achtelik, Chli, et al., 2012; S. Weiss, Scaramuzza, et al., 2011; S. Weiss and Siegwart, 2011) and have resulted in two popular open-source repositories ${ }^{910}$ Another influencing research on fusing IMU measurements with the output of PTAM was performed by Jakob Engel et al. in 2012 (Engel, Jakob et al., 2014, Engel et al., 2012), and is also available as an open-source package ${ }^{11}$. The main difference between both works is that the latter was realized using an off-board computer, since the flying platform is an AR

6 https: / / www $\cdot$ youtube. com/watch?v=Df9WhgibCQA, 2011

7 http://www.sfly.org/, Swarm of Micro Flying Robots (FP7/2007-2013)

8 http://wiki.ros.org/asctec_mav_framework, 2012

shttp://wiki.ros.org/ethzasl_sensor_fusion 2012

10 https://github.com/ethz-asl/ethzasl_msf 2012

11 https://github.com/tum-vision/tum_ardrone, 2012 
Drone, and that the scale of the map is recovered in a different manner. PTAM has also been explored as a technique to perform collision avoidance in indoors environments for wall-like obstacles in (Fu et al., 2013).

The Aerial Vision Group of the Institute for Computer Graphics and Vision of the Graz University of Technology (Wendel, Maurer, et al., 2011) is applying several 3D vision techniques to images acquired by quadrotors. Both for reconstruction purposes and for localization. A localization technique based on the PTAM algorithm for multirotors was developed in (Wendel, Irschara, et al., 2011). The technique required to obtain a 3D map of the environment from hand-held camera images. The map was specifically designed to be able to locate the camera. Their work often applies the concept of vocabulary trees (Nister et al., 2006) to asses the identification of the same image point in several images. The algorithm has the advantage of being able to manage larger feature databases, by relating them if they appear together in several images. The vocabulary tree algorithm has its origins on web crawlers and web-page indexing algorithms.

New advancements in vision-based SLAM have been done since the development of PTAM and DTAM. For instance, a collaborative SLAM approach performed by multiple multicopters (Forster, Lynen, et al., 2013). But more interestingly, two outstanding new algorithms that are based on direct-vision methods, which means that they do not employ image features, are the LSD-SLAM algorithm ${ }^{12}$ from Jakob Engel et al. (Engel, Schöps, et al., 2014; Engel et al., 2013) from the TU Munich Computer Vision group; and the SVO algorithm ${ }^{13}$ by Christian Forster et al. (Forster, Pizzoli, et al., 2014) from the ETH Zurich Robotics \& Perception Group. As their names suggest, one of them is a full SLAM method, and the other is a Visual Odometry one, both have in common the requirement that the image streams need to have a high frame-rate; so that the tracked points do not experience a big displacement between frames. However, they can both run on hardware with limited computing power. Both of these algorithms are probably going to inspire much research, in the same way that PTAM has done in the past.

It is also outstanding the highly optimized visual-inertial odometry pipeline developed by Shaojie Shen et al. (Shen, Shaojie et al., 2013; Shen, Mulgaonkar, et al., 2013) at the GRASP Laboratory of the University of Pennsylvania. In comparison to SVO, the pipeline is featured-based; but leverages IMU integration, carefully selected fast CV algorithms that decouple rotation from translation estimation (Burschka et al., 2008) and the utilization of Unscented Kalman Filters (Julier et al., 1995; NøRgaard et al., 2000, Wan et al., 2001). This pipeline has been demonstrated to be able to perform real-time state estimation while flying and being executed in a low-end on-board computing platform, on a board with a two-core Atom processor computer. In the same line of visual-inertial fusion, it is very remarkable the accomplishment of fusing IMU measurements with visual based ones jointly in a SLAM bundle adjustment optimization framework by Stefan Leutenegger et al. (Leutenegger, Furgale, et al., 2013, Leutenegger, Lynen, et al., 2014) from the ETH Zurich. Additionally, this works are complemented by the development of a commercially available Visual-Inertial Stereo camera sensor, that efficiently synchronizes IMU measurements and the image acquired data (Nikolic et al., 2014), and also by a the new Binary Robust Invariant Scalable Keypoints (BRISK) descriptor (Leutenegger, Chli, et al., 2011); which performs similarly to more computationally intensive descriptors but that is orders of magnitude faster. Although showing that this algorithm can run on real-time on limited computing hardware is still to be done, it is outstanding that it is the first algorithm of its class, and it may give way to a new family of algorithms that can optimally fuse visual-based measurements with data gathered from other sensors using Bundle Adjustment (BA); which has usually been done in the past using EKF or related frameworks.

Probably one of the best exponents of EKF based visual-inertial fusion are the works by Mourikis et al. (Mourikis and Roumeliotis, 2006; Mourikis and Roumeliotis, 2007), which developed the so-called Multi-State Constraint Kalman Filter (MSCKF). A slightly modified version of this algorithm was shown to be able to drastically improve the accuracy of the localization of an spacecraft entry and landing operation, which was simulated acquiring real data during a sounding rocket experiment (Mourikis, Trawny, et al., 2009). The MSCKF algorithm has been improved during the years still with the participation of Mourikis (Li et al., 2013), and many interesting issues have been tackled: like an approach to deal with rolling-shutter cameras during SLAM (Li et al., 2014b), and works regarding the on-line temporal calibration of visual and inertial measurements (Li et al., 2014a).

\footnotetext{
${ }^{12}$ Large-Scale Direct Monocular SLAM (LSD-SLAM), released as open-source in GitHub https://github . com/tum-v ision/lsd_slam, 2014

${ }^{13}$ Fast Semi-Direct Monocular Visual Odometry (SVO), released as open-source in GitHub https://github. com/uzh-r pg/rpg_svo, 2014
} 
After discussing recent advances in localization estimation techniques, the discussion is here focused again on the topic of multicopters. Mapping, potentially recognizing obstacles to be avoided and localizing the drone with respect to the environment are critical robotic tasks; but what state is otherwise necessary to estimate, to attain a competitive flight control, will be now discussed. Of the control approaches used with quadrotors, it is interesting to address this topic from the ones that exploit the differential flatness of quadrotor flight. A system is differentially flat if there exists a set of outputs from it which fully determine the state of the system given the values of the flat outputs and their derivatives (to a given order) ${ }^{14}$ (Fliess et al., 1995, Murray et al., 1995). When a non-linear system is differentially flat, this characteristic can be used for both: control and trajectory generation (Dunbar et al., 2003, Van Nieuwstadt et al., 1998). This concept is of great interest for multicopters, since they are highly non-linear dynamic systems. The characteristic that they are differentially flat was demonstrated and shown by Mellinger et al. in (Mellinger et al., 2011). This fact has allowed the development of very competitive control (Fritsch et al., 2012) and trajectory generation algorithms (Mellinger et al., 2011; Richter et al., 2013) for these platforms. By the property of differential flatness, and as exploited by the two latter cited papers, the state of the quadrotor can be fully determined by its linear position, speed, acceleration, jerk and jounce (or snap); and by its yaw rotation, speed and acceleration (Mellinger et al., 2011). This means, that besides localization and mapping, it is desirable for multicopter control to determine its position, speed, acceleration; attitude and rotation speed; and possibly more of their derivatives, until the orders mentioned above.

In terms of multicopter model identification, there are many unknowns to be determined: mass, moments of inertia, thrust and torque constants of the propellers, etc. The full quadrotor model identification scheme introduced by Markus Achtelik et al. (M. W. Achtelik, Lynen, Chli, et al., 2013), which was partly motivated by prior work in the ETHZ Flying Arena (Schoellig et al., 2012), can be used for system parameter estimation purposes and is able to determine the subset of them that is absolutely necessary to perform dynamics inversion based control.

In terms of attitude estimation, the issue is that competitive attitude estimators are a research topic by themselves. There are many different mathematical attitude representation methods (Shuster, 1993). Quaternions are probably the preferred rotation representation method (Kuipers, 2002), because of their lack of singularities and minimal number of parameters, but care must be taken to know which quaternion convention is used. There are two main conventions: the Hamilton convention and the JPL convention (Breckenridge, 1999), which is for instance used in this attitude estimation paper (Trawny et al., 2005). A neat explanation of quaternion conventions is given in this technical report by Joan Solà (Sola, 2012), which might be used in combination with these papers (Barfoot et al., 2011; Trawny et al., 2005) to understand the linearization of the state error of attitude states when represented by quaternions. Thus as a first step, it is very important to know which convention is used in a given paper or software library. Then, there are multiple attitude estimation methods that have been already tested. The following work presents a detailed summary of non-linear observers (Misawa et al., 1989), and a more modern overview of attitude estimation methods is presented in (Crassidis et al., 2007). The conventional way of performing attitude estimation is based on the Extended Kalman Filter (EKF) algorithm (Farrell, 1970, Lefferts et al., 1982; Schmidt, 1981), and the Multiplicative Extended Kalman Filter (MEKF) (Markley, 2003; Murrell, 1978). Popular attitude quaternion estimators are presented in (Shuster and Oh, 1981) and (Psiaki, 2000). The Unscented Kalman Filter (UKF) (Julier et al., 1995, NøRgaard et al., 2000; Wan et al., 2001) can be also used for attitude estimation and it is based on performing second order or higher approximations of the non-linear system; which differs from the EKF that is based on a first order approximation. However, many of the afore mentioned methods still put constraints on the probability density function of the tracked system. A popular estimation approach which does not make any such assumptions is the Particle Filter or Monte Carlo Method (Arulampalam et al., 2002, Doucet et al., 2001), which has been applied to attitude estimation in the following works (Cheng et al., 2004; Oshman et al., 2004).

Currently, there are three quadrotor open-source quadrotor simulators based on the Gazebo 3D rigid body simulator for robots, the first was developed by Meyer et al. (Meyer et al., 2012) from TU Darmstadt 15 , and the second is an adaption from the first by Hongrong Huang et al. ${ }^{16}$ to simulate an AR Drone 1.0 or 2.0 quadrotor. The third, the RotorS simulator framework (Furrer et al., 2016), was utilized by

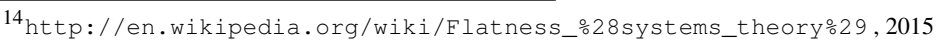

15 https://github.com/tu-darmstadt-ros-pkg/hector_quadrotor, 2012

16 https://github.com/tum-vision/tum_simulator, 2013
} 
the $E u R o C 201417$ organizers during the simulation challenge. The main feature of the RotorS simulator are that it utilizes the Gazebo physics engine, that it can be easily run and interfaced using ROS, that it provides a controller and a state estimator to help the user getting started, and that it provides an already implemented evaluation framework. Comprehensive models for quadrotors can be found in many papers, but the two following are highlighted, the hector_quadrotor simulator paper (Meyer et al., 2012), and the multicopter parameter identification described in the following paper by Markus Achletik et al. (M. W. Achtelik, Lynen, Chli, et al., 2013). Previous works by the STARMAC project, a quadrotor project that took place at the Standford University, describe aerodynamic considerations that should be taken into account by multicopter models (Hoffmann et al., 2007, H. Huang et al., 2009). A quadrotor model developed for the open-source FlightGear flight simulator is described in (Goel et al., 2009). And a model that includes many aerodynamic effects present in quadrotors was introduced in (Bouabdallah, Samir and Siegwart, Roland, 2007).

However, a very important aspect that is often not described, is the mathematical modeling of the inertial sensors themselves. The MSc thesis by Minha Park was focused on determining the sources of measurement error in MEMS (Microelectromechanical systems) based inertial sensors (Park, 2004). Gyroscopes measure the rotational speed of a rigid body with respect to an inertial frame, and are affected by noise and by a time varying drift. More concise works which give the mathematical model representations for gyroscope sensors are provided in (Abeywardena et al., 2013, Crassidis, 2006, Farrenkopf, 1978). Accelerometers measure the specific acceleration of a body, which means that they measure the acceleration of the body minus the gravity. Thus, in order to exploit their measurements, it is desirable to use a model that takes into account most, if not all, of the forces acting on the quadrotor body. Thus, the model should include aerodynamic forces among others, and this fact has been the target of recent research (Abeywardena et al., 2013; Leishman et al., 2014) which have demonstrated that the inclusion of such forces increases the overall performance of the multicopter state estimator. The authors achieved this by identifying the friction on the propellers of the multicopter as a mayor contributor to the aerodynamic friction of the aircraft (Leishman et al., 2014).

\section{E.3 Dynamic systems and filtering algorithms}

There are two sources of information that a robot can exploit to navigate, see (Koller et al., 2009, Rudy, 2003; Russell et al., 2003; Thrun, 2002). The first, is the commands it is sending to its engines or actuators. For example, a multirotor could use the rotor speed references it is sending to the ESC boards, to simulate its rigid body dynamics, see Appx. D.2 The second, is that the robot can use sensor measurements to correct the estimation of his self-motion. Both information sources are available frequently over time, in a discrete manner.

In order to reason mathematically about the motion of a robot, and be able to implement estimation algorithms; it is important to have a dynamic stochastic model of the robot. The model encodes many information about the system. For instance, the minimum required frequency of arrival of measurements and commands to the estimation module can be determined from the model. As a specific example, if the horizontal speed of a multirotor shows a response time of 3 seconds, then the minimum frequency required for the speed measurements is about $\frac{1}{3 s / 5} \approx 1.7 \mathrm{~Hz}$.

The dynamics model, also called a State-Observation model, of the robot has the following elements:

- $X_{t}$ : a set of state variables that among other things, represent the position, speed and orientation of the robot. Part of these variables may be measured but always with a varying level of uncertainty. ' $t$ ' denotes the time variable.

- $U_{t}$ : is the set of command variables that are sent to the engines of the robot.

- $O_{t}$ : denotes the set of measurements

- A transition model that specifies how the state of the robot may evolve. It is usually specified as an stochastic relationship $\mathbf{P}\left(X_{t+1} \mid X_{1: t}, U_{1: t}\right)$, between the previous values of the state and the current command $U_{t}$.

\footnotetext{
${ }^{17}$ http://www.euroc-project.eu/
} 
- A observation model that specifies the probability distribution of the measurements depending on the state $\mathbf{P}\left(O_{t} \mid X_{1: t}, O_{1: t-1}\right)$.

The term $\mathbf{P}\left(X_{t} \mid X_{1: t}, U_{1: t}\right)$ is called belief state, and it containts the probability distribution of $X_{t}$ taking into account all the prior information about the system's trajectory. It is important to note that it potentially depends on all the past values of the variables at the right. The problem is that the past values of the state and commands can grow unboundedly over time. This problem is usually solved making the 'Markov assumption', which basically states that the current state of a dynamic system depends only in a finite fixed number of previous states and commands. The simpler version of the assumption is the first-order Markov process which states that the current state depends only on the previous state and command:

$$
\mathbf{P}\left(X_{t+1} \mid X_{1: t}, U_{1: t}\right)=\mathbf{P}\left(X_{t+1} \mid X_{t}, U_{t}\right)
$$

This equation is further simplified assuming "time invariance", that is, that the robot is governed by model equations that do not themselves change over time. Both hypothesis are also applied to the observation model, obtaining:

$$
\mathbf{P}\left(O_{t} \mid X_{1: t}, O_{1: t-1}\right)=\mathbf{P}\left(O_{t} \mid X_{t}\right)
$$

The type of inference problems that can be solved using the dynamic model of the robot are:

- Filtering: it is the task of computing the belief state (the posterior distribution over the most recent state) given all the evidence to date. With the prior notation this would involve calculating, $\mathbf{P}\left(X_{t+1} \mid O_{1: t}, U_{1: t}\right)$.

- Prediction: this is the task of computing the posterior distribution over the future state, given all evidence to date. That is, to calculate $\mathbf{P}\left(X_{t+k} \mid O_{1: t}, U_{1: t}\right)$ for $k \geq 0$.

- Smoothing: this is the task of computing the posterior distribution over a past state, given all evidence up to the present. That is, to compute $\mathbf{P}\left(X_{k} \mid O_{1: t}, U_{1: t}\right)$ for some $\mathrm{k}$ such that $0 \leq k<t$.

- Most likely explanation: given a sequence of observations, we might wish to find the sequence of states that is most likely to have generated those observations. That is, to calculate $\operatorname{argmax}_{X_{1: t}} \mathbf{P}\left(X_{1: t} \mid O_{1: t}, U_{1: t}\right)$

A useful filtering algorithm needs to maintain a current state estimate and update it, rather than going back over the entire history of measurements for each new update. Otherwise, the cost of each update would increase as time goes by. A filtering algorithm with this property is called a recursive filtering algorithm. This means that it is necessary that given the posterior distribution $\mathbf{P}\left(X_{t} \mid O_{1: t}, U_{1: t}\right)$ and the new measurement $O_{t+1}$ the algorithm must be able to calculate

$$
\mathbf{P}\left(X_{t+1} \mid O_{1: t+1}, U_{1: t+1}\right)=f\left(O_{t+1}, \mathbf{P}\left(X_{t} \mid O_{1: t}, U_{1: t}\right)\right)
$$

Note the requirement to only use the posterior distribution and the new measurement, this is really important because it permits to decrease the memory requirements of the algorithm.

\section{E.3.1 Filtering in State-Observation models}

Focusing on the filtering of the dynamic system state, there exists an approach that provides a simple recursive rule for the filtering problem. The Markovian assumption underlying the State-Observation model provides a recursive two step approach that relies only in the posterior distribution $\mathbf{P}\left(X_{t} \mid O_{1: t}, U_{1: t}\right)$ and the new measurement $O_{t+1}$ to calculate $\mathbf{P}\left(X_{t+1} \mid O_{1: t+1}, U_{1: t+1}\right)$. The two steps are the following:

First there is a prediction step, that uses the state estimation and the command input to predict the state at the moment of arrival of the measurement:

$$
\begin{aligned}
\mathbf{P}\left(X_{t+1} \mid O_{1: t}, U_{1: t}\right) & =\sum_{X_{t}} \mathbf{P}\left(X_{t+1} \mid X_{t}, O_{1: t}, U_{1: t}\right) \mathbf{P}\left(X_{t} \mid O_{1: t}, U_{1: t}\right) \\
\text { (Markov assumption) } & =\sum_{X_{t}} \mathbf{P}\left(X_{t+1} \mid X_{t}, U_{t}\right) \mathbf{P}\left(X_{t} \mid O_{1: t}, U_{1: t}\right)
\end{aligned}
$$


Note that the transition model of the system is used as part of this step. This step can be understood as a convolution of the posterior probability at $t$ and the transition model to predict the state at $t+1$, that can be called prior belief state.

The first step is repeated over time whenever a state estimation is required by some module of the system. Then, when a measurement is available, the prior belief state is conditioned on the measurement to update the state estimation:

$$
\begin{aligned}
\mathbf{P}\left(X_{t+1} \mid O_{t+1}, O_{1: t}, U_{1: t}\right) & = \\
\text { (Bayes Rule }) & =\frac{\mathbf{P}\left(O_{t+1} \mid X_{t+1}, O_{1: t}, U_{1: t}\right) \mathbf{P}\left(X_{t+1} \mid O_{1: t}, U_{1: t}\right)}{\mathbf{P}\left(O_{t+1} \mid O_{1: t}, U_{1: t}\right)} \\
\text { (State-Observation model }) & =\frac{\mathbf{P}\left(O_{t+1} \mid X_{t+1}\right) \mathbf{P}\left(X_{t+1} \mid O_{1: t}, U_{1: t}\right)}{\mathbf{P}\left(O_{t+1} \mid O_{1: t}, U_{1: t}\right)}
\end{aligned}
$$

This simple recursive filtering procedure maintains the belief state over time, without keeping track of a network or a sequence of observations of growing length. This characteristic reduces both the memory requirements of the algorithm and also the number of computations to propagate the belief state over time.

This two-step process is used in the two approaches that are explained in this chapter: the Kalman Filter (KF), and the Particle Filter (PF).

As it will be explained in Sec. E.4 the Kalman Filter performs the calculations steps in Eqs. E.4 \& E.5 making the assumption that all the variables (measurements, inputs and state) can be modeled as Gaussian distributions. It is important to note that the acceptance of this assumption depends on the quality of the model. If the model explains well enough the internal dynamics of the system, then it is probable that the relationship between the state variables can be modeled with Gaussians. The Kalman Filter has the limitation that it works only with unimodal distributions, which can be a limitation in some situations.

On the other hand, the Particle Filter, Sec. E.7, implements the Eqs. E.4 \& E.5 using sampling methods. The algorithm maintains a population of particles that is updated overtime using these equations. Its main advantages are that it is easy to implement and it is easy to incorporate new sensors to the implementation. It is specially well prepared to work with sensors such as laser range finders. And a big difference with the Kalman Filter is that it can maintain multimodal distributions. In the case where the measurements are ambiguous, the population is divided between the possible sources of the measurement until new measurements and navigation data clarify which population is the correct one.

\section{E.4 Kalman filter}

The Kalman Filter (KF) is a recursive data processing algorithm that has successfully been used in a wide range of applications and specifically in many artificial intelligence and robotics problems. For instance, it can be used as a sensor data fusion algorithm in the IMU of a multirotor that combines the measurements from the gyroscopes, accelerometers and magnetometer together.

The KF, as a recursive filtering algorithm, can be understood as an implementation of the two step prediction-correction algorithm explained before, see Eqs. E.4 \& E.5. The State-Observation model explained in Sec. E.3 is stochastic and includes uncertainty in both the transition and the observation models. It is important to note that the uncertainty is specified adding white noise stochastic variables in the equations of the model. Then, these noise variables are defined with different variance values to account for the different levels of uncertainty.

In the measurement update step, Eq. E.5. the KF compares the uncertainty of the state estimation and the measurements, and combines both distributions to minimize variance of the state estimation error. During the propagation over time of the belief state using Eq. E.4 the uncertainty increases gradually due to the presence white noise in the model. And in the measurement step, the uncertainty is reduced again, providing a mechanism to bound and minimize the uncertainty of the estimation over time.

The variance values of the noise variables are selected, first taking measurements about the concerned parts of the system. This can lead sometimes to a KF that relies to much either on the measurements or in the transition model. Therefore, the variance values are further tuned to obtain a somewhat optimal filtering behavior. This is important, because in many robotic problems the KF is used to provide feedback to a controller, and a good tuning of these parameters leads to a more preferable navigation behavior of the robot. 


\section{E.4.1 The Kalman Filter algorithm}

The KF is based on a number of hypothesis on the system, measurements and different noises that are involved in the estimation problem. The KF assumes that the system is adequately modeled by a linear dynamic system:

$$
\begin{aligned}
& \text { Transition model: } \quad x_{t}=A x_{t-1}+B u_{t-1}+w_{t-1} \\
& \text { Observation model: } z_{t}=H x_{t}+v_{t}
\end{aligned}
$$

where,

- The true state of the system $x_{t}$ depends on the previous state $x_{t-1}$ trough the state matrix $A$, the command in the previous state $u_{t-1}$ trough the command matrix $B$, and the state noise $w_{t-1}$.

- The state noise is a Gaussian white noise, $w_{t} \sim \mathscr{N}\left(0, Q_{t}\right)$

- The command is stochastic with mean $\hat{u}_{t}, u_{t} \sim \mathscr{N}\left(\hat{u}_{t}, U_{t}\right)$

- The true measurement $z_{t}$ depends linearly on the state at time $t, x_{t}$, trough the observation matrix $H$. The uncertainty in the measurement is modeled through the measurement noise $v_{t}$.

- The measurement noise $v_{t}$ is a Gaussian white noise, $v_{t} \sim \mathscr{N}\left(0, R_{t}\right)$

The noise variables are independent from one another, white, Gaussian and with zero-mean. Moreover, the KF assumes that an estimate of the initial state of the system is available and that it can be represented with an independent Gaussian distribution.

$$
x_{0} \sim \mathscr{N}\left(\hat{x}_{0}, P_{0}\right)
$$

The Kalman Filter starts with an initialization step after which it alternatively performs prediction and correction steps. The algorithm itself is described in the following:

Initialization:

The filter is initialized with an estimated state, $\hat{x}_{0}$, and a state estimation error covariance, $P_{0}$.

Prediction equations:

If the equation E.4 is implemented using the state-observation model introduced before, then the following equations are obtained:

$$
\begin{gathered}
\hat{x}_{t}^{-}=A x_{t-1}^{+}+B \hat{u}_{t-1} \\
P_{t}^{-}=A P_{t-1}^{+} A^{T}+B U_{t-1} B^{T}+Q_{t-1}
\end{gathered}
$$

The set, $\left\{\hat{x}_{t+1}^{-}, P_{t+1}^{-}\right\}$, consisting of the state and the state covariance matrix at $t+1$ is called prior belief state. This step can be performed multiple times until a new measurement is acquired. As stated before, the uncertainty over the state estimation increases over time. The correction step bounds the variance of the estimation by means of the comparison of the uncertainties of the belief state and the measurements.

\section{Correction equations:}

The correction or the measurement update equations use the new measurement to update the state estimate. The measurements give information about the current state of the system. The correction equation are a version of Eq. E.5 adapted to the Kalman Filter hypothesis:

$$
\begin{gathered}
\hat{x}_{t}^{+}=x_{t}^{-}+K_{t}\left(z_{t}-H x_{t}^{-}\right) \\
P_{t+1}^{+}=\left(I-K_{t} H\right) P_{t}^{-} \\
K_{t}=P_{t}^{-} H^{T}\left(H P_{t}^{-} H^{T}+R_{t}\right)^{-1}
\end{gathered}
$$

First the measurement innovation, $\left(z_{t}-H x_{t}^{-}\right)$, is calculated. Then the uncertainty of the prior belief and from the measurement are compared to calculate the strength, $K_{t}$, with which the innovation is used to update the state estimate, $\hat{x}_{t}^{+}$, and the estimation covariance, $P_{t+1}^{+}$.

The new posterior belief is used in the next time step to compute the new prior belief. This recursive nature of KFs allows for practical implementations, because not all of the data is required to estimate the states. Also note that the filter can be used with multiple asynchronous measurement update frequencies. 
When the filter has much more confidence in the model than in the measurements, then $K_{k} \approx 0$, and then $\hat{x}_{t}^{+} \approx x_{t}^{-}+0, P_{t+1}^{+} \approx P_{t}^{-}$. To the contrary, when the filter overestimates the uncertainty of the model, then $K_{k} \approx H_{t}^{-1}$, and then $\hat{x}_{t}^{+} \approx H_{t}^{-1} z_{t}$. These two last equations are too simplified and only apply on same cases where all the states are measured.

In short, the KF calculates the belief state by performing two alternating steps:

- In the prediction step the KF calculates the prior belief. This belief consists of the prior state estimate and the prior error covariance, which describes the uncertainty in the state estimate. Given the model and the last state estimate the prediction step predicts the most likely current state of the system after a time step.

- The KF applies the correction step when it has access to a measurement. It uses the measurement to form the posterior belief. The KF computes the measurement innovation as the difference between the true measurement and a measurement prediction. It uses the kalman gain to determine to what extent the measurement innovation should be incorporated into the state estimate.

\section{E.4.2 Extended Kalman Filter}

The EKF is an extension of the KF to the case where the model dynamics are non linear:

$$
\begin{aligned}
& \text { Transition model: } x_{t}=f\left(x_{t-1}, u_{t-1}\right)+w_{t-1} \\
& \text { Observation model: } z_{t}=h\left(x_{t}\right)+v_{t}
\end{aligned}
$$

where the state matrixes are derived from the model trough derivation:

$$
\begin{aligned}
& A_{t}=\left.\frac{\partial f}{\partial x}\right|_{x_{t}} \\
& B_{t}=\left.\frac{\partial f}{\partial u}\right|_{u_{t}} \\
& H_{t}=\left.\frac{\partial h}{\partial x}\right|_{x_{t}}
\end{aligned}
$$

The algorithm follows the same steps as the KF, but utilizes the first-order approximation of the model around the current belief state at $t$ :

Initialization:

The filter is initialized with an estimated state, $\hat{x}_{0}$, and a state estimation error covariance, $P_{0}$.

Prediction equations:

If the equation E.4 is implemented using the state-observation model introduced before, then the following equations are obtained:

$$
\begin{gathered}
\hat{x}_{t}^{-}=A x_{t-1}^{+}+B \hat{u}_{t-1} \\
P_{t}^{-}=A P_{t-1}^{+} A^{T}+B U_{t-1} B^{T}+Q_{t-1} \\
A_{t}=\left.\frac{\partial f}{\partial x}\right|_{x_{t-1}^{+}, \hat{u}_{t-1}} \quad B_{t}=\left.\frac{\partial f}{\partial u}\right|_{x_{t-1}^{+}, \hat{u}_{t-1}}
\end{gathered}
$$

\section{Correction equations:}

The correction equation are the version of Eq. E.5 adapted to the Kalman Filter hypothesis:

$$
\begin{gathered}
\hat{x}_{t}^{+}=x_{t}^{-}+K_{t}\left(z_{t}-H x_{t}^{-}\right) \\
P_{t+1}^{+}=\left(I-K_{t} H\right) P_{t}^{-} \\
K_{k}=P_{t}^{-} H^{T}\left(H P_{t}^{-} H^{T}+R_{t}\right)^{-1} \\
H_{t}=\left.\frac{\partial h}{\partial x}\right|_{x_{t}^{-}}
\end{gathered}
$$

The Jacobian matrices $A_{k}$ and $H_{k}$ are evaluated at the most recent state estimates. Since the state estimates are not known in advance, the Jacobians can not be computed off-line as is the case with the KF. Thus, in the EKF case, the Jacobians have to be computed on-line, resulting in higher computational costs. 


\section{E.5 Complete simplified multirotor model, state equations}

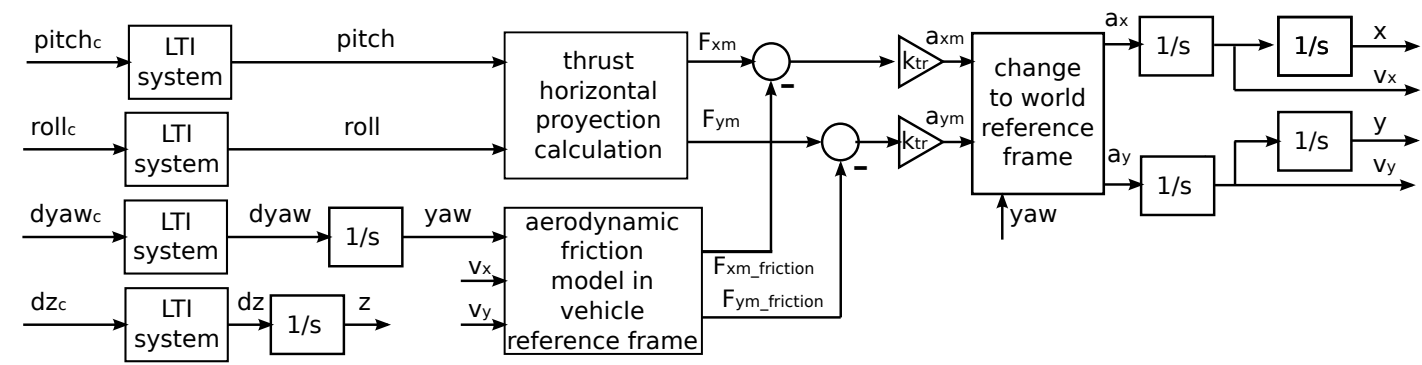

Figure E.1: Multirotor complete simplified model, all inputs are taken into account. The model multirotor is already equipped with an autopilot that controls the attitude of the aerial platform. The model also assumes that there is an altitude controller that accepts altitude speeds commands. The model roughly identifies the multirotor stabilized by the mentioned controllers, which are usually implemented in the autopilot.

In this section, the multirotor simplified model equations are shown. The model was introduced in Appx. D.5 and the model's diagram block is shown again in Fig. E.1 for convenience. The model also assumes that the multirotor's are stabilized by controllers that accept attitude and altitude speeds commands. The notation of the attitude angles is $\{\phi$ (roll), $\theta$ (pitch), $\psi$ (yaw) $\}$, and the altitude is $-z$. The equations for each group of blocks are explained separately:

\section{Input commands:}

The input commands $\left\{\theta_{c}, \phi_{c}, \dot{\psi}_{c}, \dot{z}\right\}$, are first saturated to $[-1,+1]$, and then an input gain is applied $\left\{K_{\theta}, K_{\phi}, K_{\dot{\psi}}, K_{\dot{z}}\right\}$,

$$
\begin{aligned}
\operatorname{pitch}_{c} & =K_{\theta} \text { saturation }_{-1}^{+1}\left(\theta_{c}\right) \\
\operatorname{roll}_{c} & =K_{\phi} \text { saturation }_{-1}^{+1}\left(\phi_{c}\right) \\
\operatorname{dyaw}_{c} & =K_{\dot{\psi}} \text { saturation }_{-1}^{+1}\left(\dot{\psi}_{c}\right) \\
\mathrm{dz}_{c} & =K_{\dot{z}} \text { saturation }_{-1}^{+1}(\dot{z})
\end{aligned}
$$

LTI blocks and integrators:

First each LTI block must be changed from transfer function representation to a state representation:

$$
\begin{aligned}
G_{\theta} & =\left[\begin{array}{l|c}
A_{\theta} & B_{\theta} \\
\hline H_{\theta} & 0
\end{array}\right] \\
G_{\phi} & =\left[\begin{array}{l|c}
A_{\phi} & B_{\phi} \\
\hline H_{\phi} & 0
\end{array}\right] \\
G_{\psi}=\frac{G_{\dot{\psi}}}{s} & =\left[\begin{array}{l|c}
A_{\psi} & B_{\psi} \\
\hline H_{\psi} & 0
\end{array}\right] \\
G_{z}=\frac{G_{\dot{z}}}{s} & =\left[\begin{array}{l|c}
A_{z} & B_{z} \\
\hline H_{z} & 0
\end{array}\right]
\end{aligned}
$$

Then this part of this diagram is regrouped as a multiple input multiple output (MIMO) system:

$$
\begin{aligned}
& {\left[\begin{array}{c}
x_{\theta} \\
x_{\phi} \\
x_{\psi} \\
x_{z}
\end{array}\right]_{t}=\left[\begin{array}{cccc}
A_{\theta} & 0 & 0 & 0 \\
0 & A_{\phi} & 0 & 0 \\
0 & 0 & A_{\psi} & 0 \\
0 & 0 & 0 & A_{z}
\end{array}\right]\left[\begin{array}{c}
x_{\theta} \\
x_{\phi} \\
x_{\psi} \\
x_{z}
\end{array}\right]_{t-1}+\left[\begin{array}{cccc}
B_{\theta} & 0 & 0 & 0 \\
0 & B_{\phi} & 0 & 0 \\
0 & 0 & B_{\psi} & 0 \\
0 & 0 & 0 & B_{z}
\end{array}\right]\left[\begin{array}{c}
\operatorname{pitch}_{c} \\
\operatorname{roll}_{c} \\
\operatorname{dyaw}_{c} \\
\mathrm{dz}_{c}
\end{array}\right]_{t-1}} \\
& {\left[\begin{array}{c}
\text { pitch } \\
\text { roll } \\
\text { yaw } \\
\mathrm{z}
\end{array}\right]_{t}=\left[\begin{array}{cccc}
H_{\theta} & 0 & 0 & 0 \\
0 & H_{\phi} & 0 & 0 \\
0 & 0 & H_{\psi} & 0 \\
0 & 0 & 0 & H_{z}
\end{array}\right]\left[\begin{array}{c}
x_{\theta} \\
x_{\phi} \\
x_{\psi} \\
x_{z}
\end{array}\right]_{t}}
\end{aligned}
$$


Calculation of the available thrust and aerodynamic forces:

The aerodynamic friction is identified using to parameters $c_{1}$ and $c_{2}$, and the thrust force is obtained from the equations of the rigid body model, assuming a thrust that approximately counteracts the gravity.

$$
\begin{aligned}
{\left[\begin{array}{l}
F_{x m} \\
F_{y m}
\end{array}\right]_{t} } & =\left[\begin{array}{cc}
m g & 0 \\
0 & m g
\end{array}\right]\left[\begin{array}{c}
-\cos (\text { roll }) \sin (\text { pitch }) \\
\sin (\text { roll })
\end{array}\right]_{t-1} \\
{\left[\begin{array}{l}
F_{x m_{-} \text {friction }} \\
F_{y m_{-} \text {friction }}
\end{array}\right]_{t} } & =\left[\begin{array}{cc}
\cos (\text { yaw }) & \sin (\text { yaw }) \\
-\sin (\text { yaw }) & \cos (\text { yaw })
\end{array}\right]_{t-1}\left(c_{1} \sqrt{v_{x}^{2}+v_{y}^{2}}+c_{1} c_{2}\right)_{t-1}\left[\begin{array}{l}
v_{x} \\
v_{y}
\end{array}\right]_{t-1}
\end{aligned}
$$

Calculation of the acceleration in body frame coordinates:

This equation includes the parameter that tunes the response time of the horizontal speed, $k_{t r}$ which has units of $\mathrm{kg}^{-1}$.

$$
\left[\begin{array}{l}
a_{x m} \\
a_{y m}
\end{array}\right]_{t}=\left[\begin{array}{cc}
k_{t r} & 0 \\
0 & k_{t r}
\end{array}\right]\left[\begin{array}{c}
F_{x m}-F_{x m \text { friction }} \\
F_{y m}-F_{y m} \text { friction }
\end{array}\right]_{t-1}
$$

Calculation of the speed and position:

The speed and position are derived from the integration of the acceleration, which only needs to be transformed again to world coordinates.

$$
\begin{aligned}
& {\left[\begin{array}{c}
x \\
v_{x} \\
y \\
v_{y}
\end{array}\right]_{t}=\left[\begin{array}{cccc}
\Delta t & \frac{\Delta t^{2}}{2} & 0 & 0 \\
0 & \Delta t & 0 & 0 \\
0 & 0 & \Delta t & \frac{\Delta t^{2}}{2} \\
0 & 0 & 0 & \Delta t
\end{array}\right]\left[\begin{array}{c}
v_{x} \\
a_{x} \\
v_{y} \\
a_{y}
\end{array}\right]_{t-1}} \\
& {\left[\begin{array}{c}
a_{x} \\
a_{y}
\end{array}\right]_{t}=\left[\begin{array}{cc}
\cos (\text { yaw }) & -\sin (\text { yaw }) \\
\sin (\text { yaw }) & \cos (\text { yaw })
\end{array}\right]_{t-1}\left[\begin{array}{l}
a_{x m} \\
a_{y m}
\end{array}\right]_{t-1}}
\end{aligned}
$$

\section{E.6 Testing the EKF on experimental data}

An Extended Kalman Filter EKF has been implemented using the model explained in Appx. E.5 and Appx. D.5. This EKF was implemented in collaboration with my colleague from the CVG (UPM) J. L. Sanchez-Lopez, who implemented a utilized EKF-library during his MSc. thesis (Sanchez Lopez, 2012). It is important to evaluate the EKF estimation with experimental measurements. As part of the development of the MAVwork (Mellado Bataller, I., 2012), the author, I. Mellado, of the framework performed a test showing the viability of the framework with a position controller. The AR Drone control problem was simplified using feedback information from a Vicon motion capture system, thus isolating the navigation problem from the localization/estimation problem. The video recorded during the test is available online in the website https://youtu.be/MFxfaHb7jT0. The data from the test includes: experimental input, telemetry and ground truth data (from the Vicon system). The availability of ground truth data for these test was the main reason to use them it for the evaluation of the implemented EKF. The control during this test was based on four PIDs: forward speed, lateral speed, yaw and altitude, which were adjusted from a model estimation. The controller is a trajectory controller that follows a trajectory defined by waypoints. The feedback information required for navigation was obtained from a Vicon motion capture system.

The data was recorded with a common timescale, and it includes:

1. Input/command dataset:

$\{\phi$ (roll), $\theta$ (pitch), $\dot{\psi}$ (yaw speed), $-\dot{z}$ (altitude speed) $\}$

2. Telemetry dataset: the data that the AR Drone sends to the ground station are the $\{\phi$ roll, $\theta$ pitch, $\psi$ yaw $\}$ attitude coordinates, the altitude (from the sonar) and the $\left\{v_{x m}, v_{y m}\right\}$ ground optical-flow speed estimations. The speed estimations are given in body frame coordinates ( $x$, front direction, and $y$, rightwards lateral direction).

3. Ground truth localization dataset (Vicon): position and attitude data, the $\{x, y, \psi$ (yaw) $\}$ coordinates with respect to the world frame. 


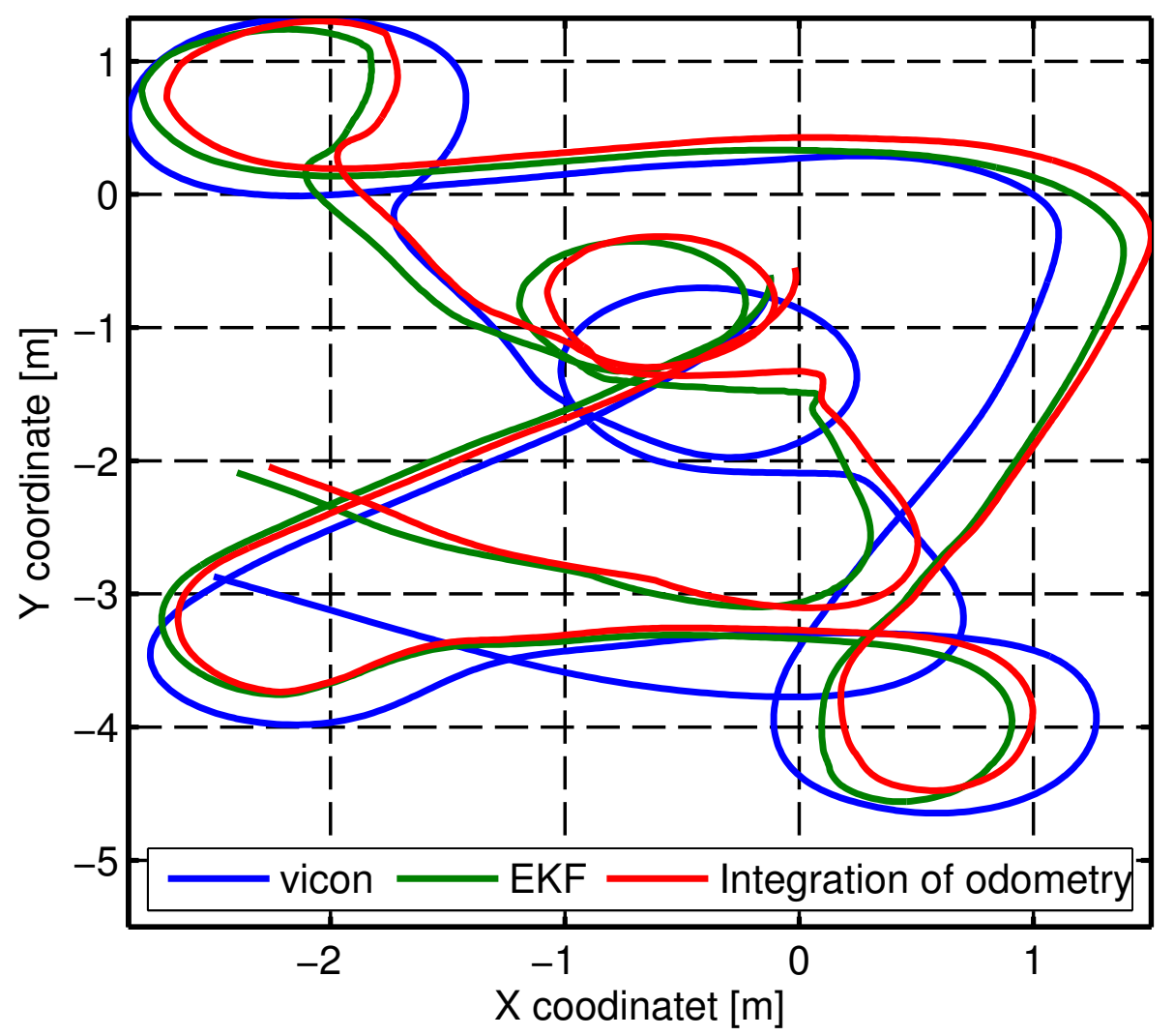

Figure E.2: Comparison of the EKF using odometry data, compared to experimental data, X-Y trajectory. The figure shows the ground truth data, the EKF estimation and the direct integration of the odometry data $\{x, y, \psi\}$. The graph shows that the EKF estimation is pretty near the integration of the odometry data.

\section{E.6.1 EKF with odometry measurements}

In the following, several graphs are shown where the EKF estimation data is either compared with the vicon data, the telemetry data, or the direct integration of the $\{x, y, \psi\}$ odometry data (that yields an estimation of the $X-Y$ trajectory). It is important to note that the EKF is only using the input dataset and the telemetry data. In this subsection, the measurements of the Vicon (the ground truth) are not used at all as input to the EKF. The take-off was eliminated from the estimation process; it was decided not to consider that part of the data for the analysis, for three reasons:

- the take-off mode of the AR Drone ignores the roll and pitch commands until the maneuver is finished,

- it helps in the analysis task to work on data that corresponds to the conditions that the identification process was aimed for,

- the take-off situation differs from the normal flying conditions and should be analyzed separately.

A comparison of the EKF running the AR Drone model versus the experimental data is shown in Fig. E.2. The figure shows the ground truth data, the EKF estimation and the direct integration of the odometry data $\{x, y, \psi\}$. It is clear that the EKF estimation is very similar to the integration of the odometry data. This makes sense, because the speed estimates are using the odometry measurements, and the EKF is tuned to have a certain level of confidence in the measurements over the model. It is worth comparing this figure to Fig. D.11. Finally the estimation error increases over time but stayed below $1 \mathrm{~m}$ during all the test, which was 40 seconds long. 


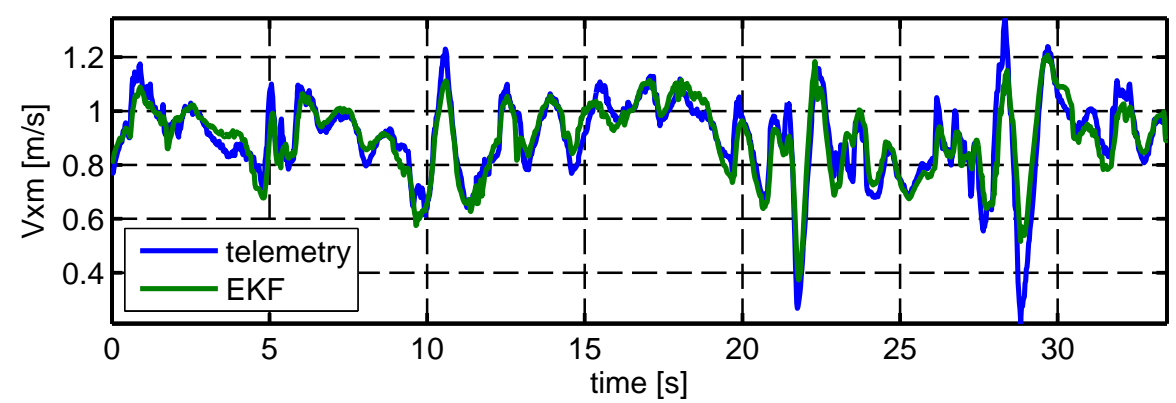

(a) Speed in the body frame, frontal speed $v_{x m}$

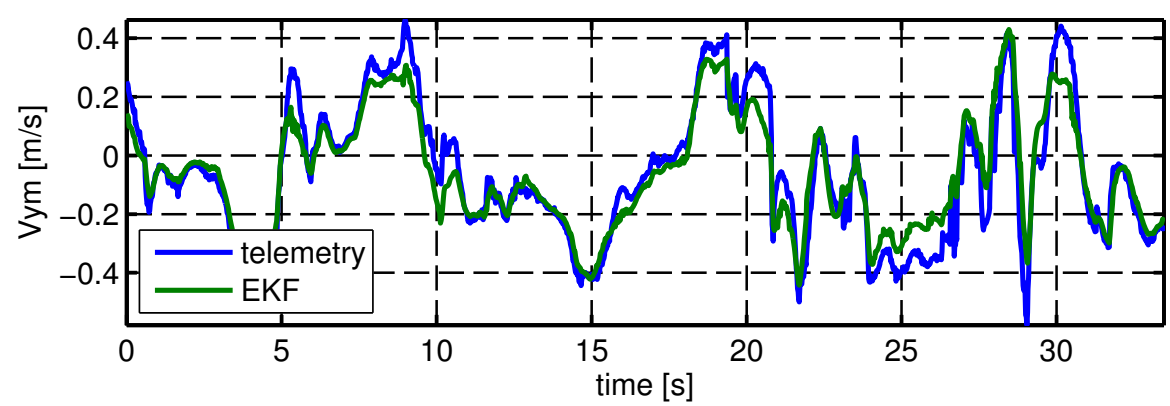

(b) Speed in the body frame, lateral speed $v_{y m}$

Figure E.3: Comparison of the EKF using odometry data, compared to experimental data, speed in the body frame. Both estimations, $v_{x m}$ and $v_{y m}$, are similar to the odometry data. But it is clear that the EKF is not completely relying in the measurements and it is performing noise filtering.

The speed estimation, shows good results, Fig. E.3. The graphs show the speed coordinates with respect to the body frame. On one hand the EKF is managing to use the measurements correctly and provides a good estimation with respect to them. On the other hand it is not completely relying on the measurements and it is performing some degree of noise filtering. Both estimations, $v_{x m}$ and $v_{y m}$, are similar to the odometry data. A really good characteristic of the estimation is that the curves in this figure have a similar appearance. Both curves change smoothly, and present similar response times.

The position coordinates $\{x, y\}$ (with respect to the world frame and the altitude are shown in Fig E.4. In some parts of the trajectory the $X$ and $Y$ estimations work really well and the EKF manages to estimate the movement of the drone correctly. More importantly the error accumulated over time increases slowly, and after 40 seconds it is only about $1 \mathrm{~m}$.

Finally, the attitude angles are shown in Fig. E.5. The pitch is much noisier than the roll estimation. This is probably due to differences in the frontal speed controller, and the lateral position controller; leading to the pitch command to be noisy. The roll estimation is good, and the pitch estimation presents an offset with respect to the telemetry data. The yaw estimation is good and has little error.

The estimation seems to be good enough to implement a controller using it as feedback. In fact the EKF shown here, was used to implement several controllers that are explained in the next chapter. Although the EKF estimate is really similar to the direct integration of the odometry measurements, the filtering process provides some advantages:

- The measurements are filtered, and their estimates present less noise than before. This is important to decrease the bad effects of noise on the derivate actions of the PID controllers.

- The EKF can generate estimates whenever needed. It provides a method to separate the sampling instants of the controller from the sampling times of each measurement.

- The EKF permits to perform data fusion, for example, to utilize the positioning data and speed measurements, or the speed estimates from different sensors. 


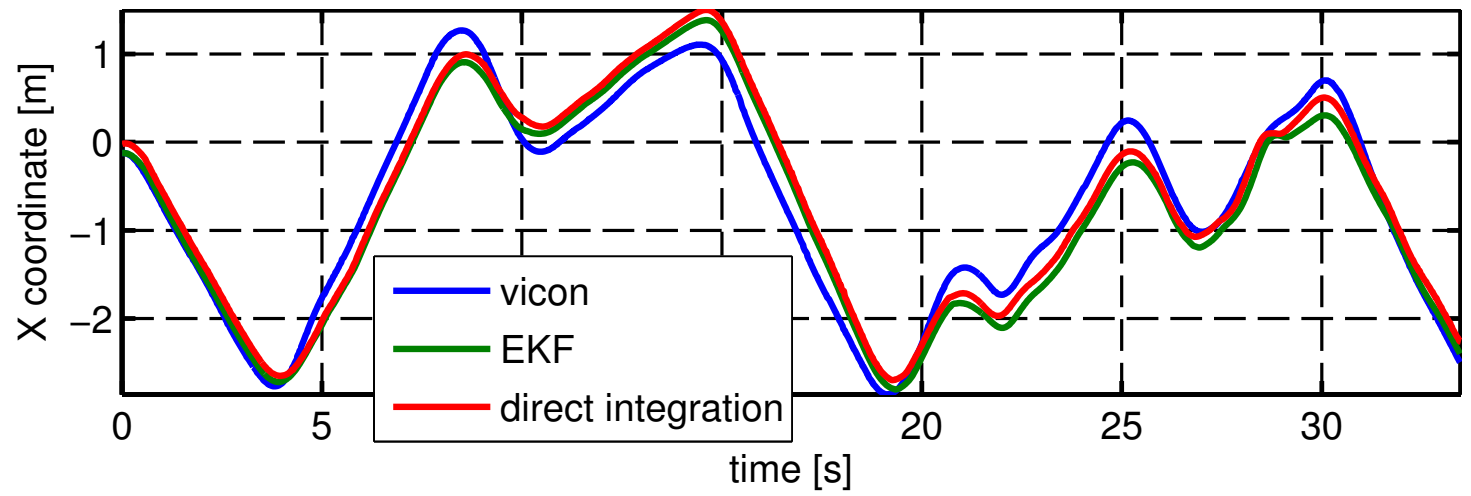

(a) X coordinate, in the world frame

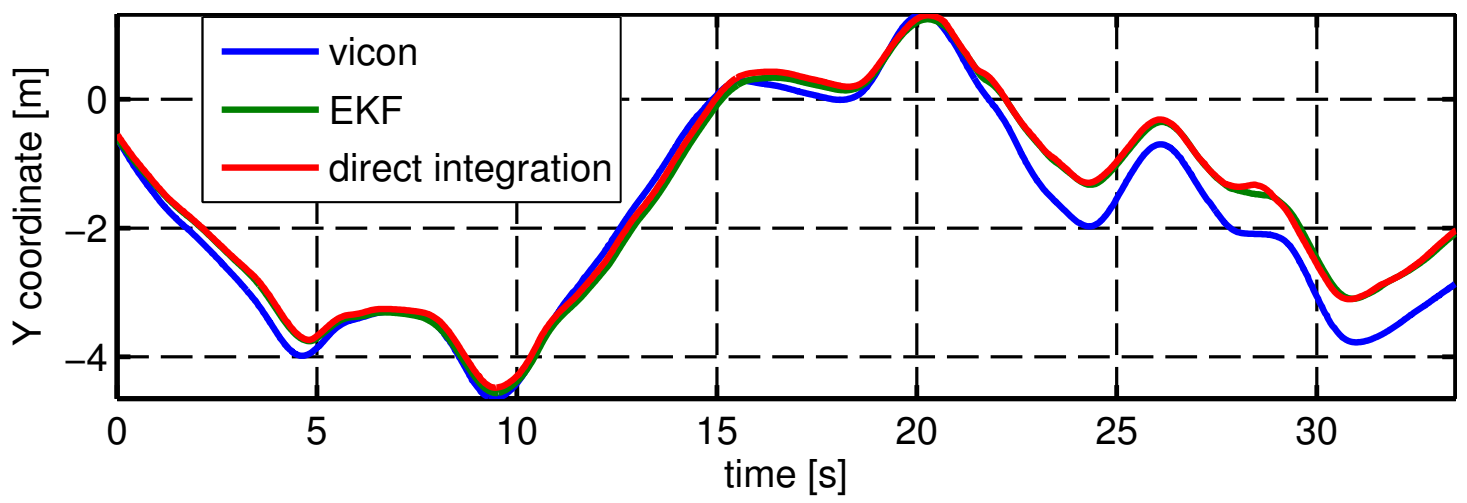

(b) Y coordinate, in the world frame

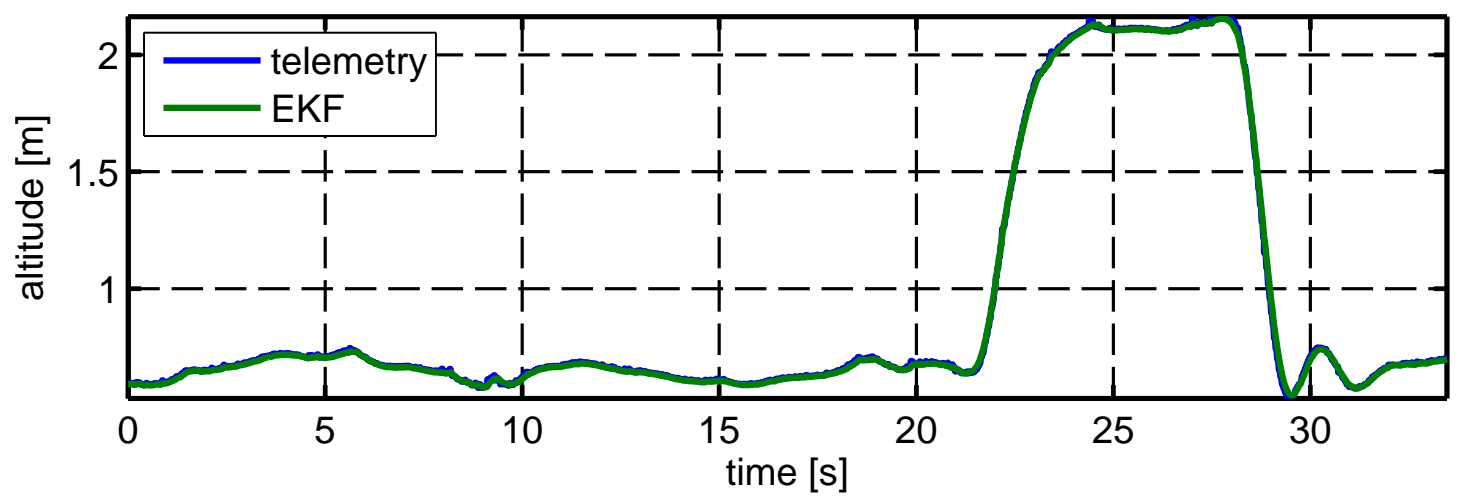

(c) Altitude

Figure E.4: Comparison of the EKF using odometry data, compared to experimental data, position coordinates. In some parts of the trajectory the $X$ and $Y$ estimations work really well and the EKF manages to estimate the movement of the drone correctly. More importantly the error accumulated over time increases slowly, and after 40 seconds it is only about $1 \mathrm{~m}$. 


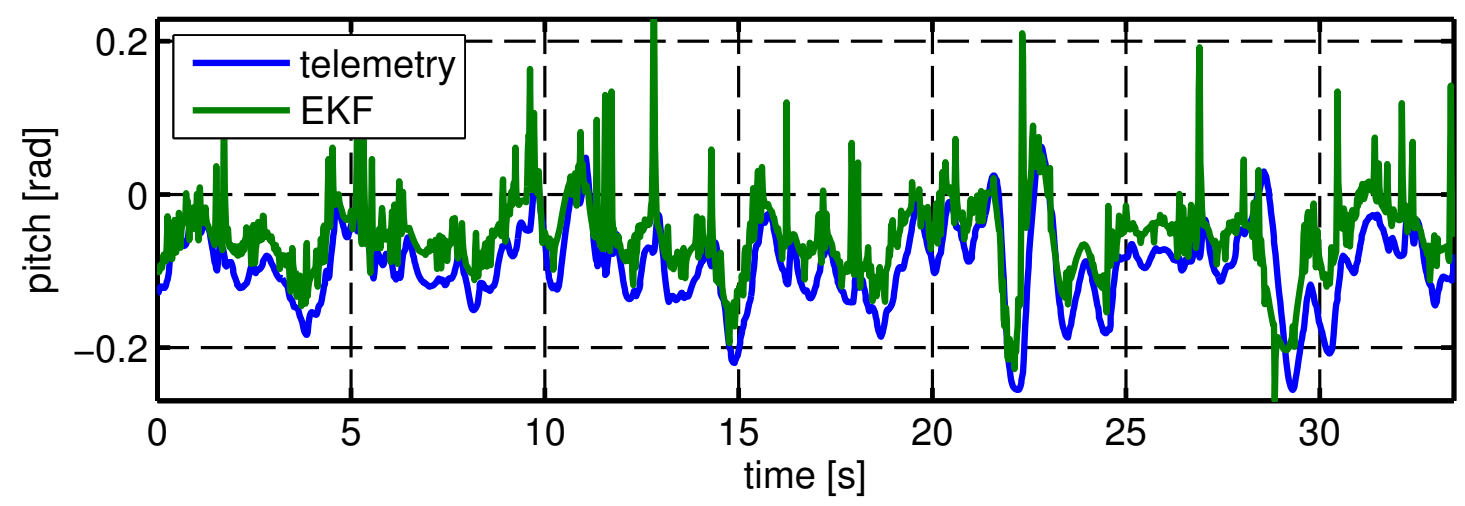

(a) Pitch angle

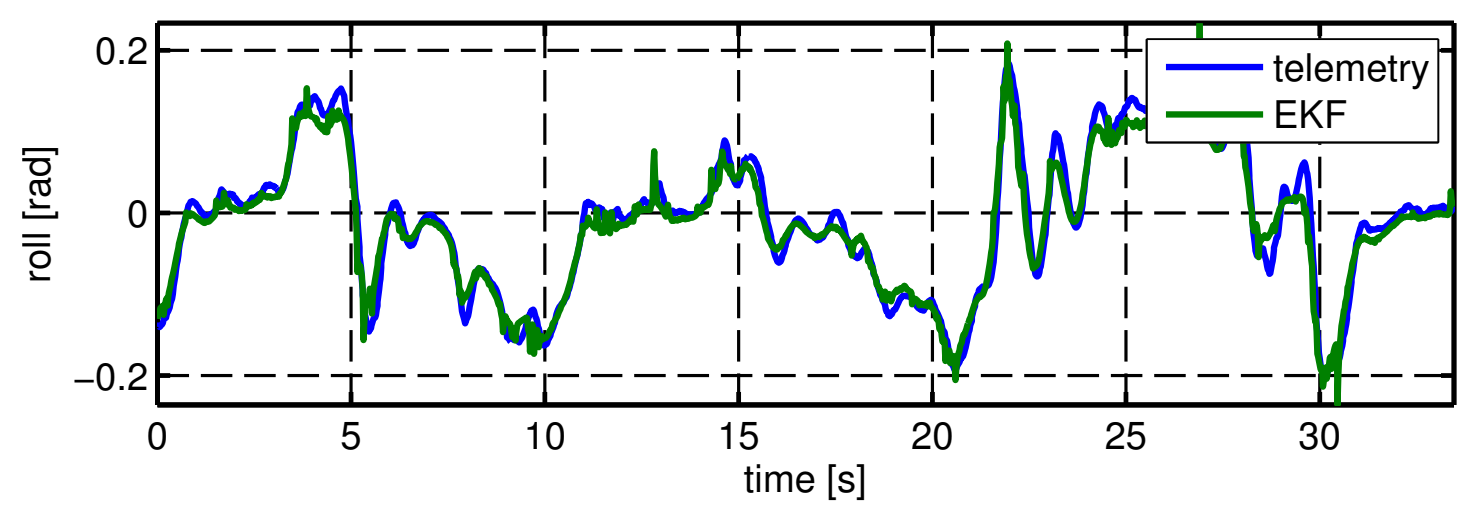

(b) Roll angle

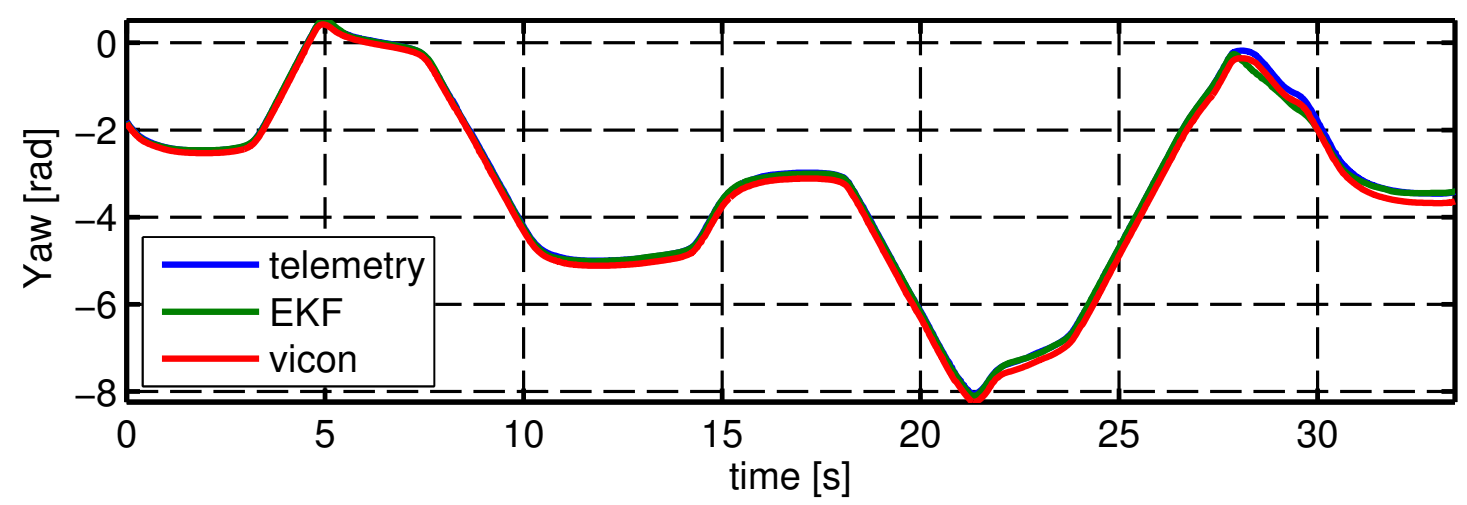

(c) Yaw angle

Figure E.5: Comparison of the EKF using odometry data, compared to experimental data, attitude angles. The pitch estimation has an offset and it is noisy. The noise in the estimation is due to the control input, which was noisy for some reason (for instance, it could be a tuning problem on the frontal speed PID). The roll and the yaw estimation show good results. The take-off part of the experiment was not taken into account for the analysis and is not shown in the graphs. 


\section{E.6.2 EKF with position measurements}

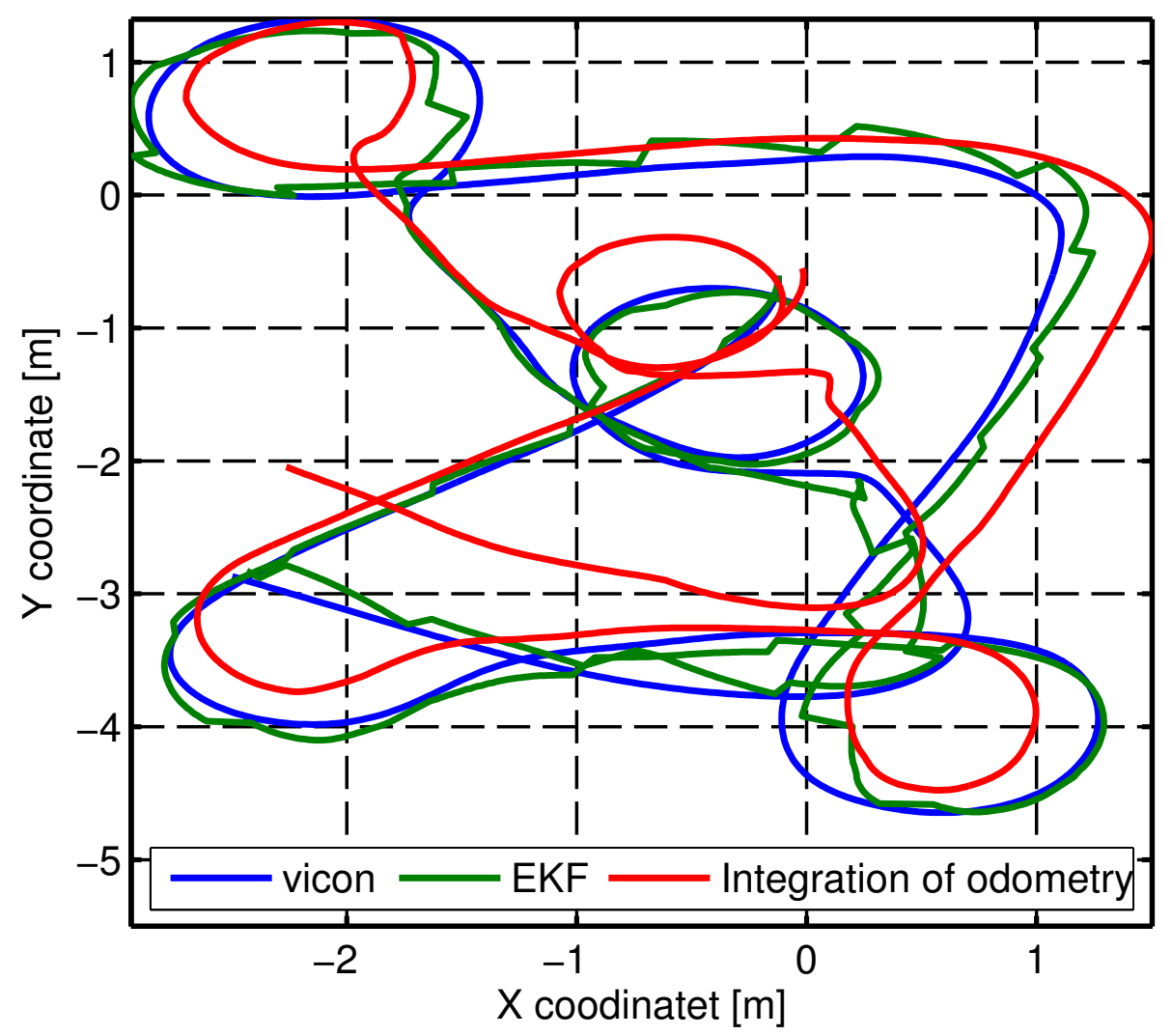

Figure E.6: Comparison of the EKF using odometry and Vicon position data, compared to experimental data, X-Y trajectory. The figure shows the ground truth data, the EKF estimation and the direct integration of the odometry data $\{x, y, \psi\}$. The graph shows that the EKF is corrected every $0.75 \mathrm{~s}$ using the Vicon data. The EKF was tuned to consider that the position measurements have little uncertainty.

In the following, several graphs are shown where the EKF estimation data is either compared with the Vicon position data, the telemetry data, or the direct integration of the odometry data, $\{x, y, \psi\}$, that yields an estimation of the $X-Y$ trajectory. It is important to note that the EKF is using the input dataset, the telemetry data and the Vicon position dataset. The telemetry measurements arrive every $60 \mathrm{~ms}$ in average, and the position measurements from the Vicon are used every $0.75 \mathrm{~s}$. The take-off was eliminated from the estimation process; it was decided not to consider that part of the data for the analysis, for three reasons:

- the take-off mode of the AR Drone ignores the roll and pitch commands until the maneuver is finished,

- it helps in the analysis task to work on data that corresponds to the conditions that the identification process was aimed for,

- the take-off situation differs from the normal flying conditions and should be analyzed separately.

The comparison of the EKF running the AR Drone model versus the experimental data is shown in Fig. E.6. The figure shows the ground truth data, the EKF estimation and the direction integration of the odometry data. The graph shows that the EKF is corrected every $0.75 \mathrm{~s}$ using the Vicon data. The EKF was tuned to consider that the position measurements have little uncertainty. During the time between position measurements the EKF estimates only with the odometry measurements. The estimation error 


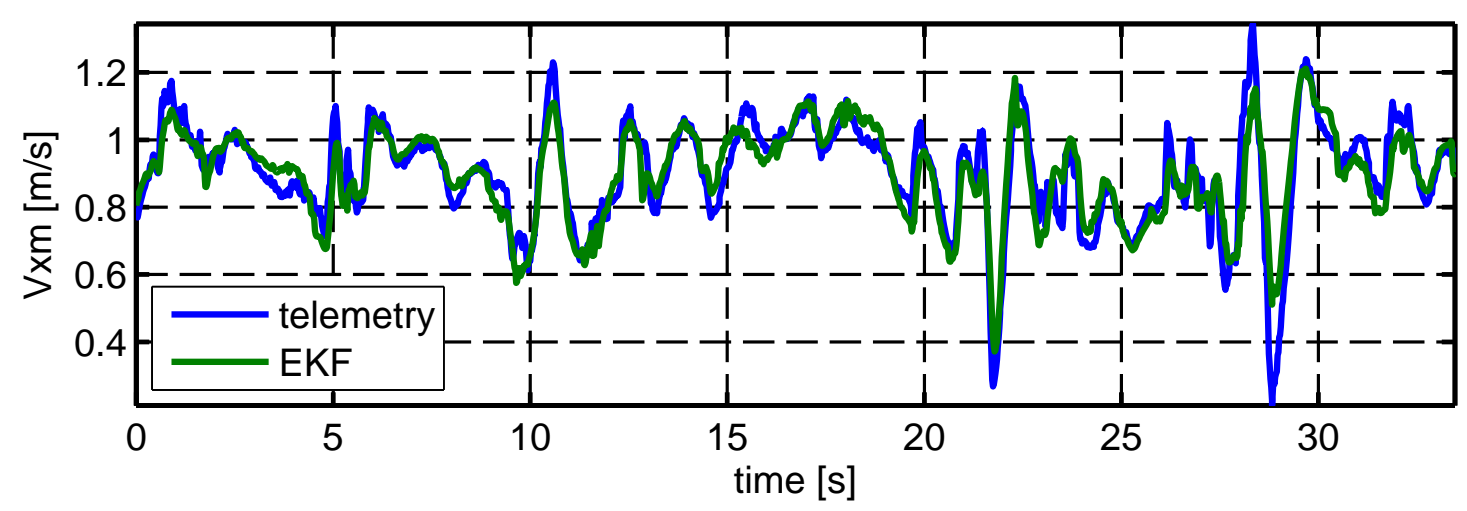

(a) Speed in body frame, frontal speed $v_{x m}$

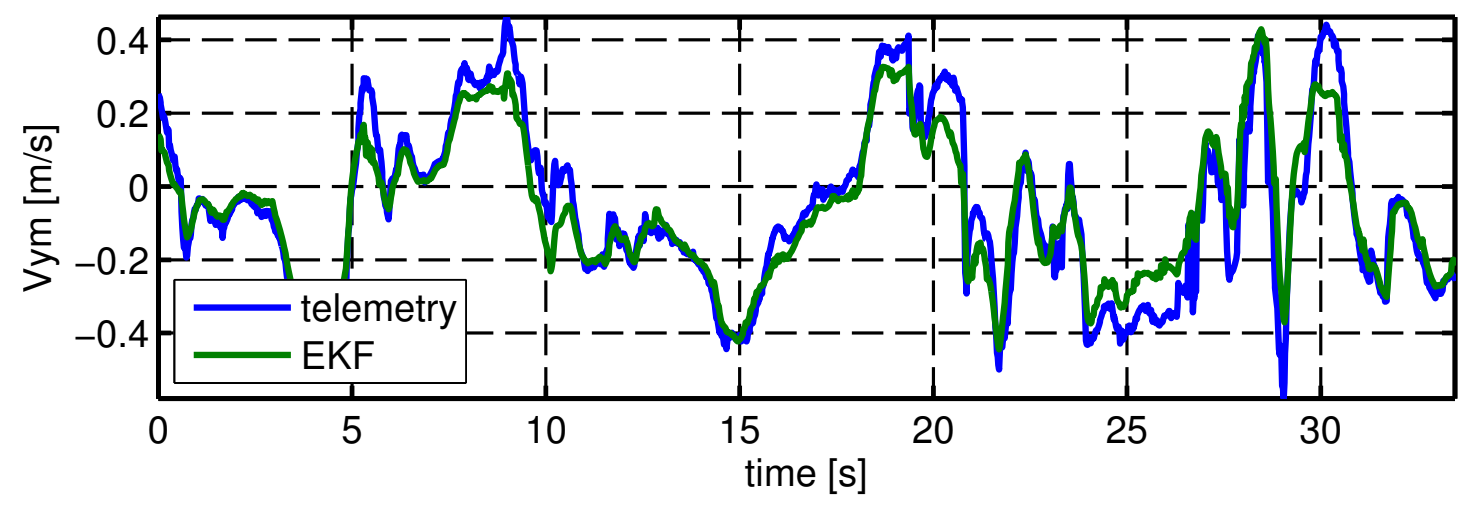

(b) Speed in body frame, laterial speed $v_{y m}$

Figure E.7: Comparison of the EKF using odometry and Vicon data, compared to experimental data, speed in the body frame. Both estimations, $v_{x m}$ and $v_{y m}$, are similar to the odometry data. It is interesting how incorporating more data on the filter (the Vicon data) is not changing visibly the speed estimation data.

stays below $0.5 \mathrm{~m}$ during all the test, which was 40 seconds long. Depending of the frequency with which the position measurements arrive the error can be smaller.

The speed estimation, shows good results, Fig. E.7. The graphs show the speed coordinates with respect to the body frame. On one hand, the EKF is managing to use the measurements correctly and providing an estimate based on them. On the other hand, it is not completely relying on the measurements and it is performing noise filtering. Both speed estimates, $v_{x m}$ and $v_{y m}$, are similar to the estimates obtained using odometry data only.

The position coordinates $\{x, y\}$, with respect to the world frame, and the altitude are shown in Fig E.8. It is clear that the position measurements increase the quality of the $X$ and $Y$ estimates. The estimation error is decreased every time that the measurements arrive. The estimation error increases between position samples, but at different rates depending on the maneuver that the drone is performing. This is due to the fact that the quality of the speed measurements is worse during turns than when the drone is navigating approximately straight on.

Finally, the attitude angles are shown in Fig. E.9 The pitch is much noisier than the roll estimation.

Again the estimation is good enough could be used to implement a controller using it as feedback. Taking into account the quality of the estimation, if the position measurements could arrive more frequently the multirotor could probably pass through narrow spaces, such as doors and hallways.

In the IMAV2012, a laser range finder (LRF) was used to derive position measurements. In order to obtain the position feedback from this sensor a particle filter was implemented, which relied on knowing the map, basically obstacles, located on the environment. The particle filter utilized as localization algorithm, with no mapping capabilities, is explained in the next section. 


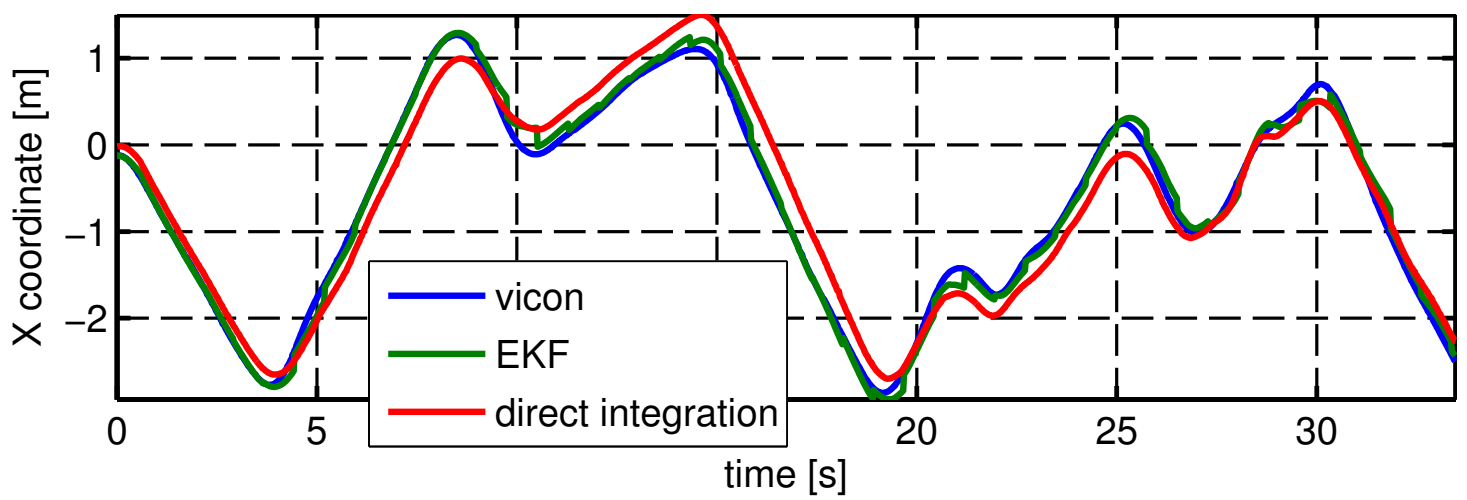

(a) $\mathrm{X}$ coordinate, in the world frame

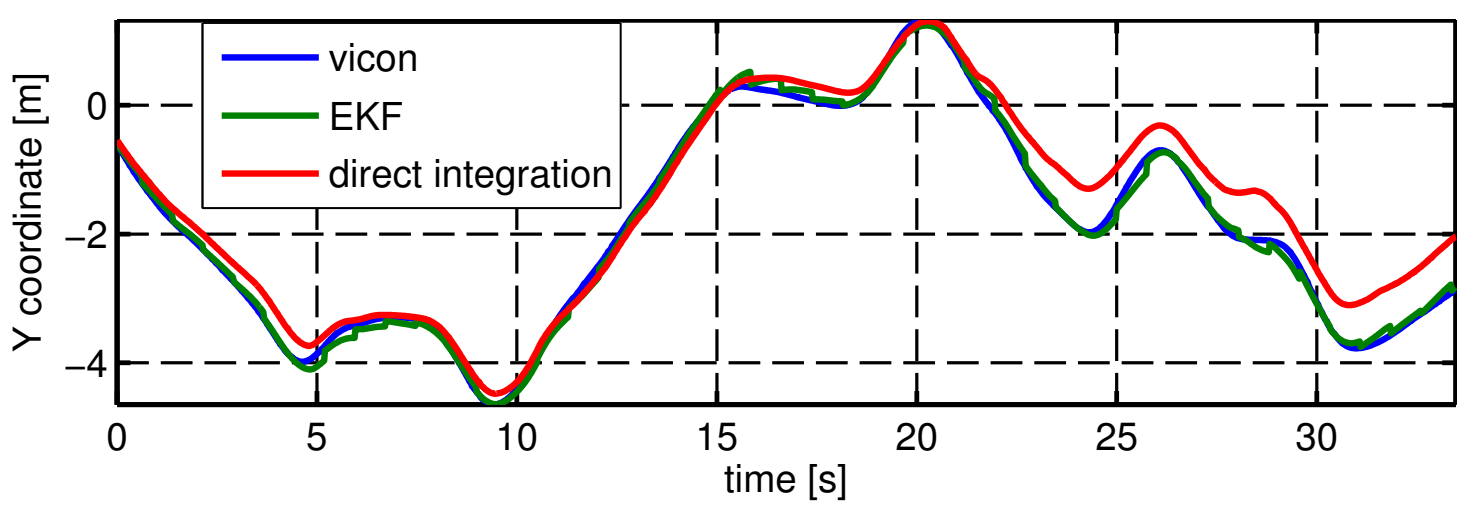

(b) Y coordinate, in the world frame

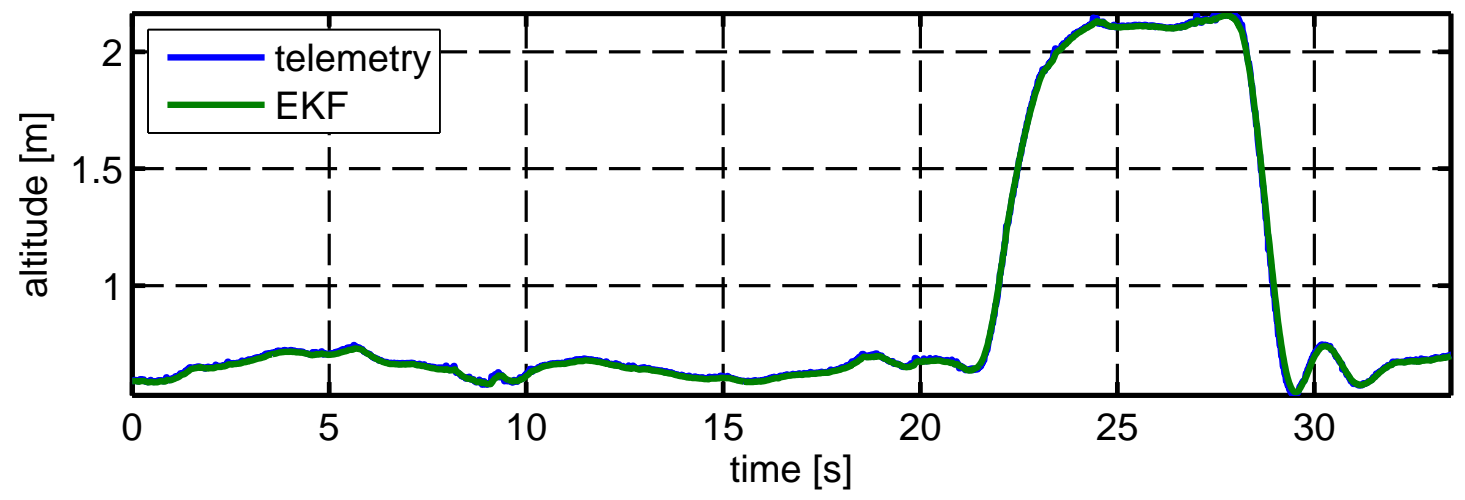

(c) Altitude

Figure E.8: Comparison of the EKF using odometry and Vicon data, compared to experimental data, position coordinates. It is clear that the position measurements increase a lot the quality of the $X$ and $Y$ estimates. The estimation error is decreased every time that the measurements arrive. The estimation error increases between position samples, but at different rates depending on the maneuver that the drone is performing. This is due to the fact that the quality of the speed measurements is worse during turns than when the drone navigates approximately straight on. It is interesting how incorporating the Vicon data is only changing the estimation at the position measurement update, and the trajectory between these updates is really similar, in shape, to the one estimated based on telemetry data only. 


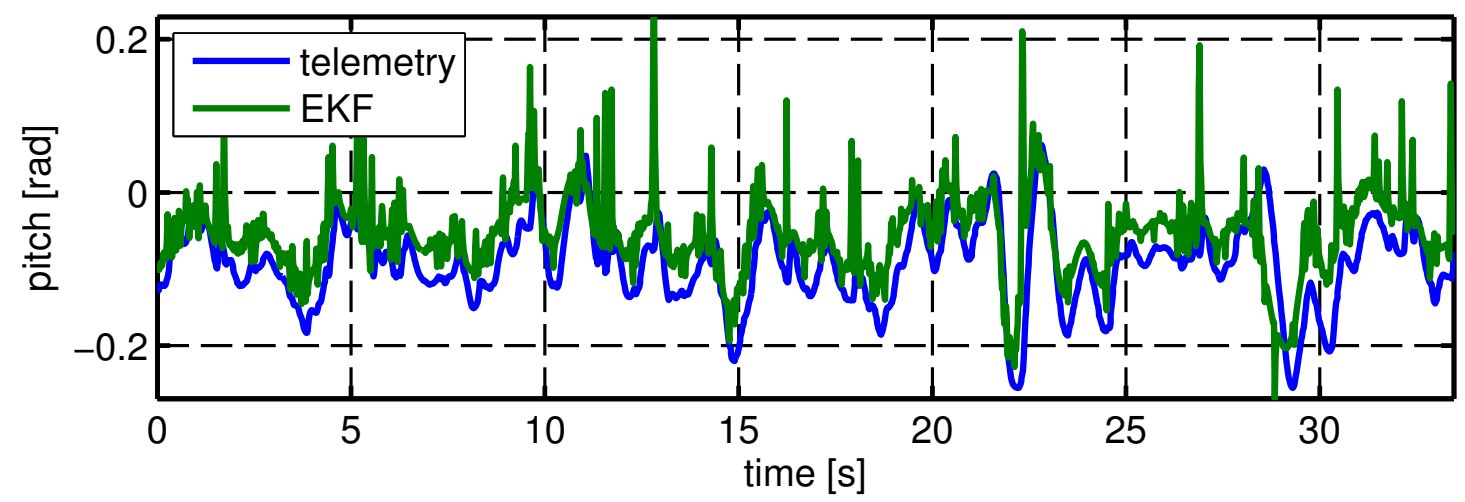

(a) Pitch angle

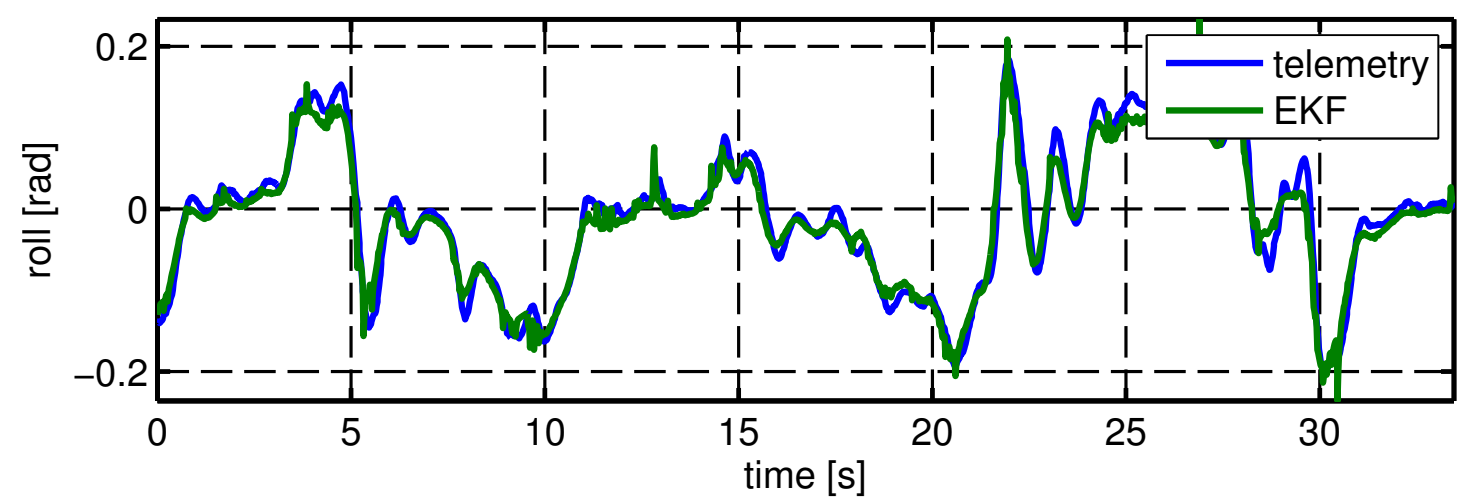

(b) Roll angle

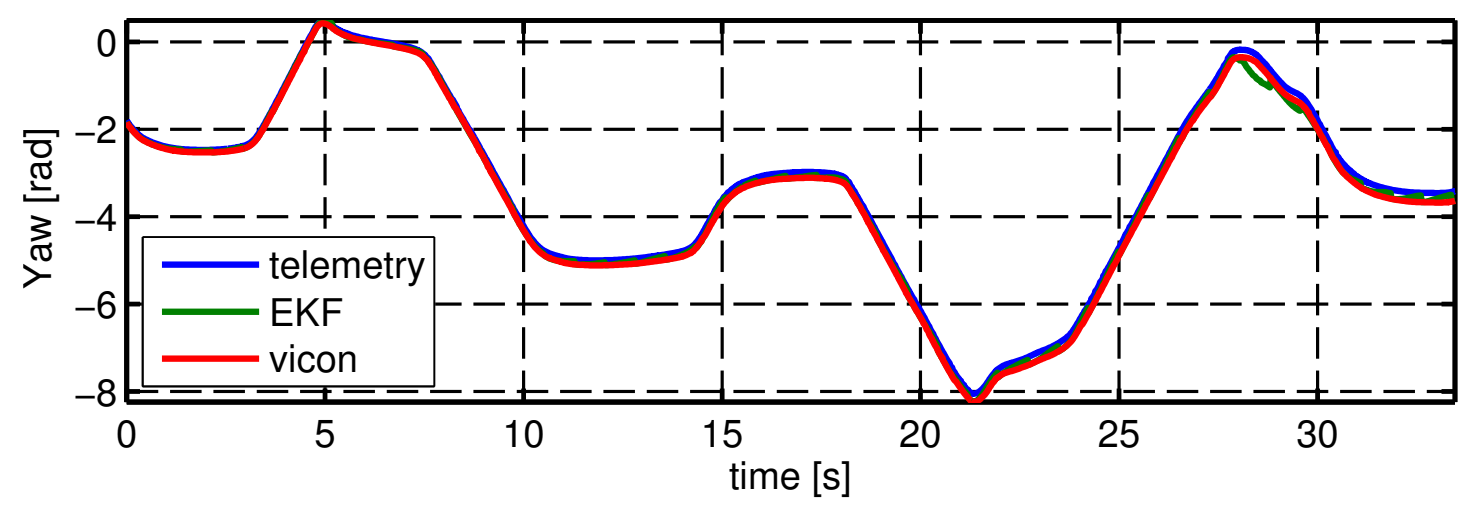

(c) Yaw angle

Figure E.9: Comparison of the EKF using odometry and Vicon data, compared to experimental data, attitude angles. The pitch estimation has an offset and it is noisy. The noise in the estimation is due to the control input, which was noisy for some reason (for instance, it could be a tuning problem of the frontal speed PID). The roll and the yaw estimation show good results. It is interesting how incorporating more data on the filter (the Vicon data) is not changing visibly the attitude estimation result. 


\section{E.7 Particle Filter}

Particle filters (PF) are recursive algorithms that use a sampling approach to the filtering problem (Koller et al., 2009, Russell et al., 2003), see Appx. E.3.1. They can be used to solve inference problems to estimate the state of a system over time. For instance they could be used to track the evolution of an illness in a patient. For this specific example, the filter would need a transition model that describes the evolution of the illness depending on various related variables; and a sensor model, that would relate the measurements (such as blood tests and the dose of medicines that the patient is taking) to the state of the patient.

Some important aspects of particle filters are:

- recursive filters need to have a way of representing the belief state, $\mathbf{P}\left(X_{t} \mid X_{1: t}, U_{1: t}\right)$. As we saw the Kalman Filter used a unimodal Gaussian distribution represented by the mean and the multivariate variance of the state variables. The PF does not make the Gaussian assumption, and it uses a population of 'particles' that keep track of the evolution of the system/robot. Each particle represents a hypothesis of the state of the system. In robotics, each particle would be a robot that can sense the environment. The particles are sometimes concentrated in separate groups, where each group would represent a possible position of the robot, with a mean value and a variance. This would represent a multimodal distribution of the state estimate, which cannot be considered using a traditional KF or EKF.

- in order to track the evolution of the system, the PF focus the set of samples/particles on the highprobability regions of the state space. To achieve this it throws away particles that are not probable, according to the measurements/observations.

- the computing time is kept manageable by bounding the total number of particles, $N_{p}$. This bounds the memory required to run the algorithm, as well as the approximate number of calculations per measurement cycle.

- The PF is based on the recursive filtering equations discussed in Appx. E.3.1, the prediction and measurement update Eqs. E.4 \& E.5.

The Particle Filter algorithm was chosen because it offers an elegant and effective way of processing LRF readings. These sensors use a laser to measure the distance from the sensor to obstacles in the environment. The direction of the laser ray is rotated in a periodic cycle using movable mirrors, thus giving a set of measurements corresponding to the distances along a horizontal plane to the surroundings of the robot. The LRF used in the present work was a Hokuyo URG-04LX-UG01 ${ }^{18}$

If the robot has a map of the environment, either a known or a learnt map; then it can compare the actual reading with the simulated measurements from each particle. If both are similar, then the particle would have a high probability or weight and would probably be replicated as part of the algorithm cycle. If they are not similar the particle would receive a low probability or weight and would probably be thrown away.

\section{E.7.1 The PF algorithm}

Particle filtering works as follows, see Alg. 6. first, a population of $N_{p}$ initial-state samples is created by sampling from the prior distribution $\mathbf{P}\left(X_{0}\right)$. Then, the update cycle is repeated for each time step:

1. Each particle is predicted forward in time by sampling its next state $x_{t+1}$, using its prior state $x_{t}$, by means of the state transition model $\mathbf{P}\left(X_{t+1} \mid x_{t}\right)$.

2. Each sample is weighted by the likelihood it assigns to the new measurements, $\mathbf{P}\left(O_{t+1} \mid x_{t+1}\right)$.

3. Then in a resampling process, a new population of $N_{p}$ samples is obtained. Each new particle is selected from the current population; the probability that a particular sample is selected is proportional to its weight. In the next measurement cycle, the particles are unweighted; the probability distribution is contained in the number of particles that there are around each region of the state space.

\footnotetext{
${ }^{18}$ http://www.hokuyo-aut.jp/02sensor/07scanner/urg_041x_ug01.html
} 


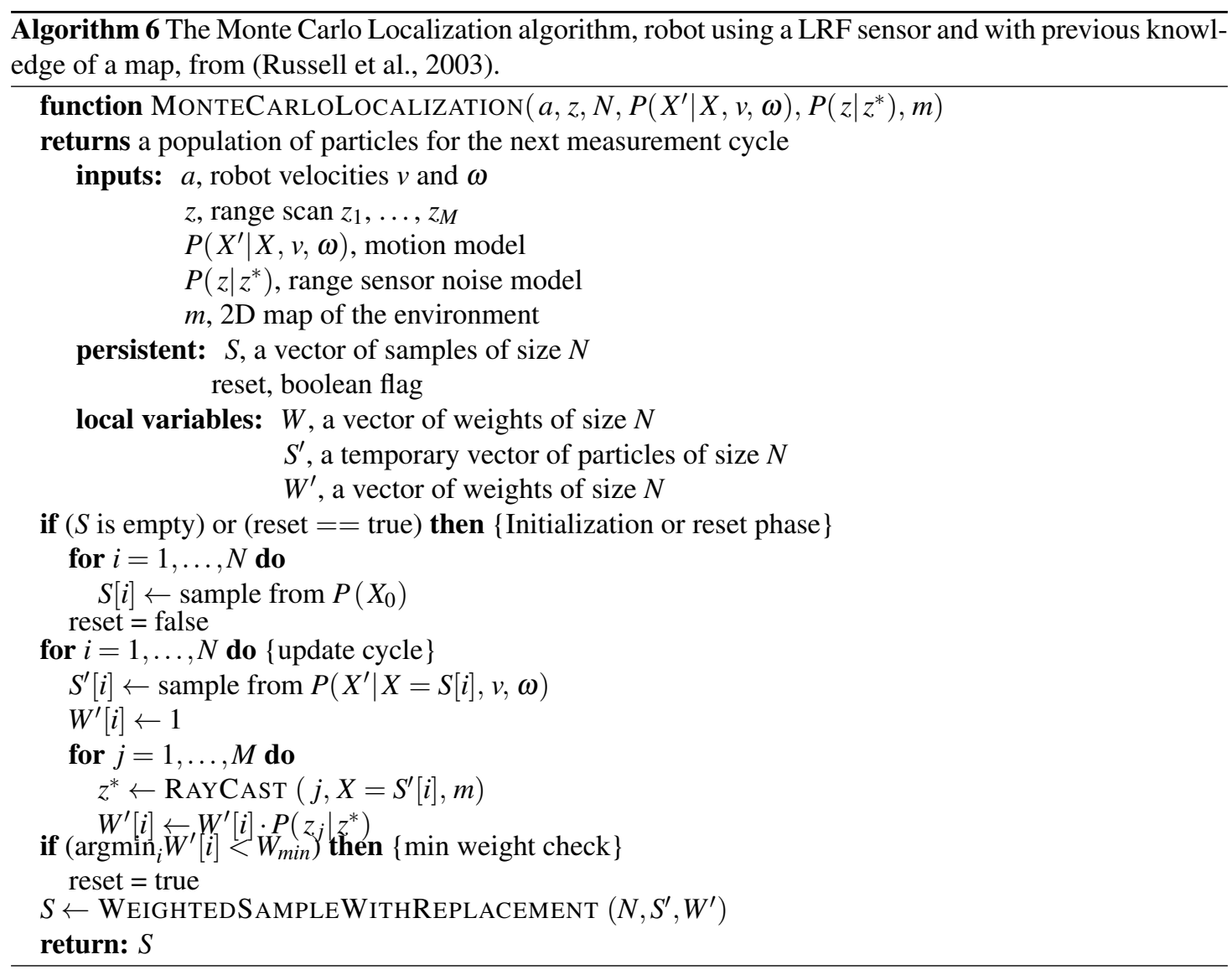

The Localization of a robot using this algorithm is called Monte Carlo Localization, MCL. It is an instance of the particle filter algorithm where the transition model and the sensor model are specified by the robot and its application. As explained before, LRF are easily modeled using a map of the robot surroundings, where the map can be learnt by the robot or known from before. The pseudocode of the MCL algorithm is shown in Alg. 6 .

\section{E.7.2 Testing the PF on experimental data}

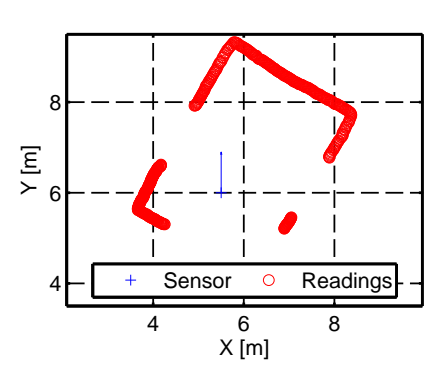

(a) Hokuyo readings

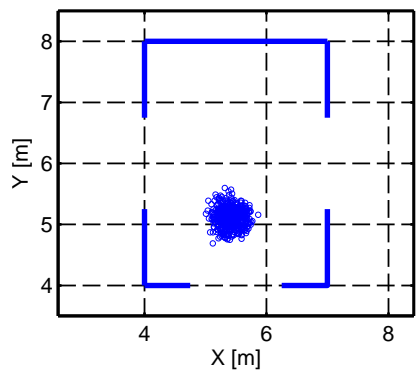

(b) Particle population

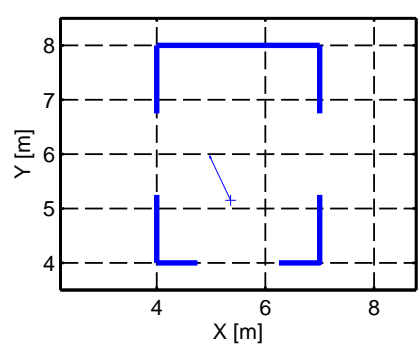

(c) Mean estimate

Figure E.10: Particle Filter on a real replica of the IMAV 2012 house, autonomy challenge. This figure shows how the Monte Carlo Localization algorithm is able to pass from a (a) laser scan, through a (b) sampling based on the particle population; to obtain (c) the mean estimated position of the sensor.

The International Micro Air Vehicle Conference and Flight Competition 2012 (IMAV 2012) competition comprises two indoors challenges. The main goal of the 'autonomy' challenge was to demonstrate 
the MAV's capabilities of fulfilling a sophisticated mission including the operation inside a small building with a covered ceiling. The small building was a one room house of $4 \times 3 \mathrm{~m}$ and $3 \mathrm{~m}$ of height. In order to make experimental tests beforehand, a full scale replica was constructed. Though, finally there was not enough time to make experimental flight tests inside the replica some measurements were taken using a Hokuyo Laser Range Finder (LRF). The data was acquired on a laptop, walking around the house with the sensor on hand. In some parts of the dataset, the sensor was moved intentionally to capture the wall above the windows and door, to be able to check the behavior of the algorithm in those cases.

In this section, using one of these datasets the results of the application of the produced implementation of the Monte Carlo Localization / Particle Filter algorithm are shown. A video is available on internet, Youtube video - Particle Filter ${ }^{19}$, where the results are shown in three graphs simultaneously. These graphs correspond to the ones shown in Fig. E.10, The left graph, (Fig.E.10(a)), shows the raw readings from the sensor, the middle one, (Fig. E.10(b)), shows the particle population of the PF, and the right graph, (Fig. E.10(c)), shows the weighted mean of the coordinates of the particles.

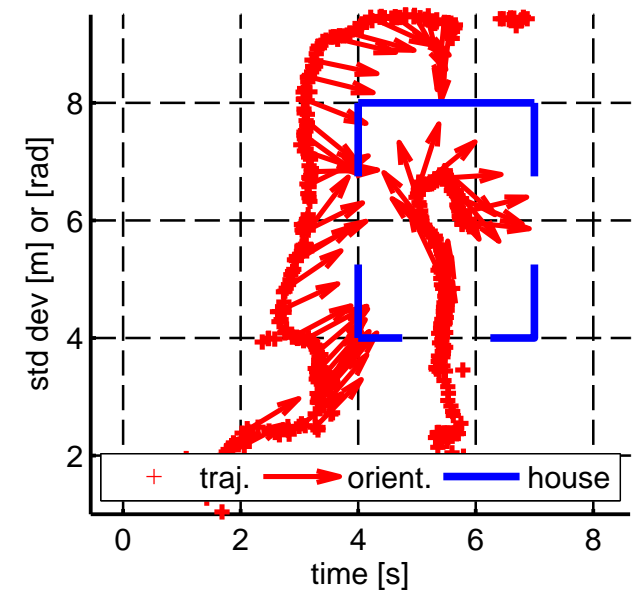

(a) Particle Filter, estimated trajectory in $\{x, y, \psi\}$

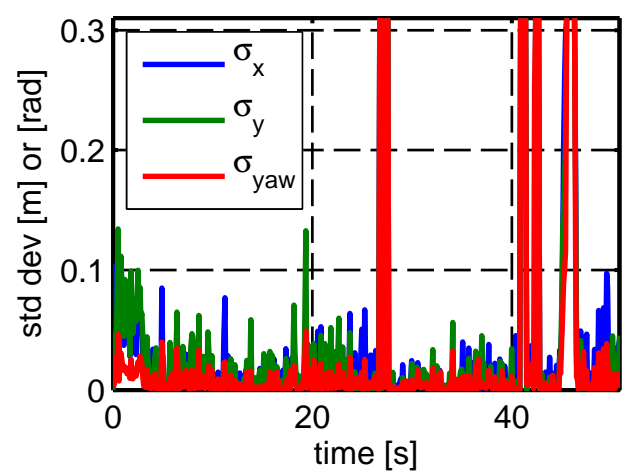

(b) Standard deviation of particle population

Figure E.11: Particle Filter on a real replica of the IMAV 2012 house, autonomy challenge. This figure shows the results using Monte Carlo Localization algorithm: (a) the trajectory estimation around the house, and (b) the standard deviation of the estimation. In the shown case, the estimation is wrong until the sensor enters the building, due to the house being symmetric on both sides. In practice, this could be avoided by starting the filter using an estimate of the initial position of the multicopter.

In Fig. E.11, the graph to the (Fig. E.11(a) left) shows the estimated trajectory of the sensor during the whole test. The graph to the (Fig. E.11(b), right) shows how the standard deviation of the estimation evolved over time.

During the acquisition, the Hokuyo LRF was looking almost always towards the house. First, the experimenter approached the house from the upper right corner of the house (with respect to how it is drawn in Fig. E.10p, then he walked to the front of the house beside the right wall, and then he entered the house through the door. As it is shown in the Fig. E.11 the particle filter estimated that the experimenter had approached the house from the lower left corner of the house, then that he walked beside the leftmost wall of the house, and then when he approached the upper wall; the filter reset the estimation. In the Alg. 6, there is a safeguard to reset the estimation when the measurements do not match well with any particle of the population. In this case the estimation is reseted, and the filter tries to locate the sensor as it did during the initialization.

Each time the standard deviation of the estimation, Fig. E.11(b) went higher than 0.3 was due to discrepancies between the measurement and the particle population:

- The first time, the filter estimated that the sensor was out of the house beyond the upper wall, but in reality the LRF was in front of the door.

\footnotetext{
${ }^{19} \mathrm{https} / / /$ youtu.be/FBx4DINpFJo
} 
- The second time, the PF assumed that he was lost because the sensor captured the upper part of the wall above the door, which is a discrepancy with regards to the map known by the filter. But in reality the estimation was correct. The PF reseted again.

- The last time the PF did not reset the estimation. The hokuyo was looking to to the area in front of the door, where there was nothing. The particles started to spread due to the lack of information in the reading. When the hokuyo looked again in the direction of the house, the particles converged again to the correct estimation without a reset.

In the IMAV2012 dynamics challenge the PF was used to locate the multirotor with respect to the vertical pylons. However, it is important to note that the measurement model used in this challenge and the dynamics challenge were different. In the dynamics challenge of the IMAV 2012, most of the space around the obstacles is empty. While the lack of a LRF measurement in both, simulated particle and real data, inside the house gives information about the position of the windows, in the dynamics challenge the same situation does not give any information. This required to use different measurement models, where the lack of measurement was treated differently. In the autonomy challenge, this event increased the weight of the particle; while in the dynamics challenge this event did not affect the weight of the particle. The weight threshold that resets the filter had to be selected manually for each challenge by evaluation the result on experimental data acquired from the replicas of the competition.

\section{E.8 Conclusions}

More details on the design and considerations leading to the development of the presented EKF and particle filter can be found in the MSc. thesis of the author (Pestana, 2012), where they were first presented. The EKF was implemented in collaboration with my colleague from the CVG (UPM) J. L. Sanchez-Lopez, who implemented a utilized EKF-library during his MSc. thesis (Sanchez Lopez, 2012).

The EKF based state estimator presented in this chapter, based on the model discussed in Appx. D.5\&E.5, requires horizontal speed estimates, for instance from the ground optical flow, in order to work properly. It differs from other research in that it fuses the attitude and altitude speeds commands and the filtered IMU measurements.

- In comparison to the work by Weiss et al. (S. Weiss, M. W. Achtelik, Chli, et al., 2012), they fuse the raw IMU measurements with visual-based pose measurements. These data are the direct readings from the three-axis accelerometers and gyroscopes from the drone's autopilot, and to be useful require the estimation of the IMU biases during flight.

- Recent research (Abeywardena et al., 2013; Leishman et al., 2014) has shown that the filtered IMU measurements in quadcopters are commonly biased due to the neglect of the propeller aerodynamic frictions which dominate all other aerodynamic frictions during normal multicopter flight.

- Arguably, there are better physical grounds to fuse the propeller thrust and torque commands than the attitude and altitude speed commands, but with the AR Drone 2.0 only the later commands $\left\{\theta_{c}, \phi_{c}, \frac{d z}{d t}, \frac{d \psi}{d t}{ }_{c}\right\}$ are available.

In spite of these differences, our odometry-based pose estimator has repeatedly allowed stable and precise quadrotor flight, and our research group has successfully used it in its participation on the International Micro Aerial Vehicle competitions, see Appx. II IMAV2012, IMAV2013 and IARC2014. The Particle Filter was specifically designed and only used to participate in the IMAV2012 competition. 


\section{E.9 Appendix Bibliography}

Abdul Shukor, S. (2013). "A geometrical-based approach to recognise structure of complex interiors". PhD thesis. University of Warwick (cit. on p. 159).

Abeywardena, D., Kodagoda, S., Dissanayake, G., and Munasinghe, R. (2013). "Improved State Estimation in Quadrotor MAVs: A Novel Drift-Free Velocity Estimator". IEEE Robotics \& Automation Magazine 20.4, pp. 32-39 (cit. on pp. 163, 181).

Achtelik, M. W., Lynen, S., Chli, M., and Siegwart, R. (2013). "Inversion based direct position control and trajectory following for micro aerial vehicles". In: Intelligent Robots and Systems (IROS), 2013 IEEE/RSJ International Conference on. IEEE, pp. 2933-2939 (cit. on pp. 162, 163).

Achtelik, M. W., Lynen, S., Weiss, S., Kneip, L., Chli, M., and Siegwart, R. (2012). "Visual-inertial SLAM for a small helicopter in large outdoor environments". In: Intelligent Robots and Systems (IROS), 2012 IEEE/RSJ International Conference on. IEEE, pp. 2651-2652 (cit. on p. 160).

Arulampalam, M. S., Maskell, S., Gordon, N., and Clapp, T. (2002). "A tutorial on particle filters for online nonlinear/non-Gaussian Bayesian tracking”. Signal Processing, IEEE Transactions on 50.2, pp. 174-188 (cit. on p. 162,.

Bachrach, A. G. (2009). "Autonomous flight in unstructured and unknown indoor environments". MA thesis. Massachusetts Institute of Technology (cit. on p. 159).

Bachrach, A., Prentice, S., He, R., and Roy, N. (2011). "RANGE-Robust autonomous navigation in GPSdenied environments”. Journal of Field Robotics 28.5, pp. 644-666 (cit. on p. 159).

Barfoot, T., Forbes, J. R., and Furgale, P. T. (2011). "Pose estimation using linearized rotations and quaternion algebra". Acta Astronautica 68.1, pp. 101-112 (cit. on p. 162).

Bills, C., Chen, J., and Saxena, A. (2011). "Autonomous MAV flight in indoor environments using single image perspective cues”. In: Robotics and Automation (ICRA), 2011 IEEE International Conference on, pp. 5776-5783 (cit. on p. 159.

Bouabdallah, Samir, Murrieri, Pierpaolo, and Siegwart, Roland (2005). "Towards Autonomous Indoor Micro VTOL”. Autonomous Robots 18 (2). 10.1007/s10514-005-0724-z, pp. 171-183 (cit. on p. 159.

Bouabdallah, Samir and Siegwart, Roland (2007). "Full control of a quadrotor". In: Intelligent robots and systems, 2007. IROS 2007. IEEE/RSJ international conference on. IEEE, pp. 153-158 (cit. on p. 163.

Breckenridge, W. (1999). "Quaternions proposed standard conventions". Jet Propulsion Laboratory, Pasadena, CA, Interoffice Memorandum IOM, pp. 343-79 (cit. on p.162).

Burschka, D. and Mair, E. (2008). "Direct pose estimation with a monocular camera". In: Robot Vision. Springer, pp. 440-453 (cit. on p. 161).

Cheng, Y. and Crassidis, J. L. (2004). "Particle filtering for sequential spacecraft attitude estimation". In: Proc. of the 2004 AIAA Guidance, Navigation, and Control Conference, p. 5337 (cit. on p. 162).

Crassidis, J. L. (2006). "Sigma-point Kalman filtering for integrated GPS and inertial navigation". Aerospace and Electronic Systems, IEEE Transactions on 42.2, pp. 750-756 (cit. on p. 163).

Crassidis, J. L., Markley, F. L., and Cheng, Y. (2007). "Survey of nonlinear attitude estimation methods". Journal of Guidance, Control, and Dynamics 30.1, pp. 12-28 (cit. on p.162).

Mellado Bataller, I. (2012). A new framework for interfacing with MAVs. https : / / github . com/ uavster/mavwork (cit. on p. 169).

Davison, A. J. (1998). "Mobile Robot Navigation Using Active Vision”. PhD thesis. University of Oxford (cit. on p. 160).

Davison, R. A. N. and J., A. (2010). "Live Dense Reconstruction with a Single Moving Camera". In: Computer Vision and Pattern Recognition (cit. on p. 160).

Doucet, A., De Freitas, N., and Gordon, N. (2001). An introduction to sequential Monte Carlo methods. Springer (cit. on p.162).

Dunbar, N. and Franz, R. (2003). "Online control customization via optimization, based control". Software-Enabled Control: Information Technology for Dynamical Systems, p. 149 (cit. on p. 162).

Engel, Jakob, Sturm, Jürgen, and Cremers, Daniel (2014). "Scale-aware navigation of a low-cost quadrocopter with a monocular camera". Robotics and Autonomous Systems 62.11, pp. 1646-1656 (cit. on p. 160 .

Engel, J., Schöps, T., and Cremers, D. (2014). "LSD-SLAM: Large-scale direct monocular SLAM". In: Computer Vision-ECCV 2014. Springer, pp. 834-849 (cit. on p. 161). 
Engel, J., Sturm, J., and Cremers, D. (2013). "Semi-dense visual odometry for a monocular camera". In: Computer Vision (ICCV), 2013 IEEE International Conference on. IEEE, pp. 1449-1456 (cit. on p. 161,.

Engel, J., Sturm, J., and Cremers, D. (2012). "Accurate figure flying with a quadrocopter using onboard visual and inertial sensing". IMU 320, p. 240 (cit. on p. 160).

Farrell, J. L. (1970). “Attitude determination by Kalman filtering”. Automatica 6.3, pp. 419-430 (cit. on p. 162,

Farrenkopf, R. (1978). "Analytic steady-state accuracy solutions for two common spacecraft attitude estimators". Journal of Guidance, Control, and Dynamics 1.4, pp. 282-284 (cit. on p. 163).

Fliess, M., Lévine, J., Martin, P., and Rouchon, P. (1995). "Flatness and defect of non-linear systems: introductory theory and examples". International journal of control 61.6, pp. 1327-1361 (cit. on p. 162.

Forster, C., Lynen, S., Kneip, L., and Scaramuzza, D. (2013). "Collaborative monocular SLAM with multiple micro aerial vehicles". In: Intelligent Robots and Systems (IROS), 2013 IEEE/RSJ International Conference on. IEEE, pp. 3962-3970 (cit. on p. 161).

Forster, C., Pizzoli, M., and Scaramuzza, D. (2014). "SVO: Fast Semi-Direct Monocular Visual Odometry". In: IEEE International Conference on Robotics and Automation (ICRA) (cit. on p. 161).

Fritsch, O., De Monte, P., Buhl, M., and Lohmann, B. (2012). "Quasi-static feedback linearization for the translational dynamics of a quadrotor helicopter". In: American Control Conference (ACC), 2012. IEEE, pp. 125-130 (cit. on p. 162).

Fu, C., Olivares-Mendez, M. A., Campoy, P., and Suarez-Fernandez, R. (2013). "UAS see-and-avoid strategy using a fuzzy logic controller optimized by Cross-Entropy in Scaling Factors and Membership Functions". In: Unmanned Aircraft Systems (ICUAS), 2013 International Conference on. IEEE, pp. 532-541 (cit. on p. 161.

Furrer, F., Burri, M., Achtelik, M., and Siegwart, R. (2016). "RotorS-A Modular Gazebo MAV Simulator Framework". In: Robot Operating System (ROS). Springer, pp. 595-625 (cit. on p. 162).

Goel, R., Shah, S. M., Gupta, N. K., and Ananthkrishnan, N. (2009). "Modeling, simulation and flight testing of an autonomous quadrotor". Proceedings of ICEAE, pp. 1-7 (cit. on p. 163).

Hartley, R. I. and Zisserman, A. (2004). Multiple View Geometry in Computer Vision. Second. Cambridge University Press, ISBN: 0521540518 (cit. on p. 160).

Henry, P., Krainin, M., Herbst, E., Ren, X., and Fox, D. (2010). "RGB-D Mapping : Using Depth Cameras for Dense 3D Modeling of Indoor Environments”. In: International Symposium on Experimental Robotics (ISER), 2010 (cit. on p. 160).

Hoffmann, G. M., Huang, H., Waslander, S. L., and Tomlin, C. J. (2007). "Quadrotor helicopter flight dynamics and control: Theory and experiment". In: Proc. of the AIAA Guidance, Navigation, and Control Conference. Vol. 2 (cit. on p. 163).

Huang, A. S., Bachrach, A., Henry, P., Krainin, M., Fox, D., and Roy, N. (2011). "Visual Odometry and Mapping for Autonomous Flight Using an RGB-D Camera". Camera (cit. on p. 160).

Huang, H., Hoffmann, G. M., Waslander, S. L., and Tomlin, C. J. (2009). "Aerodynamics and control of autonomous quadrotor helicopters in aggressive maneuvering”. In: Robotics and Automation, 2009. ICRA'09. IEEE International Conference on. IEEE, pp. 3277-3282 (cit. on p. 163).

Julier, S. J., Uhlmann, J. K., and Durrant-Whyte, H. F. (1995). "A new approach for filtering nonlinear systems”. In: American Control Conference, Proceedings of the 1995. Vol. 3. IEEE, pp. 1628-1632 (cit. on pp.161,162).

Klein, G. and Murray, D. (2007). "Parallel Tracking and Mapping for Small AR Workspaces". In: 2007 6th IEEE and ACM International Symposium on Mixed and Augmented Reality, pp. 1-10 (cit. on p. 160 .

Klein, G. and Murray, D. (2009). "Parallel Tracking and Mapping on a camera phone". In: 2009 8th IEEE International Symposium on Mixed and Augmented Reality, pp. 83-86 (cit. on p. 160).

Koller, D. and Friedman, N. (2009). Probabilistic Graphical Models: Principles and Techniques. MIT Press (cit. on pp. 158, 163, 178,.

Kuipers, J. (2002). Quaternions and Rotation Sequences: A Primer with Applications to Orbits, Aerospace and Virtual Reality (cit. on p. 162).

Leberl, F., Irschara, A., Pock, T., Meixner, P., Gruber, M., Scholz, S., Wiechert, A., and Scholz, S. (2010). "Point Clouds : Lidar versus 3D Vision". Photogrammetric Engineering \& Remote Sensing 76.10 (cit. on p. 160. 
Lefferts, E. J., Markley, F. L., and Shuster, M. D. (1982). "Kalman filtering for spacecraft attitude estimation”. Journal of Guidance, Control, and Dynamics 5.5, pp. 417-429 (cit. on p. 162).

Leishman, R. C., Macdonald, J., Beard, R. W., and McLain, T. W. (2014). "Quadrotors and accelerometers: State estimation with an improved dynamic model". Control Systems, IEEE 34.1, pp. 28-41 (cit. on pp.163,181).

Leutenegger, S., Chli, M., and Siegwart, R. Y. (2011). "BRISK: Binary robust invariant scalable keypoints”. In: Computer Vision (ICCV), 2011 IEEE International Conference on. IEEE, pp. 2548-2555 (cit. on p. 161).

Leutenegger, S., Furgale, P. T., Rabaud, V., Chli, M., Konolige, K., and Siegwart, R. (2013). "KeyframeBased Visual-Inertial SLAM using Nonlinear Optimization.” In: Robotics: Science and Systems (cit. on p.161.

Leutenegger, S., Lynen, S., Bosse, M., Siegwart, R., and Furgale, P. (2014). "Keyframe-based visualinertial odometry using nonlinear optimization". The International Journal of Robotics Research, p. 0278364914554813 (cit. on p. 161).

Li, M. and Mourikis, A. I. (2013). "High-Precision, Consistent EKF-based Visual-Inertial Odometry". International Journal of Robotics Research 32.6, pp. 690-711 (cit. on p. 161).

Li, M. and Mourikis, A. I. (2014a). "Online Temporal Calibration for Camera-IMU Systems: Theory and Algorithms”. International Journal of Robotics Research 33.7, pp. 947-964 (cit. on p. 161).

Li, M. and Mourikis, A. I. (2014b). "Vision-aided Inertial Navigation with Rolling-Shutter Cameras". International Journal of Robotics Research. in print (cit. on p. 161).

Lynen, S., Achtelik, M. W., Weiss, S., Chli, M., and Siegwart, R. (2013). "A robust and modular multisensor fusion approach applied to mav navigation”. In: Intelligent Robots and Systems (IROS), 2013 IEEE/RSJ International Conference on. IEEE, pp. 3923-3929 (cit. on p. 160).

Markley, F. L. (2003). “Attitude error representations for Kalman filtering”. Journal of guidance, control, and dynamics 26.2, pp. 311-317 (cit. on p. 162).

Matsue, A., Hirosue, W., Tokutake, H., Sunada, S., and Ohkura, A. (2005). "Navigation of Small and Lightweight Helicopter". Transactions of the Japan Society for Aeronautical and Space Sciences 48.161, pp. 177-179 (cit. on p. 159).

Mayol, W., Davison, A., and Tordoff, B. (2004). "Interaction between hand and wearable camera in 2D and 3D environments". In: British Machine Vision Conference (cit. on p. 160).

Mellinger, D. and Kumar, V. (2011). "Minimum snap trajectory generation and control for quadrotors". In: Robotics and Automation (ICRA), 2011 IEEE International Conference on. IEEE, pp. 2520-2525 (cit. on p. 162).

Meyer, J., Sendobry, A., Kohlbrecher, S., Klingauf, U., and Stryk, O. von (2012). "Comprehensive simulation of quadrotor uavs using ros and gazebo". In: Simulation, Modeling, and Programming for Autonomous Robots. Springer, pp. 400-411 (cit. on pp. 162, 163).

Michael, N., Shen, S., Mohta, K., Mulgaonkar, Y., Kumar, V., Nagatani, K., Okada, Y., Kiribayashi, S., Otake, K., Yoshida, K., et al. (2012). "Collaborative mapping of an earthquake-damaged building via ground and aerial robots". Journal of Field Robotics 29.5, pp. 832-841 (cit. on p. 159.).

Misawa, E. and Hedrick, J. (1989). "Nonlinear observers - a state-of-the-art survey". Journal of dynamic systems, measurement, and control 111.3, pp. 344-352 (cit. on p.162).

Molton, N., Davison, A., and Reid, I. (2004). "Locally Planar Patch Features for Real-Time Structure from Motion”. British Machine Vision Conference, 2004, pp. 90.1-90.10 (cit. on p. 160).

Mourikis, A. I. and Roumeliotis, S. I. (2006). A Multi-State Constraint Kalman Filter for Vision-aided Inertial Navigation. Tech. rep. www.cs.umn.edu/ mourikis/tech_reports/TR_MSCKF.pdf. Dept. of Computer Science and Engineering, University of Minnesota (cit. on p. 161).

Mourikis, A. I. and Roumeliotis, S. I. (2007). "A multi-state constraint Kalman filter for vision-aided inertial navigation". In: Robotics and Automation, 2007 IEEE International Conference on. IEEE, pp. 3565-3572 (cit. on p. 161).

Mourikis, A. I., Trawny, N., Roumeliotis, S. I., Johnson, A. E., Ansar, A., and Matthies, L. (2009). "Vision-aided inertial navigation for spacecraft entry, descent, and landing". Robotics, IEEE Transactions on 25.2, pp. 264-280 (cit. on p. 161).

Murray, R. M., Rathinam, M., and Sluis, W. (1995). "Differential flatness of mechanical control systems: A catalog of prototype systems". In: ASME International Mechanical Engineering Congress and Exposition. Citeseer (cit. on p. 162). 
Murrell, J. W. (1978). "Precision attitude determination for multimission spacecraft". AIAA paper 78, p. 1248 (cit. on p. 162).

Nikolic, J., Rehder, J., Burri, M., Gohl, P., Leutenegger, S., Furgale, P. T., and Siegwart, R. (2014). “A synchronized visual-inertial sensor system with FPGA pre-processing for accurate real-time SLAM". In: Robotics and Automation (ICRA), 2014 IEEE International Conference on. IEEE, pp. 431-437 (cit. on p.161).

Nister, D. and Stewenius, H. (2006). "Scalable Recognition with a Vocabulary Tree". In: 2006 IEEE Computer Society Conference on Computer Vision and Pattern Recognition - Volume 2 (CVPR'06). Ieee, pp. 2161-2168 (cit. on p. 161).

NøRgaard, M., Poulsen, N. K., and Ravn, O. (2000). "New developments in state estimation for nonlinear systems". Automatica 36.11, pp. 1627-1638 (cit. on pp. 161,162).

Nützi, G., Weiss, S., Scaramuzza, D., and Siegwart, R. (2011). "Fusion of IMU and vision for absolute scale estimation in monocular SLAM". Journal of intelligent \& robotic systems $61.1-4$, pp. 287-299 (cit. on p. 160).

Oshman, Y. and Carmi, A. (2004). "Estimating attitude from vector observations using a genetic algorithm-embedded quaternion particle filter". In: AIAA Guidance, Navigation, and Control Conference and Exhibit. AIAA Reston, VA, pp. 1-24 (cit. on p. 162).

Park, M. (2004). Error analysis and stochastic modeling of MEMS based inertial sensors for land vehicle navigation applications. University of Calgary, Department of Geomatics Engineering (cit. on p. 163 ).

Pestana, J. (2012). "On-board control algorithms for Quadrotors and indoors navigation". MA thesis. Spain: Universidad Politécnica de Madrid (cit. on p. 181).

Psiaki, M. L. (2000). “Attitude-determination filtering via extended quaternion estimation”. Journal of Guidance, Control, and Dynamics 23.2, pp. 206-214 (cit. on p. 162).

Richter, C., Bry, A., and Roy, N. (2013). "Polynomial trajectory planning for quadrotor flight". In: International Conference on Robotics and Automation (cit. on p. 162).

Roberts, J. F., Stirling, T., Zufferey, J.-C., and Floreano, D. (2007). "Quadrotor Using Minimal Sensing For Autonomous Indoor Flight”. In: European Micro Air Vehicle Conference and Flight Competition (EMAV2007). Toulouse, France (cit. on p. 159).

Rudy, N. (2003). "Robot Localization and Kalman Filters". MA thesis. Netherlands: Utrecht University (cit. on pp. 158, 163 .

Russell, S. and Norvig, P. (2003). Artificial Intelligence: A Modern Approach. 3rd edition. Prentice-Hall, Englewood Cliffs, NJ (cit. on pp. 158, 163, 178, 179).

Sanchez Lopez, J. L. (2012). "Path Following Control System for car-like Unmanned Ground Systems". MA thesis. Spain: Universidad Politécnica de Madrid (cit. on pp. 169,181.

Scaramuzza, D., Achtelik, M., Doitsidis, L., Fraundorfer, F., Kosmatopoulos, E., Martinelli, A., et al. (2013). "Vision-controlled micro flying robots: from system design to autonomous navigation and mapping in gps-denied environments". IEEE Robotics and Automation Magazine (cit. on p. 160.

Schmidt, S. F. (1981). "The Kalman filter-Its recognition and development for aerospace applications". Journal of Guidance, Control, and Dynamics 4.1, pp. 4-7 (cit. on p.162,.

Schoellig, A. P., Wiltsche, C., and D'Andrea, R. (2012). "Feed-forward parameter identification for precise periodic quadrocopter motions". In: American Control Conference (ACC), 2012. IEEE, pp. 43134318 (cit. on p. 162).

Shen, Shaojie, Mulgaonkar, Yash, Michael, Nathan, and Kumar, Vijay (2013). "Vision-based state estimation for autonomous rotorcraft mavs in complex environments". In: Robotics and Automation (ICRA), 2013 IEEE International Conference on. IEEE, pp. 1758-1764 (cit. on p. 161).

Shen, S., Michael, N., and Kumar, V. (2011). "Autonomous multi-floor indoor navigation with a computationally constrained MAV". Robotics and Automation (ICRA), 2011 IEEE International Conference on, pp. 20-25 (cit. on p. 159).

Shen, S. and Michael, N. (2013). "State estimation for indoor and outdoor operation with a micro-aerial vehicle”. In: Experimental Robotics. Springer, pp. 273-288 (cit. on p. 159].

Shen, S., Mulgaonkar, Y., Michael, N., and Kumar, V. (2013). "Vision-Based State Estimation and Trajectory Control Towards High-Speed Flight with a Quadrotor.” In: Robotics: Science and Systems. Citeseer (cit. on p.161).

Shuster, M. D. (1993). "A survey of attitude representations". Navigation 8.9 (cit. on p. 162).

Shuster, M. D. and Oh, S. (1981). "Three-axis attitude determination from vector observations". Journal of Guidance, Control, and Dynamics 4.1, pp. $70-77$ (cit. on p.162. 
Sola, J. (2012). "Quaternion kinematics for the error-state KF”. Laboratoire d'Analyse et d'Architecture des Systemes-Centre national de la recherche scientifique (LAAS-CNRS), Toulouse, France, Tech. Rep (cit. on p. 162).

Strasdat, H., Montiel, J., and Davison, A. (2010). "Real-time monocular SLAM: Why filter?” In: Robotics and Automation (ICRA), 2010 IEEE International Conference on, pp. 2657-2664 (cit. on p. 160).

Thrun, S. (2002). "Robotic Mapping: A Survey". In: Exploring Artificial Intelligence in the New Millenium. Ed. by G. Lakemeyer and B. Nebel. Morgan Kaufmann (cit. on pp. 158, 159,163,

Trawny, N. and Roumeliotis, S. I. (2005). "Indirect Kalman filter for 3D attitude estimation". University of Minnesota, Dept. of Comp. Sci. \& Eng., Tech. Rep 2 (cit. on p. 162).

Van Nieuwstadt, M. J. and Murray, R. M. (1998). "Real-time trajectory generation for differentially flat systems". International Journal of Robust and Nonlinear Control 8.11, pp. 995-1020 (cit. on p. 162,.

Wan, E. A. and Van Der Merwe, R. (2001). "The unscented Kalman filter". Kalman filtering and neural networks, pp. 221-280 (cit. on pp. 161, 162,

Weiss, S. M. (2012). "Vision based navigation for micro helicopters". PhD thesis. Diss., Eidgenössische Technische Hochschule ETH Zürich, Nr. 20305, 2012 (cit. on p. 160p.

Weiss, S., Achtelik, M. W., Chli, M., and Siegwart, R. (2012). "Versatile distributed pose estimation and sensor self-calibration for an autonomous MAV". In: Robotics and Automation (ICRA), 2012 IEEE International Conference on. IEEE, pp. 31-38 (cit. on pp. 160,181.

Weiss, S., Achtelik, M. W., Lynen, S., Chli, M., and Siegwart, R. (2012). "Real-time onboard visualinertial state estimation and self-calibration of mavs in unknown environments". In: Robotics and Automation (ICRA), 2012 IEEE International Conference on. IEEE, pp. 957-964 (cit. on p. 160).

Weiss, S., Scaramuzza, D., and Siegwart, R. (2011). "Monocular-SLAM-based navigation for autonomous micro helicopters in GPS-denied environments". Journal of Field Robotics 28.6, pp. 854874 (cit. on p. 160 ).

Weiss, S. and Siegwart, R. (2011). "Real-time metric state estimation for modular vision-inertial systems". In: Robotics and Automation (ICRA), 2011 IEEE International Conference on. IEEE, pp. 4531-4537 (cit. on p. 160).

Wendel, A., Irschara, A., and Bischof, H. (2011). "Natural Landmark - based Monocular Localization for MAVs". In: IEEE International Conference on Robotics and Automation (ICRA 2011) (cit. on p. 161).

Wendel, A., Maurer, M., Irschara, A., and Bischof, H. (2011). "3D Vision Applications for MAVs: Localization and Reconstruction". In: International Symposium on 3D Data Processing, Visualization and Transmission (3DPVT), 2011, pp. 3-4 (cit. on p. 161).

Winkvist, S. (2013). "Low computational SLAM for an autonomous indoor aerial inspection vehicle". PhD thesis. University of Warwick (cit. on p. 159).

Winkvist, S., Rushforth, E., and Young, K. (2013). “Towards an autonomous indoor aerial inspection vehicle”. Industrial Robot: An International Journal 40.3, pp. 196-207 (cit. on p. 159). 


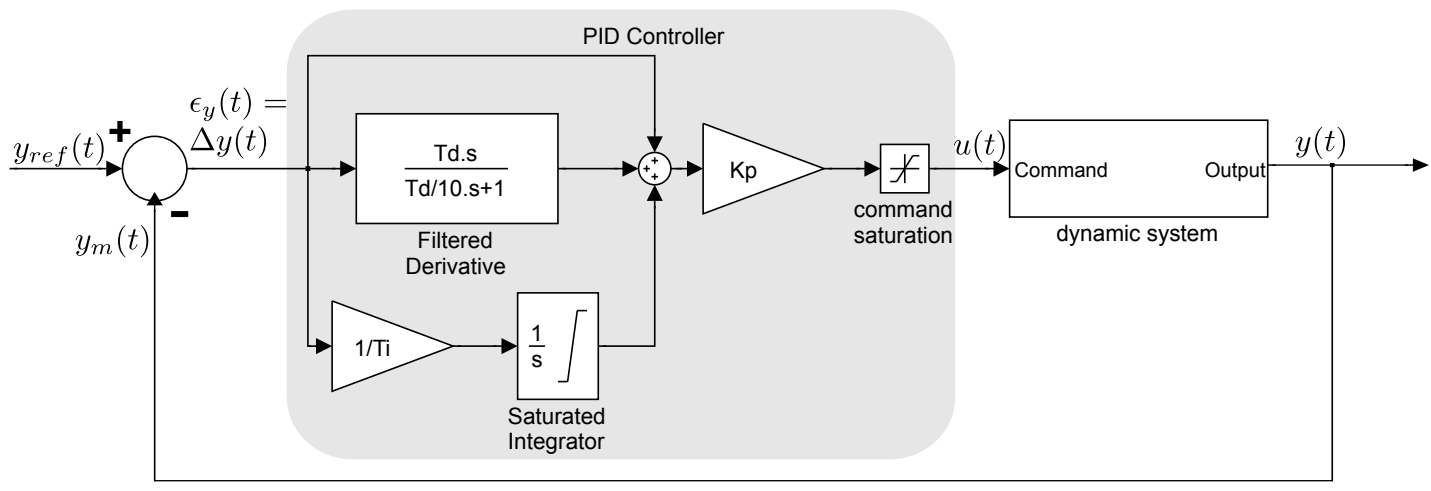

Figure F.1: Proportional-Integral-Derivative (PID) controller block diagram. The figure shows the usual feedback loop architecture for a Single Input Single Output (SISO) dynamic system. The PID has three additional features: filtering of the derivative action, anti-windup of the integral action, and saturation of the command output.

PIDs are widely used in industry, for a variety of control problems. The Fig. F.1 shows a dynamic system commanded using a feedback loop architecture with a PID controller. The variables shown in the figure are the magnitude that is sought to be automatically controlled $y(t)$, its measurement $y_{m}(t)$ and its desired reference value $y_{r e f}(t)$; and the command variable $u(t)$. The PID algorithm takes as input the tracking error, $\varepsilon_{y}=\Delta y(t)$, and outputs the command value corresponding to the following equation, 
Eq. F.1

$$
u(t)=K_{p} \cdot\left\{\varepsilon_{y}(t)+\frac{1}{T_{i}} \int \varepsilon_{y}(t) d t+T_{d} \frac{d \varepsilon_{y}(t)}{d t}\right\}
$$

PID controllers use three kind of control actions, each corresponding to one of the terms in Eq.F.1.

- proportional action, $K_{p} \varepsilon_{y}(t)$ : it is the main term of the controller as it fixes the strength of controller and is tunned by the parameter $K_{p}$. Higher values of $K_{p}$ lead to faster system response, until a limit value where the system starts to behave badly or becomes unstable. When the rest of the parameters are tuned, usually $K_{p}$ is increased to obtain the fastest response time possible.

- derivative, $K_{p} T_{d} \varepsilon_{y}(t)$ : the effect of the derivative term is to dampen oscillations, and it is tuned by the parameter $T_{d}$.

- integral, $\frac{K_{p}}{T_{i}} \int \varepsilon_{y}(t) d t$ : when there is no integral term, perturbations on the system can make that $y \neq y_{\text {ref }}$ in steady-state. The integral term can compensate for constant disturbances, non-modeled forces, etc. In the case of multirotors, the integral term on a linear speed controller can compensate slow varying wind disturbances.

The PIDs used in the controllers explained in this $\mathrm{PhD}$ thesis are implemented with the following additional features and contribute to a much more reliable behavior of the controlled system:

- Filtering of the derivative action: if the sampling time and the derivation algorithm are not well selected, this command action can output short high values. This can lead to undesired oscillations of the controlled variable. In order to solve this issue the output of the derivative action is low-pass filtered.

- Output saturation: all the dynamic systems have boundaries for the command input $u(t)$, then, the PID output is saturated to only send acceptable commands to the system. Additionally, it may be desirable to bound the control action to ensure a less aggressive behavior of the system.

- Anti-windup of the integral action: the output of the controller has to be kept between the minimum and maximum values of the command variable, which is set by the capabilities of the controlled system. When the calculated command variable is out of these limits it is saturated, and set to the nearest of them (the minimum or the maximum value). When the integral action reaches the saturation of the command variable $u(t)$, then the integration is not performed whenever it leads to values over the saturation limit. The integration only continues when its value is such that the integral value is unsaturated. 


\title{
G
}

\section{Contributions to the Open-Source Aerial Robotics Framework Aerostack}

\begin{abstract}
Aerostack is an Open-Source Aerial Robotics Framework developed at the Computer Vision Group of the Universidad Politécnica de Madrid (CVG, UPM). Its purpose is to provide a set of modules for aerial robotics, which are interfaced and tested to work properly together, with the main objectives of: (1) achieving autonomous flight of the MAV and (2) being versatile and able to perform different types of missions autonomously. Currently, it is also used as a common framework for the CVG to collaborate with the Artificial Intelligence Department of the Informatics Engineering School of the UPM (ETSII, UPM). The tasks that have been performed with Aerostack are: single-drone navigation, multi-robot navigation, object tracking and following, person tracking and following, human-machine interaction with the drone, entering buildings through windows or doors and pick and place operations with objects. As a reference of the types of missions achieved using Aerostack, this framework was used to participate in IMAV 2013, IARC 2014 and IMAV 2016, see Appx. I. and it has been utilized in performances in many public demonstrations, see Appx. J.1 \& J.2
\end{abstract}

More information about the Aerostack framework:

- Article: "Aerostack: An architecture and open-source software framework for aerial robotics." Sanchez-Lopez, Jose Luis, Ramón A. Suárez Fernández, Hriday Bavle, Carlos Sampedro, Martin Molina, Jesus Pestana, and Pascual Campoy. In Unmanned Aircraft Systems (ICUAS), 2016 International Conference on, pp. 332-341. IEEE, 2016.

- Aerostack website: https://github.com/Vision4UAV/Aerostack/wiki

- Github repository: https://github.com/Vision4UAV/Aerostack

Contributions of author to Aerostack framework:

- Flight controller module presented in Ch.2,

- Aerial robotics visual servoing module presented in Chap.3.

- State estimator module presented in Appx.E.

- Simple kinematics simulator module based on the model presented in Appx.D. 



\section{Appendix}

\section{Utilized Multirotor MAV Platforms}

This appendix describes the multirotors that have been used during the course of this $\mathrm{PhD}$ thesis, which are the Asctec Pelican, the Parrot AR Drone 1\&2 and the DJI M100. The main differences between these platforms is their degree of openness and usability:

- The easiest to use, but do not provide the possibility to program its Autopilot, of the three are the Parrot AR Drone 1\&2, described in Sec. H.2. They are also the most lightweight, even to the point of being mostly harmless. Unlike the Asctec Pelican and the DJI M100, they are not prepared to carry an onboard computer.

- The Asctec Pelican, described in Sec.H.1. os the only of these three MAVs that allows the developer to access the Autopilot and program their own software on it. It is prepared to carry an onboard computer, and has a payload capability of $1 \mathrm{~kg}$ and plenty of space to add sensors.

- While both of the other two previous quadcopters were first released around 2010, the DJI M100, described in Sec. H.3. was released in 2015. Similar to the Pelican it has around a $1 \mathrm{~kg}$ payload capability and its frame makes it easy to mount new sensors on it.

Other multicopter MAVs with which the author or his colleagues at the CVG have utilized parts of the control and trajectory planning algorithms presented in this thesis are: the UASTech LinkQuad (UAS Technologies Sweden AB Company, 2012 1 and MAVs utilizing the Pixhawk Autopilow

$\sqrt[1]{\text { https://youtu.be/gD_BfdF9OI }}$
$\sqrt[3]{\text { https://pixhawk.org/ }}$
https://www.youtube.com/playlist? list=PLd jvAEULWOEHLOGpsQe2Smuw7bplB51 jw 


\section{H.1 AscTec Pelican from Ascending Technologies}

Ascending Technologies (AscendingTechnologies, 2010), see Fig. H.1, is a German company specialized in multi-rotor flying platforms. The Asctec Pelican is a mUAV that can perform GPS navigation, with a nominal payload of $500 \mathrm{~g}$ and an endurance of about 10-15 min. The autopilot board of the Asctec Pelican has low-level stability controllers that control pitch, roll and yaw using the IMU and a magnetometer. This board can also control altitude using a pressure sensor. The Pelican quadrotor can perform altitude hold, with a precision that is enough for outdoors environments. When GPS signal are available, the autopilot can control position using the GPS signal. These enables the Pelican to make reliable position hold in outdoors missions and follow GPS waypoints. The Asctec Autopilot allows to choose between the different control modes specified previously: attitude commands and thrust or altitude speed, and GPS-based speed and heading rotation commands. The following table, Table.H.1, shows a summary of some characteristics of the Pelican quadrotor.

\begin{tabular}{c|c} 
weight & $1000-1200 \mathrm{~g}$ \\
\hline payload & $500 \mathrm{~g}$, with a payload limit of $1000 \mathrm{~g}$ \\
\hline max. speed & $2-3 \mathrm{~m} / \mathrm{s}$ \\
\hline flight time & $10-20 \mathrm{~min}$ \\
\hline sensors & IMU, magnetometer, pressure altitude sensor, GPS receiver \\
\hline other payload & Atom Processor Board $(90 \mathrm{~g})$, computer vision cameras, LIDAR
\end{tabular}

Table H.1: Asctec Pelican characteristics, from Asctec Pelican website

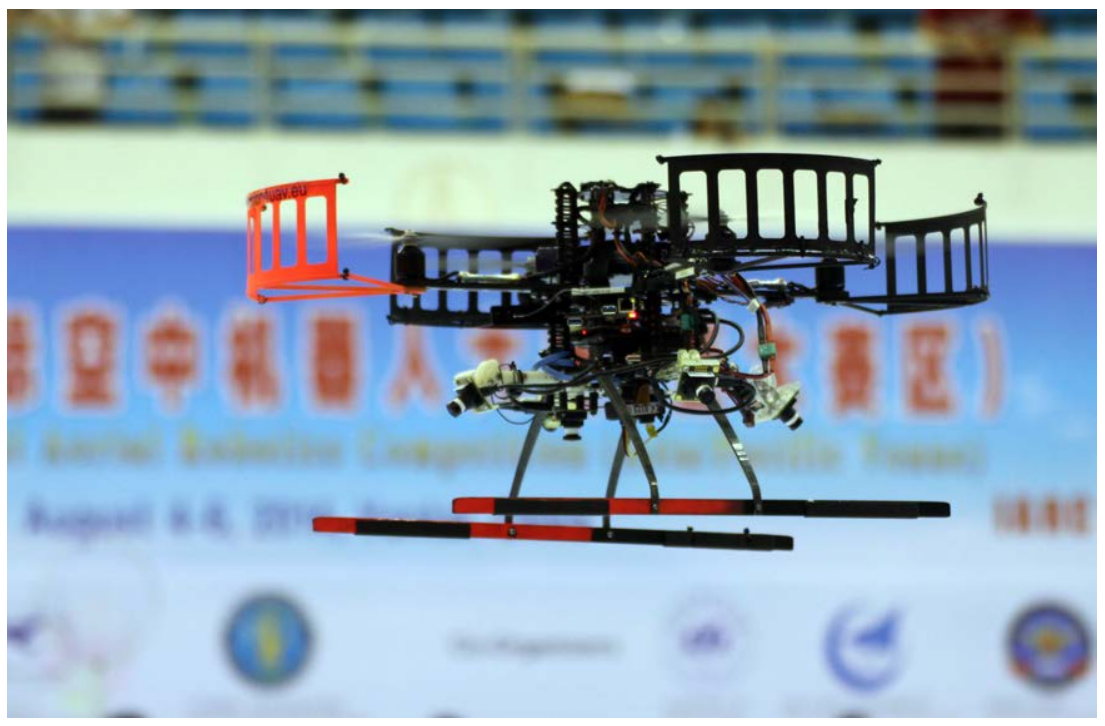

Figure H.1: CVG-UPM (CVG-UPM, 2012) Asctec Pelican quadrotor fully equipped during the CVGUPM's participation in the International Competition IARC 2014. The Pelican is equipped with 5 cameras, a ground optical flow sensor and a powerful Asctec Mastermind (multi-core i7 processor).

The CVG-UPM, has developped an interface for the Pelican to work with the CVG's Framework for interfacing with MAVs (Mellado Bataller, I., 2012), MAVWork. This framework takes care of acquiring telemetry from the Autopilot and the delivery of the flight commands. It allows to easily integrate state estimation and control methods on the onboard computer of the quadcopter and perform the necessary communications with the Autopilot seamlessly. MAVWork was successfully implemented and used for our participation in the IMAV2012 competition.

There exists, however, a more commonly used ROS alternative, which was released at around the same time in 2011-12, the asctec_mav_framework (Achtelik, Markus, 2011). 


\section{H.1.1 Asctec Pelican Multirotor Experimental Setup - IMAV 2012}

The implemented hardware architecture is inspired on the AR Drone quadrotor, which capabilities are described in (Bristeau et al., 2011). An Asctec Pelican quadrotor was equipped with a camera and an on-board computer to obtain a similar setup. The advantages of using the Asctec Pelican are that all the algorithms can run on the on-board computer, and that the vehicle can be equipped with more sensors such as cameras and Laser Range Finders.

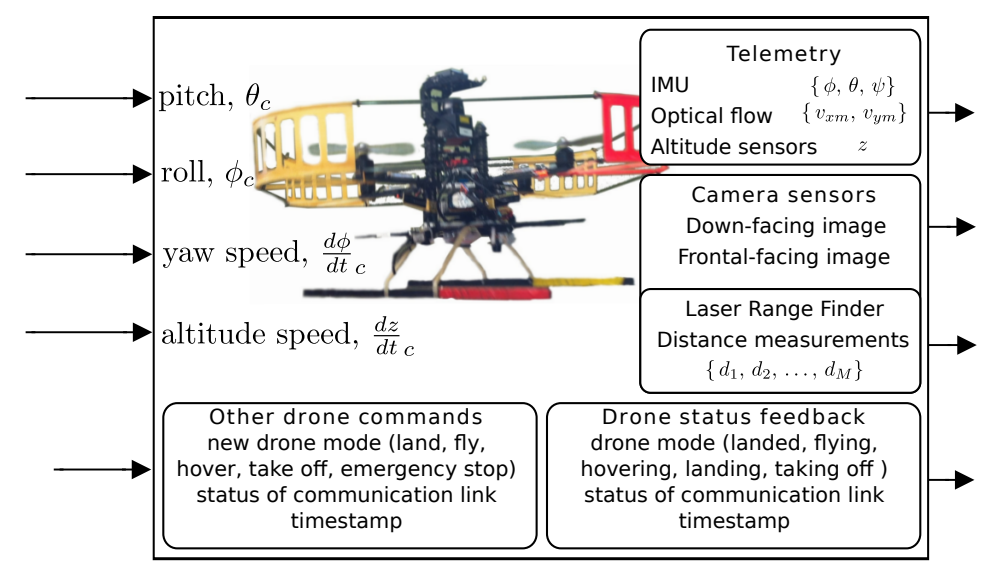

Figure H.2: Pelican on CVG Framework for interfacing with MAVs (Mellado Bataller, I., 2012), user point of view. The Pelican offers several feedback channels: the telemetry package with IMU attitude, horizontal body speed and altitude data, and camera feedback channels where each camera is identified with an ID value. The drone modes are replicated from those available on the AR Drone.

The Asctec Pelican shown in Figure H.2 is equipped with an autopilot board that stabilizes the vehicle using information from GPS (only when outdoors), IMU, pressure altimeter and magnetometer fused using a Kalman Filter. This controller is embedded, closed, unmodifiable but gains are tunable. This quadrotor has also been equipped with a series of complementary sensors: a Hokuyo Scanning Laser Range Finder for horizontal depth mapping, a sonar altitude sensor for height estimation improvement and two cameras, one of them a downward looking sensor for velocity estimation based on optical flow. The optical flow is calculated using the OpenCV implementation of the pyramidal Lucas-Kanade algorithm for some image features selected by the Shi-Tomasi method. The state estimation and control processing are exeuted onboard in an Atom Board, which has a dual core Atom 1.6 GHz processor with $1 \mathrm{~GB}$ of RAM.

\section{H.1.2 Asctec Pelican Multirotor Experimental Setup - IARC 2014}

For the IARC 2014 the Asctec Pelican was used as shown in Fig. H.1. It is equipped with 5 cameras, a ground optical flow sensor, the PX4Flow sensor (Honegger et al., 2013), and a powerful onboard computer, the Asctec Mastermind (multi-core i7 processor) ${ }^{4}$ The MAV was over its highest recommended payload, thus, providing a flight-time slightly lower than 10 minutes. The 5 computer vision cameras are looking in all directions: down, front, right, back and left. This was necessary to be able to detect the IARC 2014 ground robots in any possible direction. In addition to this equipment, the Pelican had a switch that could be triggered by the team to completely disconnect the battery from the MAV, therefore stopping al propellers and the operation of the MAV.

The communication between software modules was achieved using ROS. The utilized main software modules are now open-source and part of AeroStack (Sanchez-Lopez et al., 2016).

$\sqrt[4]{\text { http: //www.asctec.de/en/asctec-mastermind/ }}$ 


\section{H.2 Parrot AR Drone 1\&2}

The toy quadcopters AR Drone and AR Drone 2.0, see Fig. H.3, were first introduced at the Las Vegas International Consumer Electronics Show (CES) in 2010 (ARDrone@Wikipedia, 2012). The AR Drone 2.0 has very attractive features for researchers: it is practically harmless to people and thirdparty objects, it is ease of use thanks to its built-in flying modes, it has a low price, it is comparatively robust to crashes, and it is relatively safe to fly close to people.

Parrot provides an API for developers to access the functionalities of the quadcopter from a connected WiFi computer or smartphone. The parrot is relatively lightweight, around $400 \mathrm{~g}$, and has an endurance of 10-15 min, see Table. H.2. The AR Drone can perform altitude hold and position hold. It achieves altitude hold/control using an ultrasound altimeter and a pressure sensor for higher altitudes. It can perform position hold based on visual odometry algorithms based on a down-facing camera. The propellers of this quadrotor are lightweight enough to be stopped with naked hands. A more detailed description of the hardware and software on the AR Drone, along with a discussion of several issues regarding drone equipment, abilities and performance can be found on (Bristeau et al.,2011; Krajník et al., 2011).

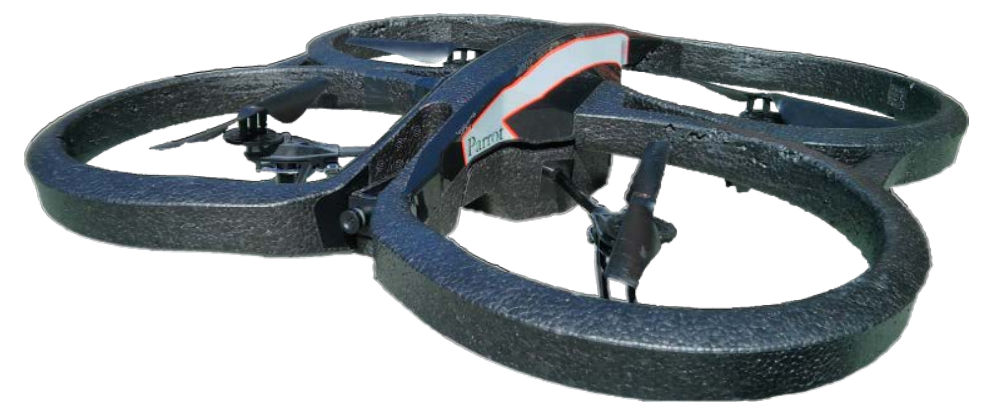

Figure H.3: Parrot AR Drone 2.0 quadcopter.

\begin{tabular}{c|c} 
weight & $380-420 \mathrm{~g}$ \\
\hline payload & $0-100 \mathrm{~g}$, with $250 \mathrm{~g}$ the drone will not take off \\
\hline max. speed & $2-3 \mathrm{~m} / \mathrm{s}$ \\
\hline flight time & $10-15 \mathrm{~min}$ \\
\hline sensors & IMU, ultrasound altitude sensor $(0-7 \mathrm{~m})$, vision-based horizontal speed estimates \\
\hline cameras & a frontal camera and a down-facing camera
\end{tabular}

Table H.2: Parrot AR Drone characteristics

The development of control applications to autonomously control the AR Drone is easier thanks to the flying modes of the AR Drone. The AR Drone can receive directly: take off, land, emergency stop, hover and "fly" commands; which change the flying mode of the drone. The land and take off commands make the drone land and take off, respectively. The emergency stop makes the drone directly switch off the propellers making the drone fall, this also happens whenever one of the propeller blades collides with an obstacle. The hover mode puts the AR Drone in position hold, meaning that the drone will stay in position without moving. The fly mode is used during autonomous flight, where the MAV accepts: pitch, roll, altitude speed and yaw speed commands.

The following is an enumeration of some APIs for the AR Drone:

- AR Drone open API platform: this is the reference platform for developing new augmented reality games based on the AR Drone. The website of this official API is https://projects.ardrone.org/.

- ardrone_autonomy ROS package: this is a well supported software that allows to use the AR Drone with ROS, Robot Operating System (Quigley et al., 2009), ardrone_autonomy ROS package ${ }^{5}$

\footnotetext{
${ }^{5}$ https://github.com/AutonomyLab/ardrone_autonomy
} 
- CVG Framework for interfacing with MAVs (Mellado Bataller, I., 2012; Mellado-Bataller, Mejias, et al., 2012, Mellado-Bataller, Pestana, et al., 2013): it is an open framework which objective is to simplify the development of control applications for quadcopters.

This Vertical Take-Off and Landing (VTOL) MAV has proven to be a realiable platform for vision based navigation algorithm prototyping. These are but just a few of the projects developed by developers and researchers:

- Autonomous flight in hallways and navigation in stairs using computer vision (Bills et al., 2011).

- Indoors navigations and surveillance applications with cooperative ground robots and the AR Drone (Faigl et al., 2010; Krajník et al., 2010).

- Autonomous flight through a forest using visual-based reactive control (Ross et al., 2013).

- Autonomous navigation using the well-known visual SLAM algorithm PTAM (Engel et al., 2012a; Engel et al., 2012b)

- Active exploration of indoor environments Mostegel et al., 2014 


\section{H.3 DJI M100 Quadcopter}

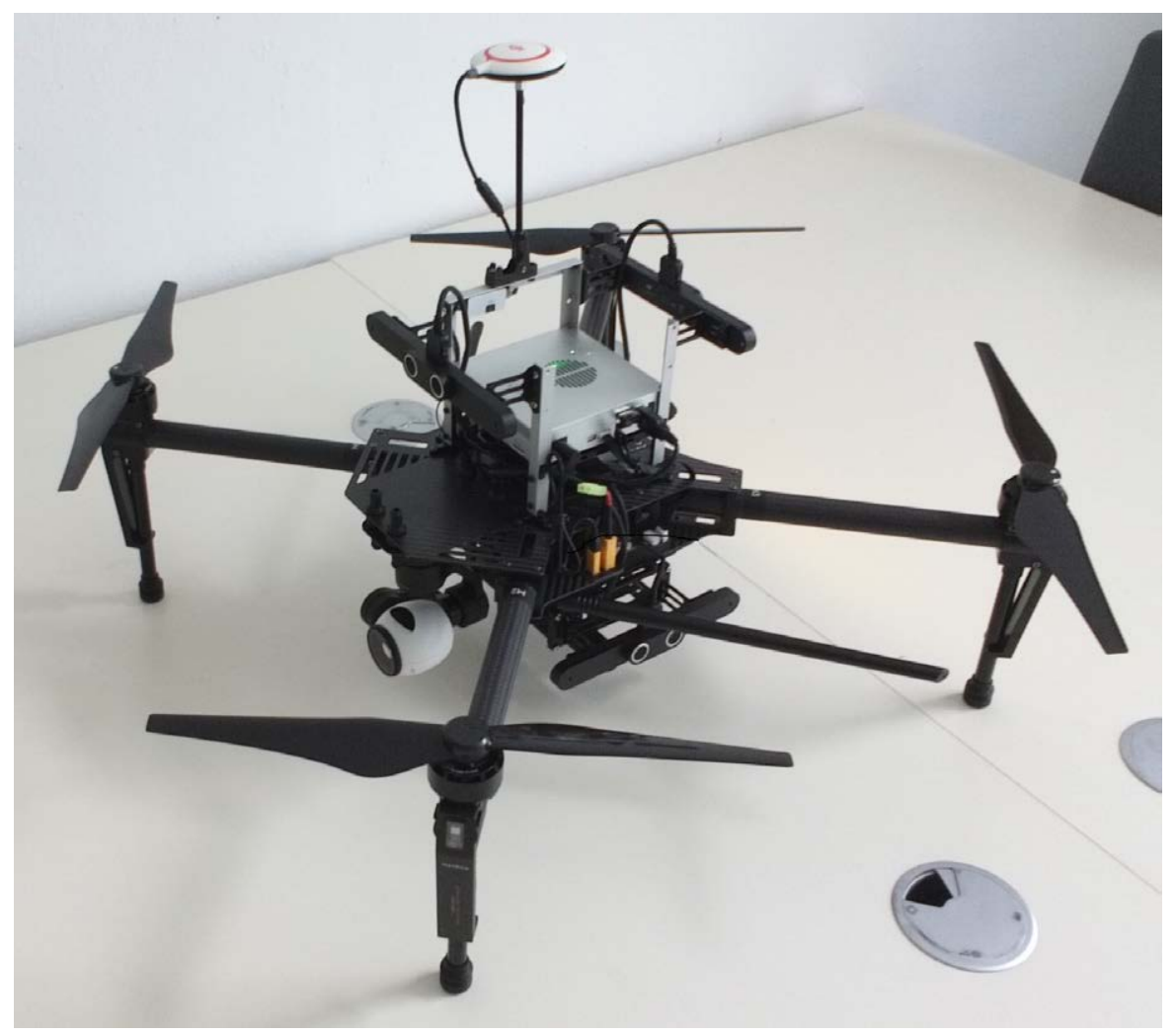

Figure H.4: Fully equipped DJI M100 quadcopter. The equipment includes the DJI Manifold onboard computer, the DJI Guidance sensing system and the DJI Zenmuse X3 gimbal camera.

This quadcopter, shown in Fig. H.5, is a developer platform from DJI, which has similar capabilities to the Asctec Pelican: attitude and thrust or altitude speed control or GPS-based flight. Therefore the main user for this quadcopter are developers aiming to work in civilian applications for MAVs. The DJI M100 is very well integrated with DJI specific sensors: DJI Zenmuse line gimbal cameras and the DJI Guidance sensor, which has 5 stereo head cameras specifically designed for obstacle detection and visual odometry. The preferred onboard computer for this quadcopter is the DJI Manifold. It has a payload capability of $1000 \mathrm{~g}$ and a flight time varying in the $10-25 \mathrm{~min}$ range. DJI offers an API to interface with the Quadcopter ${ }^{6}$, and an associated ROS node ${ }^{7}$.

\section{H.3.1 DJI M100 Multirotor Experimental Setup - DJIC 2016}

The following lists summarize the specifications and capabilities of the equipment that is available on our DJI M100 quadcopter MAV. The list only details the main characteristics of the equipment that we consider important from a technical point of view.

[Fig. H.5] DJI Matrice 100 Quadrotor:

- Equipment and payload:

- DJI Manifold Onboard Computer, 197g

- DJI Guidance Sensing System, 337g

- DJI Zenmuse X3 Gimbal and Camera, 221g

- (x1) DJI TB47D Flight Battery, 600g, 4500mah, 6-cell battery (22.2V nominal) 


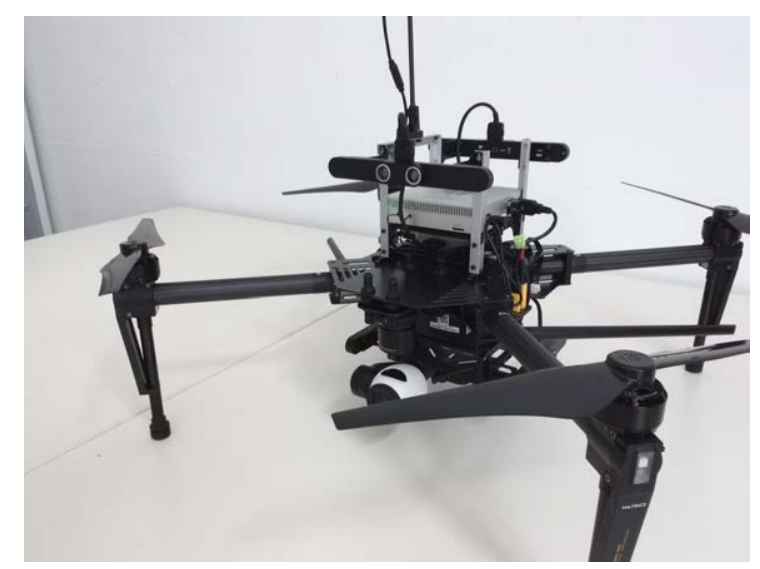

Figure H.5: Picture of our fully equipped DJI Matrice 100 Quadrotor MAV.

- Fully equipped DJI M100 quadcopter MAV:

- Weight: $3.2 \mathrm{Kg}$

- Flight-time: $\sim 10 \mathrm{~min}$

- Size, propeller tip-to-tip quadrotor diameter: 0.996m

- Autopilot Control Interfaces:

- Lowest-level: roll, pitch and yaw_rate attitude commands $\left\{\phi, \theta, \frac{d \psi}{d t}\right\}_{c}$, and thrust $T_{c}$.

- Highest-level: desired velocity $\left\{v_{x}, v_{y}, v_{z}\right\}$ and heading /yaw rotation speeds $\omega_{\psi}$.

[Fig. H.6] Onboard Computer - DJI Manifold:

- (Very similar to) Nvidia Jetson TK1 development board:

- Ubuntu 14.04 and Robot Operating System (ROS) Indigo

- Processors:

- Quad-core, 4-Plus-1 ARM Cortex-A15 MPcore Processor with NEON technology

- Low-power NVIDIA Kepler-based GeForce graphics processor

- Memory: 2GB DDR3L system RAM

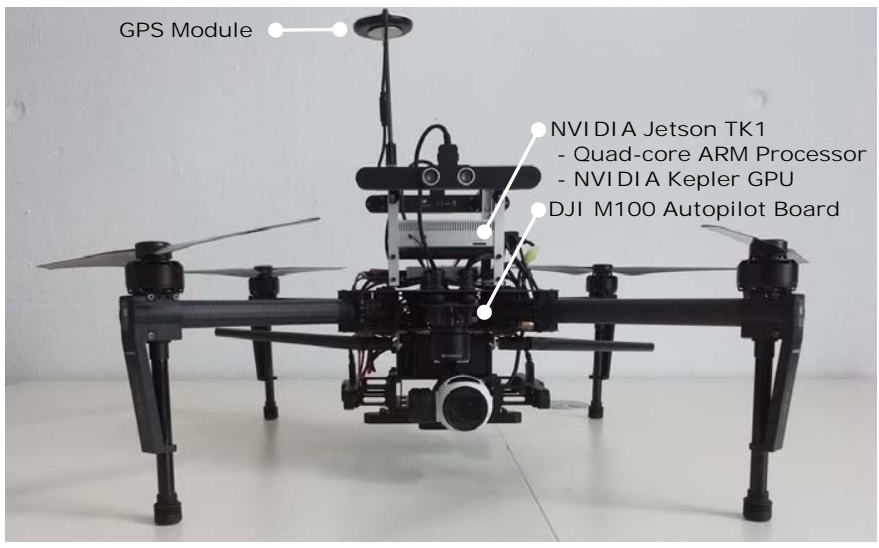

Figure H.6: MAV Hardware - Onboard Computer, Autopilot and GPS Module

[Fig. H.7] DJI Guidance Visual Sensing System:

- Embedded computing hardware: DJI Guidance Core - [Very similar to] Altera Cyclone V (low-cost SoC FPGA) - FPGA + dual Core ARM Processor.

- Sensors:

- Built-in IMU 


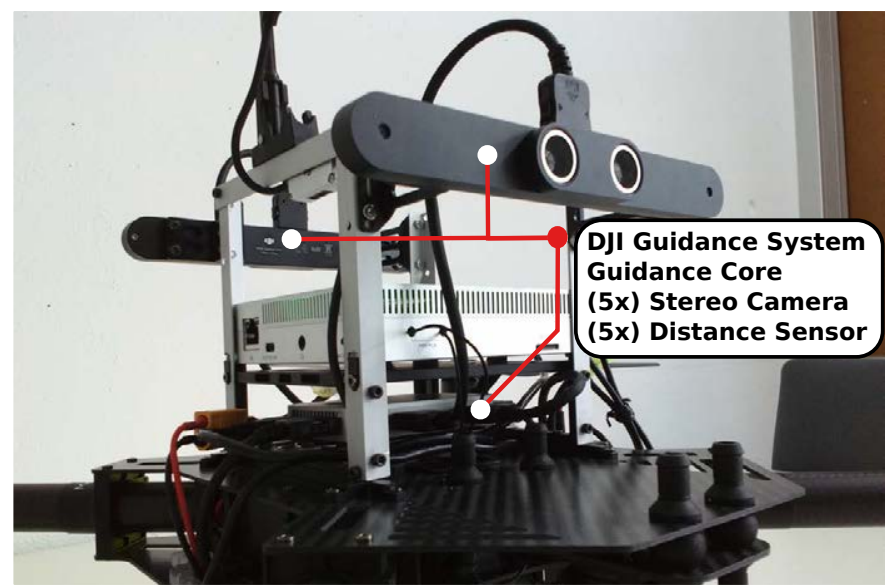

Figure H.7: MAV Hardware - Guidance Sensing System, which is composed of 5 stereo heads and an embedded processing unit.

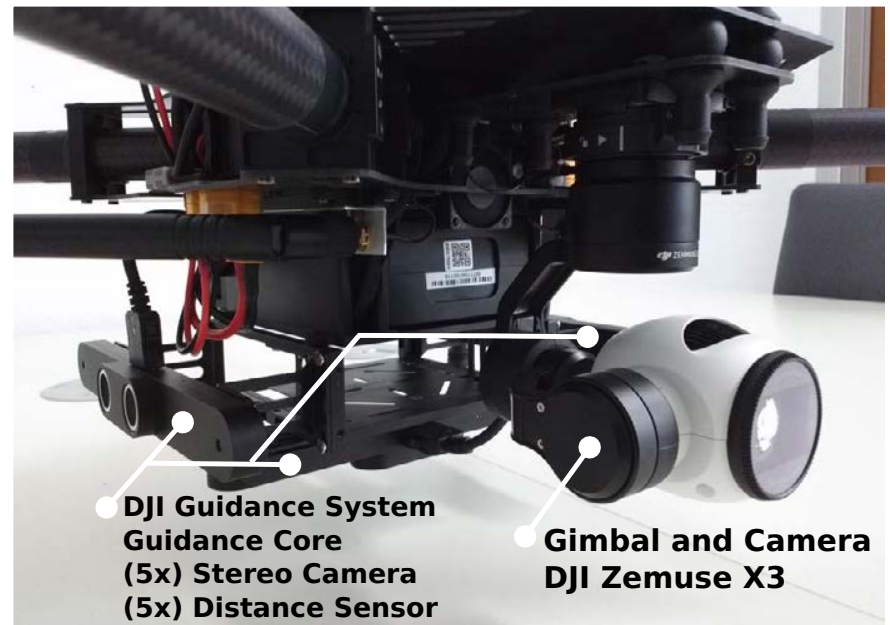

Figure H.8: MAV Hardware - Gimbal Camera and Guidance Sensing System, which is composed of 5 stereo heads and an embedded processing unit.

- (x5) Stereo camera heads: front, rear, left, right and bottom

* Built-in ultrasonic distance sensor

* Built-in stereo camera pair

- Velocity measurement (only from downwards looking stereo head), $0-16 \mathrm{~m} / \mathrm{s}$ at $2 \mathrm{~m}$ altitude

- Embedded visual-inertial odometry algorithm from DJI to estimate the motion of the MAV in GPSdenied/degraded environments. Integrates the IMU measurements with the five camera view directions (monocular or stereo depending on current conditions). The visual-inertial odometry algorithm is described in the following three scientific works (Zhou, Fang, et al., 2015; Zhou, Liu, et al., 2014, Zhou, Ye, et al., 2014).

- Acquisition rate of the sensors configured using a GUI from DJI

[Fig. H.8] DJI Zenmuse X3 Gimbal and Camera:

- Note: Max. resolution is $\sim 4 \mathrm{~K}$ standard, but the resolution available onboard: image frame is 1280 X 720 pixel (pixel format NV12)

- Gimbal position commands are directed through the Autopilot, the Gimbal is very well stabilized. 


\section{H.4 Appendix Bibliography}

AscendingTechnologies (2010). Ascending Technologies. http://www.asctec.de (cit. on p. 192).

Bills, C., Chen, J., and Saxena, A. (2011). "Autonomous MAV flight in indoor environments using single image perspective cues". In: Robotics and Automation (ICRA), 2011 IEEE International Conference on, pp. 5776-5783 (cit. on p. 195).

Bristeau, P.-J., Callou, F., Vissiere, D., Petit, N., et al. (2011). "The navigation and control technology inside the ar. drone micro uav". In: 18th IFAC World Congress. Vol. 18, pp. 1477-1484 (cit. on pp. 193. 194).

Mellado Bataller, I. (2012). A new framework for interfacing with MAVs. https: / / github . com/ uavster/mavwork (cit. on pp. 192, 193, 195,.

Engel, J., Sturm, J., and Cremers, D. (2012a). "Camera-Based Navigation of a Low-Cost Quadrocopter". In: Proc. of the International Conference on Intelligent Robot Systems (IROS) (cit. on p. 195).

Engel, J., Sturm, J., and Cremers, D. (2012b). "Accurate figure flying with a quadrocopter using onboard visual and inertial sensing". IMU 320, p. 240 (cit. on p. 195).

Faigl, J., Krajník, T., Vonásek, V., and Přeučil, L. (2010). "Surveillance Planning with Localization Uncertainty for UAVs". In: 3rd Israeli Conference on Robotics, Ariel: Ariel University Center, pages (cit. on p. 195).

Honegger, D., Meier, L., Tanskanen, P., and Pollefeys, M. (2013). “An open source and open hardware embedded metric optical flow cmos camera for indoor and outdoor applications". In: Robotics and Automation (ICRA), 2013 IEEE International Conference on. IEEE, pp. 1736-1741 (cit. on p. 193.

Krajník, T., Faigl, J., Vonásek, V., Košnar, K., Kulich, M., and Přeučil, L. (2010). "Simple, Yet Stable Bearing-Only Navigation”. Journal of Field Robotics 27.5, pp. 511-533 (cit. on p. 195).

Krajník, T., Vonásek, V., Fišer, D., and Faigl, J. (2011). “AR-Drone as a Platform for Robotic Research and Education”. In: Research and Education in Robotics: EUROBOT 2011. Heidelberg: Springer (cit. on p. 194.

Mellado-Bataller, I., Mejias, L., Campoy, P., and Olivares-Mendez, M. A. (2012). "Rapid prototyping framework for visual control of autonomous micro aerial vehicles". In: 12th International Conference on Intelligent Autonomous System (IAS-12). Jeju Island, Korea (cit. on p. 195).

Mellado-Bataller, I., Pestana, J., Olivares-Mendez, M. A., Campoy, P., and Mejias, L. (2013). "MAVwork: a framework for unified interfacing between micro aerial vehicles and visual controllers". In: Frontiers of Intelligent Autonomous Systems. Springer, pp. 165-179 (cit. on p. 195).

Mostegel, C., Wendel, A., and Bischof, H. (2014). "Active monocular localization: Towards autonomous monocular exploration for multirotor mavs". In: Robotics and Automation (ICRA), 2014 IEEE International Conference on. IEEE, pp. 3848-3855 (cit. on p. 195).

Quigley, M., Gerkey, B., Conley, K., Faust, J., Foote, T., Leibs, J., Berger, E., Wheeler, R., and Ng, A. (2009). "ROS : an open-source Robot Operating System". In: IEEE International Conference on Robotics and Automation (ICRA 2009) (cit. on p. 194).

Achtelik, Markus (2011). ROS asctec_mav_framework repository, https://github.com/ethzasl/asctec_mav_framework (cit. on p. 192).

Ross, S., Melik-Barkhudarov, N., Shankar, K. S., Wendel, A., Dey, D., Bagnell, J. A., and Hebert, M. (2013). "Learning monocular reactive uav control in cluttered natural environments". In: Robotics and Automation (ICRA), 2013 IEEE International Conference on. IEEE, pp. 1765-1772 (cit. on p. 195.

Sanchez-Lopez, J. L., Fernández, R. A. S., Bavle, H., Sampedro, C., Molina, M., Pestana, J., and Campoy, P. (2016). "Aerostack: An architecture and open-source software framework for aerial robotics". In: Unmanned Aircraft Systems (ICUAS), 2016 International Conference on. IEEE, pp. 332-341 (cit. on p. 193 .

UAS Technologies Sweden AB Company (2012). UAS Technologies Sweden AB Company website. http: / / www . uastech.com/ (cit. on p. 191).

CVG-UPM (2012). Universidad Politécnica de Madrid. Computer Vision Group. Vision for UAV Project. http: / /www.vision4uav.com (cit. on p. 192).

ARDrone@Wikipedia (2012). Parrot AR Drone article at english Wikipedia. http : / / en . wikipedia.org/wiki/Parrot_AR.Drone (cit. on p. 194).

Zhou, G., Fang, L., Tang, K., Zhang, H., Wang, K., and Yang, K. (2015). “Guidance: A Visual Sensing Platform For Robotic Applications". In: Proceedings of the IEEE Conference on Computer Vision and Pattern Recognition Workshops, pp. 9-14 (cit. on p. 198). 
Zhou, G., Liu, A., Yang, K., Wang, T., and Li, Z. (2014). “An embedded solution to visual mapping for consumer drones". In: Proceedings of the IEEE Conference on Computer Vision and Pattern Recognition Workshops, pp. 656-661 (cit. on p. 198).

Zhou, G., Ye, J., Ren, W., Wang, T., and Li, Z. (2014). “On-board inertial-assisted visual odometer on an embedded system”. In: 2014 IEEE International Conference on Robotics and Automation (ICRA). IEEE, pp. 2602-2608 (cit. on p. 198. 


\section{I}

\section{International Micro Aerial Vehicle Competitions}

This appendix provides a short description and a summary of the achieved results for each of the aerial robotics competitions in which, to this date, some of the algorithms developed during this thesis has been used. The description will be kept concise, but the link to the main website of the competition and to the competition rules, when available, are provided. The following are the competitions and the events in which the author of the thesis either participated directly and/or tutored students and/or some of the algorithms presented in this thesis were utilized by his colleagues.

- 2016 DJI Developer Challenge - DJIC 2016

- International Micro Air Vehicle Conference and Flight Competition 2016 - IMAV 2016

- European Robotics Challenges 2014 - Simulation Challenge 3 - EuRoC 2014

- International Aerial Robotics Competition 2014 - Mission 7 - IARC 2014

- International Micro Air Vehicle Conference and Flight Competition 2013 - IMAV 2013

- International Micro Air Vehicle Conference and Flight Competition 2012 - IMAV 2012

- Concurso en Ingeniería de Control 2012 - CEA 2012 


\section{I.1 2016 DJI Developer Challenge - DJIC 2016}

\section{I.1.1 External Links}

- Website: https://developer.dji.com/challenge2016/

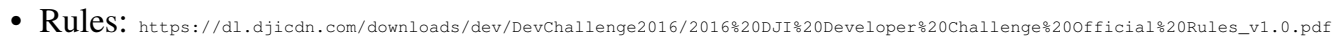

- Teams qualified for final round: https://developer.dji.com/news/2016-dji-developer-challenge-10-teams-enter-final-round/

- DJI's summary video: https://youtu.be/DIRkzH3cTAM

\section{I.1.2 Short description}

This competition was organized by DJI, a manufacturer of UAVs, mainly multicopters, and related products such as flight controllers and propulsion systems; gimbal stabilized mounts for cameras and cameras. The competition proposed an outdoor emulated search and rescue scenario. The participants had to submit a proposal and get selected to participate, and later on get qualified in successive stages of the competition in order to be able to participate in the finals. The hardware for the competition was mostly supplied by DJI, with little space to use alternatives, and included: the DJI M100 quadcopter, the DJI Guidance sensing system, the DJI Zenmuse X3 Gimbal and the DJI Manifold onboard computer.

The competition hosted only one event, in which a team from the TU Graz participated and qualified to participate in the finals. It featured an emulated search and rescue mission, whose main objectives were: (0) perform the mission completely in Autonomous mode with no interaction with the pilot, (1) take-off from a moving vehicle, (2) enter a search area and find survivors, emulated using small fiducial markers, and (3) come back to the pick-up area and land on a moving vehicle, alternatively on a static vehicle.

\section{I.1.3 Achieved Results}

Experimental MAV Platform: DJI M100 as described in Sec. H.3.1

\section{Contributions of author to results:}

- Module utilized in autonomous flight architecture: trajectory and speed planner presented in Ch. 4

- Other roles of the author as part of the team: team leader, main system integrator, experimental tester and being part of the team members participating and performing the MAV deployment in the event finals.

Achieved Results:

The competition recreated a drone Search and Rescue scenario. The "Graz Griffins" team qualified through successive rounds, from the initial $\approx 140$ teams, to be part of the last 10 teams showing their solution experimentally in the finals.

At the time of the competition our MAV could perform the following tasks autonomously:

- take-off from a moving vehicle,

- produce a map performing an overview flight of an area using only onboard computation,

- use the created map to navigate safely avoiding obstacles while adding new obstacles to the map,

- land on the static vehicle.

We were not able to integrate all these tasks in a single flight for the competition. However, overall our team showed a very innovative solution using on-board mapping based on photogrammetry algorithm 11. The on-board calculated map is converted to an obstacle-map that can be used by the presented planner to deliver trajectories that are then executed by the controller, all in the same flight and without requiring any off-board computation.

${ }^{1}$ DJIC 2016 video playlist: http://bit.ly/2faublQ 


\section{I.2 International Micro Air Vehicle Conference and Flight Competition 2016 - IMAV 2016}

\section{I.2.1 External Links}

- Website: http://www.imavs.org/2016/

- Rules: http://www.imavs.org/2016/documents/IMAV2016_Competition_rules_v2.3.pdf

- Results: http://www.imavs.org/2016/result.html

- Proceedings: http://www.imavs.org/imav2016proceedings/

- Video of proposal: https://vimeo.com/156657670

\section{I.2.2 Short description}

This competition proposed two events, an outdoor mission and an indoor mission. The choice of MAV, sensors and autonomy level are quite open; but the scoring system promotes autonomy, although manual piloting entries are also accepted, and very heavily custom lightweight MAVs. Therefore, this competitions tries to attract and bring both: robotics autonomy and new lightweight MAV designs.

The main objectives of the IMAV 2016 Indoor Competition, in which the CVG_UPM participated, were: (1) take-off, (2) enter a building, several options are available, (3) pick up and release items, (4) exit the building, (5) land, with also several options are available and (6) build a map of the environment.

\section{I.2.3 Achieved Results - Indoor Competition}

Experimental MAV Platform: custom MAV based on the Pixhawk Autopilo: 2

Contributions of author to results:

- Module utilized in autonomous flight architecture: controller presented in Ch. 2 .

Achieved Results:

At the time of the competition our MAV could perform the following tasks autonomously:

- take-off,

- enter a building through the door,

- pick up and release items,

- exit the building,

- land.

The CVG_UPM team was able to solve multiple tasks in fully autonomous mode and was the team to achieve the highest number of tasks, although in separate flights, in fully autonomous mode ${ }^{3}$ Despite its outstanding performance, the CVG team earned the $4^{\text {th }}$ position due to the scoring system leaning very heavily towards the usage of very small MAVs. 


\section{I.3 European Robotics Challenges 2014 - Simulation Challenge 3 - EuRoC 2014}

\section{I.3.1 External Links}

- Website: http://www.euroc-project.eu/index.php?id=euroc_project

- Challenge 3: http: //www. euroc-project.eu/index.php?id=challenge_3

- Rules: http://www.euroc-project.eu/index.php?id=motivation0

- Results (Simulation Challenge 3): http://www.euroc-project.eu/index.php?id=simulation_challenge3

- Publications: http://www.euroc-project.eu/index.php?id=publications

\section{I.3.2 Short description}

The European Robotics Challenges (EuRoC) have a very general motivation, they seek to accelerate the adoption of robotics by the industry. The first challenge started in 2014, and included three main event categories:

Challenge 1 - Reconfigurable Interactive Manufacturing Cell - research in advanced concepts for robotic arm manipulators.

Challenge 2 - Shop Floor Logistics and Manipulation - robotic object manipulation from mobile robots equipped with an arm manipulator.

Challenge 3 - Plant Servicing and Inspection - integration of MAVs in industrial environments.

The first stage of all three challenges was a simulation competition with precise scoring rules, evaluated using an automatic script. The 10 top qualified teams passed to the next stage, which promoted the proposal along with an industry partner of innovative high impact projects. The TU Graz participated in Challenge 3. The simulation challenge of this track required the development of algorithms for three separate tasks. These three tasks were scored separately and had the following objectives, which apply very well to MAVs:

- The development of high precision real-time vision-based localization algorithms.

- The development of high precision real-time obstacle mapping algorithms.

- The development of state-estimation, trajectory control and trajectory planning algorithms.

\section{I.3.3 Achieved Results - EuRoC 2014 - Challenge 3 - Simulation Competition}

Experimental MAV Platform: simulated MAV, prepared by competition organizers.

Contributions of author to results:

- Module utilized in autonomous flight architecture: trajectory and speed planner presented in Ch. 4

- Other roles of the author as part of the team: close collaboration with main developer of the state estimator and controller used in the flight simulation.

\section{Achieved Results:}

The developed fast real-time trajectory and speed planner ${ }^{4}$ enabled reliable obstacle avoidance and very fast trajectory execution by the simulated quadrotor that replicated the capabilities of an Asctec Firefly.

The "Graz Griffins" team achieved the $6^{\text {th }}$ position out of 21 teams, which included world renowned European universities and laboratories, in the EuRoC 2014 - Challenge 3 - Simulation Competition. This fact enabled the team to present a proposal in collaboration with a company to the next stage of EuRoC.

${ }^{4}$ EuRoC 2014 trajectory flight video: http://bit. Iy/2cQno6I 


\section{I.4 International Aerial Robotics Competition 2014 - Mission 7 - IARC 2014}

\section{I.4.1 External Links}

- Website: http://www .aerialroboticscompetition.org/index.php

- Rules - Mission 7: http://www.aerialroboticscompetition.org/rules.php

- Proceedings: http://www.aerialroboticscompetition.org/symposium2014.php

- Competitors: http://www.aerialroboticscompetition.org/competitors.php

\section{I.4.2 Short description}

The International Aerial Robotics Competition (IARC) seeks to propose high difficulty robotic challenges that are currently not solved by either universities or industry. The organization proposes a "Mission" which is yearly organized as a competition event with little changes in the rules until a participant is able to achieve the proposed objectives. Once a team is able to demonstrate a solution to the current objectives, a new "Mission" is conceived. After three decades of IARC challenges, the current "Mission" is Mission 7. Past challenges, Mission 5 and Mission 6, have already tackled MAV indoors navigation, autonomous exploration, Simultaneous Localization and Mapping (SLAM) and small object search, picking and delivery.

The current objectives proposed in Mission 7, in which the CVG_UPM participated in 2014, are extremely challenging and require the combination of: MAV odometry localization, object detection, reasoning on the behavior of a of group ground robots, autonomous decision making by the MAV and in the future also to reason on the behavior of another challenger MAV. More precisely, the flight environment is a square region where 2 sets of ground robots are navigating autonomously, 1 set are the target robots and the others are obstacles, which hold columns. The MAV has to navigate in this area avoiding obstacles and can affect the navigation direction of the target robots by interacting physically with them, which follow a pseudo-random navigation behavior. The main objective of the challenge is for the MAV to successfully redirect 4 or more target robots to exit the challenge square area through one specific border.

\section{I.4.3 Achieved Results - IARC 2014 - Asia Venue}

Experimental MAV Platform: Asctec Pelican as described in Sec. H.1.2.

Contributions of author to results:

- Modules utilized in autonomous flight architecture: controller presented in Ch. 2 and state estimator presented in Appx. E

- Other roles of the author as part of the team: system integrator, experimental tester and being part of the team members participating and performing the MAV deployment in the competition event.

\section{Achieved Results:}

At the time of the competition our MAV could perform the following tasks autonomously:

- take-off,

- navigate inside the competition area,

- ground-robot detection,

- simple ground robot avoidance technique,

- land.

Same as other teams, we were still far from achieving the interaction with the ground robots. Therefore, the CVG_UPM ${ }^{5}$ team showed similar capabilities to other contestants. Our level of development was good as a first step towards continuing our participation the next year for IARC 2015.

${ }^{5}$ IARC 2014 video playlist: https://goo.gl/bEvK3F 


\section{I.5 International Micro Air Vehicle Conference and Flight Competition 2013 - IMAV 2013}

\section{I.5.1 External Links}

- Website: http://www.imavs.org/2013/index.html

- Rules 1: http://www.imavs.org/2013/index.php/information.html

- Rules 2: http://www.imav2013.org/images/IMAV2013_General_Rules.pdf

- Results 1: http://www.imavs.org/imav2013/

- Results 2: http://www.imavs.org/2013/index.php/final-results.html

- Proceedings: http: //www.imavs.org/imav2013proceedings/

\section{I.5.2 Short description}

This competition proposed two events, an outdoor mission and an indoor mission. The choice of MAV, sensors and autonomy level are quite open; but the scoring system promotes autonomy, although manual piloting entries are also accepted, and very heavily custom lightweight MAVs. Therefore, this competitions tries to attract and bring both: robotics autonomy and new lightweight MAV designs.

The main objectives of the IMAV 2013 Indoor Mission, in which the CVG_UPM participated, were: (1) take-off, (2) cross a window, with two available options, (3) several navigation objectives through and around an area with column obstacles, (4) column obstacle detection and (5) alternative tasks such as moving landing, static landing and precise object dropping.

\section{I.5.3 Achieved Results - Indoor Mission}

Experimental MAV Platform: multi-robot solution using the AR Drone 2.0, see Appx. H.2

\section{Contributions of author to results:}

- Modules utilized in autonomous flight architecture: controller presented in Ch. 2 and state estimator presented in Appx. E integrated as part of a ROS-based multi-robot autonomous system.

- Other roles of the author as part of the team: system integrator, experimental tester and being part of the team members participating and performing the MAV deployment in the competition event.

\section{Achieved Results:}

At the time of the competition our MAV could perform the following tasks autonomously:

- take-off,

- localization achieved by placing visual fiducial markers in the competition arena,

- enter the navigation area by crossing the big window,

- multi-robot navigation around the obstacle area,

- multi-robot navigation through unknown obstacle area,

- land on static target.

The CVG_UPM showed an outstanding multi-robot autonomous solution to IMAV 2013 The team that represented the UPM and the Computer Vision Group (CVG, UPM) was awarded with: first place in "Indoor Autonomy - IMAV 2013".

${ }^{6}$ IMAV 2013 video playlist: https://goo.gl/KosWKH 


\section{I.6 International Micro Air Vehicle Conference and Flight Competition 2012 - IMAV 2012}

\section{I.6.1 External Links}

- Website: http://www.imavs.org/2012/

- Rules: http://hadmernok.hu/132_31_bekesib.pdf

- Results: http://www.imavs.org/imav2012results/

- Proceedings: http://www.imavs .org/imav2012proceedings/

- Video - AKAFlieg Summary: https: / / www - youtube.com/wat ch?v=E2 fwMWeuqGw

\section{I.6.2 Short description}

This competition proposed two events, an outdoor mission and an indoor mission. The choice of MAV, sensors and autonomy level are quite open; but the scoring system promotes autonomy, although manual piloting entries are also accepted, and very heavily custom lightweight MAVs. Therefore, this competitions tries to attract and bring both: robotics autonomy and new lightweight MAV designs.

The CVG_UPM participated in the IMAV 2012 Indoor Dynamics Competition. This challenge sought only fast navigation and obstacle avoidance objectives. The flight environment had two opposing horizontal tubes, each supported by two vertical columns on the sides, and was precisely described in the rules. The main objectives to be achieved were: (1) to perform the localization task in this environment and (2) to perform as many 8 -shaped laps around the 2 horizontal tubes as possible.

\section{I.6.3 Achieved Results - Indoor Dynamics Competition}

Experimental MAV Platform: Asctec Pelican, equipped as described in Sec. H.1.1.

\section{Contributions of author to results:}

- Modules utilized in autonomous flight architecture: controller presented in Ch. 2, state estimator and particle filter presented in Appx.E

- Other roles of the author as part of the team: system integrator, experimental tester and being part of the team members participating and performing the MAV deployment in the competition event.

\section{Achieved Results:}

At the time of the competition our MAV could perform the following tasks autonomously:

- take-off,

- localization achieved by a using a Lidar sensor combined with a particle filter,

- otherwise, odometry-based state estimation combining different sensor technologies,

- perform 8-shaped laps around the 2 horizontal tubes,

- land.

The CVG_UPM was the only team to show a fully autonomous solution to the IMAV 2012 Indoor Dynamics Competition 7 . The team that represented the UPM and the Computer Vision Group (CVG) was twice awarded with:

- the special award "Best Automatic Performance - IMAV 2012", as part of the overall results of the competitions, and

- second place in the "Indoor Flight Dynamics - Rotary Wing MAV" challenge, where the first place was obtained by a remotely piloted multirotor.

${ }^{7}$ IMAV 2012 video playlist: https://goo.gl/PRns2D 


\section{I.7 Contest on Control Engineering 2012 - CEA 2012}

\section{I.7.1 External Links}

- Website: http://intranet.ceautomatica.es/og/ingenieria-de-control/benchmark-2011-2012

- Rules: http://intranet.ceautomatica.es/sites/default/files/upload/13/files/Concursoะ20IC2012:20-。20Bases (1).pdf

- Video Summary: https: / / www . youtube.com/watch?v=uWYatwnze-A

\section{I.7.2 Short description}

The Contest on Control Engineering 2012 (in Spanish "Concurso en Ingeniería de Control 2012”), organized by the Spanish Committee of Automation, has the objective of proposing a challenge that can be solved by bachelor and master students, mainly from the field of control and automation.

By using the off-the-shelf AR Drone MAV, the students could focus on the identification, stateestimation and control tasks. This MAV was probably chosen because it provides out-of-the-box velocity and attitude estimates as part of its telemetry.

The challenge was divided in two stages: simulation and flight competition. For the simulation stage, the scoring was divided in the three main target tasks: (1) model design and identification, and (2) state-estimation and trajectory control. The scoring system in the finals evaluated the tracking error and the trajectory round-trip time. The CVG_UPM participated with 2 teams, a team of PhD students and a team of undergraduate students, tutored by the $\mathrm{PhD}$ students' team.

\section{I.7.3 Achieved Results}

Experimental MAV Platform: AR Drone 2.0 quadrotor, see Appx. H.2

\section{Contributions of author to results:}

- Module utilized in autonomous flight architecture: controller similar to presented in Ch. 2

- Other roles of the author as part of the team: tutoring the undergraduate students' team and being part of the team members participating and performing the MAV deployment in the competition event.

\section{Achieved Results:}

Two teams participated representing the Universidad Politécnica de Madrid (UPM) and the Computer Vision Group (CVG_UPM), and were awarded with:

- Undergraduate students' team of the ETSII-UPM engineering school using their own developed software supervised by J. Pestana: the first (phase 1 - simulation) and third places (final phase autonomous flight event)

- CVG_UPM PhD students' team: the second (phase 1 - simulation) and fifth places (final phase autonomous flight event). In the final phase of the competition our team achieved the fifth place, due to our controller, when compared to other teams, leaning towards low tracking error with higher round-trip times. 


\section{Appendix}

\section{Other Micro Aerial Vehicle Autonomous Flight Demonstrations}

The modules developed during the course of this $\mathrm{PhD}$ thesis have been used as part of robotics software architectures to achieve autonomous flight and task execution in several experimental autonomous flight demonstrations. Setting aside their usage as part of the participation in International MAV Competitions, which has been described in Appx. I. the presented modules have been utilized in the following autonomous flight demonstrations. 


\section{J.1 Using Trajectory Controller and/or State Estimator}

The controller presented in Chap. 2 and/or the state estimator presented in Appx. Ehave been showcased in the following live demos by the Computer Vision Group of the Universidad Politécnica de Madrid (CVG, UPM):

- CivilDRON 2017 congres 11 Madrid (Spain)

Simplified version of IMAV 2016 competition mission January 2017

- CivilDRON 2016 congres ${ }^{2}$, Madrid (Spain)

Multi-robot mission and vision-based person following

May 2016

- Semana de la Ciencia 2015 - Science Week 2015 非, Madrid (Spain)

Multi-robot mission and vision-based person following

November 2-15, 2015

- Noche Europea de los investigadores - European Researchers' Night 20155

Multi-robot mission, vision-based person following and a simple search and rescue mission, which included the usage of a multi-modal human-machine interface with the MAV.

Madrid (Spain), September 2015

- Semana de la Ciencia 2014 - Science Week 20146 Madrid (Spain)

Multi-robot mission and vision-based person following

November 2014

- Semana de la Ciencia 2013 - Science Week 201377 Madrid (Spain)

Multi-robot mission and vision-based person following

November 4-17, 2013

${ }^{1}$ CivilDRON 2017: http://www.civildron.com/

${ }^{2}$ CivilDRON 2016: http://www.aerpas.es/wp-content/uploads/2015/04/CivilDRON16_v2.pdf

${ }^{3}$ Semana de la Ciencia 2015 - Website http://bit.ly/21N9RNg

${ }^{4}$ Semana de la Ciencia 2015 - Results Report http://bit.ly/2muELHG

${ }^{5}$ European Researchers' Night 2015 - Website: http://bit.ly/2mM6a9Q

${ }^{6}$ Semana de la Ciencia 2014 - Results Report http://bit.ly/21Ng1NA

${ }^{7}$ Semana de la Ciencia 2013 - Results Report http://bit.ly/2npz2lF 


\section{J.2 Using Visual Servoing Architecture}

The aerial robotics visual servoing architecture presented in Chap. 3 has been showcased in the following live demos mostly by the CVG (UPM), and one demo by the Institute of Computer Graphics of the Graz University of Technology (ICG, TU Graz). These demonstrations have shown the MAV performing a person following task:

- CivilDRON 2016 congres 8 . Madrid (Spain)

Multi-robot mission and vision-based person following

May 2016

- Semana de la Ciencia 2015 - Science Week 2015 I0 $^{0}$ Madrid (Spain)

Multi-robot mission and vision-based person following

November 2-15, 2015

- 2015 European Robotics Week $\left.{ }^{11}\right|^{2}$, Madrid (Spain)

Vision-based person following

September 2015

- Noche Europea de los investigadores - European Researchers' Night $2015^{13}$

Multi-robot mission, vision-based person following and a simple search and rescue mission

Madrid (Spain), September 2015

- 2014 European Robotics $14 \mid 15$, Madrid (Spain)

Vision-based person following

November 2014

- Semana de la Ciencia 2014 - Science Week 2014 16 , Madrid (Spain)

Multi-robot mission and vision-based person following

November 2014

- OpenLabNight 2014 17 , ICG - TU Graz (Austria)

Vision-based person following

October 9, 2014

- Semana de la Ciencia 2013 - Science Week 2013 ${ }^{18}$, Madrid (Spain)

Multi-robot mission and vision-based person following

November 4-17, 2013

- The 11th IEEE International Symposium on Safety, Security, and Rescue Robotics (SSRR 2013) Vision-based person following

Linköping (Sweden), October 21-26, 2013

\footnotetext{
${ }^{8}$ CivilDRON 2016: http://www. aerpas.es/wp-content/uploads/2015/04/CivilDRON16_v2.pdf

${ }^{9}$ Semana de la Ciencia 2015 - Website http://bit.ly/2lN9RNg

${ }^{10}$ Semana de la Ciencia 2015 - Results Report http://bit.ly/2muELHG

${ }^{11} 2015$ European Robotics Week - Website: http://bit.ly/2mcMglt

${ }^{12}$ European Robotics Week - Website: http://bit.ly/2mT12Bh

${ }^{13}$ European Researchers' Night 2015 - Website: http://bit.1y/2mM6a9Q

${ }^{14} 2014$ European Robotics Week - Website: http://bit.ly/2mMaTIV

${ }^{15}$ European Robotics Week - Website: http://bit.1y/2mT12Bh

${ }^{16}$ Semana de la Ciencia 2014 - Results Report http://bit.ly/21Ng1NA

${ }^{17}$ OpenLabNight 2014, ICG - TU Graz, Website: http://bit. ly/2mwigu4

${ }^{18}$ Semana de la Ciencia 2013 - Results Report http://bit.ly/2npz2lF

${ }^{19}$ SSRR 2013 Demos Website http://www.ssrr-conference.org/2013/Demos.html
} 


\section{J.3 Using Trajectory and Speed Planner}

The works related to the 2016 DJI Developer Challenge, which entailed the improvement and development of the trajectory planner presented in Chap. 4, have been showcased, representing the ICG - TU Graz, in the following two public events:

- An experimental fully autonomous package delivery flight demonstration made by the ICG - TU Gra $2^{20}$ in collaboration with the company SFL Technologies as part of the roll-out of their brand new electric vehicle, the ELI. The roll-out of this vehicle and the autonomous flight demo have been described by two Austrian newspapers: Mein Bezirk ${ }^{21}$ and Kleine Zeitung ${ }^{22}$.

November 2016

- A public presentation, with no experimental flights, named Intelligent Search and Rescue Drone in the world renowned Ars Electronica Festival 2016 - DroneLab - FutureLab 23 . This festival shows modern artwork that utilizes technology as a medium of expression and tries to deliver an outstanding and open view of current technology developments to its public.

September 8-12, 2016

\footnotetext{
${ }^{20}$ Fully autonomous package delivery flight demonstration: https : / youtu . be/bxM6dls 2 wuo

${ }^{21}$ ELI roll-out - Mein Bezirk - http://bit. ly/2hott $8 t$

${ }^{22}$ ELI roll-out - Kleine Zeitung - http://bit. ly/2it6N2P

${ }^{23}$ Ars Electronica Festival 2016 - DroneLab: http://www. aec.at/radicalatoms/en/ars-electronica-dronelab/
} 


\section{Bibliography}

Abdul Shukor, S. (2013). "A geometrical-based approach to recognise structure of complex interiors". PhD thesis. University of Warwick.

Abeywardena, D., Kodagoda, S., Dissanayake, G., and Munasinghe, R. (2013). "Improved State Estimation in Quadrotor MAVs: A Novel Drift-Free Velocity Estimator". IEEE Robotics \& Automation Magazine 20.4, pp. 32-39.

Achtelik, M., Achtelik, M., Weiss, S., and Siegwart, R. (2011). "Onboard IMU and monocular vision based control for MAVs in unknown in- and outdoor environments". In: Robotics and Automation (ICRA), 2011 IEEE International Conference on, pp. 3056-3063.

Achtelik, M. W., Lynen, S., Chli, M., and Siegwart, R. (2013). "Inversion based direct position control and trajectory following for micro aerial vehicles". In: Intelligent Robots and Systems (IROS), 2013 IEEE/RSJ International Conference on. IEEE, pp. 2933-2939.

Achtelik, M. W., Lynen, S., Weiss, S., Kneip, L., Chli, M., and Siegwart, R. (2012). "Visual-inertial SLAM for a small helicopter in large outdoor environments". In: Intelligent Robots and Systems (IROS), 2012 IEEE/RSJ International Conference on. IEEE, pp. 2651-2652.

Achtelik, M., Bachrach, A., He, R., Prentice, S., and Roy, N. (2009). "Stereo vision and laser odometry for autonomous helicopters in GPS-denied indoor environments". In: SPIE Defense, Security, and Sensing. International Society for Optics and Photonics, pp. 733219-733219.

Aerosystems, B. (1999). "Civilian applications: the challenges facing the UAV industry". Air \& Space Europe.

Agrachev, A. A. and Sachkov, Y. (2004). Control theory from the geometric viewpoint. Vol. 2. Springer Science \& Business Media.

Mani Monajjemi (2012). ardrone_autonomy : A ROS Driver for ARDrone 1.0 \& 2.0. https : / / github. com / AutonomyLab/ardrone_autonomy, Autonomy Lab, Simon Fraser University.

Argyros, A., Georgiadis, P., Trahanias, P., and Tsakiris, D. (2002). "Semi-autonomous Navigation of a Robotic Wheelchair". Journal of Intelligent and Robotic Systems 34 (3), pp. 315-329.

Ars Electronica Linz Gmbh (2012). SPAXELS: DRONE SHOWS SINCE 2012. https : / / www . spaxels.at/. Accessed: 2017-03-19.

Arulampalam, M. S., Maskell, S., Gordon, N., and Clapp, T. (2002). “A tutorial on particle filters for online nonlinear/non-Gaussian Bayesian tracking”. Signal Processing, IEEE Transactions on 50.2, pp. 174-188.

AscendingTechnologies (2010). Ascending Technologies. http://www.asctec.de.

Babenko, B., Yang, M.-H., and Belongie, S. (2009). "Visual tracking with online Multiple Instance Learning". In: Computer Vision and Pattern Recognition, 2009. CVPR 2009. IEEE Conference on, pp. 983990 . 
Bachrach, A. G. (2009). “Autonomous flight in unstructured and unknown indoor environments”. MA thesis. Massachusetts Institute of Technology.

Bachrach, A., Prentice, S., He, R., and Roy, N. (2011). "RANGE-Robust autonomous navigation in GPSdenied environments". Journal of Field Robotics 28.5, pp. 644-666.

Baker, S. and Matthews, I. (2004). "Lucas-kanade 20 years on: A unifying framework". International journal of computer vision 56.3, pp. 221-255.

Barfoot, T., Forbes, J. R., and Furgale, P. T. (2011). "Pose estimation using linearized rotations and quaternion algebra". Acta Astronautica 68.1, pp. 101-112.

Bento, M. (2008). "Unmanned aerial vehicles: an overview”. Inside GNSS 3.1, pp. 54-61.

Bills, C., Chen, J., and Saxena, A. (2011). "Autonomous MAV flight in indoor environments using single image perspective cues”. In: Robotics and Automation (ICRA), 2011 IEEE International Conference on, pp. 5776-5783.

Blyenburgh, P. v. (2006). “UAV Systems : Global Review”. In: Avionics'06 Conference. Amsterdam, The Netherlands.

Bouabdallah, Samir, Murrieri, Pierpaolo, and Siegwart, Roland (2005). "Towards Autonomous Indoor Micro VTOL”. Autonomous Robots 18 (2). 10.1007/s10514-005-0724-z, pp. 171-183.

Bouabdallah, Samir and Siegwart, Roland (2007). "Full control of a quadrotor". In: Intelligent robots and systems, 2007. IROS 2007. IEEE/RSJ international conference on. IEEE, pp. 153-158.

Bouabdallah, S., Murrieri, P., and Siegwart, R. (2005). "Towards autonomous indoor micro VTOL". Autonomous Robots 18.2, pp. 171-183.

Bouabdallah, S. and Siegwart, R. (2005). "Backstepping and sliding-mode techniques applied to an indoor micro quadrotor". In: Robotics and Automation, 2005. ICRA 2005. Proceedings of the 2005 IEEE International Conference on. IEEE, pp. 2247-2252.

Bourquardez, O., Mahony, R., Guenard, N., Chaumette, F., Hamel, T., and Eck, L. (2009). "Image-Based Visual Servo Control of the Translation Kinematics of a Quadrotor Aerial Vehicle". Robotics, IEEE Transactions on 25.3, pp. 743-749.

Breckenridge, W. (1999). "Quaternions proposed standard conventions". Jet Propulsion Laboratory, Pasadena, CA, Interoffice Memorandum IOM, pp. 343-79.

Bristeau, P.-J., Callou, F., Vissiere, D., Petit, N., et al. (2011). "The navigation and control technology inside the ar. drone micro uav". In: 18th IFAC World Congress. Vol. 18, pp. 1477-1484.

Burschka, D. and Mair, E. (2008). "Direct pose estimation with a monocular camera". In: Robot Vision. Springer, pp. 440-453.

Campoy, P., Correa, J. F., Mondragón, I., Martínez, C., Olivares, M., Mejías, L., and Artieda, J. (2009). "Computer Vision Onboard UAVs for Civilian Tasks". Journal of Intelligent and Robotic Systems 54.1-3, pp. 105-135.

Castillo, P., Dzul, A., and Lozano, R. (2004). "Real-time stabilization and tracking of a four-rotor mini rotorcraft”. Control Systems Technology, IEEE Transactions on 12.4, pp. 510-516.

Castillo, P., Lozano, R., and Dzul, A. (2005). "Stabilization of a mini rotorcraft with four rotors". IEEE Control Systems Magazine 25.6, pp. 45-55.

Chaumette, F. (1998). "Potential problems of stability and convergence in image-based and position-based visual servoing". In: The confluence of vision and control. Springer, pp. 66-78.

Cheng, Y. and Crassidis, J. L. (2004). "Particle filtering for sequential spacecraft attitude estimation". In: Proc. of the 2004 AIAA Guidance, Navigation, and Control Conference, p. 5337.

Chesi, G. (2009). "Visual servoing path planning via homogeneous forms and LMI optimizations". Robotics, IEEE Transactions on 25.2, pp. 281-291.

Chesi, G. and Vicino, A. (2004). "Visual servoing for large camera displacements". Robotics, IEEE Transactions on 20.4 , pp. 724-735.

Comport, A., Mahony, R., and Spindler, F. (2011). "A visual servoing model for generalised cameras: Case study of non-overlapping cameras". In: Robotics and Automation (ICRA), 2011 IEEE International Conference on, pp. 5683-5688.

Corke, P. (2011). Robotics, vision and control: fundamental algorithms in MATLAB. Vol. 73. Springer.

Corke, P. I. et al. (1996). Visual Control of Robots: high-performance visual servoing. Research Studies Press Taunton, UK.

Cowling, I. D., Yakimenko, O. A., Whidborne, J. F., and Cooke, A. K. (2010). "Direct method based control system for an autonomous quadrotor". Journal of Intelligent \& Robotic Systems 60.2, pp. 285316. 
Crassidis, J. L. (2006). "Sigma-point Kalman filtering for integrated GPS and inertial navigation". Aerospace and Electronic Systems, IEEE Transactions on 42.2, pp. 750-756.

Crassidis, J. L., Markley, F. L., and Cheng, Y. (2007). "Survey of nonlinear attitude estimation methods". Journal of Guidance, Control, and Dynamics 30.1, pp. 12-28.

Mellado Bataller, I. (2012). A new framework for interfacing with MAVs. https: / / github . com/ uavster/mavwork

Danelljan, M., Khan, F. S., Felsberg, M., Granström, K., Heintz, F., Rudol, P., Wzorek, M., Kvarnström, J., and Doherty, P. (2014). "A low-level active vision framework for collaborative unmanned aircraft systems". In: Workshop at the European Conference on Computer Vision. Springer, pp. 223-237.

Danelljan, M., Shahbaz Khan, F., Felsberg, M., and Van de Weijer, J. (2014). “Adaptive color attributes for real-time visual tracking". In: Proceedings of the IEEE Conference on Computer Vision and Pattern Recognition, pp. 1090-1097.

Davison, A. J., Reid, I. D., Molton, N. D., and Stasse, O. (2007). "MonoSLAM: Real-time single camera SLAM". IEEE transactions on pattern analysis and machine intelligence 29.6.

Davison, A. J. (1998). "Mobile Robot Navigation Using Active Vision". PhD thesis. University of Oxford.

Davison, R. A. N. and J., A. (2010). "Live Dense Reconstruction with a Single Moving Camera". In: Computer Vision and Pattern Recognition.

Deng, H., Zhao, X., and How, Z. (2009). "A Vision-Based Ground Target Tracking System for a SmallScale Autonomous Helicopter”. Image and Graphics, International Conference on, pp. 739-743.

Dijkstra, E. W. (1959). "A note on two problems in connexion with graphs". Numerische mathematik 1.1, pp. 269-271.

DJI - Da-Jiang Innovations Science and Technology Co., Ltd (2015). DJI Matrice developer drone line. https://www.dji.com/matrice100, Accessed: 2017-03-18.

Doucet, A., De Freitas, N., and Gordon, N. (2001). An introduction to sequential Monte Carlo methods. Springer.

Driessen, B. J. and Robin, A. L. (2004). “A globally convergent tracking controller for the X4 flyer rotor craft for reference trajectories with positive thrust". Robotica 22.04, pp. 375-388.

Dunbar, N. and Franz, R. (2003). "Online control customization via optimization, based control". Software-Enabled Control: Information Technology for Dynamical Systems, p. 149.

Engel, Jakob, Sturm, Jürgen, and Cremers, Daniel (2014). "Scale-aware navigation of a low-cost quadrocopter with a monocular camera". Robotics and Autonomous Systems 62.11, pp. 1646-1656.

Engel, J., Sturm, J., and Cremers, D. (2012a). "Camera-Based Navigation of a Low-Cost Quadrocopter". In: Proc. of the International Conference on Intelligent Robot Systems (IROS).

Engel, J., Schöps, T., and Cremers, D. (2014). "LSD-SLAM: Large-scale direct monocular SLAM". In: Computer Vision-ECCV 2014. Springer, pp. 834-849.

Engel, J., Sturm, J., and Cremers, D. (2013). "Semi-dense visual odometry for a monocular camera". In: Computer Vision (ICCV), 2013 IEEE International Conference on. IEEE, pp. 1449-1456.

Engel, J., Sturm, J., and Cremers, D. (2012b). "Accurate figure flying with a quadrocopter using onboard visual and inertial sensing”. IMU 320, p. 240.

ERSG (2013). Roadmap for the integration of civil Remotely - Piloted Aircraft Systems into the European Aviation System. Tech. rep. European RPAS Steering Group.

Espiau, B., Chaumette, F., and Rives, P. (1992). "A new approach to visual servoing in robotics". ieee Transactions on Robotics and Automation 8.3, pp. 313-326.

Fahd Rafi Saad Khan, K. S. and Shah, M. (2006). "Autonomous target following by unmanned aerial vehicles”. In: Proc. SPIE. Orlando (Kissimmee), FL, USA.

Faigl, J., Krajník, T., Vonásek, V., and Přeučil, L. (2010). "Surveillance Planning with Localization Uncertainty for UAVs". In: 3rd Israeli Conference on Robotics, Ariel: Ariel University Center, pages.

Farrell, J. L. (1970). “Attitude determination by Kalman filtering”. Automatica 6.3, pp. 419-430.

Farrenkopf, R. (1978). "Analytic steady-state accuracy solutions for two common spacecraft attitude estimators". Journal of Guidance, Control, and Dynamics 1.4, pp. 282-284.

Feddema, J. T. and Mitchell, O. R. (1989). "Vision-guided servoing with feature-based trajectory generation [for robots]". Robotics and Automation, IEEE Transactions on 5.5, pp. 691-700.

Finn, R. L. and Wright, D. (2012). "Unmanned aircraft systems: Surveillance, ethics and privacy in civil applications". Computer Law \& Security Review 28.2, pp. 184-194.

Fliess, M., Lévine, J., Martin, P., and Rouchon, P. (1995). "Flatness and defect of non-linear systems: introductory theory and examples”. International journal of control 61.6, pp. 1327-1361. 
Fomena, R. T. and Chaumette, F. (2007). "Visual servoing from spheres using a spherical projection model". In: Robotics and Automation, 2007 IEEE International Conference on. IEEE, pp. 2080-2085.

Forster, C., Lynen, S., Kneip, L., and Scaramuzza, D. (2013). "Collaborative monocular SLAM with multiple micro aerial vehicles". In: Intelligent Robots and Systems (IROS), 2013 IEEE/RSJ International Conference on. IEEE, pp. 3962-3970.

Forster, C., Pizzoli, M., and Scaramuzza, D. (2014). "SVO: Fast Semi-Direct Monocular Visual Odometry". In: IEEE International Conference on Robotics and Automation (ICRA).

Fraundorfer, F., Heng, L., Honegger, D., Lee, G. H., Meier, L., Tanskanen, P., and Pollefeys, M. (2012). "Vision-based autonomous mapping and exploration using a quadrotor MAV". In: Intelligent Robots and Systems (IROS), 2012 IEEE/RSJ International Conference on. IEEE, pp. 4557-4564.

Fraundorfer, F. and Scaramuzza, D. (2012). "Visual odometry: Part II: Matching, robustness, optimization, and applications". IEEE Robotics \& Automation Magazine 19.2, pp. 78-90.

Fritsch, O., De Monte, P., Buhl, M., and Lohmann, B. (2012). "Quasi-static feedback linearization for the translational dynamics of a quadrotor helicopter". In: American Control Conference (ACC), 2012. IEEE, pp. 125-130.

Fu, C., Olivares-Mendez, M. A., Campoy, P., and Suarez-Fernandez, R. (2013). "UAS see-and-avoid strategy using a fuzzy logic controller optimized by Cross-Entropy in Scaling Factors and Membership Functions". In: Unmanned Aircraft Systems (ICUAS), 2013 International Conference on. IEEE, pp. 532-541.

Furrer, F., Burri, M., Achtelik, M., and Siegwart, R. (2016). "RotorS-A Modular Gazebo MAV Simulator Framework". In: Robot Operating System (ROS). Springer, pp. 595-625.

Garrido-Jurado, S., Muñoz-Salinas, R., Madrid-Cuevas, F. J., and Marín-Jiménez, M. J. (2014). “Automatic generation and detection of highly reliable fiducial markers under occlusion". Pattern Recognition 47.6, pp. 2280-2292.

Gillula, J. H., Huang, H., Vitus, M. P., and Tomlin, C. J. (2010a). "Design of Guaranteed Safe Maneuvers Using Reachable Sets : Autonomous Quadrotor Aerobatics in Theory and Practice". IEEE Electrical Engineering (2010), pp. 1649-1654.

Goel, R., Shah, S. M., Gupta, N. K., and Ananthkrishnan, N. (2009). "Modeling, simulation and flight testing of an autonomous quadrotor". Proceedings of ICEAE, pp. 1-7.

Guenard, N., Hamel, T., and Moreau, V. (2005). "Dynamic modeling and intuitive control strategy for an" X4-flyer"'. In: Control and Automation, 2005. ICCA'05. International Conference on. Vol. 1. IEEE, pp. 141-146.

Haag, K., Dotenco, S., and Gallwitz, F. (2015). "Correlation filter based visual trackers for person pursuit using a low-cost Quadrotor”. In: Innovations for Community Services (I4CS), 2015 15th International Conference on. IEEE, pp. 1-8.

Hamel, T. and Mahony, R. (2002a). "Visual servoing of an under-actuated dynamic rigid-body system: an image-based approach". Robotics and Automation, IEEE Transactions on 18.2, pp. 187-198.

Hamel, T. and Mahony, R. (2002b). "Visual servoing of an under-actuated dynamic rigid-body system: an image-based approach". Robotics and Automation, IEEE Transactions on 18.2, pp. 187-198.

Hartley, R. I. and Zisserman, A. (2004). Multiple View Geometry in Computer Vision. Second. Cambridge University Press, ISBN: 0521540518.

Heng, L., Honegger, D., Lee, G. H., Meier, L., Tanskanen, P., Fraundorfer, F., and Pollefeys, M. (2014). "Autonomous visual mapping and exploration with a micro aerial vehicle". Journal of Field Robotics 31.4, pp. 654-675.

Henry, P., Krainin, M., Herbst, E., Ren, X., and Fox, D. (2010). "RGB-D Mapping : Using Depth Cameras for Dense 3D Modeling of Indoor Environments". In: International Symposium on Experimental Robotics (ISER), 2010.

Hofer, M., Maurer, M., and Bischof, H. (2016). "Efficient 3D scene abstraction using line segments". Computer Vision and Image Understanding.

Hoffmann, Gabriel M, Waslander, Steven L, and Tomlin, Claire J (2008). "Quadrotor Helicopter Trajectory Tracking Control”. Electrical Engineering (2008), pp. 1-14 (cit. on p. 113).

Hoffmann, G.M., Waslander, S.L., and Tomlin, C.J. (2009). "Aerodynamics and control of autonomous quadrotor helicopters in aggressive maneuvering". In: 2009 IEEE International Conference on Robotics and Automation. Ieee, pp. 3277-3282. 
Hoffmann, G. M., Huang, H., Waslander, S. L., and Tomlin, C. J. (2007). "Quadrotor helicopter flight dynamics and control: Theory and experiment". In: Proc. of the AIAA Guidance, Navigation, and Control Conference. Vol. 2.

Hoffmann, G. M., Waslander, S. L., and Tomlin, C. J. (2006). "Distributed Cooperative Search using Information-Theoretic Costs for Particle Filters, with Quadrotor Applications”. Electrical Engineering, pp. 1-21.

Holzmann, T., Prettenthaler, R., Pestana, J., Muschick, D., Graber, G., Mostegel, C., Fraundorfer, F., and Bischof, H. (2015). "Performance Evaluation of Vision-Based Algorithms for MAVs". arXiv preprint arXiv: 1505.02247.

Honegger, D., Meier, L., Tanskanen, P., and Pollefeys, M. (2013). “An open source and open hardware embedded metric optical flow cmos camera for indoor and outdoor applications". In: Robotics and Automation (ICRA), 2013 IEEE International Conference on. IEEE, pp. 1736-1741.

Hoppe, C., Klopschitz, M., Donoser, M., and Bischof, H. (2013). "Incremental Surface Extraction from Sparse Structure-from-Motion Point Clouds.” In: BMVC, pp. 94-1.

Hoppe, C., Klopschitz, M., Rumpler, M., Wendel, A., Kluckner, S., Bischof, H., and Reitmayr, G. (2012). "Online Feedback for Structure-from-Motion Image Acquisition." In: BMVC. Vol. 2, p. 6.

Hornung, A., Wurm, K. M., Bennewitz, M., Stachniss, C., and Burgard, W. (2013). "OctoMap: An efficient probabilistic 3D mapping framework based on octrees". Autonomous Robots 34.3, pp. 189206.

Hörtner, H., Gardiner, M., Haring, R., Lindinger, C., and Berger, F. (2012). "Spaxels, Pixels in Space-A Novel Mode of Spatial Display." In: International Conference on Signal Processing and Multimedia Applications, SIGMAP, pp. 19-24.

Hua, M.-D., Hamel, T., Morin, P., and Samson, C. (2009). "A control approach for thrust-propelled underactuated vehicles and its application to VTOL drones". Automatic Control, IEEE Transactions on 54.8, pp. 1837-1853.

Huang, A. S., Bachrach, A., Henry, P., Krainin, M., Fox, D., and Roy, N. (2011). "Visual Odometry and Mapping for Autonomous Flight Using an RGB-D Camera". Camera.

Huang, H., Hoffmann, G. M., Waslander, S. L., and Tomlin, C. J. (2009). "Aerodynamics and control of autonomous quadrotor helicopters in aggressive maneuvering". In: Robotics and Automation, 2009. ICRA'09. IEEE International Conference on. IEEE, pp. 3277-3282.

Hutchinson, S and Chaumette, F (2006). "Visual servo control, Part I: Basic approaches". IEEE Robotics and Automation Magazine 13.4, pp. 82-90.

Hutchinson, S and Chaumette, F (2007). "Visual servo control, Part II: Advanced approaches". IEEE Robotics and Automation Magazine 14.1, pp. 109-118.

Intel Corporation (2014). Intel RealSense Developer Kits. https : / / click . intel . com / realsense.html, Accessed: 2017-03-18.

Intel Corporation (2016a). Intel Aero Compute Board. https : / / click . intel . com / intelaero-platform-for-uavs-compute-board.html. Accessed: 2017-03-18.

Intel Corporation (2016b). Intel Aero Ready to Fly Drone. https : / click . intel . com/intelaero-ready-to-fly-drone.html. Accessed: 2017-03-18.

James, G., Witten, D., Hastie, T., and Tibshirani, R. (2013). An introduction to statistical learning. Vol. 6. Springer.

Julier, S. J., Uhlmann, J. K., and Durrant-Whyte, H. F. (1995). “A new approach for filtering nonlinear systems”. In: American Control Conference, Proceedings of the 1995. Vol. 3. IEEE, pp. 1628-1632.

Jurdjevic, V. (1997). Geometric control theory. Cambridge university press.

Kalal, Z. (2011). "Tracking Learning Detection”. PhD thesis. University of Surrey.

Kalal, Z., Mikolajczyk, K., and Matas, J. (2012). “Tracking-Learning-Detection”. IEEE Transactions on Pattern Analysis and Machine Intelligence 34.7, pp. 1409-1422.

Karaman, S. and Frazzoli, E. (2011). "Sampling-based algorithms for optimal motion planning". The international journal of robotics research 30.7, pp. 846-894.

Kavraki, L. E., Svestka, P., Latombe, J.-C., and Overmars, M. H. (1996). "Probabilistic roadmaps for path planning in high-dimensional configuration spaces". IEEE transactions on Robotics and Automation 12.4, pp. 566-580.

Kendoul, F., Yu, Z., and Nonami, K. (2010). "Guidance and nonlinear control system for autonomous flight of minirotorcraft unmanned aerial vehicles”. Journal of Field Robotics 27.3, pp. 311-334. 
Klein, G. and Murray, D. (2007). "Parallel Tracking and Mapping for Small AR Workspaces”. In: 2007 6th IEEE and ACM International Symposium on Mixed and Augmented Reality, pp. 1-10.

Klein, G. and Murray, D. (2009). "Parallel Tracking and Mapping on a camera phone”. In: 2009 8th IEEE International Symposium on Mixed and Augmented Reality, pp. 83-86.

Kneip, L., Scaramuzza, D., and Siegwart, R. (2011). "A novel parametrization of the perspective-threepoint problem for a direct computation of absolute camera position and orientation". In: Computer Vision and Pattern Recognition (CVPR), 2011 IEEE Conference on. IEEE, pp. 2969-2976.

Koller, D. and Friedman, N. (2009). Probabilistic Graphical Models: Principles and Techniques. MIT Press.

Korodi, A., Codrean, A., Banita, L., and Volosencu, C. (2008). “Aspects regarding the object following control procedure for wheeled mobile robots”. WSEAS Trans. Sys. Ctrl. 3 (6), pp. 537-546.

Krajník, T., Faigl, J., Vonásek, V., Košnar, K., Kulich, M., and Přeučil, L. (2010). "Simple, Yet Stable Bearing-Only Navigation”. Journal of Field Robotics 27.5, pp. 511-533.

Krajník, T., Vonásek, V., Fišer, D., and Faigl, J. (2011). "AR-Drone as a Platform for Robotic Research and Education". In: Research and Education in Robotics: EUROBOT 2011. Heidelberg: Springer.

Kuipers, J. (2002). Quaternions and Rotation Sequences: A Primer with Applications to Orbits, Aerospace and Virtual Reality.

Kushleyev, A., Kumar, V., and Mellinger, D. (2012). “Towards A Swarm of Agile Micro Quadrotors”. In: Proceedings of Robotics: Science and Systems. Sydney, Australia.

Labatut, P., Pons, J.-P., and Keriven, R. (2007). "Efficient multi-view reconstruction of large-scale scenes using interest points, delaunay triangulation and graph cuts". In: 2007 IEEE 11th international conference on computer vision. IEEE, pp. 1-8.

Lapresté, J.-T. and Mezouar, Y. (2004). “A Hessian approach to visual servoing”. In: Intelligent Robots and Systems, 2004.(IROS 2004). Proceedings. 2004 IEEE/RSJ International Conference on. Vol. 1. IEEE, pp. 998-1003.

Lau, B., Sprunk, C., and Burgard, W. (2013). "Efficient grid-based spatial representations for robot navigation in dynamic environments". Elsevier RAS2013.

LaValle, S. M. (1998). "Rapidly-Exploring Random Trees: A New Tool for Path Planning".

LaValle, S. M. and Kuffner, J. J. (2001). "Randomized kinodynamic planning”. The International Journal of Robotics Research 20.5, pp. 378-400.

Leberl, F., Irschara, A., Pock, T., Meixner, P., Gruber, M., Scholz, S., Wiechert, A., and Scholz, S. (2010). "Point Clouds : Lidar versus 3D Vision”. Photogrammetric Engineering \& Remote Sensing 76.10.

Lee, D., Kim, H. J., and Sastry, S. (2009). "Feedback linearization vs. adaptive sliding mode control for a quadrotor helicopter". International Journal of control, Automation and systems 7.3, pp. 419-428.

Lee, T., Leoky, M., and McClamroch, N. H. (2010). "Geometric tracking control of a quadrotor UAV on SE (3)". In: Decision and Control (CDC), 2010 49th IEEE Conference on. IEEE, pp. 5420-5425.

Lefferts, E. J., Markley, F. L., and Shuster, M. D. (1982). "Kalman filtering for spacecraft attitude estimation”. Journal of Guidance, Control, and Dynamics 5.5, pp. 417-429.

Leishman, R. C., Macdonald, J., Beard, R. W., and McLain, T. W. (2014). "Quadrotors and accelerometers: State estimation with an improved dynamic model". Control Systems, IEEE 34.1, pp. 28-41.

Leutenegger, S., Chli, M., and Siegwart, R. Y. (2011). "BRISK: Binary robust invariant scalable keypoints”. In: Computer Vision (ICCV), 2011 IEEE International Conference on. IEEE, pp. 2548-2555.

Leutenegger, S., Furgale, P. T., Rabaud, V., Chli, M., Konolige, K., and Siegwart, R. (2013). "KeyframeBased Visual-Inertial SLAM using Nonlinear Optimization.” In: Robotics: Science and Systems.

Leutenegger, S., Lynen, S., Bosse, M., Siegwart, R., and Furgale, P. (2014). "Keyframe-based visualinertial odometry using nonlinear optimization”. The International Journal of Robotics Research, p. 0278364914554813.

Li, M. and Mourikis, A. I. (2013). "High-Precision, Consistent EKF-based Visual-Inertial Odometry". International Journal of Robotics Research 32.6, pp. 690-711.

Li, M. and Mourikis, A. I. (2014a). "Online Temporal Calibration for Camera-IMU Systems: Theory and Algorithms". International Journal of Robotics Research 33.7, pp. 947-964.

Li, M. and Mourikis, A. I. (2014b). "Vision-aided Inertial Navigation with Rolling-Shutter Cameras". International Journal of Robotics Research. in print.

Lin, F., Chen, B., Lum, K. Y., and Lee, T. (2010). "A robust vision system on an unmanned helicopter for ground target seeking and following”. In: Intelligent Control and Automation (WCICA), 2010 8th World Congress on, pp. 276-281. 
Linux Foundation (2014). Dronecode Project. https : / / www . dronecode . org/about/ Accessed: 2017-03-18.

Lippiello, V., Loianno, G., and Siciliano, B. (2011). "MAV indoor navigation based on a closed-form solution for absolute scale velocity estimation using Optical Flow and inertial data". In: Decision and Control and European Control Conference (CDC-ECC), 2011 50th IEEE Conference on, pp. 35663571 .

Liu, Y.-C. and Dai, Q.-h. (2010). “A survey of computer vision applied in aerial robotic vehicles”. In: Optics Photonics and Energy Engineering (OPEE), 2010 International Conference on. Vol. 1. IEEE, pp. 277-280.

Liu, P., Chen, A. Y., Huang, Y.-N., Han, J.-Y., Lai, J.-S., Kang, S.-C., Wu, T.-H., Wen, M.-C., and Tsai, M.-H. (2014). "A review of rotorcraft Unmanned Aerial Vehicle (UAV) developments and applications in civil engineering”. SMART STRUCTURES AND SYSTEMS 13.6, pp. 1065-1094.

Lowe, D. G. (2004). "Distinctive image features from scale-invariant keypoints". International journal of computer vision 60.2, pp. 91-110.

Lynen, S., Achtelik, M. W., Weiss, S., Chli, M., and Siegwart, R. (2013). “A robust and modular multisensor fusion approach applied to mav navigation”. In: Intelligent Robots and Systems (IROS), 2013 IEEE/RSJ International Conference on. IEEE, pp. 3923-3929.

Madani, T. and Benallegue, A. (2007). "Backstepping control with exact 2-sliding mode estimation for a quadrotor unmanned aerial vehicle”. In: Intelligent Robots and Systems, 2007. IROS 2007. IEEE/RSJ International Conference on. IEEE, pp. 141-146.

Mahony, R., Corke, P., and Chaumette, F. (2002). "Choice of image features for depth-axis control in image based visual servo control”. In: Intelligent Robots and Systems, 2002. IEEE/RSJ International Conference on. Vol. 1, 390-395 vol.1.

Mahony, R., Brasch, A., Corke, P., and Hamel, T. (2005). "Adaptive depth estimation in image based visual servo control of dynamic systems." In: Decision and Control, 2005 and 2005 European Control Conference. CDC-ECC '05. 44th IEEE Conference on, pp. 5372-5378.

Mahony, R., Corke, P., and Chaumette, F. (2002). "Choice of image features for depth-axis control in image based visual servo control”. In: Intelligent Robots and Systems, 2002. IEEE/RSJ International Conference on. Vol. 1. IEEE, pp. 390-395.

Malis, E. (2004). "Improving vision-based control using efficient second-order minimization techniques". In: Robotics and Automation, 2004. Proceedings. ICRA'04. 2004 IEEE International Conference on. Vol. 2. IEEE, pp. 1843-1848.

Markley, F. L. (2003). "Attitude error representations for Kalman filtering”. Journal of guidance, control, and dynamics 26.2, pp. 311-317.

Martínez, C., Mondragón, I. F., Campoy, P., Sánchez-López, J. L., and Olivares-Méndez, M. A. (2013). “A Hierarchical Tracking Strategy for Vision-Based Applications On-Board UAVs”. Journal of Intelligent \& Robotic Systems, pp. 1-23.

Matsue, A., Hirosue, W., Tokutake, H., Sunada, S., and Ohkura, A. (2005). "Navigation of Small and Lightweight Helicopter". Transactions of the Japan Society for Aeronautical and Space Sciences 48.161, pp. 177-179.

Mayol, W., Davison, A., and Tordoff, B. (2004). "Interaction between hand and wearable camera in 2D and 3D environments". In: British Machine Vision Conference.

Meier, L., Honegger, D., and Pollefeys, M. (2015). "PX4: A node-based multithreaded open source robotics framework for deeply embedded platforms". In: Robotics and Automation (ICRA), 2015 IEEE International Conference on. IEEE, pp. 6235-6240.

Mellado-Bataller, I., Mejias, L., Campoy, P., and Olivares-Mendez, M. A. (2012). "Rapid prototyping framework for visual control of autonomous micro aerial vehicles". In: 12th International Conference on Intelligent Autonomous System (IAS-12). Jeju Island, Korea.

Mellado-Bataller, I., Pestana, J., Olivares-Mendez, M. A., Campoy, P., and Mejias, L. (2013). “MAVwork: a framework for unified interfacing between micro aerial vehicles and visual controllers". In: Frontiers of Intelligent Autonomous Systems. Springer, pp. 165-179.

Mellinger, D. and Kumar, V. (2011). "Minimum snap trajectory generation and control for quadrotors". In: Robotics and Automation (ICRA), 2011 IEEE International Conference on. IEEE, pp. 2520-2525.

Mellinger, D., Michael, N., and Kumar, V. (2010). "Trajectory Generation and Control for Precise Aggressive Maneuvers with Quadrotors". In: Int Symposium on Experimental Robotics (2010). 
Meyer, J., Sendobry, A., Kohlbrecher, S., Klingauf, U., and Stryk, O. von (2012). "Comprehensive simulation of quadrotor uavs using ros and gazebo". In: Simulation, Modeling, and Programming for Autonomous Robots. Springer, pp. 400-411.

Mezouar, Y. and Chaumette, F. (2002). "Path planning for robust image-based control". Robotics and Automation, IEEE Transactions On 18.4, pp. 534-549.

Michael, B. Y. N., Mellinger, D., and Lindsey, Q. (2010). "The GRASP Multiple Micro UAV Testbed". IEEE Robotics \& Automation Magazine, pp. 56-65 (cit. on p. 113).

Michael, N., Shen, S., Mohta, K., Mulgaonkar, Y., Kumar, V., Nagatani, K., Okada, Y., Kiribayashi, S., Otake, K., Yoshida, K., et al. (2012). "Collaborative mapping of an earthquake-damaged building via ground and aerial robots". Journal of Field Robotics 29.5, pp. 832-841.

Misawa, E. and Hedrick, J. (1989). "Nonlinear observers - a state-of-the-art survey". Journal of dynamic systems, measurement, and control 111.3, pp. 344-352.

Mistler, V., Benallegue, A., and M'sirdi, N. (2001). "Exact linearization and noninteracting control of a 4 rotors helicopter via dynamic feedback". In: Robot and Human Interactive Communication, 2001. Proceedings. 10th IEEE International Workshop on. IEEE, pp. 586-593.

Molton, N., Davison, A., and Reid, I. (2004). "Locally Planar Patch Features for Real-Time Structure from Motion”. British Machine Vision Conference, 2004, pp. 90.1-90.10.

Mondragoón, I., Campoy, P., Olivares-Mendez, M., and Martinez, C. (2011). “3D object following based on visual information for Unmanned Aerial Vehicles”. In: Robotics Symposium, 2011 IEEE IX Latin American and IEEE Colombian Conference on Automatic Control and Industry Applications (LARC), pp. 1-7.

Mostegel, C., Wendel, A., and Bischof, H. (2014). “Active monocular localization: Towards autonomous monocular exploration for multirotor mavs". In: Robotics and Automation (ICRA), 2014 IEEE International Conference on. IEEE, pp. 3848-3855.

Mourikis, A. I. and Roumeliotis, S. I. (2006). A Multi-State Constraint Kalman Filter for Vision-aided Inertial Navigation. Tech. rep. www.cs.umn.edu/ mourikis/tech_reports/TR_MSCKF.pdf. Dept. of Computer Science and Engineering, University of Minnesota.

Mourikis, A. I. and Roumeliotis, S. I. (2007). "A multi-state constraint Kalman filter for vision-aided inertial navigation". In: Robotics and Automation, 2007 IEEE International Conference on. IEEE, pp. 3565-3572.

Mourikis, A. I., Trawny, N., Roumeliotis, S. I., Johnson, A. E., Ansar, A., and Matthies, L. (2009). "Vision-aided inertial navigation for spacecraft entry, descent, and landing". Robotics, IEEE Transactions on 25.2, pp. 264-280.

Murray, R. M., Rathinam, M., and Sluis, W. (1995). "Differential flatness of mechanical control systems: A catalog of prototype systems". In: ASME International Mechanical Engineering Congress and Exposition. Citeseer.

Murrell, J. W. (1978). "Precision attitude determination for multimission spacecraft". AIAA paper 78, p. 1248.

Nebehay, G. (2012). "Robust Object Tracking Based on Tracking-Learning-Detection”. MA thesis. Austria: Vienna University of Technology.

Nice, E. B. (2004). "Design of a four rotor hovering vehicle". PhD thesis. Cornell University.

Nikolic, J., Rehder, J., Burri, M., Gohl, P., Leutenegger, S., Furgale, P. T., and Siegwart, R. (2014). “A synchronized visual-inertial sensor system with FPGA pre-processing for accurate real-time SLAM". In: Robotics and Automation (ICRA), 2014 IEEE International Conference on. IEEE, pp. 431-437.

Nister, D. and Stewenius, H. (2006). "Scalable Recognition with a Vocabulary Tree". In: 2006 IEEE Computer Society Conference on Computer Vision and Pattern Recognition - Volume 2 (CVPR'06). Ieee, pp. 2161-2168.

NøRgaard, M., Poulsen, N. K., and Ravn, O. (2000). "New developments in state estimation for nonlinear systems". Automatica 36.11 , pp. 1627-1638.

Nützi, G., Weiss, S., Scaramuzza, D., and Siegwart, R. (2011). "Fusion of IMU and vision for absolute scale estimation in monocular SLAM". Journal of intelligent \& robotic systems 61.1-4, pp. 287-299.

Nvidia Corporation (2014). NVIDIA Jetson TK1 developer kit. https : / / www . nvidia . com / object/jetson-tk1-embedded-dev-kit.html. Accessed: 2017-03-18.

Kalal, Z (2011). OpenTLD object image tracker source repositories: (2011) Kalal, Z (PhD Thesis); Matlab OpenTLD implementation https://github.com/zk00006/OpenTLD 
Nebehay, G (2012). OpenTLD object image tracker source repositories: (2012) Nebehay, G(Msc. Thesis); C++ OpenTLD implementation https://github.com/gnebehay/OpenTLD.

Chauvin, R (2012). OpenTLD object image tracker source repositories: (2013) Chauvin, $R$; $C++R O S$ OpenTLD wrapper https://github.com/Ronan0912/ros_opentld

Oshman, Y. and Carmi, A. (2004). "Estimating attitude from vector observations using a genetic algorithm-embedded quaternion particle filter". In: AIAA Guidance, Navigation, and Control Conference and Exhibit. AIAA Reston, VA, pp. 1-24.

Pajares, G. (2015). "Overview and Current Status of Remote Sensing Applications Based on Unmanned Aerial Vehicles (UAVs)". Photogrammetric Engineering \& Remote Sensing 81.4, pp. 281-330.

Park, M. (2004). Error analysis and stochastic modeling of MEMS based inertial sensors for land vehicle navigation applications. University of Calgary, Department of Geomatics Engineering.

Parrot SA (2014). Parrot BEBOP 2. https: / / www.parrot.com/us/Drones/Parrot-bebop2. Accessed: 2017-03-18.

Parrot SA (2016). Parrot S.L.A.M. dunk. https : / / www . parrot . com / us / business solutions/parrot-slamdunk. Accessed: 2017-03-14.

Pestana, J., Mellado-Bataller, I., Fu, C., Sanchez-Lopez, J. L., Mondragon, I. F., and Campoy, P. (2012). “A Visual Guided Quadrotor for IMAV 2012 Indoor Autonomy Competition and Visual Control of a Quadrotor for the IMAV 2012 Indoor Dynamics Competition”. International Micro Air Vehicle (IMAV), 2012 Conference and Flight Competition.

Pestana, J. (2012). "On-board control algorithms for Quadrotors and indoors navigation". MA thesis. Spain: Universidad Politécnica de Madrid.

Pestana, J., Mellado-Bataller, I., Fu, C., Sanchez-Lopez, J. L., Mondragon, I. F., and Campoy, P. (2013). "A general purpose configurable navigation controller for micro aerial multirotor vehicles". In: Unmanned Aircraft Systems (ICUAS), 2013 International Conference on. IEEE, pp. 557-564 (cit. on p. 113.

Pestana, J., Mellado-Bataller, I., Sanchez-Lopez, J. L., Fu, C., Mondragón, I. F., and Campoy, P. (2014). "A general purpose configurable controller for indoors and outdoors gps-denied navigation for multirotor unmanned aerial vehicles". Journal of Intelligent \& Robotic Systems 73.1-4, pp. 387-400.

Pestana, J., Prettenthaler, R., Holzmann, T., Muschick, D., Mostengel, C., Fraundorfer, F., and Bischof, H. (2015). "Graz griffins' solution to the european robotics challenges 2014". In: Proceedings of Austrian Robotics Workshop 2015, pp. 11-12.

Pestana, J., Sanchez-Lopez, J. L., Campoy, P., and Saripalli, S. (2013). "Vision based gps-denied object tracking and following for unmanned aerial vehicles". In: 2013 IEEE International Symposium on Safety, Security, and Rescue Robotics (SSRR). IEEE, pp. 1-6.

Pestana, J., Sanchez-Lopez, J. L., Puente, P. de la, Carrio, A., and Campoy, P. (2015). “A Vision-based Quadrotor Multi-robot Solution for the Indoor Autonomy Challenge of the 2013 International Micro Air Vehicle Competition”. Journal of Intelligent \& Robotic Systems, pp. 1-20 (cit. on p. 113).

Pestana, J., Sanchez-Lopez, J. L., Saripalli, S., and Campoy, P. (2014). “Computer vision based general object following for GPS-denied multirotor unmanned vehicles". In: 2014 American Control Conference. IEEE, pp. 1886-1891.

Possegger, H., Mauthner, T., and Bischof, H. (2015). "In defense of color-based model-free tracking". In: Proceedings of the IEEE Conference on Computer Vision and Pattern Recognition, pp. 2113-2120.

Psiaki, M. L. (2000). “Attitude-determination filtering via extended quaternion estimation”. Journal of Guidance, Control, and Dynamics 23.2, pp. 206-214.

Qualcomm Technologies, Inc. (2015). Snapdragon Flight Kit. https: / / developer. qualcomm . com/hardware/snapdragon-flight Accessed: 2017-03-18.

Quaritsch, M., Stojanovski, E., Bettstetter, C., Friedrich, G., Hellwagner, H., Rinner, B., Hofbaur, M., and Shah, M. (2008). "Collaborative Microdrones: Applications and Research Challenges". In: Proceedings of the Second International ICST Conference on Autonomic Computing and Communication Systems. Gent, BELGIUM: Icst.

Quigley, M., Gerkey, B., Conley, K., Faust, J., Foote, T., Leibs, J., Berger, E., Wheeler, R., and Ng, A. (2009). "ROS : an open-source Robot Operating System". In: IEEE International Conference on Robotics and Automation (ICRA 2009).

Raffo, G. V., Ortega, M. G., and Rubio, F. R. (2010). “An integral predictive/nonlinear Hinf control structure for a quadrotor helicopter”. Automatica 46.1, pp. 29-39. 
Richter, Charles, Bry, Adam, and Roy, Nicholas (2013). "Polynomial trajectory planning for aggressive quadrotor flight in dense indoor environments". In: Proceedings of the International Symposium on Robotics Research (ISRR).

Richter, C., Bry, A., and Roy, N. (2013). "Polynomial trajectory planning for quadrotor flight". In: International Conference on Robotics and Automation.

Rives, P. and Azinheira, J. R. (2004). "Linear structures following by an airship using vanishing point and horizon line in a visual servoing scheme". In: Robotics and Automation, 2004. Proceedings. ICRA'04. 2004 IEEE International Conference on. Vol. 1. IEEE, pp. 255-260.

Roberts, J. F., Stirling, T., Zufferey, J.-C., and Floreano, D. (2007a). "Quadrotor Using Minimal Sensing For Autonomous Indoor Flight". In: European Micro Air Vehicle Conference and Flight Competition (EMAV2007). Toulouse, France.

Achtelik, Markus (2011). ROS asctec_mav_framework repository, https://github.com/ethzasl/asctec_mav_framework.

Willow Garage, S. A. I. L. (2007). Robot Operating System (ROS). http: / /www.ros.org/wiki/.

Ross, S., Melik-Barkhudarov, N., Shankar, K. S., Wendel, A., Dey, D., Bagnell, J. A., and Hebert, M. (2013). "Learning monocular reactive uav control in cluttered natural environments". In: Robotics and Automation (ICRA), 2013 IEEE International Conference on. IEEE, pp. 1765-1772.

Rudol, P., Wzorek, M., Conte, G., and Doherty, P. (2008). "Micro unmanned aerial vehicle visual servoing for cooperative indoor exploration". In: Aerospace, 2008 IEEE Conference on.

Rudy, N. (2003). "Robot Localization and Kalman Filters". MA thesis. Netherlands: Utrecht University.

Rumpler, M., Tscharf, A., Mostegel, C., Daftry, S., Hoppe, C., Prettenthaler, R., Fraundorfer, F., Mayer, G., and Bischof, H. (2016). "Evaluations on multi-scale camera networks for precise and geo-accurate reconstructions from aerial and terrestrial images with user guidance". Computer Vision and Image Understanding.

Russell, S. and Norvig, P. (2003). Artificial Intelligence: A Modern Approach. 3rd edition. Prentice-Hall, Englewood Cliffs, NJ.

S., L., A., S., M, S., and D’Andrea, R. (2010). “A Simple Learning Strategy for High-Speed Quadrocopter Multi-Flips”. In: 2010 IEEE International Conference on Robotics and Automation (ICRA 2010), pp. 1642-1648.

Sanchez Lopez, J. L. (2012). "Path Following Control System for car-like Unmanned Ground Systems". MA thesis. Spain: Universidad Politécnica de Madrid.

Sanchez-Lopez, J. L., Fernández, R. A. S., Bavle, H., Sampedro, C., Molina, M., Pestana, J., and Campoy, P. (2016). "Aerostack: An architecture and open-source software framework for aerial robotics". In: Unmanned Aircraft Systems (ICUAS), 2016 International Conference on. IEEE, pp. 332-341.

Saripalli, S. and Sukhatme, G. S. (2007). "Landing a Helicopter on a Moving Target". In: Proceedings of IEEE International Conference on Robotics and Automation. Rome, Italy, pp. 2030-2035.

Savkin, A. and Teimoori, H. (2008). "Bearings-only guidance of an autonomous vehicle following a moving target with a smaller minimum turning radius". In: Decision and Control, 2008. CDC 2008. 47th IEEE Conference on, pp. 4239-4243.

Scaramuzza, D., Achtelik, M., Doitsidis, L., Fraundorfer, F., Kosmatopoulos, E., Martinelli, A., et al. (2013). "Vision-controlled micro flying robots: from system design to autonomous navigation and mapping in gps-denied environments". IEEE Robotics and Automation Magazine.

Scaramuzza, D. and Fraundorfer, F. (2011). "Visual odometry [tutorial]". IEEE robotics \& automation magazine 18.4, pp. 80-92.

Schmidt, S. F. (1981). "The Kalman filter-Its recognition and development for aerospace applications". Journal of Guidance, Control, and Dynamics 4.1, pp. 4-7.

Schoellig, A. P., Wiltsche, C., and D'Andrea, R. (2012). "Feed-forward parameter identification for precise periodic quadrocopter motions". In: American Control Conference (ACC), 2012. IEEE, pp. 43134318.

Schölling, A., Augugliaro, F., Lupashin, S., and D’Andrea, R. (2010). "Synchronizing the Motion of a Quadrocopter to Music". In: IEEE International Conference on Robotics and Automation ICRA, pp. 3355-3360.

Schramm, F., Geffard, F., Morel, G., and Micaelli, A. (2007). "Calibration free image point path planning simultaneously ensuring visibility and controlling camera path". In: Robotics and Automation, 2007 IEEE International Conference on. IEEE, pp. 2074-2079. 
Shah, S., Dey, D., Lovett, C., and Kapoor, A. (2017). Aerial Informatics and Robotics Platform. Tech. rep. MSR-TR-2017-9. Microsoft Research.

Shen, Shaojie, Mulgaonkar, Yash, Michael, Nathan, and Kumar, Vijay (2013). "Vision-based state estimation for autonomous rotorcraft mavs in complex environments". In: Robotics and Automation (ICRA), 2013 IEEE International Conference on. IEEE, pp. 1758-1764.

Shen, S., Michael, N., and Kumar, V. (2011). "Autonomous multi-floor indoor navigation with a computationally constrained MAV". Robotics and Automation (ICRA), 2011 IEEE International Conference on, pp. 20-25.

Shen, S. and Michael, N. (2013). "State estimation for indoor and outdoor operation with a micro-aerial vehicle”. In: Experimental Robotics. Springer, pp. 273-288.

Shen, S., Mulgaonkar, Y., Michael, N., and Kumar, V. (2013). "Vision-Based State Estimation and Trajectory Control Towards High-Speed Flight with a Quadrotor.” In: Robotics: Science and Systems. Citeseer.

Shuster, M. D. (1993). “A survey of attitude representations”. Navigation 8.9.

Shuster, M. D. and Oh, S. (1981). "Three-axis attitude determination from vector observations". Journal of Guidance, Control, and Dynamics 4.1, pp. 70-77.

Sola, J. (2012). "Quaternion kinematics for the error-state KF". Laboratoire d'Analyse et d'Architecture des Systemes-Centre national de la recherche scientifique (LAAS-CNRS), Toulouse, France, Tech. Rep.

Strasdat, H., Montiel, J., and Davison, A. (2010). "Real-time monocular SLAM: Why filter?" In: Robotics and Automation (ICRA), 2010 IEEE International Conference on, pp. 2657-2664.

Şucan, I. A., Moll, M., and Kavraki, L. E. (2012). "The Open Motion Planning Library". IEEE Robotics \& Automation Magazine.

Tahri, O. and Chaumette, F. (2005a). "Point-based and region-based image moments for visual servoing of planar objects". Robotics, IEEE Transactions on 21.6, pp. 1116-1127.

Tahri, O., Mezouar, Y., Chaumette, F., and Corke, P. (2010). "Decoupled Image-Based Visual Servoing for Cameras Obeying the Unified Projection Model”. Robotics, IEEE Transactions on 26.4, pp. 684-697.

Tahri, O. (2004). "Utilisation des moments en asservissement visuel et en calcul de pose". PhD thesis. Rennes 1.

Tahri, O. and Chaumette, F. (2005b). "Point-based and region-based image moments for visual servoing of planar objects". Robotics, IEEE Transactions on 21.6, pp. 1116-1127.

Tahri, O., Chaumette, F., and Mezouar, Y. (2008). "New decoupled visual servoing scheme based on invariants from projection onto a sphere". In: Robotics and Automation, 2008. ICRA 2008. IEEE International Conference on. IEEE, pp. 3238-3243.

Tahri, O. and Mezouar, Y. (2010). "On visual servoing based on efficient second order minimization". Robotics and Autonomous Systems 58.5, pp. 712-719.

Tahri, O., Mezouar, Y., Chaumette, F., and Corke, P. (2009). "Generic decoupled image-based visual servoing for cameras obeying the unified projection model". In: Robotics and Automation, 2009. ICRA'09. IEEE International Conference on. IEEE, pp. 1116-1121.

Teuliere, C., Eck, L., and Marchand, E. (2011). "Chasing a moving target from a flying UAV". In: 2011 IEEE/RSJ International Conference on Intelligent Robots and Systems. IEEE, pp. 4929-4934.

(2012). The Stanford/Berkeley Testbed of Autonomous Rotorcraft for Multi-Agent Control (STARMAC) project. http://hybrid.eecs.berkeley.edu/starmac/.

Thrun, S. (2002). "Robotic Mapping: A Survey". In: Exploring Artificial Intelligence in the New Millenium. Ed. by G. Lakemeyer and B. Nebel. Morgan Kaufmann.

Trawny, N. and Roumeliotis, S. I. (2005). "Indirect Kalman filter for 3D attitude estimation". University of Minnesota, Dept. of Comp. Sci. \& Eng., Tech. Rep 2.

Triggs, B., McLauchlan, P. F., Hartley, R. I., and Fitzgibbon, A. W. (1999). "Bundle adjustment - a modern synthesis". In: International workshop on vision algorithms. Springer, pp. 298-372.

UAS Technologies Sweden AB Company (2012). UAS Technologies Sweden AB Company website. http://www. uastech.com/

Valenti, M., Bethke, B., Fiore, G., How, J. P., and Feron, E. (2006). "Indoor multi-vehicle flight testbed for fault detection, isolation, and recovery". In: Proceedings of the AIAA Guidance, Navigation, and Control Conference and Exhibit, Keystone, CO. Vol. 63, p. 64.

Van Blyenburgh, P. (1999). "UAVs: an overview". Air \& Space Europe 1.5, pp. 43-47.

Van Nieuwstadt, M. J. and Murray, R. M. (1998). "Real-time trajectory generation for differentially flat systems". International Journal of Robust and Nonlinear Control 8.11, pp. 995-1020. 
CVG-UPM (2012a). Universidad Politécnica de Madrid. Computer Vision Group. Vision for UAV Project. http://www.vision4uav.com

Wan, E. A. and Van Der Merwe, R. (2001). "The unscented Kalman filter". Kalman filtering and neural networks, pp. 221-280.

Watts, A. C., Ambrosia, V. G., and Hinkley, E. A. (2012). "Unmanned aircraft systems in remote sensing and scientific research: Classification and considerations of use”. Remote Sensing 4.6, pp. 1671-1692.

Weiss, S. M. (2012). "Vision based navigation for micro helicopters". PhD thesis. Diss., Eidgenössische Technische Hochschule ETH Zürich, Nr. 20305, 2012.

Weiss, S., Achtelik, M. W., Chli, M., and Siegwart, R. (2012). "Versatile distributed pose estimation and sensor self-calibration for an autonomous MAV". In: Robotics and Automation (ICRA), 2012 IEEE International Conference on. IEEE, pp. 31-38.

Weiss, S., Achtelik, M. W., Lynen, S., Chli, M., and Siegwart, R. (2012). "Real-time onboard visualinertial state estimation and self-calibration of mavs in unknown environments". In: Robotics and Automation (ICRA), 2012 IEEE International Conference on. IEEE, pp. 957-964.

Weiss, S., Scaramuzza, D., and Siegwart, R. (2011). "Monocular-SLAM-based navigation for autonomous micro helicopters in GPS-denied environments". Journal of Field Robotics 28.6, pp. 854874.

Weiss, S. and Siegwart, R. (2011). "Real-time metric state estimation for modular vision-inertial systems". In: Robotics and Automation (ICRA), 2011 IEEE International Conference on. IEEE, pp. 4531-4537.

Welch, G. and Bishop, G. (2005). European Civil Unmanned Air Vehicle Roadmap, Volume 3 Strategic Research Agenda. Tech. rep. European Civil UAV FP5 R\&D Program Members.

Wendel, A., Irschara, A., and Bischof, H. (2011b). "Natural Landmark - based Monocular Localization for MAVs". In: IEEE International Conference on Robotics and Automation (ICRA 2011).

Wendel, A., Maurer, M., Irschara, A., and Bischof, H. (2011). “3D Vision Applications for MAVs: Localization and Reconstruction”. In: International Symposium on 3D Data Processing, Visualization and Transmission (3DPVT), 2011, pp. 3-4.

ARDrone@Wikipedia (2012). Parrot AR Drone article at english Wikipedia. http : / / en . wikipedia.org/wiki/Parrot_AR.Drone

Winkvist, S. (2013). "Low computational SLAM for an autonomous indoor aerial inspection vehicle". $\mathrm{PhD}$ thesis. University of Warwick.

Winkvist, S., Rushforth, E., and Young, K. (2013). "Towards an autonomous indoor aerial inspection vehicle". Industrial Robot: An International Journal 40.3, pp. 196-207.

Yanmaz, E., Hayat, S., Scherer, J., and Bettstetter, C. (2014). "Experimental performance analysis of twohop aerial 802.11 networks". In: 2014 IEEE Wireless Communications and Networking Conference (WCNC). IEEE, pp. 3118-3123.

Zhang, H. and Ostrowski, J. (1999). "Visual servoing with dynamics: control of an unmanned blimp". In: Robotics and Automation, 1999. Proceedings. 1999 IEEE International Conference on. Vol. 1, 618623 vol.1.

Zhou, G., Fang, L., Tang, K., Zhang, H., Wang, K., and Yang, K. (2015a). “Guidance: A Visual Sensing Platform For Robotic Applications". In: Proceedings of the IEEE Conference on Computer Vision and Pattern Recognition Workshops, pp. 9-14.

Zhou, G., Fang, L., Tang, K., Zhang, H., Wang, K., and Yang, K. (2015b). “Guidance: A visual sensing platform for robotic applications". In: IEEE Conference on Computer Vision and Pattern Recognition Workshops, 2015 IEEE CVPR Workshop, pp. 9-14.

Zhou, G., Liu, A., Yang, K., Wang, T., and Li, Z. (2014). “An embedded solution to visual mapping for consumer drones". In: Proceedings of the IEEE Conference on Computer Vision and Pattern Recognition Workshops, pp. 656-661.

Zhou, G., Ye, J., Ren, W., Wang, T., and Li, Z. (2014). "On-board inertial-assisted visual odometer on an embedded system". In: 2014 IEEE International Conference on Robotics and Automation (ICRA). IEEE, pp. 2602-2608.

Zingg, S., Scaramuzza, D., Weiss, S., and Siegwart, R. (2010). "MAV Navigation through Indoor Corridors Using Optical Flow". In: Robotics and Automation (ICRA), 2010 IEEE International Conference on.

Zufferey, J.-C., Beyeler, A., and Floreano, D. (2010). "Autonomous flight at low altitude with visionbased collision avoidance and GPS-based path following”. In: Proceedings of the IEEE International Conference on Robotics and Automation (ICRA). Anchorage, Alaska: IEEE. 